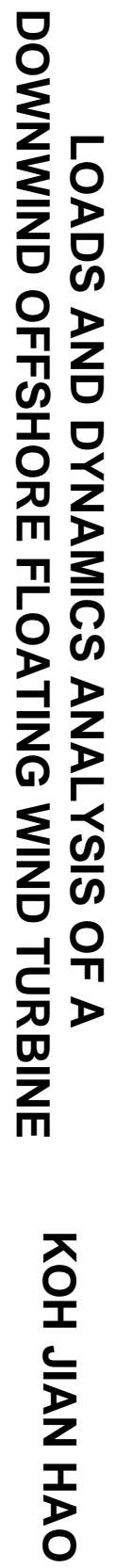

LOADS AND DYNAMICS ANALYSIS OF A DOWNWIND OFFSHORE FLOATING WIND TURBINE

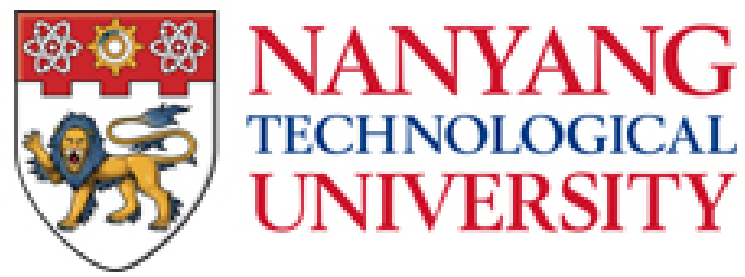

KOH JIAN HAO

SCHOOL OF MECHANICAL AND AEROSPACE ENGINEERING

NANYANG TECHNOLOGICAL UNIVERSITY

2017

$\stackrel{\text { ก }}{\stackrel{v}{\ominus}}$ 


\section{LOADS AND DYNAMICS ANALYSIS OF A DOWNWIND OFFSHORE FLOATING WIND TURBINE}

KOH JIAN HAO

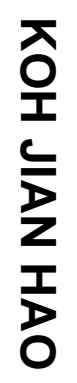

SCHOOL OF MECHANICAL AND AEROSPACE ENGINEERING

A thesis submitted to Nanyang Technological University in partial fulfillment of the requirements for the degree of Doctor of Philosophy (Mechanical Engineering) 


\section{ABSTRACT}

Current offshore wind turbine designs are basic concepts using standard upwind land-based wind turbines 'marinised' using a platform from the offshore oil and gas industry with additional anticorrosion and structural stiffness. This design conservatism is present to avoid significant changes to the proven technology of land-based wind turbines and offshore foundations, to assure technical feasibility and economic viability for short term development. This study aims to tackle some of the technical challenges and gaps identified for implementing downwind floating wind turbines.

Using data obtained from open-sea testing of the $1 / 6.5^{\text {th }}$ scale prototype of the SWAY hybrid tension-leg spar-type floating wind turbine, a FAST model of the SWAY system was built and validated using an improved version of FAST numerical simulation tool.

The author developed a novel meshing and modeling method to conduct computational fluid dynamics analysis efficiently for high Reynolds number flow over bluff bodies that can produce reasonable predictions of turbulent wake profile.

A new tower influence model was proposed by the author based on the computational fluid dynamics analysis conducted for different 18 test cases generated to analyze the flow across a wind turbine tower. The new tower influence model proposed by the author showed significant improvement over the models used in FAST and Bladed.

Similarly, 9 test cases were generated to analyze the flow across a wind turbine nacelle based on each permutation of smooth- and sharp-edged nacelles, and cylindrical or cuboid shaped nacelles. The simulation and experiment results showed very good comparisons over various flow parameters. Key findings were noted that support in the development of nacelle influence model. 


\section{ACKNOWLEDGEMENT}

The author of this report would like to express his sincere gratitude and appreciation to the following:

- Associate Professor Ng Yin Kwee Eddie, supervisor of the PhD program, for his advices, support and understanding. The author obtained continuous academic and non-academic help, ideas and encouragement that spurred him to work on more profound and extensive research. The author is privileged to have worked under his supervision.

- Dr. Kelvin Tan, industrial supervisor from DNV GL of the JointIndustry-Project in Offshore Renewables, for his invaluable suggestions and guidance. He provided the author an industrial perspective to the study. In addition, the author appreciates the help and support from the staff of DNV GL.

- $\quad$ Energy Research Institute @ NTU (ERI@N), for supporting the author in the PhD program. ERI@N's involvement allowed the author to be part of the Joint Industry Program on Offshore Renewables and provided the opportunity for the author to do a 2-month research exchange at National Wind Technology Centre in Boulder, Colorado. Special mentions go to Mr. James Moses and Dr. Seri Lee for providing their endless support in administrative coordination.

- Dr. Amy Robertson, Senior Engineer from National Wind Technology Centre (NWTC), National Renewable Energy Laboratory (NREL), for her technical advice and guidance. As the leader of the SWAY validation project, Dr. Robertson helped the author to settle into the work at NREL and guided him closely along throughout the project. Her cordiality also helped the author to adapt to living in Colorado. 
- Dr. Jason Jonkman, Senior Engineer from NWTC, NREL, for his technical support in the FAST tool. Dr. Jonkman is one of the main developers of the FAST tool and the author is privileged to work closely with him to pick up new knowledge and skills in developing wind turbine models.

- Dr. Fredrick Driscoll, Senior Engineer from NWTC, NREL, for his assistance in post-processing of the experimental data from the SWAY wind turbine. His experience and knowledge in the data acquisition systems and data management allowed the author to swiftly obtain useful data to be used for model validation.

- Mr. Jørgen Jorde, Chief Technology Officer in SWAY AS, for supporting the project efforts and allowing the author to use SWAY's proprietary data for model validation. Mr. Jorde has always been prompt in providing solutions to the author queries on the SWAY wind turbine specifications.

- Professor Tetsuro Tamura, Supervisor of the research exchange project, for providing valuable information to the SWAY project. He actively engaged the author in discussions and research tasks that helped the author to progress in this research project. Professor Tamura's hospitality also helped the author settle down in Japan quickly.

- The author's family and friends who have provided encouragement and support throughout his $\mathrm{PhD}$ candidature. 


\section{TABLE OF CONTENT}

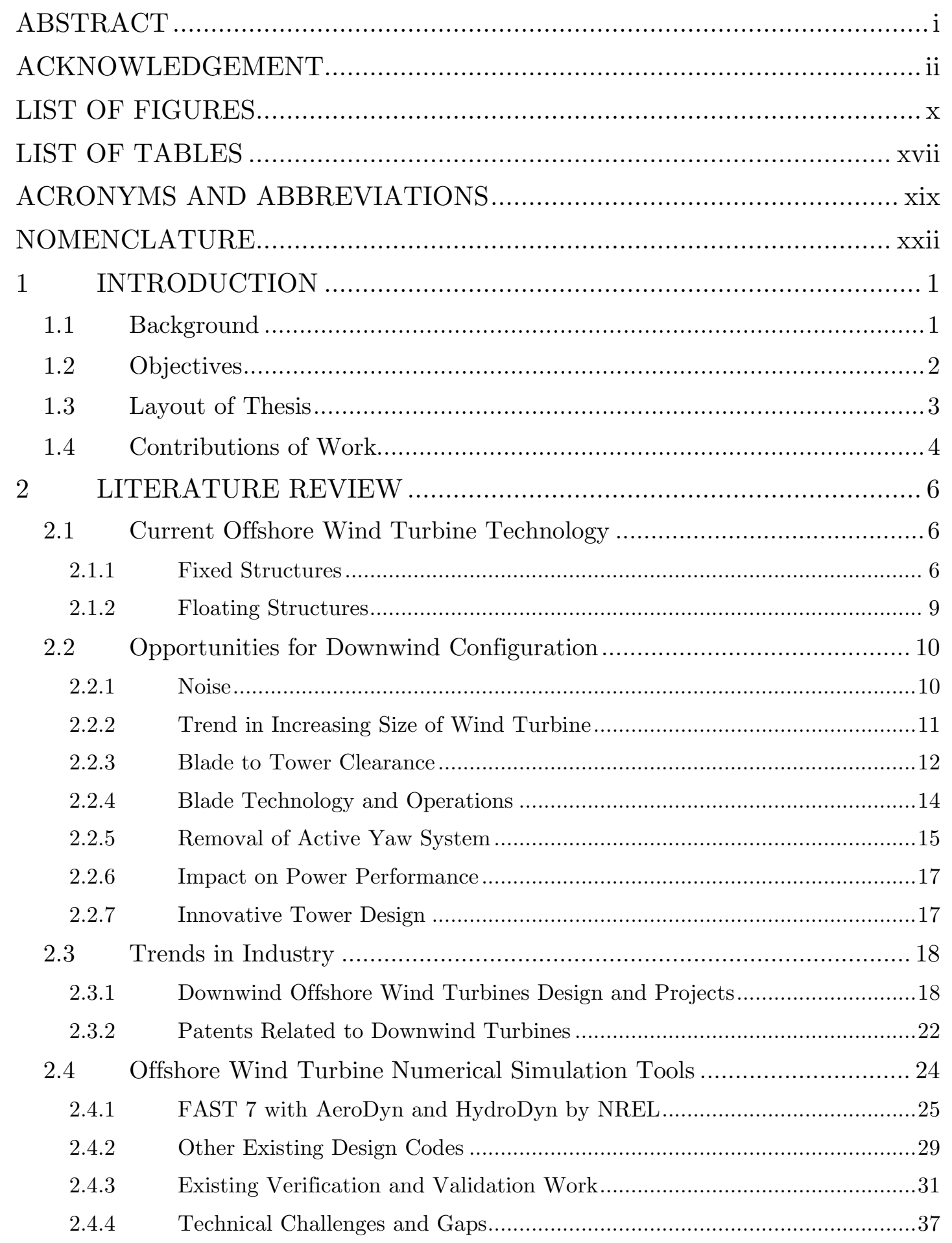




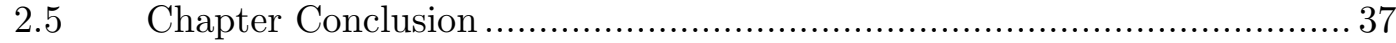

3 TECHNICAL BACKGROUND .......................................... 39

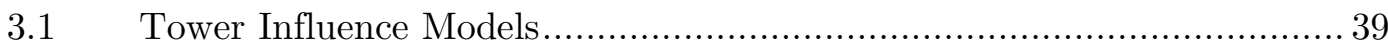

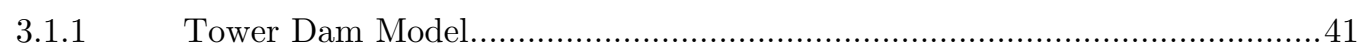

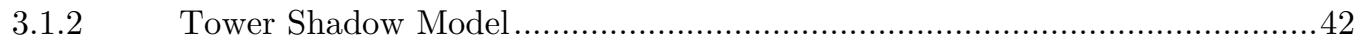

3.2 Nacelle Influence Models ........................................................... 47

3.3 Computational Fluid Dynamics and Turbulence Modeling .................... 48

3.3.1 Scales of Turbulent Motion ....................................................................... 49

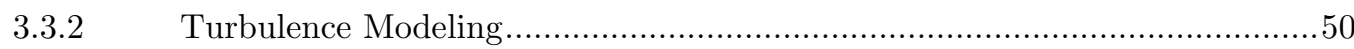

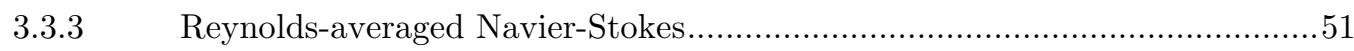

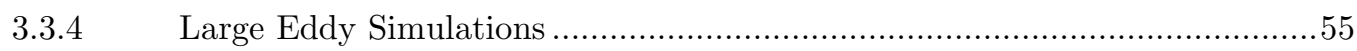

3.3.5 Embedded Large Eddy Simulation (ELES) ….............................................57

4 SWAY WIND TURBINE VALIDATION STUDY ....................... 59

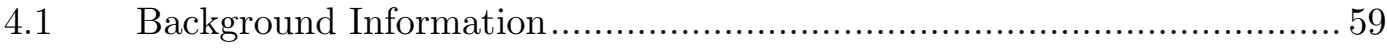

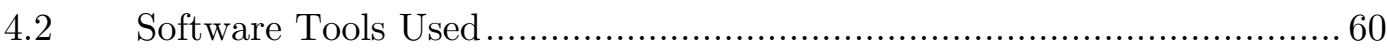

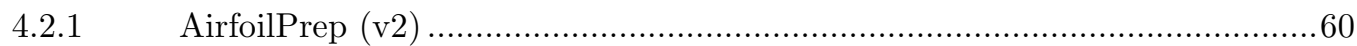

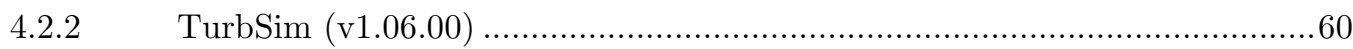

4.3 Code Improvement and Modification................................................... 61

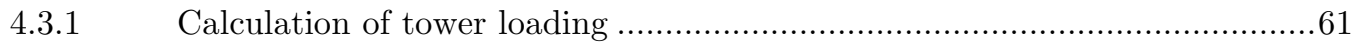

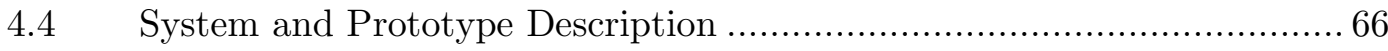

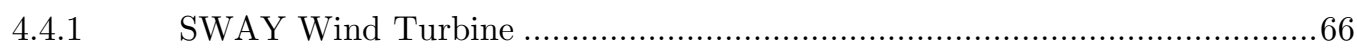

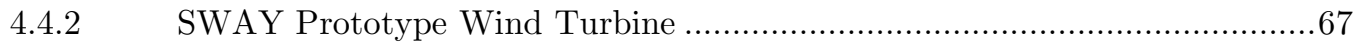

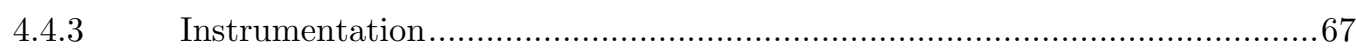

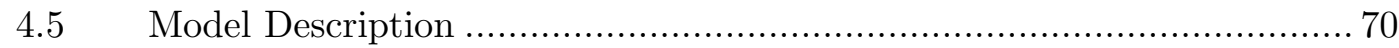

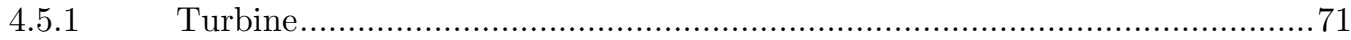

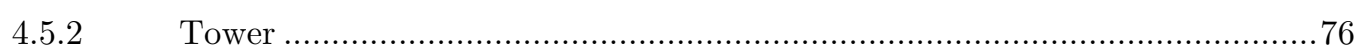

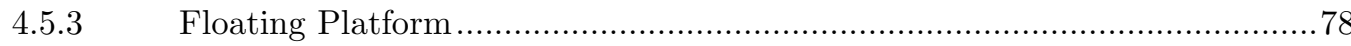

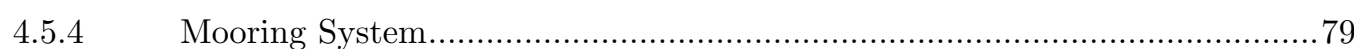

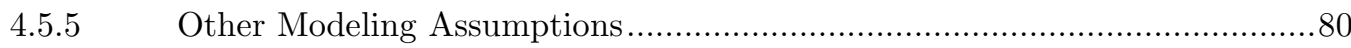

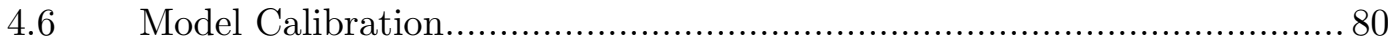

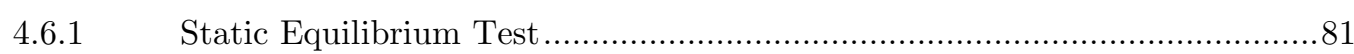

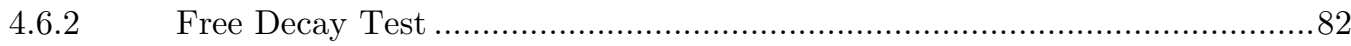

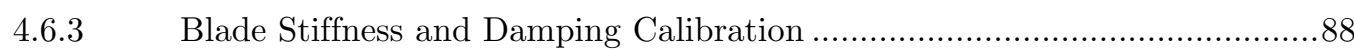

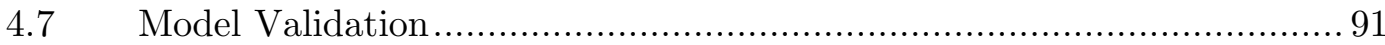

4.7.1 Non-operating Conditions …………....................................................... 91

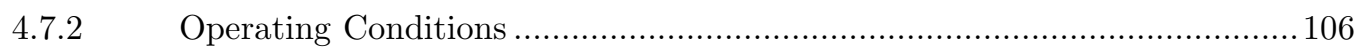




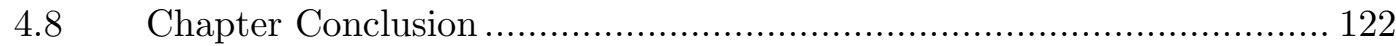

5 TOWER/NACELLE INFLUENCE MODEL ...........................126

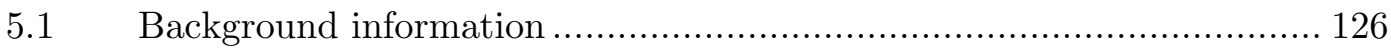

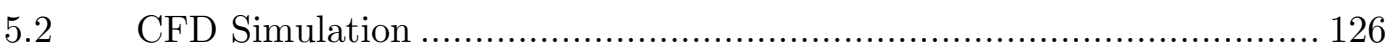

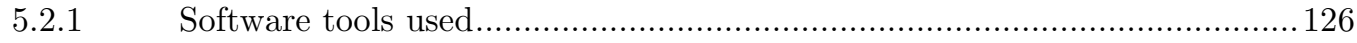

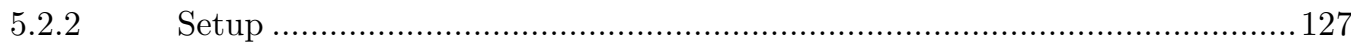

5.3 General Tower and Nacelle Design ................................................. 128

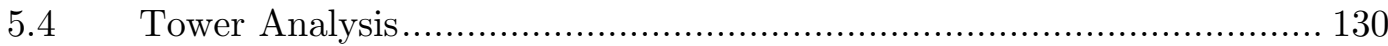

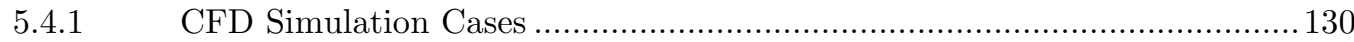

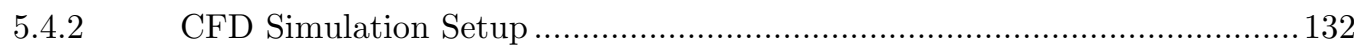

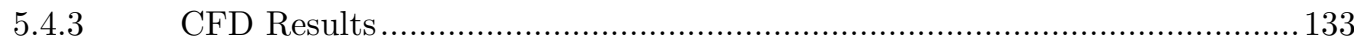

5.4.4 Comparison with Existing Tower Influence Model ….................................150

5.4.5 Development of New Tower Influence Model.............................................. 153

5.5 Nacelle Analysis ....................................................................... 162

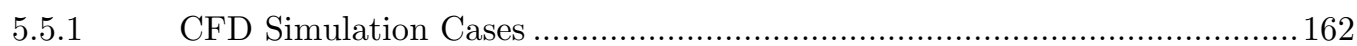

5.5.2 CFD Simulation Setup (Case Specific) ……........................................... 163

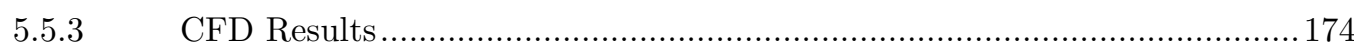

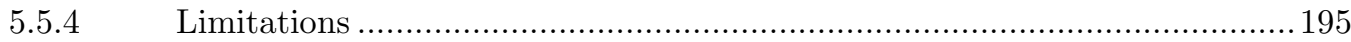

5.6 Chapter Conclusion ................................................................... 196

$6 \quad$ CONCLUSION AND FUTURE WORK …................................198

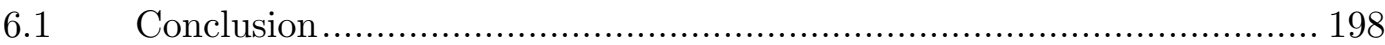

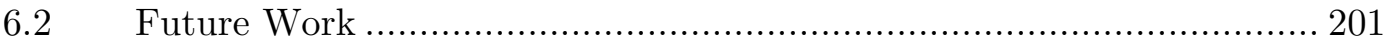

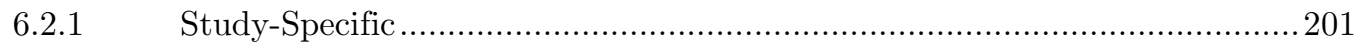

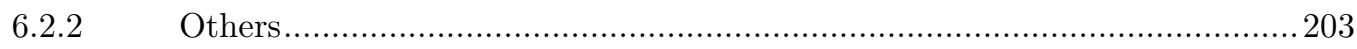

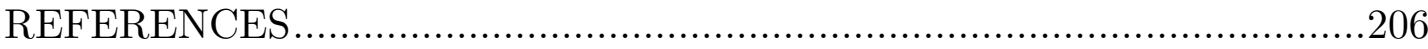

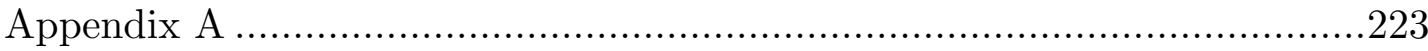

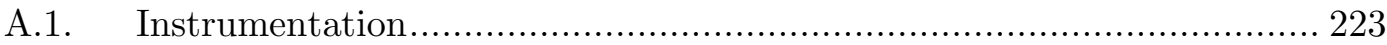

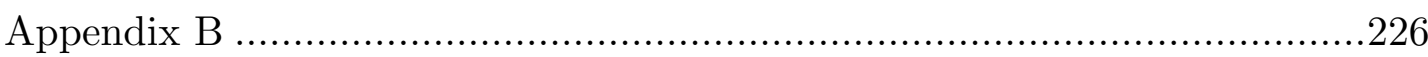

B.1. WAMIT Setup Log File ................................................................. 226

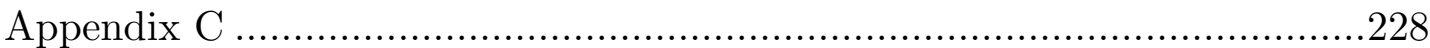

C.1. Airfoil-Data File - Cylinder.dat.................................................. 228

C.2. Airfoil-Data File - Transition.dat .................................................. 228

C.3. Airfoil-Data File - Main 1.dat .................................................... 230

C.4. Airfoil-Data File - Main 2.dat ...................................................... 232

C.5. Airfoil-Data File - Main 3.dat ........................................................ 234 
Appendix D

.237

D.1. $\quad$ Tower Case 1 - Normalized X Velocity …......................................... 237

D.2. Tower Case 1 - Normalized Y Velocity ............................................. 238

D.3. Tower Case 2 - Normalized X Velocity .............................................. 239

D.4. $\quad$ Tower Case 2 - Normalized Y Velocity .......................................... 240

D.5. Tower Case 3 - Normalized X Velocity .......................................... 241

D.6. Tower Case 3 - Normalized Y Velocity .......................................... 242

D.7. Tower Case 4 - Normalized X Velocity ................................................ 243

D.8. $\quad$ Tower Case 4 - Normalized Y Velocity ............................................ 244

D.9. Tower Case 5 - Normalized X Velocity …….................................... 245

D.10. Tower Case 5 - Normalized Y Velocity ........................................... 246

D.11. Tower Case 6 - Normalized X Velocity .............................................. 247

D.12. Tower Case 6 - Normalized Y Velocity ......................................... 248

D.13. Tower Case 7 - Normalized X Velocity …........................................ 249

D.14. Tower Case 7 - Normalized Y Velocity ............................................. 250

D.15. Tower Case 8 - Normalized X Velocity ............................................... 251

D.16. Tower Case 8 - Normalized Y Velocity .............................................. 252

D.17. Tower Case 9 - Normalized X Velocity .......................................... 253

D.18. Tower Case 9 - Normalized Y Velocity .............................................. 254

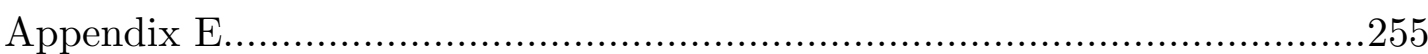

E.1. $\quad$ FFT of $\boldsymbol{C l}$ for Tower Case 1 in $\log$ and Linear Scale ........................ 255

E.2. $\quad$ FFT of $\boldsymbol{C l}$ for Tower Case 2 in Log and Linear Scale ........................ 255

E.3. FFT of $\boldsymbol{C l}$ for Tower Case 3 in Log and Linear Scale ........................ 256

E.4. FFT of $\boldsymbol{C l}$ for Tower Case 4 in Log and Linear Scale ......................... 256

E.5. FFT of $\boldsymbol{C l}$ for Tower Case 5 in Log and Linear Scale ........................ 257

E.6. $\quad$ FFT of $\boldsymbol{C l}$ for Tower Case 6 in $\log$ and Linear Scale ......................... 257

E.7. $\quad$ FFT of $\boldsymbol{C l}$ for Tower Case 7 in Log and Linear Scale ......................... 258

E.8. FFT of $\boldsymbol{C l}$ for Tower Case 8 in $\log$ and Linear Scale ........................ 258

E.9. FFT of $\boldsymbol{C l}$ for Tower Case 9 in Log and Linear Scale .......................... 259

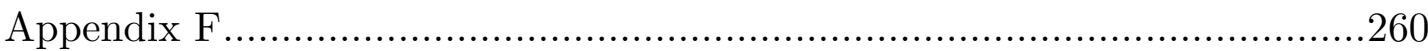

F.1. $\quad \boldsymbol{v} \boldsymbol{x}$ vs $\boldsymbol{y}$ Plot of Various $\boldsymbol{x}$ Values for Tower Case 1.......................... 260

F.2. $\quad \boldsymbol{v} \boldsymbol{x}$ vs $\boldsymbol{y}$ Plot of Various $\boldsymbol{x}$ Values for Tower Case 2......................... 260

F.3. $\quad \boldsymbol{v} \boldsymbol{x}$ vs $\boldsymbol{y}$ Plot of Various $\boldsymbol{x}$ Values for Tower Case 3........................... 261

F.4. $\quad \boldsymbol{v} \boldsymbol{x}$ vs $\boldsymbol{y}$ Plot of Various $\boldsymbol{x}$ Values for Tower Case 4.............................2 261 


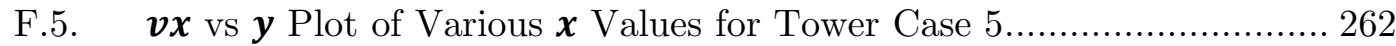

F.6. $\quad \boldsymbol{v} \boldsymbol{x}$ vs $\boldsymbol{y}$ Plot of Various $\boldsymbol{x}$ Values for Tower Case 6........................... 262

F.7. $\quad \boldsymbol{v} \boldsymbol{x}$ vs $\boldsymbol{y}$ Plot of Various $\boldsymbol{x}$ Values for Tower Case $7 \ldots \ldots \ldots \ldots \ldots \ldots \ldots \ldots . . . . . . . . . . . . .263$

F.8. $\quad \boldsymbol{v} \boldsymbol{x}$ vs $\boldsymbol{y}$ Plot of Various $\boldsymbol{x}$ Values for Tower Case 8........................... 263

F.9. $\quad \boldsymbol{v} \boldsymbol{x}$ vs $\boldsymbol{y}$ Plot of Various $\boldsymbol{x}$ Values for Tower Case 9.......................... 264

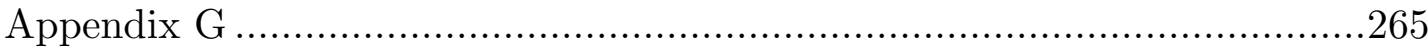

G.1. Cuboid Nacelle Case 1 (Sharp) ……................................................. 265

G.2. Cuboid Nacelle Case 10 (Smooth) ..................................................... 265

G.3. Cuboid Nacelle Case 2 (Sharp) .......................................................... 266

G.4. Cuboid Nacelle Case 11 (Smooth) …............................................ 266

G.5. Cuboid Nacelle Case 3 (Sharp) …................................................. 267

G.6. Cuboid Nacelle Case 12 (Smooth) ................................................... 267

G.7. Cuboid Nacelle Case 4 (Sharp) ...................................................... 268

G.8. Cuboid Nacelle Case 13 (Smooth) …............................................ 268

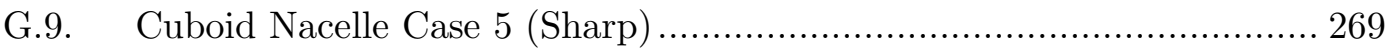

G.10. Cuboid Nacelle Case 14 (Smooth) ...................................................... 269

G.11. Cuboid Nacelle Case 6 (Sharp) ….................................................. 270

G.12. Cuboid Nacelle Case 15 (Smooth) ….............................................. 270

G.13. Cuboid Nacelle Case 7 (Sharp) …................................................. 271

G.14. Cuboid Nacelle Case 16 (Smooth) ..................................................... 271

G.15. Cuboid Nacelle Case 8 (Sharp) …............................................... 272

G.16. Cuboid Nacelle Case 17 (Smooth) …............................................. 272

G.17. Cuboid Nacelle Case 9 (Sharp) …................................................. 273

G.18. Cuboid Nacelle Case 18 (Smooth) ...................................................... 273

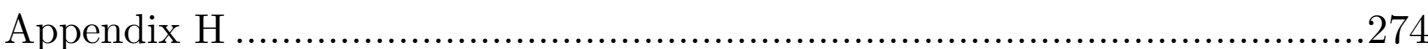

H.1. Cylinder Nacelle Case 1 (Sharp) ...................................................... 274

H.2. Cylinder Nacelle Case 10 (Smooth) …............................................ 274

H.3. Cylinder Nacelle Case 2 (Sharp) ....................................................... 275

H.4. Cylinder Nacelle Case 11 (Smooth) .................................................... 275

H.5. Cylinder Nacelle Case 3 (Sharp) ........................................................ 276

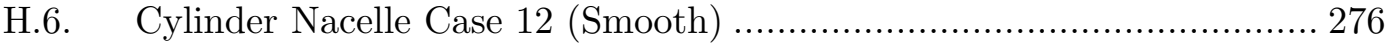

H.7. Cylinder Nacelle Case 4 (Sharp) ......................................................... 277

H.8. Cylinder Nacelle Case 13 (Smooth) .................................................. 277

H.9. Cylinder Nacelle Case 5 (Sharp) ...................................................... 278 
H.10. Cylinder Nacelle Case 14 (Smooth) …............................................ 278

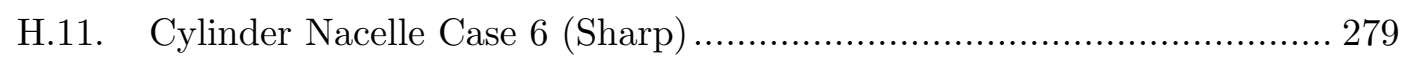

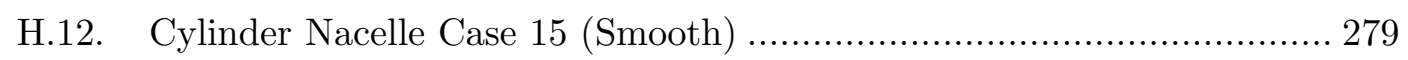

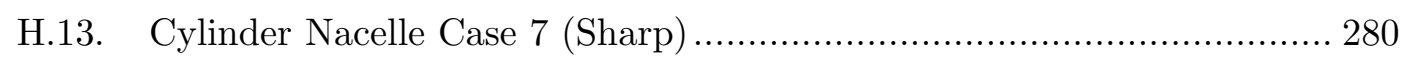

H.14. Cylinder Nacelle Case 16 (Smooth) .................................................... 280

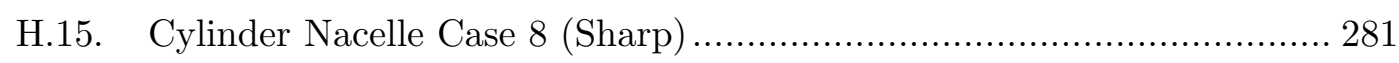

H.16. Cylinder Nacelle Case 17 (Smooth) .................................................... 281

H.17. Cylinder Nacelle Case 9 (Sharp) ..................................................... 282

H.18. Cylinder Nacelle Case 18 (Smooth) ................................................... 282 


\section{LIST OF FIGURES}

Figure 2.1 Fixed structures for offshore wind turbines .......................... 7

Figure 2.2 Floating structures for offshore wind turbines ........................ 8

Figure 2.3 Growth in size of wind turbines since 1980 and prospects

(International Energy Agency, 2013) ......................................................12

Figure 2.4 Rotor blade tip and tower clearance (Jamieson, 2011b) ............13

Figure 2.5 Overview of FAST Modules for Coupled Aero-hydro-servo-elastic

Simulation (J. Jonkman \& Buhl, 2007) ............................................26

Figure 2.6 OC3 and OC4 offshore wind system designs modeled (Robertson,

Jonkman, Musial, Vorpahl, \& Popko, 2013) ..........................................32

Figure 3.1 Reference frame for tower dam and shadow model...................40

Figure 3.2 Bladed combined model regions (Garrad Hassan \& Partners Ltd.,

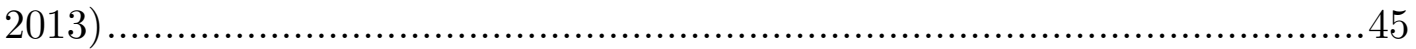

Figure 3.3 Energy cascade process (Davidson, 2011) ............................49

Figure 3.4 Kolmogorov's energy spectrum (adapted from Davidson (2011))50

Figure 3.5 Resolved and modeled regions for different turbulence models (adapted from Eggenspieler (2011)) ..........................................51

Figure 4.1 Simulation flow chart for calculation of tower loading .............64

Figure 4.2 Undisturbed wind inflow along the rotor and tower ................65

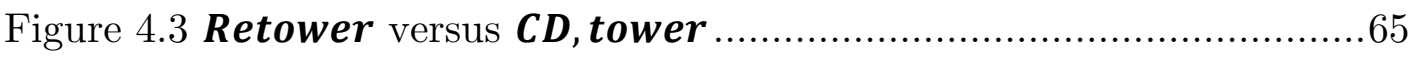

Figure 4.4 SWAY floating wind turbine ........................................6 67

Figure 4.5 Profile of the tower, spreader beam, and tension rod ................68

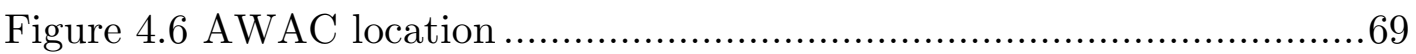

Figure 4.7 Tower wave height sensor ....................................... 70

Figure 4.8 Coordinate systems and platform DOF ............................. 71

Figure 4.9 Illustration of Airfoil Region........................................... 72

Figure $4.10 \boldsymbol{C l}$, airfoil data of blade sections ..................................73

Figure $4.11 \boldsymbol{C d}$, airfoil data of blade sections ...................................73 
Figure 4.12 Cm, airfoil data of blade sections ....................................74

Figure 4.13 Tension line versus tension rod .....................................79

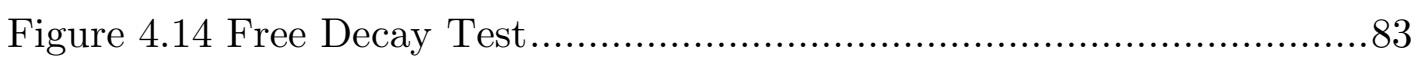

Figure 4.15 Roll motion in roll free decay............................................84

Figure 4.16 Power spectral density (PSD) versus frequency of roll DOF (enlarged on right)

Figure 4.17 Illustration of physical motion of first and second peak frequency .86

Figure 4.18 PSD versus frequency of tension rod motion (enlarged view on right) .86

Figure 4.19 Pitch motion in pitch free decay .87

Figure 4.20 PSD versus frequency of pitch DOF (enlarged view on right) ..88 Figure $4.21 \mathrm{FFT}$ analysis of flapwise blade loads at wind speed $=4.35 \mathrm{~m} / \mathrm{s}$ for FAST and experiment response .89

Figure 4.22 FFT analysis of edgewise blade loads at wind speed $=4.35 \mathrm{~m} / \mathrm{s}$ for FAST and experiment response ............................................. 90

Figure 4.23 Summary of non-operational test cases..............................92

Figure 4.24 Velocity inflow comparison (Non-Op Case 1) ........................95

Figure 4.25 Velocity inflow comparison (Non-Op Case 2) ........................96

Figure 4.26 Wave height comparison (Non-Op Case 1) ............................98

Figure 4.27 Wave height comparison (Non-Op Case 2) ..........................99

Figure 4.28 Summary of results for Non-Op Case 1 ............................... 102

Figure 4.29 Summary of results for Non-Op Case 2 .............................. 103

Figure 4.30 Rotor speed and blade pitch angle for operating cases........... 107

Figure 4.31 Summary of operational test cases ................................... 107

Figure 4.32 Velocity inflow comparison (Op Case 1) ............................. 110

Figure 4.33 Velocity inflow comparison (Op Case 2) ........................... 111

Figure 4.34 Wave height comparison (Op Case 1) ................................. 112

Figure 4.35 Wave height comparison (Op Case 2) ................................ 113 


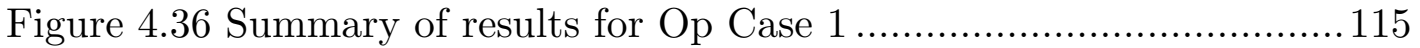

Figure 4.37 Summary of results for Op-Case 2 ................................. 116

Figure 4.38 FFT analysis of the flapwise loading on Blade 2 for Op Case 1 120

Figure 4.39 FFT analysis of the edgewise loading on Blade 2 for Op Case 1 .120

Figure 4.40 FFT analysis of the flapwise loading on Blade 2 for Op Case 2 121

Figure 4.41 FFT analysis of the edgewise loading on Blade 2 for Op Case 2

Figure 5.1 Tower Model 132

Figure 5.2 Tower Full Domain and Surface Mesh 133

Figure 5.3 Zoom-in Views of Tower Mesh 133

Figure 5.4 Reference Frame for Tower Cases 134

Figure 5.5 Instantaneous Normalized X Velocity for Tower Case 2:

Retower $=4.61 \mathrm{E}+06$ 135

Figure 5.6 Instantaneous Normalized Y Velocity for Tower Case 2:

Retower $=4.61 \mathrm{E}+06$ 135

Figure 5.7 Instantaneous Vorticity $\mathrm{Z}$ for Tower Case 2: Retower = $4.61 \mathrm{E}+06$ 136

Figure 5.8 Mean Normalized X Velocity for Tower Case 2: Retower = $4.61 \mathrm{E}+06$ 136

Figure 5.9 Mean Normalized X Velocity for Tower Case 2: Retower = $4.61 \mathrm{E}+06$ 137

Figure 5.10 CD, towervs Flow Time 138

Figure 5.11 CL, tower vs Flow Time.... 139

Figure 5.12 FFT of $\boldsymbol{C l}$ data for Tower Case 1 in Log and Linear Scale..... 140 Figure 5.13 FFT of $\boldsymbol{C l}$ data for Tower Case 2 in Log and Linear Scale..... 141 
Figure 5.14 Comparison graph between experimental and simulation data for $C D$, mean vs Retower

Figure 5.15 Comparison graph between experimental and simulation data for $\boldsymbol{S t}$ vs Retower 145

Figure $5.16 \boldsymbol{C P}$ vs $\boldsymbol{\theta}$ 146

Figure 5.17 Cp, base vs Retower 147

Figure $5.18 \boldsymbol{C p}$, base $-\boldsymbol{C p}, \boldsymbol{m i n}$ vs Retower 148

Figure 5.19 Separation Angle vs Retower (Method 1) 149

Figure 5.20 Separation Angle vs Retower (Method 2) 149

Figure 5.21 Mean Normalized Velocity Comparison for $\boldsymbol{x}=\mathbf{1}$ and $\boldsymbol{x}=\mathbf{3} 150$ Figure 5.22 Tower Case $2($ Retower $=4.61 \mathrm{E}+06)$ Mean Normalized X Velocity Model Comparison 152

Figure 5.23 Tower Case $2($ Retower $=4.61 \mathrm{E}+06)$ Mean Normalized Y Velocity Model Comparison........................................................... 153

Figure 5.24 Flow Field Zones ….................................................. 154

Figure $5.25 \boldsymbol{v} \boldsymbol{x}$ vs $\boldsymbol{y}$ Plot of Various $\boldsymbol{x}$ Values for Tower Case 2 $($ Retower $=4.61 \mathrm{E}+06)$ 155

Figure $5.26 \boldsymbol{v} \boldsymbol{x}$ vs $\boldsymbol{y}$ Plot of Various Retower Values for Tower Cases ... 156

Figure $5.27 \boldsymbol{y} \boldsymbol{v} \boldsymbol{x}=\mathbf{1}$ vs $\boldsymbol{x}$ Plot for Various Retower Values 157

Figure $5.28 \boldsymbol{v} \boldsymbol{x}, \boldsymbol{y}=\mathbf{0}$ vs $\boldsymbol{x}$ Plot for Various Retower Values 159

Figure 5.29 Tower Case 2 (Retower $=4.61 \mathrm{E}+06)$ Mean Normalized X Velocity Model Comparison 161

Figure 5.30 Tower Case $2($ Retower $=4.61 \mathrm{E}+06)$ Mean Normalized Y Velocity Model Comparison 161

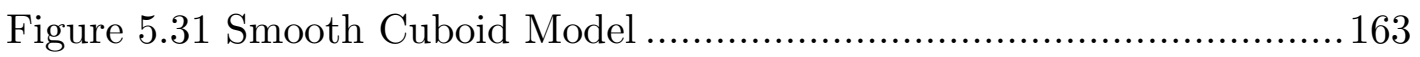

Figure 5.32 Smooth Cuboid Mesh.................................................... 163

Figure 5.33 Smooth Cuboid Cross-Section View................................... 164

Figure 5.34 Smooth Cuboid Cross-Section View of Boundary Layer ......... 164

Figure 5.35 Sharp Cuboid Model ................................................. 166 
Figure 5.36 Sharp Cuboid Mesh ........................................................ 166

Figure 5.37 Sharp Cuboid Cross-Section View...................................... 167

Figure 5.38 Sharp Cuboid Cross-Section View of Boundary Layer ............ 167

Figure 5.39 Smooth Cylinder Model ............................................... 169

Figure 5.40 Smooth Cylinder Mesh...................................................... 169

Figure 5.41 Smooth Cylinder Cross-Section View ............................... 170

Figure 5.42 Smooth Cylinder Cross-Section View of Boundary Layer ....... 170

Figure 5.43 Sharp Cylinder Model................................................ 172

Figure 5.44 Sharp Cylinder Mesh ..................................................... 172

Figure 5.45 Sharp Cylinder Cross-Section View .................................. 173

Figure 5.46 Sharp Cylinder Cross-Section View of Boundary Layer.......... 173

Figure 5.47 Reference Frame for Nacelle Cases .................................... 175

Figure 5.48 Mean Normalized X Velocity for Cuboid Nacelle Case 2: Recub $=6.71 \mathrm{E}+06$ 177

Figure 5.49 Mean Normalized Y Velocity for Cuboid Nacelle Case 2: Recub $=6.71 \mathrm{E}+06$ 177

Figure 5.50 Mean Normalized Velocity Vectors for Cuboid Nacelle Case 2:

$\boldsymbol{R e c u b}=6.71 \mathrm{E}+06$ 178

Figure 5.51 Instantaneous Normalized Velocity Vectors for Cuboid Nacelle

Case $2: \boldsymbol{R e c u b}=6.71 \mathrm{E}+06$ 178

Figure 5.52 Mean Normalized Velocity X on YZ Plane for Cuboid Nacelle Case 2: $\boldsymbol{R e c u b}=6.71 \mathrm{E}+06$ 179

Figure 5.53 Mean Normalized X Velocity for Cuboid Nacelle Case 11:

$\boldsymbol{R e c u b}=6.71 \mathrm{E}+06$ 179

Figure 5.54 Mean Normalized Y Velocity for Cuboid Nacelle Case 11:

$\boldsymbol{R e c u b}=6.71 \mathrm{E}+06$ 180

Figure 5.55 Mean Normalized Velocity Vectors for Cuboid Nacelle Case 11:

$\boldsymbol{R e c u b}=6.71 \mathrm{E}+06$ 180 
Figure 5.56 Instantaneous Normalized Velocity Vectors for Cuboid Nacelle

Case 11: $\boldsymbol{R e c u b}=6.71 \mathrm{E}+06$ 181

Figure 5.57 Mean Normalized Velocity X on YZ Plane for Cuboid Nacelle

Case 11: $\boldsymbol{R e c u b}=6.71 \mathrm{E}+06$ 181

Figure 5.58 Mean Normalized X Velocity for Cylinder Nacelle Case 2: $\boldsymbol{R e c y \boldsymbol { l }}$ $=6.71 \mathrm{E}+06$ 182

Figure 5.59 Mean Normalized Y Velocity for Cylinder Nacelle Case 2: Recyl $=6.71 \mathrm{E}+06$ 182

Figure 5.60 Mean Normalized Velocity Vectors for Cylinder Nacelle Case 2: $\boldsymbol{R e c y l}=6.71 \mathrm{E}+06$ 183

Figure 5.61 Instantaneous Normalized Velocity Vectors for Cylinder Nacelle

Case $2: \mathfrak{R} \boldsymbol{c y l}=6.71 \mathrm{E}+06$ 183

Figure 5.62 Mean Normalized Velocity X on YZ Plane for Cylinder Nacelle Case 2: $\mathfrak{R} \boldsymbol{c u b}=6.71 \mathrm{E}+06$ 184

Figure 5.63 Mean Normalized X Velocity for Cylinder Nacelle Case 11: $\mathfrak{R} \boldsymbol{c y \boldsymbol { l }}$ $=6.71 \mathrm{E}+06$ 184

Figure 5.64 Mean Normalized Y Velocity for Cylinder Nacelle Case 11: $\mathfrak{R} \boldsymbol{c y \boldsymbol { l }}$ $=6.71 \mathrm{E}+06$ 185

Figure 5.65 Mean Normalized Velocity Vectors for Cylinder Nacelle Case 11: $\mathfrak{R} \boldsymbol{c y l}=6.71 \mathrm{E}+06$ 185

Figure 5.66 Instantaneous Normalized Velocity Vectors for Cylinder Nacelle Case 11: $\mathfrak{R} \boldsymbol{c y l}=6.71 \mathrm{E}+06$ 186

Figure 5.67 Mean Normalized Velocity X on YZ Plane for Cylinder Nacelle Case 11: $\mathfrak{R} \boldsymbol{c u} \boldsymbol{b}=6.71 \mathrm{E}+06$ 186

Figure $5.68 \boldsymbol{v} \boldsymbol{x}$ vs $\boldsymbol{y}$ Plot of Various $\boldsymbol{R} \boldsymbol{e}$ Values for Sharp Cylinder Cases 187 Figure $5.69 \boldsymbol{v} \boldsymbol{x}$ vs $\boldsymbol{y}$ Plot of Various $\boldsymbol{R e}$ Values for Smooth Cylinder Cases 188

Figure $5.70 \boldsymbol{v} \boldsymbol{x}$ vs $\boldsymbol{y}$ Plot of Various $\boldsymbol{R} \boldsymbol{e}$ Values for Sharp Cuboid Cases.. 189 
Figure $5.71 \boldsymbol{v} \boldsymbol{x}$ vs $\boldsymbol{y}$ Plot of Various $\boldsymbol{R} \boldsymbol{e}$ Values for Smooth Cuboid Cases 190

Figure 5.72 Comparison graph between experimental and simulation data for $C D, c y l$ vs Recyl. 191

Figure 5.73 Comparison graph between experimental and simulation data for $\boldsymbol{C D}, \boldsymbol{c} \boldsymbol{y} \boldsymbol{l}$ vs Fineness ratio

Figure 5.74 Comparison graph between experimental and simulation data for $\boldsymbol{C} \boldsymbol{P}$ vs normalized distance (diameter) from leading edge of cylinder.... 193 Figure 5.75 Comparison graph between experimental and simulation data for Reattachment length vs $\boldsymbol{R e c y l}$ .194

Figure 5.76 Mean velocity with fineness ratio=3 cylinder at $\boldsymbol{R e} \boldsymbol{c y} \boldsymbol{l}=1.00 \mathrm{E}+04$ from experiment (Hiroshi et al., 2005) ....................... 195 Figure 5.77 Mean velocity with fineness ratio=2.5 cylinder at

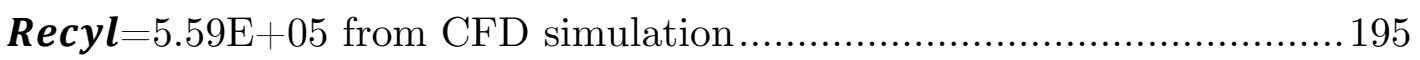




\section{LIST OF TABLES}

Table 2.1 Cost trend exponent of all components of a wind turbine as it upsizes from 5 to $20 \mathrm{MW}$ in the power output (Ashuri, 2012) ..................16 Table 2.2 Mass trend exponent of all components of a wind turbine as it upsizes from 5 to $20 \mathrm{MW}$ in the power output (Ashuri, 2012) ...................16

Table 2.3 List of patents related to downwind wind turbines....................22

Table 2.4 List of offshore wind modeling tools used in OC4 (Robertson, Jonkman, Vorpahl, et al., 2014) ....................................................24

Table 2.5 FAST v7 and FAST v8 ...............................................29

Table 4.1 SWAY 1:6.5 Scale Wind Turbine Prototype Specifications ........67

Table 4.2 Turbine Gross Properties ................................................... 74

Table $4.3 \mathrm{Hub}$ and Nacelle Gross Properties .......................................74

Table 4.4 Blade Gross Properties ................................................... 75

Table 4.5 Blade-Distributed Mass Properties ........................................75

Table 4.6 Blade-Distributed Aerodynamic Properties.............................76

Table 4.7 Gross Tower Properties ..................................................77

Table 4.8 Tower Distributed Properties ............................................77

Table 4.9 Platform Gross Properties ........................................................78

Table 4.10 Mooring System Properties ...................................................80

Table 4.11 Results of Static Equilibrium Analysis...................................82

Table 4.12 System Eigenfrequencies ................................................ 91

Table 4.13 TurbSim Wind Inflow Properties for Non-Operating Case ........93

Table 4.14 Comparison of Wind Properties for Non-Op Case 1 ................96

Table 4.15 Comparison of Wind Properties for Non-Op Case 2 .................96

Table 4.16 Mean and Standard Deviation for DOFs for the Non-Op Case 1 100

Table 4.17 Mean and Standard Deviation for DOFs for the Non-Op Case 2 100

Table 4.18 TurbSim Wind Inflow Properties for Operating Cases............. 108

Table 4.19 Comparison of Wind Properties for Op Case 1 ......................109 
Table 4.20 Comparison of Wind Properties for Op Case 2 ...................... 109

Table 4.21 Mean and Standard Deviation for DOFs for the Op Case 1 ....114

Table 4.22 Mean and Standard Deviation for DOFs for the Op Case 2 ....114

Table 4.23 Blade Frequencies (Hz) Observed in Experiment and Simulation

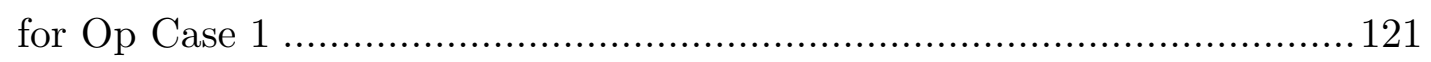

Table 4.24 Blade Frequencies (Hz) Observed in Experiment and Simulation

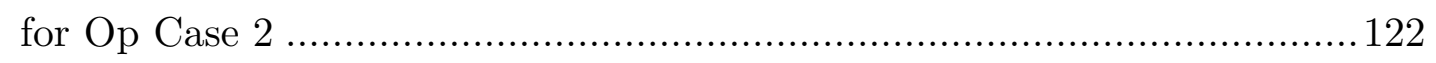

Table 5.1 Summary of Modeling Setup and Parameters.......................... 127

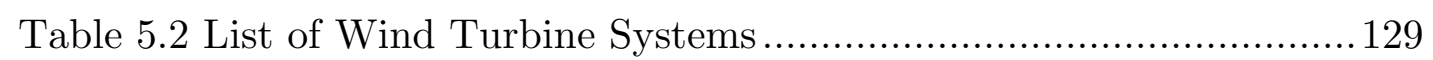

Table 5.3 Parameters for Tower Test Cases ........................................ 130

Table 5.4 Vortex Shedding Frequency, mean CL,tower and mean

CD, tower for Tower Cases ............................................................... 142

Table 5.5 Literatures Used for Tower Results Comparison ....................... 143

Table 5.6 Parameters for Nacelle Test Cases (Cylinder and Cuboid Shaped)

Table 5.7 Literatures Used for Nacelle Results Comparison ..................... 191

Table A.1 Data Summary of Deployment Period ..................................2225 


\section{ACRONYMS AND ABBREVIATIONS}

\begin{tabular}{|c|c|}
\hline ADAMS & - Automatic Dynamic Analysis of Mechanical Systems \\
\hline $\mathrm{AWAC}$ & - Acoustic Doppler Wave and Current Profiler \\
\hline BEM & - Blade Element Momentum \\
\hline CFD & - Computational Fluid Dynamics \\
\hline $\mathrm{CG}$ & - Center of Gravity \\
\hline DAS & - Data Acquisition System \\
\hline DNS & - Direct Numerical Simulation \\
\hline DNV & - Det Norske Veritas \\
\hline DNV GL & - Det Norske Veritas and Germanischer Lloyd \\
\hline $\mathrm{DOF}$ & - Degree-of-Freedom \\
\hline ELES & - Embedded Large Eddy Simulations \\
\hline ERI@N & - Energy Research Institute @ NTU \\
\hline ESDU & - Engineering Sciences Data Unit \\
\hline FAST & - Fatigue, Aerodynamics, Structures, and Turbulence \\
\hline FFT & - Fast Fourier Transform \\
\hline GL & - Germanischer Lloyd \\
\hline GPS & - Global Positioning System \\
\hline HAWC2 & $\begin{array}{l}\text { - Horizontal Axis Wind turbine simulation Code 2nd } \\
\text { generation }\end{array}$ \\
\hline
\end{tabular}




\begin{tabular}{|c|c|}
\hline IEA & - International Energy Agency \\
\hline IEC & - International Electrotechnical Commission \\
\hline ISO & - International Standards Organization \\
\hline JONSWAP & - Joint North Sea Wave Observation Project \\
\hline LES & - Large Eddy Simulations \\
\hline MARIN & - Maritime Research Institute Netherlands \\
\hline MBS & - Multi-Body System \\
\hline MRU & - Motion Reference Unit \\
\hline MSC & - MacNeal-Schwendler Corporation \\
\hline MSL & - Mean Sea Level \\
\hline NASA & - National Aeronautics and Space Administration \\
\hline NREL & - National Renewable Energy Laboratory \\
\hline NTU & - Nanyang Technological University \\
\hline NWTC & - National Wind Technology Center \\
\hline OC3 & - Offshore Code Comparison Collaboration \\
\hline OC4 & - Offshore Code Comparison Collaboration Continuation \\
\hline OC4 & $\begin{array}{l}\text { - Offshore Code Comparison Collaboration Continuation, } \\
\text { with Correlation }\end{array}$ \\
\hline PM & - Pierson-Moskowitz \\
\hline PSD & - Power Spectral Density \\
\hline RANS & - Reynolds-averaged Navier-Stokes \\
\hline
\end{tabular}


SGS

- Subgrid-Scale

SST

- Shear Stress Transport

TLP

- Tension-Legged Platform

WALE

- Wall-Adapting Local Eddy-viscosity

WAMIT

- Wave Analysis at Massachusetts Institute of Technology 


\section{NOMENCLATURE}

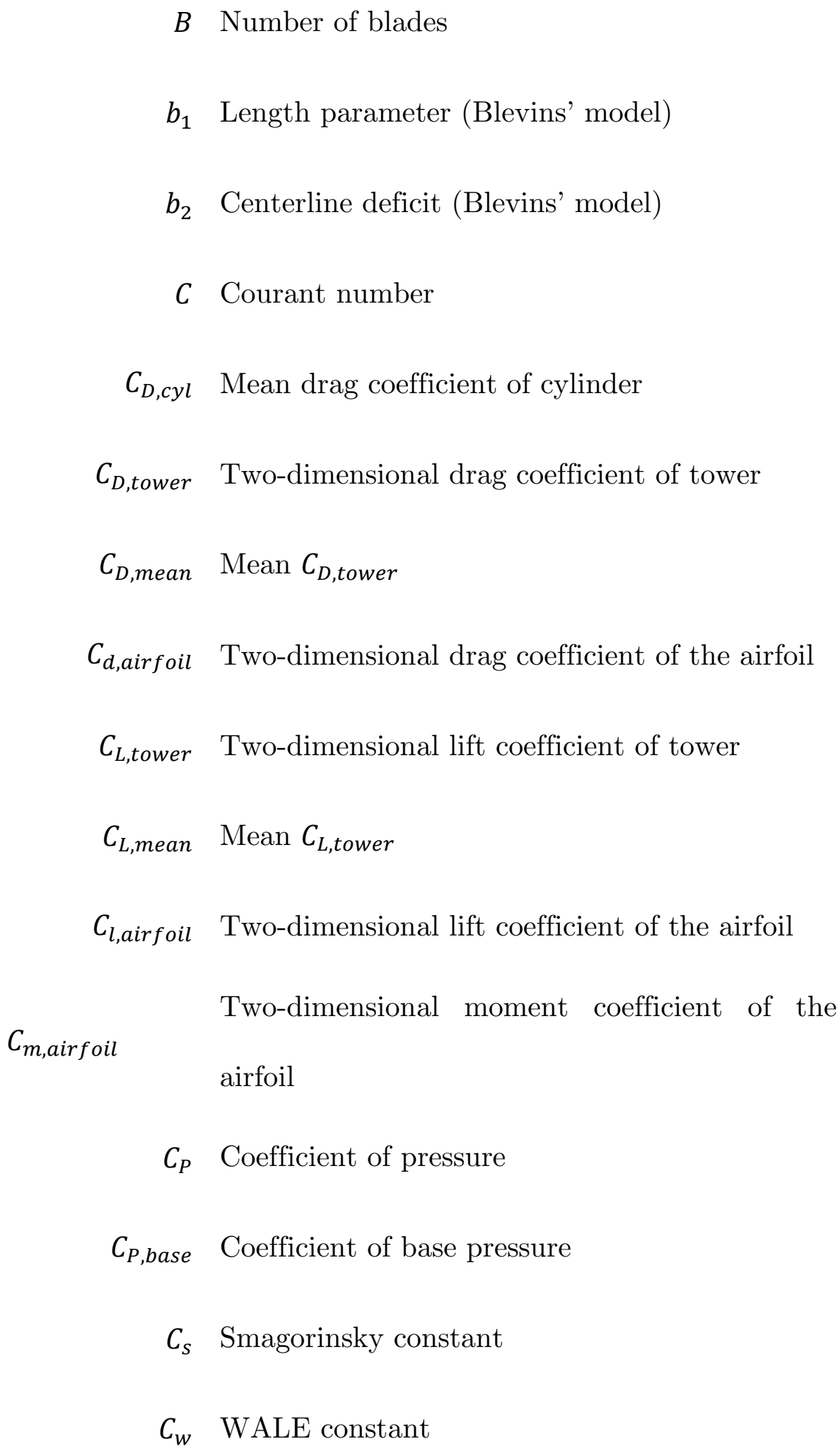

$B$ Number of blades

$b_{1} \quad$ Length parameter (Blevins' model)

$b_{2}$ Centerline deficit (Blevins' model)

C Courant number

$C_{D, c y l} \quad$ Mean drag coefficient of cylinder

$C_{D, \text { tower }}$ Two-dimensional drag coefficient of tower

$C_{D, \text { mean }}$ Mean $C_{D, \text { tower }}$

$C_{d, a i r f o i l}$ Two-dimensional drag coefficient of the airfoil

$C_{L, t o w e r}$ Two-dimensional lift coefficient of tower

$C_{L, \text { mean }}$ Mean $C_{L, \text { tower }}$

$C_{l, \text { airfoil }}$ Two-dimensional lift coefficient of the airfoil

$C_{m, \text { airfoil }}$

Two-dimensional moment coefficient of the airfoil

$C_{P} \quad$ Coefficient of pressure

$C_{P, \text { base }}$ Coefficient of base pressure

$C_{s} \quad$ Smagorinsky constant

$C_{w}$ WALE constant 
$D$ Tower diameter m

$D_{c y l}$ Cylinder diameter m

Radial distance from the yaw axis to the point

$d$ of interest normalized by tower radius (Powles' $m$ model)

Distance to the closest wall (Smargorinsky-

$d \quad m$

Lilly model)

$F_{\text {Prandtl }}$ Prandtl correction factor for tip and hub losses

$f_{\text {hub }}$ Hub loss model

$f \quad$ Frequency of vortex shedding $\quad \mathrm{Hz}$

$J_{m}$ Momentum deficit (Madsen's model)

K Kinematic momentum (Madsen's model)

$k \quad$ Turbulence kinetic energy

$J$

$L_{s} \quad$ Mixing length $m$

$p$ Pressure $\quad P a$

$R_{\text {hub }}$ Radius of rotor hub m

$R e_{c u b}$ Reynolds number of cylinder

$R e_{c y l}$ Reynolds number of cuboid

$R e_{\text {tower }}$ Reynolds number of tower 
$r$ Local radius of blade element or annulus

$m$

St Strouhal Number

$s_{i j} \quad$ Strain-rate tensor

$s^{-1}$

$t$ Time

$S$

$t_{i j} \quad$ Viscous stress tensor (CFD)

$N m^{-2}$

Dimensionless velocity in tower influence model $u$ or $v_{x}$

$(x)$

$u_{i} \quad$ Fluid velocity in $i$ component (CFD) $\quad \mathrm{ms}^{-1}$

$V_{0}$ Free stream wind velocity $\quad \mathrm{ms}^{-1}$

Velocity of tower element (Tower loading

$V_{\text {tower }} \mathrm{ms}^{-1}$ model)

$V_{x}$ Wind velocity in $x$ direction $\quad m s^{-1}$

$V_{x, \infty} \quad x$ - component of free stream wind velocity $\quad \mathrm{ms}^{-1}$

$V_{y}$ Wind velocity in $y$ direction $\quad \mathrm{ms}^{-1}$

$V_{y, \infty} y$ - component of free stream wind velocity $\quad \mathrm{ms}^{-1}$

Dimensionless velocity in tower influence model $v$ or $v_{y}$ $(y)$

Fractional decrease in velocity due to tower $v_{d}$ shadow

xxiv 
$W_{c u b}$ Width of the cuboid m

\author{
Wake width parameter normalized to tower \\ w \\ radius (Powles' model) \\ Wake width reference parameter (Powles' \\ $w_{\text {ref }}$ \\ model) \\ X Dimensional longitudinal position \\ $m$ \\ Longitudinal position normalized by tower \\ $x$ \\ diameter, cylinder width or cuboid width \\ $x_{b}$ Offset constant (Blevin's Model) \\ $x_{\text {offset }}$ Offset constant (Bak's Model) \\ Longitudinal position normalized by tower \\ $x_{r}$ \\ radius \\ $x_{\text {ref }} \quad x$ reference length (Powles' model) \\ $Y$ Dimensional transversal position \\ $m$ \\ Transversal position normalized by tower \\ $y_{r}$ \\ diameter, cylinder width or cuboid width \\ $Z \quad z$ position of tower node (Tower loading model) $\quad m$
}




\section{Greek Letters}

Velocity deficit at the wake center normalized

$\Delta$ with the free stream wind velocity (Powles' model)

$\Delta$ Local grid scale ((Smargorinsky-Lilly model) $\quad m$

$\Delta_{\text {ref }}$ Velocity deficit reference parameter

$\Delta_{t} \quad$ Time step

$S$

$\Delta_{x} \quad$ Length interval

$m$

$\varepsilon$ Viscous dissipation

$J \mathrm{~kg}^{-1} \mathrm{~s}^{-1}$

Virtual or eddy kinematic viscosity

$\varepsilon_{0}$

(Schlichting and Gerten model)

$\mathrm{kg} \mathrm{m}^{-1} \mathrm{~s}^{-1}$

$\eta$ Dimensional coordinate (Madsen's model)

$\theta \quad$ Reference angle on tower

$\circ$

$\ell_{n} \quad$ Length scale

$\kappa$ von Kármán constant

$\mu$ Dynamic viscosity

$N s m^{-2}$

$\mu_{t}$ Eddy (or turbulent) viscosity

$N s m^{-2}$

$v_{n}$ Velocity scale

$m s^{-1}$

$v_{T} \quad$ Kinetic eddy viscosity

$m^{2} s^{-1}$ 
$\rho \quad$ Fluid density

$\sigma$ Empirical constant (Madsen's model)

$v$ Kinematic viscosity

$\tau_{i j}^{S} \quad$ Subgrid-scale Reynolds stress

$\tau_{n}$ Time scale

$\varphi$ Angle of relative wind to the blade

$\omega$ Specific dissipation rate $k g m^{-3}$

$m^{2} s^{-1}$

$N m^{-2}$

$S$

$\circ$

$s^{-1}$ 


\section{INTRODUCTION}

\subsection{Background}

For the majority of countries in the world, non-renewable energy sources such as coal, natural gas, oil and nuclear are used as the primary source of energy (International Energy Agency, 2014a). The burning of fossil fuels that result in massive amounts of carbon dioxide emissions have been widely accepted to cause adverse environmental effects such as global warming. A substantial redirection of energy policies is vital to reduce the harm and extend of climate change in the world (Saidur, Islam, Rahim, \& Solangi, 2010). While fossil fuels are subjected to price volatility due to high dependence on political and social-economic events across the world, various countries are in search for low-cost energy sources and technologies which will allow them to be more energy independent.

Renewable energy is a key pillar in low-carbon energy supply mix. With many strong driving forces, these renewable energy resources have generated huge amount of interest and shown the fastest growth among other energy sources (British Petroleum, 2015). With the support of global subsidies and cost reductions, renewables resources is expected to gain significant share of the world power generation (International Energy Agency, 2014b).

For the past four decades, wind power is the leading source of renewable energy and has become potentially one of the major sources of energy of the $21^{\text {st }}$ century (Leung \& Yang, 2012). On a percentage basis, wind energy has also been one of the world's fastest growing energy source due to its reliability and cost-effectiveness (Watson, Hill, Courtney, \& Goldman, 2005). One of the key reasons for the growth of wind energy is due to advantages in the economics and competitiveness compared to the other sources of energy (World Wind Energy Association, 2009). 
The majority of the growth of wind energy was attributed to land-based wind power projects (Global Wind Energy Council, 2014). As such, favorable lands with good wind resource will be steadily taken up, a trend being observed in Europe (Wood, 2010). In the United States, the majority of the land-based wind resources are located in areas with low population (Musial \& Ram, 2010). Like many other countries, a main challenge to future development of wind energy is the insufficient transmission-line capacity to highly populated areas (load centers) near the coast line (Musial \& Butterfield, 2004).

Offshore wind energy is one of the key research and development directions in the wind energy sector (Perveen, Kishor, \& Mohanty, 2014). Musial, Butterfield, and Ram (2006) suggested that offshore wind energy has the potential to become a major source of energy in the United States. Some of the reasons explained by Musial et al. (2006) and Shikha, Bhatti, and Kothari (2003) to site turbines offshore are: (1) location (could be near highly populated coastal loads where onshore wind energy is not available); (2) higher wind speeds; (3) lower turbulence; (4) reduced visual impact; (5) reduced noise impact.

\subsection{Objectives}

The main objectives of this work were:

- To build a reliable aero-hydro-servo-elastic FAST numerical model of the SWAY prototype downwind floating wind turbine (developed and built by SWAY A/S);

- To verify, calibrate and validate the FAST numerical model of the SWAY prototype downwind floating wind turbine with open sea test data;

- To modify and improve the FAST numerical code based on validation analysis;

- To conduct a computational fluid dynamics (CFD) analysis to analyze the flow fields around tower and nacelle; and 
- Compare and improve/develop correction models for tower and nacelle influence using CFD simulations over various wind speed, size and shapes.

\subsection{Layout of Thesis}

Chapter 2 consists of a thorough literature review to support the motivation of this work. It begins with the discussion of current offshore wind systems, foundation and floating platform concepts, followed by detailed discussion on the motivation for the downwind design investigation and selection, including existing academic research and industry trends supporting this design concept. The chapter concludes with discussion on the technical challenges and gaps, and the areas that will be taken on in this study.

Chapter 3 provides key technical background on various engineering topics that are relevant to the latter chapters of the report. This includes details of the current tower and nacelle influence models used in various wind turbine simulation tools. The engineering background on turbulence modeling for CFD is briefly presented.

Chapter 4 presents the work of using the open-water test data of the SWAY prototype wind turbine to calibrate and validate a FAST floating offshore downwind turbine model. The chapter provides details of the code improvements and modifications, modeling strategies, and the development of a FAST model of the SWAY prototype wind turbine, including justification of the modeling assumptions and tuning of system parameters. The key findings calibration and validation studies are presented together with the limitations of the FAST model and other potential problematic areas.

Chapter 5 discusses the improvement and development of the tower and nacelle influence models that can be used in the numerical simulation tools. The chapter describes the details on the general and model specific CFD simulation setup for the tower and nacelle model. The CFD results are 
compared with available experimental results from literature and existing simplified models. The chapter concludes with the proposed improved mode and describes key findings and limitation of this study.

Chapter 6 concludes the thesis, with discussions on the potential developments that can be carried out in continuation from this work.

\subsection{Contributions of Work}

During the course of this study, the author has contributed to some significant findings and progress in this area of research. These contributions include:

1) Improved the FAST numerical simulation tool to include loading due to wind flow over the tower. It has been shown that wind loading on the tower is important for loads and dynamics analysis of floating turbines.

2) Developed a reliable aero-hydro-servo-elastic FAST numerical model of the SWAY prototype downwind floating wind. The details of the model inputs presented in this study can be used by the research community for further study.

3) Calibrated, verified and validated the simulation results of the SWAY model in FAST numerical simulation tool. The publications produced for this work were the first few of its kind where results from a similarly scaled floating wind turbine model was compared with open sea test data.

4) Developed a novel meshing and modeling method to conduct computational fluid dynamics analysis robustly and efficiently for high Reynolds number flow over bluff bodies. Reasonable predictions of turbulent wake profile were achieved for different type of flows that includes, (1) flow around a circular cylinder, (2) axial flow across a 3D cylinder, (2) axial flow across a 3D cuboid.

5) Developed a new semi-empirical representation of the tower influence model that showed significant improvement over the models used previously. 
6) Produced good comparative results for flow across the cylindrical between the experiment and CFD simulations. While no experiment comparison was done with cuboid shaped models and smooth cylinder models, it is very likely that similar computation mesh, CFD setup and turbulence models can yield results that are reasonably representative of actual flow conditions. Key findings were also noted that support in the development of nacelle influence models.

A number of published journal paper, conference papers and proceedings, and reports have been authored by the author and the contents may be reiterated in this thesis. The publications are listed below:

1) Koh, J. H., Robertson, A., Jonkman, J., Driscoll, F., \& Ng, E. Y. K. (2013). Building and Calibration of a FAST Model of the SWAY Prototype Floating Wind Turbine. Paper presented at the International Conference on Renewable Energy Research and Applications (ICRERA) 2013, Madrid, Spain. doi: 10.1109/ICRERA.2013.6749860

2) Koh, J. H., Robertson, A., Jonkman, J., Driscoll, F., \& Ng, E. Y. K. (2015). Validation of SWAY Wind Turbine Response in FAST, with a Focus on the Influence of Tower Wind Loads. Paper presented at The Twenty-fifth International Offshore and Polar Engineering Conference.

3) Koh, J. H., \& Ng, E. Y. K. (2016). Downwind offshore wind turbines: Opportunities, trends and technical challenges. Renewable and Sustainable Energy Reviews, 54, 797-808. doi: 10.1016/j.rser.2015.10.096

4) Koh, J. H., Ng, E. Y. K., Robertson, A., Jonkman, J., \& Driscoll, F. (2016). Validation of a FAST Model of the SWAY Prototype Floating Wind Turbine (pp. Medium: ED; Size: 64 p.): ; National Renewable Energy Lab. (NREL), Golden, CO (United States). NREL/TP--500061744, U.S. Department of Energy. Contract no: DE-AC36-08G028308. doi: $10.2172 / 1259950$ 


\section{LITERATURE REVIEW}

\subsection{Current Offshore Wind Turbine Technology}

Most offshore wind projects built in recent years are focused on fixed offshore wind turbines at depths of less than $50 \mathrm{~m}$. This is expected due to the mature technology in fixed foundations (Perveen et al., 2014), assurance in technical feasibility and economic viability (Henderson et al., 2004). Floating wind turbines can be used in deeper water and allow offshore wind turbines to be installed in more areas. It is also possible that the larger turbines may be transported, deployed and decommissioned at a lower cost (Henderson et al., 2004; Myhr, Bjerkseter, Ågotnes, \& Nygaard, 2014).

Offshore environment is generally more challenging as foundations and floating platform need to account for turbine weight and height, the nature of the sea bed, the depth of water, loads and dynamics of the system, and expected storm conditions (Sun, Huang, \& Wu, 2012). In current offshore wind turbine designs, many are basic concepts of using standard land-based wind turbines and 'marinised' using a platform from the offshore oil and gas industry with additional anti-corrosion and structural stiffness. Each type of support structures has its advantages and disadvantages, and is selected based on numerous factors.

Offshore wind turbine support structures used commercially and developmental projects can generally be classified accordingly.

\subsubsection{Fixed Structures}

In shallow water below $50 \mathrm{~m}$ in water depth, five fixed types of structures have been used, as shown in Figure 2.1: 


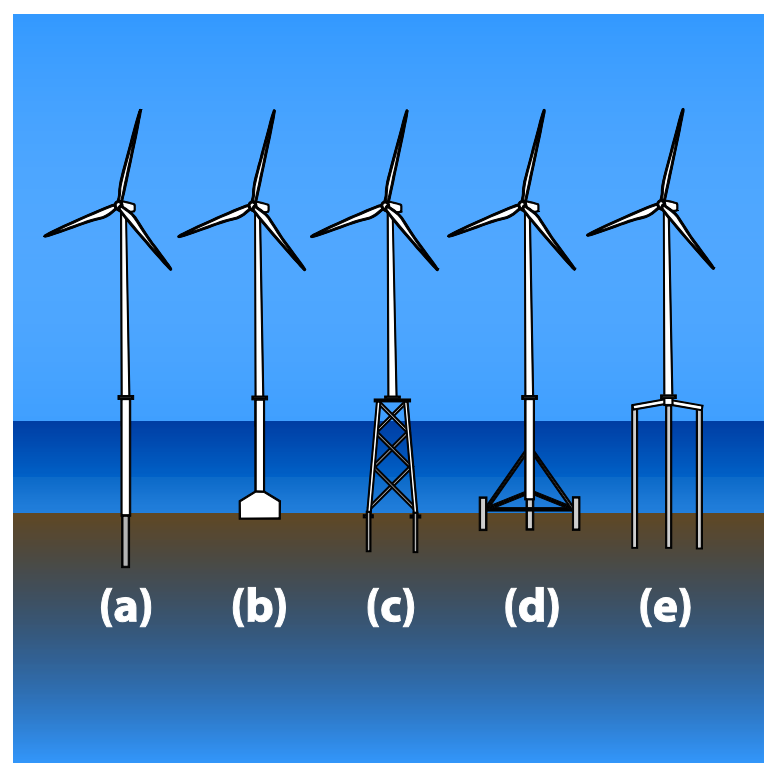

Figure 2.1 Fixed structures for offshore wind turbines

Monopile (Figure 2.1a) - This is simplest and cheapest type of foundation that is used commonly in the North Sea. The structure is a steel or concrete tube driven into the seabed to a depth of 0 to $25 \mathrm{~m}$ (Det Norske Veritas AS, 2014). The tower of the turbine is usually connected to the monopile with a transition piece. The monopile structure has been used in many major offshore wind projects, such as the London Array offshore wind farm (London Array Limited, 2013), Horns Rev 1-2 (DONG energy, 2015; Vattenfall, 2010), DanTysk offshore wind farm (DanTysk Offshore Wind GmbH, 2012) and Anholt offshore wind farm (DONG energy, 2013).

Gravity-based (Figure 2.1b) - A gravity-based foundation is normally fabricated in reinforced concrete with ballast and it uses its own weight to anchor to the seabed at water depth between 0 to $25 \mathrm{~m}$ (Det Norske Veritas AS, 2014). While the seabed needs to be dredged and leveled, a large base area with scour protection is commonly used to counteract the moments produced by the system. The gravity-based foundation have been used in the Vindeby offshore wind farm (world's first offshore wind farm) (Vølund, 2005) and the Nysted offshore wind farm (DONG energy, 2008).

Jacket (Figure 2.1c) - Often used in water depths of about 20-50 m (Det Norske Veritas AS, 2014), jacket structure usually consist of a three or four- 
legged lattice structure made from tubular steel. These structures experience low wave loads and are similar to those used in offshore oil and gas industries with design and performance well understood. The jacket structure have been used in the Thornton Bank Phase 2 and 3 offshore wind farm (C-Power, 2011) and Ormonde offshore wind farm (Vattenfall, 2012).

Tripod (Figure 2.1d) - A tripod structure is a relatively lightweight threelegged steel structure where the frame is submerged in the water. This provides good stability and stiffness to the entire system and can be located in water depth between 20 to $50 \mathrm{~m}$ (Det Norske Veritas AS, 2014). This structure has only been used in more recent offshore wind farm projects such as the Alpha Ventus (Deutsche Offshore-Testfeld und Infrastruktur GmbH \& Co. KG (DOTI), 2010), Trianel Windpark Borkum (Areva, 2013) and Global Tech 1 (Global Tech I Offshore Wind GmbH, 2014) wind farm.

Tripile (Figure 2.1e) - The patented tripile structure has been developed by Bard Engineering GmbH that is suitable for water depths between 30 to 50 m (Hansen \& Schoenefeldt, 2007). The three-legged steel support frame extends from the foundation to above the water level, before connecting to the tower. Currently, it has only been used in the Bard Offshore 1 wind farm.

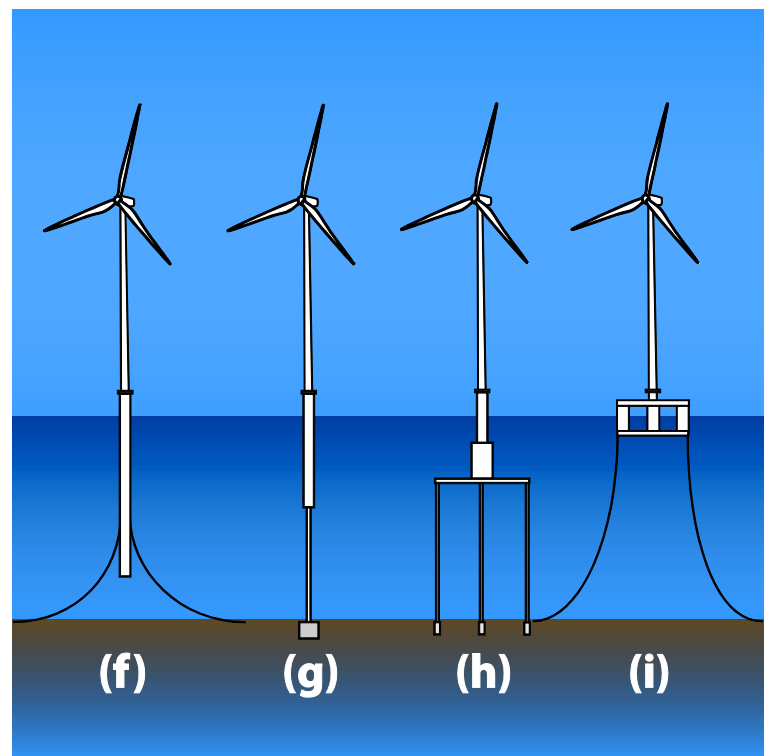

Figure 2.2 Floating structures for offshore wind turbines 


\subsubsection{Floating Structures}

For water depths above $50 \mathrm{~m}$, cost of fixed structures becomes expensive, which results in the interest to develop floating structures as the offshore wind industry develops. To date, floating structures remains in the research and development stage. The following floating structures (illustrated in Figure 2.2) have been used in prototype and full-scale testing.

Spar Type (Figure 2.2f) - A spar buoy is normally made up of a long closed steel cylinder, which is made compliant by catenary mooring lines and anchors. The buoyancy force causes the cylinder to float upright while the system is stabilized by ballast at the base. Statoil worked with Siemens Wind Energy to install the world's first full-scale floating spar type wind turbine at the coast off Norway. The 2.3 MW wind turbine has a draft of $100 \mathrm{~m}$ and is installed at a depth of $200 \mathrm{~m}$ (Statoil ASA, 2012).

Tension-legged platform (TLP) (Figure 2.2g and 2h) - The floating platform is partially submerged below the sea surface and fixed to slim tension $\operatorname{rod}(\mathrm{s})$ or line(s) that is(are) anchored to the seabed. The platform needs to have significant buoyancy to support the turbine and maintain tension in the legs under various sea and wind conditions. A single tension leg spar platform wind turbine was developed and tested by SWAY A/S through a prototype installed in Norway (Koh, Robertson, Jonkman, Driscoll, \& Ng, 2013; SWAY AS, 2013). Multiple tension leg platform has been demonstrated through a prototype developed by Blue H Group Technologies Ltd, where a system was deployed in $113 \mathrm{~m}$ water depth and $21.3 \mathrm{~km}$ off the coast of Southern Italy (Blue H Group Technology Ltd, 2013).

Semi-submersible (Figure 2.2i) - The semi-submersible structure contains column-stabilized units designed in a triangular or rectangular formation that provide buoyancy and stability. The structure is typically in place with catenary mooring lines. Floating wind turbine developer Principle Power installed WindFloat (Roddier, Cermelli, Aubault, \& Weinstein, 2010), a 2 
MW prototype of the semi-submersible concept off the coast of Portugal in 2011 .

\subsection{Opportunities for Downwind Configuration}

Design conservatism is observed in the current offshore wind turbines. The key reason to this is to avoid major changes to the proven technology for onshore systems, which helps to reduce the capital cost in the initial implementation. However, exportation of onshore technology directly to the offshore environment may not be entirely advantageous as the weight penalty of conventional rotor designs becomes more significant offshore (Moe, 2007). There are opportunities in new designs or configurations (Chen, Ponta, \& Lago, 2011), which can potentially lower the cost of energy in a less restrictive offshore environment.

Downwind wind turbines played an important role in pioneering the design of wind turbines. In 1941, the Smith-Putnam wind turbine was the first megawatt-size wind turbine that was considered to be a success technically, and helped to initiate economic feasibility study for large wind turbines (Johnson, 2001; Vargo, 1974). Several sized experimental wind turbines dominated the wind industry between 1950 s to 1970 s with notable downwind systems such the Ulrich Hutter Allgaier StGW-34 wind turbine and National Aeronautics and Space Administration (NASA) wind turbines (Le Gourieres, 2014). These helped to pave foundations to the technology development and initial deployment of large wind turbines.

The introduction of active yaw systems spurred the growth of upwind rotors. As the offshore wind energy industry grows, this section discusses some of the factors and opportunities in selecting a downwind configuration for offshore wind turbines.

\subsubsection{Noise}

Noise consideration was one of the key reasons for turbine manufacturers and designers to select the upwind configuration on land-based turbines (C. 
P. Butterfield, Musial, \& Jonkman, 2007). Analytical acoustic studies conducted on wind turbines indicated coherently that downwind rotors generate more noise compared to upwind rotors (Madsen, Johansen, Sørensen, Larsen, \& Hansen, 2007; Sescu \& Afjeh, 2012) and experimental (Hubbard, Grosveld, \& Shepherd, 1983; Hubbard \& Shepherd, 1982, 1990; Kelley, McKenna, \& Hemphill, 1985; McNerney, van Dam, \& Yen-Nakafuji, 2003). The additional noise source of downwind rotors is a result of the interaction of the turbine blades with the aerodynamic wake of the tower.

Offshore wind farms are generally located further away from populated areas. Therefore, the issue of noise to human population is likely eliminated. However, marine life may be affected during installation and operation of the offshore wind turbine (Kapsali \& Kaldellis, 2012), which may require further study.

\subsubsection{Trend in Increasing Size of Wind Turbine}

Current developments of wind turbine technology are leading to larger wind turbines rated $5 \mathrm{MW}$ or more, as larger wind turbines generally benefit economically from an overall cost reduction per rated MW (Ashuri, 2012) and reduction of environment impact by producing greener energy (Caduff, Huijbregts, Althaus, Koehler, \& Hellweg, 2012). While on-shore wind turbines is be limited by the available transportation infrastructure and erection equipment, offshore wind turbines face lesser issues and are likely to continue the trend for even larger wind turbines (Musial \& Butterfield, 2004). The largest wind turbines installed for testing and commercial usage includes the Vestas V164 8MW, Enercon E126 7.5MW and Samsung S7.0 171 7MW (Windpower Monthly, 2016). With recent works on development of wind turbine blades designed for 13.2 MW systems (Griffith \& Ashwill, 2011) and initiatives from industries (Chayesteh, 2014; Gamesa Corporación Tecnológica, 2014) and organizations such as the European Wind Energy Association (European Wind Energy Association, 2011, 2013) and International Energy Agency (IEA) (International Energy Agency, 2013), 
wind turbines rated between $10 \mathrm{MW}$ to $20 \mathrm{MW}$ are projected to be designed and tested by the end of this decade (illustrated in Figure 2.3).

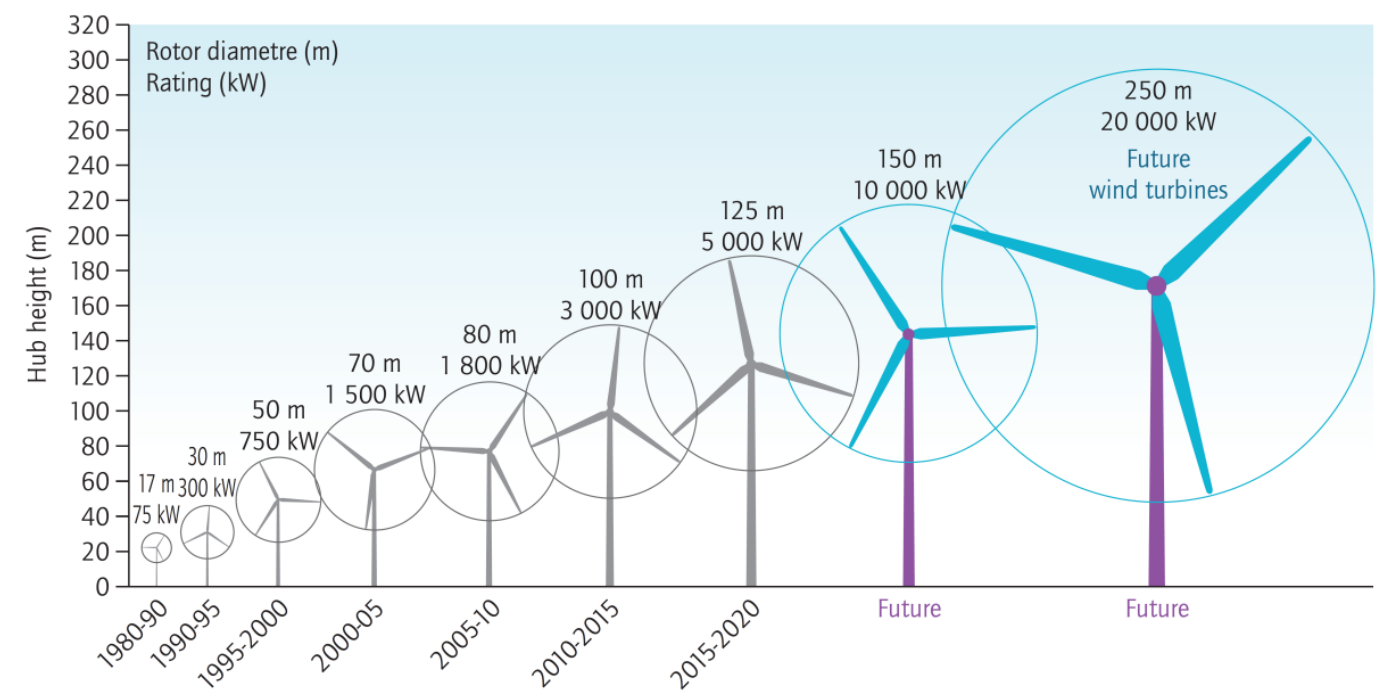

Figure 2.3 Growth in size of wind turbines since 1980 and prospects (International Energy Agency, 2013)

The growth in the size of traditional upwind wind turbines are met with challenges that need to be dealt with. These technical issues include manufacturing technology, higher weight induced structural loads, higher aerodynamic loads on blades, increased loading on gearbox and nacelle, and blade to tower clearance. A downwind configuration wind turbine provides opportunities to mitigate some of these issues, and these are discussed in details in the Section 2.2.3 and 2.2.4.

\subsubsection{Blade to Tower Clearance}

While the majority of wind turbines are in the upwind configuration, designers have constantly taken note of providing a safe clearance between the blade and the tower surface as advised by IEC standards (International Electrotechnical Commission, 2014) and other researchers (Laino, 2001). This clearance may also diminish over time as a result creep/fatigue of composite-material (Jin et al., 2009; John, David, \& Daniel, 2013). The methods used to improve clearance of an upwind rotor include (illustrated in Figure 2.4): 
- $\quad$ Coning of blades - Blades are connected to the hub at a small angle out of the rotor plane, shown as angle $\gamma$.

- Tilting of shaft axis - The shaft axis is connected to the system at a small angle out of the horizontal, shown as angle $\beta$.

- $\quad$ Curved blades - Blades are designed and manufactured with out-ofplane curvature resulting in higher clearance.

- Overhang distance - Increasing the overhang distance increases the rotor-tower clearance.

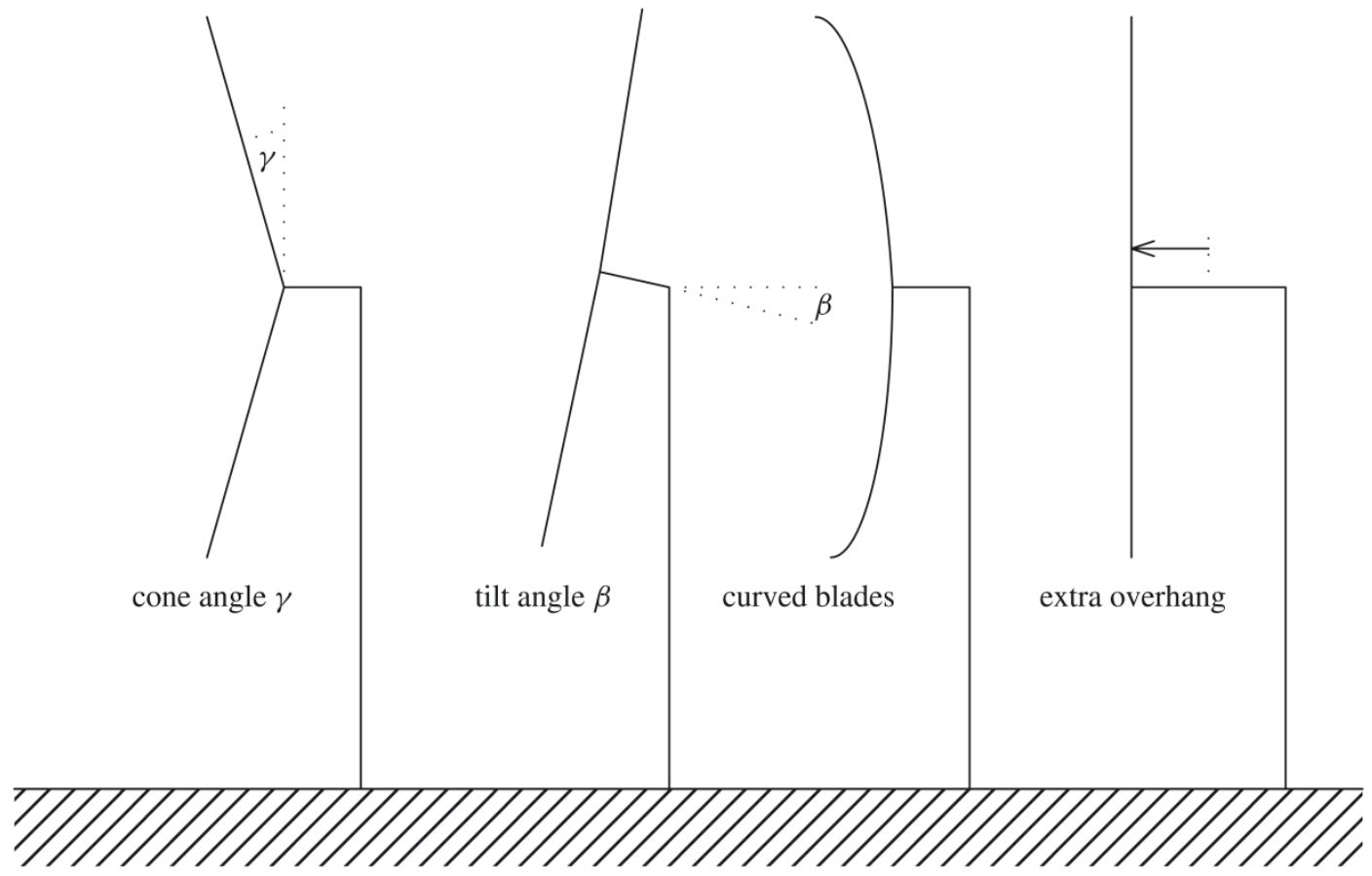

Figure 2.4 Rotor blade tip and tower clearance (Jamieson, 2011b)

Although the above options are available and may be used concurrently, they can only be used to a limited extent. For example, tilting beyond 4 or 5 degrees may introduce unwanted cyclic loads while coning results in a moment at the blade root due to centrifugal forces on the blade elements that may have adverse effect in over-speed condition. Coning and tilting options also results in a slight reduction of power or require marginally longer blades to maintain power (GE Wind Energy LLC, 2006; Jamieson, 2011b). Curved blades and increasing overhang distance are costly options 
while the latter causes increased loads on nacelle structure and rotor bearings (Jamieson, 2011b).

Since the wind thrust loads deflects the blades in the downwind direction, having a downwind configuration eliminates issues with blade to tower

clearance (Janajreh, Talab, \& Macpherson, 2010). The average blade to tower clearance would now increase (instead of decreasing in the upwind configuration) with increasing blade loads (C. P. Butterfield et al., 2007).

\subsubsection{Blade Technology and Operations}

Usage of more flexible blades has been introduced by wind turbine designers and researchers (Hoogedoorn, Jacobs, \& Beyene, 2010; Jamieson, 2009; Kristiansen, 2014; Reiso \& Muskulus, 2013; Siegfriedsen, 2007). However, this poses engineering challenges to providing a safe blade to tower clearance for the wind turbine system. As discussed in Section 2.2.3, this issue would be eliminated if a downwind configuration is used. As a result, usage of flexible blades opens opportunities to reduce blade material, and reduce loads to the blades, hub, and gearbox (Ahlström, 2006; Cheney, 1997; Hoogedoorn et al., 2010; Jamieson, 2009; Tangler, 2000; Verelst, 2009; Wright). A downwind configuration can also take advantage of centrifugal forces on the blades during turbine operation to reduce the blade root bending moments (Manwell, McGowan, \& Rogers, 2009).

Other blade operation methods have been suggested for downwind rotors. High speed rotor operations as suggested by Jamieson (2009, 2011b) helps to further justify the choice of using downwind rotors as an integrated design concept, potentially resulting in a 15 to $20 \%$ reduction in wind turbine capital cost. Two-bladed turbines was also suggested to be integrated to the design of offshore turbines due to the lighter weight rotor (S. Butterfield, Musial, \& Jonkman, 2005; De Vries, 2011; Kaldellis \& Zafirakis, 2012; Snieckus, 2014b) and the option of using teetering hub that further reduces rotor loads (Bergami, Madsen, \& Rasmussen, 2014). Pre-curved blades have 
also been suggested to reduce the mass and the bending moments on the blades (Loth, Steele, Ichter, Selig, \& Moriarty, 2012; Steele et al., 2013).

Individual pitch control of wind turbine blades was introduced for upwind wind turbines to reduce loading and fatigue of blades (Bossanyi, 2003, 2005; Larsen, Madsen, \& Thomsen, 2005; Namik \& Stol, 2010; Selvam, 2007; Selvam, Kanev, van Wingerden, van Engelen, \& Verhaegen, 2009; Van Engelen \& Van der Hooft, 2005; van Solingen \& van Wingerden, 2015). It has also been proposed by researchers for two other possible reasons for downwind rotors. First, individual pitch control can be used for yawing of wind turbines when it is not operating (motor-driven mode) and for better yaw stability during operation (Navalkar, Wingerden, \& Kuik, 2014; Wenxin \& Karl, 2007). Second, individual pitch control may also be used to mitigate the loading effects of velocity deficit in the wake of the tower, which helps to reduce fatigue loading on the wind turbine blades (Kittilä, 2012; Li, Sun, Xing, \& Chen, 2012).

\subsubsection{Removal of Active Yaw System}

According to studies done by the Energy Research Centre at the Netherlands (Engels, Obdam, \& Savenije, 2009), the yaw system can amounts to about $1.3-5 \%$ of the entire upwind wind turbine cost for a land-based system. In addition, a cost trend analysis by Ashuri (2012) also showed that the cost and mass trend exponent of the yaw system is the second highest component in the entire wind turbine as detailed in Table 2.1 and Table 2.2. This indicates that the cost and mass of the yaw system is heavily influenced by up-scaling of wind turbine system. 
Table 2.1 Cost trend exponent of all components of a wind turbine as it upsizes from 5 to $20 \mathrm{MW}$ in the power output (Ashuri, 2012)

\begin{tabular}{llll}
\hline Cost element & $\begin{array}{l}\text { Trend } \\
\text { exponent }\end{array}$ & Cost element & $\begin{array}{l}\text { Trend } \\
\text { exponent }\end{array}$ \\
\hline Tower & 3.22 & Electrical Interface & 1.75 \\
Yaw system & 2.97 & Installation & 1.75 \\
Low speed shaft & 2.89 & Scour protection & 1.75 \\
Blade & 2.66 & Generator & 1.75 \\
Pitch Mechanism & 2.66 & Power electronics & 1.75 \\
Warranty & 2.55 & Electrical connection & 1.75 \\
Marinization & 2.55 & Permits & 1.75 \\
Main bearing & 2.44 & Port and staging & 1.75 \\
Hub & 2.39 & Levelized replacement & 1.75 \\
Decommissioning & 2.22 & Hydraulic and cooling & 1.75 \\
Gearbox & 2.19 & HSS and brake & 1.75 \\
Bedplate & 1.95 & Nacelle cover & 1.69 \\
Platform and railing & 1.95 & Nose cone & 1.18 \\
O \& M & 1.85 & Access equipment & Fixed \\
Foundation system & 1.75 & Control and safety & Fixed \\
\hline
\end{tabular}

Table 2.2 Mass trend exponent of all components of a wind turbine as it upsizes from 5 to $20 \mathrm{MW}$ in the power output (Ashuri, 2012)

\begin{tabular}{llll}
\hline Mass element & $\begin{array}{l}\text { Trend } \\
\text { exponent }\end{array}$ & Mass element & $\begin{array}{l}\text { Trend } \\
\text { exponent }\end{array}$ \\
\hline Main bearing & 3.52 & Gearbox & 1.98 \\
Yaw system & 3.32 & Bedplate & 1.95 \\
Tower & 3.22 & Platform and railing & 1.95 \\
Low speed shaft & 2.89 & HSS and brake & 1.75 \\
Blade & 2.65 & Hydraulic and cooling & 1.75 \\
Pitch bearing & 2.59 & Nacelle cover & 1.69 \\
Pitch system & 2.54 & Generator & 1.61 \\
Hub & 2.39 & Nose cone & 1.18 \\
\hline
\end{tabular}

An upwind configuration rotor on a multi-megawatt wind turbine uses an active yaw system to track the optimal wind inflow direction. While many yaw controlled turbines are intrinsically stable at specific tip speed ratio, downwind turbines tend to respond faster to changes in wind direction, reducing the time in yawed conditions (Snel \& Schepers, 1995). Improved yaw stability in downwind rotor as compared with upwind rotor has been observed in testing and simulation of turbines (Bracchi, 2014; Bracchi \& Krogstad, 2012).

When utilizing a downwind configuration, there is a potential of removal of the active yaw system on the wind turbine as the system is able to passively 
yaw into the wind. Direct cost reduction is achieved with the removal of the active yaw system and lower operating and maintenance cost. A mass reduction to the rotor-nacelle system is technically beneficial in an offshore wind turbine, particularly in floating turbines, and also brings down the indirect cost of other components of the system.

\subsubsection{Impact on Power Performance}

The effects of tower shadow and non-uniform in-flow is generally small (Viterna \& Janetzke, 1982), and the variation in rotor power between and upwind and downwind rotor has been predicted to be less than $5 \%$ (Glasgow, Miller, \& Corrigan, 1981). Based on the computational work (Frau, Kress, Chokani, \& Abhari, 2015) and experimental work (Kress, Chokani, \& Abhari, 2015) (1:160 scale model) on the Hitachi 2MW wind turbine, downwind rotors experienced higher power and thrust when compared with corresponding upwind configurations. It was deemed that having the nacelle resulted in higher axial velocities ahead of the rotor and higher flow incidences on the blade (Kress, Chokani, Abhari, et al., 2015). In addition, if the rotor was tilted to increase the blade to tower clearance for a floating wind turbine, a downwind rotor is expected to have a better power performance as the floating system pitches in the direction of the wind. This is because error of the angle between the rotor axis and the wind inflow increases for an upwind system but decreases for a downwind system.

\subsubsection{Innovative Tower Design}

Wind turbine tower shape and design in the recent decades have streamlined to cylindrical and tubular towers. Using a downwind configuration and eliminating issues with blade to tower clearance provides designers opportunity to develop innovative tower designs that reduce cost and loads to the system.

Full-height lattice towers for wind turbine were commonly used by pioneering on-shore wind turbines. Some researchers suggest using full lattice towers for offshore wind turbines due to its advantages such as 
weight savings and omission of the expensive transition piece for conventional fixed support structures (Long \& Moe, 2012; Muskulus, 2012). Investigations comparing the effects of tubular and lattice towers on downwind rotors have also been carried out (Reiso, 2010; Reiso, Hagen, \& Muskulus, 2013).

Usage of tower fairing have been proposed and analyzed by a number of inventors (Adrian, 2012; Loth \& Selig, 2014) and researchers (O'Connor, Loth, \& Selig, 2013, 2015; O’Connor, 2014; Reiso \& Muskulus, 2013; Wilmshurst, Powles, \& Wilson, 1985). Tower fairing devices have shown to reduce the drag forces on the tower and also reduce the wake size behind the tower. This reduces tower loads and the tower shadow effect on the wind turbine blades, which is one of the pertaining issues with downwind rotors that is discussed further in Section 3.1.2.

\subsection{Trends in Industry}

\subsubsection{Downwind Offshore Wind Turbines Design and Projects}

While majority of offshore wind turbines today are upwind in configuration, there have been an increased number of downwind offshore wind turbines that were designed and tested in the recent years. This section discusses some of the downwind offshore wind turbine projects conducted by the industry.

\subsubsection{2-B Energy}

2-B Energy plans to manufacture and test its $6 \mathrm{MW}$ two-bladed downwind fixed wind turbine onshore, at Eemshaven harbor in the Netherlands, and offshore, at the Methil project in Scotland (Miller, 2014). The Dutch firm, with the backing of a funding consortium from UK and Scottish governments (reNEWS, 2014), aims to build and test the onshore turbine in 2015 and 2 offshore turbines one year later. The 140-m-rotor-diameter wind turbine also features a 3-legged full truss structure with helicopter landing access (2-B Energy). 


\subsubsection{Aerodyn Energiesystems GmbH / Ming Yang Wind Power}

Ming Yang Wind Power installed 6 MW two-bladed downwind fixed wind turbine in China at late 2014 and connected the turbine to the grid in early 2015 (Weston, 2015). The wind turbine is expected to go on further trials and testing over the next two years. This 140-m-rotor-diameter wind turbine designed by Aerodyn Energiesystems GmbH (Aerodyn Energiesystems GmbH, 2014a) uses its super-compact drive turbine technology that is characterized by a single rotor bearing, a two-stage planetary gearbox and a permanent magnet generator (Mingyang Wind Power, 2012). The manufacturer has also claimed the two-bladed downwind configuration is designed to deal with conditions in Chinese waters (Smith, 2014).

Ming Yang Wind Power has also signed agreements with Norway's Marin Energy Test Centre to build a similar turbine at the Karmoy turbine demonstration project in south-west Norway (China Ming Yang Wind Power Group Limited, 2014; Snieckus, 2014c). This effectively sets up the downwind wind turbine to prove its performance and solution to one of the most difficult marine environments (Snieckus, 2015).

Aerodyn Energiesystems GmbH has also designed a downwind floating wind turbine system, known as SCD Nezzy. The $8 \mathrm{MW}$ two-bladed wind turbine is claimed to reduce significant cost by having concrete foundation design guided leaning profiled tower and self-adjusting downwind rotor, floating platform stabilized by three flexible floaters and installation without crane ships (Aerodyn Energiesystems GmbH, 2014b). The 168-m-rotor-diameter wind turbine is also designed to be deployed in water depths between 40 to $200 \mathrm{~m}$, having features such as guyed tubular steel tower, droplet-shaped tower and Y-shape hollow concrete support structure (Snieckus, 2014a; Vries, 2014).

\subsubsection{Fukushima Floating Offshore Wind Farm Demonstration Project (Fukushima FORWARD)}


The Fukushima FORWARD project (Fukushima Offshore Wind Consortium, 2013, 2014) funded by the Ministry of Economy, Trade and Industry in Japan is conducted by the Fukushima offshore wind consortium. This consists of Marubeni Corporation (Project integrator), the University of Tokyo (Technical advisor), Mitsubishi Corporation, Mitsubishi Heavy Industries, Japan Marine United Corporation, Mitsui Engineering \& Shipbuilding, Nippon Steel \& Sumitomo Metal Corporation, Ltd., Hitachi Ltd., Furukawa Electric Co., Ltd., Shimizu Corporation and Mizuho Information \& Research. This project focuses on solving technical challenges of floating offshore wind turbines, collaboration with fishery industry, marine navigation safety and environmental assessment (Mizuno, 2014).

The first phase of the project involved construction, installation and testing of a $2 \mathrm{MW}$ downwind wind turbine with a compact semi-submersible floater from Hitachi Ltd. (Inamura, Shigenaga, \& Kiyoki, 2013; Matsunobu et al., 2009). This phase aims to minimize floater motion, safety and power generation efficiency.

\subsubsection{Goto Floating Offshore Wind Turbine Demonstration Project}

The Goto Floating Offshore Wind Turbine Demonstration Project was launched by the Ministry of Environment in Japan. The project is scheduled from September 2010 to March 2016 involved the installation of two floating wind turbines (Utsunomiya, Shiraishi, Sato, Inui, \& Ishida, 2014). The first wind turbine was a 1:2-scale model $100 \mathrm{~kW}$ downwind wind turbine that was installed on 11 June 2012 as the first grid-connected floating offshore wind turbine in Japan. The floating wind turbine, which consists of a hybrid-spar platform (precast pre-stress concrete and ring-stiffened steel) with three mooring chain, withstood two separated severe typhoons, Bolaven (international designation: 1215) and Sanba (international designation: 1216) with no damaged sustained (Ishida et al., 2013; Utsunomiya, Sato, Yoshida, Ookubo, \& Ishida, 2013). The second wind 
turbine, a 2 MW downwind type was installed on 18 October 2013. Both downwind turbines were developed and manufactured by Hitachi Ltd. Technical feasibility, met-ocean measurement and the surveys for the environmental impact assessment have been made through the demonstration project.

\subsubsection{Hitachi Ltd.}

Hitachi Ltd. has installed at least $152 \mathrm{MW}$ downwind wind turbines on fixed foundations (Hitachi Ltd, 2013). It has also been focused in developing a 5 MW downwind wind turbine for offshore environment (Saeki, Sano, Kato, Owada, \& Yoshida, 2014; Saeki, Tobinaga, Sugino, \& Shiraishi, 2014). Construction of a demonstration system began and the turbine was installed in March 2015 (Publicover, 2014). The company is also expected to supply 445 MW downwind wind turbines for the 220-MW Murakami offshore project (Foster, 2015).

\subsubsection{SWAY A/S}

The SWAY turbine developed by SWAY A/S is a three-bladed downwind floating wind turbine that utilizes a single tension leg spar platform. The whole wind turbine system, including the tower, rotates about the yaw mechanism located at the universal joint at the bottom of the tower. The wind turbine has individual blade-pitch control, and the nacelle is fixed to the tower at an optimal angle such that the rotor axis is oriented parallel to the wind when the tower pitches during operation. Some of the key components in the system are the unique spreader beams and tension cables, which help to stiffen the tower, reduce fatigue loads and allow the tower to carry a larger turbine (Borgen, 2007; SWAY AS, 2013). A 1:6.5 scale prototype was launched and tested off the coast of Norway in May 2012 (Koh et al., 2013). The full-scale SWAY wind turbine is designed to have a rated power of 2.5 to $10 \mathrm{MW}$, rotor diameter up to a $124 \mathrm{~m}$ diameter rotor and tower up to $210 \mathrm{~m}$ in length. 
Full details of the SWAY turbine is provided and discussed in Chapter 4 .

\subsubsection{Patents Related to Downwind Turbines}

To identify technological trends for downwind turbines, a patent search was done to highlight some of the design and development concepts. Table 2.3 shows a list of patents recently published related to downwind wind turbines that were retrieved (Adrian, 2012; Azegami, Hasegawa, Matsunobu, Sakamoto, \& Yanagibashi, 2012; Azegami, Sakamoto, Yanagibashi, \& Matsunobu, 2014; Borgen, 2007; Carter, 2001; De, Duffey, \& Picot, 2012; Drake, 2005; Edzard, 1999; Lauritsen, 2004; Lefranc, 2011; Liu, 2012; Loth \& Selig, 2014; Matsunobu, Saeki, Hasegawa, \& Kato, 2012; Nygaard \& Grorud, 2010; Sato, Asano, Utsunomiya, \& Yoshida, 2014; Scholte-Wassink, 2011; Siegfriedsen, 2007, 2013; Stoer, 2011; Weaver, 2014).

Table 2.3 List of patents related to downwind wind turbines

\begin{tabular}{|c|c|c|c|c|}
\hline Inventor & Patent Title & Patent No. & Year & Main Novelty/Claim \\
\hline H. Edzard & $\begin{array}{l}\text { Windkraftanlage } \\
\text { mit leeseitig } \\
\text { angeordnetem Rotor } \\
\text { (Wind turbine with } \\
\text { rotor downwind } \\
\text { arranged) }\end{array}$ & DE 29912737 & 1999 & $\begin{array}{l}\text { Crescent-shaped } \\
\text { curved blades that } \\
\text { reduce direct } \\
\text { interaction between } \\
\text { tower and blades } \\
\text { mast. Passive wind } \\
\text { tracking. }\end{array}$ \\
\hline J. W. Carter & $\begin{array}{l}\text { Wind-driven } \\
\text { electric generator } \\
\text { apparatus of the } \\
\text { downwind type with } \\
\text { flexible } \\
\text { changeable-pitch } \\
\text { blades }\end{array}$ & US 6327957 & 2001 & $\begin{array}{lr}\text { Wind-driven } & \text { yaw } \\
\text { system with } & \text { flexible } \\
\text { blades capable of } \\
\text { downwind } & \text { coning. } \\
\text { Airflow } & \text { responsive } \\
\text { device } & \text { inside } \\
\text { nacelle } & \text { that } \\
\text { controls } & \text { changing } \\
\text { the pitch of blades } \\
\text { depending on airflow } \\
\text { conditions. }\end{array}$ \\
\hline P. Lauritsen & $\begin{array}{l}\text { Floating offshore } \\
\text { wind power } \\
\text { installation }\end{array}$ & $\begin{array}{l}\text { US } \\
20040103655\end{array}$ & 2004 & $\begin{array}{l}\text { New floating } \\
\text { foundation concept } \\
\text { that allows at least } \\
\text { one downwind wind } \\
\text { turbine. }\end{array}$ \\
\hline D. G. Drake & $\begin{array}{l}\text { Downstream } \\
\text { turbine }\end{array}$ & US 6979175 & 2005 & $\begin{array}{l}\text { Downwind } \\
\text { turbine designed to } \\
\text { respond to high } \\
\text { winds and gyroscopic } \\
\text { precession, } \\
\text { allows for automatic } \\
\text { control of the rotor } \\
\text { speed. }\end{array}$ \\
\hline
\end{tabular}




\begin{tabular}{|c|c|c|c|c|}
\hline Inventor & Patent Title & Patent No. & Year & Main Novelty/Claim \\
\hline E. Borgen & \begin{tabular}{lrr} 
Device for a wind \\
power & \multicolumn{2}{c}{ station } \\
placed & in deep \\
water & &
\end{tabular} & US 7156037 & 2007 & $\begin{array}{l}\text { Innovative floating } \\
\text { tower and foundation } \\
\text { concept for downwind } \\
\text { wind turbine. }\end{array}$ \\
\hline $\begin{array}{l}\text { S. } \\
\text { Siegfriedsen }\end{array}$ & $\begin{array}{lc}\text { Wind } & \text { turbine } \\
\text { comprising } & \\
\text { elastically } & \\
\text { flexible } & \text { rotor } \\
\text { blades } & \end{array}$ & $\begin{array}{l}\text { US } \\
20070098555\end{array}$ & 2007 & $\begin{array}{l}\text { Downwind turbine } \\
\text { comprising } \\
\text { elastically flexible } \\
\text { rotor blades. }\end{array}$ \\
\hline $\begin{array}{ll}\text { T. } & \text { A. } \\
\text { Nygaard, } & \text { C. } \\
\text { Grorud } & \end{array}$ & $\begin{array}{lr}\text { Downwind } & \text { power } \\
\text { plant, and } & \text { a } \\
\text { method } & \text { for } \\
\text { operating } & \text { a } \\
\text { downwind } & \text { power } \\
\text { plant } & \end{array}$ & $\begin{array}{l}\text { US } \\
20100202881\end{array}$ & 2010 & $\begin{array}{lr}\text { Method } & \text { for } \\
\text { supporting } & \text { an } \\
\text { offshore downwind } \\
\text { power plant using } \\
\text { guy wires. }\end{array}$ \\
\hline R. D. Stoer & $\begin{array}{l}\text { Windkraftanlage } \\
\text { mit reduziertem } \\
\text { Turmschatteneffekt } \\
\text { (Wind turbine with } \\
\text { reduced tower } \\
\text { shadow effect) }\end{array}$ & $\begin{array}{l}\mathrm{DE} \\
102010009435\end{array}$ & 2011 & $\begin{array}{l}\text { New tower feature } \\
\text { with an orbiting } \\
\text { scroll design to } \\
\text { reduce tower shadow } \\
\text { effect. }\end{array}$ \\
\hline $\begin{array}{l}\text { H. Scholte- } \\
\text { Wassink }\end{array}$ & $\begin{array}{l}\text { Floating offshore } \\
\text { wind farm, a } \\
\text { floating offshore } \\
\text { wind turbine and a } \\
\text { method for } \\
\text { positioning a } \\
\text { floating offshore } \\
\text { wind turbine }\end{array}$ & $\begin{array}{l}\text { US } \\
20110074155\end{array}$ & 2011 & $\begin{array}{lr}\text { Method } & \text { for } \\
\text { positioning } & \text { a } \\
\text { floating } & \text { offshore } \\
\text { wind } & \text { turbine } \\
\text { suitable } & \text { for } \\
\text { downwind turbines }\end{array}$ \\
\hline M. Lefranc & $\begin{array}{l}\text { Floating wind } \\
\text { power apparatus }\end{array}$ & $\begin{array}{l}\text { US } \\
20110006539\end{array}$ & 2011 & $\begin{array}{l}\text { Design of a floating } \\
\text { unit that supports } \\
\text { at least } \text { three } \\
\text { rotors, with at } \\
\text { least one downwind } \\
\text { turbine. }\end{array}$ \\
\hline $\begin{array}{l}\text { K. Azegami, } \\
\text { T. Hasegawa, } \\
\text { T. } \\
\text { Matsunobu, } \\
\text { K. Sakamoto, } \\
\text { T. } \\
\text { Yanagibashi }\end{array}$ & $\begin{array}{lr}\text { Offshore } & \text { downwind } \\
\text { wind } & \text { turbine } \\
\text { system } & \end{array}$ & $\begin{array}{l}\text { WO } \\
2012105034\end{array}$ & 2012 & $\begin{array}{lr}\text { Method } & \text { of } \\
\text { installation } & \text { of } \\
\text { multiple } & \text { offshore } \\
\text { downwind } & \text { wind } \\
\text { turbines at } & \text { various } \\
\text { depths. } & \end{array}$ \\
\hline $\begin{array}{l}\text { T. } \\
\text { Matsunobu, } \\
\text { M. Saeki, T. } \\
\text { Hasegawa, H. } \\
\text { Kato }\end{array}$ & $\begin{array}{l}\text { Downwind type wind } \\
\text { turbine }\end{array}$ & $\begin{array}{l}\text { US } \\
20120057976\end{array}$ & 2012 & 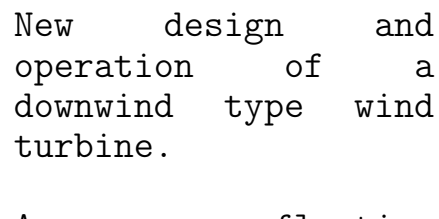 \\
\hline K. S. Liu & $\begin{array}{l}\text { Device of floating } \\
\text { wind turbine } \\
\text { capable of } \\
\text { counterbalancing } \\
\text { torques therein }\end{array}$ & $\begin{array}{l}\text { US } \\
20120269628\end{array}$ & 2012 & $\begin{array}{l}\text { A floating } \\
\text { foundation with two } \\
\text { turbines } \\
\text { counterbalances that } \\
\text { torques. }\end{array}$ \\
\hline $\begin{array}{l}\text { B. A. De, T. } \\
\text { Duffey, } \\
\text { Picot }\end{array}$ & $\begin{array}{l}\text { Downwind turbine } \\
\text { with free yaw } \\
\text { system }\end{array}$ & $\begin{array}{l}\text { WO } \\
2012123019\end{array}$ & 2012 & $\begin{array}{l}\text { New devices and } \\
\text { methods suitable for } \\
\text { optimizing free yaw } \\
\text { control of downwind }\end{array}$ \\
\hline
\end{tabular}




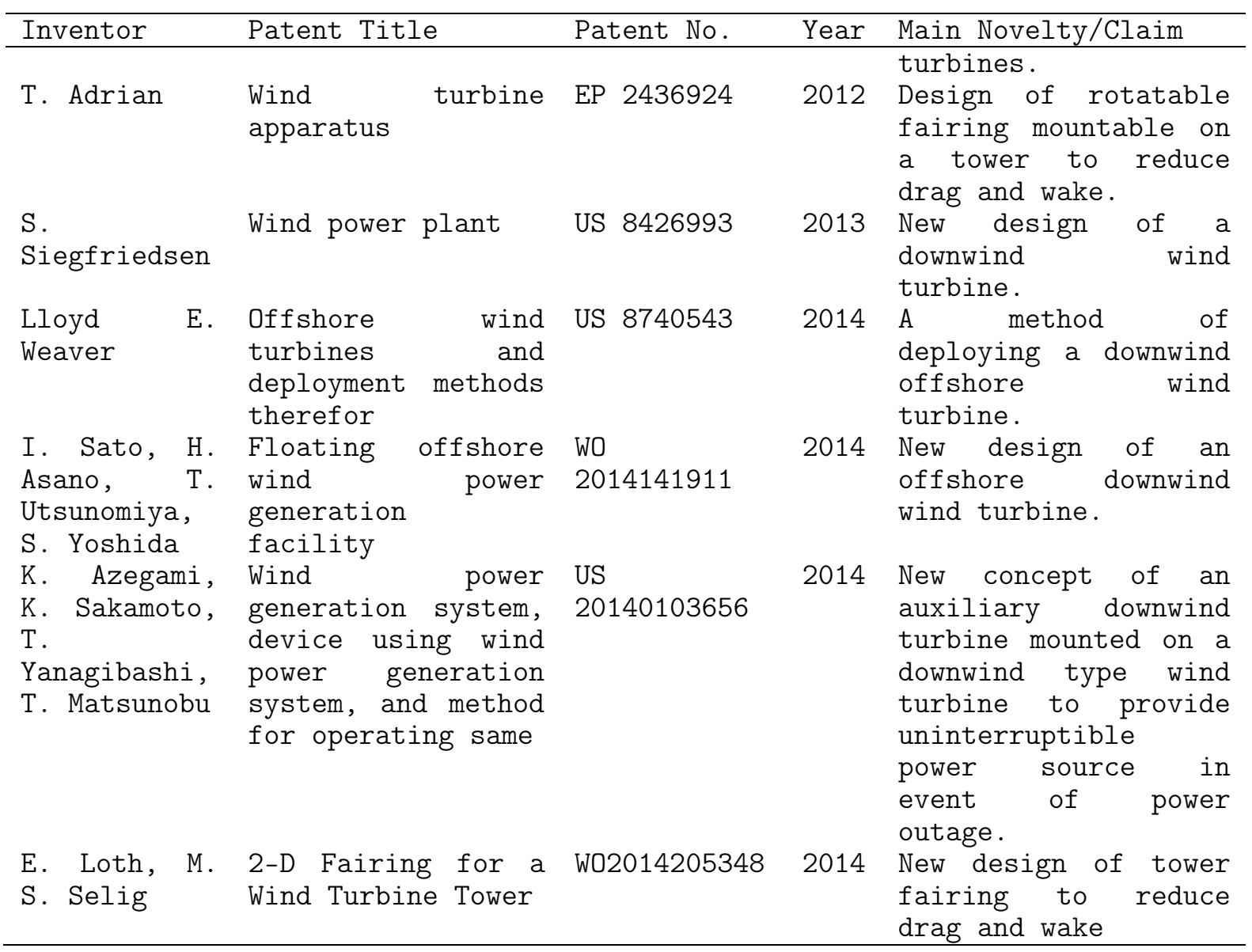

\subsection{Offshore Wind Turbine Numerical Simulation Tools}

Reliable and state-of-the-art simulation tools that are able to comprehensively model the loads and dynamics of offshore wind turbines are needed for designing the wind turbine system. Currently, there are a number of simulation codes developed by research institutes, universities and commercial entities that are capable of modeling offshore wind turbines. A list of codes used in the IEA Wind Offshore Code Comparison Collaboration Continuation (OC4) is shown in Table 2.4.

Table 2.4 List of offshore wind modeling tools used in OC4 (Robertson, Jonkman, Vorpahl, et al., 2014)

\begin{tabular}{ll}
\hline Code & Code Developer \\
\hline FAST & National Renewable Energy Laboratory (NREL) \\
FAST v8 & NREL \\
CHARM3D + FAST & Texas A\&M University + NREL \\
OPASS + FAST & Centro Nacional de Energias Renovables + NREL \\
University of Ulsan & University of Ulsan + NREL \\
+ FAST & \\
Bladed & DNV GL \\
Bladed 4.5 & DNV GL
\end{tabular}




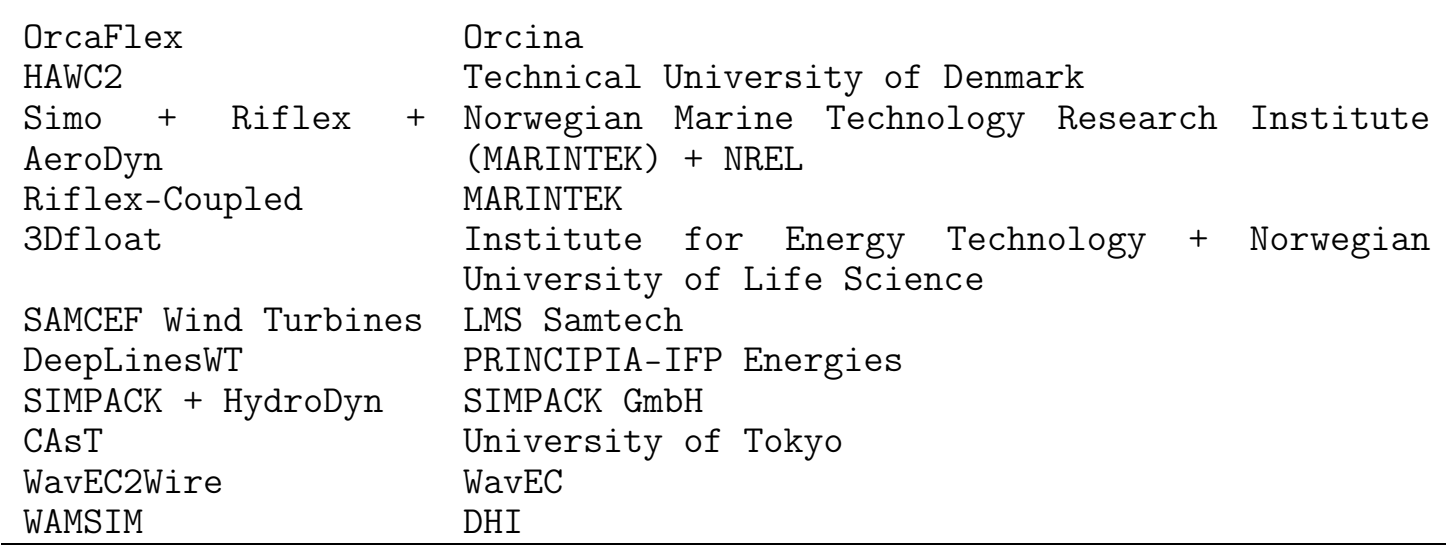

This section presents an overview of the current status of three different codes, with an emphasis on the FAST code which is used by the author. Two other commonly used codes, Automatic Dynamic Analysis of Mechanical Systems (ADAMS) and Bladed are discussed briefly. Detailed description of other simulation codes and analysis of strength and weaknesses is described in Cordle, Jonkman, and Hassan (2011), the OC3 (J. Jonkman \& Musial, 2010) and OC4 (Robertson, Jonkman, Musial, Popko, \& Vorpahl, 2014) reports.

This is followed by describing details on existing verification and validation work of current code-to-code and code-to-measurement comparison studies. Technical gaps in the usage of these simulation tools for downwind offshore wind turbines are presented at the end of the section.

\subsubsection{FAST 7 with AeroDyn and HydroDyn by NREL}

The FAST tool was originally developed through a subcontract between NREL and Oregon State University for dynamic analysis of conventional fixed-bottom wind turbines. In recent years, additional modules and capabilities for coupled dynamic analysis of floating wind turbines has been largely developed by J. Jonkman and Buhl (2007).

An overview of the FAST 7 modules is illustrated in Figure 2.5. 


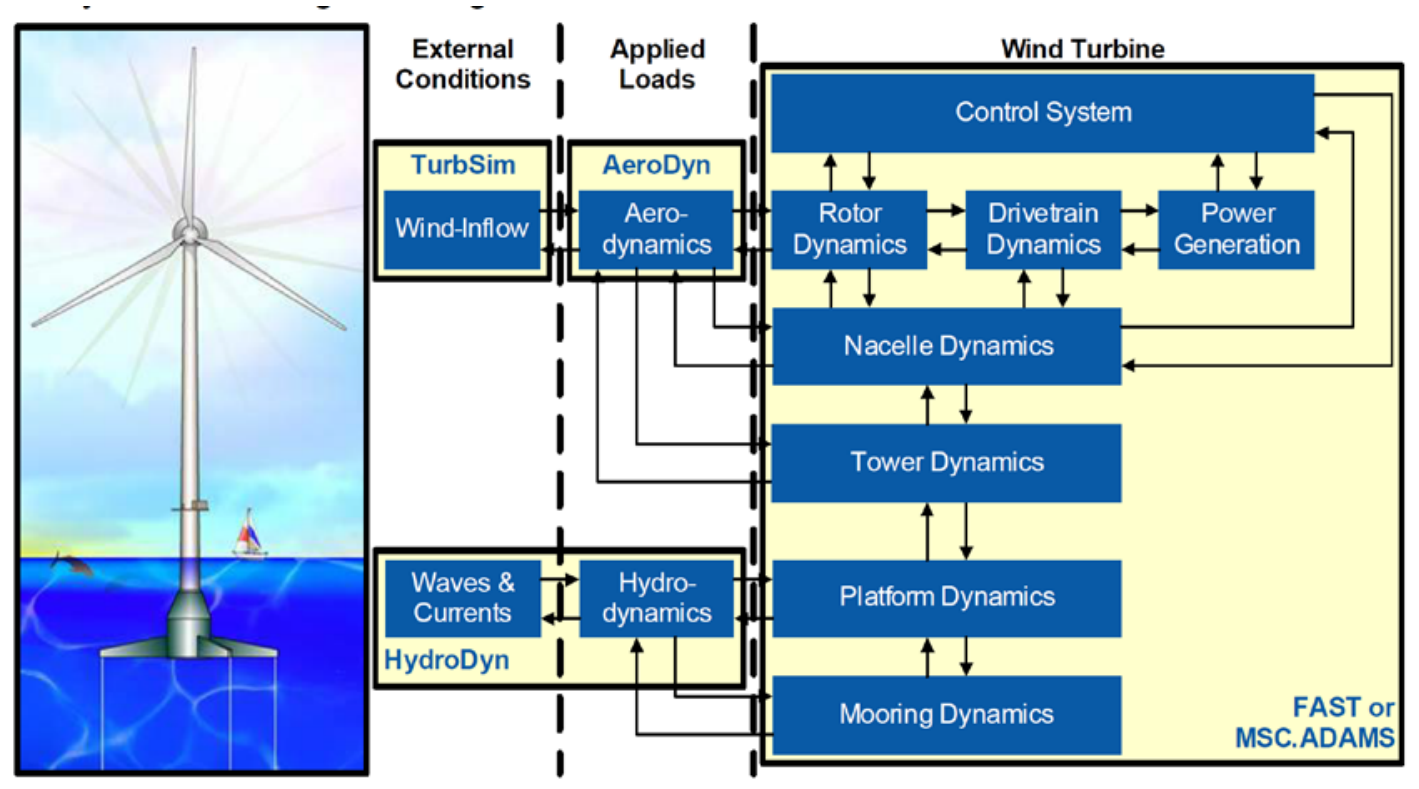

Figure 2.5 Overview of FAST Modules for Coupled Aero-hydro-servo-elastic Simulation (J. Jonkman \& Buhl, 2007)

A number of reasons led the author to use this simulation tool over others. One of the key advantages of the FAST code is that simulation tool and source code is publicly available, and is being used extensively by the research and industrial community. The support available is augmented by the collaboration work of the author with Dr. Jonkman during a research exchange at NREL (Section 4). In addition, the modules in FAST code are open-source, and can be manually coupled with a number of other dynamic analysis programs increasing the flexibility of customization and user development.

\subsubsection{Structural Dynamics}

This FAST version employs a combined modal and multi-body system (MBS) approach for modeling the structural dynamics of offshore wind systems. The blades and tower are modeled using a nonlinear beam model that retains only a few modes (assuming moderate deflections), with two flap-wise bending modes and one edge-wise bending mode per blade and two fore-aft and two side-to-side bending modes for the tower. A separate finite 
element method pre-processor BModes developed by Bir (2007) is used to compute the coupled mode shapes.

The tower is cantilevered on the floating platform, which has six degrees-offreedom (DOF) rigid-body motion, and the drivetrain is modeled using rigidbody rotation with a linear rotational spring and damper system. A detailed description of the theory, code and numerical solution techniques can be found in the FAST Theory Manual (J. Jonkman, 2014), FAST User Guide's (J. Jonkman \& Buhl, 2005) and National Wind Technology Center (NWTC) website.

\subsubsection{Aerodynamics}

AeroDyn is developed by Windward Engineering, which is further improved and maintained by NREL. AeroDyn is strip-theory-based horizontal-axis wind turbine aerodynamics analysis code that has the option of using either blade element momentum (BEM) theory (used in this study) or generalized dynamic wake theory. AeroDyn includes a model of unsteady airfoil aerodynamics, including dynamic stall. In the analysis, Prandtl's tip and hub loss corrections were applied.

In the publicly available version of FAST v7 and AeroDyn 13, the drag forces from the wind on the tower are not calculated.

Details of the AeroDyn module are discussed in the AeroDyn User's Guide (Laino \& Hansen, 2002), AeroDyn Theory Manual (Moriarty \& Hansen, 2005) and Addendum to the User's Guides for FAST, A2AD, and AeroDyn (B. J. Jonkman \& Jonkman, 2013).

\subsubsection{Hydrodynamics}

HydroDyn is a module coupled to FAST that computes the hydrodynamic equations as part of the hydro-elastic solution. The module was developed by J. Jonkman (2009) and full details can be found in his dissertation (J. Jonkman, 2007). The hydrodynamic loading accounts for (1) linear 
hydrostatic restoring, (2) nonlinear viscous drag from Morison's equation, (3) added mass and damping contributions from linear wave radiation (including free-surface memory effects), and (4) incident wave excitation from linear diffraction. Airy wave theory with free-surface corrections is used to calculate the wave kinematics for the module. Both regular and irregular waves can be introduced, with irregular waves defined using either a Joint North Sea Wave Observation Project (JONSWAP) spectrum, PiersonMoskowitz (PM) spectrum, or a user-defined wave spectrum. Some of the structures currently supported by HydroDyn include spar type, barge, TLP and multi-member bodies such as jacket and tripod structure.

To obtain the hydrodynamic parameters required by HydroDyn, radiation and diffraction problems are solved using the boundary integral equation method in the frequency domain for a platform of arbitrary shape using WAMIT (Wave Analysis at Massachusetts Institute of Technology), a program used for computing wave loads and dynamics of ships and offshore structures developed by researchers at Massachusetts Institute of Technology ("WAMIT User Manual Version 7.0," 1999).

\subsubsection{Mooring lines}

Within HydroDyn, a quasi-static mooring system module is implemented to solve the nonlinear mooring-line restoring forces. It is able to account for taut or catenary lines, the weight of the line in fluid, elastic stretching, and seabed friction. However, it does not account for line bending stiffness or hydrodynamic inertia and damping. Full details of the quasi-static mooring line module can be found in Jonkman's works (J. Jonkman, 2007), (J. Jonkman, 2009).

\subsubsection{FAST v8}

During the course of the thesis work by the author, FAST v8 was released by NREL in September 2014, improving the overall modularity of the software tool. As some of the features of FAST v7 have yet to be included 
in FAST v8, both tools are currently being used and supported by NREL. A brief outline on the differences on the software framework is described in Table 2.5. Further details can be found in the "Guide to Changes in FAST v8" (B. J. Jonkman \& Jonkman, 2016).

Table 2.5 FAST v7 and FAST v8

\begin{tabular}{|c|c|}
\hline FAST v7 & FAST v8 \\
\hline $\begin{array}{l}\text { - Limited range of modeling } \\
\text { fidelity }\end{array}$ & $\begin{array}{l}\text { - Framework allowing modules to be } \\
\text { exchanged }\end{array}$ \\
\hline $\begin{array}{l}\text { Development of new modules of } \\
\text { higher fidelity }\end{array}$ & $\begin{array}{l}\text { - Solution driven by structural } \\
\text { solver } \\
\text { - Separate module interface and } \\
\text { coupler }\end{array}$ \\
\hline $\begin{array}{l}\text { - Inability to isolate a given } \\
\text { model }\end{array}$ & $\begin{array}{llr}\text { - Modules that can be called by } \\
\text { separate driver programs } & \text { or } \\
\text { interfaced together to form a } \\
\text { coupled solution }\end{array}$ \\
\hline $\begin{array}{l}\text { - Dependent spatial discretization } \\
\text { and time steps across modules }\end{array}$ & $\begin{array}{l}\text { - Library of spatial elements and } \\
\text { mesh-to-mesh } \\
\text { - } \quad \text { Data } \\
\text { interpolation/extrapolation } \\
\text { time }\end{array}$ \\
\hline $\begin{array}{l}\text { - Inability to linearize } \text { all } \\
\text { system equations }\end{array}$ & $\begin{array}{l}\text { - Tight coupling with options for } \\
\text { operating-point determination and } \\
\text { linearization }\end{array}$ \\
\hline - Focus on single turbine & $\begin{array}{l}\text { - Dynamic allocation of modules } \\
\text { for wind-plant simulation }\end{array}$ \\
\hline $\begin{array}{l}\text { - "Spaghetti code" due to unclear } \\
\text { data transfer and global data }\end{array}$ & $\begin{array}{l}\text { - Modularization } \quad \text { with } \\
\text { encapsulation }\end{array}$ \\
\hline $\begin{array}{l}\text { - Limited number of developers due } \\
\text { to code size \& complexity }\end{array}$ & $\begin{array}{l}\text { - Modularization of code into } \\
\text { separate } \\
\text { - Programmer's handbook explaining } \\
\text { code development requirements and } \\
\text { best practices }\end{array}$ \\
\hline $\begin{array}{l}\text { - } \quad \text { Potentially poor } \\
\text { accuracy and stability }\end{array}$ & $\begin{array}{l}\text { - Multiple coupling schemes and } \\
\text { integration/solver options }\end{array}$ \\
\hline
\end{tabular}

\subsubsection{Other Existing Design Codes}

\subsubsection{ADAMS by MSC Software Corporation}

ADAMS (Automatic Dynamic Analysis of Mechanical Systems) is a commercial code used for general-purpose MBS dynamics analysis developed by MacNeal-Schwendler Corporation (MSC) Software Corporation (Cordle et al., 2011). Models of wind turbines for ADAMS can be generated from FAST code (Laino \& Hansen, 2001). 
The ADAMS code uses an MBS representation which allows for higher number of structural configurations and DOF as compared with FAST. The blades, tower and drivetrain can be modeled as flexible components consisting of a series of rigid bodies with mass and inertia attached by flexible joints with linear stiffness and damping. Additional features includes modeling torsional DOF in the blades and tower, flap/twist coupling in the blades, mass offsets in the blades and tower, and pitch actuator dynamics. The ADAMS code interfaces with AeroDyn, HydroDyn and mooring line module in FAST to calculate aerodynamic forces, hydrodynamic forces and mooring line tensions respectively.

\subsubsection{Bladed by DNV GL}

Another software commonly used in the industry is the DNV GL Bladed originally developed by Germanischer Lloyd (GL) Garrad Hassan (Garrad Hassan \& Partners Ltd., 2013). Similar to FAST, structural dynamics are calculated using MBS and modal representation. Modal properties of blade and towers are computed using a finite element method representation of the body as a Timoshenko beam. The modes are coupled to equations of motions and a Craig-Bampton style modes is used to reduce the DOFs in the support structure. The support structure can be modeled with large rotations and displacement as it is connected a reference frame by a free joint and constrained by mooring-line forces (Cordle et al., 2011).

Similar to AeroDyn in FAST, the aerodynamics forces are calculated using BEM theory with corrections or dynamic wake model. Dynamic stall is also accounted for using the Beddoes-Leishman model. Hydrodynamic forces are calculated in Bladed using Morison's equation. Linear wave kinematics is calculated using Airy wave theory with free-surface corrections and irregular sea state implemented using a JONSWAP spectrum or a user-defined waveenergy spectrum, similar to HydroDyn in FAST. In addition, Bladed is able to calculate nonlinear waves kinematics using stream-function theory. 
Mooring line forces are modeled in non-linear relationships between the displacement of the platform and the restoring force from the mooring line. However, these relationships are calculated and implemented separately by the user with a stiffness matrix.

\subsubsection{Existing Verification and Validation Work}

Presently, efforts are primarily focused on verifying these tools through code-to-code comparisons. Code-to-code comparisons can only identify differences between codes developed by different software tools but are unable to determine if the results are accurate in comparison to experiment or actual conditions. Therefore, there is a logical shift in research focus to perform code-to-measurement comparisons, with many difficulties due to the limited availability and access to measurement data. This remaining section discusses some of the reputable and notable code-to-code and code-to-measurement work conducted. 


\subsubsection{Code-to-Code Comparison}

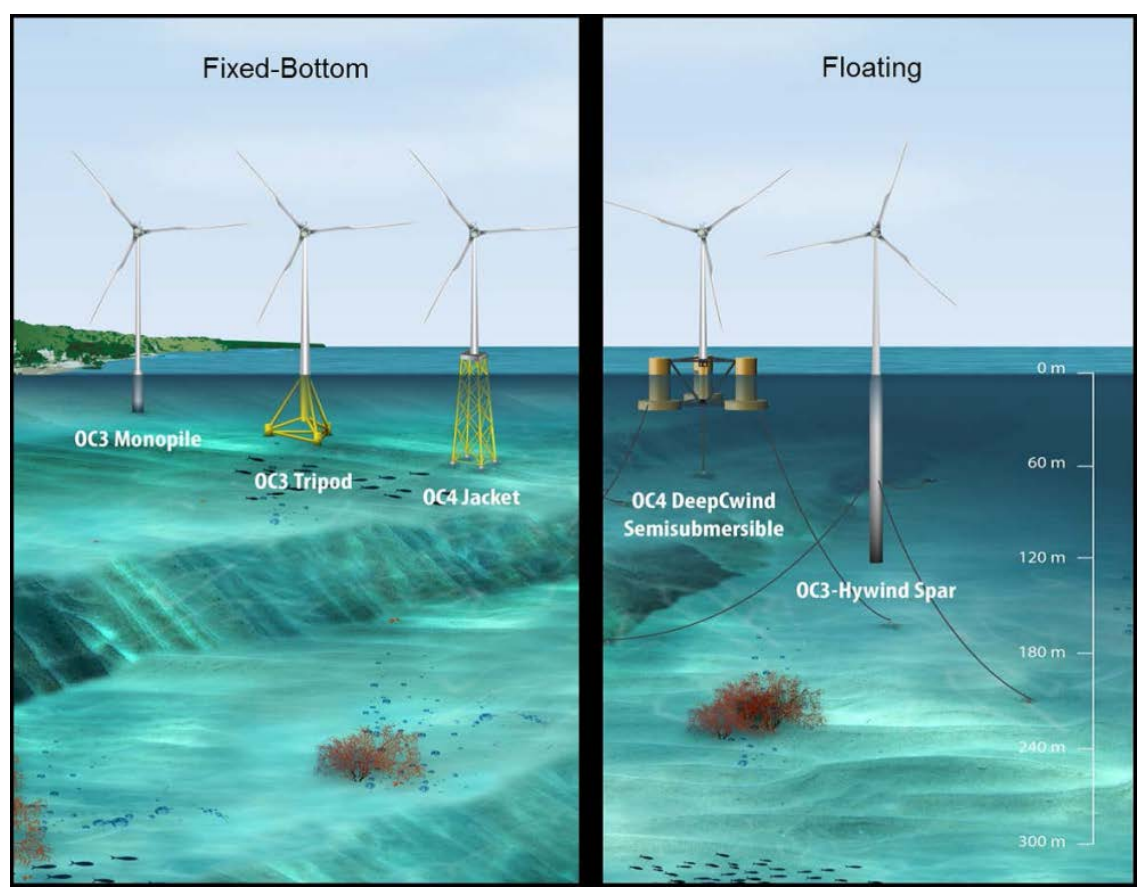

Figure 2.6 OC3 and OC4 offshore wind system designs modeled (Robertson, Jonkman, Musial, Vorpahl, \& Popko, 2013)

Offshore Code Comparison Collaboration (OC3) for IEA Task 23

The OC3 project, which operates under Subtask 2 of the IEA Wind Task 23, was established to test newly developed codes. The project was conducted from 2004 to 2009 through a technical exchange of international participants from universities, research institutions, and industry across the United States of America, Germany, Denmark, the United Kingdom, Spain, the Netherlands, Norway, Sweden, and Korea (J. Jonkman \& Musial, 2010). Most of the aero-hydro-servo-elastic codes developed then were tested within OC3 project.

Using the NREL offshore 5-MW baseline wind turbine, the task was split into four different phases. Phase I tested a fixed monopile with a rigid foundation in $20 \mathrm{~m}$ water depth, Phase II introduced flexibility to the foundation to represent soil-pile interactions, Phase III tested a fixed tripod substructure at a water depth of $45 \mathrm{~m}$ and Phase IV tested floating sparbuoy in deep water with depth $320 \mathrm{~m}$ (Figure 2.6). 
With the codes at its early development phase in the past, such verification work led to huge improvements in model accuracy and limitations. The comparisons and lessons learnt helped identify weakness in model and areas of improvements. Results were crucial as advancement in offshore wind industry depended heavily on the accuracy of the codes. While comparisons in all phases have generally agreed very well, details for the comparison, differences and findings can be found in the full extensive report by J. Jonkman and Musial (2010).

\section{Offshore Code Comparison Collaboration Continuation (OC4) for}

\section{IEA Task 30}

The OC4 project was formed under the IEA Wind Task 30 in 2010 focused in continuing the work done in OC3 project. There have also been additional participants whom each have their expertise and contribution to the projects. Phase I investigates wind turbine coupled simulations with a fixed jacket support structure while Phase II investigates a floating semisubmersible platform. The analysis is of much interest due to the lack of research in the local vibration phenomena experienced by jacket support structure and the complex hydrodynamics of the semisubmersible platform.

In Phase I, many participants have been able to verify their codes and methodologies developed for a jacket support, which was particularly beneficial to newly developed tools such as the 3DFloat by Norwegian University of Life Sciences and OneWind by Fraunhofer Institute for Wind Energy and Energy System Technology. Extensive results of Phase I of the OC4 project is published in the paper by Popko et al. (2012); Popko et al. (2014) and Robertson, Jonkman, Musial, et al. (2014).

In Phase II, various simulation codes were used by the participants to model the coupled dynamic response of a 5-MW wind turbine installed on a floating semisubmersible in $200 \mathrm{~m}$ water depth. A total of 21 different load cases were modeled, and the differences in modeling approaches were evident 
in the simulation results. This helped to draw conclusions to the importance of mooring dynamics to the mooring loads, non-linear drift forces, and difference between global (at the platform level) and local (at the member level) hydrodynamic modeling of viscous drag of the substructure. Similar to OC3, the process helped to improve simulation codes and standard of offshore wind turbine modeling. Extensive results of Phase II of the OC4 project is published in the paper by Robertson, Jonkman, Musial, et al. (2014); Robertson et al. (2013); Robertson, Jonkman, Vorpahl, et al. (2014)

\subsubsection{Code-to-Measurement Comparison}

\section{Laboratory Scale Testing}

In a laboratory test conducted at Maritime Research Institute Netherlands (MARIN) led by the DeepCwind consortium, a $1 / 50^{\text {th }}$-scale model of the NREL 5-MW reference wind turbine was mounted on a spar, TLP and semisubmersible floating platform (Browning \& Goupee, 2012; Coulling, Goupee, Robertson, Jonkman, \& Dagher, 2013; Goupee, Koo, Kimball, Lambrakos, \& Dagher, 2014; Koo, Goupee, Kimball, \& Lambrakos, 2014; Prowell, Stewart, \& Goupee, 2013; Stewart, Lackner, \& Goupee, 2012). The experimental results were scaled up and compared with the full-scale FAST model, with focus on the global motions, wind excitation and damping effects, nacelle acceleration, tower loads and mooring loads.

The approach for the studies was to conduct a hammer test to obtain the structural natural frequency, followed by a free decay test to calibrate the model to account for differences in the mooring systems and nonlinear viscous damping. The calibrated model was then subjected to the test conditions. In general, the responses compared well between the experiment and the simulation, especially for wind-only loadings. For wave-only loadings, the mean drift and second-order difference-frequency responses present in test data are not simulated by FAST, resulting in differences in the simulation and test results. The combined wind and wave cases showed 
a fairly good agreement between the simulation and test data due to FAST's strong ability to model the aerodynamic loadings. However, there appears to be significant discrepancies in damping behavior between the experiment and the FAST simulation. Differences between each floating platform are also noted in details in the papers.

A number of combined wind and wave laboratory tests of floating wind turbine systems have also been conducted by separate research organizations. Ren, Li, and Ou (2012) tested a $1 / 60^{\text {th }}$-scale TLP based on a 5-MW wind turbine system at a joint wind tunnel and wave flume laboratory at Harbin Institute of Technology in 2012. Tomasicchio et al. (2014) tested a $1 / 40^{\text {th }}$-scale TLP and spar buoy based on a 5-MW wind turbine system at the Danish Hydraulic Institute off-shore wave basin within the European Union-Hydralab IV Integrated Infrastructure Initiative. Tang, Song, and Wang (2015) tested a $1 / 40^{\text {th }}$-scale semi-submersible system based on a 1.5 MW wind turbine system in a wind/wave tank at Tianjing University.

\section{Large/Full Scale Testing}

Almost all published studies to date on code-to-measurement validation work are conducted in a controlled laboratory setup. This is due to the limited testing in open-sea and availability of data to large/full-scale tests. Several experimental floating substructures of floating offshore wind turbine have or are currently being tested in open-sea: Statoil Hywind (Spar), SWAY (Spar), Blue H (TLP), Poseidon (Semisubmersible) and Principle Power WindFloat in Europe, Fukushima FORWARD (Semisubmersible), Goto Floating Offshore Wind Turbine Demonstration Project (Spar) and WindLens Floater (Semisubmersible) in Japan and DeepCwind (Semisubmersible) floating turbine in the US. Other than the SWAY testing (presented in this thesis) and the Hywind testing (presented after the author's contribution in described in Section 4), the remaining test projects 
did not produce any publicly available documentations regarding the technical details and data of the test results.

Results of the Statoil Hywind testing and numerical simulation comparison have only recently been published by Skaare et al. (2014) and Driscoll et al. (2016). Skaare et al. (2014) modeled the system using SIMO/RIFLEX/HAWC2 analysis tool and compared for one case each for below and above rated wind speed of the wind turbine. The global motions, tower bending moments, mooring line tensions, power production, rotor speed and blade pitch angle comparison between the measured and simulated responses showed good agreement. It was concluded that the analysis tool is suitable for confirming designs which have similar characteristics as the Hywind concept. Driscoll et al. (2016) modeled the system using FAST analysis tool and compared 8 time series at operating conditions with varying mean wind speeds, wave and current conditions. Good agreement was also found between measured \& simulated responses, and the project is still progressing to improve the blade and mooring models, and analysis of additional test cases.

\section{Offshore Code Comparison Collaboration Continuation, with} Correlation (OC5) project

As described above, code-to-code comparisons can only help to identify differences in codes but are unable to conclude if the solution is accurate when compared to real systems. The Offshore Code Comparison Collaboration Continuation, with Correlation (OC5) project (Robertson, 2015) is 4-year project initiated by IEA Wind Research Task 30 in 2014 with the aims to validated the tools through comparison with actual measurements of physical models. The project is planned for three different fixed and floating structures, (1) monopile (tank testing), (2) semisubmersible (tank testing), (3) jacket/tripod (open sea testing). Results for Phase I of the project has recently been published by (Robertson et al., 2015), highlighting the importance of higher-order theory in modeling of the 
hydrodynamic loadings. Further work is planned for more analysis of the monopole, including CFD simulations.

\subsubsection{Technical Challenges and Gaps}

As observed in Section 2.4.3, validation of the tools has been hampered by the scarcity of experimental data for floating offshore wind turbines with which to compare simulated results. Calibration and validation work on models with open-water test data will be valuable to the development of aero-hydro-servo-elastic numerical tools as such tests are performed in realistic environmental conditions. In addition, larger-than-laboratory-scale testing will also yield results with less scaling issues as it is able to show the complete overall system characteristic behavior more accurately.

The offshore wind systems used in these comparison studies in Section 2.4.3 considered fixed and floating wind turbines with upwind configuration. While the comparison between these codes led to huge improvements to modeling capabilities in upwind offshore turbines, no comparison models have yet to be suggested for downwind offshore turbines. This limits the development of the modeling capabilities for downwind turbines, especially in the accuracy in representing the tower shadow and nacelle effect on the wind turbine blades.

\subsection{Chapter Conclusion}

The design conservatism observed in the current offshore wind turbines meant that there is opportunity in improving the design of wind turbines specifically for offshore conditions. Implementing a downwind configuration for offshore wind turbine may prove to be decisive in lowering the cost of energy. With the trend of increasing size of wind turbines, technical challenges such as higher loads on the structure, rotor, and gearbox, and blade to tower clearance issue may be mitigated with a downwind rotor. A downwind configuration also provides new prospects in tower and blade design, such as flexible blades, high speed rotors, tower fairing and fullheight lattice towers. Direct cost reduction can be achieved with the 
removal of active yaw system if enough confidence is gained in the yawing the system with individual pitch control.

In the past decade, increasing number of wind turbine manufacturing companies and government-supported initiatives has also looked at the usage of downwind turbines in the offshore environment. 2-B Energy has received financial support to build and test their two-bladed downwind turbines, while SWAY A/S has tested their prototype floating spar-type turbine in Norway. Companies such as Aerodyn Energiesysteme GmbH, Ming Yang Wind Power, and Hitachi Ltd. has built and tested full-sized downwind offshore turbines that are connected to the grid. Governmentsupported projects such as the Fukushima FORWARD and Goto Floating Offshore Wind Turbine Demonstration Project that were initiated to study feasibility of floating wind turbines have also tested full-scale downwind systems.

Technical challenges and gaps for offshore wind turbine numerical simulation tools have also been highlighted. Tower shadow effect on the blades for large downwind turbines can be studied in further details. Accuracy in representing the tower shadow and nacelle effect can be improved in wind turbine simulation codes. Tackling these challenges and research gap will eventually increase the confidence and adoption of downwind offshore wind turbines to the market. 


\section{TECHNICAL BACKGROUND}

\subsection{Tower Influence Models}

One of the main challenges for a downwind rotor is the tower shadow effect, whereby a mean velocity deficit and turbulent unsteady vortices behind the tower causes an impulsive loading on the blade when it passes behind the tower (Glasgow et al., 1981). Experiment tests have been used to understand the tower shadow effects (Brown, Graham, \& Giannakidis, 2000; Graham \& Brown, 1999; Munduate, Coton, \& Galbraith, 2004; Powles, 1983; Reiso, Muskulus, \& Moe, 2011; Wilmshurst et al., 1985) but are commonly faced with issues such as scaling effects and achieving comparable Reynolds numbers $(R e)$, especially for larger wind turbines. High fidelity models using computational fluid dynamics have been used to compare rotor-tower interactions for upwind and downwind rotor (Brown et al., 2000; Janajreh, Qudaih, Talab, \& Ghenai, 2010; Janajreh, Talab, et al., 2010; Zahle, Sorensen, \& Johansen, 2009; Zhao, Sheng, \& Afjeh, 2014). These simulations are computationally expensive and not practical for a wind turbine designer to use for analysis on different design load cases.

To represent both the effects of tower shadow (affecting downwind rotors) and tower dam (affecting upwind rotors) effects, wind turbine simulation tools use empirical models that involve relatively simple potential flow and empirical equations that result in wind velocity adjustments. The basic equations are shown below:

$$
\begin{aligned}
& V_{x}(x, y)=\alpha V_{x, \infty}(x, y) \\
& V_{y}(x, y)=\beta V_{y, \infty}(x, y)
\end{aligned}
$$

where $\alpha$ and $\beta$ are the adjustment fraction that is typically a function of different parameters, $V_{x}(x, y)$ and $V_{y}(x, y)$ are the $x$ - and $y$-components of the adjusted wind speed, $V_{x, \infty}(x, y)$ and $V_{y, \infty}(x, y)$ are the $x$ - and $y$ - 
components of the free stream wind speed. The reference frame can be referred to in Figure 3.1.

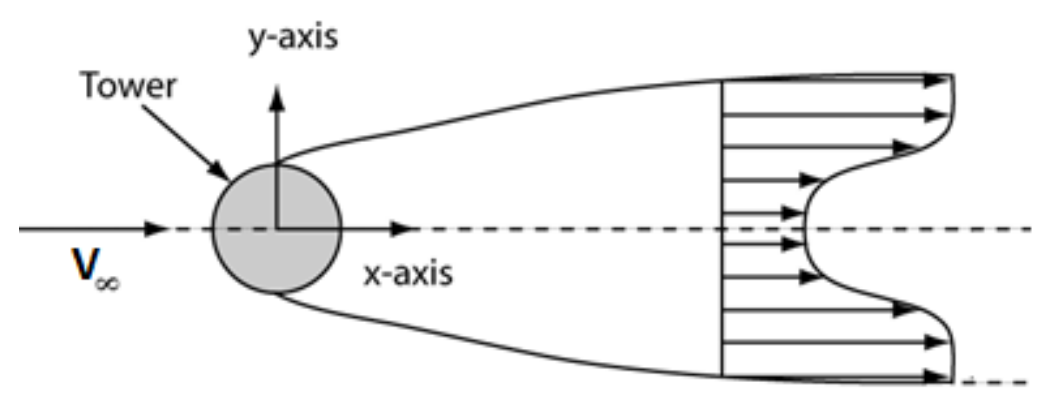

Figure 3.1 Reference frame for tower dam and shadow model

These models generally describe a Gaussian or cosine square shaped profile for the mean velocity deficit. These models are developed from two different engineering fields. Models developed by the wind energy and engineering field include those developed by Powles (Powles, 1983) (implemented in Bladed by DNV GL and FAST by NREL), Blevins (Blevins, 2001). Models based on fluid dynamics field include those developed by Schlichting and Gertsen (Schlichting \& Gersten, 2000), and Madsen et.al (Madsen et al., 2007) (implemented in HAWC2 (Horizontal Axis Wind turbine simulation Code 2nd generation) by Technical University of Denmark).

Input parameters in the models may include wake width, drag coefficient of tower and velocity. When implemented, these models are combined with wind inflow conditions that can be steady or turbulent in nature. Such an approach use is necessary as it is less computationally expensive compared to the high fidelity models such as CFD simulations.

These models are capable of representing the time-averaged velocity field, but the input parameters may need to be determined separately, and the time-dependent turbulence and vortex shedding phenomenon caused by the tower geometry are not accounted for. The models prescribed below are applicable only for a single cylindrical structure. 


\subsubsection{Tower Dam Model}

For simulation tools such as FAST, Bladed and HAWC2, tower dam effect is commonly represented by potential flow field. The general equation can be represented by:

$$
\begin{gathered}
V_{x}=V_{0}\left(1-\frac{x_{r}^{2}-y_{r}^{2}}{\left(x_{r}^{2}+y_{r}^{2}\right)^{2}}\left(\frac{D}{2}\right)^{2}\right) \\
u=1-\frac{x_{r}^{2}-y_{r}^{2}}{\left(x_{r}^{2}+y_{r}^{2}\right)^{2}} \\
V_{y}=V_{0} \frac{-2 x_{r} y_{r}}{\left(x_{r}^{2}+y_{r}^{2}\right)^{2}}\left(\frac{D}{2}\right)^{2} \\
v=\frac{-2 x_{r} y_{r}}{\left(x_{r}^{2}+y_{r}^{2}\right)^{2}}
\end{gathered}
$$

where $V_{0}$ is the free stream wind velocity, $D$ is the tower diameter $x_{r}$ and $y_{r}$ are the longitudinal and transversal position normalized by tower radius, $V_{x}$ and $V_{y}$ are the $\mathrm{x}$ - and $\mathrm{y}$ - velocity, $u$ and $v$ are the $x$-and $y$-velocity normalized by the free stream wind velocity.

In FAST, users are allowed to use a modified potential flow model by Bak, Madsen, and Johansen (2001) takes into account the effect of the drag of the cylinder.

$$
\begin{gathered}
V_{x}=V_{0}\left(1-\frac{x_{c}^{2}-y_{r}^{2}}{\left(x_{c}^{2}+y_{r}^{2}\right)^{2}}\left(\frac{D}{2}\right)^{2}+\frac{C_{d}}{2 \pi} \frac{x_{c}}{x_{c}^{2}+y_{r}^{2}}\left(\frac{D}{2}\right)\right) \\
u=1-\frac{x_{r}^{2}-y_{r}^{2}}{\left(x_{r}^{2}+y_{r}^{2}\right)^{2}}+\frac{C_{d}}{2 \pi} \frac{x_{c}}{x_{c}^{2}+y_{r}^{2}} \\
V_{y}=V_{0}\left(\frac{-2 x_{r} y_{r}}{\left(x_{r}^{2}+y_{r}^{2}\right)^{2}}\left(\frac{D}{2}\right)^{2}+\frac{C_{d}}{2 \pi} \frac{y_{r}}{x_{c}^{2}+y_{r}^{2}}\left(\frac{D}{2}\right)\right)
\end{gathered}
$$




$$
v=\frac{-2 x_{r} y_{r}}{\left(x_{r}^{2}+y_{r}^{2}\right)^{2}}+\frac{C_{d}}{2 \pi} \frac{y_{r}}{x_{c}^{2}+y_{r}^{2}}
$$

where $C_{d}$ is the tower drag coefficient, and $x_{c}=x_{r}+x_{\text {offset }}$ where $x_{\text {offset }}$ is an offset constant that can be set by the user. Bak et al. (2001) determined $x_{\text {offset }}=0.1$ for a best fit to Parkinson and Jandali (1970) works that made use of conformal mapping.

\subsubsection{Tower Shadow Model}

There are two main approaches taken to account for the wake behind the cylinder: models developed by the wind energy and engineering field, and models based on fluid dynamics field.

\subsubsection{Wind Energy and Engineering Approach}

In the wake of the tower $(x>0)$ where $y<1$ (this means that the wake has a constant width of the tower diameter D), Bak et al. (2001) defined the formulation as:

$$
\begin{gathered}
u=1-\frac{x_{r}^{2}-y_{r}^{2}}{\left(x_{r}^{2}+y_{r}^{2}\right)^{2}}+\frac{C_{d}}{2 \pi} \frac{x_{c}}{x_{c}^{2}+y_{r}^{2}}-4 \sqrt{1-y_{r}^{2}} \\
v=\frac{-2 x_{r} y_{r}}{\left(x_{r}^{2}+y_{r}^{2}\right)^{2}}+\frac{C_{d}}{2 \pi} \frac{y_{r}}{x_{c}^{2}+y_{r}^{2}}
\end{gathered}
$$

The assumption that the wake width does not increase with downwind position is not realistic and this is rarely used in simulations (Note: Bladed does allow users to choose a constant width wake). Therefore, the most popular approach is account for the tower shadow effect is to implement a velocity deficit model based on the work of Powles (1983).

The Powles (1983) model defines the velocity deficit using a cosine square function:

$$
v_{d}=\Delta \cos ^{2}\left(\frac{\pi y_{r}}{2 w}\right)
$$


where $v_{d}$ is the fractional decrease in wind velocity behind the tower, $\Delta$ is the velocity deficit at the wake center normalized with the free stream wind velocity and $w$ is the wake width parameter normalized to tower radius, where $w=\frac{W}{(D / 2)} \cdot \Delta$ and $w$ is further defined as:

$$
\begin{gathered}
\Delta=\Delta_{r e f} \sqrt{\frac{x_{r}}{x_{r e f}}} \\
w=w_{r e f} \sqrt{\frac{x_{r e f}}{x_{r}}}
\end{gathered}
$$

where $\Delta_{r e f}$ and $w_{r e f}$ are reference parameters for velocity deficit and wake width defined by the users at a fixed reference length $x_{\text {ref }}$ at a downwind distance from the yaw axis normalized to the tower radius.

The Powles (1983) model is implemented with slight variations for different wind turbine simulation codes.

In FAST, the Powles (1983) model is modeled in two different ways. The first model used in FAST (termed as FAST-old) developed from the equations described in the AeroDyn Theory Manual 2005 (Moriarty \& Hansen, 2005):

$$
\begin{gathered}
V_{d, F A S T-\text { old }}=\left\{\begin{array}{cc}
\Delta \cos ^{2}\left(\frac{\pi y_{r}}{2 w}\right) & |y| \leq \frac{w}{2} \& x_{r}>0 \\
0 & |y|>\frac{w}{2} \text { or } x_{r} \leq 0
\end{array}\right. \\
V_{x}=V_{x, \infty}\left(1-v_{d}\right) \\
V_{y}=V_{\mathrm{y}, \infty}\left(1-v_{d}\right)
\end{gathered}
$$

where $V_{x}$ and $V_{y}$ are the $x$ - and $y$-components of the wind speed reduced by the deficit fraction, $V_{x, \infty}$ and $V_{y, \infty}$ are the $x$ - and $y$-components of the free stream wind speed. The wake is symmetric about its centerline and it is 
assumed that there is no effect on the vertical component of wind speed. Users are required to define $\Delta_{\text {ref }}, w_{\text {ref }}$ and $x_{\text {ref }}$ parameters. This model cannot be combined with the tower dam model described earlier.

The more recently updated model in FAST (termed as FAST-new) is obtained directly from the source code of AeroDyn 14:

$$
V_{d, F A S T-n e w}=\left\{\begin{array}{cc}
\frac{C_{d}}{\sqrt{d}} \cos ^{2}\left(\frac{\pi y_{r}}{2 \sqrt{d}}\right) & \left|y_{r}\right| \leq \sqrt{d} \\
0 & \left|y_{r}\right|>\sqrt{d}
\end{array}\right.
$$

where $d$ is the radial distance from the yaw axis to the point of interest normalized by tower radius $\left(\sqrt{x_{r}^{2}+y_{r}^{2}}\right)$. This approach accounts for the velocity increase on the sides of the tower through drag coefficient term $C_{d}$. The velocity deficit term is also determined by $\frac{C_{d}}{\sqrt{d}}$.

$$
\begin{gathered}
V_{x}=\left\{\begin{array}{cc}
V_{x, \infty}\left(1-v_{d}\right) & x>0 \\
V_{x, \infty}(u) & x \leq 0
\end{array}\right. \\
V_{y}=V_{y, \infty}(v)
\end{gathered}
$$

When used in combination with the tower dam model, the FAST-new model replaces the $x$-direction wind speed when $x_{r}>0$ while the potential flow model is used for all $y$-direction wind speed and for $x$-direction wind speed when $x_{r} \leq 0$. Users are required to define $C_{d}$ and $x_{\text {offset }}$ parameters.

In Bladed 4.5, the tower shadow model is exactly similar to FAST-old. However, it is implemented differently with the tower dam model. 


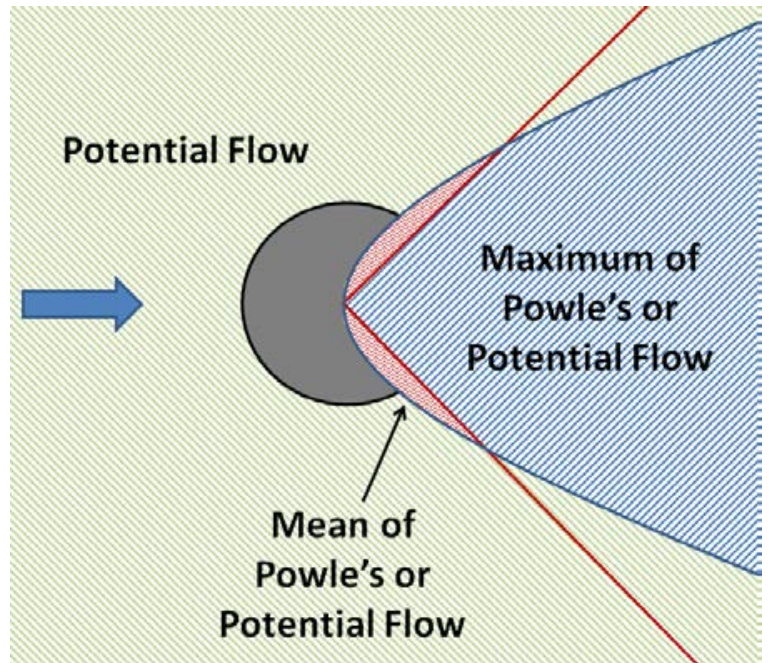

Figure 3.2 Bladed combined model regions (Garrad Hassan \& Partners Ltd., 2013)

From the wind inflow through forward of the $45^{\circ}$ line, an average of the potential flow model and Powles (1983) model is used whenever the Powles (1983) model is in effect, otherwise only the potential flow model is used. For region rearwards of the $45^{\circ}$ line, whichever method that results in the largest perturbation is used. The combined model is in effect for both $x$ direction and wind speed and $y$-direction wind speed. Users are required to define $\Delta_{\text {ref }}, w_{\text {ref }}, x_{\text {ref }}$ and $x_{\text {offset }}$ parameters.

\subsubsection{Fluid Dynamics Approach}

Blevins (2001) introduced a decaying Gaussian function to describe the wake behind a cylinder. While both Powles (1983) and Blevins (2001) models have some similarities in using the square-root law to describe the wake, the key difference is that Blevins (2001) introduced a parameter $x_{b}$ that represents the upstream location of the virtual origin of the wake. The model is defined as:

$$
\begin{gathered}
b_{1}=0.23 \sqrt{C_{d} D\left(x+x_{b}\right)} \\
b_{2}=1.02 V_{o} \sqrt{\frac{C_{d} D}{x+x_{b}}}
\end{gathered}
$$




$$
V(x, y)=V_{o}\left(1-b_{2} \exp \left(-0.69 y^{2} / b_{1}^{2}\right)\right)
$$

where $x$ and $y$ are the longitudinal and transversal position normalized by tower diameter, half-width $b_{1}$ is the distance from the centerline to the position where half the centerline velocity deficit is obtained. Users are required to define $C_{d}$ and $x_{b}$.

Another wake model that originates from boundary layer theory was suggested by Schlichting and Gersten (2000). The model was originally based on interior flow over a flat plate under the influence of friction.

$$
V(X, Y)=V_{o}\left(\frac{C_{d}}{4 \sqrt{\pi}} \sqrt{\frac{V_{0} D}{\varepsilon_{o}}}\left(\frac{X}{D}\right)^{-\frac{1}{2}} \exp \left(-\frac{Y^{2} V_{o}}{4 X \varepsilon_{o}}\right)\right), X>3 D
$$

where $X$ and $Y$ are the dimensional longitudinal and transversal positions and $\varepsilon_{o}$ is the virtual or eddy kinematic viscosity of the fluid. However, this model is only valid for $X>3 D$. Users are required to input $C_{d}$ and $\varepsilon_{o}$

In HAWC2, Madsen et al. (2007) implemented a tower shadow model by adapting the two dimensional jet wake model based on the work of Reichardt (1942) and Görtler (1942). This wake model that is based on the boundary layer solution for a jet flowing into a fluid at rest is defined as:

$$
\begin{gathered}
V_{x}(x, \eta)=\frac{\sqrt{3}}{2} \sqrt{\frac{K \sigma}{x}}\left(1-\tanh ^{2} \eta\right) \\
V_{y}(x, \eta)=\frac{\sqrt{3}}{4} \sqrt{\frac{K}{x \sigma}}\left\{2 \eta\left(1-\tanh ^{2} \eta\right)-\tanh \eta\right\} \\
J_{m}=\frac{V_{o}^{2} D}{2} \frac{\rho}{\pi}\left(\frac{1}{8}+\frac{16}{3 \pi}\right) C_{d}^{2}
\end{gathered}
$$

where $K$ is the kinematic momentum where $K=J_{m} / \rho, \rho$ is the mass density of fluid, $J_{m}$ is the momentum deficit behind the tower, $\eta$ is a 
dimensional coordinate where $\eta=\sigma y / x, \sigma=7.67$ is an empirical constant determined by experiments from Reichardt (1942). This model is used independently of the tower dam model described in Section 3.1.1.

\subsection{Nacelle Influence Models}

In an upwind configuration, the wind meets the hub of the rotor before impacting the wind turbine blades, and often, the distance of the hub to the blades is rather small. In both Bladed and FAST, the effects are corrected in using Prandtl's hub loss correction model.

This theory introduces a correction factor to the induced velocity field, $F_{\text {Prandtl }}$, and is expressed by:

$$
F_{\text {prandtl }}=\frac{2}{\pi} \cos ^{-1} e^{-f_{\text {hub }}}
$$

The hub-loss model corrects the induced velocity resulting from vortex shed near the hub of the rotor. The hub-loss model uses similar representation as the Prandtl tip-loss model where:

$$
f_{h u b}=\frac{B}{2} \frac{r-R_{h u b}}{r \sin \varphi}
$$

where $B$ is number of blades, $r$ is local radius of blade element or annulus, $R_{\text {hub }}$ is radius of rotor hub and $\varphi$ is angle of relative wind to the blade.

However, in a downwind configuration, the wind meets the nacelle of the wind turbine before impacting the wind turbine blades and the losses are likely to differ from those modeled in Prandtl's hub loss correction model. In both FAST and Bladed, the Prandtl's hub loss correction model is typically not used and the effects of the nacelle on the wind flow are not accounted for by any other theories or models. 


\subsection{Computational Fluid Dynamics and Turbulence Modeling}

As high fidelity CFD simulations are computationally expensive, it is not practically possible to combine them with wind turbine simulation tools and perform numerous simulations with long durations that are required by standards and guidelines. However in this study, CFD simulations are used in Section 5 to improve and develop empirical tower shadow and nacelle influence models that are simplified and faster to compute.

The background information of the CFD and turbulence modeling are widely available and produced in many books and publications. For this section, most background materials are referenced and cross-checked from books of reputable authors (Ferziger \& Peric, 2012; Pope, 2000; Wilcox, 2006) whom have vast amount of experience in CFD.

For both laminar and turbulent flows, the governing laws for fluid motion are described by the Navier-Stokes equation that is based on conservation of mass, momentum and energy, developed over a century ago. For an incompressible, constant-property flow where the flow of gases is below the Mach number of 0.3 (utilized in this study), density of the fluid $\rho$ is constant, the equation for conservation of mass and momentum are:

$$
\begin{gathered}
\frac{\partial u_{i}}{\partial x_{i}}=0 \\
\rho \frac{\partial u_{i}}{d t}+\rho u_{j} \frac{\partial u_{i}}{\partial x_{j}}=-\frac{\partial p}{\partial x_{i}}+\frac{\partial t_{j i}}{\partial x_{j}}
\end{gathered}
$$

where $u_{i}(x, y, z)$ is the fluid velocity, $x_{i}(i=1,2,3)$ or $(x, y, z)$ is the spatial coordinates of the domain, $t$ is time, $p$ is pressure and $t_{i j}$ is the viscous stress tensor defined by

$$
t_{i j}=2 \mu s_{i j}
$$


where $\mu$ is the molecular viscosity and $s_{i j}$ is the strain-rate tensor.

In a CFD simulation process, it involves determining the body of interest in a domain that is divided into cells (termed as the mesh), material and flow properties, boundary conditions, convergence criteria and solving the NavierStokes equation numerically using various numerical methods, algorithm and solver models.

\subsubsection{Scales of Turbulent Motion}

The concept of energy cascade was introduced by Richardson (1922), whereby turbulence can be considered to be made up of eddies of different sizes, and through the cascade process, kinetic energy is transferred (by inviscid processes) from larger eddies to smaller eddies as they are unstable, and the smallest eddies breakup by viscous action. The rate of kinetic energy transfer for each eddy size is constant and is defined as viscous dissipation $\varepsilon$, which is energy per unit time and unit mass.

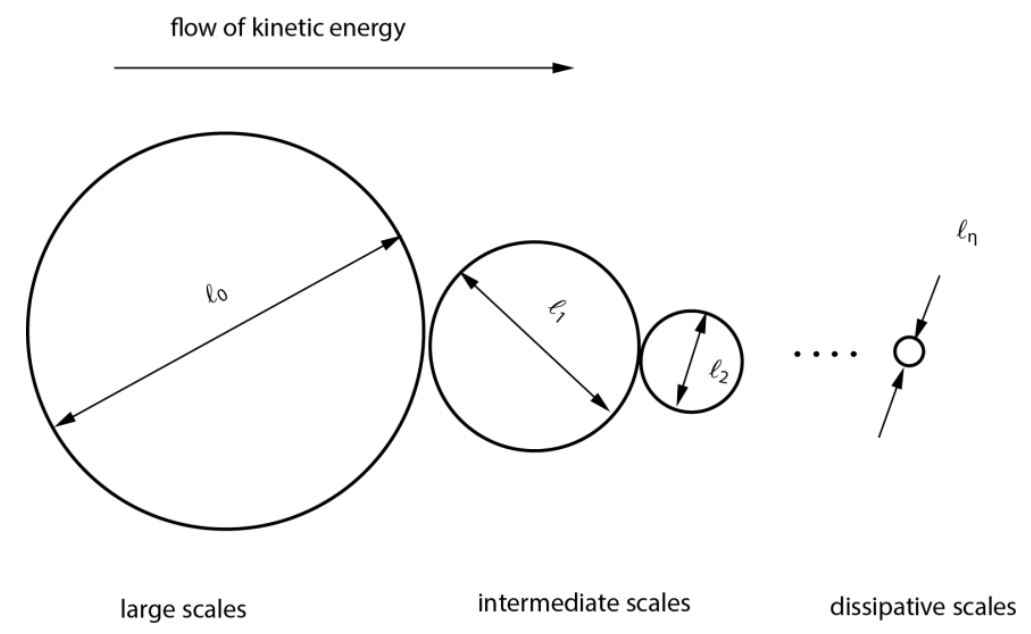

Figure 3.3 Energy cascade process (Davidson, 2011)

When the process reaches the smallest eddies, the viscous stresses in the fluid become significant and kinetic energy is dissipated into thermal energy. The smallest scales are termed as Kolmogorovs' dissipation scales (Kolmogorov, 1941) with velocity scale $v_{\eta}$, length scale $\ell_{\eta}$ and time scale $\tau_{\eta}$ - These scales are assumed to be determined by kinematic viscosity $v$ and dissipation $\varepsilon$, which can be expressed as: 


$$
v_{\eta}=(v \varepsilon)^{1 / 4}, \ell_{\eta}=\left(\frac{v^{3}}{\varepsilon}\right)^{1 / 4}, \tau_{\eta}=\left(\frac{v}{\varepsilon}\right)^{1 / 2}
$$

There are three main regions on the energy spectra, the integral length scale, the Taylor micro-scale (or inertia sub-range) and the Kolmogorovs' dissipation scale. The energy cascade, where energy is transferred from larger to smaller eddies until the $R e$ is sufficiently small for stability of the eddy motion, can be visualized in the Kolmogorovs' energy spectrum (Figure $3.4)$.

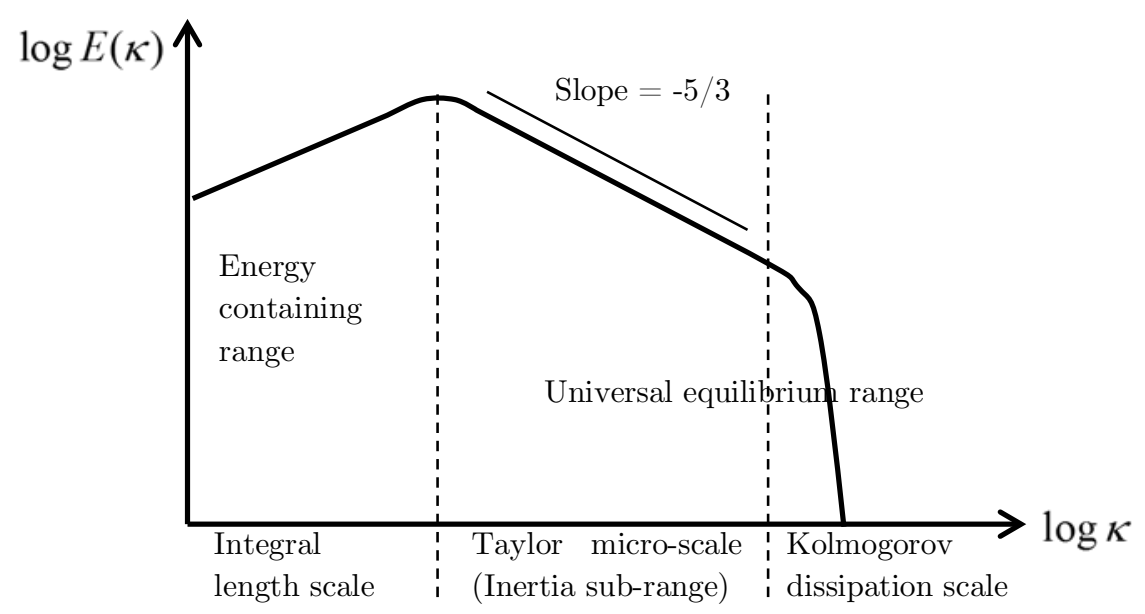

Figure 3.4 Kolmogorov's energy spectrum (adapted from Davidson (2011))

\subsubsection{Turbulence Modeling}

The Navier-Stokes equation is a relatively simple but powerful set of formulation that can describe fluid flow accurately in full details. However, its ability to describe a turbulence velocity field from the smallest to largest time and length scales results in an overload of information in solving the equations, resulting in an extremely complex problem to solve directly.

Solving the Navier-Stokes equations without any turbulence model is termed as direct numerical simulation (DNS). While this approach is gives the best accuracy, it is computationally expensive even with the recent developments and growth of computational power. More importantly, its application is usually limited to low or moderate $R e$ as the number of degrees of freedom 
of a 3-D flow increases by $R e^{9 / 4}$, consequently increasing the memory requirements and computational time (Mohseni, Kosović, Shkoller, \& Marsden, 2003). Therefore, turbulence modeling is used to represent the scales of the flow that are not resolved.
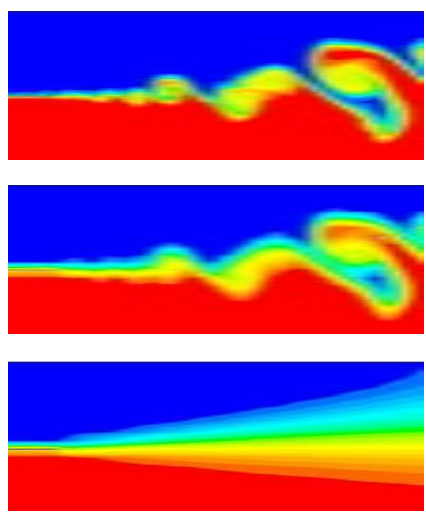

LES

Figure 3.5 Resolved and modeled regions for different turbulence models (adapted from Eggenspieler (2011)).

\subsubsection{Reynolds-averaged Navier-Stokes}

An approach to solve the Navier-Stokes equation is by applying time (for steady flow) or ensemble averaging (for unsteady flow). Both of this process is termed as Reynolds averaging and the resulting equations are called Reynolds-averaged Navier-Stokes (RANS) equations.

The averaged continuity and momentum equations from Eq. (3.31) and (3.32) are:

$$
\begin{gathered}
\frac{\partial \bar{u}_{i}}{\partial x_{i}}=0 \\
\rho \frac{\partial \bar{u}_{i}}{d t}+\rho \bar{u}_{j} \frac{\partial \bar{u}_{i}}{\partial x_{j}}=-\frac{\partial \bar{p}}{\partial x_{i}}+\frac{\partial}{\partial x_{j}}\left(2 \mu S_{i j}-\rho \overline{u_{i}^{\prime} u_{j}^{\prime}}\right)
\end{gathered}
$$

The quantity $-\rho \overline{u_{i}^{\prime} u_{j}^{\prime}}$ is known as the Reynolds-stress tensor and the specific Reynolds-stress tensor is termed as 


$$
\tau_{i j}=-\overline{u_{i}^{\prime} u_{j}^{\prime}}
$$

The fundamental problem of turbulence is to determine a method to compute $\overline{u_{i}^{\prime} u_{j}^{\prime}}$, which is commonly termed as the closure problem, due to a larger number of unknowns than equations available.

The Reynolds-averaged approach to turbulence modeling commonly employs the Boussinesq hypothesis, where:

$$
-\rho \overline{u_{i}^{\prime} u_{j}^{\prime}}=2 \mu_{T} S_{i j}-\frac{2}{3} \rho k \delta_{i j}
$$

where the turbulence kinetic energy, $k$ is defined as

$$
k=\frac{1}{2} \overline{u_{i}^{\prime} u_{i}^{\prime}}
$$

and $\mu_{T}$ is the dynamic eddy viscosity, assumed (as an approximation) as an isotropic scalar quantity.

The turbulence models developed for RANS equation is used to compute the Reynolds stresses tensor. Many of such models have been developed such as the linear and non-linear eddy viscosity models, and Reynolds stress models. In this study, the two-equation $k-\varepsilon, k-\omega$ and $k-\omega$ shear-stress transport (SST) models are discussed in further details.

\subsubsection{1 $k-\varepsilon$ model}

The standard $k-\varepsilon$ semi-empirical model is one of the most widely used turbulence model for practical engineering flow calculations developed by Jones and Launder (1972) and improved by Launder and Sharma (1974). Earlier contributions by Davydov (1961), Harlow and Nakayama (1968), Hanjalic (1970), and others cited by Launder and Spalding (1972) are significant in the development of the model.

The eddy (or turbulent) viscosity $\mu_{t}$ is computed by: 


$$
\mu_{T}=\rho C_{\mu} \frac{k^{2}}{\varepsilon}
$$

The turbulence kinetic energy $k$ is derived from:

$$
\frac{\partial}{\partial t}(\rho k)+\frac{\partial}{\partial x_{i}}\left(\rho k u_{i}\right)=\frac{\partial}{\partial x_{j}}\left[\left(\mu+\frac{\mu_{t}}{\sigma_{k}}\right) \frac{\partial k}{\partial x_{j}}\right]+\rho \tau_{i j} \frac{\partial k}{\partial x_{j}}-\rho \varepsilon
$$

The rate of dissipation of turbulence kinetic energy $\varepsilon$ is:

$$
\frac{\partial}{\partial t}(\rho \varepsilon)+\frac{\partial}{\partial x_{i}}\left(\rho \varepsilon u_{i}\right)=\frac{\partial}{\partial x_{j}}\left[\left(\mu+\frac{\mu_{t}}{\sigma_{\varepsilon}}\right) \frac{\partial \varepsilon}{\partial x_{j}}\right]+C_{\varepsilon 1} \frac{\varepsilon}{k} \rho \tau_{i j} \frac{\partial k}{\partial x_{j}}-C_{e 2} \rho \frac{\varepsilon^{2}}{k}
$$

where $C_{\mu}=0.09, C_{\varepsilon 1}=1.44, C_{\varepsilon 2}=1.92, \sigma_{k}=1.0, \sigma_{e}=1.3$.

The $k-\varepsilon$ model is relatively simple, stable (in terms of convergence) and accurate for fully turbulent flow application. However, it has been showed by many studies (Rodi and Scheuerer (1986), Wilcox (1988), Wilcox (1993), Henkes (1996)), the $k-\varepsilon$ model may not be adequate for flows with adverse pressure gradient, boundary layer separation, swirls and over curved surface.

\subsubsection{2 $k-\omega$ model}

The $k-\omega$ model was initially proposed by Kolmogorov (1942) as a twoequation model. This model was also formulated by Saffman (1970) without prior knowledge of Kolmogorov's work and further developed by many others cited by Wilcox (2006). While the equations of the $k-\omega$ model has evolved and improved over the years, the following model description is primarily based on Wilcox (2006) that is utilized in the CFD solver (ANSYS Fluent) that is used in this study (ANSYS, 2013).

The turbulence kinetic energy $k$ is derived from:

$$
\frac{\partial}{\partial t}(\rho k)+\frac{\partial}{\partial x_{i}}\left(\rho k u_{i}\right)=\frac{\partial}{\partial x_{j}}\left[\Gamma_{k} \frac{\partial k}{\partial x_{j}}\right]+G_{k}-Y_{k}+S_{k}
$$


The specific dissipation rate $\omega$ is derived from:

$$
\frac{\partial}{\partial t}(\rho \omega)+\frac{\partial}{\partial x_{i}}\left(\rho \omega u_{i}\right)=\frac{\partial}{\partial x_{j}}\left[\Gamma_{\omega} \frac{\partial \omega}{\partial x_{j}}\right]+G_{\omega}-Y_{\omega}+S_{\omega}
$$

where $G_{k}$ represents the generation of turbulence kinetic energy due to mean velocity gradients, $G_{\omega}$ represents the generation of $\omega, \quad \Gamma_{k}$ and $\Gamma_{\omega}$ represent the effective diffusivity of $k$ and $\omega, Y_{k}$ and $Y_{\omega}$ represent the dissipation of $k$ and $\omega$ due to turbulence, and $S_{k}$ and $S_{\omega}$ are user-defined source terms. The details of the equations and calculation of the above terms can be found in the ANSYS Fluent Theory Guide (ANSYS, 2013), and Wilcox (2006)'s book.

The $k-\omega$ model is accurate in predicting attached boundary layers, mildly separated and in its treatment of the viscous near-wall region, and in its accounting for the effects of streamwise pressure gradients. However, the disadvantage is that it does not simulate non-turbulent free-stream boundaries well due to the requirements on the boundary conditions on $\omega$.

\subsubsection{3 $k-\omega$ shear stress transport (SST) model}

As discussed earlier, both $k-\varepsilon$ and $k-\omega$ model have their respective strength and weakness, and the $k-\omega$ SST model was developed by Menter (1994) to blend both models. The blending function is designed to activate the standard $k-\omega$ model in the near-wall region and transit to the transformed $k-\varepsilon$ model away from the surface. This allows the $k-\omega$ SST model to be more accurate and reliable for a larger range of flow types. Details on the formulation and the application of the blending functions is available from Menter (1994)'s work and the ANSYS Fluent Theory Guide (ANSYS, 2013). 


\subsubsection{Large Eddy Simulations}

In LES, only small eddies are modeled with a turbulence model, making it more accurate than RANS. Time averaging is not used as compared to the RANS approach. Instead a low-pass filter is applied to the velocity field such that the large grid scales motions are resolved while subgrid-scale motions are modeled.

Assuming incompressible flow, the filtered Navier-Stokes equation is denoted as:

$$
\frac{\partial\left(\rho \bar{u}_{i}\right)}{\partial t}+\overline{u_{j}} \frac{\partial\left(\rho \overline{u_{i}}\right)}{\partial x_{j}}=-\frac{\partial(\bar{p})}{\partial x_{i}}+\mu \frac{\partial^{2} \bar{u}_{i}}{\partial x_{j} \partial x_{j}}-\frac{\partial \tau_{i j}^{s}}{\partial x_{j}}
$$

where $\tau_{i j}^{s}$ is the subgrid-scale (SGS) Reynolds stress (or residual-stress tensor) given by,

$$
\tau_{i j}^{s}=\rho\left(\overline{u_{i} u_{j}}-\bar{u}_{i} \bar{u}_{j}\right)
$$

The Boussinesq hypothesis is usually used to calculate the deviatoric part of the SGS stress where,

$$
\tau_{i j}^{s}-\frac{1}{3} \tau_{k k} \delta_{i j}=-2 \mu_{t} \overline{S_{i j}}
$$

where $\mu_{t}$ is the SGS turbulent viscosity. The isotropic part of the SGS is not modeled by the turbulence model, and usually neglected or added to the filtered pressure term. The $\overline{S_{i j}}$ is the rate-of-strain tensor for the resolved scale,

$$
\overline{S_{i j}}=\frac{1}{2}\left(\frac{\partial \overline{u_{i}}}{\partial x_{j}}+\frac{\partial \overline{u_{j}}}{\partial x_{i}}\right)
$$

The SGS models provide approximation to the SGS Reynolds stress that contains local averages of the small scale field. While many SGS models have been developed and utilized by researchers, two models available in 
ANSYS Fluent are described here, the Smargorinsky-Lilly model and the Wall-adapting local eddy-viscosity (WALE) model (used in this study).

\subsubsection{Smargorinsky-Lilly model}

The Smargorinsky-Lilly model is the earliest and most commonly used that is developed by Smagorinsky (1963).

The SGS turbulent viscosity is modeled by

$$
\mu_{t}=\rho L_{s}^{2}|\bar{S}|
$$

where $L_{s}$ is the mixing length for SGS, and $|\bar{S}|=\sqrt{2 \overline{S_{i j} S_{i j}}}$. ANSYS Fluent (ANSYS, 2013) models the mixing length using

$$
L_{s}=\min \left(\kappa d, C_{s} \Delta\right)
$$

where $\kappa$ is the von Kármán constant, $d$ is the distance to the closest wall, $C_{s}$ is the Smagorinsky constant (default at 0.1 ), and $\Delta$ is the local grid scale computed using the volume of the computational cell $V$ where $\Delta=V^{1 / 3}$ . The disadvantage of using this simple model is that the value of $C_{s}$ is not a universal constant, and various values have been proposed by other researchers.

\subsubsection{WALE model}

The WALE model was developed by (Nicoud \& Ducros, 1999) proposes to model the SGS turbulent viscosity is modeled by

$$
\mu_{t}=\rho L_{s}^{2} \frac{\left(S_{i j}^{d} S_{i j}^{d}\right)^{3 / 2}}{\left(\overline{S_{i j}} \overline{S_{i j}}\right)^{5 / 2}+\left(S_{i j}^{d} S_{i j}^{d}\right)^{5 / 4}}
$$

where $L_{s}$ and $S_{i j}^{d}$ is defined in ANSYS Fluent (ANSYS, 2013) as

$$
L_{s}=\min \left(\kappa d, C_{w} \Delta\right)
$$




$$
S_{i j}^{d}=\frac{1}{2}\left(\bar{g}_{i j}^{2}+\bar{g}_{j i}^{2}\right)-\frac{1}{3} \delta_{i j} \bar{g}_{k k}^{2}, \bar{g}_{i j}=\frac{\partial \overline{u_{i}}}{\partial x_{j}}
$$

The recommended value of the WALE constant, $C_{w}$ is 0.325 .

The key advantage and difference with the Smagorinsky-Lilly model is that the WALE model can return a zero turbulent viscosity for laminar shear flows, and therefore allowing correct treatment of the laminar flows in the domain.

\subsubsection{Embedded Large Eddy Simulation (ELES)}

Due to its high computational costs, Large Eddy Simulations (LES) are difficult to apply to industrial CFD simulations cases and particularly to large Reynolds flow conditions. High mesh resolution close to the wall is the key reason for limiting the usage of LES. Therefore, this limit LES application to low Reynolds flows or flows where wall boundary layers are not critical.

To overcome this issue, hybrid RANS-LES models such as the ScaleAdaptive Simulation and Detached Eddy Simulation have been developed. These models rely on flow instabilities in the CFD simulation to generate turbulent structures in large separated flow region. RANS and LES zones are determined automatically through flow criterion set by the hybrid models. In some cases where generation of turbulent structures is required and/or having clear distinction between RANS and LES zones is desired, a zonal RANS-LES model can be used.

In ANSYS Fluent, this formulation is known as Embedded LES (ELES) (Cokljat, Caridi, Link, Lechner, \& Menter, 2009), where the domain can be predefined during the grid/mesh generation phase as different models for each zone (RANS or LES portion). ELES is not a new turbulence model, and instead serves as an infrastructure for flexible implementation of existing turbulence models in different portions of the flow field. Therefore, the advantages and disadvantages are applicable to each individual models 
selected in the simulation. Modeled turbulence in RANS is converted to resolve turbulence at the RANS-LES interface using the Vortex Method (Mathey, Cokljat, Bertoglio, \& Sergent, 2006). 


\section{SWAY WIND TURBINE VALIDATION STUDY}

\subsection{Background Information}

As part of a collaboration of NREL and SWAY AS, NREL installed scientific wind, wave, and motion measurement equipment on the SWAY prototype system. The equipment enhanced SWAY's data collection and allowed SWAY to verify the concept and NREL to validate a model of the SWAY design in an open-water condition. Nanyang Technological University (NTU), in collaboration with NREL, conducted the validation wok. Under the funding and support from Energy Research Institute@ NTU, the author worked at National Renewable Energy Laboratory (NREL), National Wind Technology Center (NWTC) as a visiting scholar from 06 May 2012 to 12 July 2012. The author continued to collaborate with NREL after the visit to conclude the validation work.

The author was primarily involved in the work for this entire study. Dr. Amy Robertson acted as supervisor and reviewer of the validation work conducted by the author. Dr. Jason Jonkman supported the author by providing guidance to the FAST tool and sharing knowledge and skills in developing wind turbine models. Dr. Fredrick Driscoll supported the author by retrieving and processing the raw data collected from the measurement equipment to datasets that can be used for the study.

This work is focused on the effort to use the open-water test data of the SWAY prototype wind turbine to calibrate and validate a FAST floating offshore downwind turbine model. This is the first time that the FAST tool has been compared to a real floating wind turbine system deployed in open water, which has a number of challenges compared to tank testing.

The chapter is organized as follows. Section 4.2 describes the software tools used in this study while Section 4.3 shows the changes made to the software for this study. Section 4.4 shows a description of the SWAY wind turbine 
and the instrumentations used on the prototype model. Section 4.5 presents the properties prescribed in FAST and modeling assumptions used in this study. Section 4.6 discusses the calibration work on the FAST model, including system mass/inertia calibration using static and free decay tests, and blade stiffness and damping calibration. Section 4.7 covers the validation process of studying the system responses in non-operating conditions and operating conditions. Section 4.8 provides a summary of the conclusions drawn from this chapter.

\subsection{Software Tools Used}

In this study, FAST version 7 (v7.02.00d-bjj) was used as described in Section 2.4.1. A newer version of FAST (v8) was made available in September 2014 that included new capabilities for modeling offshore wind systems, but the original work in this project was initiated before this release.

In addition, additional tools were used to generate model and simulation inputs.

\subsubsection{AirfoilPrep (v2)}

AirfoilPrep (v2) is a spreadsheet developed by NREL for users to generate airfoil data files required by AeroDyn. In this study, it was applied to generate 360-degree data of the airfoil data input files using the Viterna and Janetzke (1982) method. The spreadsheet was also used to interpolate the aerodynamic coefficients for other span locations and to apply threedimensional delayed stall corrections caused by blade rotation using the method proposed by Du and Selig (1998) and Eggers and Digumarthi (1992).

\subsubsection{TurbSim (v1.06.00)}

The TurbSim stochastic inflow turbulence code is able to generate a fullfield flow with various wind spectral models, turbulence models, coherent turbulence structures and wind profiles. This includes models specified by 
the IEC standards as well as non-IEC models that simulate flow instabilities associated with nocturnal boundary layer flows. The key purpose of the code is to provide the wind turbine designer simulated inflow turbulence environment to drive wind turbine design codes. The details for the use of the TurbSim code is available in TurbSim User's Guide (B. J. Jonkman \& Kilcher, 2012).

\subsection{Code Improvement and Modification}

\subsubsection{Calculation of tower loading}

During the initial runs of the simulation cases, it was noted that wind loading on the tower may be important in the analysis of floating turbines - particularly for the SWAY prototype that has a large tower relative to the size of the rotor - especially in conditions where the turbine blades are not operating. To account for the tower drag, changes were made to FAST to calculate the wind loading on each tower element for each simulation time-step. The approach here assumes that the tower is a cylinder, either tapered or un-tapered, and does not apply to multi-member or lattice-type towers.

Several strategies in accounting for wind loading on towers are available from industrial standards and other researchers. For example, according to the International Standards Organization (ISO) 4354 (2009) standard, the total wind load can be a combination of three components. The first is the mean component that is due to the mean wind force. The second is the fluctuating (background) component, which results from the unstable nature of flow around bluff bodies including in buffeting, flow separation, reattachments and vortex shedding. Other transient loads can also be induced by the motion of the structure caused by the wind such as galloping or flutter. The third is the "resonant" component, which results in a phenomenon called lock-in (Holmes, 2007) or vortex-induced vibration, which occurs when the frequency of vortex shedding is similar to the frequency motion of the body. 
For the majority of structures, the resonant component is small, and as suggested by Scruton (1981) and ISO 4354 (2009), the assumption of static loads due to wind is reasonably adequate unless the structure is large, lightweight and lightly damped. Although this assumption may fully apply to a wind turbine tower, because of difficulties and uncertainties in quantifying the fluctuating component in a time-domain simulation, only the mean component of the wind force with effects of natural free-stream turbulence is considered in this code improvement.

The code improvement is summarized in the simulation flow chart illustrated in Figure 4.1. First, FAST checks the platform input file for the platform load model (PtfmModel) value set by the user. If the value (4) is set to include tower loading, FAST reads the tower drag coefficient and tower diameter values from the tower input file. Next, FAST checks the type of wind inflow data used. If TurbSim-generated wind inflow data are used, FAST obtains the lowest Z-position of the wind data defined as GridBase. As a result, the wind inflow for the region below GridBase is undefined, as shown in Figure 4.2. It is common practice to only define wind inflow data for the expected region of turbine blade motion, as generation of a larger area of wind inflow is limited by computational cost and limited memory.

Next, for every simulation time-step and tower node, FAST checks the position of the tower node termed as $Z$ and retrieves the undisturbed wind velocity from the wind inflow data if the position is equal or more than the GridBase value. If the position is less than the GridBase value, the wind inflow data for the undefined region (which is needed for the tower drag calculations) are obtained by assuming that the wind profile from the GridBase to MSL follows the power law (Eq. (4.1)). The exponent $\alpha=$ 0.140 is recommended by the IEC 61400-3 (2009) for offshore wind conditions. 


$$
U_{z}=U_{\text {Gridbase }}\left(\frac{Z}{\text { GridBase }}\right)^{0.140}
$$

With the wind inflow data obtained, the magnitude of the drag force per unit length is calculated using Eq. (4.2) added vectorially along the relative wind direction to the overall platform and tower loads calculation for every time-step.

$$
D_{\text {tower }}=\frac{1}{2} \rho\left(\overline{\left(V_{0}-V_{\text {tower }}\right)}\right)^{2} C_{D, \text { tower }} D
$$

$D_{\text {tower }}$ is the drag force per unit length of the tower element, $\rho$ is the density of air, $\left(\overline{\left(V_{0}-V_{\text {tower }}\right)}\right)^{2}$ is the magnitude of the difference between the undisturbed inflow velocity (with turbulence) and the velocity of the tower element at the tower element location $V_{\text {tower }}, C_{D \text {,tower }}$ is the drag coefficient of the tower element, and $D$ is the diameter of the tower element.

It is also commonly known that factors such as aspect ratio, surface roughness, turbulence intensity, and $R e$ may affect the $C_{D, \text { tower }}$ for circular cylinders. For example, Roshko (1961) and Wieselsberger (1921) described the various flow regimes, the occurrence of the critical $R e$, and drag coefficient values for a circular cylinder with smooth surface across different Re. Scruton, Rogers, Menzies, and Scorer (1971) showed that reduction in drag coefficient occurs for circular cylinder of finite aspect ratio with a single free end. The surface roughness effect on lowering the critical $R e$ range is also shown in Engineering Sciences Data Unit' ESDU 80025 (1986).

Because of the high variability and numerous factors, the drag coefficient of the tower was designed to be manually inputted by the user. This gives the user a certain degree of control, which can be useful in accounting for wind loads for non-tubular towers or utilizing drag coefficients prescribed specifically for individual tower designs or standards such as the ISO 4354 (2009) and EN 1991-1-4 (2005). Otherwise, the user can use the default Re$C_{D, \text { tower }}$ table (plotted in Figure 4.2 and used in this study), which is 
applicable for a circular smooth cylinder of high aspect ratio. The data is obtained from the work of Roshko (1961) and Wieselsberger (1921). The Re of the tower is calculated based on Eq. (4.3).

$$
R e_{\text {tower }}=\frac{\overline{\left(V_{0}-V_{\text {tower }}\right)} \times D}{v}
$$

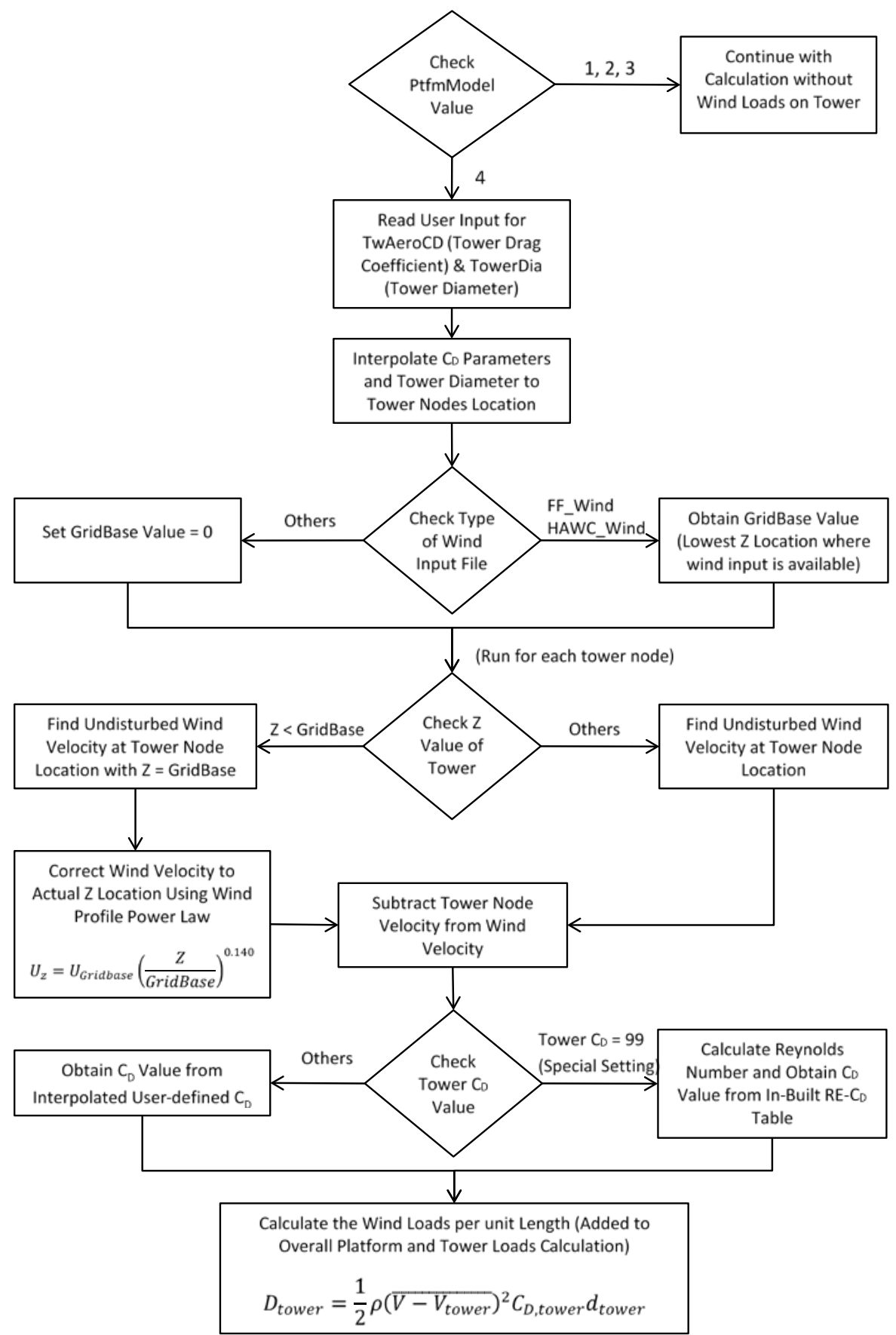

Figure 4.1 Simulation flow chart for calculation of tower loading 


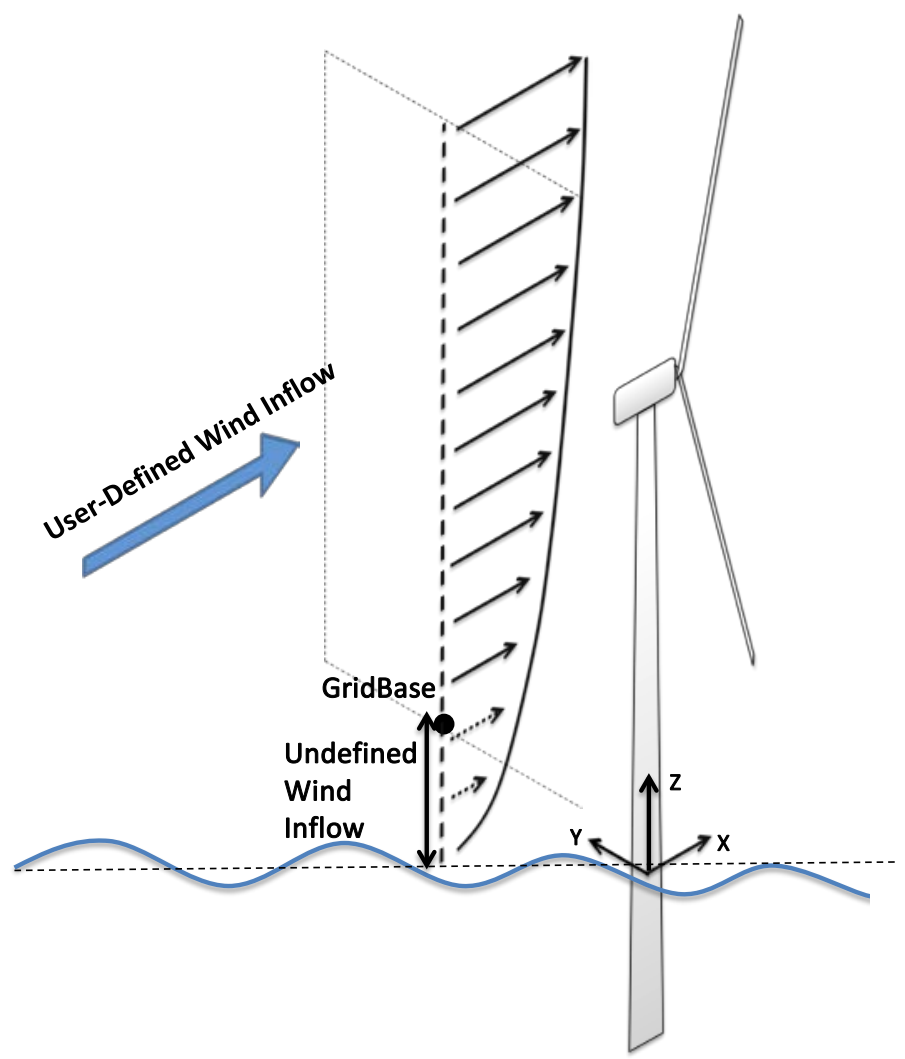

Figure 4.2 Undisturbed wind inflow along the rotor and tower

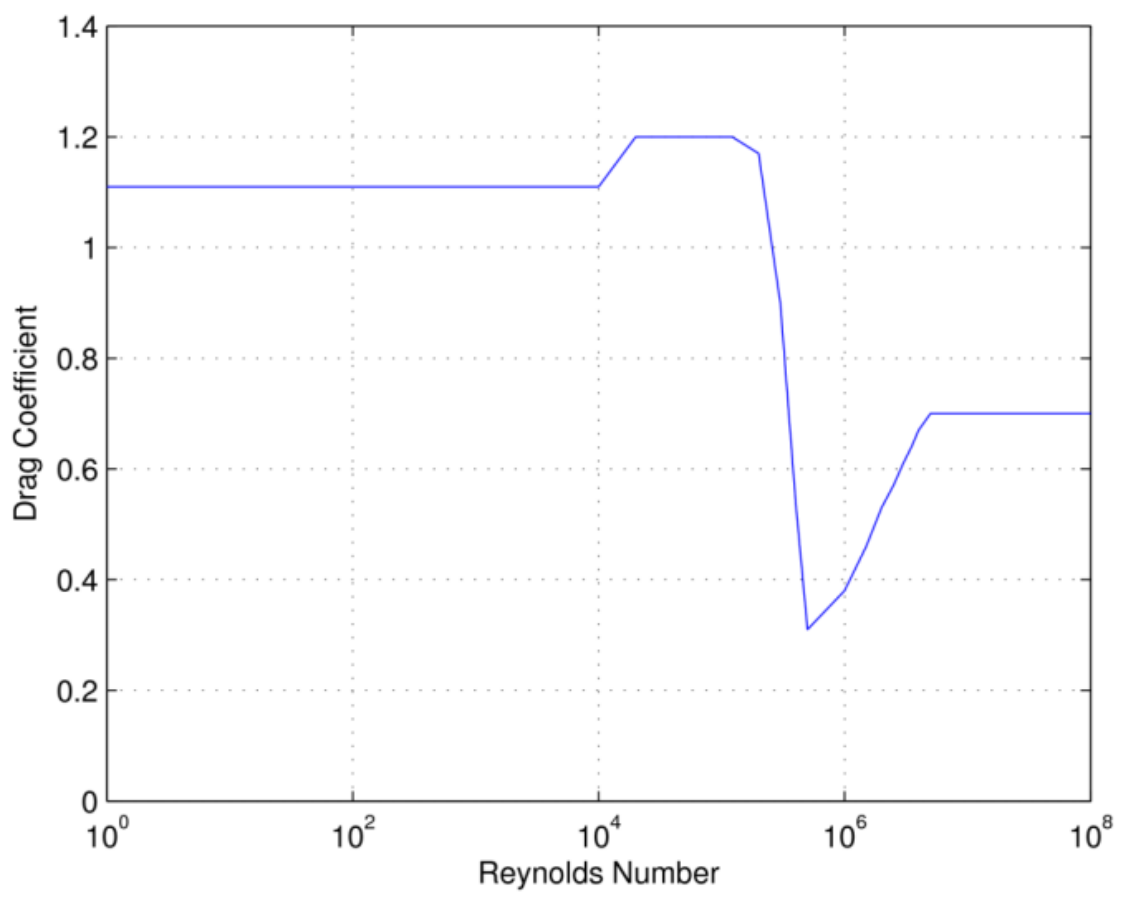

Figure 4.3 $R e_{\text {tower }}$ versus $C_{D, \text { tower }}$ 


\subsection{System and Prototype Description}

\subsubsection{SWAY Wind Turbine}

The SWAY spar-type floating wind turbine as shown in Figure 4.4 has a three-bladed downwind configuration that uses a tension rod system for station-keeping and maintaining the hydrostatic stability of the wind turbine. The bottom of the spar structure is fixed to the tension rod with a universal joint. The other end of the tension rod is attached to a large steel mass (gravity anchor) on the seafloor with a universal joint. A downwind configuration allows the possibility of using a passive yaw system that eliminates the cost that would be incurred by having an active yaw system in an upwind design. The wind turbine system, including the tower, yaws about the yaw mechanism located at the universal joint at the bottom of the tower. In addition, the blade clearance from the tower, which is a concern for larger turbines, is less of a concern for the downwind SWAY turbine.

The wind turbine has individual blade-pitch control, and the nacelle is fixed to the tower at an optimal angle so that the rotor axis is oriented horizontally (parallel to the wind) when the tower pitches during operation. Some of the key components in the system are unique spreader beams and tension cables, which help stiffen the tower, reduce fatigue loads, and allow the tower to carry a larger turbine.

The full-scale SWAY wind turbine is designed to have a rated power of 2.5$10 \mathrm{MW}$, a rotor diameter up to $190 \mathrm{~m}$, and a substructure up to $300 \mathrm{~m}$ in length. 


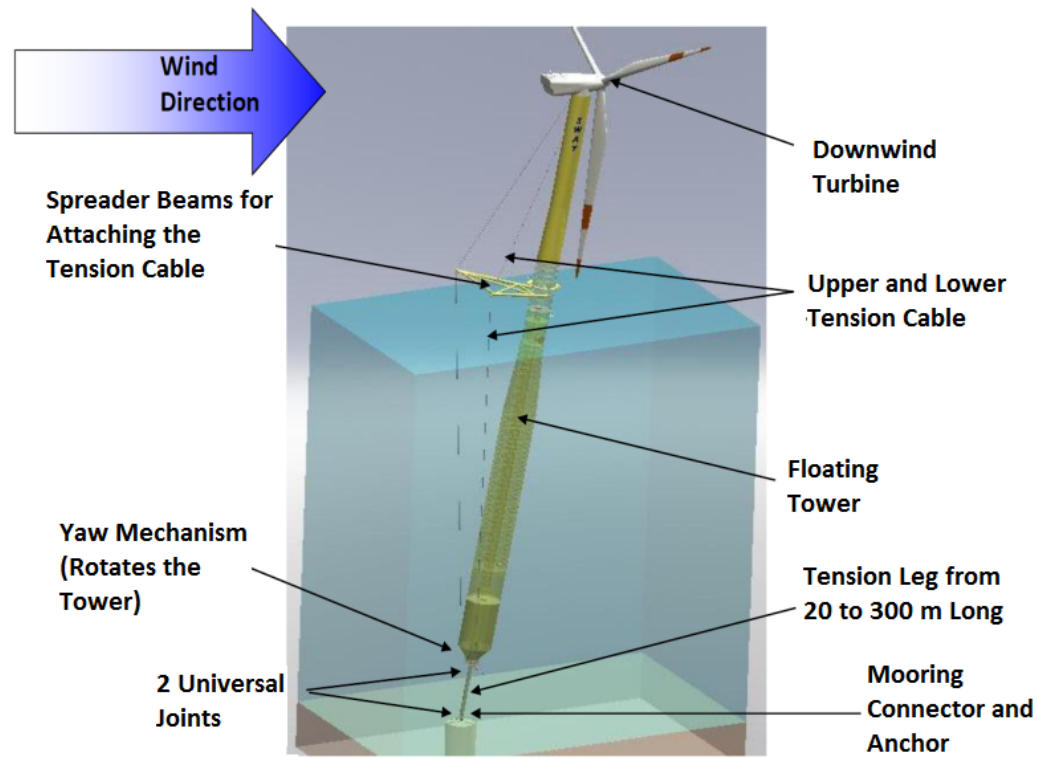

Figure 4.4 SWAY floating wind turbine

\subsubsection{SWAY Prototype Wind Turbine}

A $1 / 6.5^{\text {th }}$-scale prototype of the SWAY spar-type floating wind turbine was deployed in Hjeltefjorden, east of Øygarden in Hordaland, Norway, in May 2012 (www.sway.no). Table 4.1 provides the key specifications of the prototype wind turbine.

Table 4.1 SWAY 1:6.5 Scale Wind Turbine Prototype Specifications

\begin{tabular}{l|ll}
\hline Support & Structure & \\
Manufacturer & SWAY 1/6.5 & \\
Make, Model of Turbine & Scale Prototype & \\
Production Year of Turbine & 2011 & \\
Rotation Axis & Horizontal & \\
Orientation & Downwind & \\
Number of Blades & 3 & \\
Rotor Diameter & $14.9 \quad \mathrm{~m} \quad$ (after extension in \\
& 2012 ) & \\
Hub Height & $\sim 13 \mathrm{~m}$ & \\
Control & Individual Pitch Control \\
Tower Type & Tubular & \\
Floater Type & Spar Buoy & \\
Cut-In Wind Speed & $2 \mathrm{~m} / \mathrm{s}$ & \\
Rated Wind Speed & $8 \mathrm{~m} / \mathrm{s}$ \\
Cut-Out Wind Speed & $16 \mathrm{~m} / \mathrm{s}$ & \\
\hline
\end{tabular}

\subsubsection{Instrumentation}

Figure 4.5 shows the profile of the tower, spreader beam, and tension rod. In FAST, the blue region is modeled as the wind turbine tower while the red 
region is modeled as the platform. Yellow circles indicate the type and locations of the instruments.

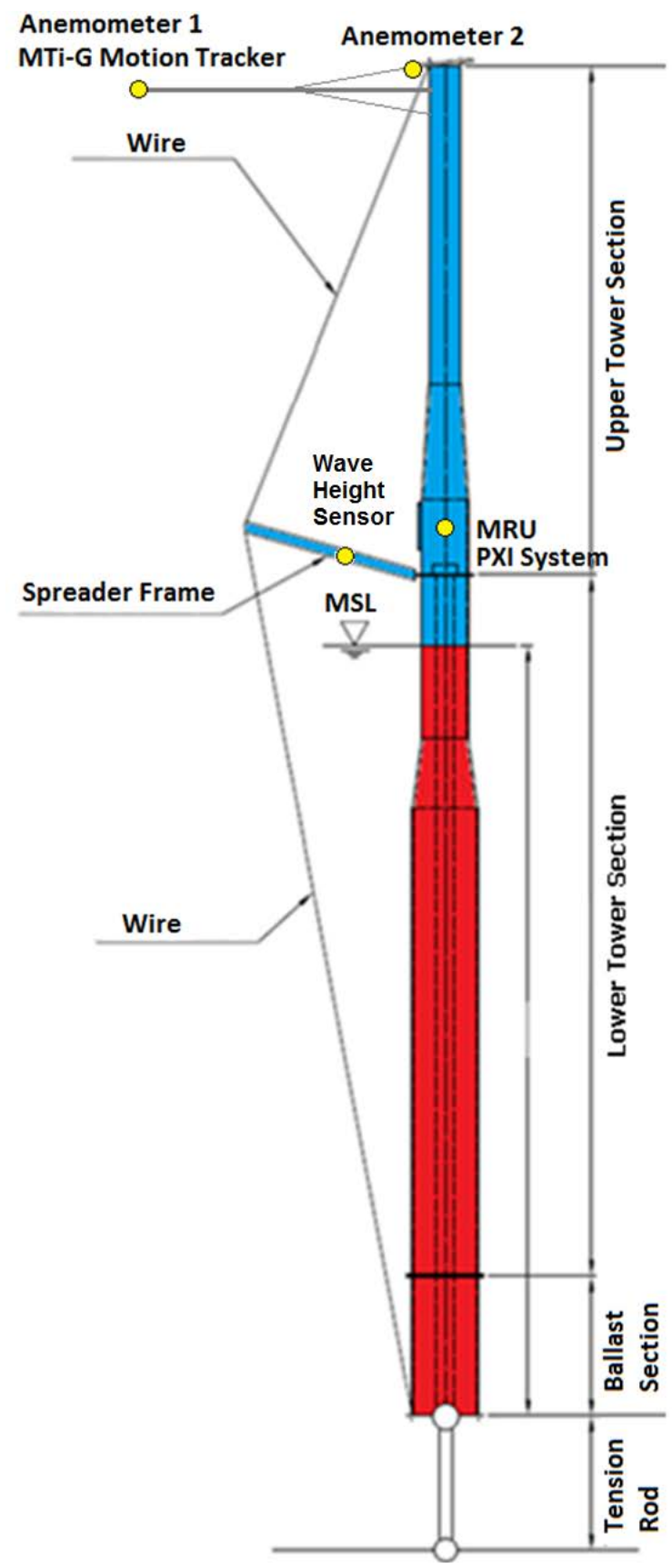

Figure 4.5 Profile of the tower, spreader beam, and tension rod

Profiles of the water velocity and directional wave spectra were measured by a Nortek $600-\mathrm{kHz}$ AWAC mounted on the seafloor approximately $12.5 \mathrm{~m}$ north-northeast of the turbine at a depth of about $20 \mathrm{~m}$. Due to installation 
limitations, the AWAC was located in an area close to bedrock and contour regions shown in Figure 4.6. Data from the AWAC were internally recorded and averaged to 1-hour readings.

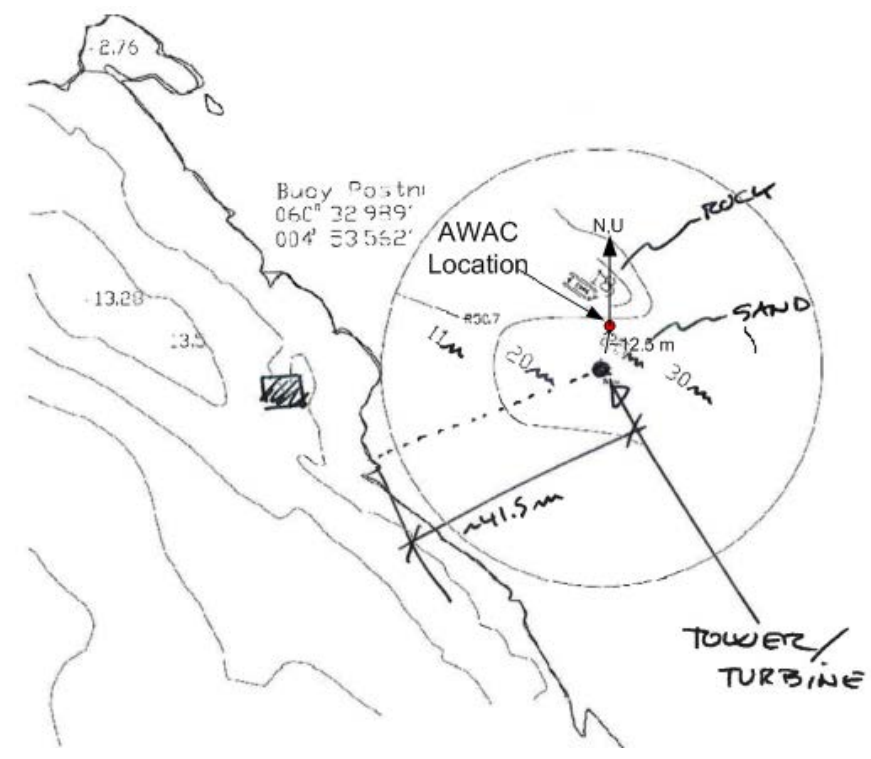

Figure 4.6 AWAC location

A tower wave height sensor measured the wave height at the tower base using an ultrasonic transducer. The measurement was manually adjusted to account for the heave motion of the system. Also, the measurement may have been influenced by reflected and radiated waves by the platform. The location and orientation of the transducer is shown in Figure 4.7. The advantage of using the tower wave height signal is its sample rate of $100 \mathrm{~Hz}$, which is useful to generate and analyze the wave spectra. 


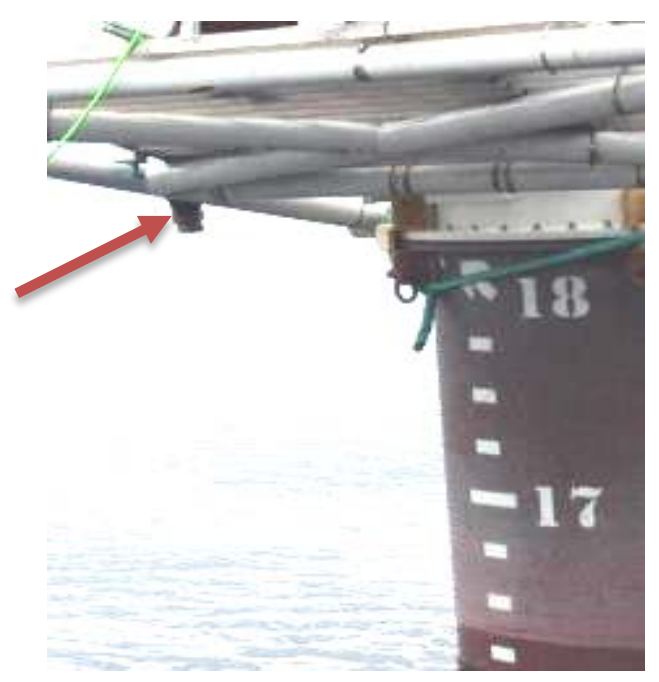

Figure 4.7 Tower wave height sensor

Further details on the instrumentation, measurement equipment and data collection process are described in Appendix A.

\subsection{Model Description}

This section details the methodology used to create a model of the SWAY demonstration system in FAST. While the system has a pitch offset in its static equilibrium position, the tower of the FAST model was built in a vertical configuration with a shaft tilt. All heights and values listed in this section assume the tower is vertical.

The translational DOFs of the platform in the $\mathrm{X}, \mathrm{Y}$, and $\mathrm{Z}$ directions are called surge, sway, and heave; and rotations about the $\mathrm{X}, \mathrm{Y}$, and $\mathrm{Z}$ axes are called roll, pitch, and yaw. Each DOF can be turned on or off individually. Figure 4.8 shows the coordinate systems and platform DOF terminology used in this chapter; the origin is located at mean sea level (MSL) and dimensions described in this chapter are referenced to this inertial frame coordinate system unless otherwise stated. 


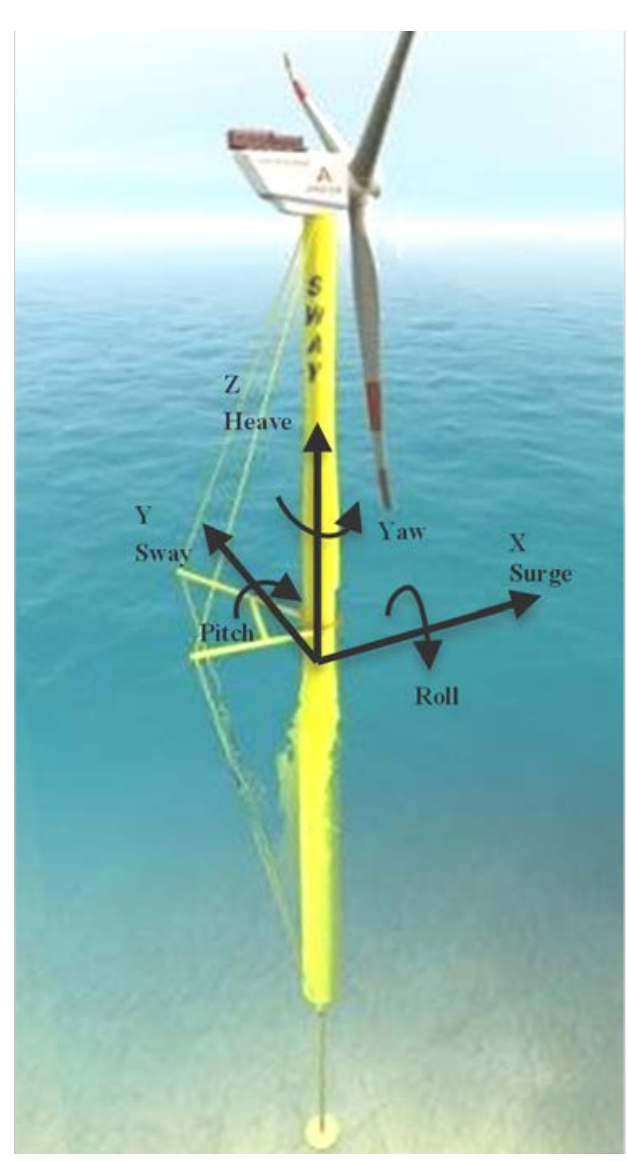

Figure 4.8 Coordinate systems and platform DOF

\subsubsection{Turbine}

The turbine blades that were mounted on the rotor are OLW620_R1 profiles produced by Olsen Wings. The blade gross properties were obtained with estimation from a technical report developed by GL Garrad Hassan (Nichols, 2011), which was engaged by SWAY AS for design calculations. The original blade length was designed at $6.2 \mathrm{~m}$ and used in the analysis in the GL Garrad Hassan report. However, during the final installation, a $1 \mathrm{~m}$ cylinder extension was added to the root of the blade to increase the rotor radius.

The blade, as shown in Figure 4.9, is made of three different blade profiles: a cylinder near the root of the blade, a transition airfoil, and a main airfoil. However, airfoil data were only available for the 2D main airfoil section (lift, drag, and moment coefficients $\left(C_{l, \text { airfoil }}, C_{d, \text { airfoil }}\right.$, and $\left.C_{m, \text { airfoil }}\right)$. To obtain the drag coefficient of the cylinder, $R e$ near the root of the blade was 
calculated at the operating condition of the wind turbine. The $C_{d \text {,airfoil }}$ value was then obtained by looking up the appropriate value on a drag coefficient versus $R e$ curve for cylinders. Using the blending function in AirfoilPrep, the transition airfoil properties were produced by linear interpolation of the $C_{l, a i r f o i l}, C_{d, a i r f o i l}$, and $C_{m \text {,airfoil }}$ of the cylinder and the main airfoil with equal weighting. Although the transition airfoil region might be subjected to error due to the assumptions made, the majority of the load on the blade is generated by the outside half of the blade, where blade data was available.

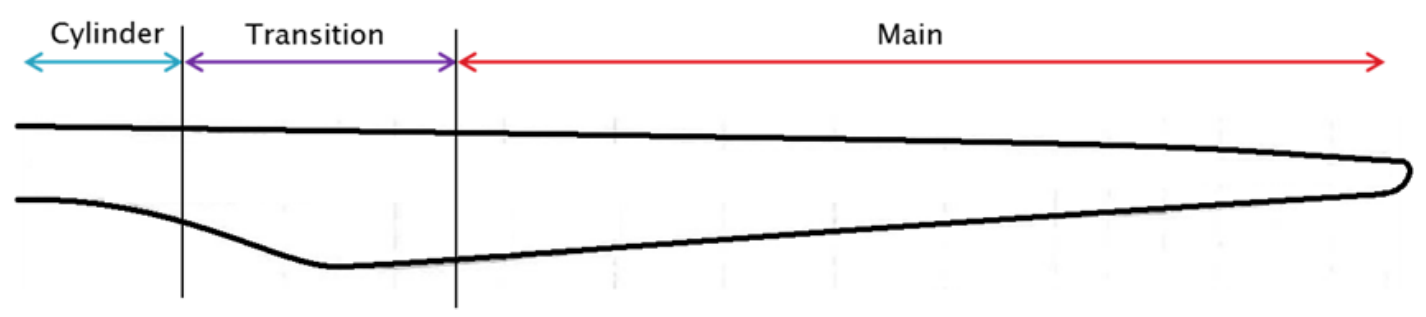

Figure 4.9 Illustration of Airfoil Region

To improve the accuracy of the main airfoil, it was further divided into three sections: Main 1, Main 2, and Main 3. 3D-delayed stall corrections due to blade rotation were applied in AirfoilPrep. The $C_{l, a i r f o i l}, C_{d, \text { airfoil }}$, and $C_{m \text {,airfoil }}$ properties for the cylinder, transition, and sections Main 1, Main 2, and Main 3 are plotted in Figure 4.10, Figure 4.11 and Figure 4.12 respectively while Appendix A contains the numerical tables for the airfoil data. The blade mode shapes were derived separately using BModes. 


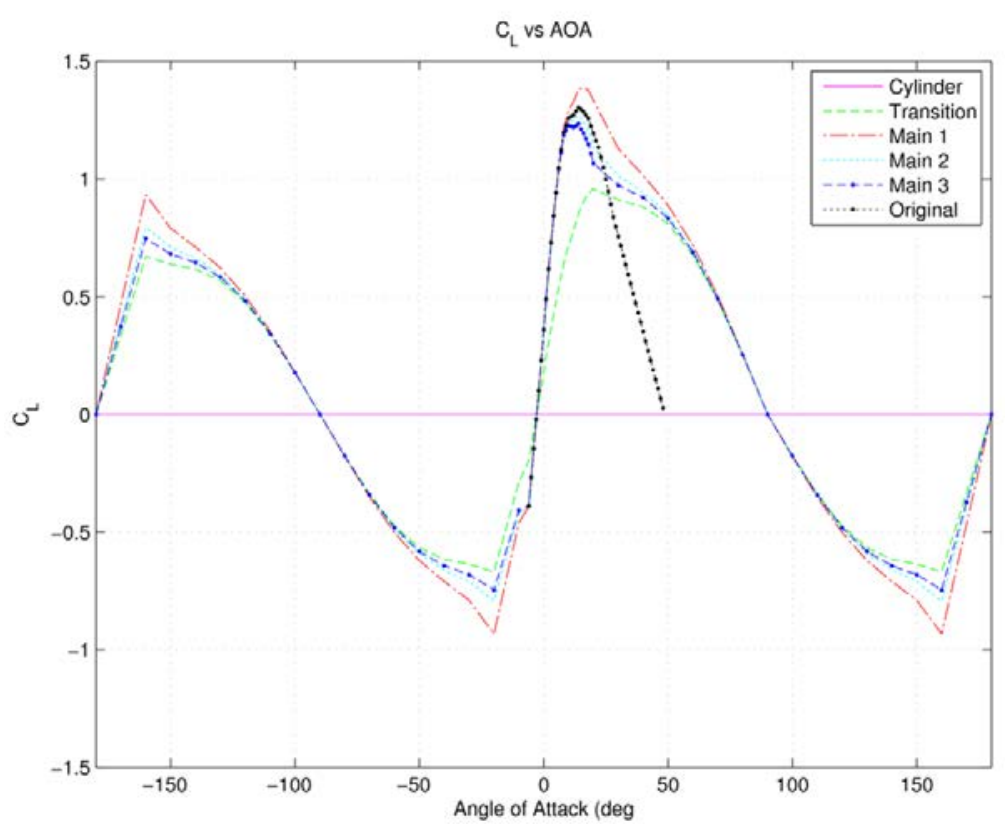

Figure $4.10 C_{l, a i r f o i l}$ data of blade sections

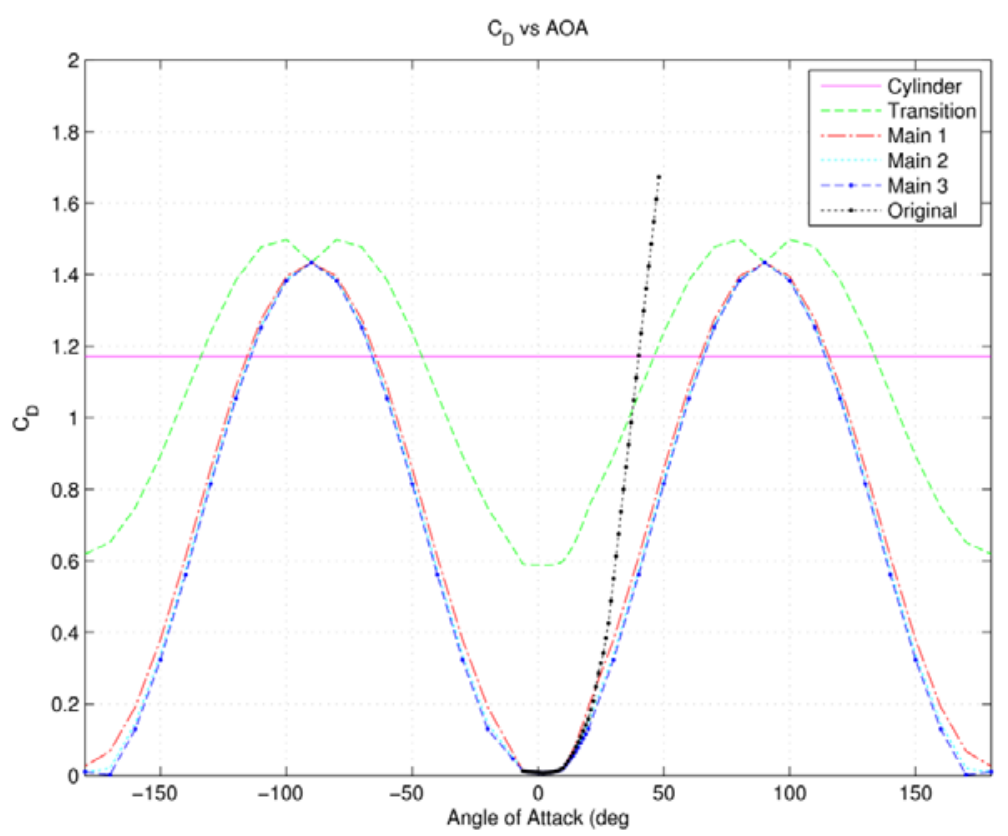

Figure $4.11 C_{d, a i r f o i l}$ data of blade sections 


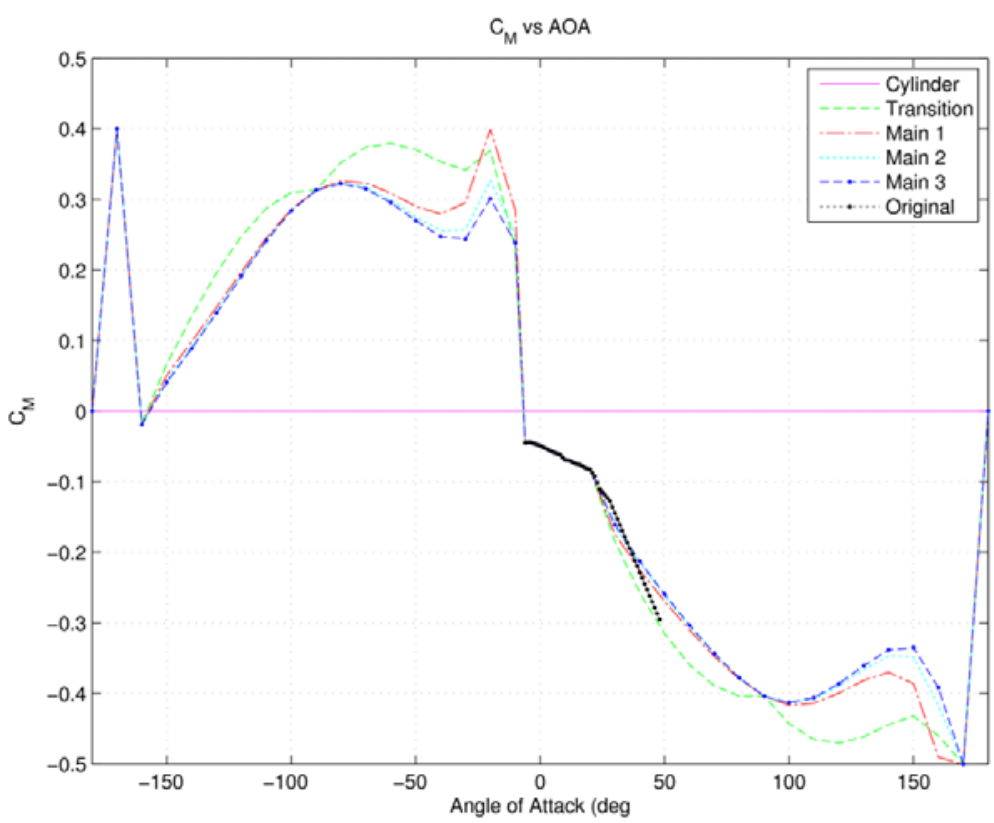

Figure 4.12 $C_{m \text {,airfoil }}$ data of blade sections

The turbine gross properties, hub and nacelle gross properties, blade gross properties, blade-distributed mass properties and blade-distributed aerodynamic properties are shown in Table 4.2, Table 4.3, Table 4.4,

Table 4.5, and Table 4.6. The values are the original properties provided by SWAY design documents ((Nichols, 2011), (Holmas, 2010a), (Holmas, 2010b)) .

Table 4.2 Turbine Gross Properties

\begin{tabular}{l|l}
\hline Rotor orientation, configuration & Downwind, three-bladed \\
Rotor, hub diameter & $14.9 \mathrm{~m}, 0.5 \mathrm{~m}$ \\
Hub height above MSL & $13.133 \mathrm{~m}$ \\
Height of tower-top flange above MSL & $12.500 \mathrm{~m}$ \\
Overhang, shaft tilt, pre-cone & $0.72275 \mathrm{~m}, 5^{\circ}, 4^{\circ}$ \\
Vertical distance along tower centerline & $0.587 \mathrm{~m}$ \\
between tower top and shaft & \\
Total tower-top mass & $1,294.60$ kilograms (kg) \\
\hline
\end{tabular}

Table 4.3 Hub and Nacelle Gross Properties

\begin{tabular}{l|l}
\hline Nacelle mass & $910 \mathrm{~kg}$ \\
Nacelle center of mass (above tower) & $0.45 \mathrm{~m}$ \\
Nacelle center of mass (downwind) & $0.2815 \mathrm{~m}$ \\
Nacelle center of mass (lateral) & $-0.0392 \mathrm{~m}$ \\
Hub mass & $134 \mathrm{~kg}$ \\
Hub inertia about rotor axis & $75 \mathrm{~kg} \mathrm{~m}{ }^{2}$ \\
Generator inertia about high speed shaft & $0.00777 \mathrm{~kg} \mathrm{~m}$ \\
Gearbox ratio & 74.77 \\
\hline
\end{tabular}


Table 4.4 Blade Gross Properties

\begin{tabular}{|c|c|}
\hline Blade length & $\begin{array}{l}6.2 \mathrm{~m}+1 \mathrm{~m} \\
\text { (extension) }\end{array}$ \\
\hline $\begin{array}{l}\text { Blade mass } \\
\text { Location of blade center of mass (measured from } \\
\text { blade root) }\end{array}$ & $\begin{array}{l}83.534 \mathrm{~kg} \\
2.762 \mathrm{~m}\end{array}$ \\
\hline $\begin{array}{l}\text { Blade first mass moment of inertia (w.r.t. Root) } \\
\text { Blade second mass moment of inertia (w.r.t. Root) }\end{array}$ & $\begin{array}{l}230.690 \mathrm{~kg} \mathrm{~m} \\
912.556 \mathrm{~kg} \mathrm{~m} \mathrm{~m}^{2}\end{array}$ \\
\hline
\end{tabular}

Table 4.5 Blade-Distributed Mass Properties

\begin{tabular}{|c|c|c|c|c|c|}
\hline $\begin{array}{l}\text { Radiu } \\
\text { s (m) }\end{array}$ & AeroCent ${ }^{a}$ & $\begin{array}{l}\text { Structural } \\
\text { Twist } \\
\text { (deg) }\end{array}$ & $\begin{array}{l}\text { Mass } \\
(\mathrm{kg} / \mathrm{m})\end{array}$ & $\begin{array}{l}\text { Flapwise } \\
\text { Stiffness } \\
\left(\mathrm{Nm}^{2}\right)\end{array}$ & $\begin{array}{l}\text { Edgewise } \\
\text { Stiffness } \quad\left(\mathrm{Nm}^{2}\right)\end{array}$ \\
\hline 0.00 & 0.25000 & 38.88 & 11.667 & $3.74 \mathrm{E}+06$ & $3.74 \mathrm{E}+06$ \\
\hline 1.00 & 0.25000 & 38.88 & 16.917 & $3.74 E+06$ & $3.74 E+06$ \\
\hline 1.10 & 0.25000 & 35.49 & 16.68 & $4.60 E+06$ & $4.60 E+06$ \\
\hline 1.45 & 0.25000 & 28.30 & 15.849 & $2.39 E+06$ & $2.39 E+06$ \\
\hline 1.70 & 0.24153 & 22.81 & 15.255 & $1.06 \mathrm{E}+06$ & 1. $33 \mathrm{E}+06$ \\
\hline 1.95 & 0.23307 & 18.66 & 14.661 & $8.83 E+05$ & 1. $47 \mathrm{E}+06$ \\
\hline 2.20 & 0.22460 & 15.49 & 14.068 & $6.53 E+05$ & $1.52 \mathrm{E}+06$ \\
\hline 2.45 & 0.24600 & 13.02 & 13.474 & $4.37 E+05$ & 1. $32 E+06$ \\
\hline 2.70 & 0.25100 & 11.06 & 12.88 & $3.29 \mathrm{E}+05$ & $1.15 \mathrm{E}+06$ \\
\hline 3.20 & 0.24100 & 8.17 & 11.693 & $2.09 \mathrm{E}+05$ & $8.45 E+05$ \\
\hline 3.70 & 0.22300 & 6.16 & 10.506 & 1. $30 \mathrm{E}+05$ & $5.93 E+05$ \\
\hline 4.20 & 0.20500 & 4.68 & 9.319 & $8.31 E+04$ & $4.20 E+05$ \\
\hline 4.70 & 0.19000 & 3.56 & 8.131 & $3.89 E+04$ & $2.11 E+05$ \\
\hline 5.20 & 0.18000 & 2.67 & 6.944 & $2.81 E+04$ & $1.61 \mathrm{E}+05$ \\
\hline 5.70 & 0.17300 & 1.96 & 5.757 & $2.13 E+04$ & $2.55 \mathrm{E}+05$ \\
\hline 5.95 & 0.16900 & 1.65 & 5.163 & $9.54 \mathrm{E}+03$ & 1. $14 \mathrm{E}+05$ \\
\hline 6.20 & 0.16500 & 1.37 & 4.57 & $8.11 \mathrm{E}+03$ & $9.73 E+04$ \\
\hline 6.45 & 0.16000 & 1.12 & 3.976 & $6.63 E+03$ & $7.95 E+04$ \\
\hline 6.70 & 0.15000 & 0.88 & 3.382 & $4.87 E+03$ & $5.84 E+04$ \\
\hline 6.95 & 0.15000 & 1.17 & 2.789 & $2.58 \mathrm{E}+03$ & $3.10 \mathrm{E}+04$ \\
\hline 7.00 & 0.15000 & 1.44 & 2.67 & $1.01 \mathrm{E}+03$ & 1. $21 \mathrm{E}+04$ \\
\hline 7.05 & 0.15000 & 1.94 & 2.551 & $1.01 \mathrm{E}+03$ & 1. $21 \mathrm{E}+04$ \\
\hline 7.10 & 0.15000 & 2.44 & 2.433 & $1.01 E+03$ & 1. $21 \mathrm{E}+04$ \\
\hline 7.15 & 0.15000 & 3.14 & 2.314 & 1. $01 E+03$ & 1. $21 E+04$ \\
\hline 7.20 & 0.15000 & 4.00 & 2.195 & $3.26 \mathrm{E}+00$ & $3.91 \mathrm{E}+01$ \\
\hline
\end{tabular}


Table 4.6 Blade-Distributed Aerodynamic Properties

\begin{tabular}{|c|c|c|c|c|c|c|}
\hline $\begin{array}{l}\text { Node } \\
\text { (m) }\end{array}$ & Radius & $\begin{array}{l}\text { Aerodynamic } \\
\text { (deg) }\end{array}$ & Twist & $\begin{array}{l}\text { Chord } \\
\text { (m) }\end{array}$ & Length & Airfoil Table \\
\hline 0.75 & & 38.88 & & 0.33 & & Cylinder.dat \\
\hline 1.3 & & 37.185 & & 0.33 & & Cylinder.dat \\
\hline 1.525 & & 31.895 & & 0.33 & & Cylinder.dat \\
\hline 1.825 & & 25.555 & & 0.365 & & Transition.dat \\
\hline 2.075 & & 20.735 & & 0.445 & & Transition.dat \\
\hline 2.325 & & 17.075 & & 0.535 & & Transition.dat \\
\hline 2.575 & & 14.255 & & 0.6 & & Transition.dat \\
\hline 2.825 & & 12.04 & & 0.625 & & Main 1.dat \\
\hline 3.2 & & 9.615 & & 0.605 & & Main 1.dat \\
\hline 3.7 & & 7.165 & & 0.55 & & Main 1.dat \\
\hline 4.2 & & 5.42 & & 0.49 & & Main 1.dat \\
\hline 4.7 & & 4.12 & & 0.435 & & Main 1.dat \\
\hline 5.2 & & 3.115 & & 0.39 & & Main 2.dat \\
\hline 5.7 & & 2.315 & & 0.35 & & Main 2.dat \\
\hline 6.075 & & 1.805 & & 0.325 & & Main 2.dat \\
\hline 6.325 & & 1.51 & & 0.31 & & Main 2.dat \\
\hline 6.575 & & 1.245 & & 0.285 & & Main 2.dat \\
\hline 6.825 & & 1 & & 0.255 & & Main 3.dat \\
\hline 7.075 & & 1.025 & & 0.22 & & Main 3.dat \\
\hline 7.225 & & 1.305 & & 0.195 & & Main 3.dat \\
\hline 7.275 & & 1.69 & & 0.185 & & Main 3.dat \\
\hline 7.325 & & 2.19 & & 0.175 & & Main 3.dat \\
\hline 7.375 & & 2.79 & & 0.165 & & Main 3.dat \\
\hline 7.425 & & 3.57 & & 0.155 & & Main 3.dat \\
\hline
\end{tabular}

\subsubsection{Tower}

One limitation of FAST is that for a floating structure, all parts below the waterline are considered rigid. Therefore, the platform and any portion of the tower that is under water cannot have flexibility. A key issue was therefore to locate where the tower transitions to the platform. Another issue is that the tension wires and spreader-beam system generates an asymmetric stiffening of the tower in the fore and aft directions, whereas the current version of FAST is only able to model symmetric properties for the tower. For this work, the tower-bending flexibility was considered negligible because the wind turbine was freely floating and no significant bending moments were applied at the bottom of the structure. For this reason, the 
tower was modeled as a rigid structure, and the transition point from tower to platform was set at MSL as a first step in getting a working model.

Many components in the design files ((Nichols, 2011), (Holmas, 2010a), (Holmas, 2010b)) - such as instrumentation equipment, service pipes, and a work platform-increase the complexity of building the tower parameters. Because it is impossible to place point masses for these components, calculations were done to account for all the components and obtain the tower properties in Table 4.7 and Table 4.8 .

Table 4.7 Gross Tower Properties

\begin{tabular}{l|l}
\hline Tower height (from MSL) & $12.5 \mathrm{~m}$ \\
Tower base elevation above MSL & $0 \mathrm{~m}$ \\
Total mass & $2449.817 \mathrm{~kg}$ \\
\hline
\end{tabular}

Table 4.8 Tower Distributed Properties

\begin{tabular}{ll}
\hline $\begin{array}{l}\text { Height } \\
(\mathrm{m})\end{array}$ & $\begin{array}{l}\text { Mass } \\
(\mathrm{kg} / \mathrm{m})\end{array}$ \\
\hline 0.00000 & 156.1300 \\
1.10000 & 156.1300 \\
1.10001 & 456.1300 \\
1.60000 & 456.1300 \\
1.61251 & 824.6413 \\
2.10000 & 824.6413 \\
2.10001 & 524.6413 \\
2.80000 & 524.6413 \\
2.80001 & 224.6413 \\
3.20000 & 224.6413 \\
3.20001 & 161.4219 \\
5.70000 & 161.4219 \\
5.70001 & 105.7788 \\
10.90000 & 105.7788 \\
10.90001 & 181.7788 \\
11.90000 & 181.7788 \\
11.90001 & 105.7788 \\
12.50000 & 105.7788 \\
\hline &
\end{tabular}




\subsubsection{Floating Platform}

The floating platform is modeled as a tapered cylinder in FAST. The floating platform model starts from MSL with a constant diameter of $1.0 \mathrm{~m}$ to the depth of $1.9 \mathrm{~m}$, and it tapers to a diameter of $1.4 \mathrm{~m}$ to the depth of $3.4 \mathrm{~m}$. It extends further with a constant diameter of $1.4 \mathrm{~m}$ to the platform base. Table 4.9 summarizes the overall properties of the structure.

Table 4.9 Platform Gross Properties

\begin{tabular}{|c|c|}
\hline $\begin{array}{l}\text { Depth to platform base below MSL (total draft) } \\
\text { Elevation to platform top (tower base) above MSL } \\
\text { Effective platform diameter } \\
\text { Effective platform normalized hydrodynamic viscous } \\
\mathrm{C}_{\mathrm{D}} \\
\text { Platform mass, including ballast } \\
\text { Displacement } \\
\text { Center of mass (CM) below MSL along platform } \\
\text { centerline } \\
\text { Platform roll inertia about } \mathrm{CM}^{\mathrm{b}} \\
\text { Platform pitch inertia about } \mathrm{CM}^{\mathrm{b}}\end{array}$ & $\begin{array}{l}16.4 \mathrm{~m} \\
0 \mathrm{~m} \\
1.4 \mathrm{~m} \\
0.6 \\
18,608.36 \mathrm{~kg} \\
23.216 \mathrm{~m}^{3} \\
12.614 \mathrm{~m} \\
3973.057 \mathrm{E}+03 \mathrm{~kg} \mathrm{~m} \mathrm{~m}^{2} \\
4373.057 \mathrm{E}+03 \mathrm{~kg} \mathrm{~m}\end{array}$ \\
\hline $\begin{array}{l}\text { a The taper in the platform below MSL (which can b } \\
\text { 3) was ignored } \\
\text { b Platform roll and pitch inertia were adjusted du } \\
\text { of the free-decay test. }\end{array}$ & observed in Figure \\
\hline
\end{tabular}

A WAMIT model was built as part of this study to generate the hydrostatic and hydrodynamic matrices required by HydroDyn to account for the radiation and diffraction loads on the body. This model was discretized with 640 panels over one quarter of the body with two planes of symmetry, and it was built using exact design dimensions of the platform that is submerged in the water. In addition, the HydroDyn model includes the viscous drag term of Morison's equation with a normalized drag coefficient of 0.6. This drag coefficient was determined by estimating the $R e$ of the flow over a cylindrical spar, and the flow was found to fall within the post-critical region. The details on the setup of the WAMIT model can be referred in the log file presented in Appendix B. 


\subsubsection{Mooring System}

As shown in Figure 4.13, the SWAY turbine uses a tension rod with universal joints instead of typical slack or taut mooring lines, which are commonly used for floating offshore platforms. Doing so provides restoring forces for the motion in surge, sway, roll, pitch, and heave.

As described in Section 2.4.1, FAST has a quasi-static mooring line model. To represent the tension rod in the FAST model, the tension rod is modeled as a taut mooring line. This assumption is valid, as the SWAY wind turbine should only generate tension forces in the design conditions. To ensure the robustness of the simulation, the fairlead and anchor tension for compressive forces ( $<0$ conditions) were monitored. Mass and thickness properties of the tension rod were used for the mooring-line model. While the specification for the tension rod stiffness was not available from turbine design data, an initial stiffness value was determined from the tension rod geometry and materials. This resulted in an overly stiff mooring line and caused divergence issues in the code. The stiffness was then readjusted to allow for a solution to be computed. The properties of the mooring system are described in Table 4.10 .

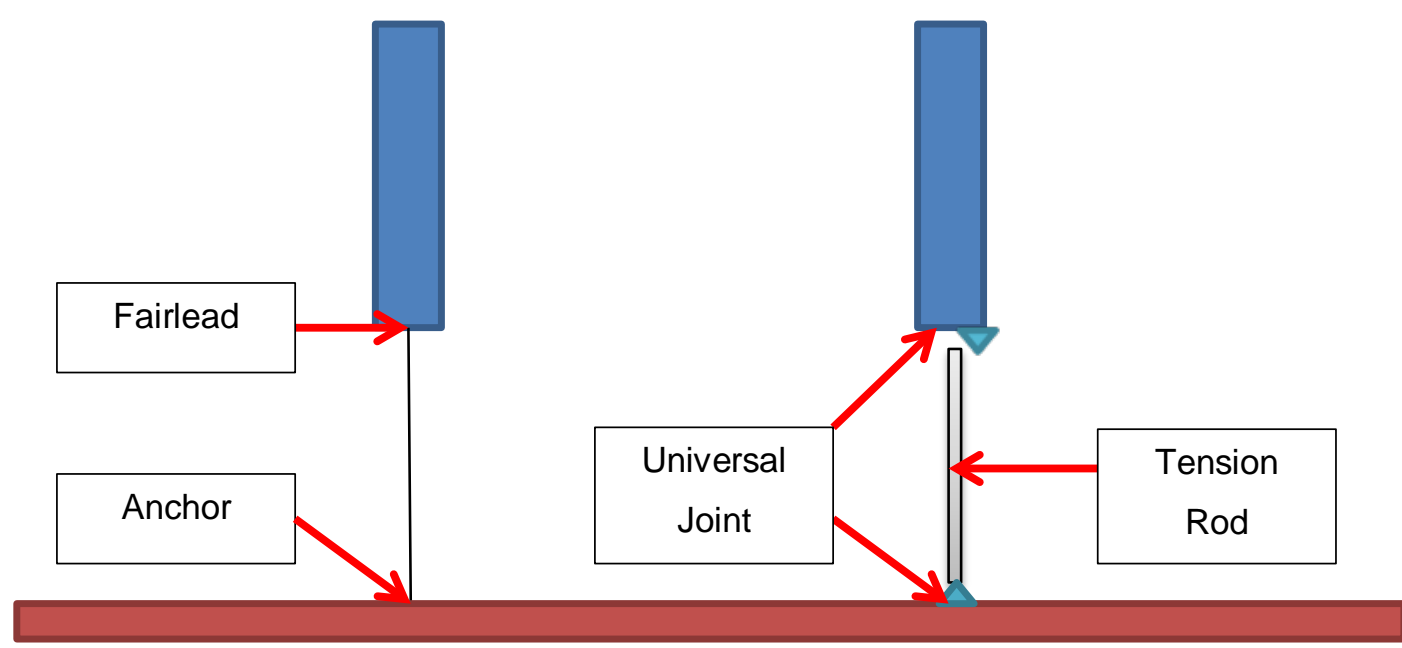

Figure 4.13 Tension line versus tension rod 
Table 4.10 Mooring System Properties

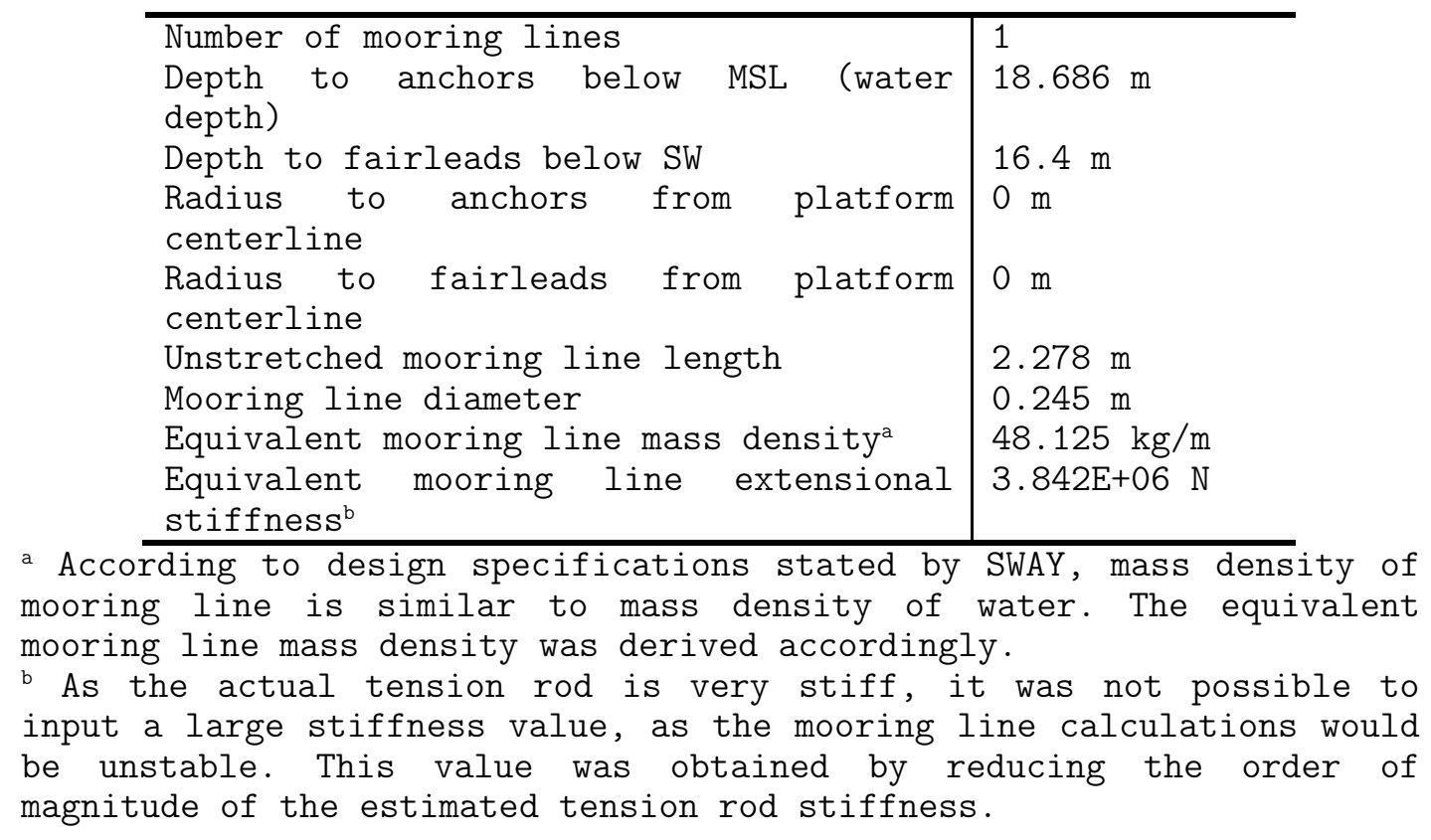

\subsubsection{Other Modeling Assumptions}

The yawing of the wind turbine occurs at the yaw mechanism, which is located at the bottom of the spar, as opposed to a conventional yaw bearing located at the nacelle. A reasonable amount of yaw damping arises from aerodynamic drag, hydrodynamic drag, and friction forces of the yaw bearing at the two universal joints. From the experimental data, minimal yaw movements were observed, and no yaw free-decay test were conducted to quantity the amount of yaw damping. Thus, the nacelle and platform yaw DOF were switched off to simplify the model. This restricts the yaw motion of the wind turbine and allows the model to work without extensive code changes to FAST.

\subsection{Model Calibration}

For this study, most of the wind turbine dimensions and blade properties were provided by SWAY AS. However, a few areas of uncertainties created the need for some calibration of the FAST model to match the recorded response behavior. One issue was the lack of complete information on the weight and location of components added to the tower and turbine. Such appurtenances include instrumentation added for measurements and other 
equipment. To address these uncertainties, the mass distribution of the tower and platform were adjusted as were their mass moments of inertia. Calibration was performed first using data obtained from a center of gravity (CG) test that was performed for the whole system before deployment. This information was used to tune the center of gravity of the FAST model, which is detailed in Section 4.6.1. A static equilibrium test was conducted to check for abnormalities in the model. Next, free-decay tests performed with the structure in the water were used to tune the mass moments of inertia of the platform, as detailed in Section 4.6.2.

From previous work on the DeepCWind validation (Stewart et al., 2012), it was seen that the hydrodynamic damping of FAST can be underestimated. Therefore, an additional quadratic global platform damping matrix was used to better represent the damping of the system. This calibration was also performed using the free-decay tests.

Finally, since modifications were done to the original blade design, a complete set of the as-built blade properties were not available. Therefore, the blade stiffness and damping properties needed to be calibrated to the asbuilt properties. This calibration process is discussed in Section 4.6.3.

\subsubsection{Static Equilibrium Test}

A series of static equilibrium tests were conducted after deployment of the prototype in the ocean. Testing was conducted during calm conditions with a mean wind speed of $1.7 \mathrm{~m} / \mathrm{s}$ and minimum and maximum values of 0.25 $\mathrm{m} / \mathrm{s}$ and $5 \mathrm{~m} / \mathrm{s}$ respectively. The ocean was predominately flat with very small wind-generated capillary waves. The current velocity ranged from 6 to $8 \mathrm{~cm} / \mathrm{s}$ based on hourly averages. The turbine did not operate during the tests and it was initially expected that the calm conditions had minimal impact on the experimental data. Therefore, a static-equilibrium simulation was initially carried out with no wind-wave-current conditions on the FAST model to obtain the natural equilibrium position of the turbine. 
In earlier work on the SWAY turbine (Koh et al., 2013), minor adjustments were made to the nacelle CG and the turbine overhang values in FAST to calibrate the mean pitch offset to $1^{\circ}$ (compared to an experimental value of $0.98^{\circ}$ ) and the mean surge offset to $0.287 \mathrm{~m}$ (compared to an experimental value of $0.34 \mathrm{~m})$. The sway, heave, and roll offsets were negligible. In a further analysis (Koh, Robertson, Jonkman, Driscoll, \& Ng, 2015), these minor adjustments were found to be unnecessary. Two other cases with very low wind conditions at $1 \mathrm{~m} / \mathrm{s}$ mean wind speed (upwind and downwind direction) at hub height were simulated. The results are shown in Table 4.11 .

Table 4.11 Results of Static Equilibrium Analysis

\begin{tabular}{l|lllll}
\hline Mean Offset & Experiment & $\begin{array}{l}\text { FAST } \\
\text { wind) }\end{array}$ & $\begin{array}{l}\text { (no } \\
\text { FAST } \\
\text { downwind) }\end{array}$ & $\begin{array}{l}\text { FAST } \\
\text { upwind) }\end{array}$ \\
\hline Pitch (degree) & 0.98 & 1.79 & 1.95 & -1.64 \\
Surge (m) & 0.34 & 0.51 & 0.56 & -0.031 \\
\hline
\end{tabular}

The system's equilibrium position is fairly sensitive to the environmental conditions, as can be seen by the change in surge and pitch offset for a 1 $\mathrm{m} / \mathrm{s}$ upwind versus downwind condition. Since the static equilibrium of the prototype was calculated in varying wind conditions, it would be difficult to know what the static offsets would be without wind, and therefore the previous calibration work to achieve the correct static offsets were removed and the model was reverted to the original properties provided by the manufacturer.

\subsubsection{Free Decay Test}

Further calibration of the model was performed using free-decay test data. After turbine deployment and installation of the NREL instrumentation, five free-decay tests were conducted (Figure 4.14) on the SWAY prototype by displacing the system and allowing it to return to equilibrium: two in the roll direction, and one each for the pitch, surge, and sway directions. Ropes were attached to the nacelle and the base of the tower to perform the displacement. The ropes were held with a quick release system and pulled 
onshore (nearby to the prototype installation) to set the system to the desired initial displacement of the system.
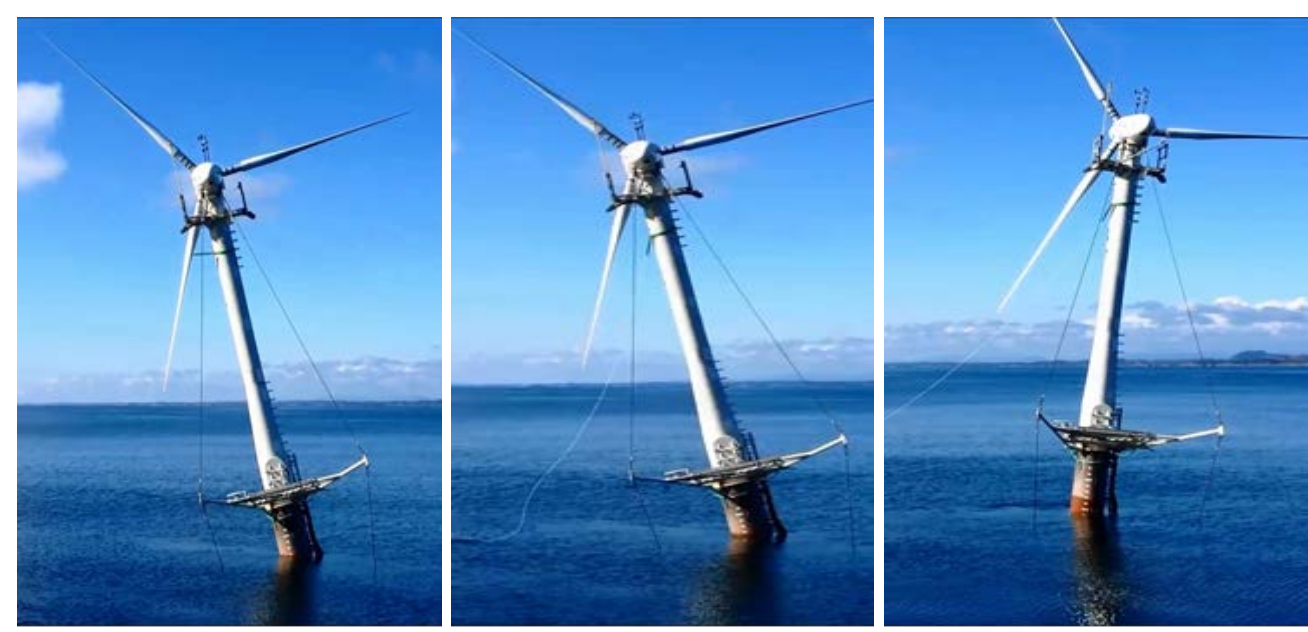

Figure 4.14 Free Decay Test

Testing was conducted in the same conditions as the static equilibrium test were conducted, and the calm conditions were expected to have minimal impact on the experimental data, as the predominant motion of the system will be due to the free-decay conditions. Therefore, the free-decay simulations were conducted in no wind, wave or current conditions.

In an ideal condition, only one DOF would be excited at a time in a freedecay test to identify the frequency and damping of that DOF. However, during the actual test, the primary DOF of interest in each test was strongly coupled with other DOFs. For example, during a roll free-decay test, the sway DOF was heavily coupled while the pitch and surge of the system experienced small motions. Nonetheless, the results collected were sufficient to allow for manual tuning of the mass moment of inertia and hydrodynamic viscous damping of the platform to calibrate the natural frequency and response of the system. Of five free-decay tests, only one roll free-decay test and one pitch free-decay test were used for calibration.

\subsubsection{Roll Free-Decay}

The initial displacements (surge, sway, and heave) and rotations (roll and pitch) from the roll free-decay test were used as initial conditions in the 
FAST model. A free-decay simulation was then run, and the platform inertia and roll term (diagonal-only) in the damping matrix was tuned so that the roll period and magnitude matched the experimental data.

The experiments exhibited an average platform roll period of 45.6 seconds (s) while the FAST simulations had an average period of $44.0 \mathrm{~s}$ after calibration of the mass moment inertia of the platform and platform damping matrix. It is noted that both the experimental and simulated roll periods varied slightly throughout the entire free-decay test (the first experimental oscillation period was $42.9 \mathrm{~s}$ ). The roll motion of the system after tuning is shown in Figure 4.15. The simulated motion follows the experimental motion quite closely. Significant deviations of the period and amplitude occur after the fourth oscillation when the magnitude of oscillation decreases.

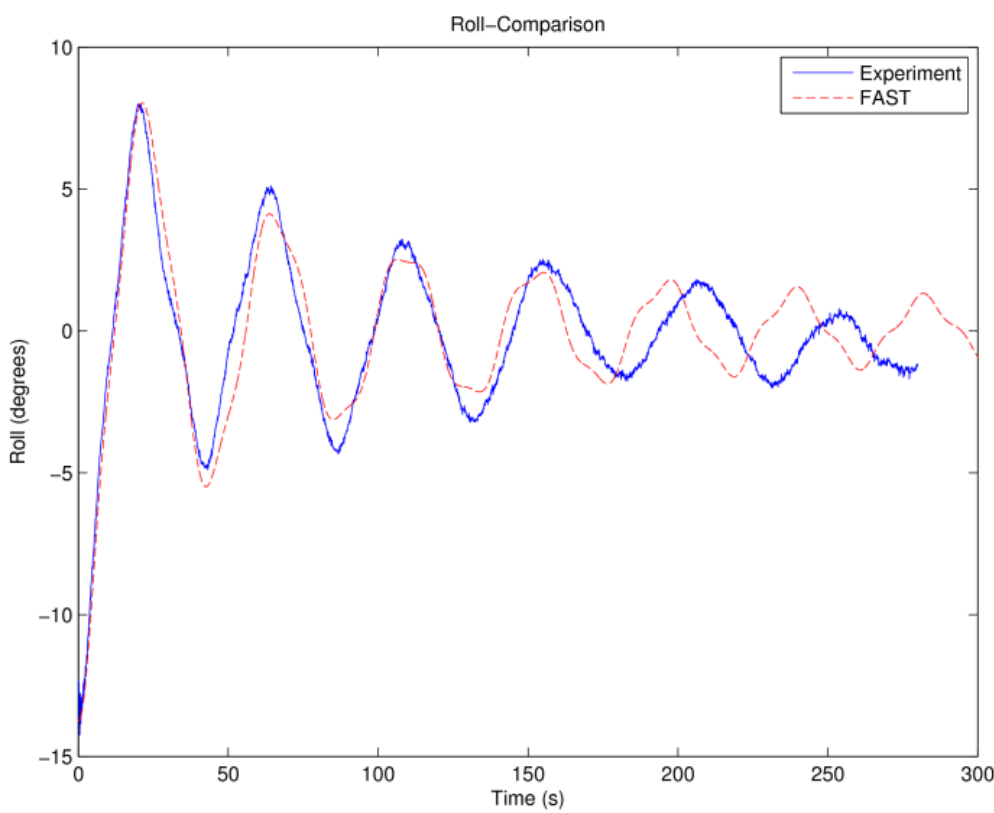

Figure 4.15 Roll motion in roll free decay

A fast Fourier transform (FFT) was carried out for the first $280 \mathrm{~s}$ of experimental time and simulation time to analyze the data in the frequency domain. Figure 4.16 shows the FFT results and enlarged views around the main peaks. The peak frequency of the experimental and simulated results occurs at values of about $0.02143 \mathrm{~Hz}$ and $0.02333 \mathrm{~Hz}$, which correspond to periods of $46.86 \mathrm{~s}$ and $43.48 \mathrm{~s}$ respectively. This is the roll frequency of the 
whole system and the calibration was deemed sufficient due to the low resolution in the experiment data.
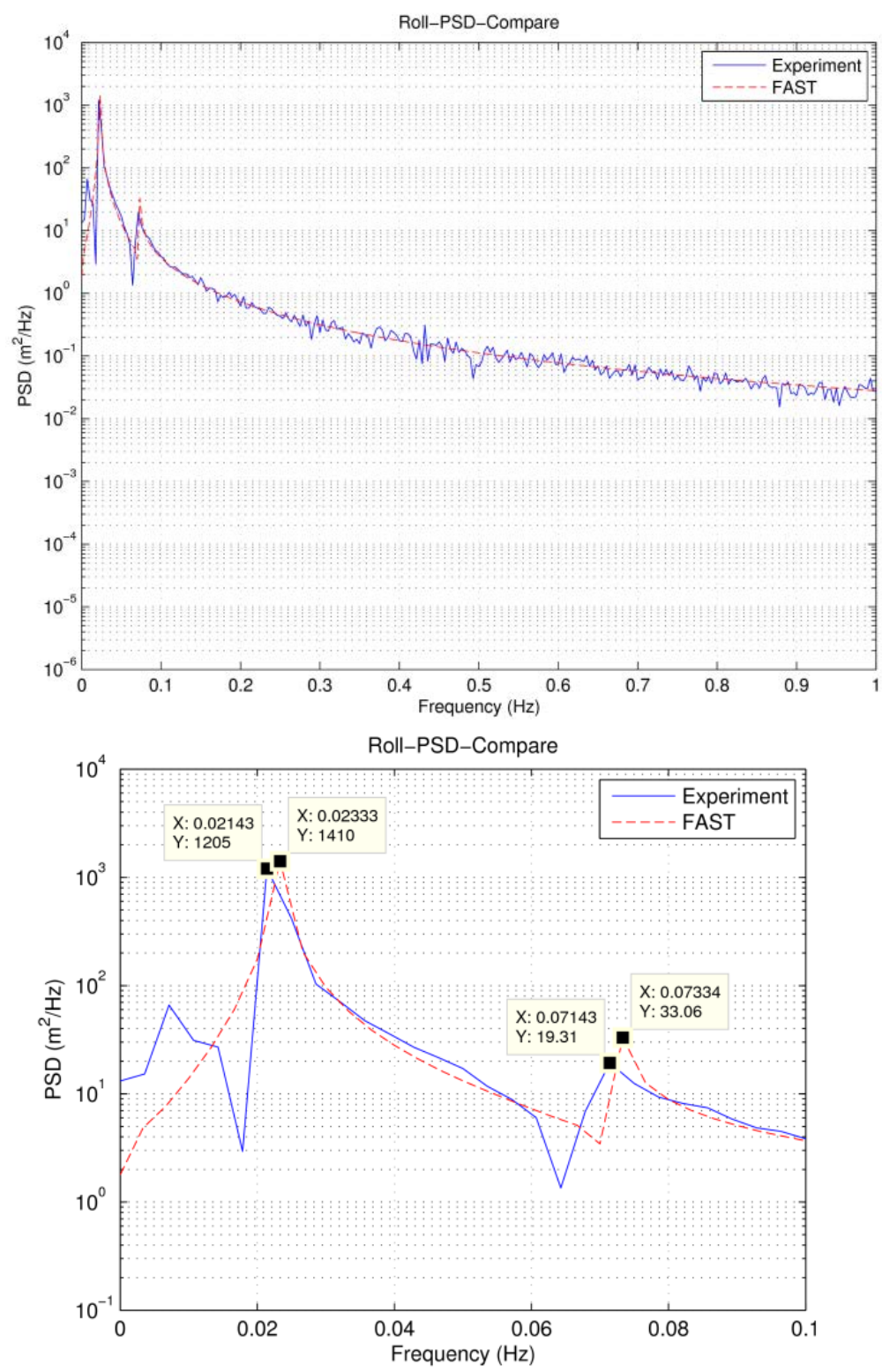

Figure 4.16 Power spectral density (PSD) versus frequency of roll DOF (enlarged on right)

A second peak frequency was also observed for experimental and simulated results occurring at values of about $0.07143 \mathrm{~Hz}$ and $0.07334 \mathrm{~Hz}$ respectively. The second peak frequency is caused by the flexibility of the universal joint between the spar structure and tension rod, which allows both to rotate about this joint. An illustration of the physical motion of the first and second peak frequency is depicted in Figure 4.17. This is verified by 
analyzing the measured angle of the tension rod with a motion plot and FFT analysis.

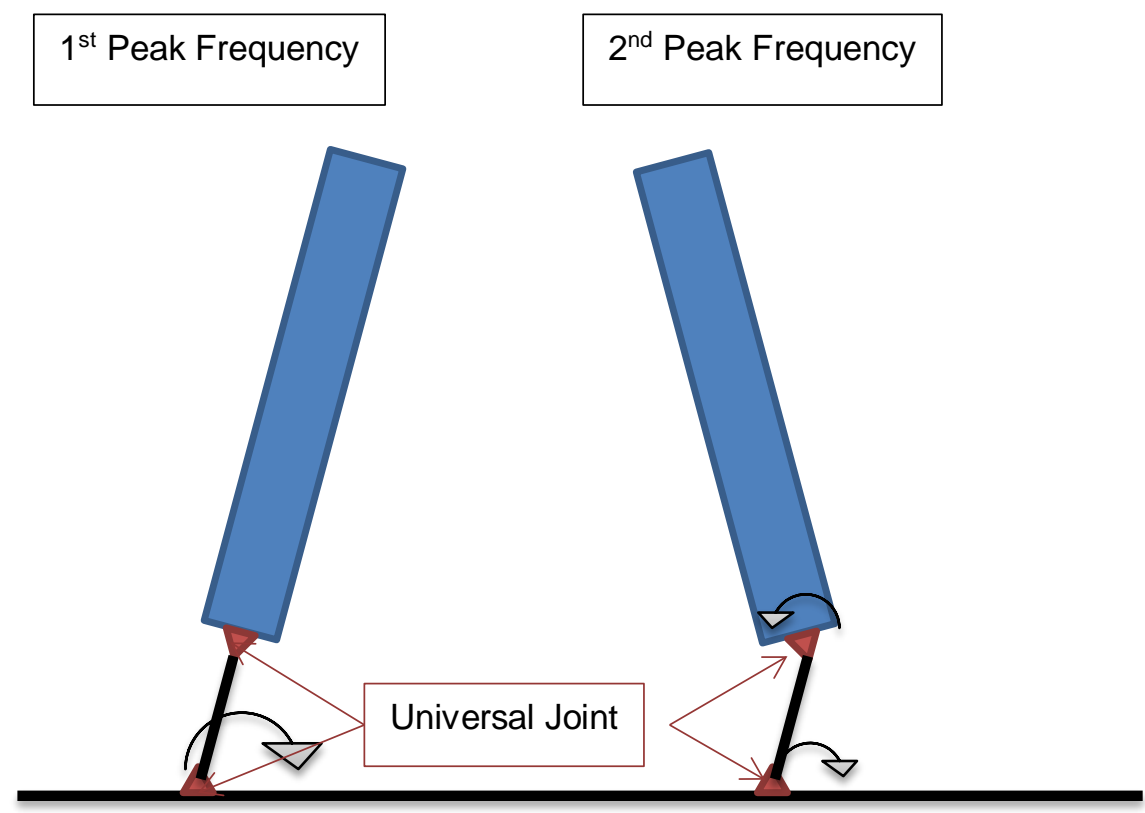

Figure 4.17 Illustration of physical motion of first and second peak frequency Figure 4.18 shows the FFT results, indicating the primary frequency is $0.0681 \mathrm{~Hz}$ and $0.07168 \mathrm{~Hz}$ from the experimental data and FAST analysis respectively, which corresponds closely to the second peak shown in the FFT results in Figure 4.16.
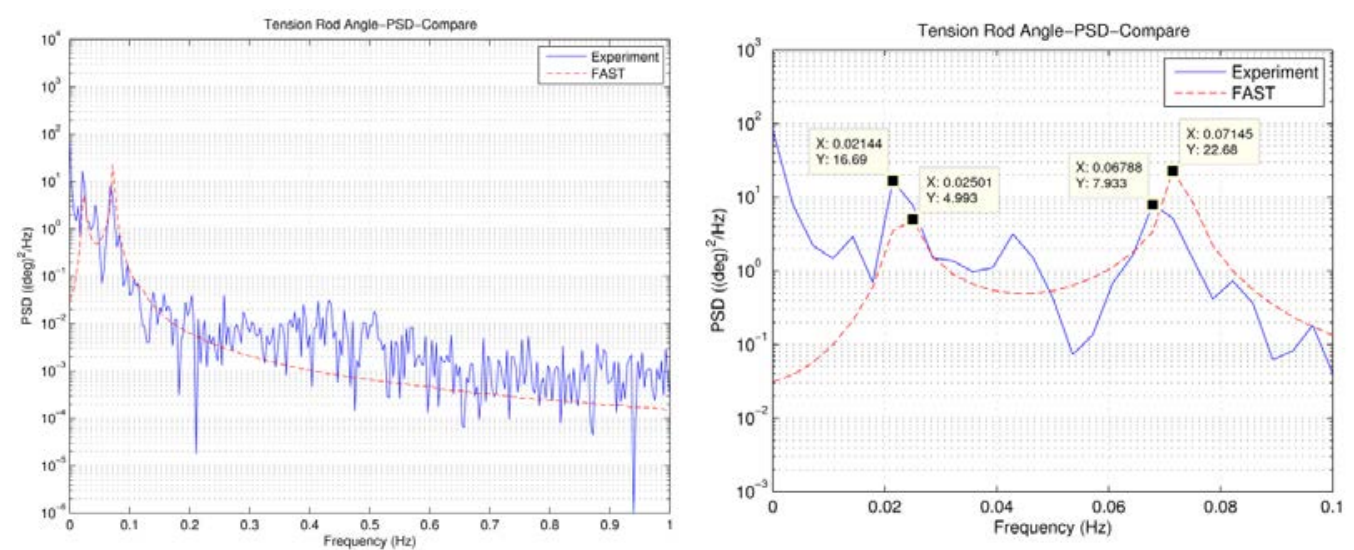

Figure 4.18 PSD versus frequency of tension rod motion (enlarged view on right)

\subsubsection{Pitch Free-Decay}

Next, initial displacements and rotations were set in the FAST model to match the starting conditions for the pitch free-decay test. The simulated 
response in FAST was used to tune the platform inertia and damping matrix in the pitch direction (diagonal-term) so that the pitch period matched that of the experimental data.

The experiment had an average platform pitch period of 44.6 s. After calibration, the FAST simulation had an average period of 42.9 s. Similarly, both the experimental and simulated period varied slightly throughout the entire free- decay test. For the first oscillation, the experimental period of the pitch motion was $42.5 \mathrm{~s}$ while the simulation period was $43.7 \mathrm{~s}$. The pitch offset was similar (at about $1^{\circ}$ ) for the experimental and simulation results. The motion plots are shown in Figure 4.19. The simulated motion follows the experimental motion rather closely. Noticeable deviations for period and amplitude occur after the fifth oscillation when the magnitude of oscillation has decreased over time.

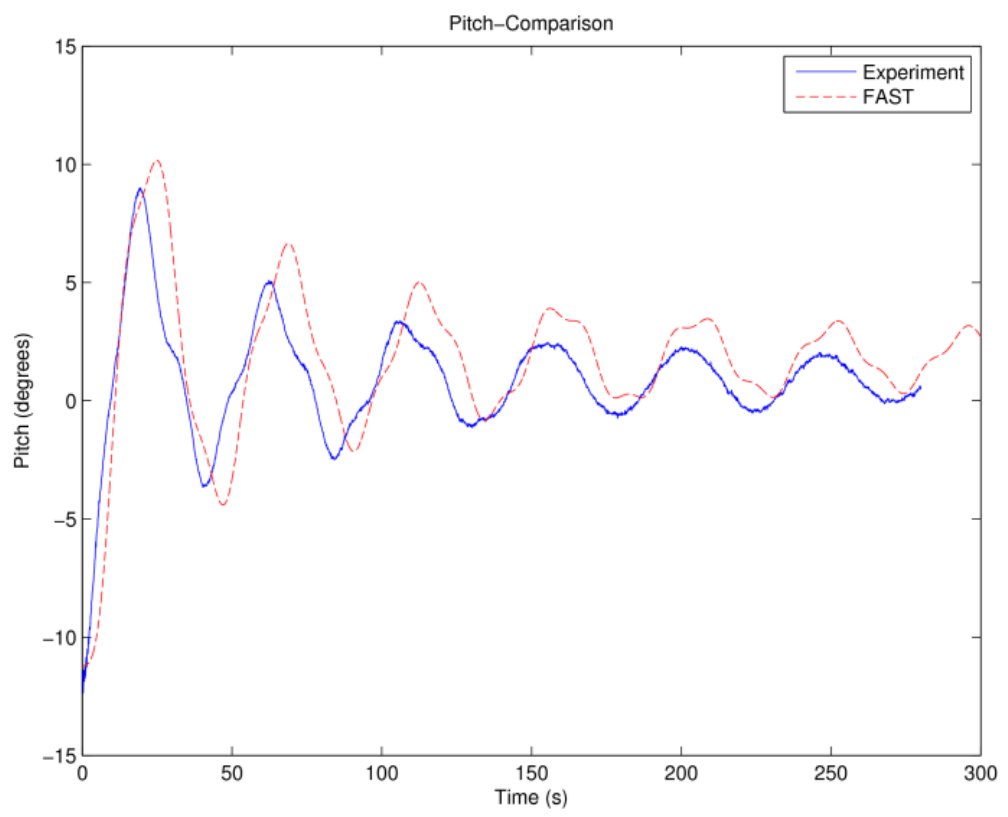

Figure 4.19 Pitch motion in pitch free decay

An FFT was carried out for the first $300 \mathrm{~s}$ of experimental and simulation time. Figure 4.20 shows the FFT results and enlarged views around the main peaks. The peak frequency of the measured and simulated pitch occurs at values of about $0.02143 \mathrm{~Hz}$ and $0.02333 \mathrm{~Hz}$, which corresponds to periods of $46.66 \mathrm{~s}$ and $42.86 \mathrm{~s}$. This frequency is the pitch frequency of the whole system. A second peak frequency is also observed for measured and 
simulated pitch occurring at values of about $0.07143 \mathrm{~Hz}$ and $0.07 \mathrm{~Hz}$. As with the roll free-decay analysis, the second peak frequency is caused by the flexibility of the universal joint between the spar structure and tension rod.

Due to the small time length and sampling rate $(10 \mathrm{~Hz})$ of the test results, the range and resolution of the frequency data is limited. This error is significant at lower frequencies. For example, for the pitch free-decay test, the next frequency higher than $0.02333 \mathrm{~Hz}$ is $0.02667 \mathrm{~Hz}$, whereby the period would have decreased to 37.5 s. Nonetheless, the FFT analysis suggests matching frequency trends between experimental and simulation results.
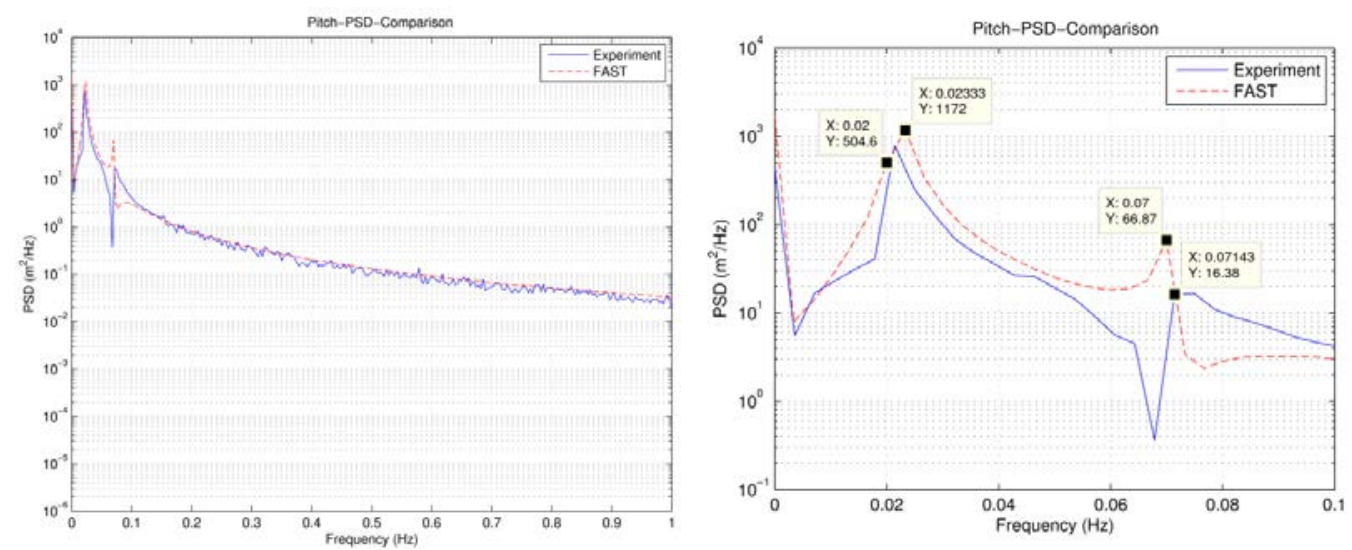

Figure 4.20 PSD versus frequency of pitch DOF (enlarged view on right)

\subsubsection{Blade Stiffness and Damping Calibration}

As discussed in Section 4.5.1, the blade properties were obtained from a GL Garrad Hassan technical report (Nichols, 2011), which did not consider a blade extension that was added later. The blade properties therefore needed to be calibrated to match those of the installed system.

The blade flapwise and edgewise blending loads were analyzed using one 10minute (min) test case with unsteady wind conditions at a mean wind speed 
of $4.35 \mathrm{~m} / \mathrm{s}$ at anemometer height ${ }^{1}$. Inconsistent results were seen for the magnitude of the edgewise loads for Blade 2 as compared to Blades 1 and 3. Figure 4.21 shows the FFT analysis of the flapwise blade loads while Figure 4.22 shows the FFT analysis of the edgewise blade loads for Blade 1 from the experimental data. In the flapwise direction, the FFT analysis indicates that the first flapwise modal frequency is about $4.156 \mathrm{~Hz}$ and the second flapwise modal frequency is about $10.18 \mathrm{~Hz}$. In the edgewise direction, the FFT analysis shows that the first edgewise modal frequency was about 6.072 $\mathrm{Hz}$, with another peak detected at about $4.113 \mathrm{~Hz}$ which is associated with the first flapwise modal frequency.

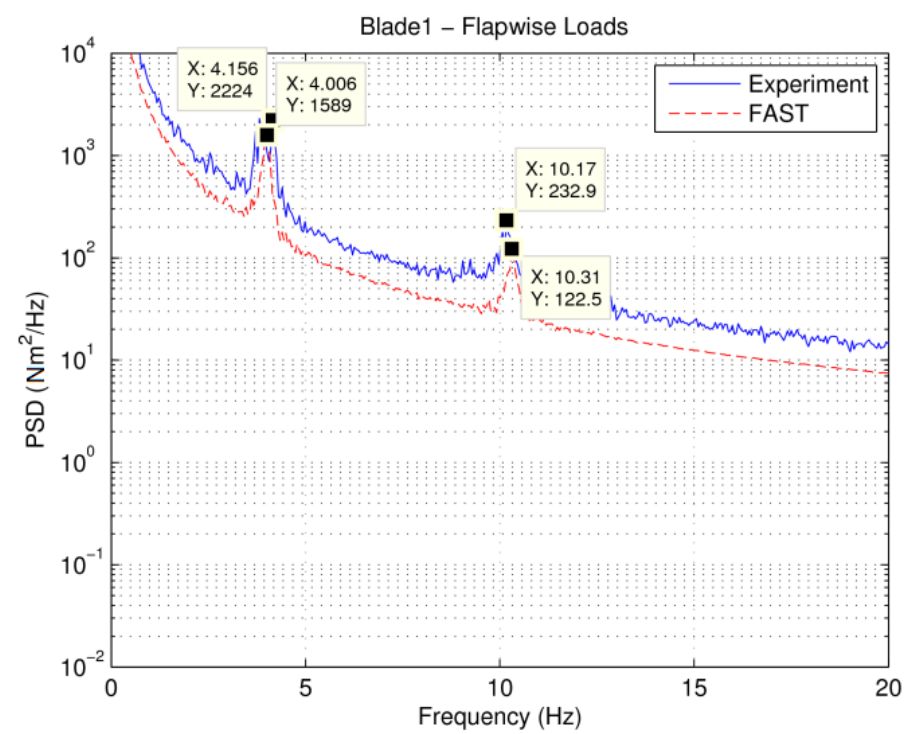

Figure 4.21 FFT analysis of flapwise blade loads at wind speed $=4.35 \mathrm{~m} / \mathrm{s}$ for FAST and experiment response

${ }^{1}$ Rotational speed of blades for wind speed at $4.35 \mathrm{~m} / \mathrm{s}$ were at 0.03181 radians per second or 0.3038 RPM. 


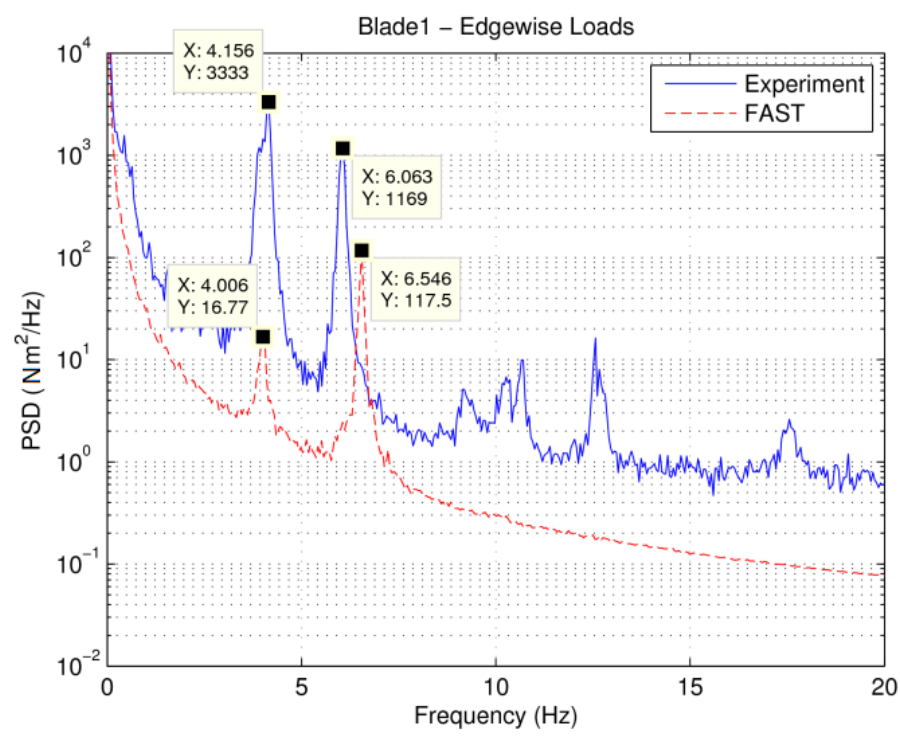

Figure 4.22 FFT analysis of edgewise blade loads at wind speed $=4.35 \mathrm{~m} / \mathrm{s}$ for FAST and experiment response

To calibrate the blade modal parameters, the test case was simulated in FAST, and the blade flapwise modal stiffness tuner for the first and second modes was adjusted with factors of 1.15 and 0.6 respectively. The second factor is too large to be considered practical, and ideally the properties of the blade should be re-examined. However, for this analysis, our focus is on the global motions, and so this activity was not undertaken. FAST does not provide an edgewise modal stiffness tuner, but results are nonetheless compared.

After tuning, the first flapwise modal frequency was about $4.006 \mathrm{~Hz}$ while the second flapwise modal frequency was about $10.31 \mathrm{~Hz}$. Even without tuning (edgewise modal stiffness tuner is not available in FAST), the FFT analysis shows a good comparison for the edgewise modal frequency with experimental data. The first edgewise modal frequency was about $6.546 \mathrm{~Hz}$ and the first peak at $4.006 \mathrm{~Hz}$ is influenced by the first flapwise model frequency and was ignored.

Higher magnitude in the experiment data of the FFT analysis was observed for the flapwise blade loads in Figure 4.21, and more significantly for edgewise blade loads presented in Figure 4.22. 
Table 4.12 System Eigenfrequencies

\begin{tabular}{l|l|l}
\hline Degree of Freedom & $\begin{array}{l}\text { Experimental } \\
\text { Frequency }(\mathrm{Hz})\end{array}$ & $\begin{array}{l}\text { Calibrated Model } \\
\text { Frequency (Hz) }\end{array}$ \\
\hline Roll (1 ${ }^{\text {st }}$ peak) & 0.02143 & 0.02333 \\
Roll (2 ${ }^{\text {nd }}$ peak) & 0.07143 & 0.07334 \\
Pitch (1 $1^{\text {st }}$ peak) & 0.02143 & 0.0233 \\
Pitch (2 peak) & 0.07143 & 0.07 \\
Blade $-1^{\text {st }}$ flapwise & 4.156 & 4.006 \\
Blade $-2^{\text {nd }}$ flapwise & 10.18 & 10.31 \\
Blade $-1^{\text {st }}$ edgewise & 6.072 & 6.546 \\
\hline
\end{tabular}

\subsection{Model Validation}

Once the FAST model had been calibrated, the accuracy of the model could be validated against the remaining available test data. This validation procedure is reviewed in this section. First, two non-operational data sets are used (Section 4.7.1), and validation was then performed for two operational cases (Section 4.7.2).

\subsubsection{Non-operating Conditions}

\subsubsection{Selection of Test Cases}

Given the large number of data sets recorded, it is important to start the validation work with simple test cases that have stable conditions over an extended period of time. Therefore, two 10-min data sets (Non-Op Case 1 and 2) were selected with the following criteria for the ease of modeling in FAST:

1. Instruments operating without abnormalities

2. Zero or very low rotor speed values

3. Mean wind speed of the preceding 10-min window has less than $5 \%$ in variation from the selected test case

4. Mean wind direction of the preceding 10-min window has less than $5^{\circ}$ in variation from the selected test case.

The criteria are focused on (1) reducing any transient motion generated by the conditions before the actual test case and (2) maintaining consistency of the wind throughout the test period. The wave height measurements were 
recorded as an average over either 20 minutes or 1 hour, and we therefore wanted to choose a 10-min data window that would not differ considerably from the 1-hour average.

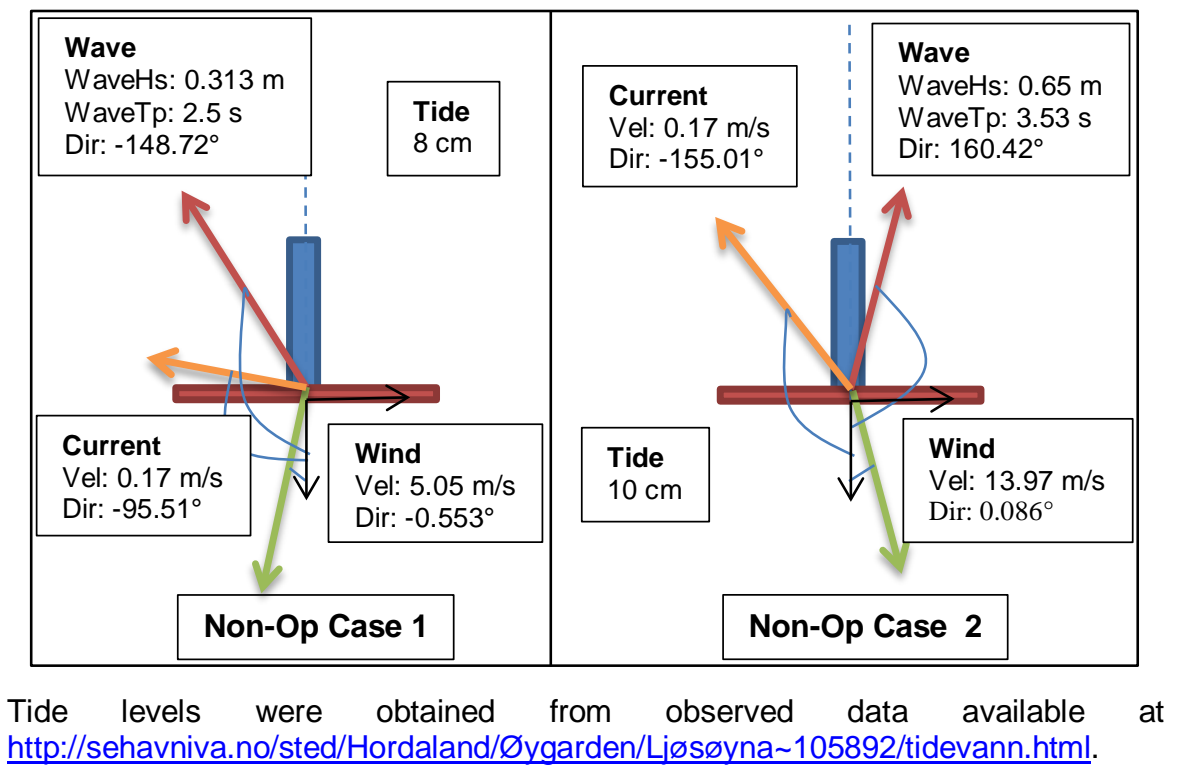

Figure 4.23 Summary of non-operational test cases

The two 10-min test cases that were selected have mean wind speeds of 5 $\mathrm{m} / \mathrm{s}$ and $14 \mathrm{~m} / \mathrm{s}$ with corresponding significant wave heights of $0.31 \mathrm{~m}$ and $0.65 \mathrm{~m}$. The wind, wave, and current conditions are summarized in Figure 4.23. The accuracy of these data recordings was checked by comparing it to other data sets of similar conditions to ensure the recorded data was consistent.

\subsubsection{Simulation Procedure}

The system was first set at an equilibrium position calculated from a simulation of the static equilibrium. The FAST simulation was conducted for a simulation time of $1,200 \mathrm{~s}$ (20 minutes), and the first 10 minutes of the simulation were discarded to remove any initial transient behavior.

\subsubsection{Wind}

The wind inflow files used by AeroDyn were generated using TurbSim with the settings shown in Figure 4.14. The mean wind speed and turbulence 
intensity were obtained from the sonic anemometer readings at the boom. Note that the minor yaw error (shown as wind direction in Figure 4.23) is a result of the system being a free-yaw system. This error was represented as a yaw offset in FAST. The analysis time was set at $1200 \mathrm{~s}$, to allow for 10 minutes of data after any initial transient behavior had died out. The vertical and horizontal grid-point sizes were set so that they were approximately the mean chord length of the blade.

Table 4.13 TurbSim Wind Inflow Properties for Non-Operating Case

\begin{tabular}{|c|c|}
\hline & Non-Op Case 2 \\
\hline $\begin{array}{lll}\text { Vertical grid-point } & \text { matrix } \\
\text { dimension } & \end{array}$ & 51 \\
\hline $\begin{array}{l}\text { Horizontal grid-point matrix } \\
\text { dimension }\end{array}$ & 63 \\
\hline Hub height $[\mathrm{m}]$ & 13.133 \\
\hline Grid height [m] & 20.000 \\
\hline Grid width [m] & 25.000 \\
\hline Turbulence model & $\begin{array}{c}\text { IEC Kaimal spectral normal } \\
\text { turbulence model }\end{array}$ \\
\hline $\begin{array}{l}\text { Turbulence intensity } \\
\text { Reference wind speed }[\mathrm{m} / \mathrm{s}]\end{array}$ & \begin{tabular}{c|c}
$25.856 \%$ & $13.715 \%$ \\
5.0460 & 13.970 \\
\end{tabular} \\
\hline $\begin{array}{l}\text { Reference height of anemometer } \\
\text { [m] }\end{array}$ & 12.500 \\
\hline Wind profile type & $\begin{array}{c}\text { IEC, Power law on rotor disk, } \\
\text { logarithmic elsewhere }\end{array}$ \\
\hline Power law exponent & 0.140 \\
\hline Surface roughness length & $0.030^{a}$ \\
\hline
\end{tabular}
61400-3 for offshore conditions.

The turbulence model was assumed to follow the IEC Kaimal spectral normal turbulence model (B. J. Jonkman \& Kilcher, 2012), and the turbulence intensity and reference wind speed were derived from sonic anemometer readings of wind speeds from the four test cases. The power law exponent and surface roughness length are recommended values from the IEC 61400-3 (2009).

Since the wind input file was generated in TurbSim with no mean crosswind component as explained previously, it is important to verify the statistical properties of the wind inflow, particularly the crosswind component of the system. 
Figure 4.24 and Figure 4.25 show the time domain comparison of the wind inflow velocity for downwind and crosswind directions for both FAST and the experimental data. The spikes observed in the experimental data in these figures are assumed to be due to errors in the sensor or data logger. The horizontal lines in the graphs represent the mean value of the wind inflow velocity. In both cases, the mean and standard deviation of the wind inflow velocity for the experiment reveal good comparison with the wind inflow generated by TurbSim. As the FAST data are obtained at the hub height while the experimental data were obtained at the boom height, this height difference led to a slightly larger mean for the experiment data. Summaries of the measured and simulated wind properties for both test cases are shown in Table 4.14 and Table 4.15. 

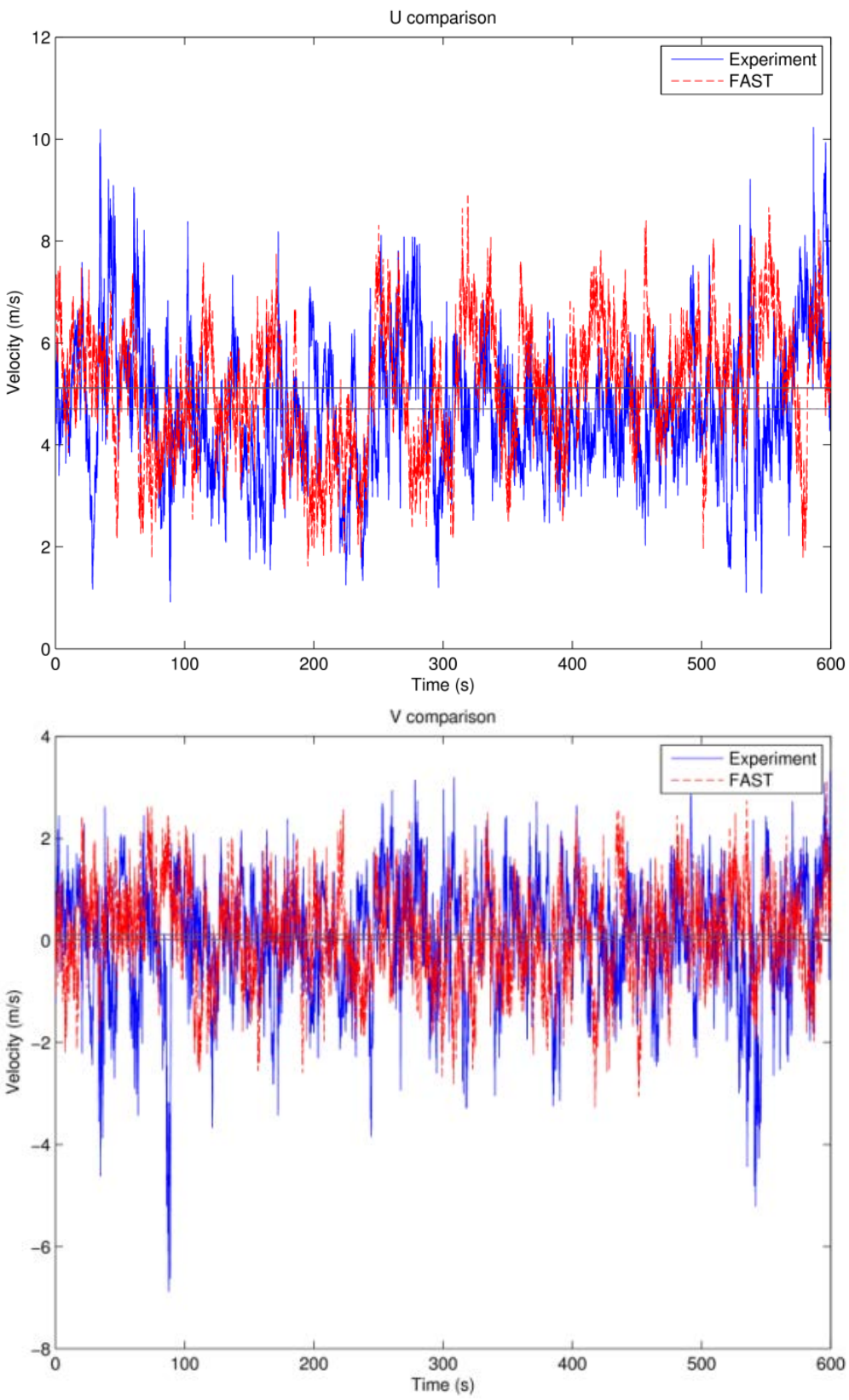

Figure 4.24 Velocity inflow comparison (Non-Op Case 1) 

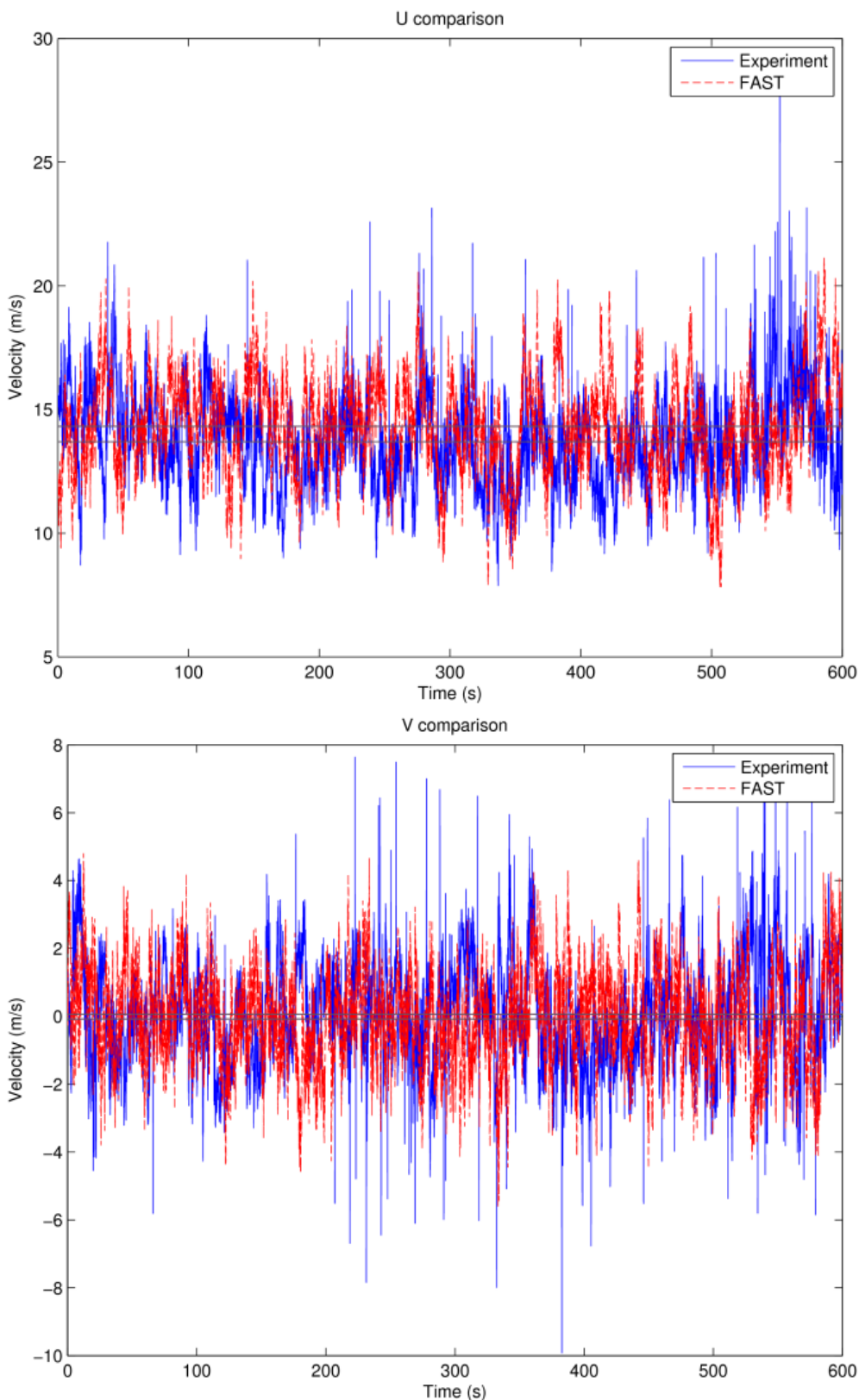

Figure 4.25 Velocity inflow comparison (Non-Op Case 2)

Table 4.14 Comparison of Wind Properties for Non-Op Case 1

\begin{tabular}{l|llllll}
\hline & Mean (Exp) & Mean (FAST) & $\begin{array}{l}\text { Std. } \\
\text { (Exp) }\end{array}$ & Dev. & $\begin{array}{l}\text { Std. } \\
\text { (FAST) }\end{array}$ & Dev. \\
\hline $\mathrm{U}(\mathrm{m} / \mathrm{s})$ & 4.7014 & 5.1131 & 1.2985 & 1.2453 & \\
$\mathrm{~V}(\mathrm{~m} / \mathrm{s})$ & 0.0218 & 0.1170 & 1.1573 & 0.9159 & \\
\hline
\end{tabular}

Table 4.15 Comparison of Wind Properties for Non-Op Case 2

\begin{tabular}{l|lllll}
\hline & Mean (Exp) & Mean (FAST) & $\begin{array}{l}\text { Std. } \\
\text { Dev. (Exp) }\end{array}$ & $\begin{array}{l}\text { Std. } \\
\text { (FAST) }\end{array}$ & Dev. \\
\hline $\mathrm{U}(\mathrm{m} / \mathrm{s})$ & 13.6939 & 14.3237 & 1.8012 & 1.9356 & \\
$\mathrm{~V}(\mathrm{~m} / \mathrm{s})$ & 0.0642 & -0.0761 & 1.5433 & 1.4118 & \\
\hline
\end{tabular}




\subsubsection{Wave}

In the initial FAST simulation runs, the irregular waves were simulated in FAST by assuming that the energy distribution follows the PM spectrum (the JONSWAP spectrum was not used because of the less pronounced peak shown in Figure 4.26 and Figure 4.27). The wave parameters used in the FAST simulation were obtained from the 1-hr averaged data observed by the AWAC; however, the wave spectrum was not represented well, particularly in the lower frequency region. The wave and current data available from the AWAC were averaged to 1-hr readings, so there will be inaccuracy in the simulation results, as wave and current properties may differ significantly within the 1-hr sampling period. Also the wave conditions at the system location may be bi-modal/multi-modal in nature, which means they cannot be represented from the 1-hr averaged data from the AWAC, because it only returns the peak frequency and period.

Therefore, a user-defined wave spectrum was entered into FAST from the wave spectrum obtained from the adjusted 10-min wave height data with the tower wave height sensor. The mean wave direction was obtained from the 1-hr averaged data observed by the AWAC. This process was performed for both cases and the time- and frequency-domain comparisons of the waves generated in FAST and the experimental data are included in Figure 4.26 and Figure 4.27. The wave spectrums in these figures do not match perfectly because only the latter 10 minutes of the waves generated from 20 minutes in FAST were processed. The time histories are also not expected to match because phases of the simulated wave time history are random. 

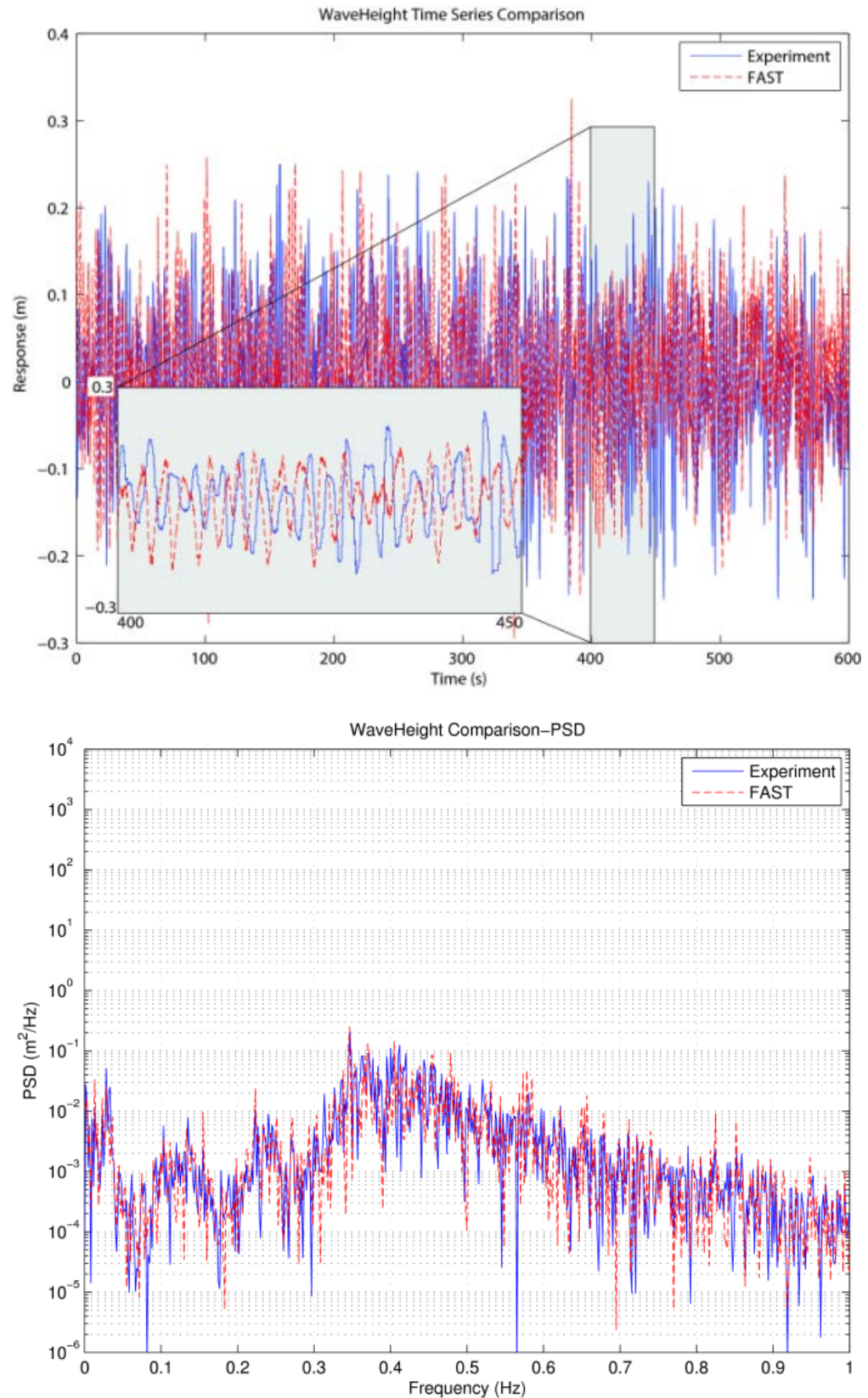

Figure 4.26 Wave height comparison (Non-Op Case 1) 

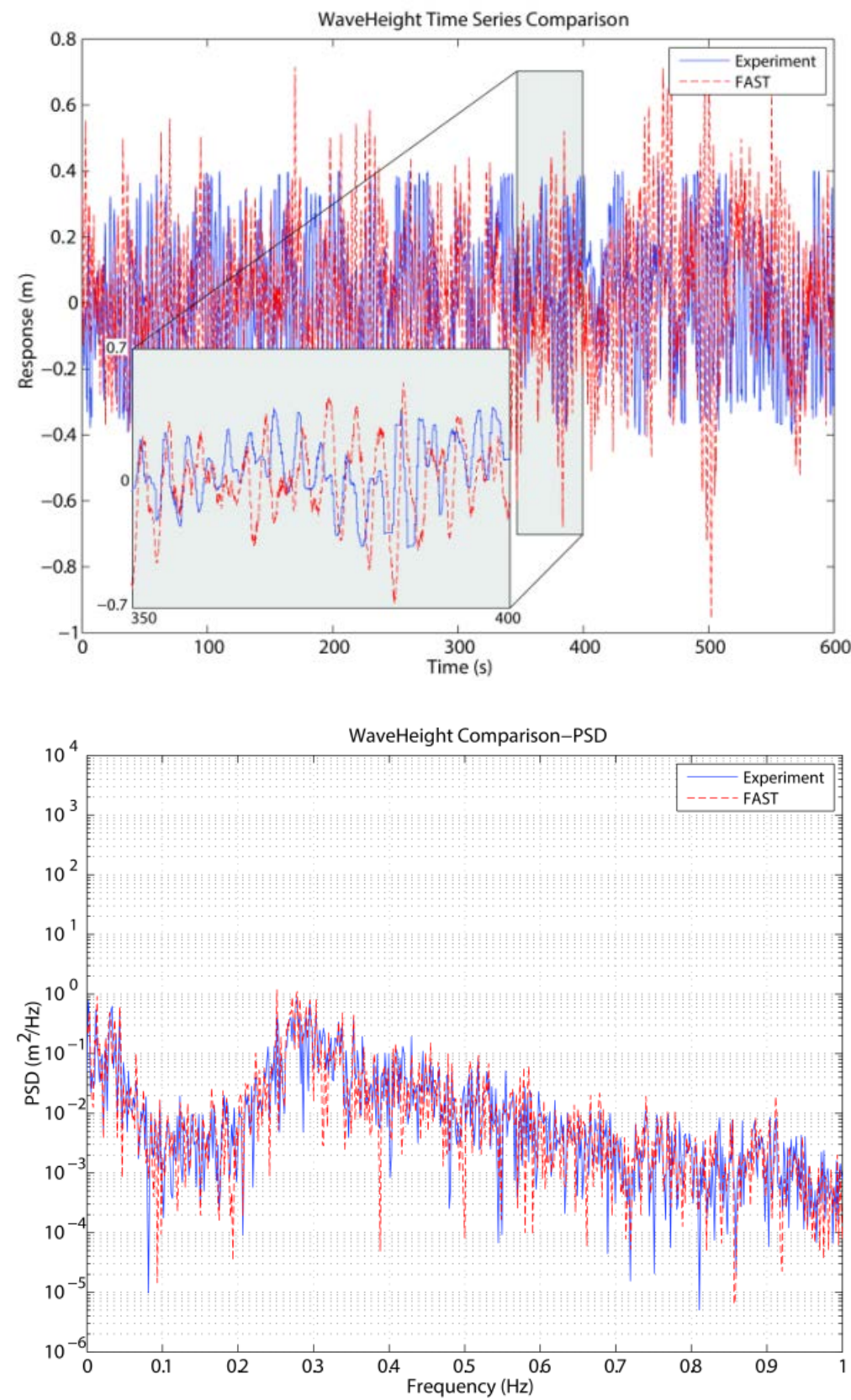

Figure 4.27 Wave height comparison (Non-Op Case 2)

\subsubsection{Results}

Table 4.16 and Table 4.17 show the mean and standard deviation values for the pitch, surge, roll, and sway motions of the wind turbine over the 10-min period for Non-Op Case 1 and Non-Op Case 2 respectively. The corresponding power spectral density (PSD) plots are shown in Figure 4.28 and Figure 4.29. 
Table 4.16 Mean and Standard Deviation for DOFs for the Non-Op Case 1

\begin{tabular}{l|lll}
\hline DOFs & Experiment & $\begin{array}{l}\text { FAST } \\
\text { Tower Load) }\end{array}$ & $\begin{array}{l}\text { FAST } \\
\text { Tower Load) }\end{array}$ \\
\hline Pitch (Mean) & $2.38470^{\circ}$ & $3.12673^{\circ}$ & $2.23870^{\circ}$ \\
Pitch (Std) & $0.81103^{\circ}$ & $0.86218^{\circ}$ & $0.60963^{\circ}$ \\
Surge (Mean) & $1.81859 \mathrm{~m}$ & $0.92096 \mathrm{~m}$ & $0.64828 \mathrm{~m}$ \\
Surge (Std) & $0.22220 \mathrm{~m}$ & $0.26364 \mathrm{~m}$ & $0.18665 \mathrm{~m}$ \\
Roll (Mean) & $-0.19097^{\circ}$ & $-0.01335^{\circ}$ & $-0.00286^{\circ}$ \\
Roll (Std) & $0.822535^{\circ}$ & $0.30514^{\circ}$ & $0.270413^{\circ}$ \\
Sway (Mean) & $1.54107 \mathrm{~m}$ & $-0.01370 \mathrm{~m}$ & $-0.01166 \mathrm{~m}$ \\
Sway (Std) & $0.25466 \mathrm{~m}$ & $0.08878 \mathrm{~m}$ & $0.07700 \mathrm{~m}$ \\
\hline
\end{tabular}

Table 4.17 Mean and Standard Deviation for DOFs for the Non-Op Case 2

\begin{tabular}{l|lll}
\hline DOFs & Experiment & $\begin{array}{l}\text { FAST } \\
\text { Tower Load) }\end{array}$ & $\begin{array}{l}\text { (with } \\
\text { Tower Load) }\end{array}$ \\
\hline Pitch (Mean) & $10.73092^{\circ}$ & $7.35102^{\circ}$ & $5.13869^{\circ}$ \\
Pitch (Std) & $2.02462^{\circ}$ & $2.20998^{\circ}$ & $1.87538^{\circ}$ \\
Surge (Mean) & $2.84368 \mathrm{~m}$ & $2.18756 \mathrm{~m}$ & $1.52206 \mathrm{~m}$ \\
Surge (Std) & $0.52778 \mathrm{~m}$ & $0.61528 \mathrm{~m}$ & $0.52537 \mathrm{~m}$ \\
Roll (Mean) & $-1.86967^{\circ}$ & $-0.12633^{\circ}$ & $-0.10568^{\circ}$ \\
Roll (Std) & $2.04729^{\circ}$ & $0.65069^{\circ}$ & $0.63543^{\circ}$ \\
Sway (Mean) & $1.02630 \mathrm{~m}$ & $0.04125 \mathrm{~m}$ & $0.03307 \mathrm{~m}$ \\
Sway (Std) & $0.56352 \mathrm{~m}$ & $0.18503 \mathrm{~m}$ & $0.17650 \mathrm{~m}$ \\
\hline
\end{tabular}

\section{Tower Loading vs. No Tower Loading}

For the Non-Op Case 1, one can observe from Table 4.16 that the mean pitch increased from $2.24^{\circ}$ without tower loading to $3.13^{\circ}$ with tower loading, and the mean surge also increased from $0.65 \mathrm{~m}$ to $0.92 \mathrm{~m}$. The standard deviation of pitch and surge increased from $0.61^{\circ}$ to $0.86^{\circ}$ and 0.19 $\mathrm{m}$ to $0.26 \mathrm{~m}$ respectively. For the Non-Op Case 2, one can observe from Table 4.17 that the mean pitch increased from $5.14^{\circ}$ without tower loading to $7.35^{\circ}$ with tower loading and the mean surge also increased from $1.52 \mathrm{~m}$ to $2.19 \mathrm{~m}$. The standard deviations of pitch and surge increased from $1.88^{\circ}$ to $2.21^{\circ}$ and $0.53 \mathrm{~m}$ to $0.62 \mathrm{~m}$ respectively. These data show wind loading does have a significant effect on the dynamics of the SWAY turbine.

The wind is more dominant in the surge/pitch direction of the system and there are minor crosswind effects on the system. The minor crosswind effects are reflected in the small increases in the mean and standard deviation values of the roll and sway values presented in Table 4.16 and Table 4.17. 
From Figure 4.28 and Figure 4.29, one can observe that the inclusion of the wind loading on the tower however does not affect the PSD plots significantly. Only minor increases throughout the entire range of frequency for all DOFs are seen, because the wind inflow generated from TurbSim is random and generally decays with increasing frequency.

\section{Experiment vs. Simulation}

When comparing the simulated data with experimental data, it can be observed from Table 4.16 and Table 4.17 that simulating the wind loading on the tower increased the accuracy of the results for the surge and pitch component in the non-operating cases.

For both cases, it can be observed from Table 4.16 and Table 4.17 that the roll and sway simulation data significantly deviated from the experimental observations. This could be due to either (1) un-modeled current or wave components coming from the non-dominant direction or (2) un-modeled cross-wind components.

The difference in magnitude between simulation and experiment for the PSD plots in the Non-Op Case 1 and 2 is quite significant, and it may be due to experimental noise in the measurements. In particular, the pitch and roll PSD plots are similar in the lower frequencies, but the magnitude is much larger for the experiment in the higher frequencies. This lack of damping in the higher frequencies is unusual and the reason for it is not understood at this point, but it could be a consequence of compliance in the universal joint, flexibility of the system (whereas the simulations assume the body to be rigid), or complexity of the wind and wave conditions (such as reflected wave patterns or currents). 

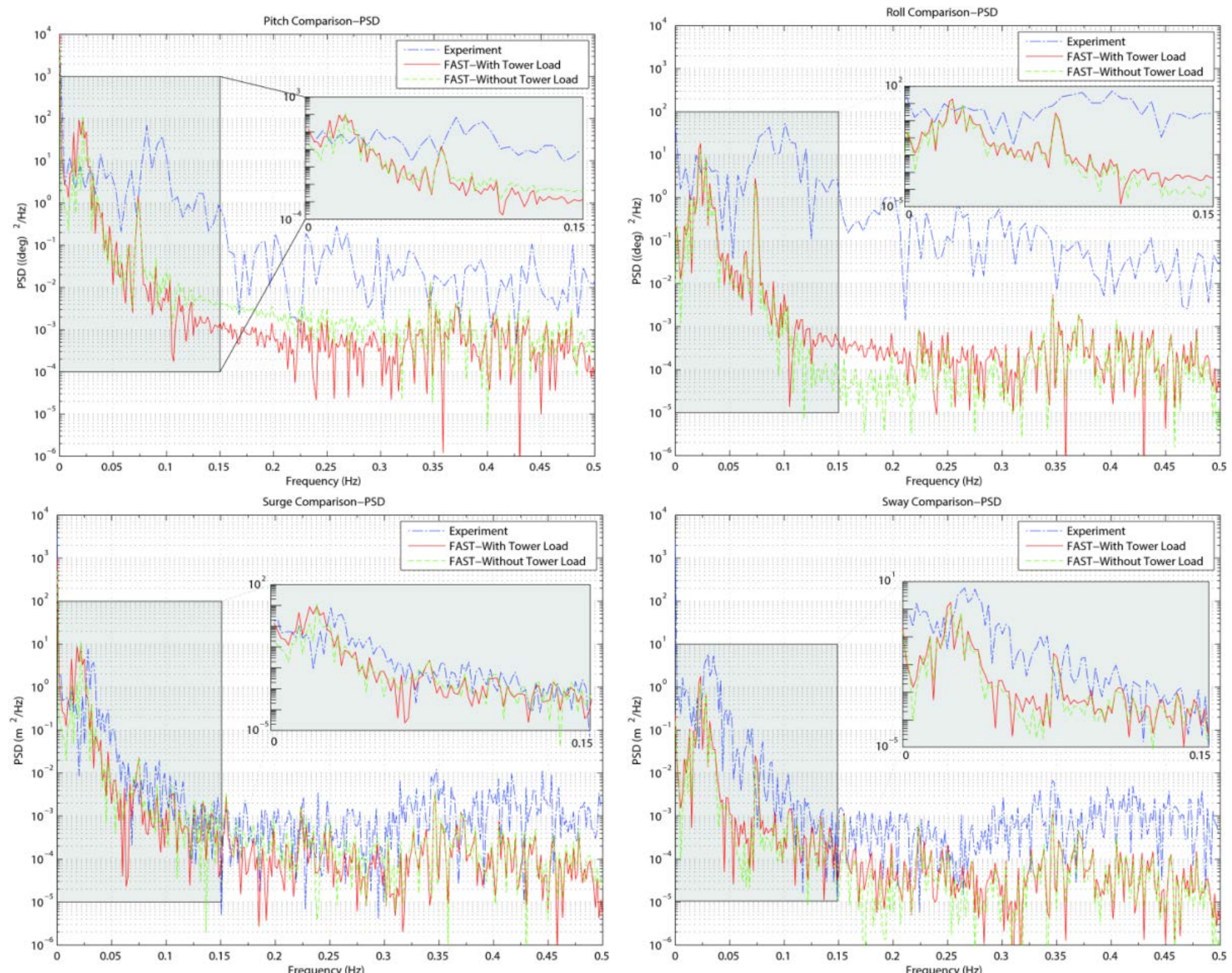

Figure 4.28 Summary of results for Non-Op Case 1 

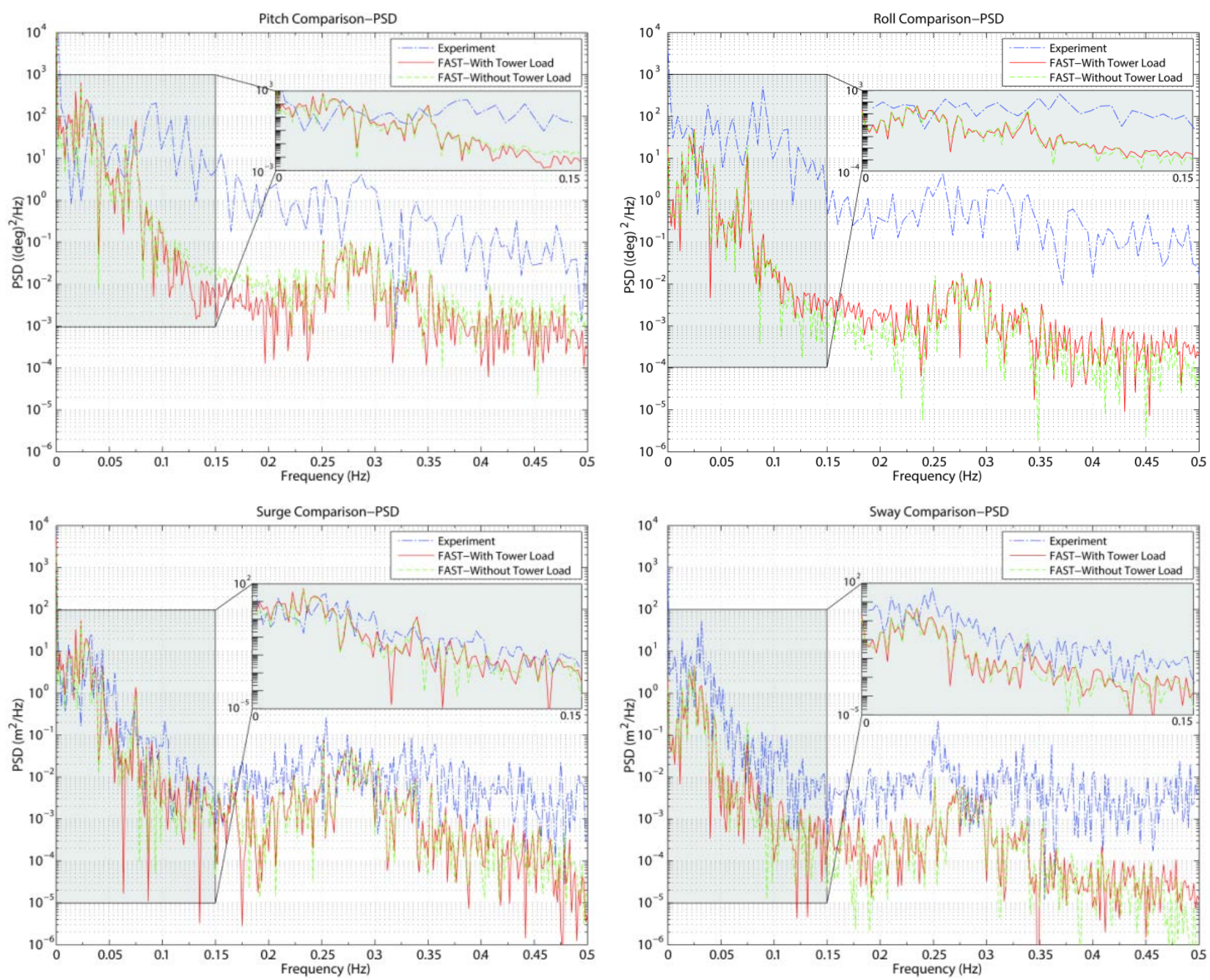

Figure 4.29 Summary of results for Non-Op Case 2 
In the frequency domain for the Non-Op Case 1, the simulated data indicates significant frequency peaks at $0.023 \mathrm{~Hz}, 0.073 \mathrm{~Hz}$, and $0.347 \mathrm{~Hz}$ (see Figure 4.28). The $0.023-\mathrm{Hz}$ and $0.347-\mathrm{Hz}$ frequency peaks are aligned with the wave conditions, where the wave spectrum suggests corresponding peaks at $0.028 \mathrm{~Hz}$ and $0.347 \mathrm{~Hz}$. The first pitch/roll natural frequency of the system is also near $0.02 \mathrm{~Hz}$. The $0.073-\mathrm{Hz}$ frequency peak is then the second roll/pitch natural frequency, which was discussed in Section 4.6.2. For the experimental data, the peaks occur at about $0.03 \mathrm{~Hz}, 0.082 \mathrm{~Hz}$, and $0.34 \mathrm{~Hz}$, and they are less defined but are observable in the PSD plots. The natural frequencies of the system have shifted a bit, and this is likely due to a change in tidal conditions.

For the Non-Op Case 2, the simulated data reveal significant frequency peaks at $0.025 \mathrm{~Hz}, 0.075 \mathrm{~Hz}$, and $0.278 \mathrm{~Hz}$ (see Figure 4.29). The 0.025- $\mathrm{Hz}$ and $0.278-\mathrm{Hz}$ frequency peaks are influenced by the wave conditions as observed in the wave spectrum, showing corresponding peaks at $0.030 \mathrm{~Hz}$ and $0.278 \mathrm{~Hz}$. The $0.025 \mathrm{~Hz}$ peak is also near the first pitch natural frequency of the system, whereas the $0.075-\mathrm{Hz}$ frequency peak is close to the second pitch natural frequency. For the experimental data, the peaks at about $0.03 \mathrm{~Hz}$ and $0.25 \mathrm{~Hz}$ are influenced by the wave conditions, while the natural frequencies of the system have shifted to about 0.03 and $0.089 \mathrm{~Hz}$.

\subsubsection{Simulation Issues and Assumptions}

Some further reasons for the differences between the experimental and simulated results are reviewed here.

For an offshore floating wind turbine, the wind loads on the tower are expected to contribute significantly to the response of the system. The first round of simulations were run using the original version of FAST v7 and AeroDyn 13 that does not account for these tower loads. After making the code changes described in Section 4.3, wind loads are accounted for and the difference in the results is shown in 4.7.2.6. 
During the model building stage, the transition from tower to platform was set at MSL, as discussed previously. The simulations were conducted at MSL even though the tide level changes for different data sets. It is not possible to directly represent this tidal variation within FAST without making large changes to the input files, and tidal variation was therefore ignored in the analysis. The tide level, however, could have a strong influence on the system behavior, as it will change the displacement of the structure and therefore the stiffness in the mooring line, which will in turn affect the pitch/roll motion of the structure.

After the de-commissioning of the turbine in December 2013, some water was detected in one of the built-in ballast tanks positioned just below MSL (assumed to be emptied in the FAST model). As a result, the water in the ballast tank may have raised the center of gravity above what has been assumed, which in turn causes an increase of the pitch and roll periods.

The tower was modeled as a rigid body, which could result in un-modeled dynamics of the system. Additionally, the stiffness of the flexible body is larger in the fore-aft direction than side-side due to the positioning of the tension wires and spreader beams, which is not accounted for in the rigid model.

The mean wave and current direction data provided from the AWAC were averaged to 1-hr readings, which may cause inaccuracy in the simulation results as wave and current properties may differ significantly within the 1hr sampling period. Also, the AWAC was located about $12.5 \mathrm{~m}$ from the wind turbine system in areas close to rock and varying seabed conditions. These circumstances reduced the confidence in the wave and current data obtained. The FAST simulation was therefore conducted in no current conditions.

From the wave verification results, it is also observed that the wave spectrum at the SWAY location may be bi-modal and multi-directional, 
which is likely due to the close proximity of the system to the shore. From both the AWAC and the tower wave height sensor, it was only possible to define the dominant peak period, significant wave height and wave direction but not these values for all the different wave modes. Even if the full wave definitions were available, FAST v7 does not allow users to represent multidirectional waves.

\subsubsection{Operating Conditions}

\subsubsection{Selection of Test Case}

After validation of non-operational conditions, validation was performed for two operating conditions: one 5-min test condition in control region II (Op Case 1) and one 10-min operational test case in control region III (Op Case 2). Op Case 1 was used instead of a usual 10-min test case due to the short duration of consistent rotor speed with no blade pitch angle changes. Op Case 2 was selected due to the stable wind velocity and rotor speed over an extended period of time. As the control algorithm from the real system was not available in the numerical model, this test case was also selected due to its limited variations in blade pitch angle to minimize the effects of the lack of control system. For Op Case 1, the system operated at an average rotor speed of $22.27 \mathrm{RPM}$ with an average wind speed of $3.53 \mathrm{~m} / \mathrm{s}$ measured at the anemometer height. For Op Case 2, the system had an average rotor speed of 33.67 RPM with a mean wind speed of $7.32 \mathrm{~m} / \mathrm{s}$. The measured time series data of the rotor speed and blade pitch angle are shown in Figure 4.30, while the wind, wave and current conditions are included in Figure 4.31. 

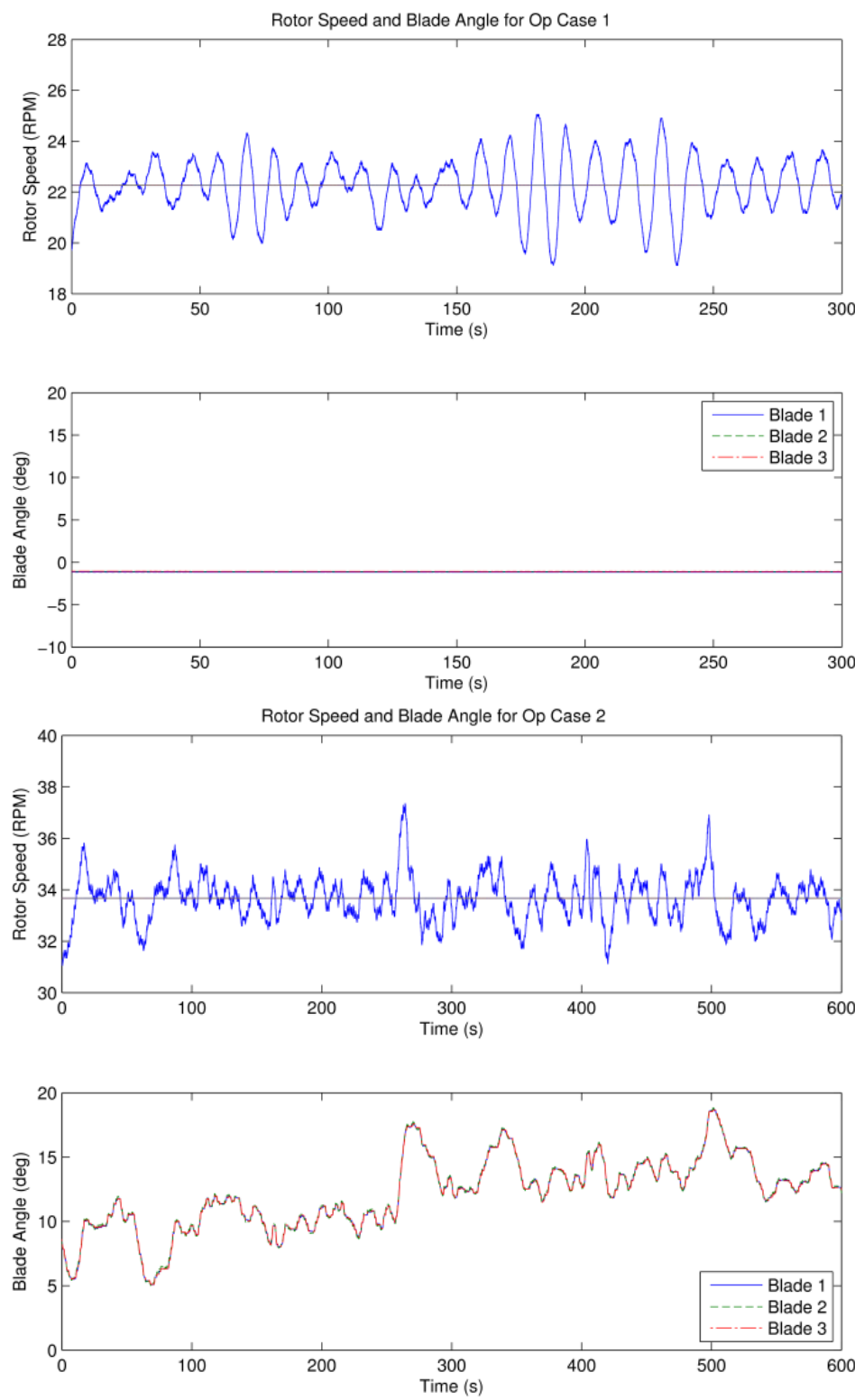

Figure 4.30 Rotor speed and blade pitch angle for operating cases

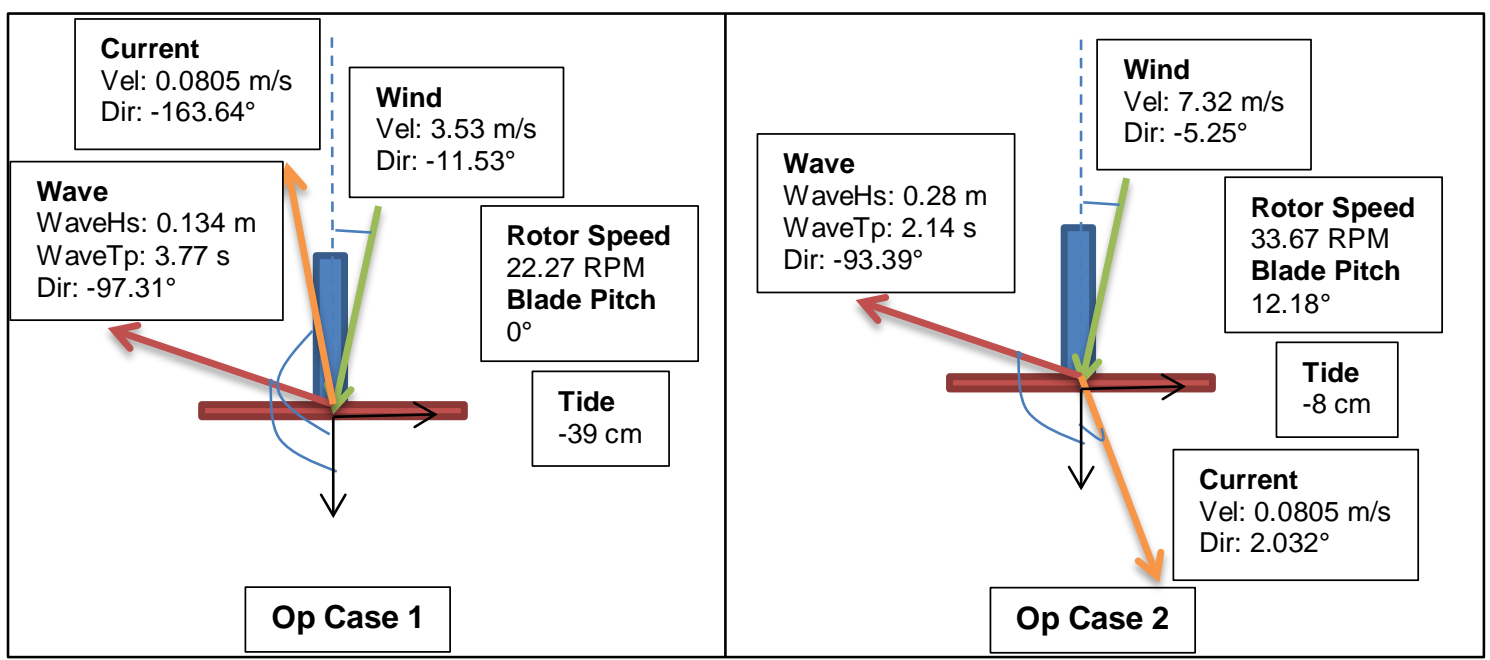

Figure 4.31 Summary of operational test cases 


\subsubsection{Simulation Procedure}

The system was first set at an equilibrium position calculated from a simulation of the static equilibrium. For the region II test case, the FAST simulation was conducted for a simulation time of $600 \mathrm{~s}$ (10 minutes), and the first five minutes of the simulation were discarded. For the region III test case, the simulation was conducted for a simulation time of $1,200 \mathrm{~s}$ (20 minutes), and the first 10 minutes of the simulation were discarded. This is to remove any initial transient behavior.

\subsubsection{Wind}

As with the non-operating cases, the wind inflow file used by AeroDyn was generated using TurbSim with the settings shown in Table 4.18. These settings were based on measurements obtained from the ultrasonic anemometer attached to the boom. The usable analysis time was set at $600 \mathrm{~s}$ for the region II test case and $1200 \mathrm{~s}$ for the region III test case. The turbulence model was assumed to follow the IEC Kaimal spectral normal turbulence model, and the turbulence intensity and reference wind speed were derived from sonic anemometer readings of wind speed at $12.5 \mathrm{~m}$.

Table 4.18 TurbSim Wind Inflow Properties for Operating Cases

\begin{tabular}{|c|c|}
\hline & Op Case 2 \\
\hline $\begin{array}{lll}\text { Vertical grid-point } & \text { matrix } \\
\text { dimension } & & \end{array}$ & 51 \\
\hline $\begin{array}{l}\text { Horizontal grid-point matrix } \\
\text { dimension }\end{array}$ & 61 \\
\hline Hub height $[\mathrm{m}]$ & 13.133 \\
\hline Grid height $[\mathrm{m}]$ & 20.000 \\
\hline Grid width [m] & 25.000 \\
\hline Turbulence model & $\begin{array}{c}\text { IEC Kaimal spectral normal turbulence } \\
\text { model }\end{array}$ \\
\hline Turbulence intensity & \begin{tabular}{l|l}
$15.311 \%$ & $12.087 \%$ \\
\end{tabular} \\
\hline Reference wind speed $[\mathrm{m} / \mathrm{s}]$ & 3.527 \\
\hline $\begin{array}{l}\text { Reference height of anemometer } \\
{[\mathrm{m}]}\end{array}$ & 12.500 \\
\hline Wind profile type & $\begin{array}{c}\text { IEC, Power law on rotor disk, } \\
\text { logarithmic elsewhere }\end{array}$ \\
\hline Power law exponent & 0.140 \\
\hline Surface roughness length [m] & 0.030 \\
\hline
\end{tabular}


As with the non-operating cases, the mean and standard deviation of the wind inflow velocity for the experiment shows good comparison with the wind inflow generated by TurbSim, with a slightly larger mean for the experimental data. Summaries of the measured and simulated wind properties for both test cases is shown in Table 4.19 and Table 4.20, and Figure 4.32 and Figure 4.33 show the time domain comparison for the wind inflow velocity for downwind and crosswind directions for both FAST and experimental data.

Table 4.19 Comparison of Wind Properties for Op Case 1

\begin{tabular}{l|llll}
\hline & Mean (Exp) & Mean (FAST) & Std. Dev. (Exp) & Std. Dev. (FAST) \\
\hline U (m/s) & 3.5071 & 3.5814 & 0.5240 & 0.3929 \\
V (m/s) & -0.6216 & -0.7900 & 0.5082 & 0.3965 \\
\hline
\end{tabular}

Table 4.20 Comparison of Wind Properties for Op Case 2

\begin{tabular}{l|llll}
\hline & Mean (Exp) & Mean (FAST) & Std. Dev. (Exp) & Std. Dev. (FAST) \\
\hline U (m/s) & 7.1336 & 7.3681 & 0.8922 & 0.8525 \\
V (m/s) & -0.7231 & -0.5817 & 0.6683 & 0.6344 \\
\hline
\end{tabular}



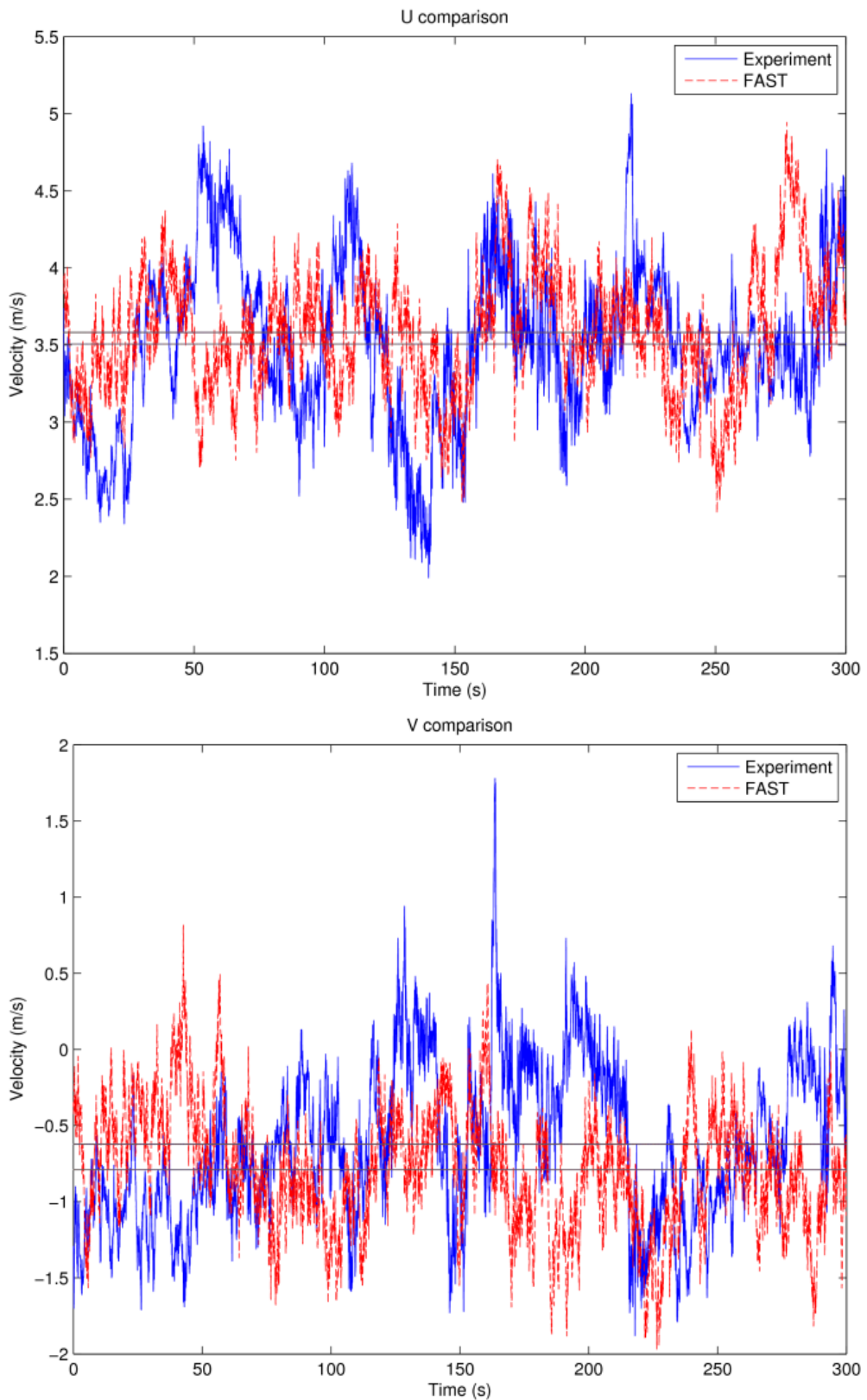

Figure 4.32 Velocity inflow comparison (Op Case 1) 

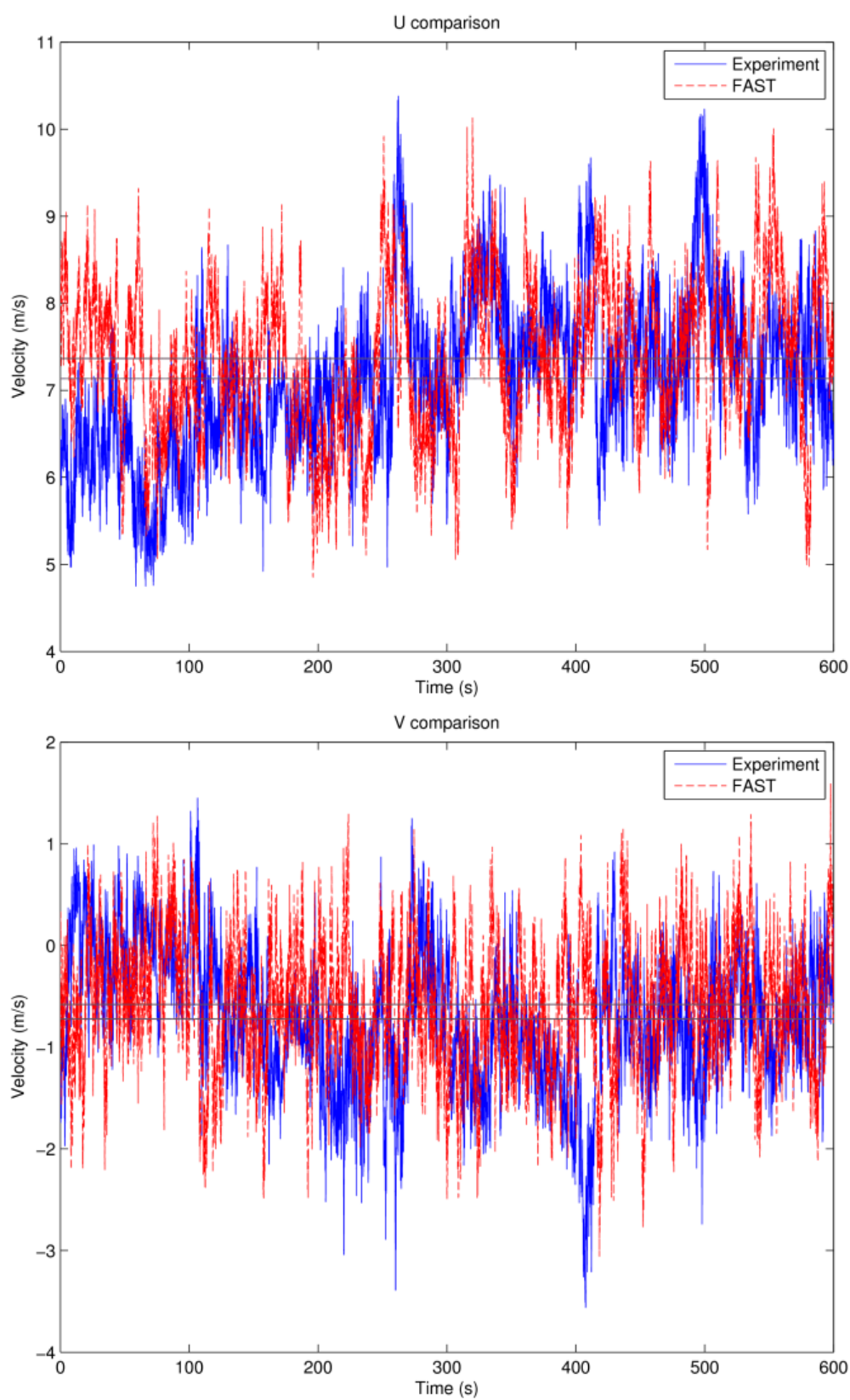

Figure 4.33 Velocity inflow comparison (Op Case 2)

\subsubsection{Waves}

For each simulation case, a user-defined wave spectrum was inputted into FAST from 5-min wave height data for Op Case 1 and 10-min wave height data for Op Case 2, which were obtained from the tower wave height sensor. The mean wave direction was obtained from the 1-hr averaged data observed by the AWAC. This process was done for both cases; the timeand frequency-domain comparisons of the waves generated in FAST and the experimental data are shown in Figure 4.34 and Figure 4.35. 

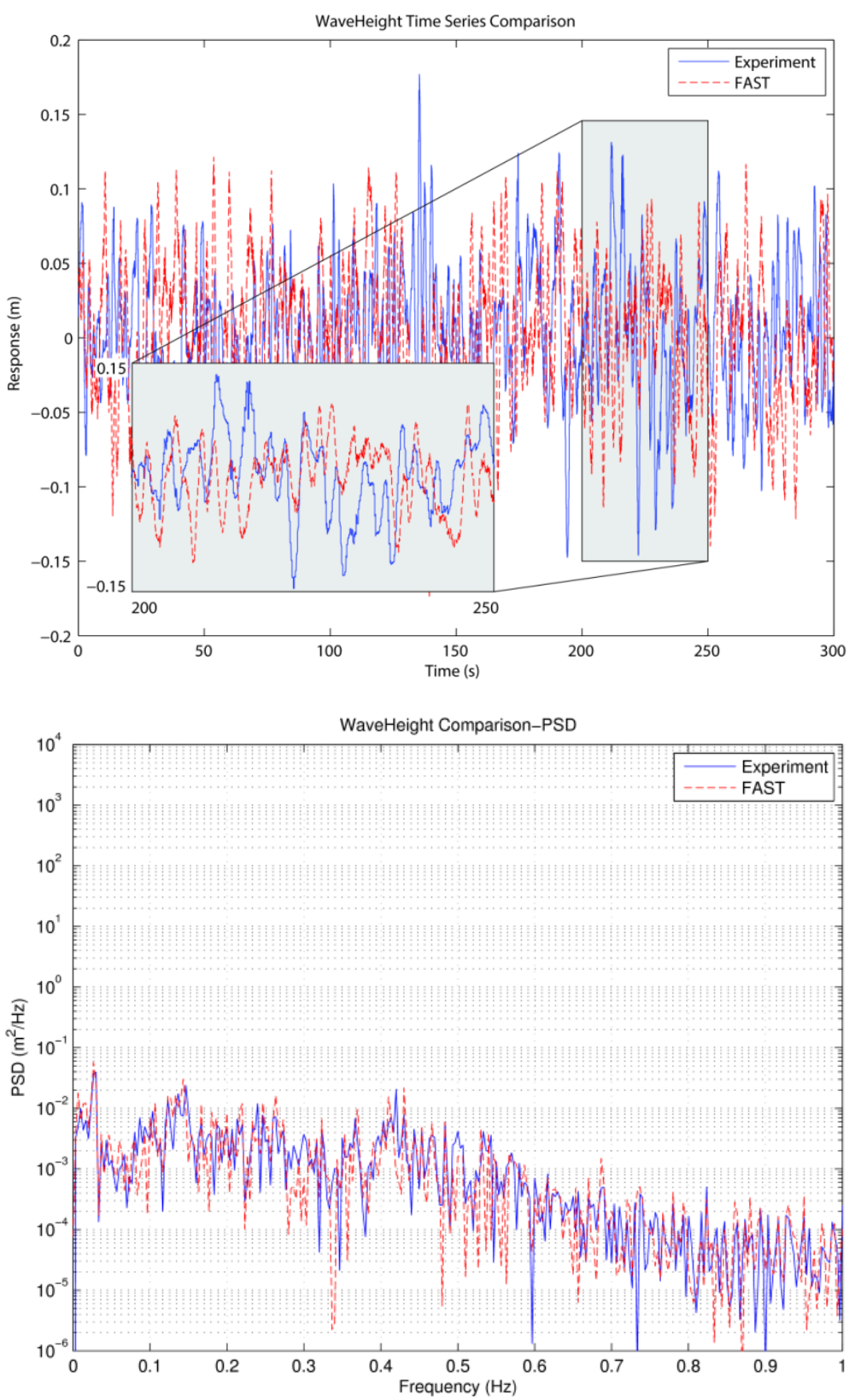

Figure 4.34 Wave height comparison (Op Case 1) 

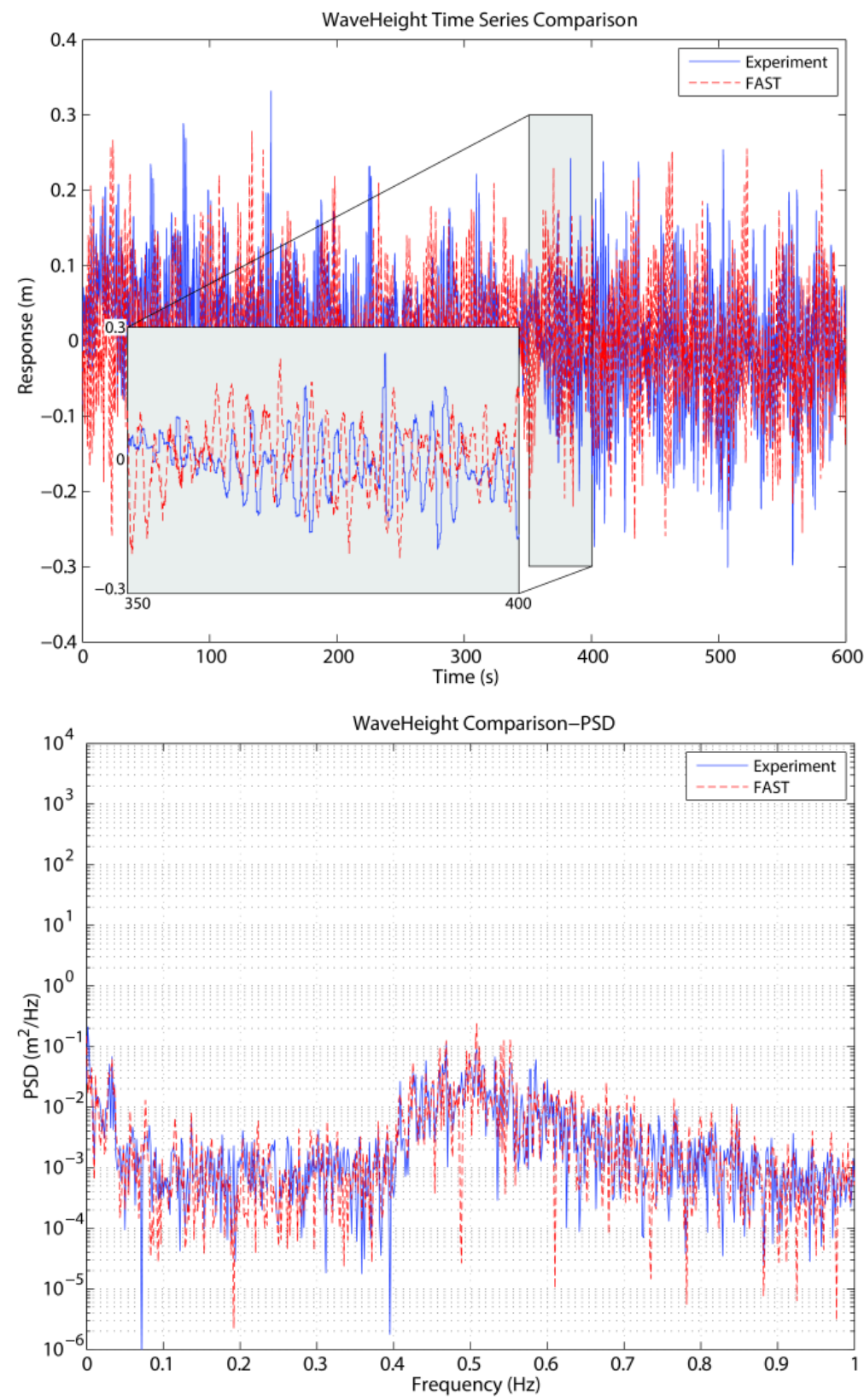

Figure 4.35 Wave height comparison (Op Case 2)

\subsubsection{Simulation Assumptions}

In addition to the issues discussed in Section 4.7.1.5 in the non-operating cases, it was assumed for the FAST simulations that the rotor speed operates at a constant rotor speed and blade pitch angle for both cases. Constant values were used because control algorithms for the blade pitch and generator speed were unavailable. The lack of control will result in significant differences between the simulation and experiment. 


\subsubsection{Results}

Table 4.21 and Table 4.22 show the mean and standard deviation values for the pitch, surge, roll, and sway motions of the wind turbine over the 5min period for Op Case 1 and the 10-min period for Op Case 2. The corresponding PSD plots are shown in Figure 4.36 and Figure 4.37.

Table 4.21 Mean and Standard Deviation for DOFs for the Op Case 1

\begin{tabular}{l|lll}
\hline DOFs & Experiment & $\begin{array}{l}\text { FAST } \\
\text { Tower Load) }\end{array}$ & $\begin{array}{l}\text { (with } \\
\text { Tower Load) }\end{array}$ \\
\hline Pitch (Mean) & $11.33967^{\circ}$ & $7.87317^{\circ}$ & $7.32953^{\circ}$ \\
Pitch (Std) & $1.35566^{\circ}$ & $1.033019^{\circ}$ & $0.93576^{\circ}$ \\
Surge (Mean) & $1.13291 \mathrm{~m}$ & $2.47827 \mathrm{~m}$ & $2.31766 \mathrm{~m}$ \\
Surge (Std) & $0.42099 \mathrm{~m}$ & $0.27074 \mathrm{~m}$ & $0.23966 \mathrm{~m}$ \\
Roll (Mean) & $0.84450^{\circ}$ & $-2.89152^{\circ}$ & $-2.77953^{\circ}$ \\
Roll (Std) & $0.53510^{\circ}$ & $0.88706^{\circ}$ & $0.85878^{\circ}$ \\
Sway (Mean) & $-3.86009 \mathrm{~m}$ & $-0.12162 \mathrm{~m}$ & $-0.08808 \mathrm{~m}$ \\
Sway (Std) & $0.16942 \mathrm{~m}$ & $0.29393 \mathrm{~m}$ & $0.288766 \mathrm{~m}$ \\
\hline
\end{tabular}

Table 4.22 Mean and Standard Deviation for DOFs for the Op Case 2

\begin{tabular}{l|lll}
\hline DOFs & Experiment & $\begin{array}{l}\text { FAST } \\
\text { Tower Load) }\end{array}$ & $\begin{array}{l}\text { (with } \\
\text { Tower Load) }\end{array}$ \\
\hline Pitch (Mean) & $12.90595^{\circ}$ & $13.95103^{\circ}$ & $12.69992^{\circ}$ \\
Pitch (Std) & $1.05561^{\circ}$ & $2.63459^{\circ}$ & $2.54336^{\circ}$ \\
Surge (Mean) & $6.76051 \mathrm{~m}$ & $4.03822 \mathrm{~m}$ & $3.69268 \mathrm{~m}$ \\
Surge (Std) & $0.34020 \mathrm{~m}$ & $0.72369 \mathrm{~m}$ & $0.71435 \mathrm{~m}$ \\
Roll (Mean) & $1.63395^{\circ}$ & $-1.43630^{\circ}$ & $-1.30943^{\circ}$ \\
Roll (Std) & $0.66471^{\circ}$ & $0.98300^{\circ}$ & $1.03791^{\circ}$ \\
Sway (Mean) & $0.31973 \mathrm{~m}$ & $-0.32309 \mathrm{~m}$ & $-0.29564 \mathrm{~m}$ \\
Sway (Std) & $0.19840 \mathrm{~m}$ & $0.18473 \mathrm{~m}$ & $0.19001 \mathrm{~m}$ \\
\hline
\end{tabular}



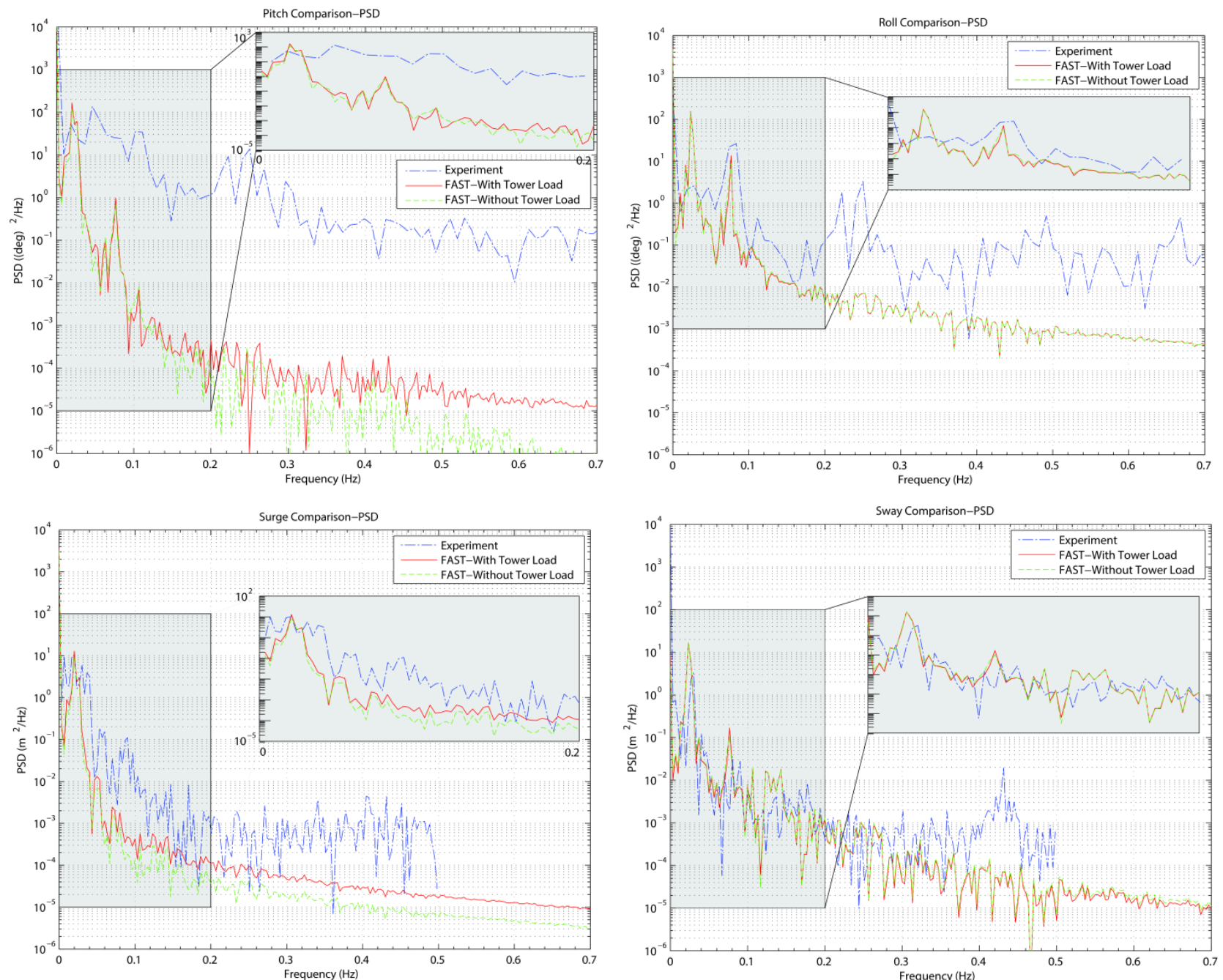

Figure 4.36 Summary of results for Op Case 1 

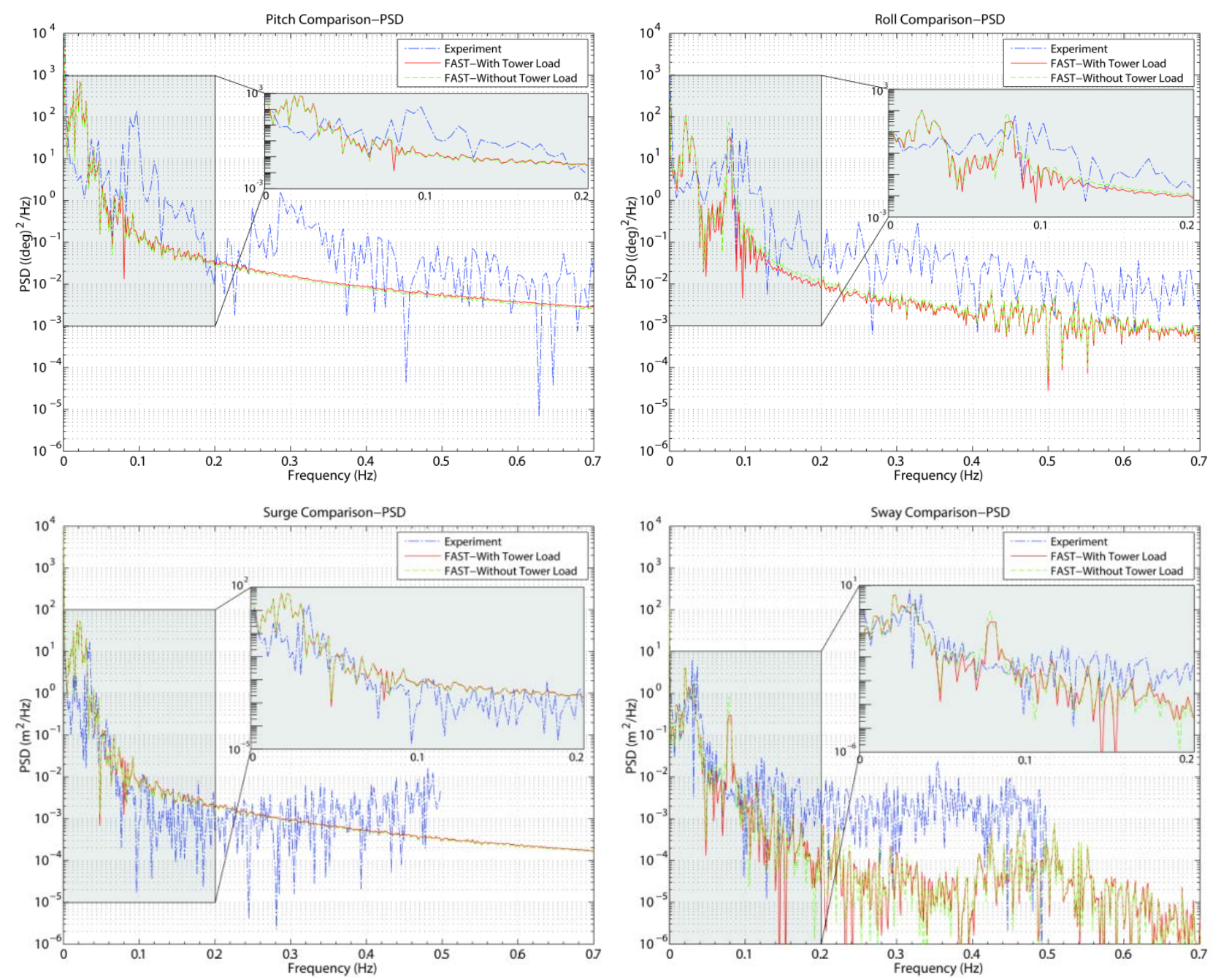

Figure 4.37 Summary of results for Op-Case 2 


\section{Tower Loading vs. No Tower Loading}

For Case 1 in Table 4.21, it can be observed that mean pitch increased from $7.33^{\circ}$ without tower loading to $7.87^{\circ}$ with tower loading, and the mean surge increased from $2.32 \mathrm{~m}$ to $2.48 \mathrm{~m}$. The standard deviation of pitch and surge increased from $0.61^{\circ}$ to $86^{\circ}$ and from $0.19 \mathrm{~m}$ to $026 \mathrm{~m}$ respectively. Similarly for Op Case 2 in Table 4.22 , the mean pitch increased from $12.70^{\circ}$ to $13.95^{\circ}$ and the mean surge increased from 3.69 to $4.04 \mathrm{~m}$ upon introducing wind loading to the tower. This increase is less significant than it was in the NonOp cases, as the dominant loading of the wind turbine system occurs on the wind turbine blades.

Similar to the non-operating cases, the wind is more dominant in the surge/pitch direction of the system and there are minor crosswind effects on the system. This is reflected in the small increases in the mean and standard deviation values of the roll and sway values shown in Table 4.21 and Table 4.22. The inclusion of the wind loading on the tower does not affect the PSD plots significantly in Figure 4.36 and Figure 4.37.

\section{Experiment vs. Simulation}

When comparing the simulated data with experimental data, it can be observed from Table 4.21 and Table 4.22 that simulating the wind loading on the tower increased the accuracy of the results for the surge and pitch component for the operational cases. The improvement of accuracy is less evident in the results in the non-operational cases. Also, for the Op Case 2 in Table 4.22, the standard deviations for pitch and surge were less for the experiment than they were for the simulation. This is probably because the control system in the experiment reduces the variations of the thrust loads on the rotor, which again reduces the tower motions. This is confirmed by

the better comparison of results from Op Case 1 in Table 4.21, which was in control region II. 
For both cases, Table 4.21 and Table 4.22 show that the roll and sway simulation data significantly deviate from the experimental observations. The reason for these deviations are similar to those for the non-operating case, in which no current conditions were set for the simulation and wave directions for the different wave modes were not represented well. In addition, from the experimental data for Op Case 1, a larger mean value was observed for sway $(-3.86 \mathrm{~m})$ than roll $\left(0.84^{\circ}\right)$, and a smaller mean value was observed for surge $(1.13 \mathrm{~m})$ than pitch $\left(11.34^{\circ}\right)$. This leads the author to suspect that the surge and sway data that are derived from the GPS measurements may contain GPS drift errors.

In the frequency domain for the Op Case 1, the simulated data show significant frequency peaks at $0.023 \mathrm{~Hz}$ and $0.077 \mathrm{~Hz}$ (see Figure 4.36). The $0.023-\mathrm{Hz}$ frequency peak is influenced by the natural frequency of the system and the wave conditions, where the wave spectrum in Figure 4.26 showed a corresponding peak at $0.03 \mathrm{~Hz}$. The $0.077-\mathrm{Hz}$ frequency peak is due to the second natural frequency of the entire system in roll and pitch DOFs, as discussed in Section 4.6.2. For the experimental data, a peak occurred at about $0.02 \mathrm{~Hz}$ for all DOFs in the PSD plots that was due to wave conditions. An additional peak occurred for the roll and pitch DOFs at 0.250 $\mathrm{Hz}$ and for the surge and sway DOFs at $0.431 \mathrm{~Hz}$. These additional peaks were not observed in the simulation; they are also likely due to the wave conditions. The second natural frequency of the system shifted to about 0.09 $\mathrm{Hz}$.

For the Op Case 2, the simulated data for all DOFs shows a significant frequency peak around $0.022 \mathrm{~Hz}$, which corresponds to the wave spectrum in Figure 4.27 with a peak at $0.033 \mathrm{~Hz}$. In the simulated data, another peak was more evident for the roll and sway DOFs around $0.08 \mathrm{~Hz}$. For the experimental data, a peak occurred at about $0.35 \mathrm{~Hz}$ for all DOFs that was due to wave conditions. Additional peak for roll and pitch DOFs at about $0.085 \mathrm{~Hz}$ was due to the natural frequency of the system. The wave 
frequency peak at about $0.5 \mathrm{~Hz}$ in Figure 4.27 influenced the sway and surge DOFs plots, but the observation is limited by the range of frequencies due to the sampling rate of the sensor.

\section{Blade Load Observations}

The loading on the blades was analyzed in the frequency domain using a FFT. Figure 4.38 to Figure 4.41 show the FFT analysis and comparison of the flapwise and edgewise loading on Blade 2. Table 4.23 and Table 4.24 list the blade frequencies observed in the analysis.

In the flapwise direction, $1 \mathrm{P}, 2 \mathrm{P}$ and $3 \mathrm{P}$ frequencies were detected in the FFT plots in both experiment and simulation results. The first and second flapwise modal frequencies calibrated and analyzed in Section 4.6 .3 were also observed.

For the edgewise loading, the $1 \mathrm{P}$ frequency and the first edgewise modal frequency analyzed in Section 4.6.3 were detected in the FFT analysis.

While the frequencies showed fairly good correlation between experiment and simulation, the magnitude of the PSD was quite different. It is likely that the tower shadow model does not accurately simulate the tower blade loads that occurring when the blade passes behind the tower. Other possibilities include that either the units of the blade loads were incorrect or the sensor connection was poor. 

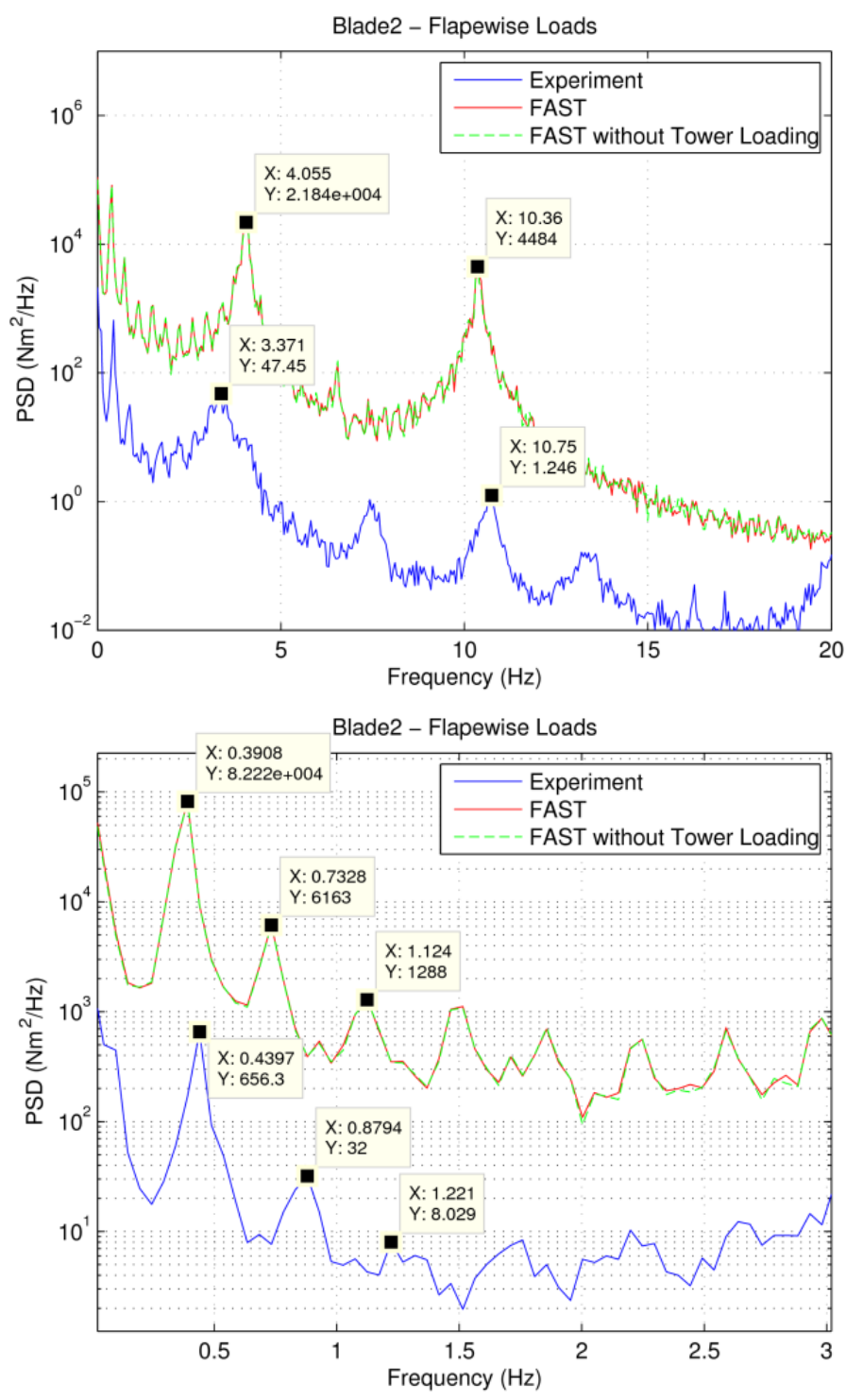

Figure 4.38 FFT analysis of the flapwise loading on Blade 2 for Op Case 1

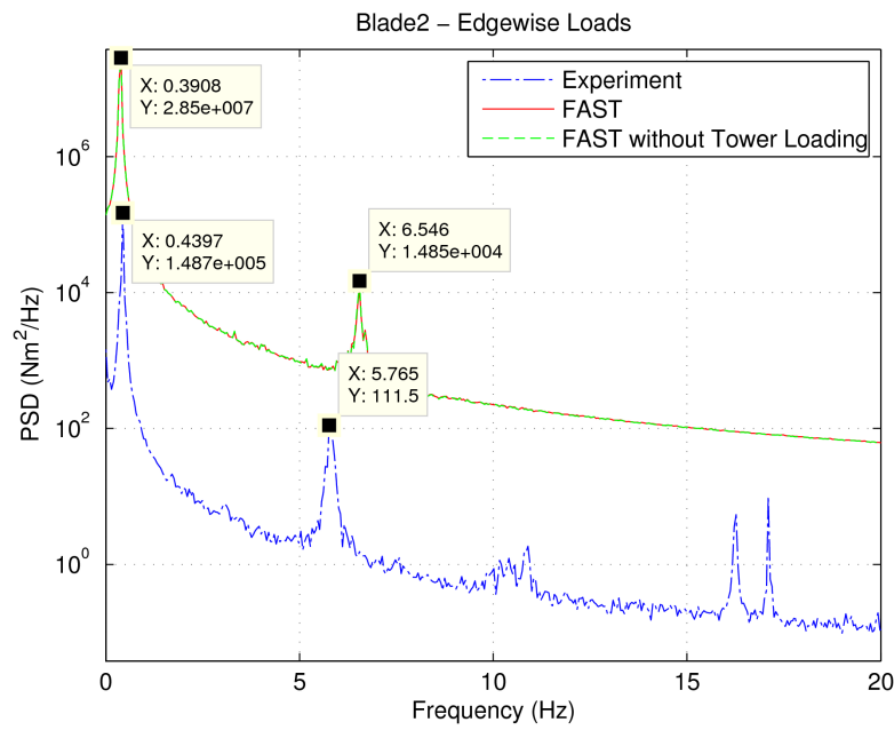

Figure 4.39 FFT analysis of the edgewise loading on Blade 2 for Op Case 1 
Table 4.23 Blade Frequencies (Hz) Observed in Experiment and Simulation for Op Case 1

\begin{tabular}{l|llllll}
\hline & $1 P$ & $2 P$ & $3 P$ & $\begin{array}{l}\text { Flapwise } \\
1^{\text {st }} \text { Mode }\end{array}$ & $\begin{array}{l}\text { Flapwise } \\
2^{\text {nd }} \text { Mode }\end{array}$ & $\begin{array}{l}\text { Edgewise } \\
1^{\text {st }} \text { Mode }\end{array}$ \\
\hline FAST & 0.3908 & 0.7328 & 1.124 & 4.055 & 10.36 & 6.546 \\
Experiment & 0.4397 & 0.8794 & 1.221 & 3.371 & 10.75 & 5.765 \\
\hline
\end{tabular}
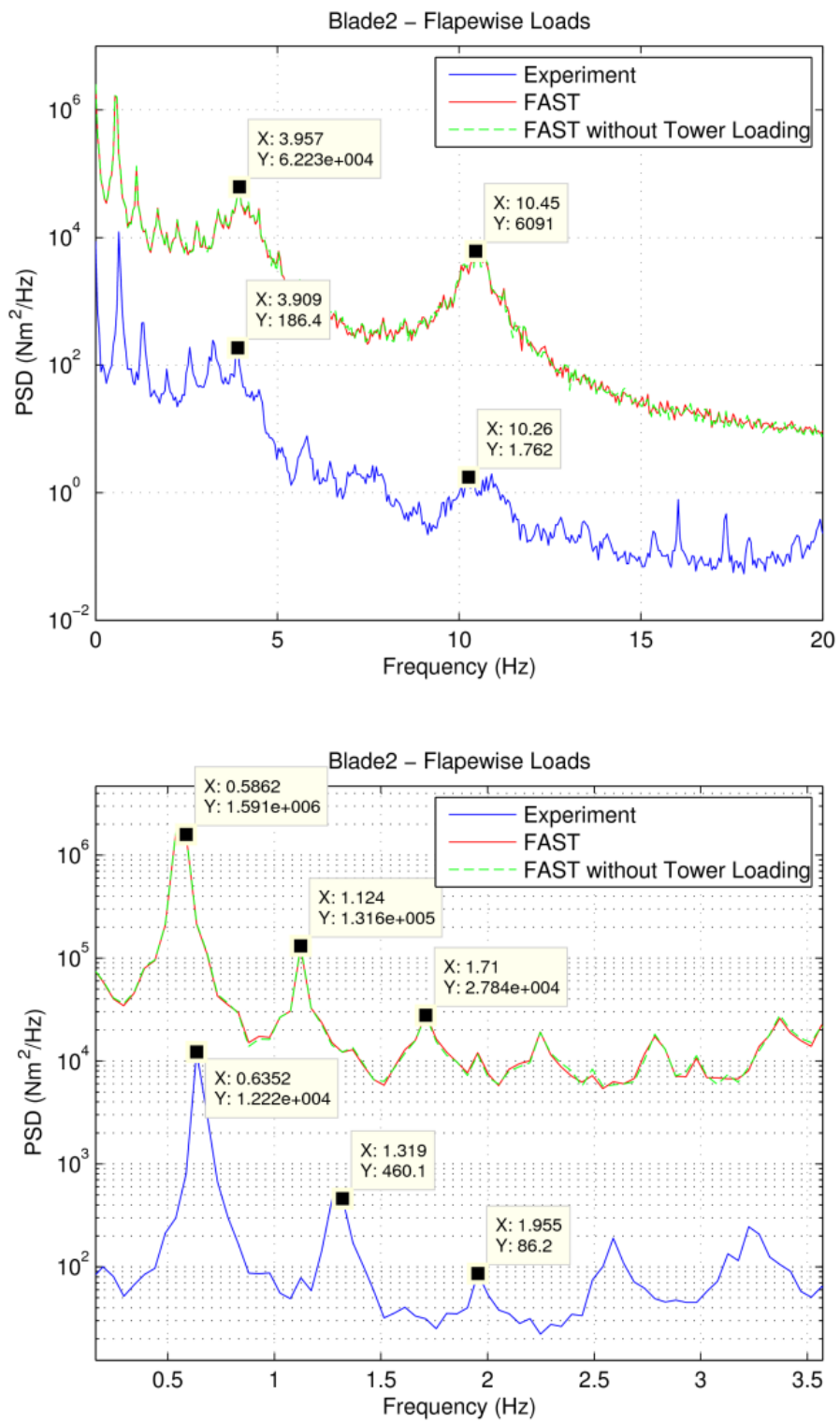

Figure 4.40 FFT analysis of the flapwise loading on Blade 2 for Op Case 2 


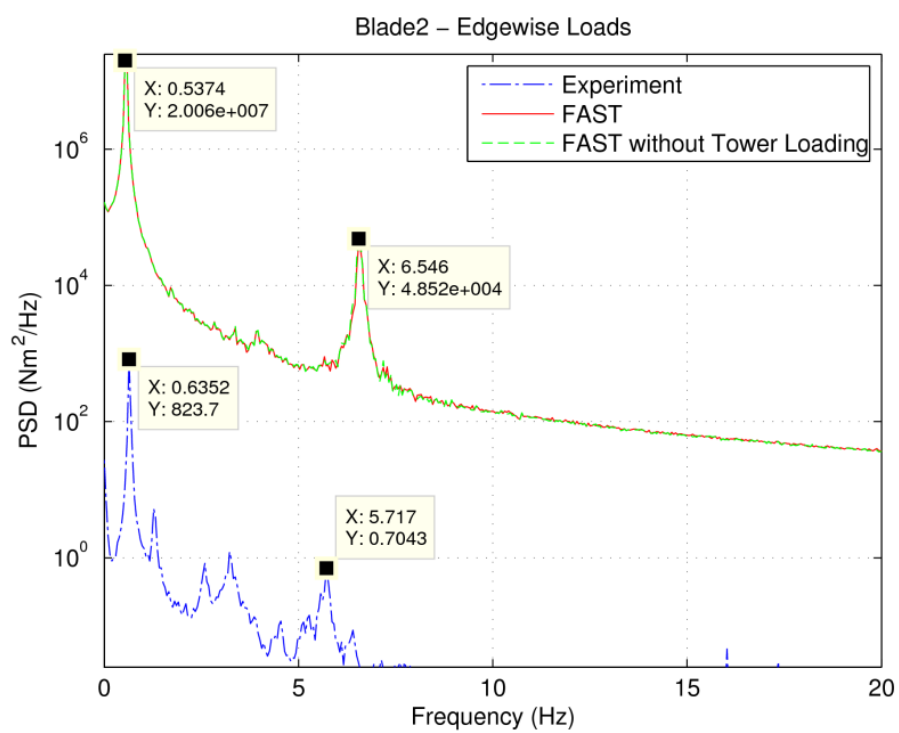

Figure 4.41 FFT analysis of the edgewise loading on Blade 2 for Op Case 2

Table 4.24 Blade Frequencies (Hz) Observed in Experiment and Simulation for Op Case 2

\begin{tabular}{l|llllll}
\hline & $1 P$ & $2 P$ & $3 P$ & $\begin{array}{l}\text { Flapwise } \\
1^{\text {st }} \text { Mode }\end{array}$ & $\begin{array}{l}\text { Flapwise } \\
2^{\text {nd }} \text { Mode }\end{array}$ & $\begin{array}{l}\text { Edgewise } \\
1^{\text {st }} \text { Mode }\end{array}$ \\
\hline FAST & 0.5862 & 1.124 & 1.710 & 3.957 & 10.45 & 6.546 \\
Experiment & 0.6352 & 1.319 & 1.955 & 3.909 & 10.26 & 5.717 \\
\hline
\end{tabular}

\subsection{Chapter Conclusion}

In this chapter, a FAST model of the spar-type $1 / 6.5^{\text {th }}$-scale prototype SWAY system was built with design descriptions made available by SWAY AS. Different modeling strategies and assumptions were used to enable the model to work without major changes to the FAST tool.

Changes were made to the FAST code to account for the wind loading on each tower element in each simulation time-step. While the improvements were focused on tubular tower designs, code users have the option to manually input the drag coefficient of the tower or to use the in-built $R e_{\text {tower }}-\mathrm{C}_{\mathrm{D} \text {,tower }}$ table applicable for a circular smooth tower. 
The FAST model was calibrated to account for the design changes made to the prototype blades and the incomplete design parameters that resulted in uncertainties in the mass distribution of the floating system. A static equilibrium test was conducted to calibrate the offsets of the system. One roll free-decay test and one pitch free-decay test were used to calibrate the mass moment of inertia and platform damping matrix. After calibration, the comparison of the FAST simulations and experiment results matched with minor discrepancies because of the simplifying assumptions made in modeling the wind turbine. The flapwise modal frequencies of the blades were tuned by analyzing the blade loading during non-operational mode at a mean wind speed of $4.35 \mathrm{~m} / \mathrm{s}$.

To validate the model, two non-operating test cases and two operating test cases (one in Control Region II and one in Control Region III) were selected from the available data sets. From the results, it was shown that wind loading on the tower did have a significant effect on the dynamics of the wind turbine system when it was not operating in high wind speeds and a less significant effect when the system is operating, as the dominant wind loading occurs on the turbine blades. Including the wind loading on the tower increased the accuracy of the simulation results in comparison with the experimental results, particularly in the wind-loading direction.

For the blade loads, the flapwise and edgewise bending structural frequencies as well as the $1 \mathrm{P}, 2 \mathrm{P}$, and $3 \mathrm{P}$ frequencies were similar for both experiment and simulation results. However, there were significant differences in the magnitude of the loads. It is likely that the tower shadow model does not accurately simulate the tower blade loads that occurring when the blade passes behind the tower. Other possibilities include that either the units of the blade loads were incorrect or the sensor connection was poor.

While there were some good correlation of the simulation results and experiment, there were differences due to a number of reasons. First, the 
inability to model frictional damping in the universal joints of the system and the inability to simulate the disturbed fluid field around the platform also contributed to discrepancies between measured and simulated results. Second, the FAST model was unable to model the wave conditions accurately, due to both the limited wave data available and limitations of FAST in representing the multi-directional nature of the waves. The nearshore location of the turbine created very complicated wave forms due to reflection of the waves from the shore. Third, tidal variations were excluded in the model due to the inability of FAST to simulate them easily without major changes to the input files. Fourth, current conditions were excluded due to the reduced confidence of the $\mathrm{AWAC}$ readings that resulted from its location and data-averaging frequency. Lastly, no control system was included for the operational case. Other uncertainties/assumptions in the modeling and issues with setting up the instrumentation on the prototype might have also contributed to the errors observed.

Although mixed results were observed in comparing the system behavior in the experiment and FAST simulations, this study was useful in building competencies, learning and understanding the key issues and challenges in an open-sea validation study, and identifying some limitations in the modeling approach. Unlike laboratory testing, the complex nature of the environment and the inability to fully characterize its influence on the system is one of the key challenges in validating a model using open-sea data.

Future work may look at quantifying the assumptions and estimating the resulting errors. A simple control system may be considered, and the model fidelity may be increased to reduce assumptions. Some changes to the FAST tool might include altering the mooring-line model to better represent the tension-rod system, addressing the ability to model different tide levels, including flexibility, and including the capability in HydroDyn to model multi-directional waves. However, the complexity of the present location of 
the SWAY system may preclude the ability to model the wave conditions accurately even with multi-directional waves, due to the inability to measure the conditions.

Finally, while this chapter has largely focused on the validation work that was performed, some of the significant benefits of this project were related to the instrumentation work performed. Significant hands-on experience was gained in installing instrumentation in an offshore environment, and interfacing with other instrumentation and DAS systems. 


\section{TOWER/NACELLE INFLUENCE MODEL}

\subsection{Background information}

As part of the Tokyo Institute of Technology International Research Opportunities Program and nomination by the College of Engineering of NTU, the author did a research exchange focusing on the development and improvement tower/nacelle influence model at the Tamura Laboratory from 02 Feb 2014 to 30 April 2014.

The author conducted this work under the supervision of Professor Tetsuro Tamura at the Tamura Laboratory, Department of Environmental Science and Technology, Interdisciplinary Graduate School of Science and Engineering. The laboratory focuses on areas of research in turbulence dynamics and modeling, wind engineering, as well as computational fluid dynamics (CFD) analysis of urban and rural flow.

The key objectives of this section are:

- $\quad$ Perform CFD analysis to analyze the flow fields around various tower and nacelle;

- Compare current correction models with CFD simulations over various wind speed, size and shapes; and

- Improve and develop new empirical/semi-empirical correction models.

\subsection{CFD Simulation}

\subsubsection{Software tools used}

The CFD analysis was performed using the ANSYS 15.0. The ANSYS is a multi-disciplinary engineering simulation software that is widely used in industries and academia. The fluid analysis tool, ANSYS Fluent is capable of predicting fluid dynamics with high level of confidence and robustness. 
ANSYS DesignModeler was used to build the geometrical model before generating the domain meshes in ANSYS Meshing and ANSYS ICEM CFD. The processor used was ANSYS Fluent 15.0 and results were post-processed in ANSYS CFD-POST and Tecplot software. The numerical simulations were conducted on Tsubame 2.5 supercomputer located in Tokyo Institute of Technology and the High Performance Computing Centre in NTU.

\subsubsection{Setup}

A summary of the modeling setup and parameters common to all simulation cases is shown in Table 5.1.

Table 5.1 Summary of Modeling Setup and Parameters

\begin{tabular}{|c|c|}
\hline Solver & $\begin{array}{l}\text { Pressure-based, Transient (suitable for } \\
\text { incompressible flows) }\end{array}$ \\
\hline $\begin{array}{l}\text { Turbulence Model } \\
\text { LES Model }\end{array}$ & $\begin{array}{l}\text { Embedded Large Eddy Simulation (hybrid) } \\
\text { WALE Model }\end{array}$ \\
\hline RANS Model & $\begin{array}{l}\text { k-omega SST (with Low-Re Correction } \\
\text { Curvature Correction) }\end{array}$ \\
\hline $\begin{array}{l}\text { RANS/LES Interface } \\
\text { Treatment }\end{array}$ & Vortex Method \\
\hline $\begin{array}{l}\text { Wall Boundary } \\
\text { Conditions }\end{array}$ & No Slip \\
\hline Material Properties & $\begin{aligned} & \text { Air - } \text { Density }=1.225 \mathrm{~kg} / \mathrm{m}^{3} \\
& \text { Viscosity }=1.7894 \times 10^{-5} \mathrm{~kg} / \mathrm{ms}\end{aligned}$ \\
\hline $\begin{array}{l}\text { Pressure-Velocity } \\
\text { Coupling }\end{array}$ & $\begin{array}{l}\text { Pressure-Implicit with Splitting of Operators } \\
\text { (PISO) }\end{array}$ \\
\hline Spatial Discretization & $\begin{array}{l}\text { Gradient: Least Square Cell Based } \\
\text { Pressure: Standard } \\
\text { Momentum, Turbulent KE, Specific Dissipation } \\
\text { Rate: 2nd Order Upwind }\end{array}$ \\
\hline Transient Formulation & $\begin{array}{l}\text { Bounded Second Order } \\
\text { accuracy and stability) }\end{array}$ \\
\hline
\end{tabular}

For this study, it is essential to keep the computational costs manageable and to have ability to control in assigning the RANS and LES zones in the 
mesh. Therefore, the ELES approach is used for all CFD simulations in this study.

PISO was selected for the pressure-velocity coupling method as it allows for larger time-steps in transient flow conditions and increases the efficiency during the iteration of the momentum balance equation. Also, the gradients are computed using the least square cell based method due to it being the least expensive method provided that the mesh is of good quality and structured. For improved accuracy and stability, the bounded second order implicit formulation was used.

\subsection{General Tower and Nacelle Design}

In order to determine the type of test model to be built for the CFD analysis, available design information about onshore (for smaller turbines) and offshore wind turbine that are currently in production and planned for production were obtained and analyzed.

Table 5.2 shows a consolidated list of small to large wind turbine systems and their specifications. While much of the wind turbine specifications are proprietary in nature, a number of key information can be gathered from this data set:

- Most towers deploy a circular, tubular structure;

- Most towers are tapered, but the amount of tapering cannot be determined, and in some cases are insignificant;

- Nacelle shapes are dominated by cylindrical and cuboid shapes with slight variations between manufactures; and

- The nacelle edge profile can be either smooth or sharp.

Using this information as a guide, the models for the tower and nacelle are built for the CFD simulation and discussed in their respective sections. 
CHAPTER 5 TOWER/NACAELLE INFLUENCE MODEL

Table 5.2 List of Wind Turbine Systems

\begin{tabular}{|c|c|c|c|c|c|c|c|c|c|c|c|c|}
\hline Manuf acturer & Model & $\begin{array}{l}\text { Rated } \\
\text { (kW) }\end{array}$ & Power & $\begin{array}{l}\text { Rotor } \\
\text { Diameter } \\
\text { (m) }\end{array}$ & $\begin{array}{l}\text { Tower } \\
\text { Shape }\end{array}$ & $\begin{array}{l}\text { Tower } \\
\text { Diameter } \\
\text { (Base) (m) }\end{array}$ & $\begin{array}{l}\text { Tower } \\
\text { Diameter } \\
\text { (Top) (m) }\end{array}$ & $\begin{array}{l}\text { Nacelle } \\
\text { Shape }\end{array}$ & $\begin{array}{l}\text { Nacelle } \\
\text { Height (m) }\end{array}$ & $\begin{array}{l}\text { Nacelle } \\
\text { Length } \\
\text { (m) }\end{array}$ & $\begin{array}{l}\text { Nacelle } \\
\text { Width (m) }\end{array}$ & Edge Profile \\
\hline AOC & AOC $15 / 50$ & & 50 & & Circular & & & Cylinder & & & & Smooth \\
\hline Bergey & Excel 10 & & 10 & & Lattice & & & Cylinder & & & & Smooth \\
\hline Endurance WP & $X-29$ & & 225 & & Circular & & & Cuboid & & & & Smooth \\
\hline Endurance WP & E-3120 & & 50 & & Circular & & & Cylinder & & & & Smooth \\
\hline Enercon & E126/7580 & & 7580 & 127 & Circular & 4.5 & 4.1 & Cylinder & & & & Round \\
\hline Gaia-Wind & Gaia-Wind 133 & & 11 & & Circular & & & Cuboid & & & & Smooth \\
\hline Gamesa & $\mathrm{G} 128 / 5000$ & & 5000 & 128 & Circular & & & Cuboid & 4 & 12.5 & 4 & Sharp \\
\hline GE Energy & $4.1-113$ & & 4100 & 113 & Circular & & & Cuboid & & & & Sharp \\
\hline Goldwind & GW100/2500 & & 2500 & 100 & Circular & & & Cuboid & & & & Round \\
\hline Goldwind & GW70/1500 & & 1500 & 70 & Circular & & & Cylinder & & & & Round \\
\hline Hitachi & HWT $2.0-80$ & & 2000 & 80 & Circular & & & Cuboid & 11.5 & 4.9 & 3.5 & Round \\
\hline Hitachi & HWT $5.0-126$ & & 5000 & 126 & & & & Cuboid & & & & Round \\
\hline Senvion & $6.2 \mathrm{M} 126$ & & 6150 & 126 & Circular & & & Cuboid & & & & Sharp \\
\hline Senvion & 6.2M152 & & 6150 & 152 & Circular & & & Cuboid & & & & Sharp \\
\hline Senvion & $5 \mathrm{M}$ & & 5075 & 126 & Circular & 6 & 5.5 & Cuboid & 6 & 18 & 6 & Sharp \\
\hline Siemens & SWT $-3.6-120$ & & 3600 & 120 & Circular & & & Cuboid & & & & Sharp \\
\hline Siemens & SWT $-4.0-130$ & & 4000 & 130 & Circular & & & Cuboid & 4 & 14 & 4.2 & Sharp \\
\hline Siemens & SWT-6.0-154 & & 6000 & 154 & Circular & & & Cylinder & 6.5 & 15 & 6.5 & Round \\
\hline Siemens & SWT-7.0-154 & & 7000 & 154 & Circular & & & Cylinder & & & & Round \\
\hline Sinovel & SL $3000 / 105$ & & 3000 & 105 & Circular & & & Cuboid & & & & Round \\
\hline Sinovel & SL $3000 / 90$ & & 3000 & 90 & Circular & & & Cuboid & & & & Round \\
\hline Sinovel & SL $3600 / 116$ & & 3600 & 116 & Circular & & & Cuboid & & & & Round \\
\hline Sinovel & SL 5000 & & 5000 & 128 & Circular & & & Cuboid & & & & Round \\
\hline Sinovel & SL 6000 & & 6000 & 128 & Circular & & & Cuboid & & & & Round \\
\hline Vergnet & GEV HP & & 1000 & & Circular & & & Cuboid & & & & Sharp \\
\hline Vestas & V112-3.3 MW & & 3,300 & 112 & Circular & 4.5 & & Cuboid & 3.4 & 12.8 & 4 & Round \\
\hline Vestas & V164-8.0 MW & & 8000 & 164 & Circular & 6.5 & 4.5 & Cuboid & 8 & 20 & 8 & Round \\
\hline
\end{tabular}




\subsection{Tower Analysis}

\subsubsection{CFD Simulation Cases}

From the information gathered in Section 5.3, 18 test cases were generated to analyze the flow around a wind turbine tower. The cylinder diameter of $0.5 \mathrm{~m}, 3.5 \mathrm{~m}$ and $5.5 \mathrm{~m}$ which are representative of small, medium and large wind turbines were used.

While the usage of a higher aspect ratio maybe more desirable and realistic to actual application, the simulation using a high $R e$ number requires a highly refined mesh and is computationally expensive. Therefore, only aspect ratios of 1 and 3 were used to check if aspect ratio values are critical to the results. Case 1 to 9 has a cylinder shaped profile with an aspect ratio of 3 while case 10 to 18 has a cylinder shaped profile with an aspect ratio of 1 . The parameters for each of the test cases are shown in Table 5.3.

Table 5.3 Parameters for Tower Test Cases

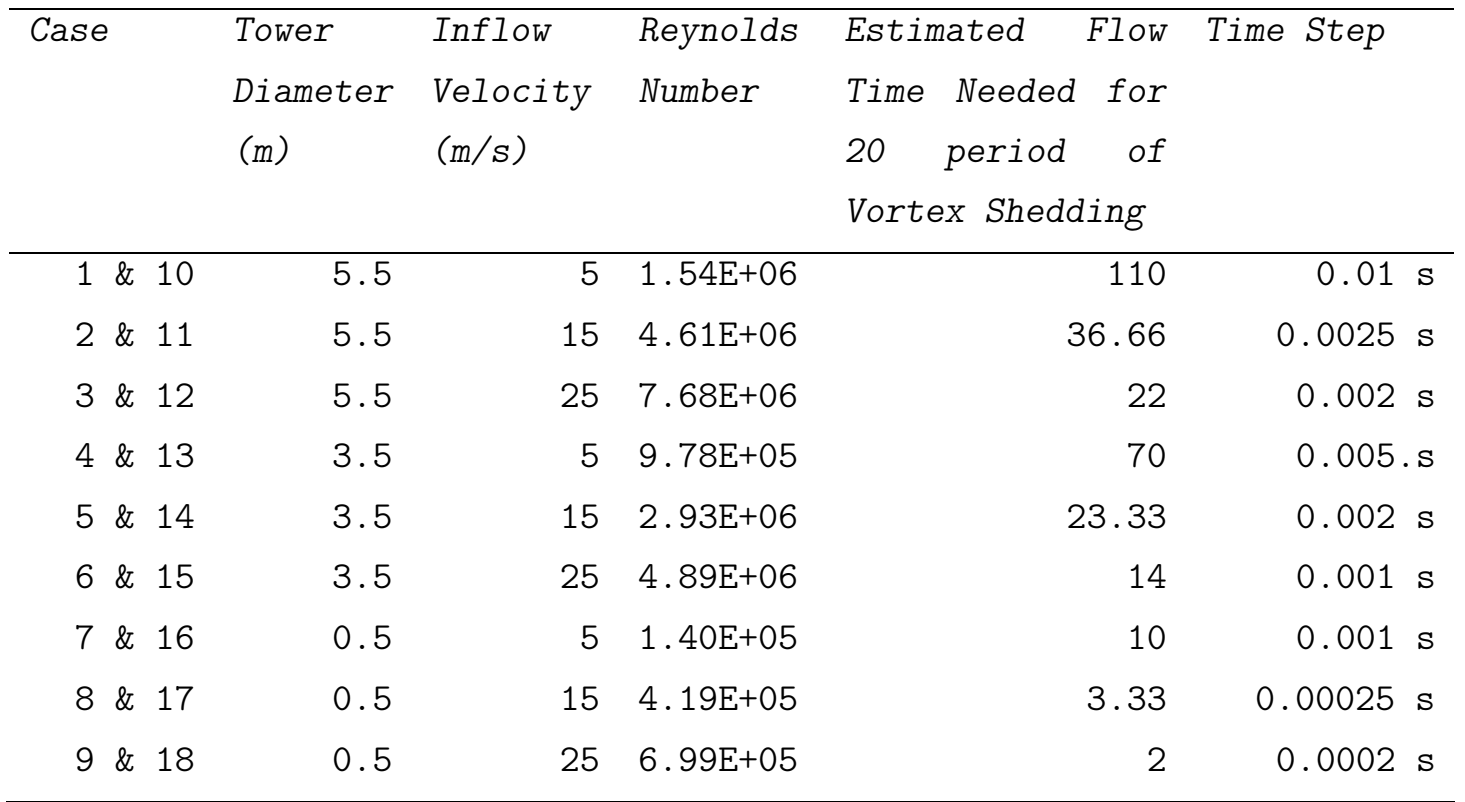

The $R e_{\text {tower }}$ of the flow is calculated based on Eq. (4.4).

$$
\operatorname{Re}_{\text {tower }}=\frac{V_{0} D}{v}
$$


where $v$ is the kinematic viscosity of air.

The estimated flow time was calculated based on the predicted duration for twenty (20) periods of vortex shedding. The frequency of the vortex shedding was estimated using a Strouhal Number $(S t)$ of 0.2 . The $S t$ is calculated based on Eq. (4.5).

$$
S t=\frac{f D}{V}
$$

where $f$ is the frequency of vortex shedding, $D$ is the diameter of the cylinder and $V$ is the velocity of the fluid.

\subsubsection{Mesh and Time Step Validation Check}

A typical methodology to validate the mesh will be to conduct a mesh sensitivity test, which includes various mesh resolutions being simulated for each test case and observing the sensitivity of the results. However, due to the large amount of test cases with various $\mathfrak{R}_{\text {tower }}$ numbers, it will take extensive resources to (1) create a different CFD mesh for each test case and (2) to conduct the mesh sensitivity test.

Instead of creating numerous meshes, the time step value was used to control the accuracy of the simulation and to ensure the convergence of the CFD simulation. To ensure a consistent approach across all test cases, the time step was evaluated based on the Courant-Fredric's-Lowy condition on the surface mesh at the wall as a general guide.

The Courant-Fredric's-Lowy condition follows the equation:

$$
C=\frac{V_{0} \Delta t}{\Delta x} \leq C_{\max }
$$

where $C$ is the Courant number, $\Delta t$ is the time step, $\Delta x$ is the length interval of the computational cell. The value of $C_{\max }=1$ was used as a guide to estimate the time step. 


\subsubsection{CFD Simulation Setup}
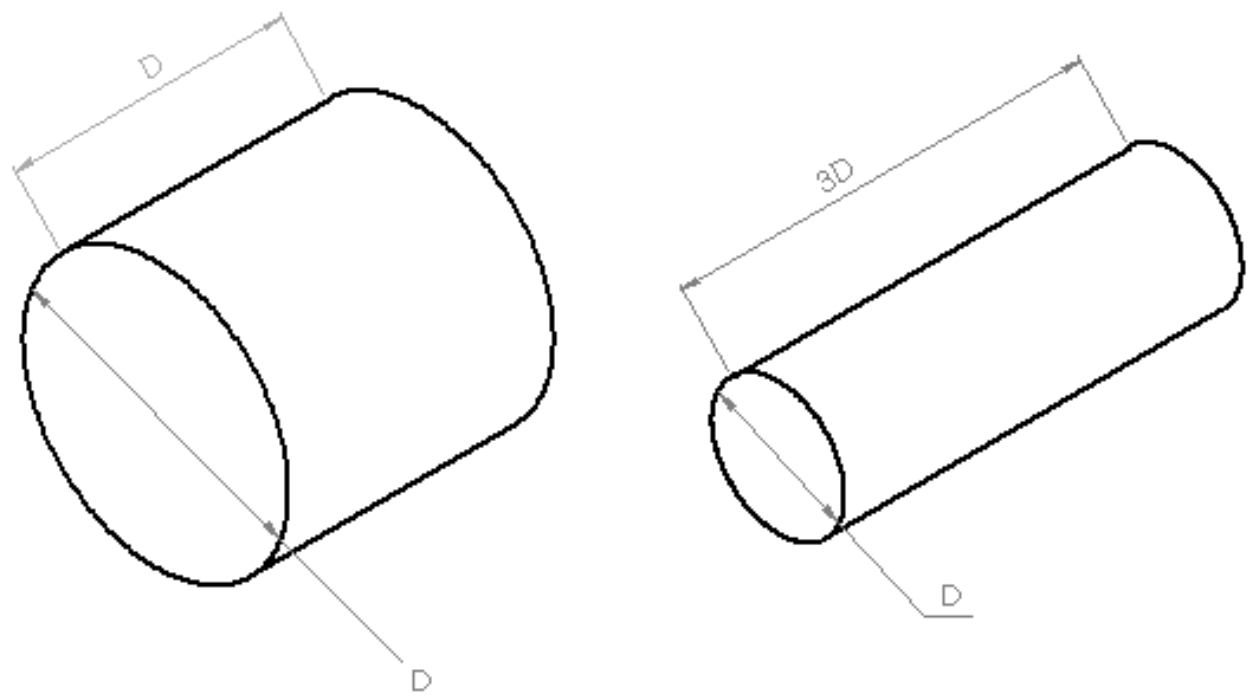

Figure 5.1 Tower Model

The mesh comprises of a fully structured configuration utilizing hexahedron cells. The tower model is shown in Figure 5.1, and the surface mesh and the cross-section of the mesh are shown in Figure 5.2 and the enlarged view is shown in Figure 5.3. In Figure 5.3, the light grey zones; which include a coarser mesh domain and the refined boundary layer were modeled as RANS zones while the dark grey zone, which includes the refined domain were modeled as LES zones. The coarse RANS zone had a $136 \times 40$ mesh and the boundary layer RANS zone had a $362 \times 30$ mesh. The inner circular LES zone had a $362 \times 100$ mesh while the LES zone in the wake affected sector had a $90 \times 100$ mesh. The tower section with an aspect ratio of 1 had 10 elements across and a total of 597,000 elements while the tower section with aspect ratio of 3 had 45 elements across and a total of 2686500 elements.

The green boundary was defined as a velocity inlet, red boundary as a pressure outlet, while the blue zone was defined as the RANS-LES interface as shown in Figure 5.2. Both remaining sides of the mesh domain were defined as symmetry boundary condition. 


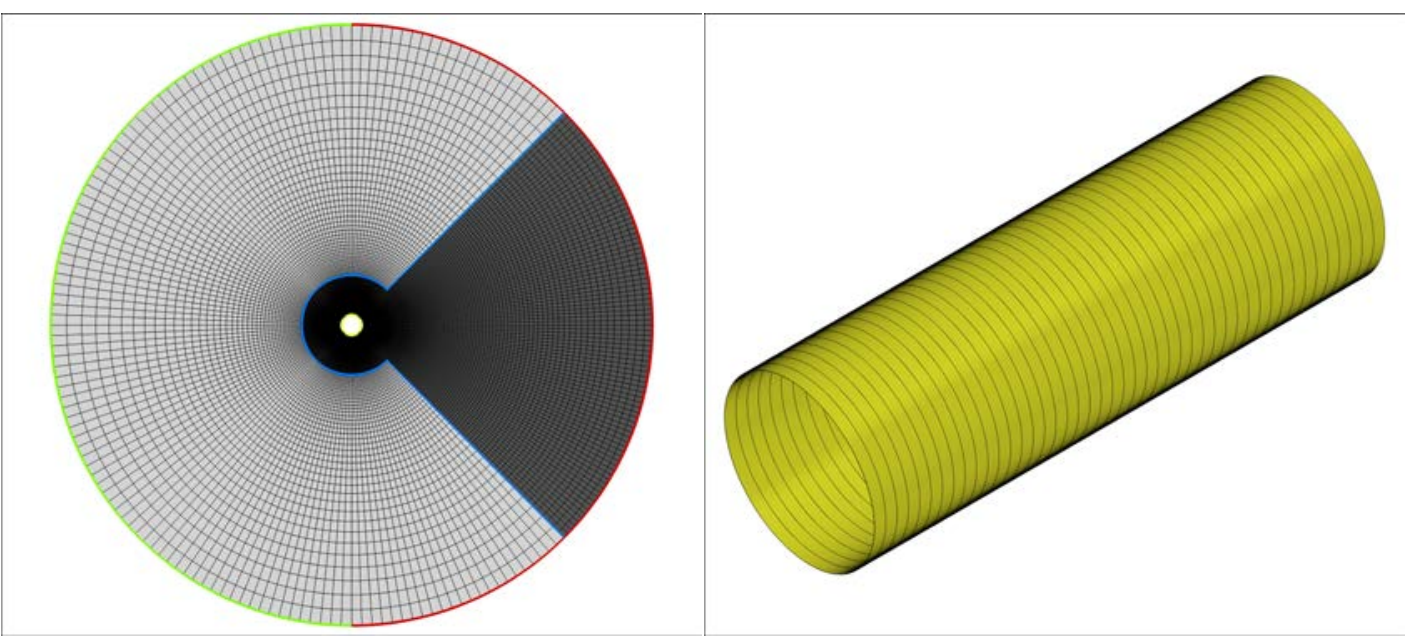

Figure 5.2 Tower Full Domain and Surface Mesh
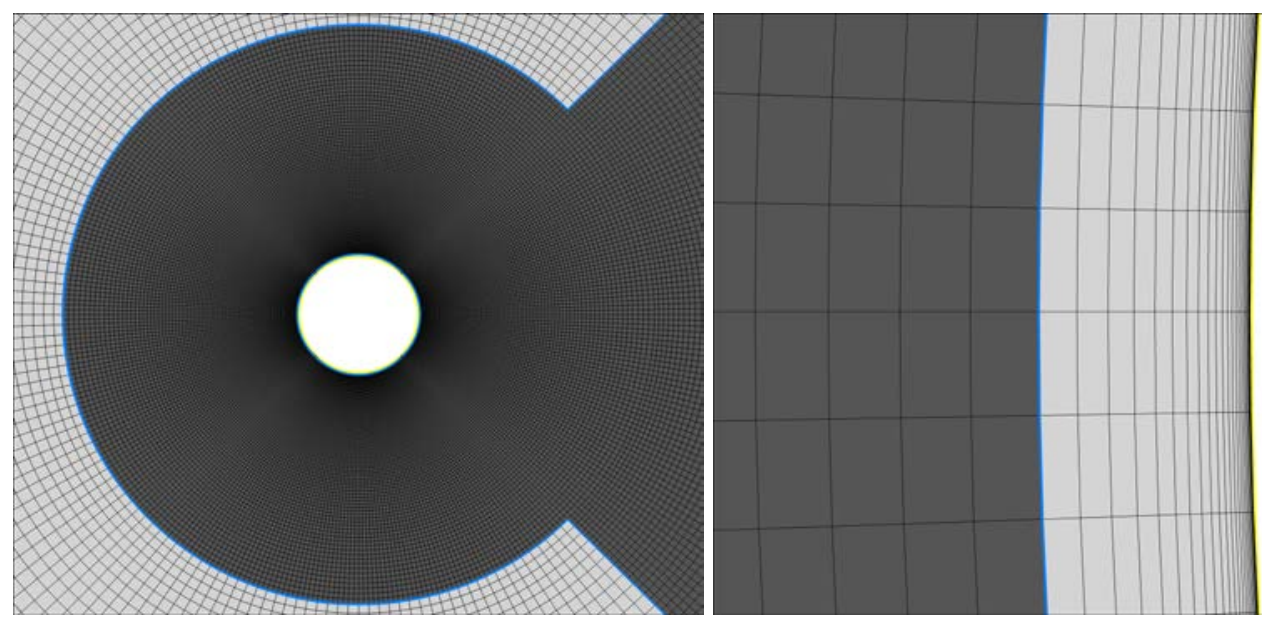

Figure 5.3 Zoom-in Views of Tower Mesh

\subsubsection{CFD Results}

The CFD simulation results were derived from the mid-plane of the cylinder. To avoid the transient conditions resulting from the initialization of the simulation, the time-averaged results were based on the averaged results over the last 10 simulated periods of vortex shedding, where each simulation ran for at least 20 vortex shedding periods.

Figure 5.4 describes the reference frame used for the tower results discussion below. 


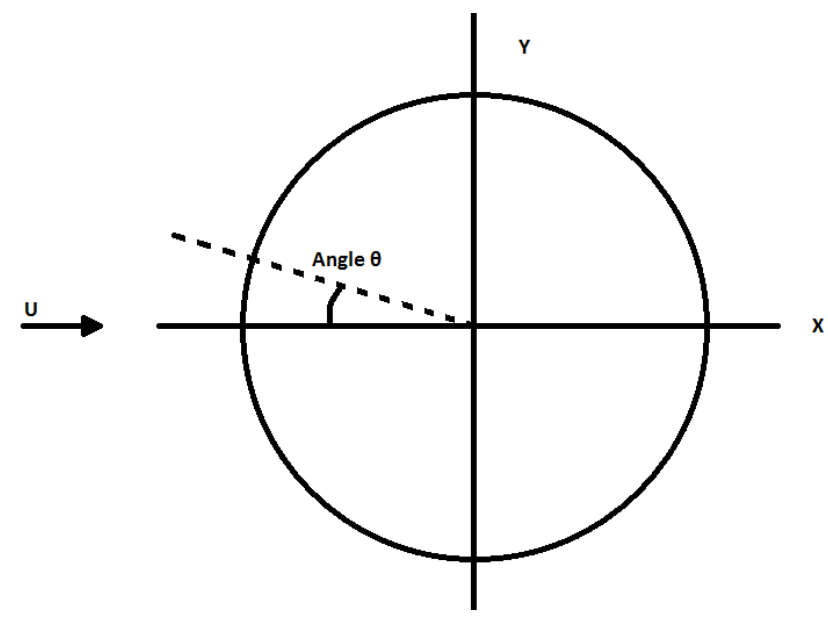

Figure 5.4 Reference Frame for Tower Cases

The origin of the reference frame is located at the center of tower. The normalized $\mathrm{X}$ displacement $x$ is parallel and follows the direction of the inflow wind while the normalized $\mathrm{Y}$ displacement $y$ is perpendicular to the axial direction of the tower. The reference angle along the tower is referred to as $\theta$ and starts clockwise from the stagnation point.

\subsubsection{General Flow Characteristics}

Some general flow characteristics have been observed in all the simulated cases. Figure 5.5, Figure 5.6 and Figure 5.7 shows the instantaneous normalized X and Y velocity, and vorticity, while Figure 5.8 and Figure 5.9 shows the time-averaged normalized $\mathrm{X}$ and $\mathrm{Y}$ velocity $\left(v_{x}\right.$ and $\left.v_{y}\right)$ for Case $2\left(R e_{\text {tower }}=5.46 \mathrm{E}+06\right)$. The figures for the remaining cases are presented in Appendix D.

The wind velocity was reduced upwind of the tower before being sped up along the side of the tower. The fluid flow was in the post-critical regime and experiences turbulent separation of flow with shear layer instabilities being observed. The downstream wake was turbulent, and periodic vortex shedding were observed, which is similar to Roshko (1961) observations. Velocity deficit and mean reversed flow were observed behind the tower, and the flow recovers gradually downstream. 


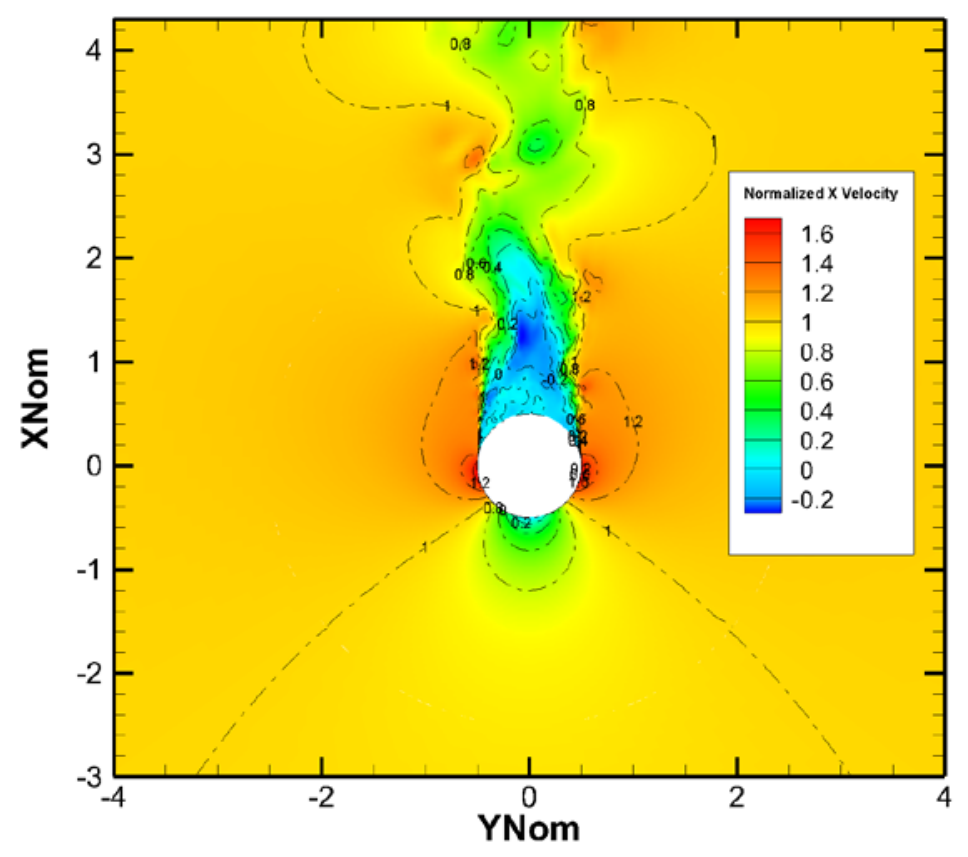

Figure 5.5 Instantaneous Normalized X Velocity for Tower Case 2: $\boldsymbol{R e}_{\text {tower }}=$ $4.61 \mathrm{E}+06$

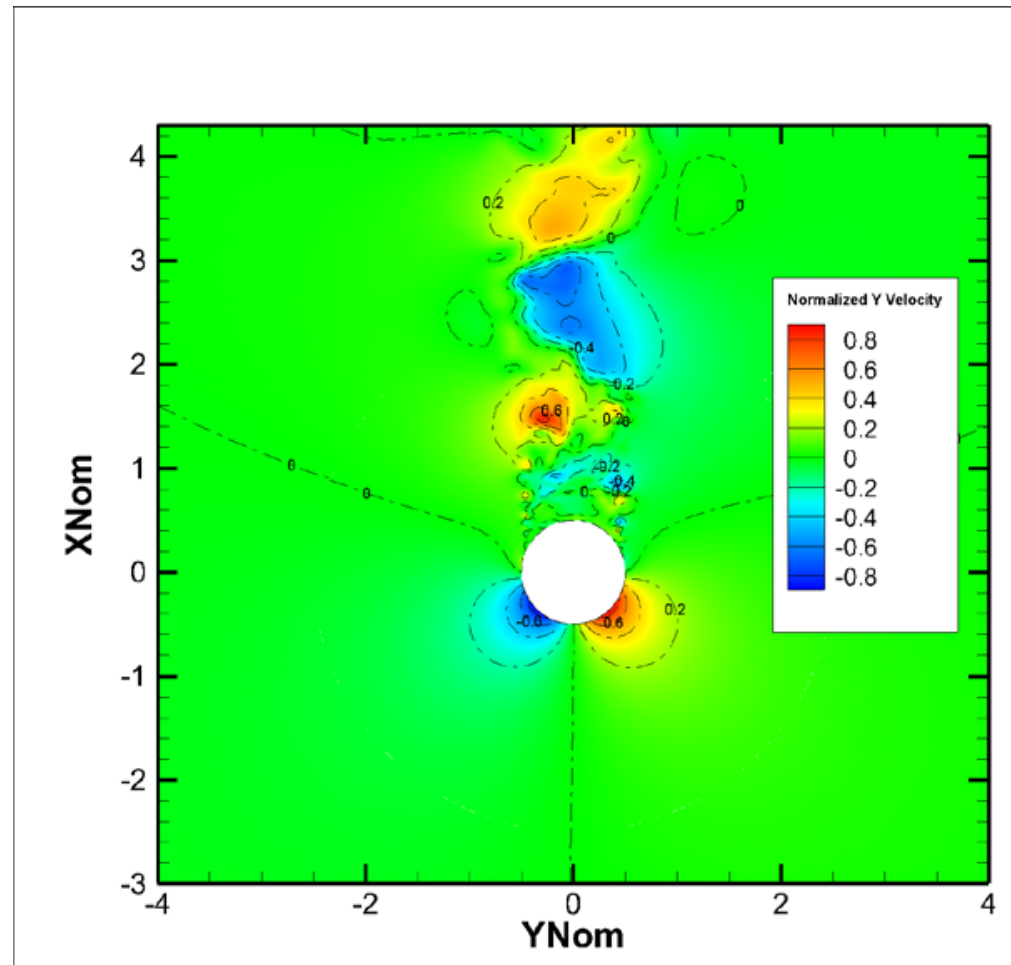

Figure 5.6 Instantaneous Normalized Y Velocity for Tower Case 2: $\boldsymbol{R e}_{\text {tower }}=$ $4.61 \mathrm{E}+06$ 


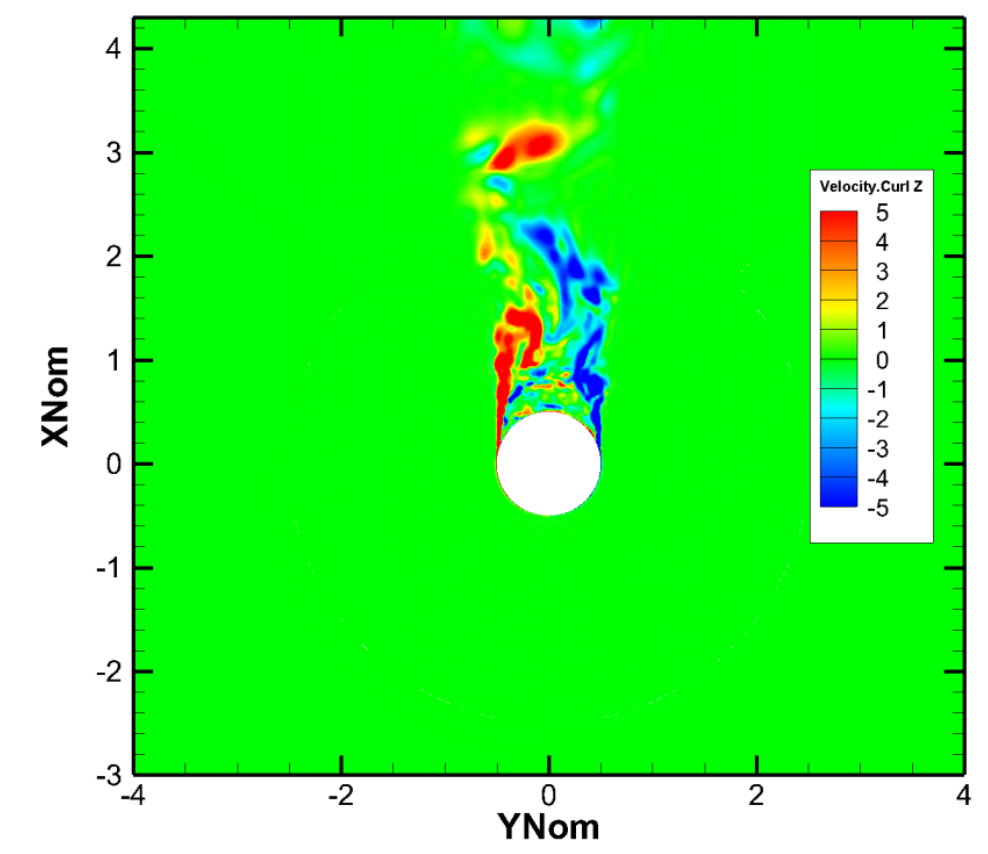

Figure 5.7 Instantaneous Vorticity $\mathrm{Z}$ for Tower Case 2: $R e_{\text {tower }}=4.61 \mathrm{E}+06$

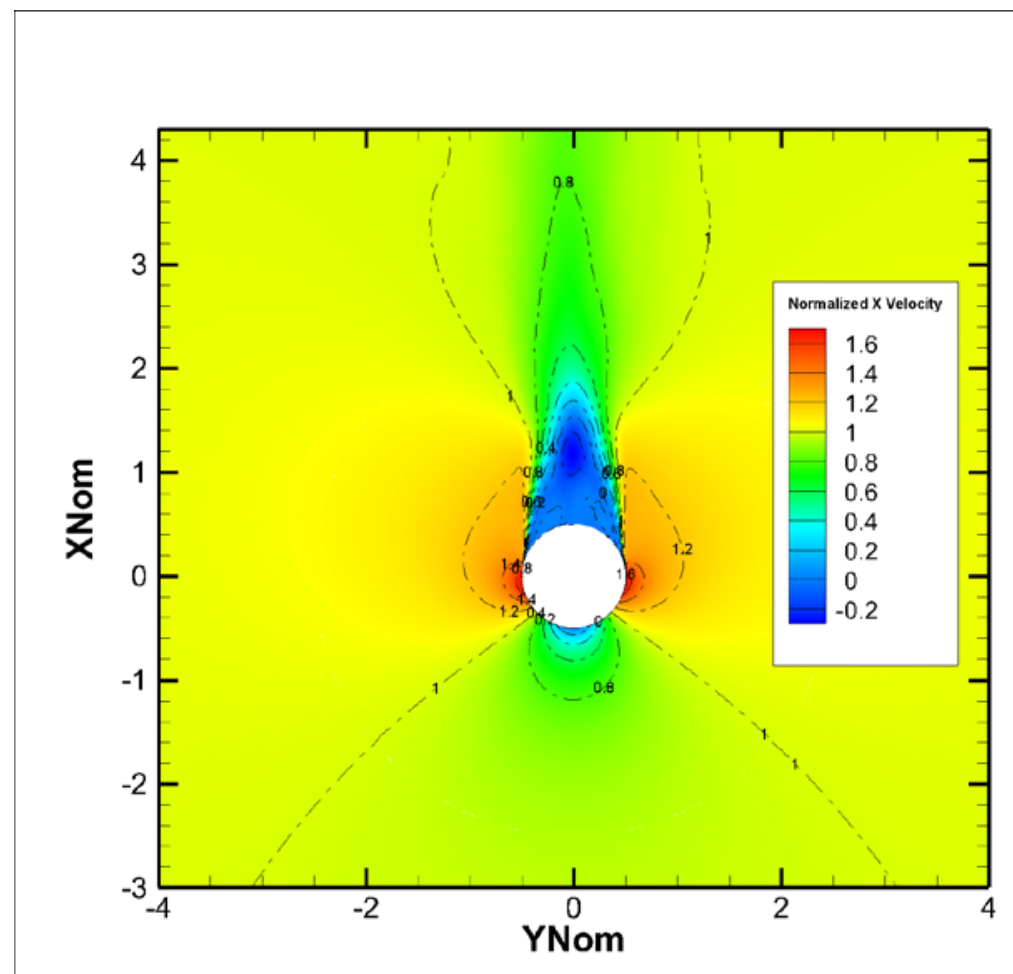

Figure 5.8 Mean Normalized X Velocity for Tower Case 2: $R e_{\text {tower }}=4.61 \mathrm{E}+06$ 


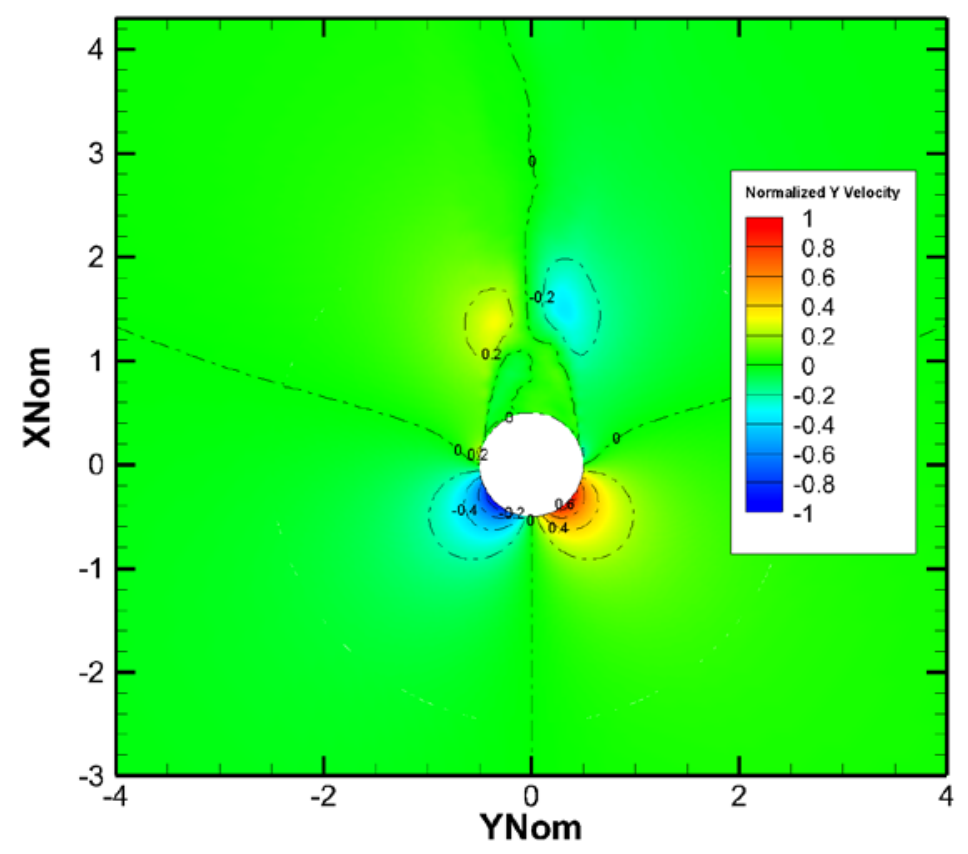

Figure 5.9 Mean Normalized X Velocity for Tower Case 2: $R e_{\text {tower }}=4.61 \mathrm{E}+06$

\subsubsection{Comparison of Different Aspect Ratios}

Figure 5.10 shows the $C_{D \text {,tower }}$ vs flow time graph for Case 1 to Case 9 . The red line shows the averaged $C_{D, \text { tower }}$ value along the cross-section of the midplane of the cylinder over the last 10 vortex shedding periods. It was observed that the $C_{D \text {,tower }}$ value in the initial part of the simulation was very low and it took approximately one quarter of the total flow time for the $C_{D \text {,tower }}$ value to stabilize. The minor fluctuations from the averaged $C_{D, \text { tower }}$ value were due to the vortex shedding of the flow behind the cylinder. 


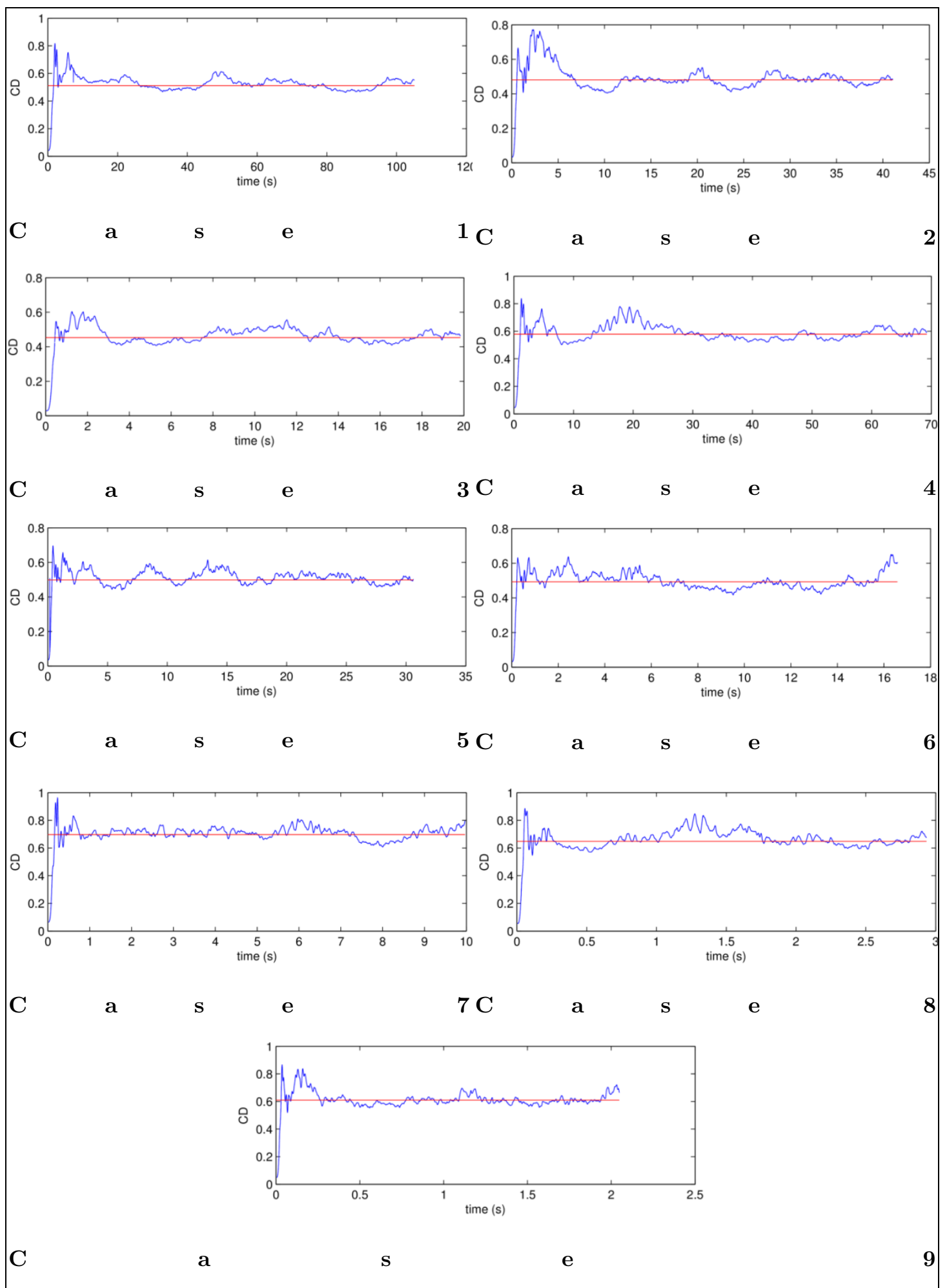

Figure 5.10 $C_{D, \text { tower }}$ vs Flow Time

Figure 5.11 shows the $C_{L, \text { tower }}$ vs flow time graph for Case 1 to Case 9. The red line shows the averaged $C_{L, \text { tower }}$ value (close to zero for all cases) along the cross-section of the mid-plane of the cylinder over last 10 vortex shedding periods. The fluctuations from the averaged $C_{L, t o w e r}$ value followed 
a regular sinusoidal function due to the vortex shedding of the flow behind the cylinder. The amplitude of the fluctuations was noted to decrease with increasing $R e_{\text {tower }}$.

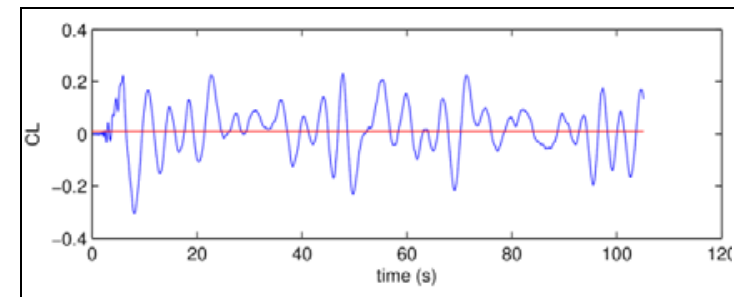

Case 1

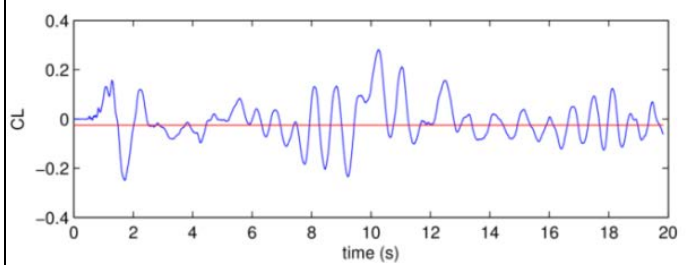

Case 3

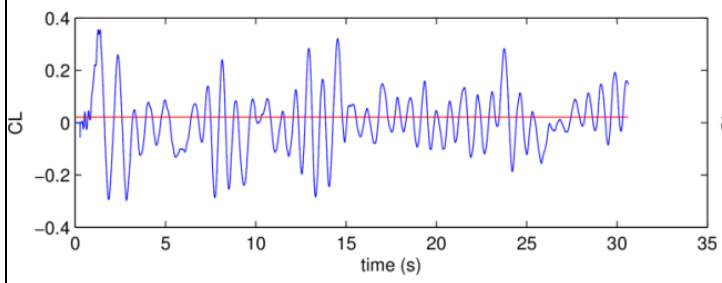

Case 5

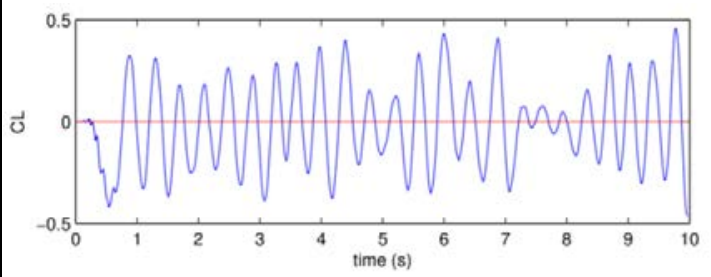

Case 7

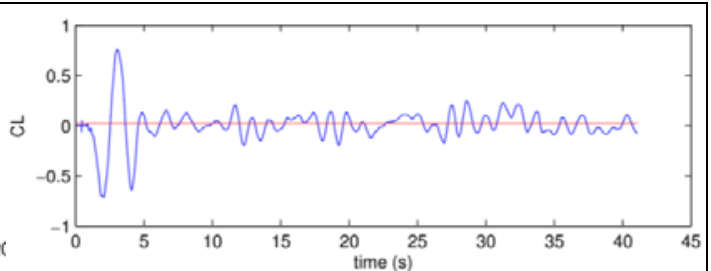

Case 2

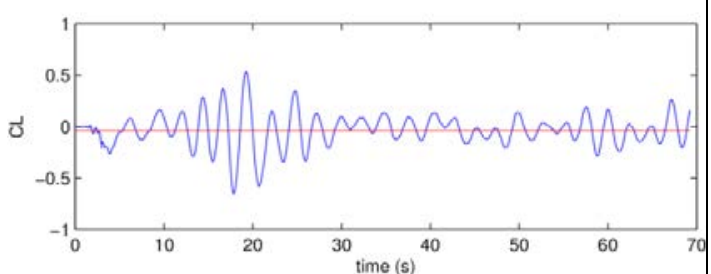

Case 4

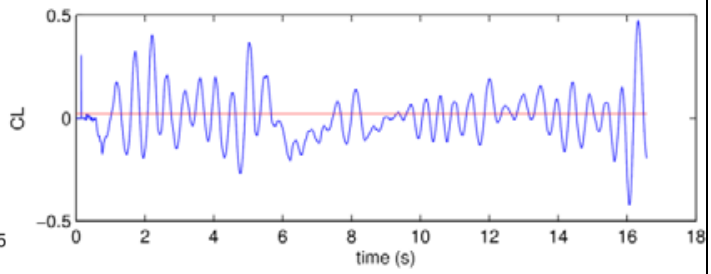

Case 6

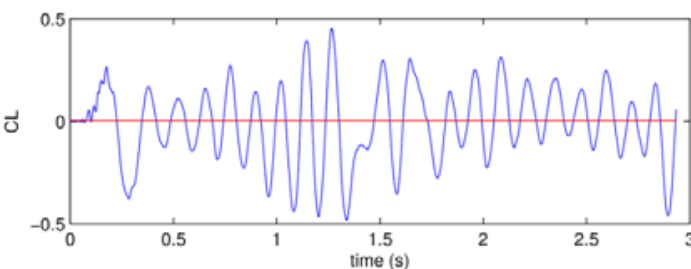

Case 8

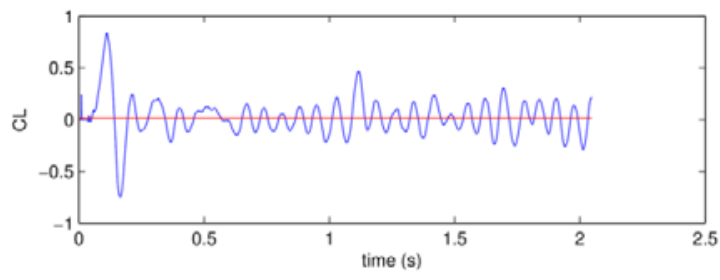

Case 9

Figure 5.11 $C_{L, t o w e r}$ vs Flow Time 
From the time series $C_{L, \text { tower }}$ data, a FFT analysis was done to obtain the vortex shedding frequencies for all the test cases. An example of the FFT analysis for Case 1 is shown in Figure 5.12. The remaining FFT plots can be found to in Appendix E.
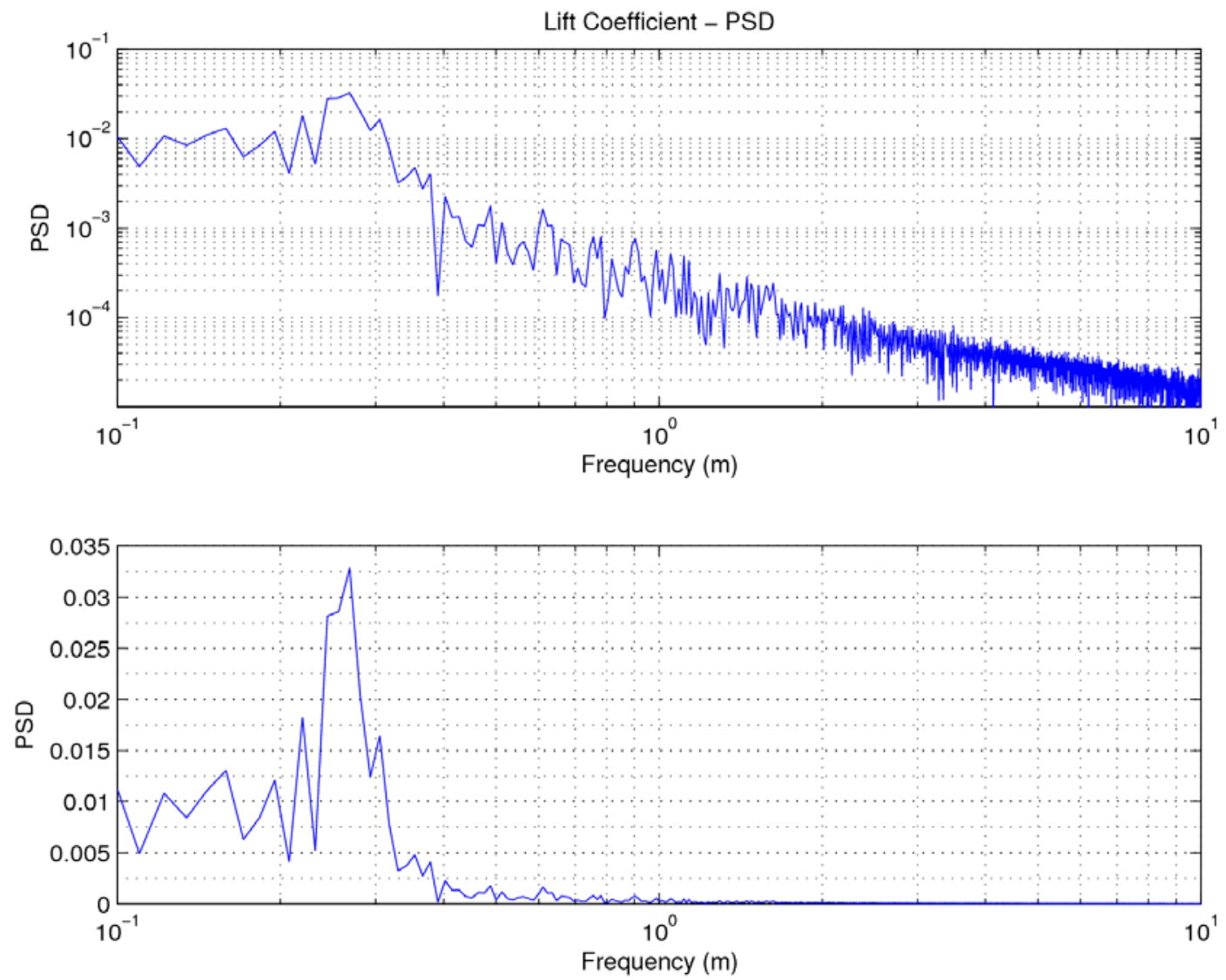

Figure 5.12 FFT of $C_{l}$ data for Tower Case 1 in Log and Linear Scale

For most cases, only one strong peak was observed during the FFT analysis.

However, Case 2 (as shown in Figure 5.13) had multiple peaks and this may be a result of shear layer instability for high $R e_{\text {tower }}$ flow. 

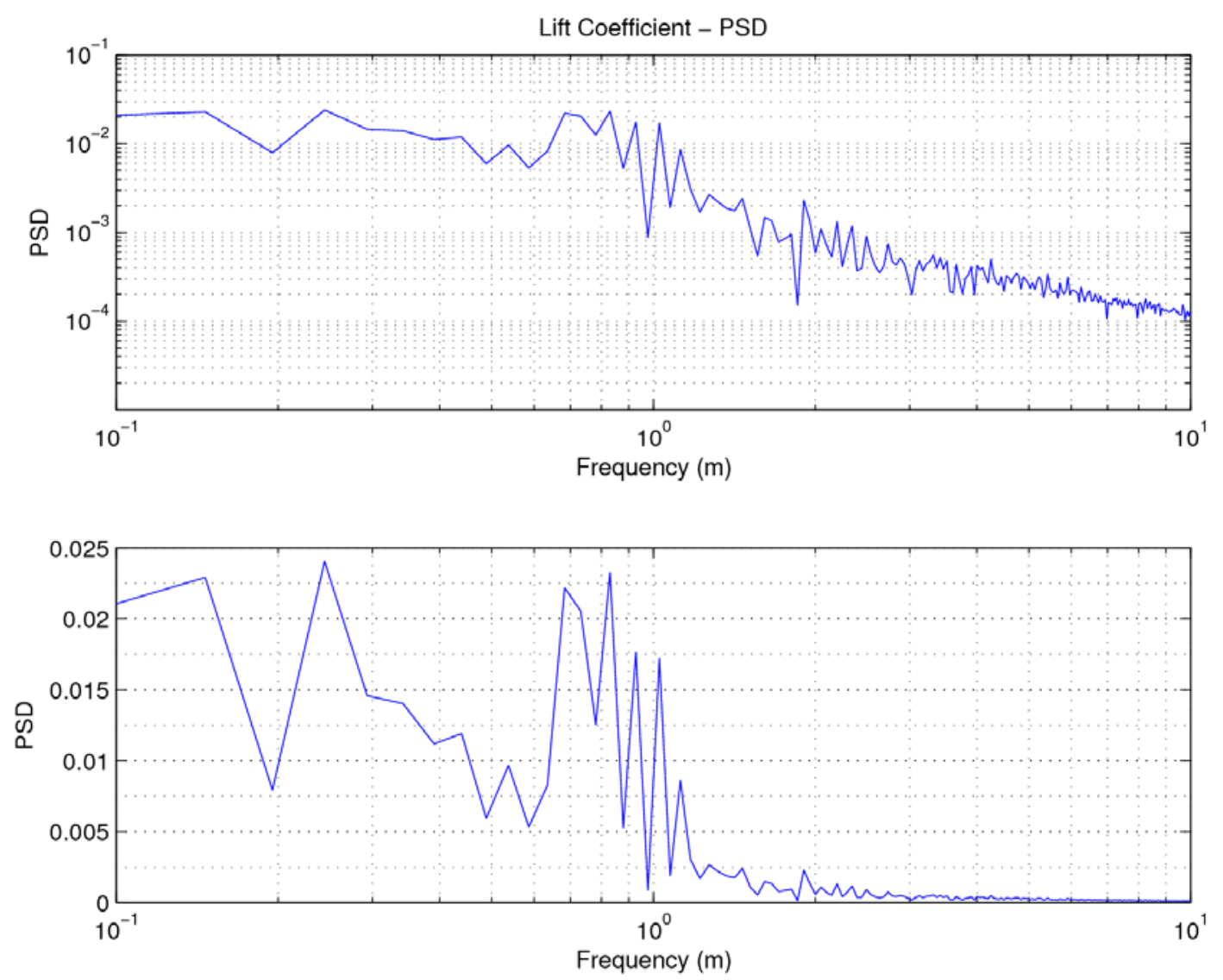

Figure 5.13 FFT of $C_{l}$ data for Tower Case 2 in Log and Linear Scale

Results for Case 10 to Case 18 were obtained by applying similar postprocessing methodology. Table 5.4 shows a summary of the results of vortex shedding frequency, mean $C_{L, \text { tower }}\left(C_{L, \text { mean }}\right)$ and mean $C_{D, \text { tower }}\left(C_{D, \text { mean }}\right)$ by comparing the cases of aspect ratio of 1 and 3. The results of Case 1 to Case 9 were similar with Case 10 to Case 18 with minor deviations within $5 \%$. Case 4(13) and Case 5(14) showed more deviations with different aspect ratio. As a result, it will be more conservative to use cases of aspect ratio of 3 for further analysis. 
Table 5.4 Vortex Shedding Frequency, mean $C_{L, \text { tower }}$ and mean $C_{D, t o w e r}$ for Tower Cases

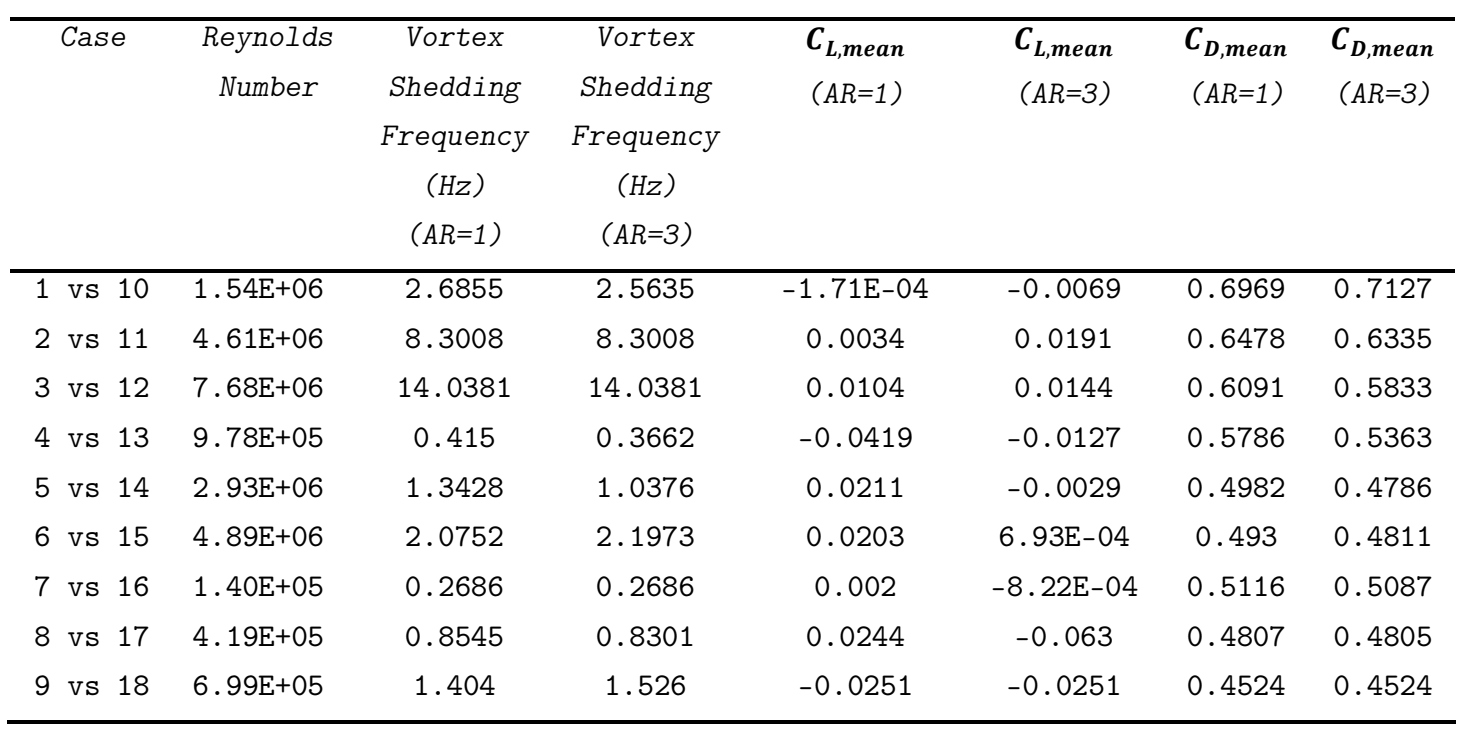

\subsubsection{Comparison with Results from Literature}

There have been a numerous number of experiments and CFD simulations conducted over the past decades on the flow over a circular cylinder. Most are based on low $R e_{\text {tower }}$ flow that can be conducted physically in laboratory-sized wind and water tunnels or computationally using CFD simulations. High $R e_{\text {tower }}$ flow experiments, where $R e_{\text {tower }}$ range between $10^{5}-10^{6}$, are less commonly seen as they often involve using a high speed, pressurized and/or large wind tunnel. Due to the complexity of the flow, only a small number of numerical simulations have been conducted to predict high $R e_{\text {tower }}$ flows.

In Table 5.5, the commonly cited literatures that were used for the comparison in this study are described. The later subsections show the data comparison with the simulation results in this study. 
Table 5.5 Literatures Used for Tower Results Comparison

\begin{tabular}{|c|c|c|c|c|c|c|}
\hline Author & $\begin{array}{l}\text { Experiment } \\
\text { Type }\end{array}$ & Re Number & $\begin{array}{l}\text { Mach } \\
\text { No. }\end{array}$ & $\begin{array}{l}\text { Turbulence } \\
\text { Intensity }\end{array}$ & $\begin{array}{l}\text { Model } \\
\text { Dimension }\end{array}$ & $\begin{array}{l}\text { Comparison } \\
\text { Data Used }\end{array}$ \\
\hline Wieselsberger (1921) & $\begin{array}{l}\text { Wind tunnel with } \\
\text { weight } \\
\text { measurement }\end{array}$ & $4.2-8 \times 10^{5}$ & $<0.1$ & - & $\begin{array}{l}0.05 \mathrm{~mm} \mathrm{-} \\
300 \mathrm{~mm}\end{array}$ & $C_{D, \text { mean }}$ \\
\hline $\begin{array}{l}\text { Delany and Sorensen } \\
(1953)\end{array}$ & $\begin{array}{l}\text { Wind tunnel with } \\
\text { force measurement } \\
\text { and pressure probe }\end{array}$ & $10^{4}-2 \times 10^{6}$ & $<0.36$ & - & $\begin{array}{ll}25.4 & \mathrm{~mm}, \\
101.6 \mathrm{~mm}, \\
304.8 \mathrm{~m}\end{array}$ & $C_{D, \text { mean }}, S t$ \\
\hline Roshko (1961) & $\begin{array}{l}\text { Pressurized wind } \\
\text { tunnel with hot } \\
\text { wire anemometer } \\
\text { and pressure } \\
\text { transducers }\end{array}$ & $\begin{array}{l}3.5 \times 10^{6}- \\
8.4 \times 10^{6}\end{array}$ & $<0.25$ & - & $\begin{array}{l}457.2 \mathrm{~mm} \mathrm{X} \\
2591 \quad \mathrm{~mm} \\
\text { (with and } \\
\text { without } \\
\text { splitter plate) }\end{array}$ & $C_{p}, C_{p, \text { base }}, S t$ \\
\hline Achenbach (1968) & $\begin{array}{l}\text { Pressurized wind } \\
\text { tunnel with } \\
\text { pressure } \\
\text { transducers and } \\
\text { skin friction probe }\end{array}$ & $\begin{array}{l}6 \times 10^{4}- \\
5 \times 10^{6}\end{array}$ & $<0.1$ & $0.7 \%$ & $\begin{array}{l}150 \mathrm{~mm} \quad \mathrm{X} \\
500 \mathrm{~mm}\end{array}$ & $\begin{array}{l}C_{p}, \quad C_{D, \text { mean }}, \\
\text { separation angle } \\
\text { and skin friction. }\end{array}$ \\
\hline $\begin{array}{l}\text { Jones Jr, Cincotta, } \\
\text { and Walker (1969) }\end{array}$ & $\begin{array}{l}\text { Pressurized wind } \\
\text { tunnel with force } \\
\text { and pressure } \\
\text { transducers }\end{array}$ & $\begin{array}{l}3.6 \times 10^{5}- \\
1.87 \times 10^{7}\end{array}$ & $<0.6$ & $0.17 \%$ & $\begin{array}{l}914 \mathrm{~mm} \quad \mathrm{X} \\
5182 \mathrm{~mm}\end{array}$ & $\begin{array}{l}C_{p}, \quad C_{p, \text { base }}, \\
C_{D, \text { mean }}, S t\end{array}$ \\
\hline $\begin{array}{l}\text { James, Paris, and } \\
\text { Malcolm (1980) }\end{array}$ & $\begin{array}{ll}\text { Pressurized } & \text { wind } \\
\text { tunnel } & \text { with } \\
\text { pressure } & \\
\text { transducers } & \end{array}$ & $\begin{array}{l}1.5 \times 10^{5}- \\
1.1 \times 10^{7}\end{array}$ & $<0.29$ & & $\begin{array}{l}152.4 \mathrm{~mm}, \\
316.5 \mathrm{~mm}, \\
451.6 \mathrm{~mm}\end{array}$ & $\begin{array}{l}C_{p}, \quad C_{p, \text { base }}, \quad S t, \\
\text { Seperation angle }\end{array}$ \\
\hline Schewe (1983) & $\begin{array}{l}\text { Pressurized wind } \\
\text { tunnel }\end{array}$ & $\begin{array}{l}2.3 \times 10^{4}- \\
7.1 \times 10^{6}\end{array}$ & & $<0.4 \%$ & $60 \mathrm{~mm} \times 600$ & $C_{D, \text { mean }}, S t$ \\
\hline $\begin{array}{l}\text { Cantwell and Coles } \\
\text { (1983) }\end{array}$ & $\begin{array}{ll}\text { Wind tunnel with } \\
\text { flying hot wire } \\
\text { anemometer and } \\
\text { pressure } & \\
\text { transducers } & \end{array}$ & $10^{3}-10^{6}$ & $<0.25$ & $<1 \%$ & $\begin{array}{l}101.4 \mathrm{~mm} \mathrm{X} \\
2970 \mathrm{~mm}\end{array}$ & $\begin{array}{lr}C_{p}, \quad C_{D, \text { mean }}, S t, \\
\text { Mean flow } \\
\text { velocities }\end{array}$ \\
\hline $\begin{array}{l}\text { Shih, Wang, Coles, } \\
\text { and Roshko (1993) }\end{array}$ & $\begin{array}{ll}\text { Pressurized } & \text { wind } \\
\text { tunnel } & \text { with } \\
\text { pressure } & \\
\text { transducers } & \end{array}$ & $\begin{array}{l}3 \times 10^{5}- \\
8 \times 10^{6}\end{array}$ & $<0.29$ & $0.04-0.08 \%$ & $\begin{array}{l}316 \mathrm{~mm} \mathrm{X} \\
2600 \quad \mathrm{~mm} \\
\text { with various } \\
\text { roughness }\end{array}$ & $\begin{array}{l}C_{p}, \quad C_{p, \text { base }}, \\
C_{D, \text { mean }}, S t, \\
\text { separation angle }\end{array}$ \\
\hline
\end{tabular}




\section{$C_{D, \text { mean }}$ vs $\operatorname{Re}_{\text {tower }}$}

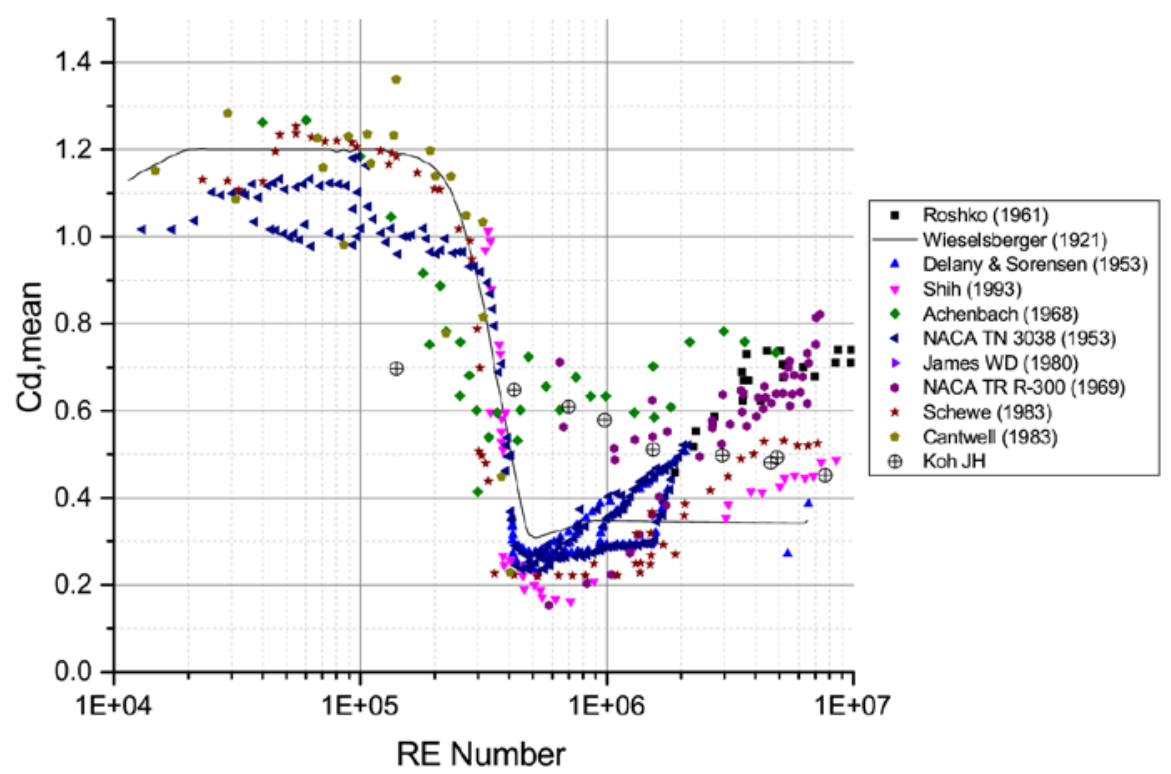

Figure 5.14 Comparison graph between experimental and simulation data for $C_{D, \text { mean }}$ vs $\boldsymbol{R e}_{\text {tower }}$

The spread of values in the post-critical regime of the flow was generally quite large due to sensitivities to the surface roughness and flow turbulence on $C_{D \text {,mean }}$. When comparing the $C_{D \text {,mean }}$ vs $R e_{\text {tower }}$ data in Figure 5.14 , the simulation results in the post-critical regime stayed within the spread of $C_{D, \text { mean }}$ values and compared reasonably well with the experimental data. For Case $7\left(R e_{\text {tower }}=1.40 \mathrm{E}+05\right)$, a much lower $C_{d \text {,mean }}$ was predicted as the flow has transited to a turbulent separation in the CFD simulation.

\section{St vs Re}

The spread of values in the critical regime of the flow was generally quite large, with some of the experimental results showing a spike in $S t$ values of up to 0.48. Utilizing the frequency data obtained from the $C_{l}$ FFT plots, $S t$ was calculated using Equation (4.5). The simulation results did not show a spike in $S t$ value in the critical regime, but remained relatively constant with a slight trend of increasing $S t$ number with increasing $R e_{\text {tower }}$ within the range of 0.242 and 0.336 . There was a slight offset with the experimental 
data, but similar trends were observed. The results are presented in Figure 5.15 .

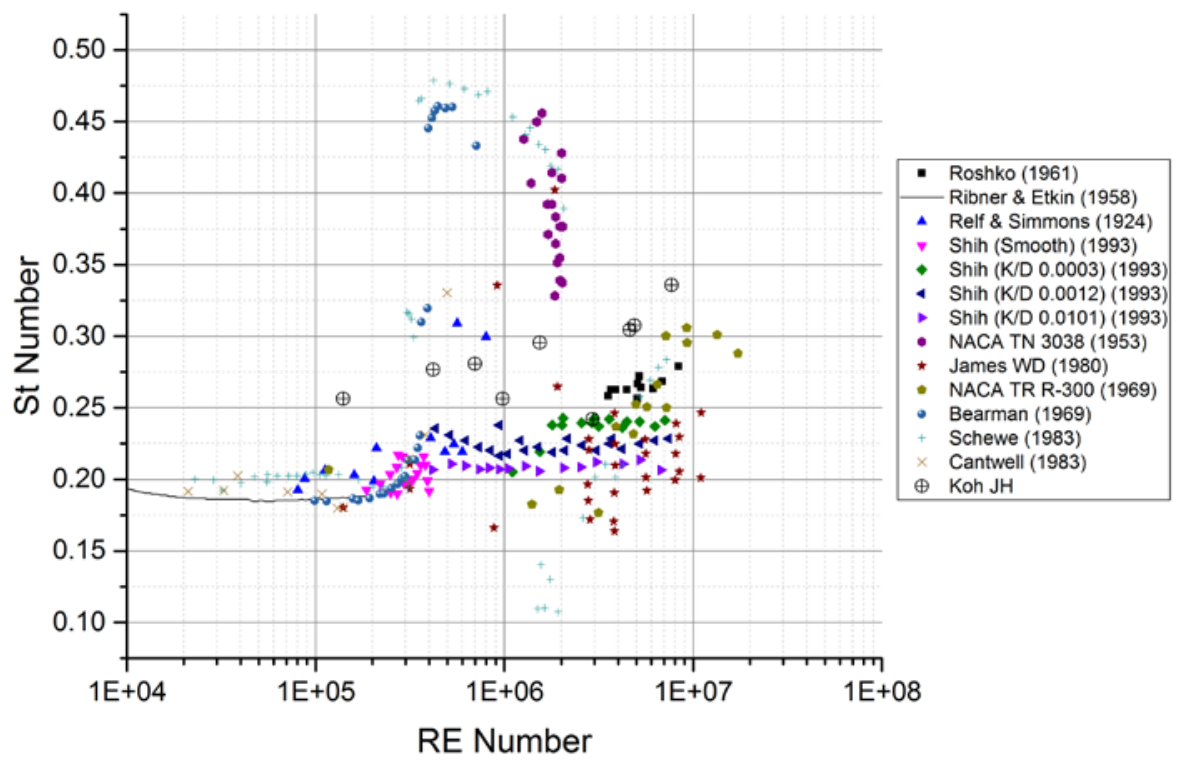

Figure 5.15 Comparison graph between experimental and simulation data for $S t$ vs $\boldsymbol{R e}_{\text {tower }}$

\section{$C_{P}$ vs $\theta$}

Figure 5.16 shows the $C_{P}$ vs $\theta$ along cylinder graph for Case 1 to Case 9 in comparison with experiment results in similar range of $R e_{\text {tower }}$. Note that Case 2 and Case 6 have been compared together within one of the subgraphs. The simulation results showed similar graph shape and trends with the experiment results. The simulation data compared reasonably well and stayed within spread of values of the experiment data. 

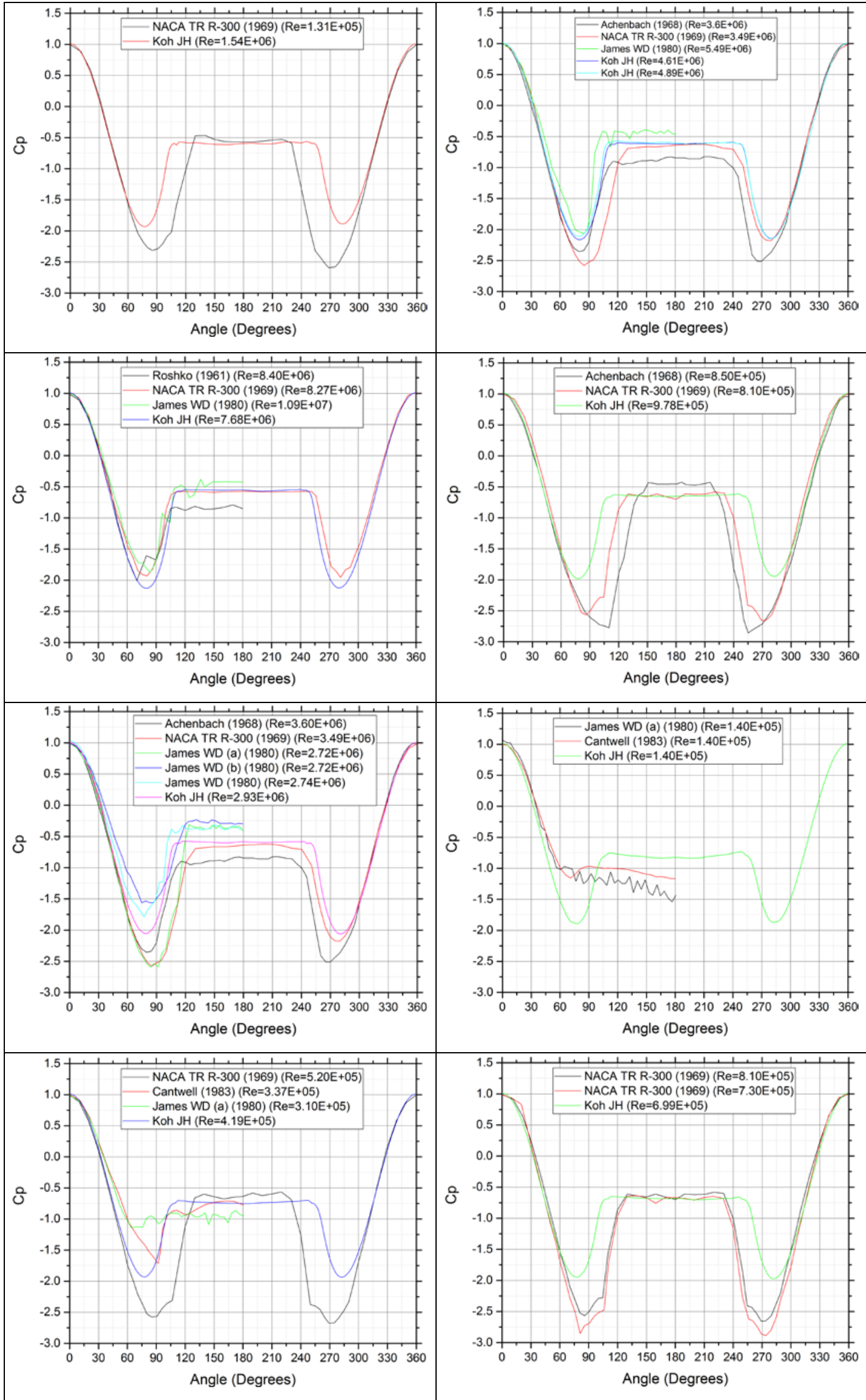

Figure $5.16 C_{P}$ vs $\theta$ 


\section{$C_{p, b a s e} v s R_{\text {tower }}$}

The spread of values in the critical and post-critical regime of the flow was generally quite large for the experiment results. When comparing the $C_{p \text {,base }}$ vs $R e_{\text {tower }}$ data in Figure 5.17, the simulation results followed the NACA TR R-300 results closely and do not displace any strong variation across the $R e_{\text {tower }}$ values. For Case $7\left(R e_{\text {tower }}=1.40 \mathrm{E}+05\right)$, a much higher $C_{p, \text { base }}$ was predicted as the flow has transited to a turbulent separation in the CFD simulation.

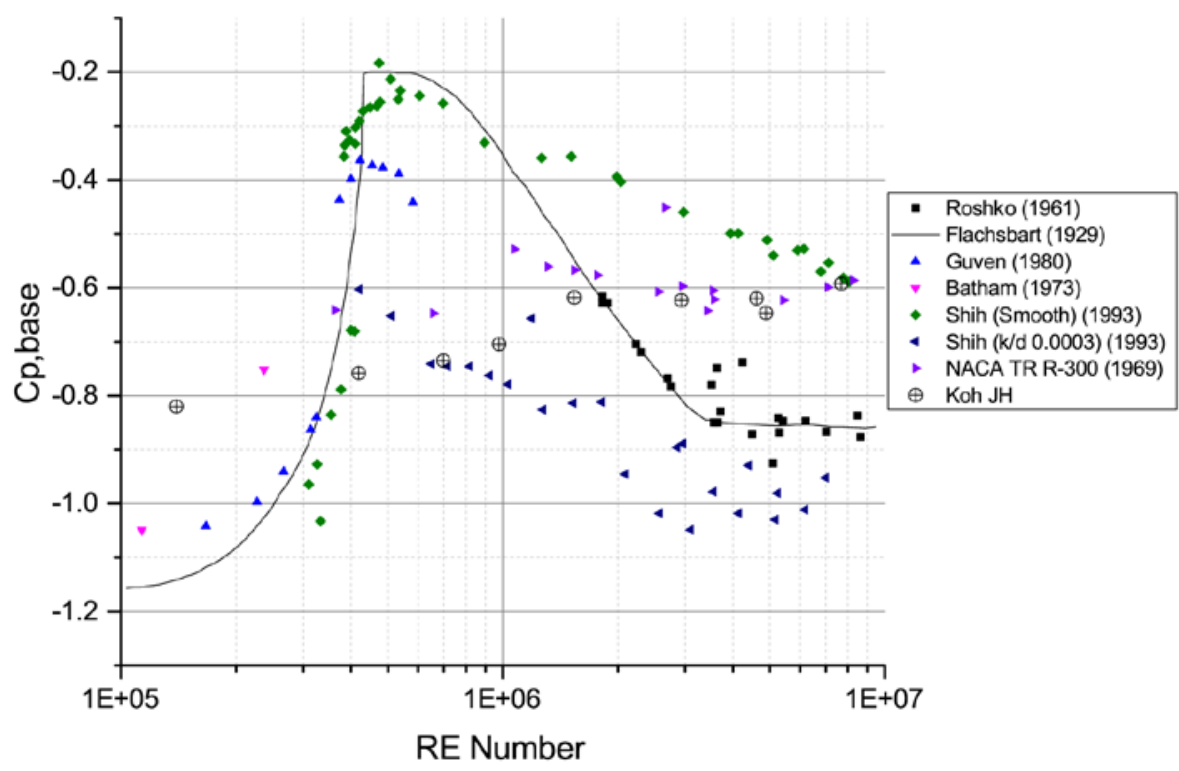

Figure $5.17 C_{p, b a s e}$ vs $R_{\text {tower }}$

$C_{p, b a s e}-C_{p, \min }$ vs $R_{\text {tower }}$

The pressure rise $C_{p, \text { base }}-C_{p, \min }$ is plotted against $R e_{\text {tower }}$ in Figure 5.18, which is used to determine flow separation for some experiment cases. As observed in the experiments, pressure rise was highly dependent on the surface roughness. The pressure rise was independent of $R e_{\text {tower }}$ for the rough cases, whereas the smooth cases showed a reduced pressure rise for higher $R e_{\text {tower }}$. The simulation results showed that the pressure rise was independent of $R e_{\text {tower }}$, with the magnitude approximately average of both smooth and rough cases from the experiment. 


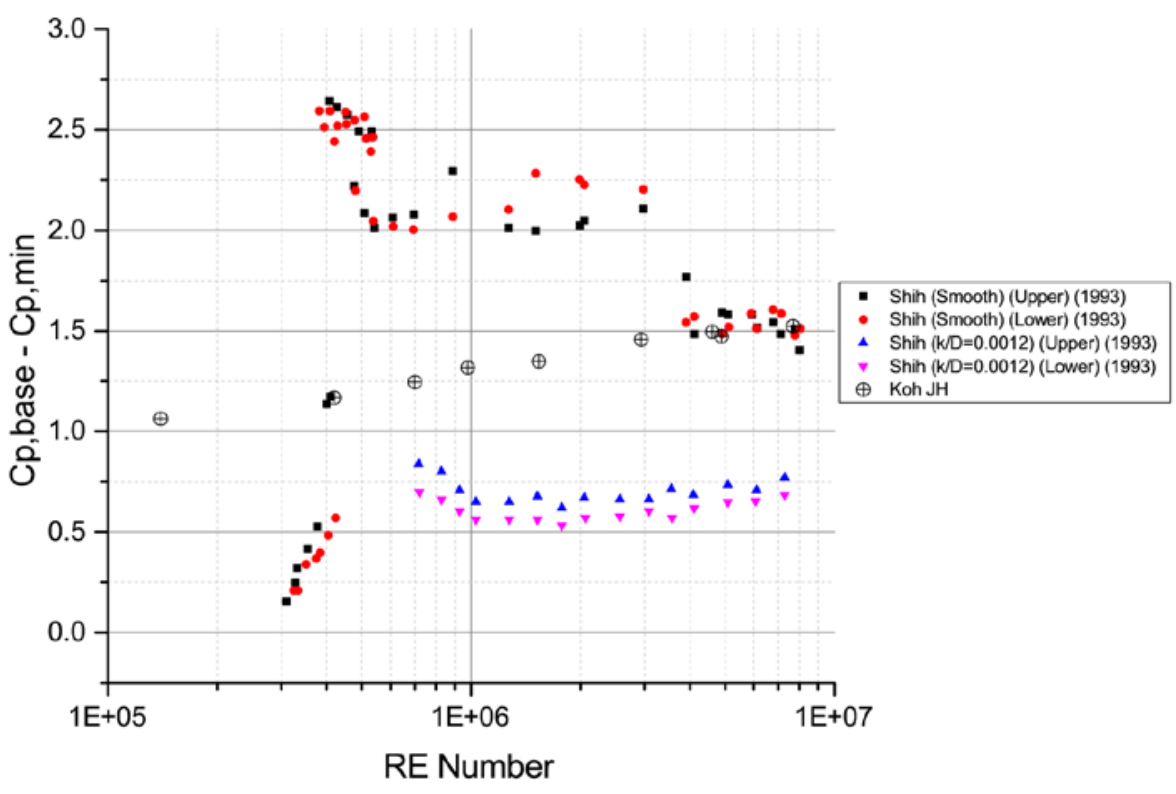

Figure $5.18 C_{p, b a s e}-C_{p, \min }$ vs $R e_{\text {tower }}$

\section{Separation Angle vs $\boldsymbol{R e}_{\text {tower }}$}

The separation angle of experimental data gathered was typically estimated using two different methodologies due to measurement challenges. For practical purposes, the first method defines the separation angle as the location where $C_{p}$ has risen half way from $C_{p, \min }$ to $C_{p, \text { base }}$. Experiment results using this method are compared with the simulation results in Figure 5.43 .

The second method is to measure the local shear stresses at the wall using a skin friction probe, in which the separation angle is indicated by when the skin friction becomes zero. Experiment results using this method were compared with the simulation results in Figure 5.20.

For Case $7\left(R e_{\text {tower }}=1.40 \mathrm{E}+05\right)$, a much higher separation angle was predicted as the flow has transited to a turbulent separation in the CFD simulation. The simulated separation angle data was more coherent with experiment results from Shih et al. (1993), and also showed similar trends against experiment results with surface roughness models in the lower $R e_{\text {tower }}$ regions from $R e_{\text {tower }}=4.0 \mathrm{E}+05$ to $2.0 \mathrm{E}+06$. It is suspected that the 
inner RANS at the wall surface may have introduced a numerical/artificial roughness in the CFD simulation, which results in this trend.

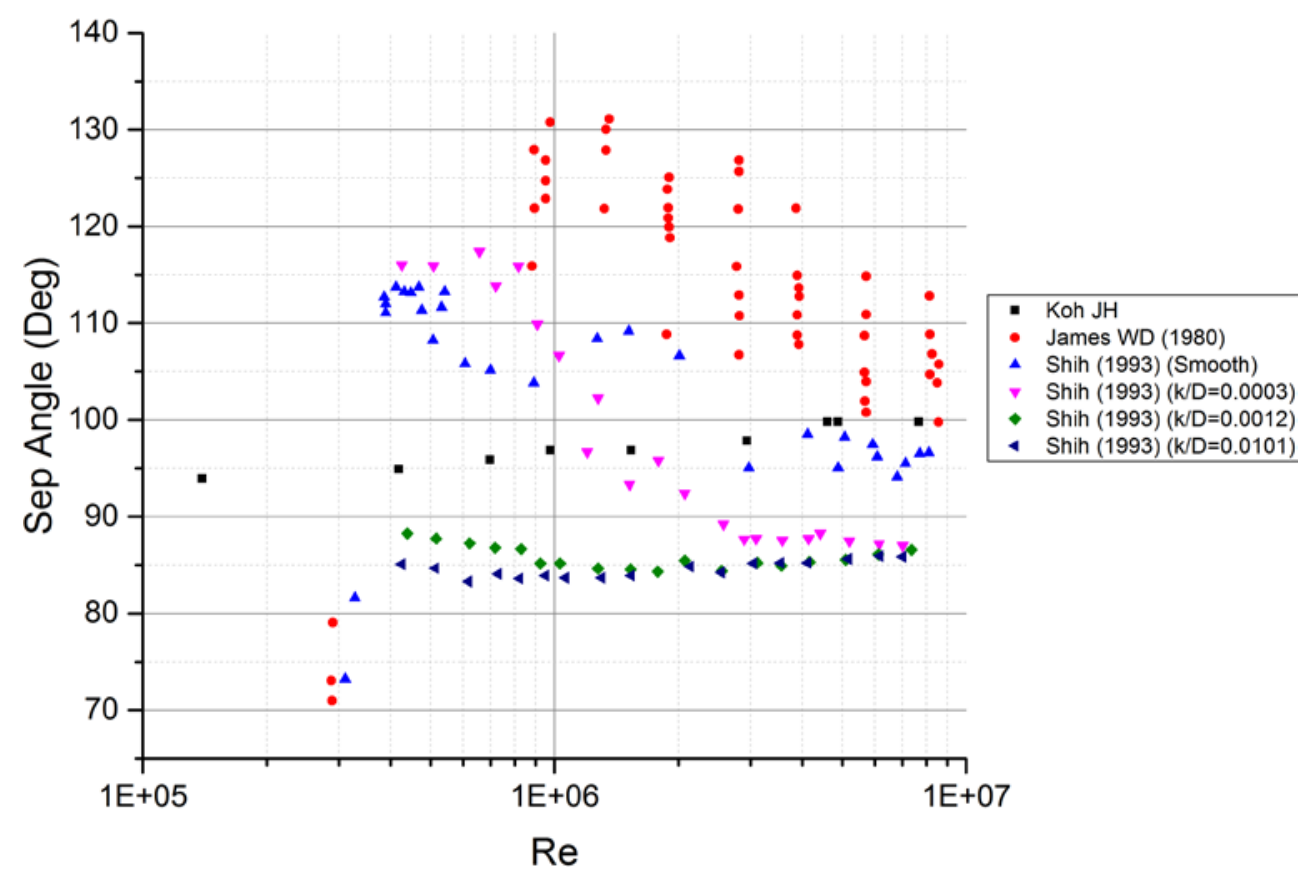

Figure 5.19 Separation Angle vs $\boldsymbol{R e}_{\text {tower }}$ (Method 1)

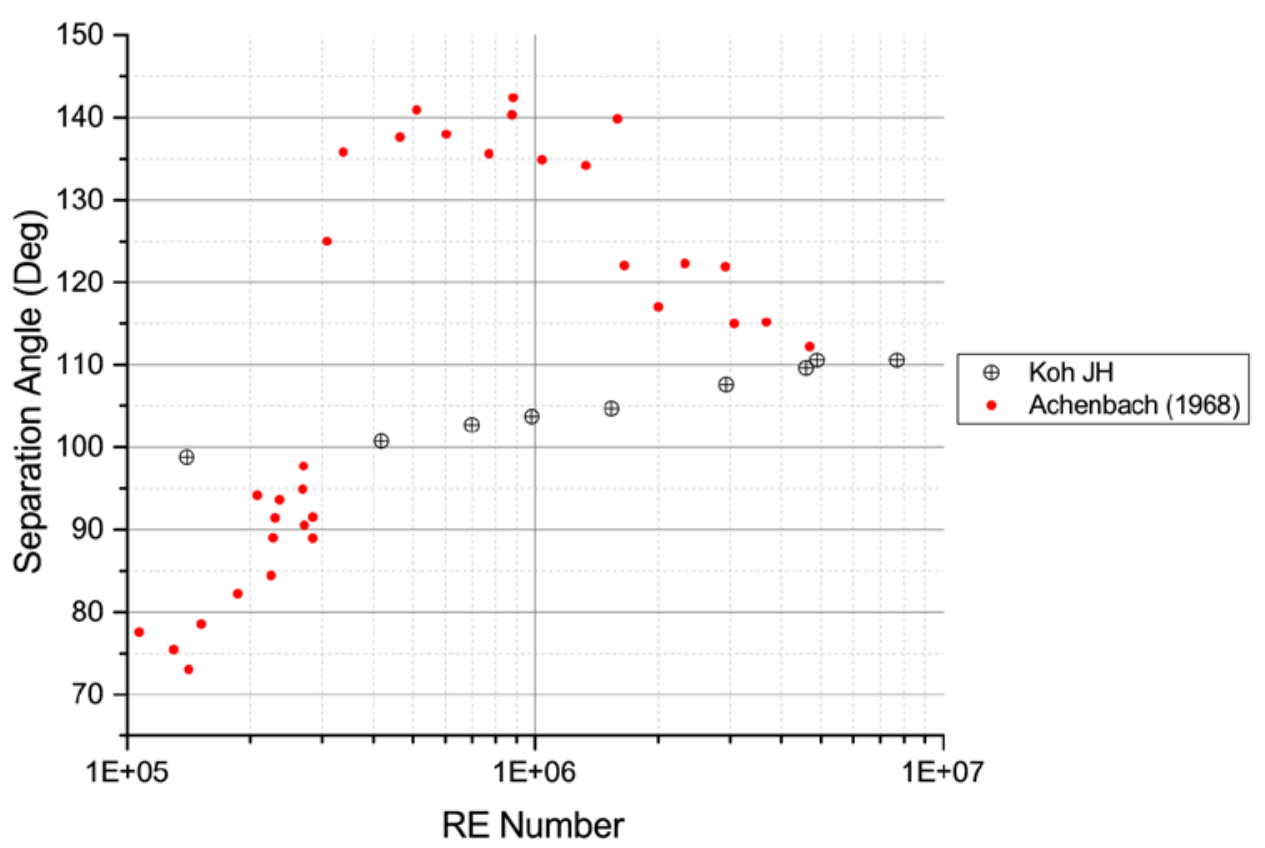

Figure 5.20 Separation Angle vs $\boldsymbol{R e}_{\text {tower }}$ (Method 2) 
Mean Velocity at $x=1$ and $x=3$

In Cantwell and Coles (1983) study, mean velocity information was published for $x=1$ and $x=3$ for $R e_{\text {tower }}=1.40 E+05$. This dataset showed good comparison with the CFD results of Case 7, and Figure 5.21 shows the mean normalized $\mathrm{X}$ velocity $\left(v_{x}\right)$ and mean normalized $\mathrm{Y}$ velocity $\left(v_{y}\right)$ plots.
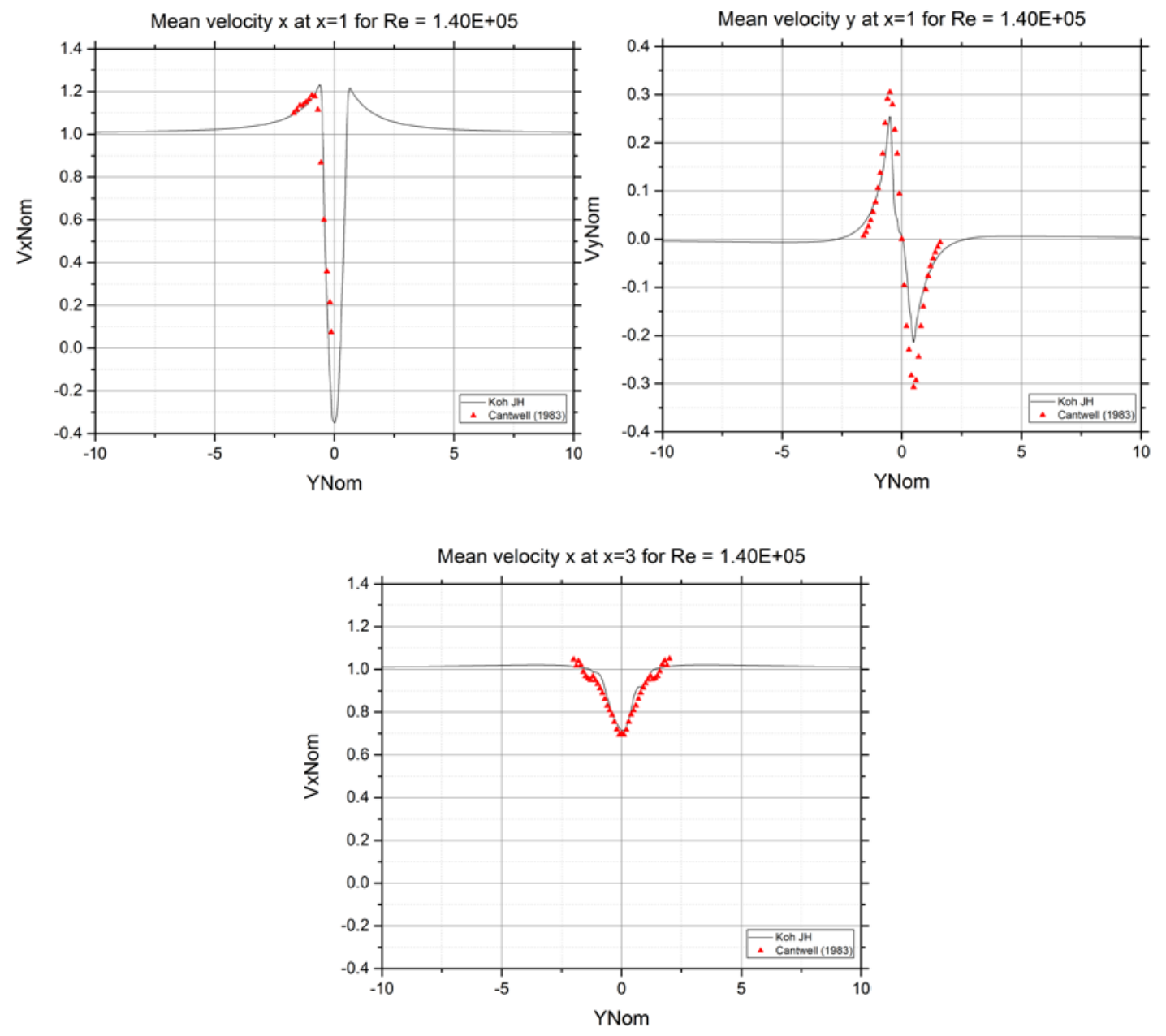

Figure 5.21 Mean Normalized Velocity Comparison for $x=1$ and $x=3$

\subsubsection{Comparison with Existing Tower Influence Model}

In Section 5.4.3.3, the CFD simulation and experiment results generally showed good comparison, which gives the author confidence to utilize the 
flow field information from the CFD simulation to compare with existing tower influence models and make improvements on them.

Based on the tower influence models used for FAST, Bladed and HAWC2 as described in Section 3.1, the velocity flow field for the CFD simulation and the existing models for Tower Case 2 are presented in Figure 5.22 for $v_{x}$ Figure 5.23 for $v_{y}$.

For $x<0$, the simulated flow followed closely to a typical potential flow over a cylinder, and the potential models used in the FAST and Bladed models showed good comparison with the CFD results for both velocity directions. The potential model in the Bladed's formulation compared slightly better in the absence of Bak et al. (2001) correction model.

For $x>0$, the simulated flow had a narrow wake and flow deficit occurred behind the tower, reaching negative mean $v_{x}$ of more than -0.2 . The flow also sped up along the side of the tower where $v_{x}$ exceeded 1.6. Due to the potential flow formulation in the FAST and Bladed models, $v_{x}$ speed up along the side of the tower was observed but the flow deficit is limited to $v_{x} \geq 0$. For the HAWC2 model, negative mean $v_{x}$ was observed but speeds up along the side of the tower is not present.

Due to the vortex shedding occurring in the wake of the tower, mean $v_{y}$ of magnitude of more than 0.2 were observed at approximately $x=1.5$. This flow characteristics was not observed in the models, and the HAWC2 formulation for $v_{y}$ provided by Madsen et al. (2007) is likely to be incorrect as illogical results were obtained.

The remaining cases (less the $\mathrm{HAWC} 2$ formulation) can be referred to in Appendix D. 


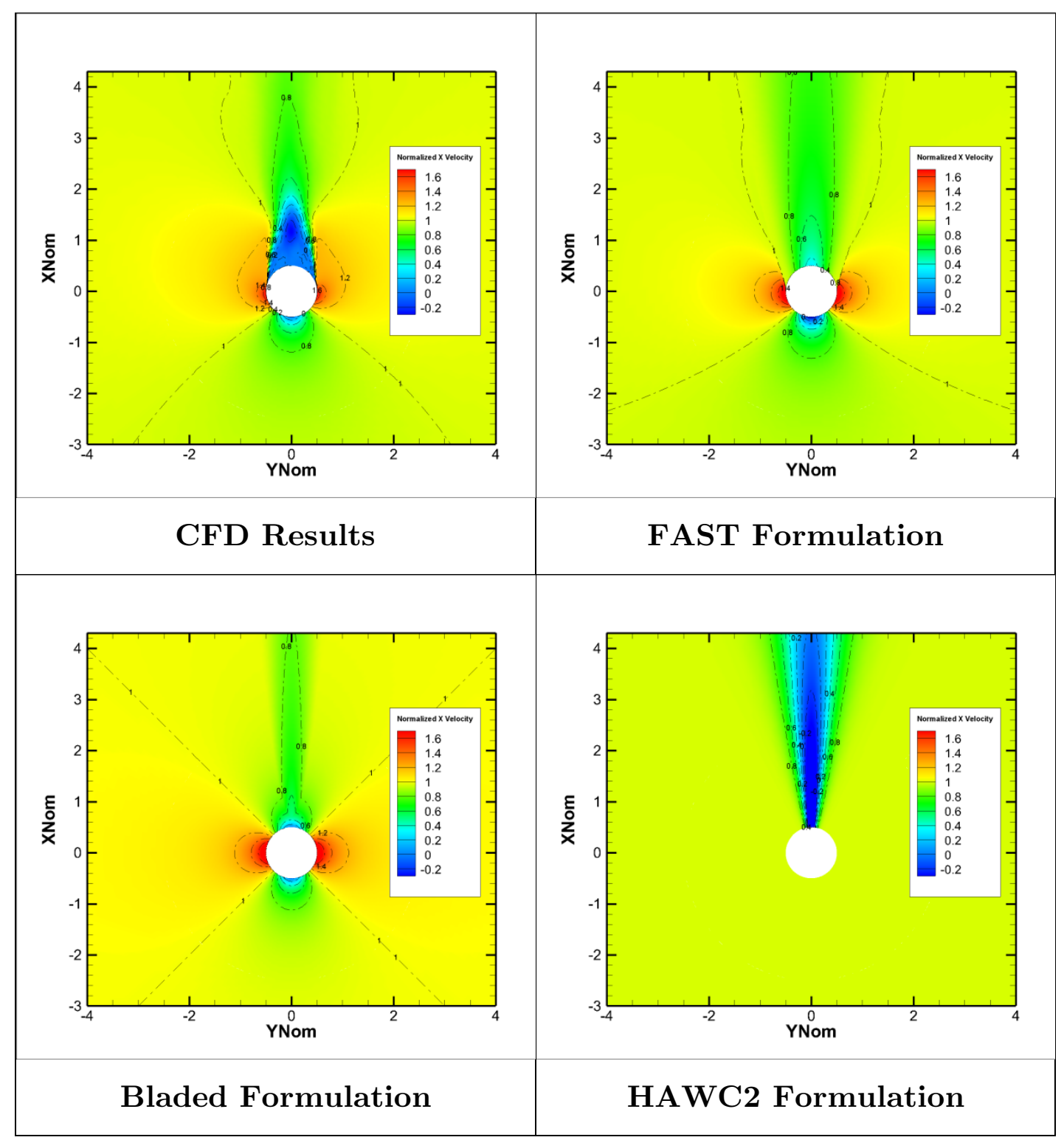

Figure 5.22 Tower Case $2\left(\operatorname{Re}_{\text {tower }}=4.61 \mathrm{E}+06\right)$ Mean Normalized X Velocity Model Comparison 


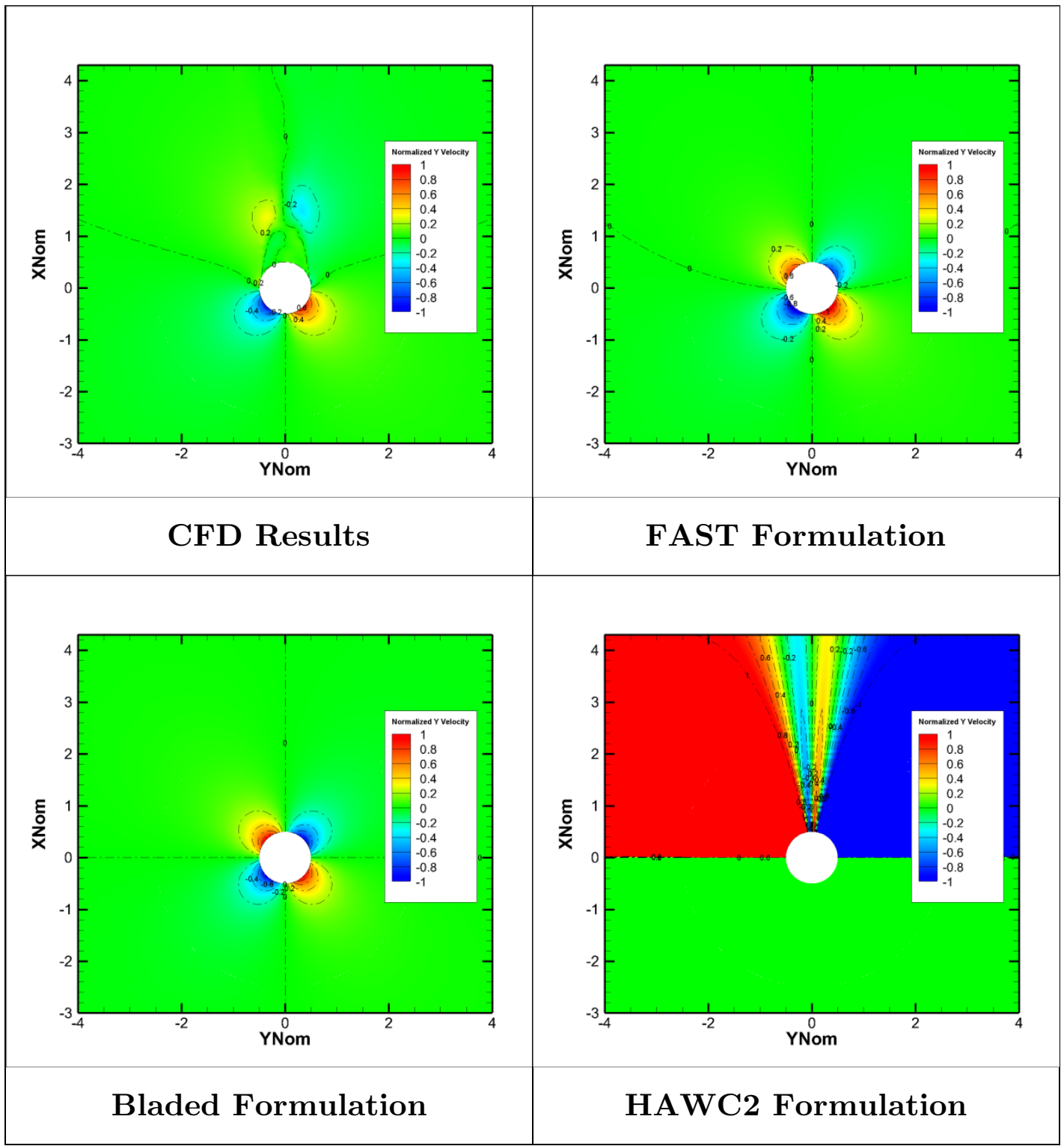

Figure 5.23 Tower Case $2\left(\operatorname{Re}_{\text {tower }}=4.61 \mathrm{E}+06\right)$ Mean Normalized Y Velocity Model Comparison

\subsubsection{Development of New Tower Influence Model}

After noting the strengths and weaknesses of various models as described in Section 5.4.4, this section describes the development of the new tower influence model suggested by the author that are based on the CFD simulation results.

The proposed model segregates the flow field into three main zones as shown in Figure 5.24: (1) $x<0$, termed as the potential flow zone, (2) $0 \leq x<0.5$, termed as the transition zone, (3) $x \geq 0.5$, termed as the wake zone. The 
wake zone is further subdivided into two specific regions, termed as the wake speed-up region and the wake deficit region.

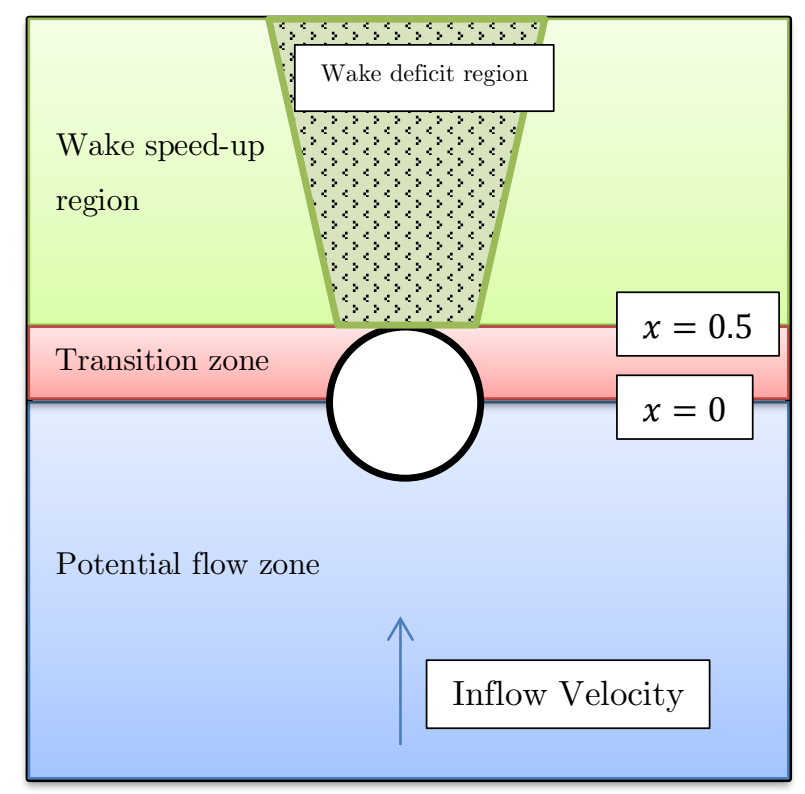

Figure 5.24 Flow Field Zones

Due to the good comparison shown in Section 5.4.4, the model proposed to be used in the potential flow zone is similar to the tower dam model described in 3.1.1. Therefore:

For $x<0$,

$$
\begin{gathered}
V_{x}=V_{0}\left(1-\frac{x^{2}-y^{2}}{\left(x^{2}+y^{2}\right)^{2}}\left(\frac{D}{2}\right)^{2}\right) \\
v_{x}=1-\frac{x^{2}-y^{2}}{\left(x^{2}+y^{2}\right)^{2}} \\
V_{y}=V_{0} \frac{-2 x y}{\left(x^{2}+y^{2}\right)^{2}}\left(\frac{D}{2}\right)^{2} \\
V_{y}=\frac{-2 x y}{\left(x^{2}+y^{2}\right)^{2}}
\end{gathered}
$$


where $x$ and $y$ are the longitudinal and transversal position normalized by tower radius, $u$ and $v$ is the $x$ - and $y$-velocity normalized by the free stream wind velocity.

For the wake zone, further analysis of the simulated flow field is required. The flow field for $v_{x}$ is described first.

Figure 5.25 shows the $v_{x}$ vs $y$ plot for various $x$ values for Tower Case 2 . The plots for the remaining cases can be referred to in Appendix F. Two key characteristics needs to be taken into account in the improved model, (1) flow speed-up near the cylinder, (2) flow deficit of $v_{x}<0$ for certain $x$ values.

Figure 5.26 shows the $v_{x}$ vs $y$ plot for various $R e_{\text {tower }}$, which illustrates the $R e_{\text {tower }}$ dependency. It can be observed that for $x<1$, the speed-up effect was inversely proportional to $R e_{\text {tower }}$. This $R e_{\text {tower }}$ dependency became less visible for $x>1$. For the flow deficit and recovery, there was no significant evidence of any $R e_{\text {tower }}$ dependency.

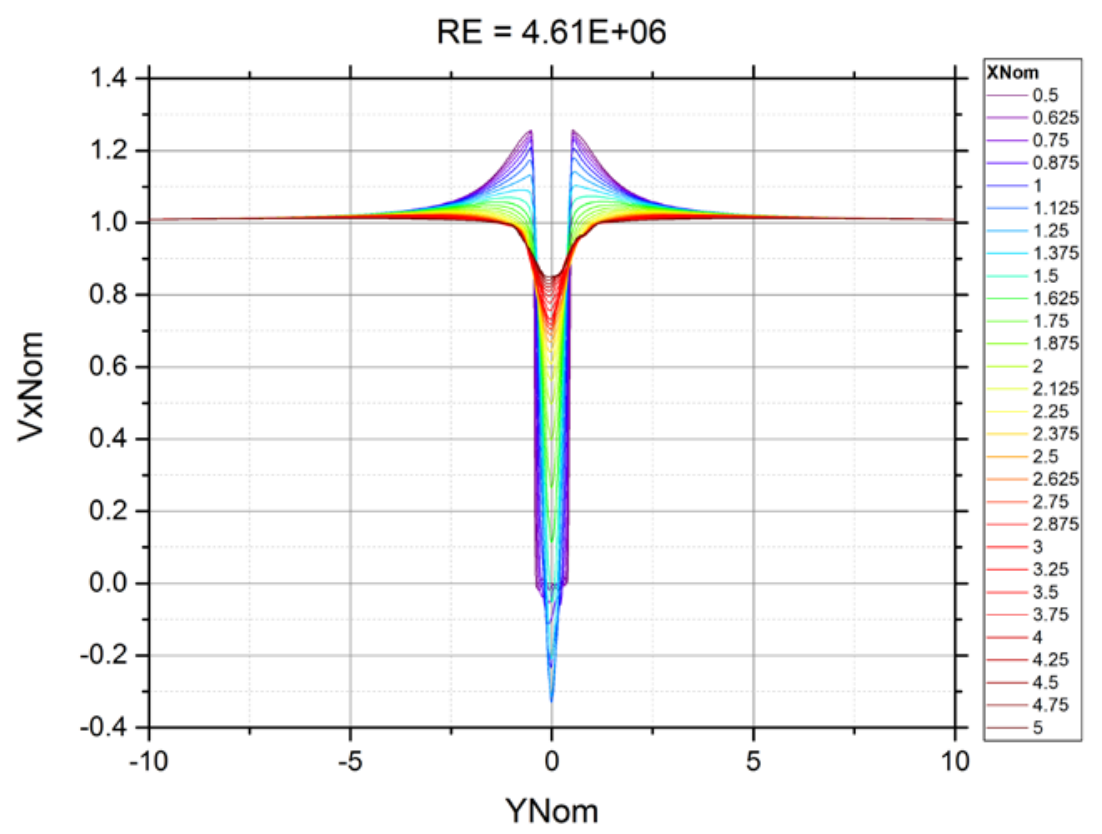

Figure 5.25 $v_{x}$ vs $y$ Plot of Various $x$ Values for Tower Case 2 $\left(R e_{\text {tower }}=4.61 \mathrm{E}+06\right)$ 


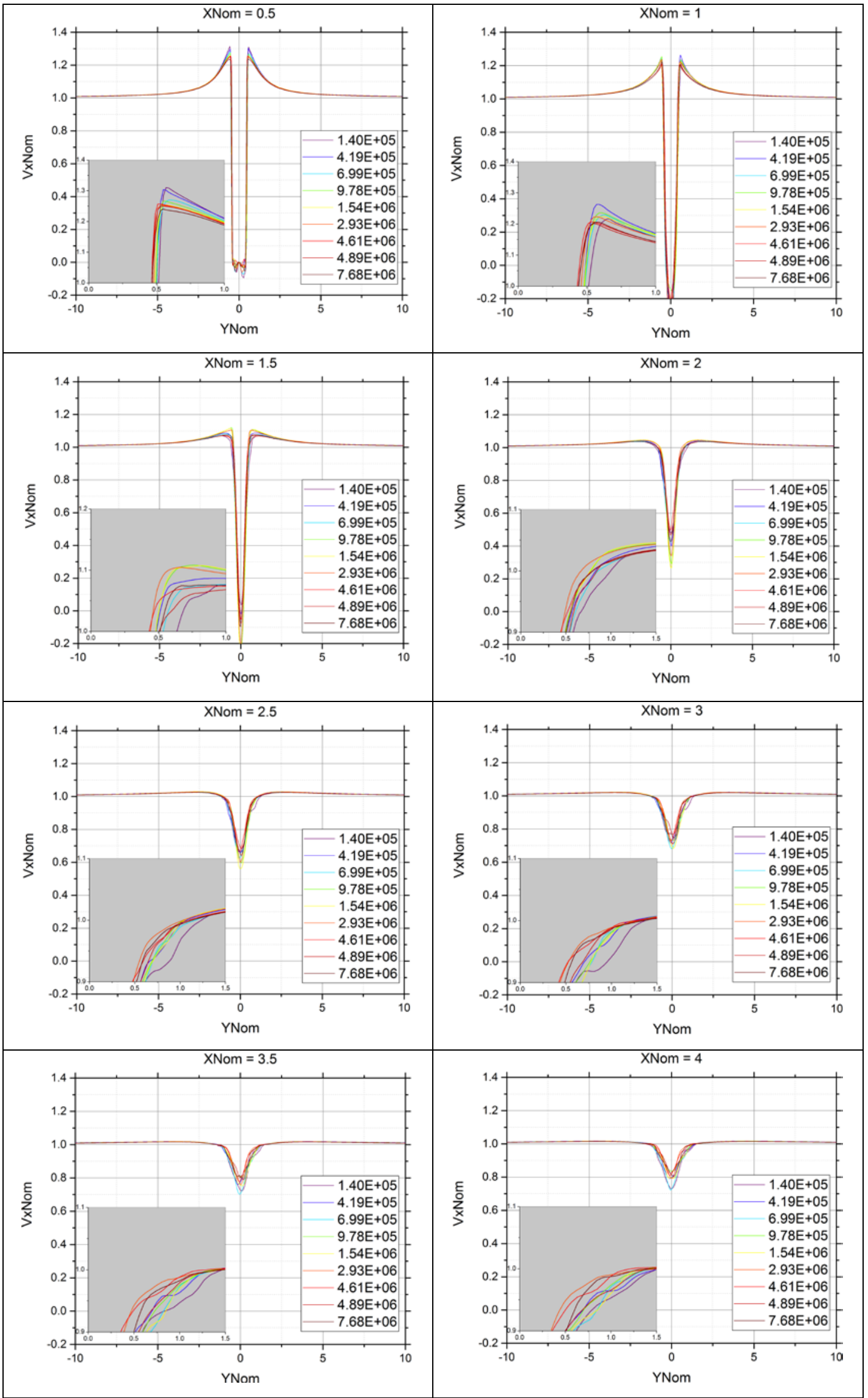

Figure 5.26 $v_{x}$ vs $y$ Plot of Various $R e_{\text {tower }}$ Values for Tower Cases 
The proposed model for the wake zone is subdivided into two regions as it is challenging to have a single empirical formulation to cover both the wake speed-up region and the wake deficit region. As such, it is critical to determine the $y$ position where $v_{x}=1$, termed as $y_{v_{x}=1}$. Using the data from Figure 5.25 and the remaining figures in Appendix F, average magnitude of $y_{v_{x}=1}$ is obtain by averaging values from both sides of the cylinder and plotted in Figure 5.27.

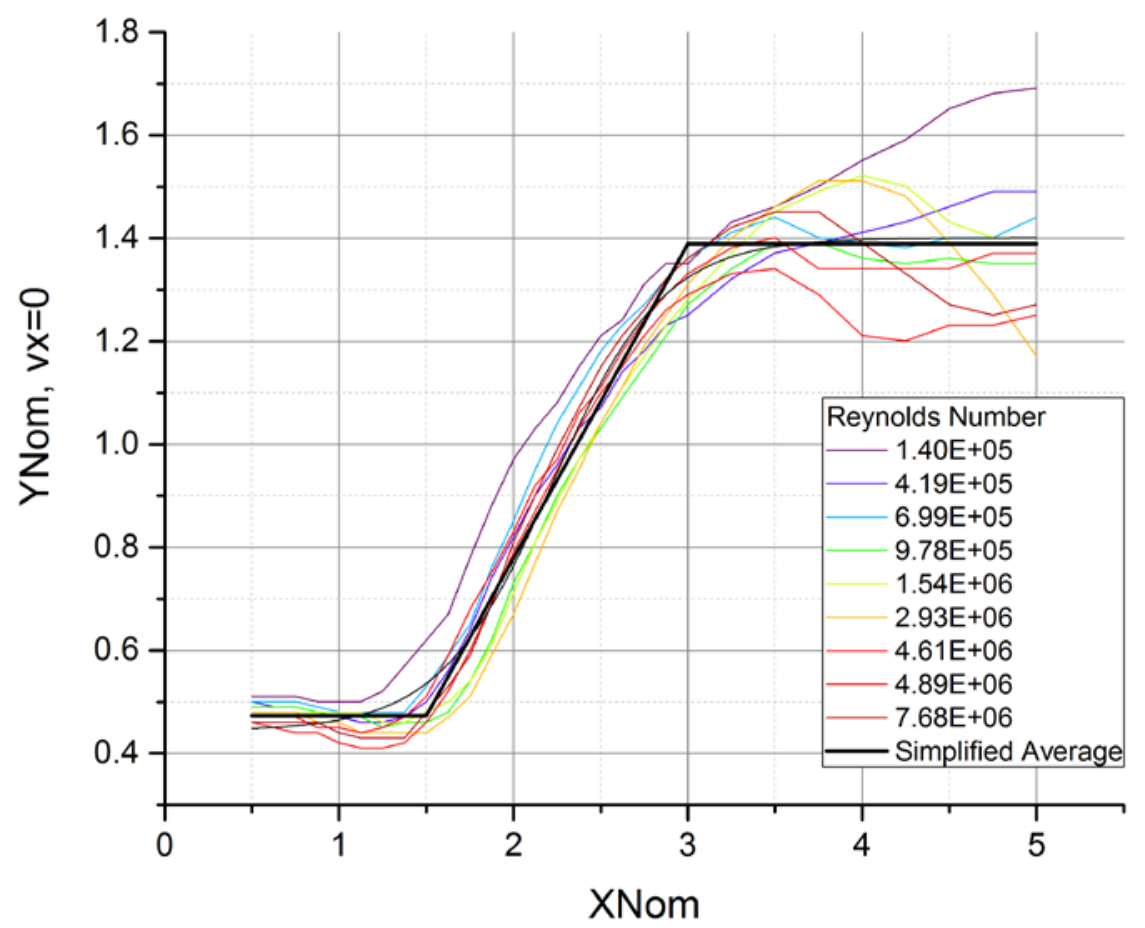

Figure 5.27 $y_{v_{x}=1}$ vs $x$ Plot for Various $R_{\text {tower }}$ Values

As there was no significant evidence of any $R e_{\text {tower }}$ dependency, $y_{v_{x}=1}$ can be represented by a simplified piecewise empirical function (shown in Figure 5.27) as:

$$
y_{v_{x}=1}=\left\{\begin{array}{ccc}
0.4733 & 0.5 \leq x<1.5 \\
0.6107 x-0.4427 & \text { for } & 1.5 \leq x<3 \\
1.3894 & 3 \leq x<5
\end{array}\right\}
$$

The constant values in Equation (4.11) are obtained based on the average $y_{v_{x}=1}$ for all simulation cases for the respective $x$ range. The formulation for $1.5 \leq x<3$ is based on a standard linear function. 
For the wake speed-up region $|y| \geq y_{v_{x}=1}$, the proposed formulation for $v_{x}$ is:

$$
v_{x}=\frac{f_{1}}{2\left[y+\left(1-y_{v_{x}=1}\right)\right]^{f^{f_{2}}}}-\frac{f_{1}}{2\left[y+\left(1-y_{v_{x}=1}\right)\right]^{e^{f_{3}}}}+1 \quad \text { for } \quad 0.5 \leq x<5
$$

where $f_{1}$ is an adjustment factor which includes the $R e_{\text {tower }}$ dependency that corrects the amount of additional speed up for $0.5 \leq x<1 . f_{2}$ controls the rate at which the flow returns to unity along $y . f_{3}$ controls the shape of the speed up.

$$
\begin{aligned}
& f_{1}=\frac{1.710 x+0.7522}{\sqrt{\lg \left(\mathrm{R}_{\text {tower }}\right)}} \quad \text { where } \max \left\{f_{1}\right\}=1 \\
& f_{2}=\left\{\begin{array}{ccc}
-0.29808+1.1899 x & & 0.5 \leq x<0.8863 \\
1.07816-0.3629 x & \text { for } & 0.8863 \leq x<4.05955 \\
0.12498-0.1281 x & & 4.05955 \leq x<5
\end{array}\right\} \\
& f_{3}=5.4582 e^{-x}-0.08598 x
\end{aligned}
$$

In the wake deficit region, one of the critical parameter required is the $v_{x}$ along $y=0$, termed as $v_{x, y=0}$ for various $R e_{\text {tower }}$, which is illustrated in Figure 5.28. 


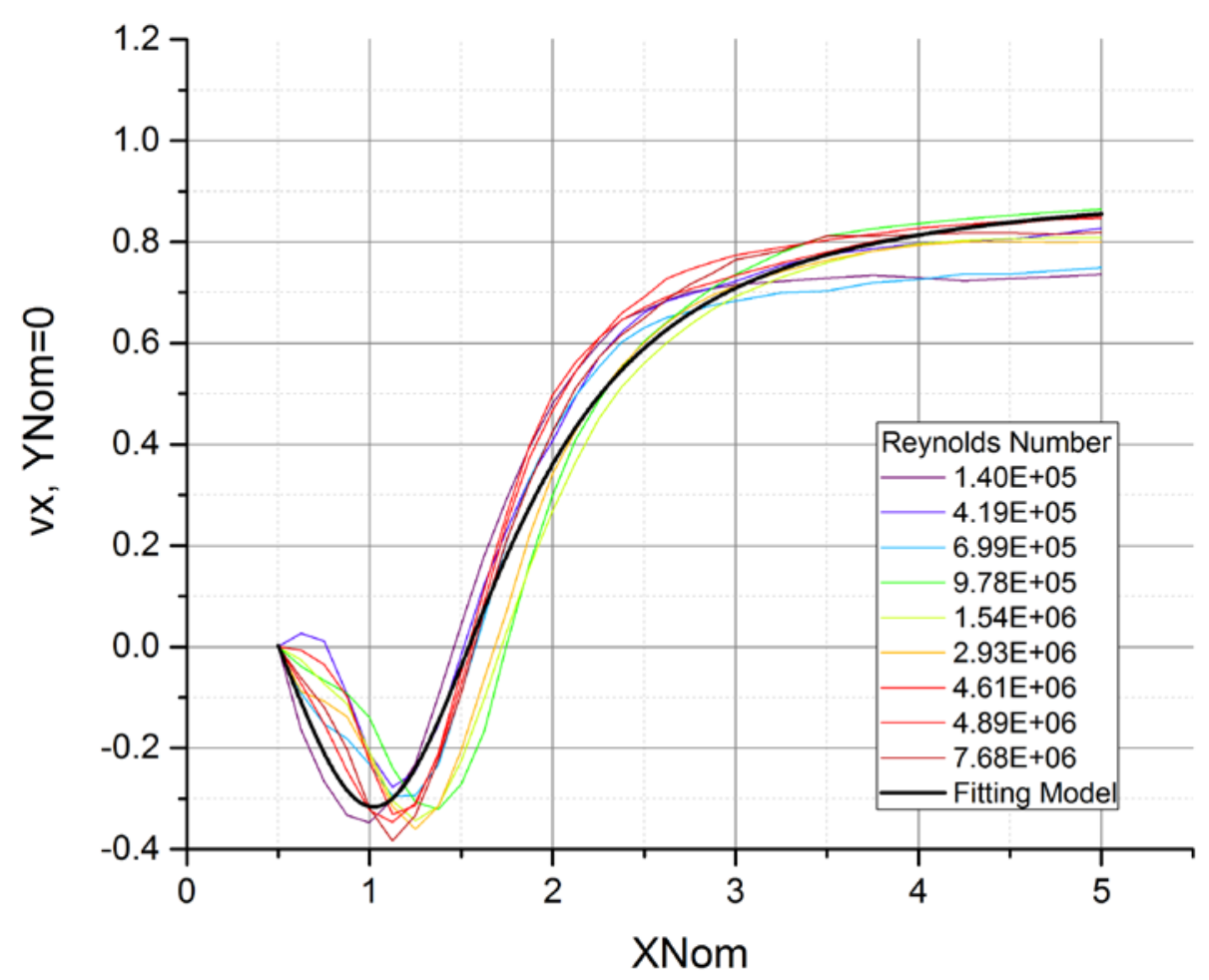

Figure $5.28 v_{x, y=0}$ vs $x$ Plot for Various $R e_{\text {tower }}$ Values

As there was no significant evidence of any $R e_{\text {tower }}$ dependency, $v_{x, y=0}$ can be represented by a Lorentzian function (shown in Figure 5.28) as:

$$
v_{x, y=0}=b_{1}+\left(\frac{2 b_{2}}{\pi}\right) \times\left(\frac{b_{3}}{4\left(x-b_{4}\right)^{2}+b_{3}^{2}}\right) \quad \text { for } \quad 0.5 \leq x<5
$$

Where $b_{1}=0.91284, b_{2}=-3.40917, b_{3}=1.76615, b_{4}=1.02254$

For the wake deficit region, a cubic function is used to represent $v_{x}$ for $|y|<y_{v_{x}=1}:$

$$
v_{x}=c_{1}+c_{2} y^{2}+c_{3} y^{3}
$$

Where $c_{1}, c_{2}$ and $c_{3}$ can be deduced by solving linear equations (shown in matrix form):

$$
\left[\begin{array}{ccc}
1 & 0 & 0 \\
0 & 2 y_{v_{x}=1} & 3 y_{v_{x}=1}^{2} \\
1 & y_{v_{x}=1} 2 & y_{v_{x}=1} 3
\end{array}\right]\left[\begin{array}{c}
c_{1} \\
c_{2} \\
C_{3}
\end{array}\right]=\left[\begin{array}{c}
v_{x, y=0} \\
{\frac{d\left(v_{x}\right)}{d y}}_{y=y_{v_{x}=1}} \\
1
\end{array}\right]
$$


$\frac{d\left(v_{x}\right)}{d y}$ can be represented by:

$$
\frac{d\left(v_{x}\right)}{d y}=-\frac{f_{1} e^{f_{2}}}{2\left[y+\left(1-y_{v_{x}=1}\right)\right]^{1+e^{f_{2}}}}+\frac{f_{1} e^{f_{3}}}{2\left[y+\left(1-y_{v_{x}=1}\right)\right]^{1+e^{f_{3}}}} \quad \text { for } \quad 0.5 \leq x<5
$$

Eq. (4.12) to (4.19) is valid for $y \geq 0$, and the equations can be applied to $y<0$ by obtaining its magnitude, using the assumption of symmetric flow on both side of the tower for $v_{x}$.

For mean $v_{y}$ in the wake deficit region, the mean flow field can be presented by a two-dimensional log-normal function for each side of the cylinder as:

$$
v_{y}=\left\{\begin{array}{ll}
A \exp \left(-\left(\frac{\ln \left(x / x_{o}\right)^{2}}{2 \sigma_{x}^{2}}+\frac{\ln \left(y / y_{o}\right)^{2}}{2 \sigma_{y}^{2}}\right)\right) & y<0 \\
-A \exp \left(-\left(\frac{\ln \left(x / x_{o}\right)^{2}}{2 \sigma_{x}^{2}}+\frac{\ln \left(y / y_{o}\right)^{2}}{2 \sigma_{y}^{2}}\right)\right) & \text { for } \quad y>=0
\end{array} \text { and } 0 \leq x<5\right\}
$$

Where $A=0.29366, \sigma_{x}=0.30023, \sigma_{y}=0.58612, x_{0}=1.71353$

$y_{0}=0.42614$

It is extremely unlikely that the blades of the wind turbine will travel through the transition zone where $0 \leq x<0.5$ due to the proximity to the tower. Therefore, a simple $x$-displacement weighted average between the potential flow zone and the wake zone is used to represent the mean flow field.

The comparison between the CFD results and the improved formulation for Tower Case 2 is shown Figure 5.29 and Figure 5.30. The remaining test cases results comparisons are presented in Appendix D. 


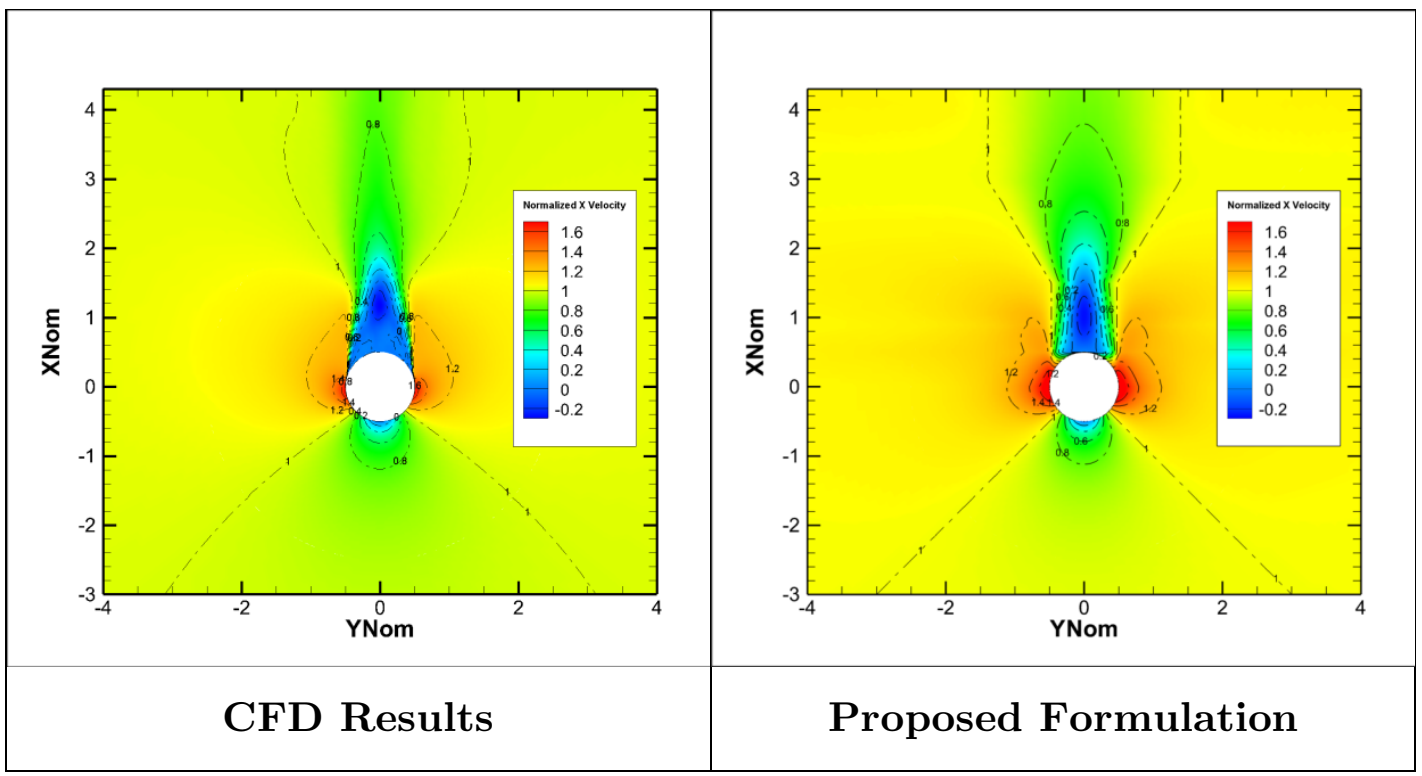

Figure 5.29 Tower Case $2\left(\operatorname{Re}_{\text {tower }}=4.61 \mathrm{E}+06\right)$ Mean Normalized X Velocity Model Comparison

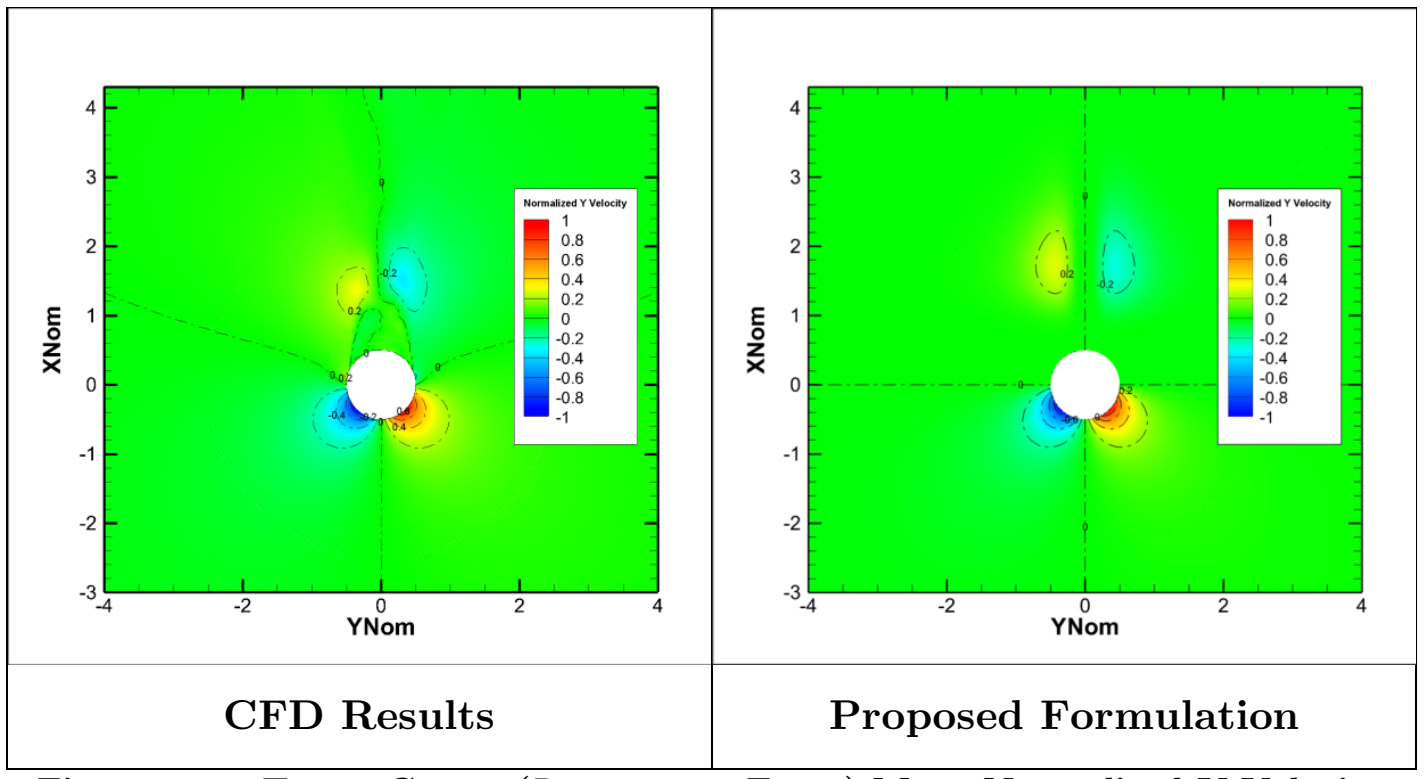

Figure 5.30 Tower Case $2\left(\operatorname{Re}_{\text {tower }}=4.61 \mathrm{E}+06\right)$ Mean Normalized Y Velocity Model Comparison

As observed, the new tower influence model proposed by the author showed significant improvement over the models used in FAST and Bladed. The amount of flow speed up along the side of the tower is dependent on $R e_{\text {tower }}$, and the negative $v_{x}$ behind the tower is also present, which better represents the tower shadow effect. In addition, the new formulation can now model the mean $v_{y}$ trends that are present due to vortex shedding in the wake of the tower. 


\subsection{Nacelle Analysis}

\subsubsection{CFD Simulation Cases}

From the study conducted in Section 5.3, 9 test cases were generated to analyze the flow across a wind turbine nacelle based on each permutation of smooth- and sharp-edged nacelles, and cylindrical or cuboid shaped nacelles. This results in a total of 36 test cases.

The fineness ratio is defined as the ratio of the length of the body to its maximum width. A fineness ratio of 2.5 is used for both the cylinder and the cuboid. The parameters for each test case are shown in Table 5.6.

The $R e$ of the flow for cylinder and cuboid nacelle are calculated based on Eq. (4.21) and Eq. (4.22)

$$
\begin{gathered}
R e_{c y l}=\frac{V_{0} \times D_{c y l}}{v} \\
R e_{c u b}=\frac{V_{0} \times W_{c u b}}{v}
\end{gathered}
$$

where $D_{c y l}$ is the diameter of the cylinder and $W_{c u b}$ is the width of the cuboid.

Table 5.6 Parameters for Nacelle Test Cases (Cylinder and Cuboid Shaped)

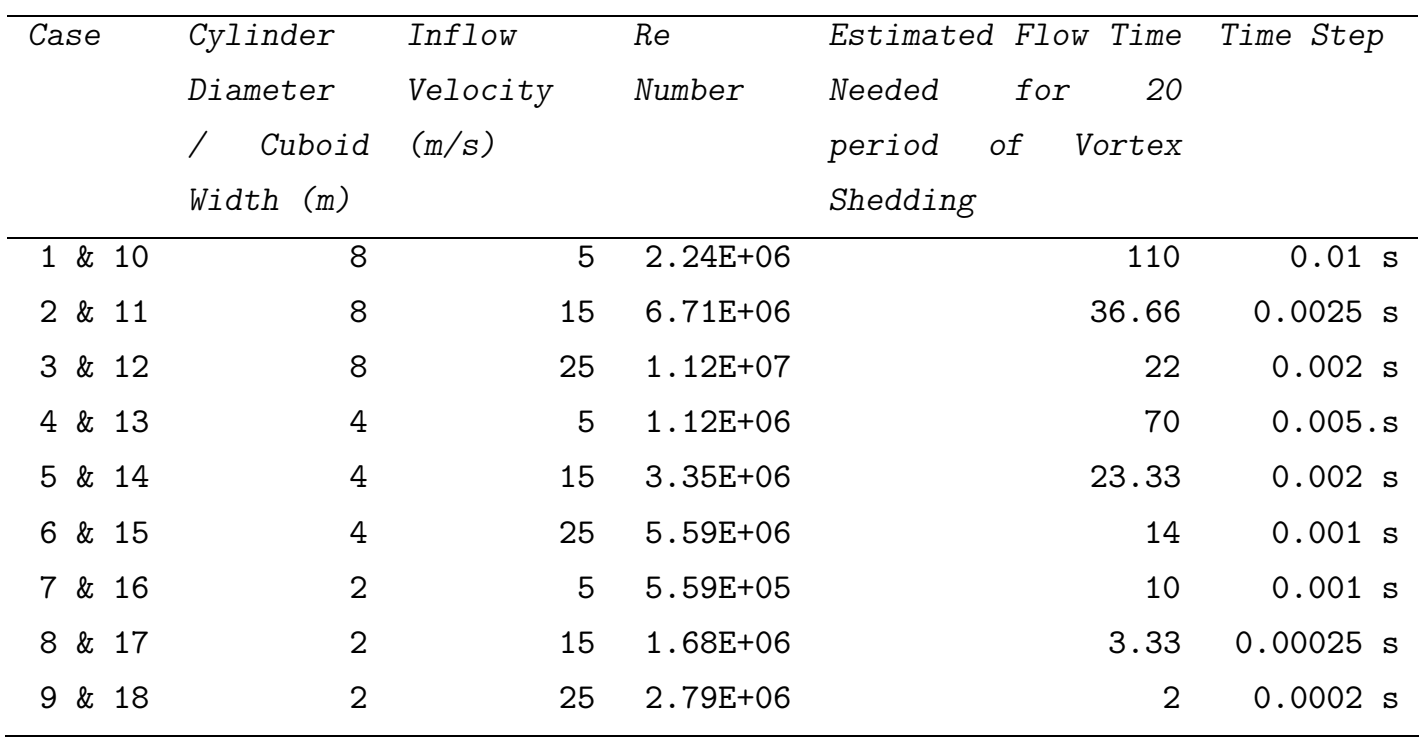


The sharp shaped cases are numbered between $1-9$ while the smooth shaped cases are numbered between $10-18$. The cylinder diameter of $0.5 \mathrm{~m}$, $3.5 \mathrm{~m}$ and $5.5 \mathrm{~m}$ are representative of small, medium and large wind turbines.

\subsubsection{CFD Simulation Setup (Case Specific)}

\subsubsection{Cuboid Shaped (Smooth)}
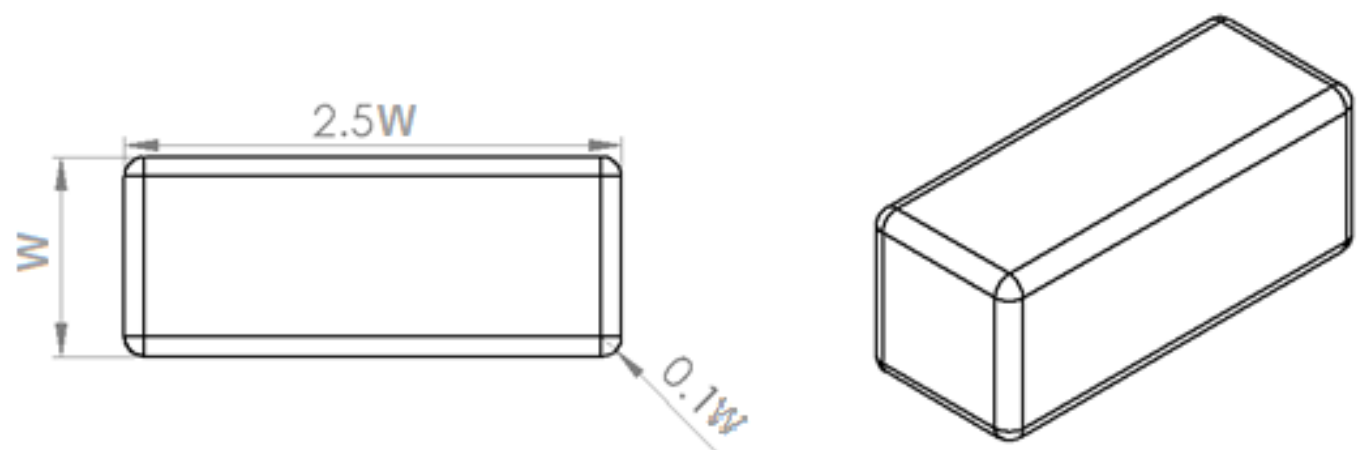

Figure 5.31 Smooth Cuboid Model
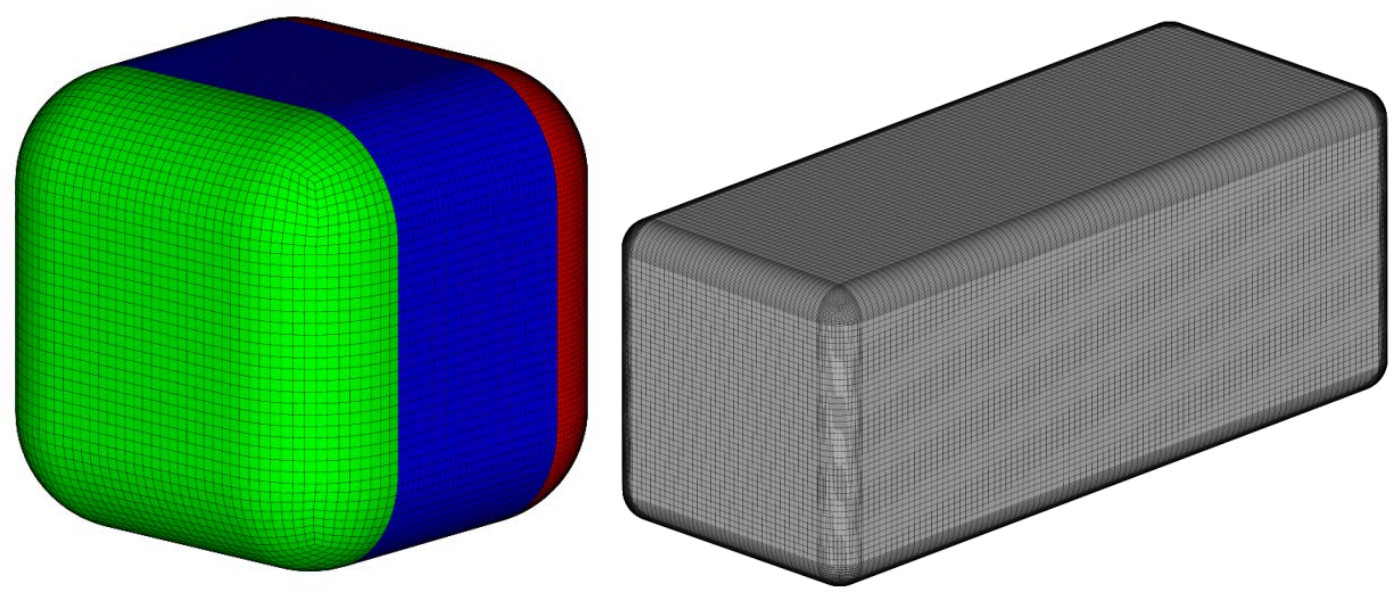

Figure 5.32 Smooth Cuboid Mesh 


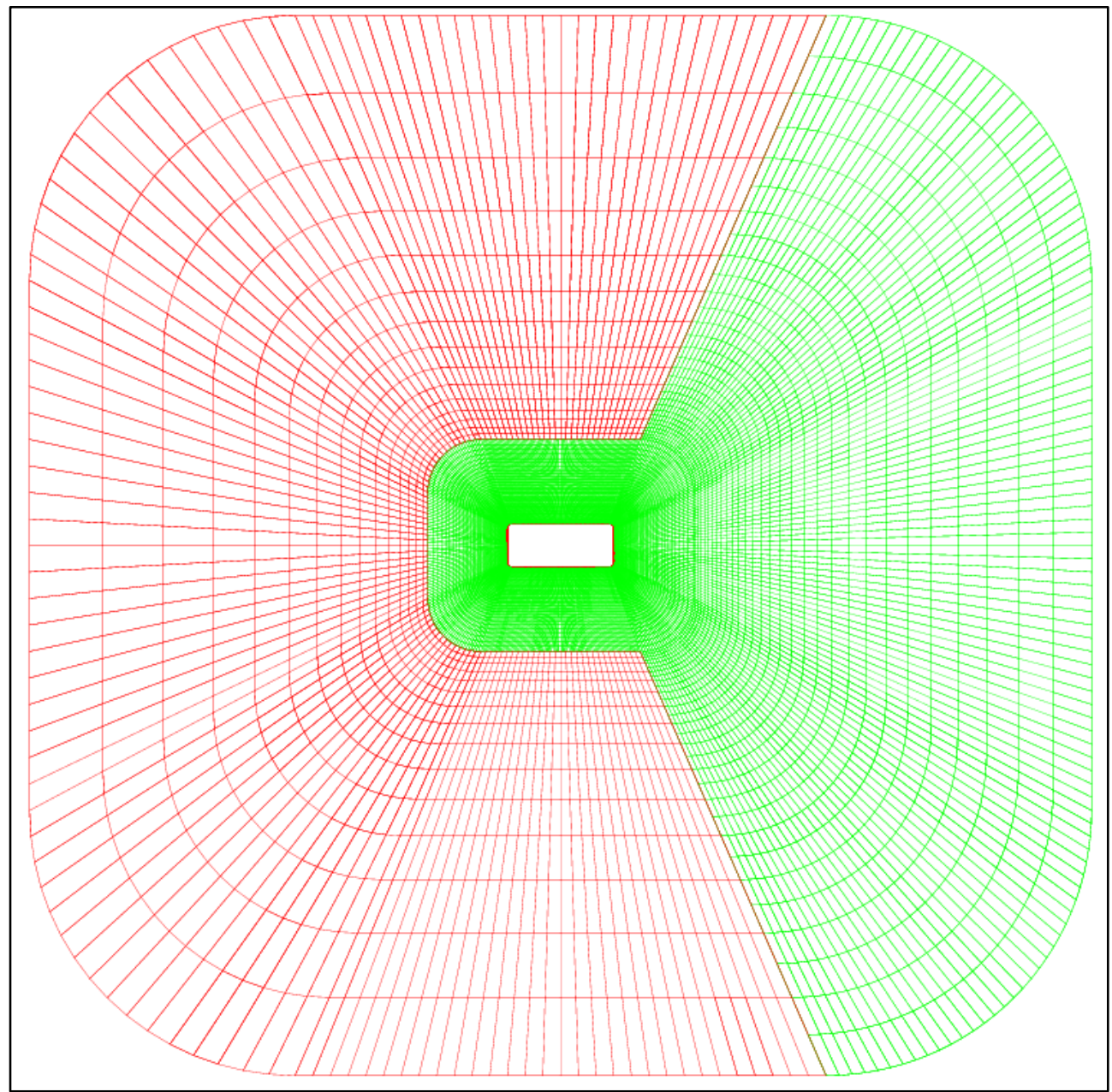

Figure 5.33 Smooth Cuboid Cross-Section View

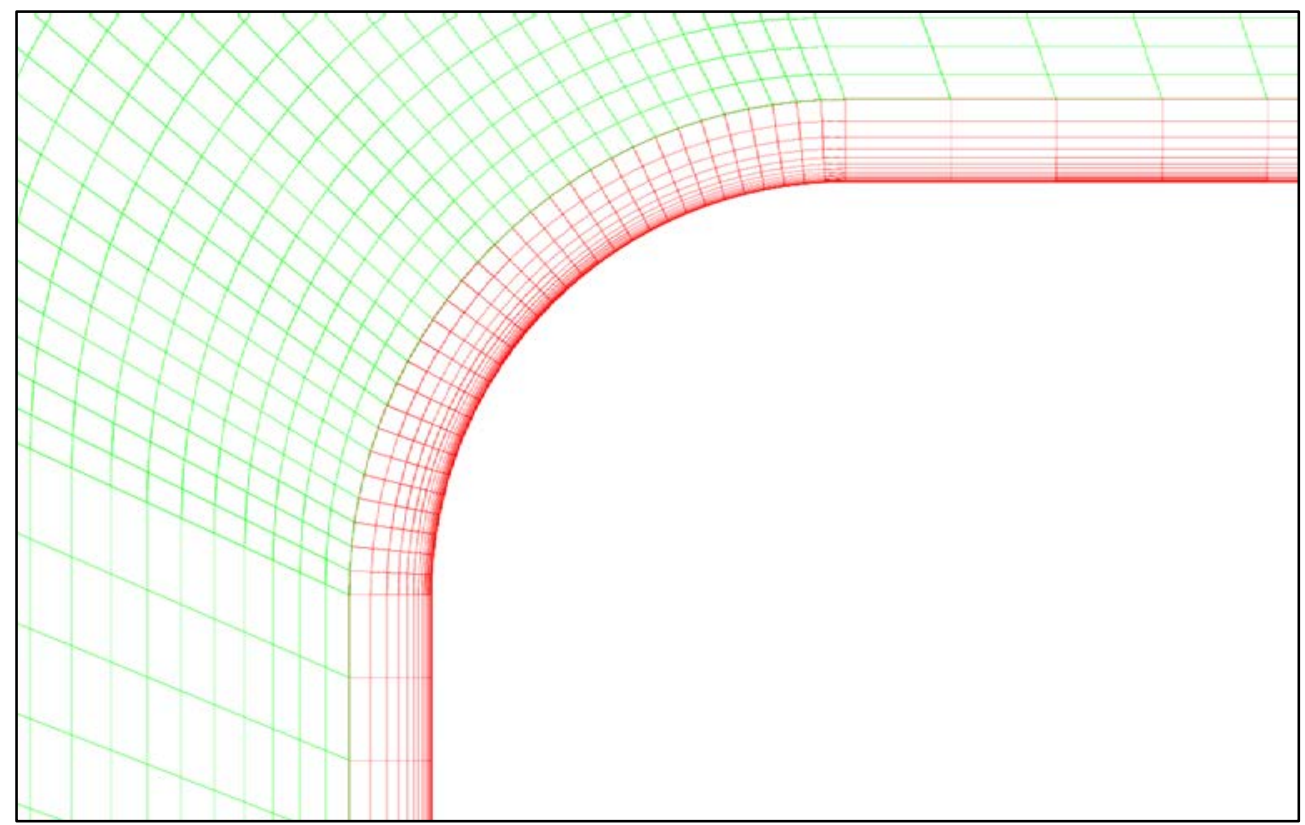

Figure 5.34 Smooth Cuboid Cross-Section View of Boundary Layer 
The dimension of the smooth cuboid was $2.5 W_{c u b} \times W_{c u b} \times W_{c u b}$ (length of the cuboid nacelle is termed as $L_{c u b}=2.5 W_{c u b}$ ) and the edges were smoothed with a corner radius of $0.1 W_{c u b}$ shown in Figure 5.31. The mesh extended $12.5 W_{c u b}$ towards the front, rear and the sides from the center of the cuboid. The mesh was shown on the left of Figure 5.32 and the enlarged view of the cuboid wall was shown on the right of Figure 5.32. In Figure 5.32 , the green zone was defined as a velocity inlet, the red zone was defined as a pressure outlet, while the blue zone was defined as symmetry boundary condition.

The mesh was a fully structured configuration utilizing hexahedron cells.

The cross-section of the mesh domain is shown in Figure 5.33, and the red zones, which included the coarser domain and the refined boundary layer (Figure 5.34) was the RANS zones while the green zones which included the refined domain was the LES zones. Due to the complexity of description of the entire mesh, a brief description of the mesh is presented below.

The flat mesh faces along the length of the cuboid had a $90 \times 40$ mesh face, while the flat mesh faces along the width of the cuboid had a $40 \times 40$ mesh face. The curved mesh faces along the length of the cuboid has a $90 \times 32$ mesh face, while the curved mesh faces along the width of the cuboid had a $40 \times 32$ mesh face. The four corner faces had a $32 \times 32$ mesh face. The mesh was extruded in all directions to the limits of domain boundary, with the outer RANS zone having $\frac{1}{4}$ the cell density of the inner LES zone and the inner RANS zone. A total 3580560 elements was used for the smooth cuboid nacelle mesh. 


\subsubsection{Cuboid Shaped (Sharp)}
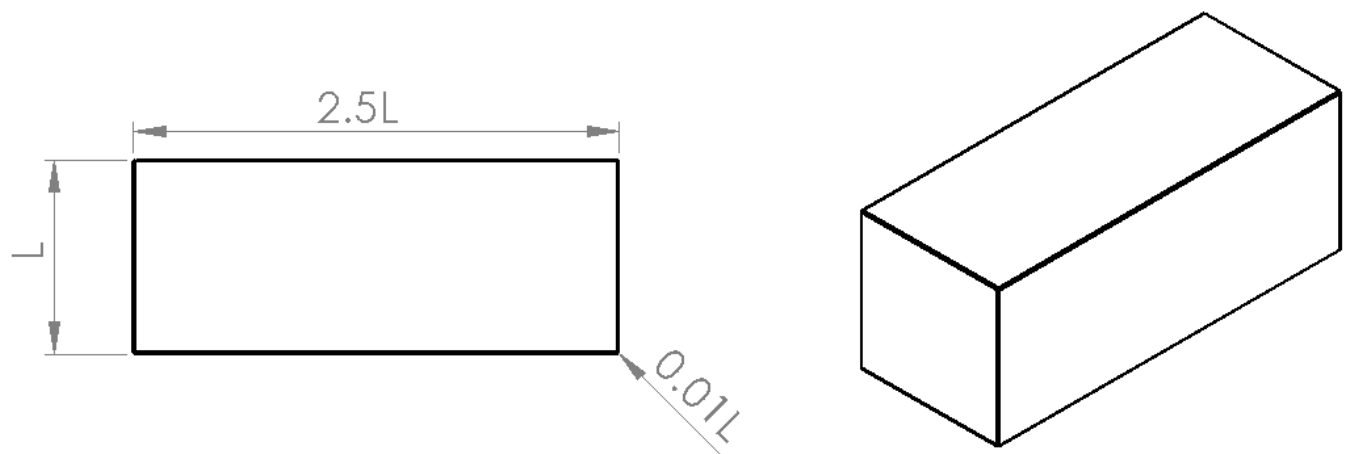

Figure 5.35 Sharp Cuboid Model

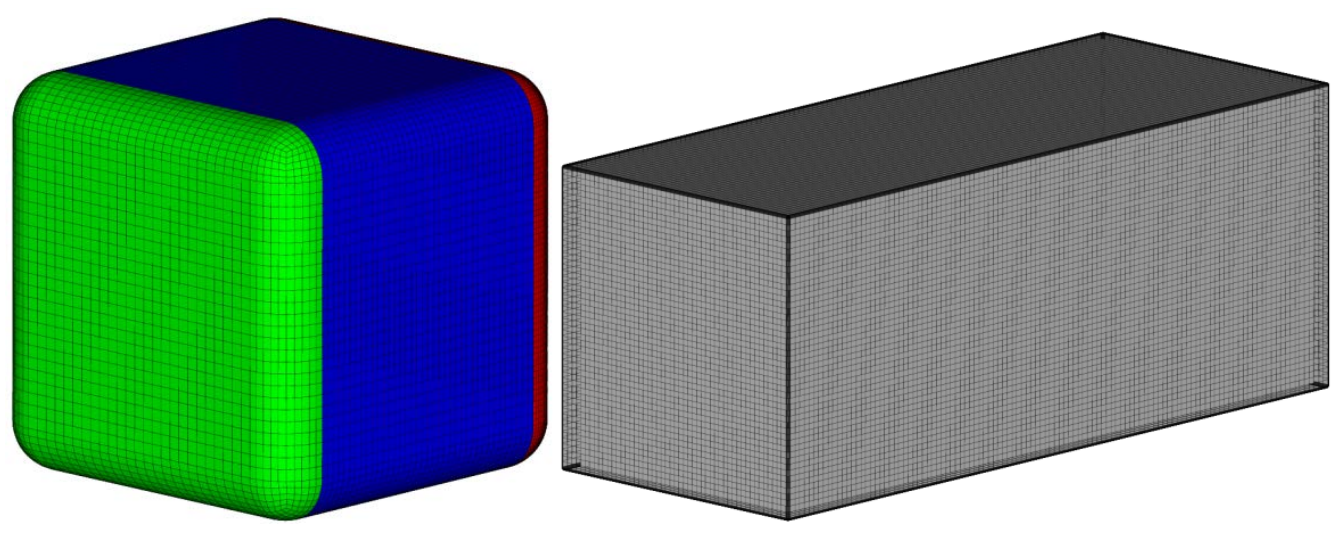

Figure 5.36 Sharp Cuboid Mesh 


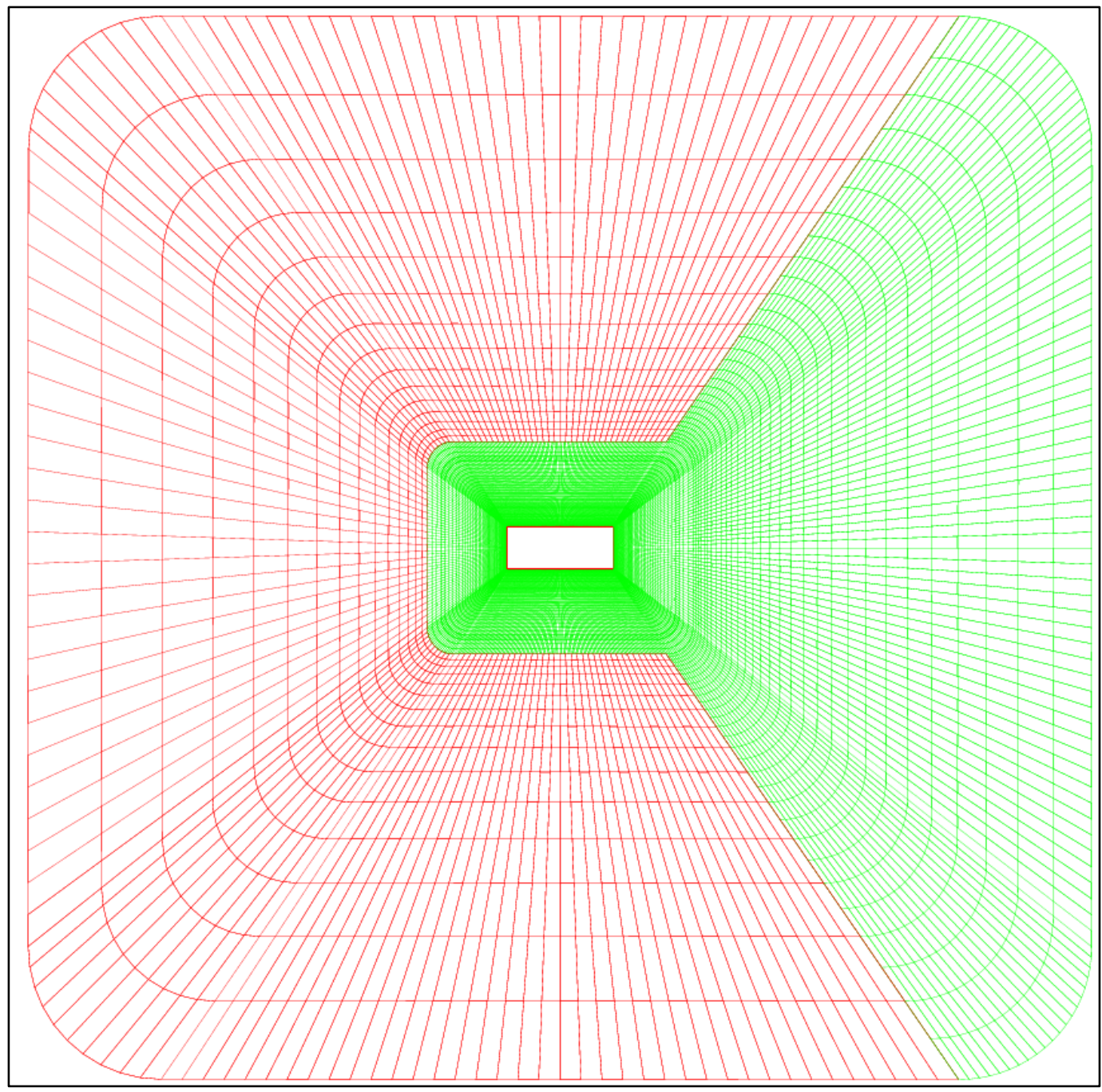

Figure 5.37 Sharp Cuboid Cross-Section View

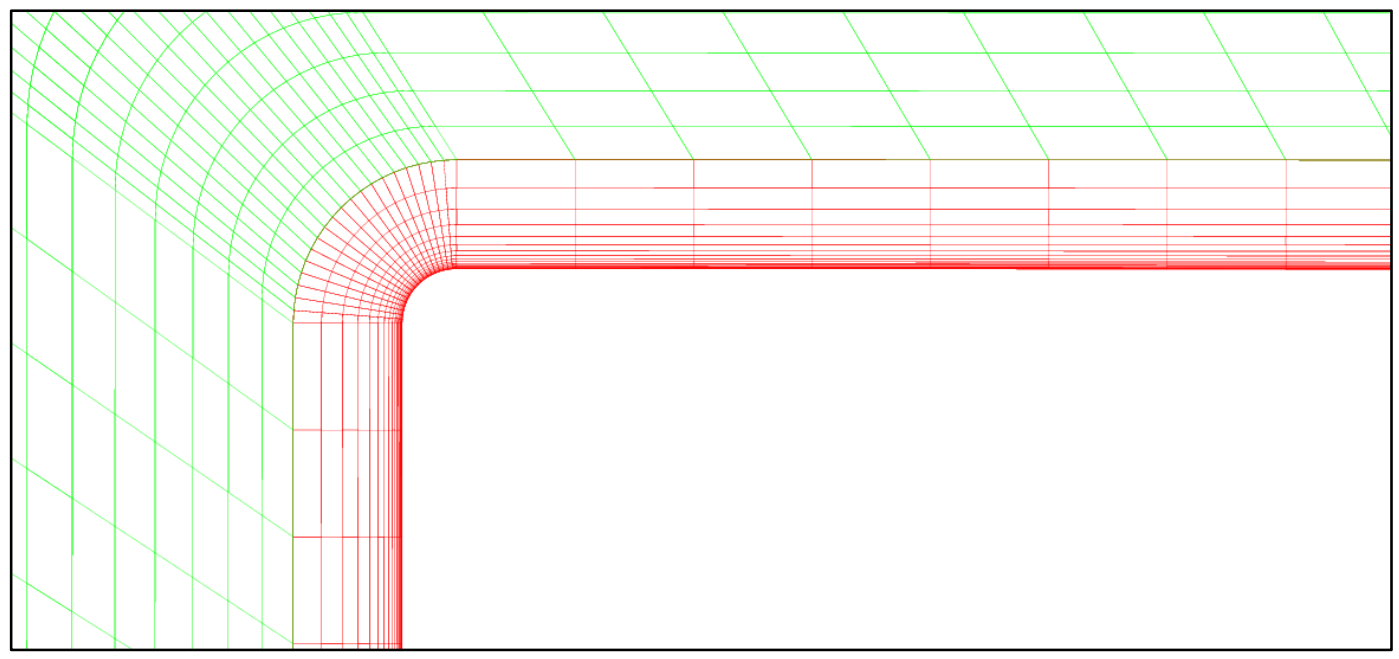

Figure 5.38 Sharp Cuboid Cross-Section View of Boundary Layer 
The dimension of the sharp cuboid was $2.5 W_{c u b} \times W_{c u b} \times W_{c u b}$ and the edges were smoothed with a corner radius of $0.01 W_{c u b}$ shown in Figure 5.35. The corner radius was incorporated mainly to prevent convergence problem due to sharp corners in the geometry. The mesh extended $12.5 W_{\text {cub }}$ towards the front, rear and the sides from the centre of the cuboid. The mesh is shown on the left of Figure 5.36 and the enlarged view of the cuboid wall is shown on the right of Figure 5.36. In Figure 5.36, the green zone was defined as a velocity inlet, the red zone was defined as a pressure outlet, while the blue zone was defined as symmetry boundary condition.

The mesh was a fully structured configuration utilizing hexahedron cells.

The cross-section of the mesh domain is shown in Figure 5.37, and the red zones, which included the coarser domain and the refined boundary layer (Figure 5.38) was the RANS zones while the green zones which included the refined domain was the LES zones. Due to the complexity of description of the entire mesh, a brief description of the mesh is presented below.

The flat mesh faces along the length of the cuboid had a $114 \times 50$ mesh face, while the flat mesh faces along the width of the cuboid had a $50 \times 50$ mesh face. The curved mesh faces along the length of the cuboid had a $114 \times 20$ mesh face, while the curved mesh faces along the width of the cuboid had a $40 \times 20$ mesh face. The four corner faces had a $20 \times 20$ mesh face. The mesh was extruded in all directions to the limits of domain boundary, with the outer RANS zone having $\frac{1}{4}$ the cell density of the inner LES zone and the inner RANS zone. A total 3652075 elements was used for the sharp cuboid nacelle mesh. 


\subsubsection{Cylinder Shaped (Smooth)}
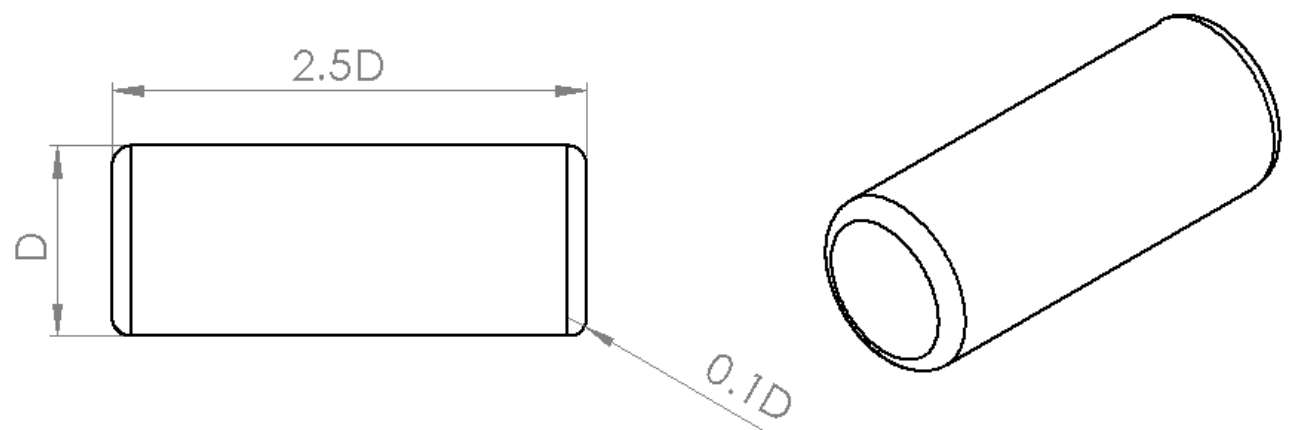

Figure 5.39 Smooth Cylinder Model

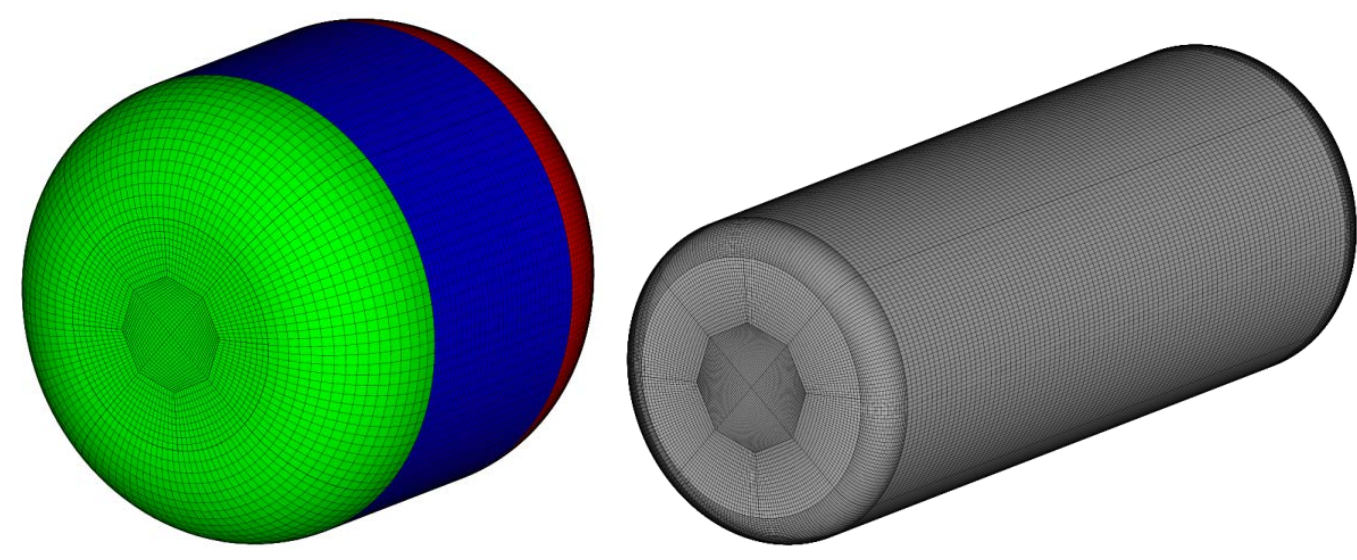

Figure 5.40 Smooth Cylinder Mesh 


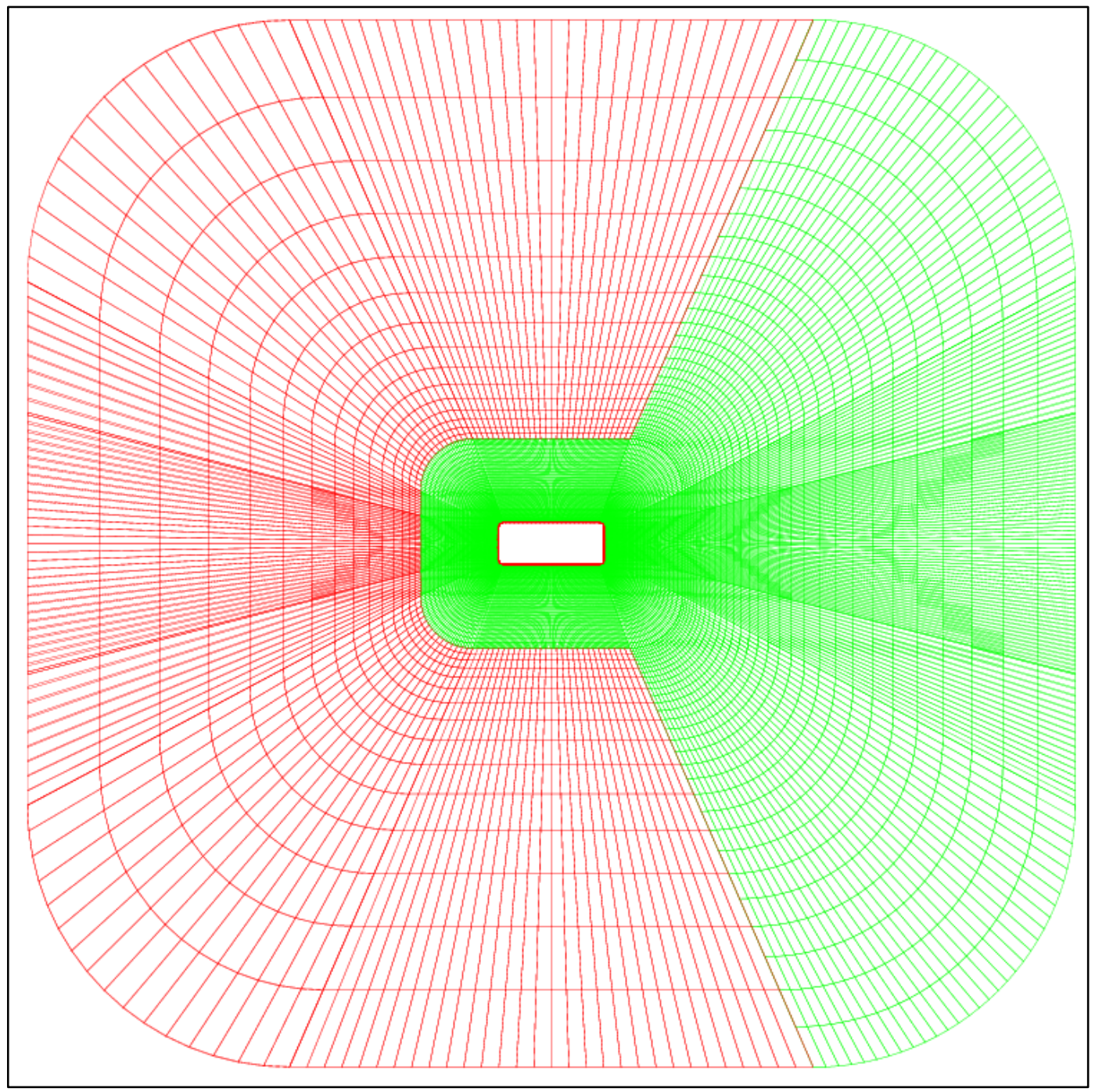

Figure 5.41 Smooth Cylinder Cross-Section View

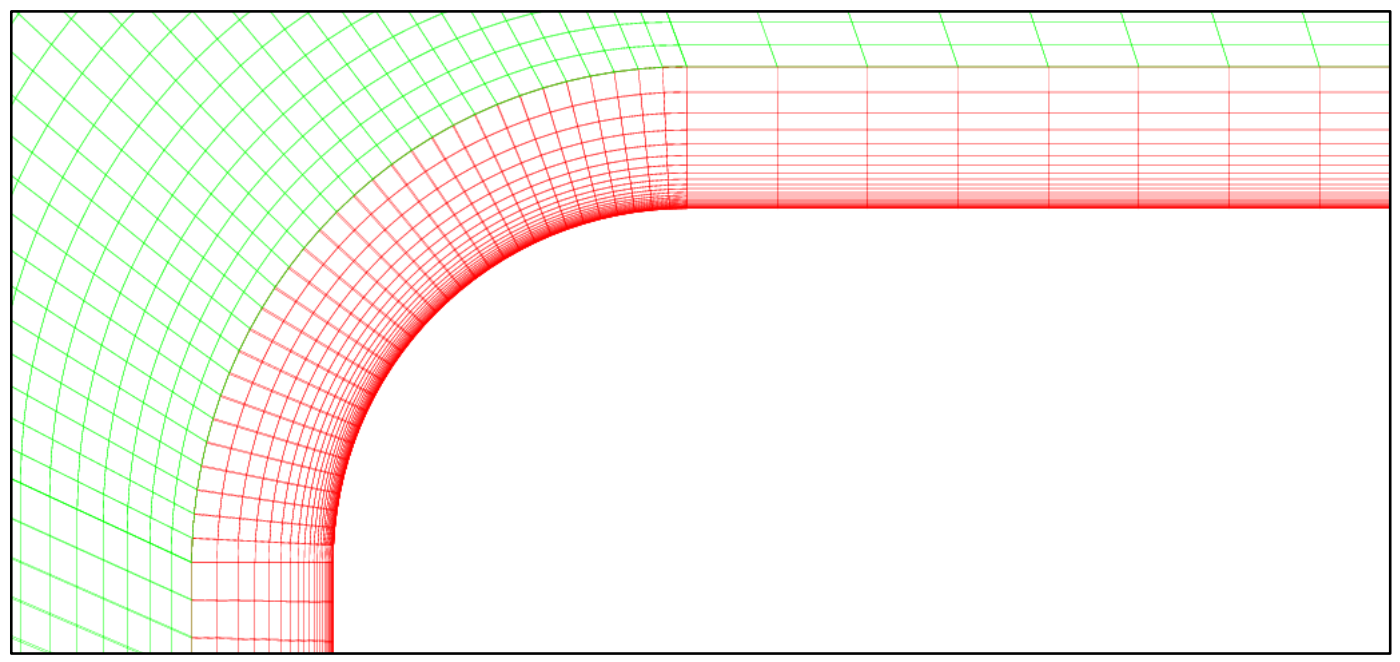

Figure 5.42 Smooth Cylinder Cross-Section View of Boundary Layer 
The dimension of the smooth cylinder was $2.5 D_{c y l} \times D_{c y l}$ (length of the cylinder nacelle is termed as $\left.L_{c y l}=2.5 W_{c y l}\right)$ and the edges were smoothed with a corner radius of $0.1 D_{c y l}$ shown in Figure 5.39. The mesh extended $12.5 D_{c y l}$ towards the front, rear and the sides from the centre of the cuboid. The mesh is shown on the left of Figure 5.40 and the enlarged view of the cuboid wall is shown on the right of Figure 5.40. In Figure 5.40, the green zone was defined as a velocity inlet, the red zone was defined as a pressure outlet, while the blue zone was defined as symmetry boundary condition.

The mesh was a fully structured configuration utilizing hexahedron cells.

The cross-section of the mesh domain is shown in Figure 5.41, and the red zones, which included the coarser domain and the refined boundary layer (Figure 5.42) was the RANS zones while the green zones which included the refined domain was the LES zones. Due to the complexity of description of the entire mesh, a brief description of the mesh is presented below.

The mesh faces along the length of the cylinder had a $90 \times 240$ mesh face, while the flat mesh faces along the diameter of the cylinder was divided into two mesh faces, consisting of a $240 \times 240$ mesh face that transited to a $18 \times 240$ mesh face. The curved edges along the circumference of the cylinder have a $32 \times 240$ mesh face. The mesh was extruded in all directions to the limits of domain boundary, with the outer RANS zone having $\frac{1}{4}$ the cell density of the inner LES zone and the inner RANS zone. A total 4804500 elements was used for the smooth cylinder nacelle mesh. 


\subsubsection{Cylinder Shaped (Sharp)}

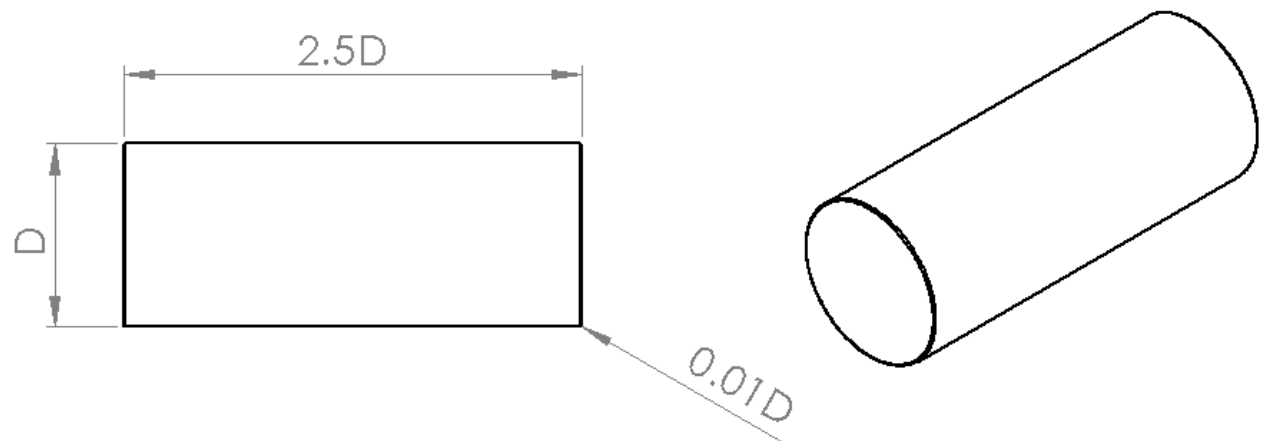

Figure 5.43 Sharp Cylinder Model

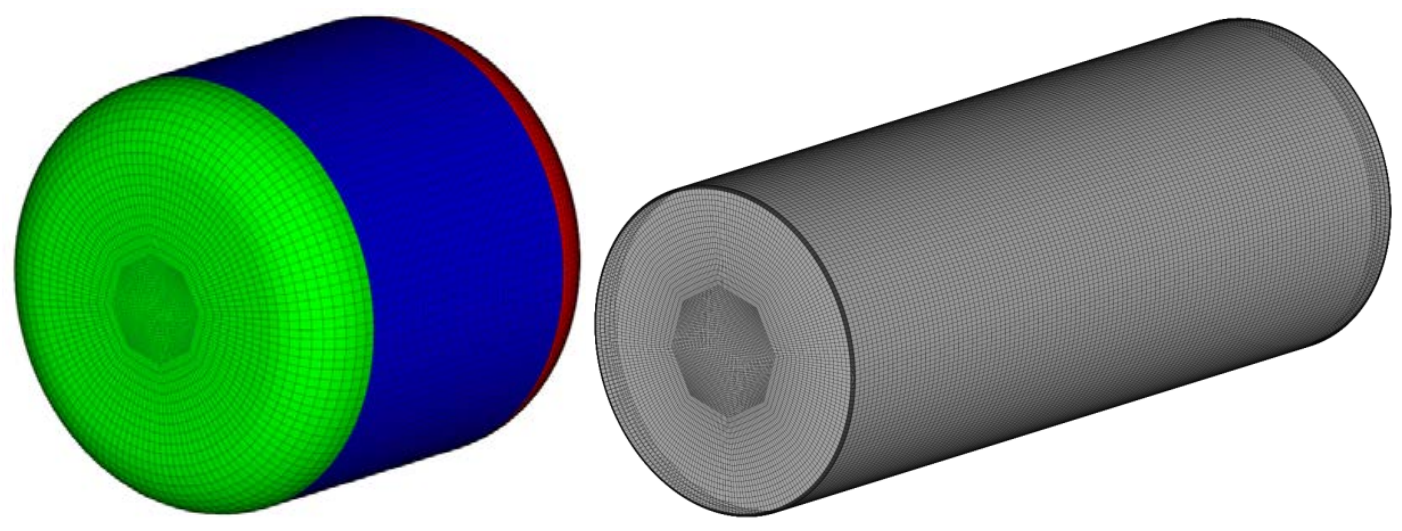

Figure 5.44 Sharp Cylinder Mesh 


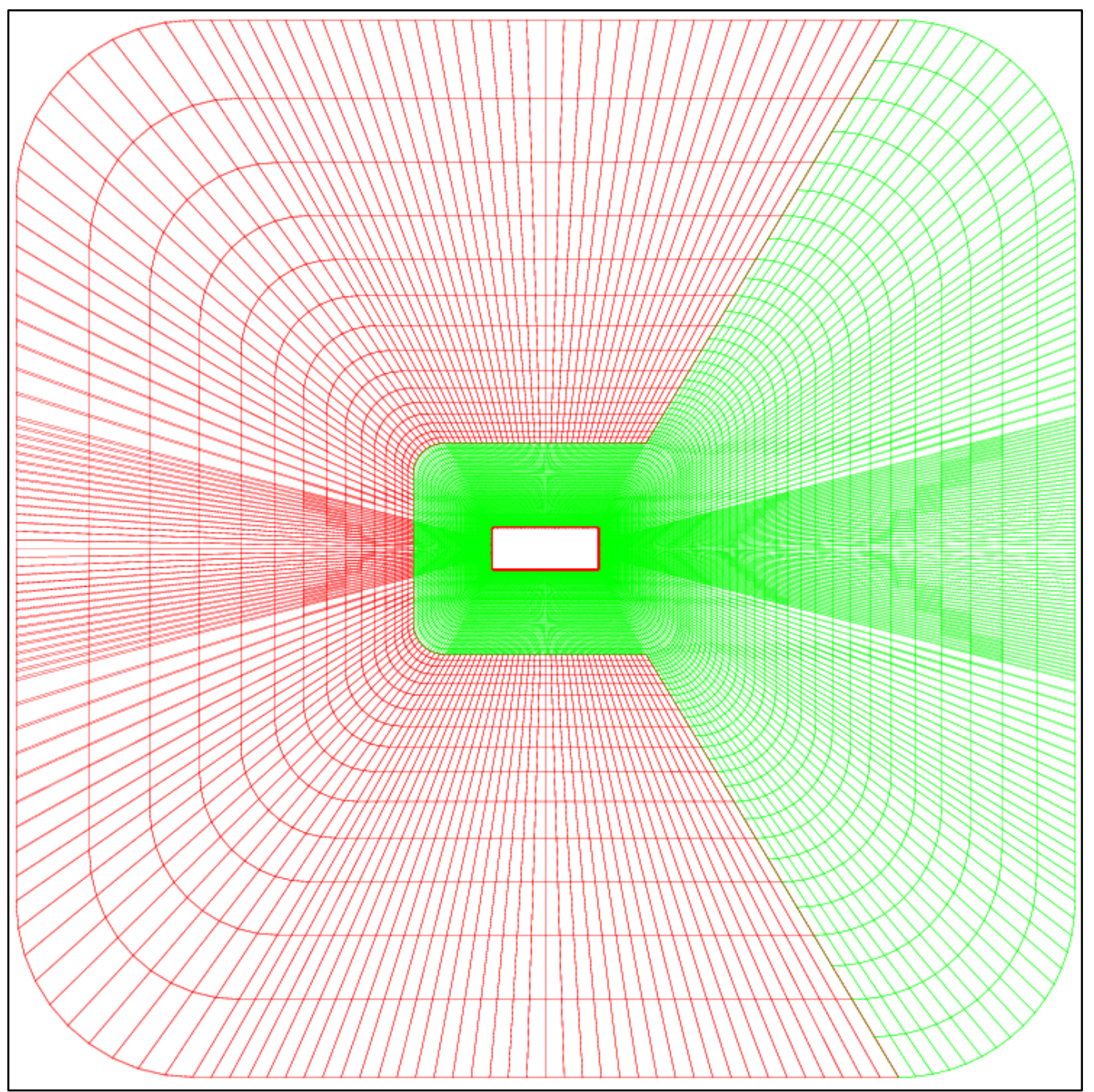

Figure 5.45 Sharp Cylinder Cross-Section View

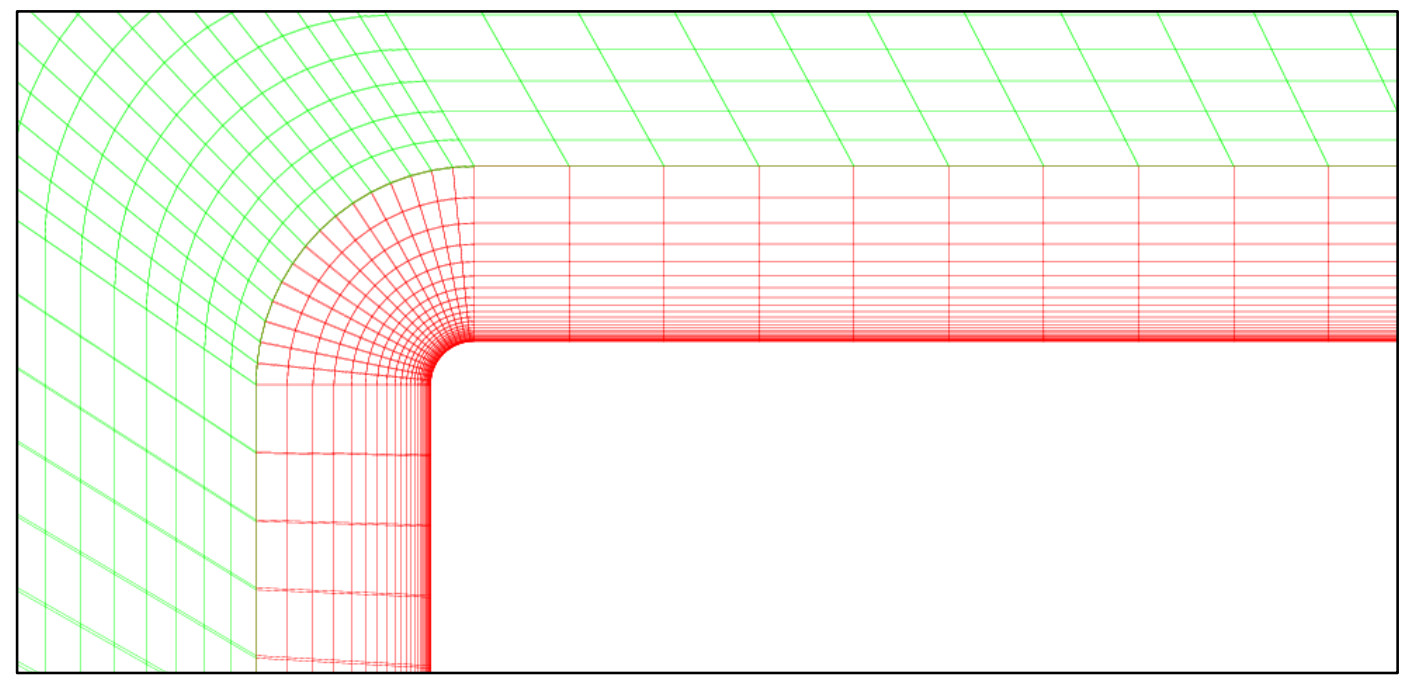

Figure 5.46 Sharp Cylinder Cross-Section View of Boundary Layer 
The dimension of the sharp cylinder was $2.5 D_{c y l} \times D_{c y l}$ and the edges were smoothed with a corner radius of $0.01 D_{c y l}$ shown in Figure 5.43. The corner radius was incorporated mainly to prevent convergence problem due to sharp corners in the geometry. The mesh extended $12.5 D_{c y l}$ towards the front, rear and the sides from the centre of the cuboid. The mesh is shown on the left of Figure 5.44 and the enlarged view of the cuboid wall is shown on the right of Figure 5.44. In Figure 5.44, the green zone was defined as a velocity inlet, the red zone was defined as a pressure outlet, while the blue zone was defined as symmetry boundary condition.

The mesh is a fully structured configuration utilizing hexahedron cells.

The cross-section of the mesh domain is shown in Figure 5.45, and the red zones, which included the coarser domain and the refined boundary layer (Figure 5.46) was the RANS zones while the green zones which included the refined domain was the LES zones. Due to the complexity of description of the entire mesh, a brief description of the mesh is presented below.

The mesh faces along the length of the cylinder had a $114 \times 240$ mesh face, while the flat mesh faces along the diameter of the cylinder was divided into two mesh faces, consisting of a $240 \times 240$ mesh face that transited to a $18 \times 240$ mesh face. The curved edges along the circumference of the cylinder had a $16 \times 240$ mesh face. The mesh was extruded in all directions to the limits of domain boundary, with the outer RANS zone having $\frac{1}{4}$ the cell density of the inner LES zone and the inner RANS zone. A total 4539300 elements was used for the sharp cylinder nacelle mesh.

\subsubsection{CFD Results}

Due to limitation on computation resources, it was not possible to run all test cases for 20 vortex shedding periods. Therefore, in order to avoid the transient conditions resulting from the initialization of the simulation, the time-averaged results were based on the averaged results over the latter half of the simulation period of each test case. 
Figure 5.47 describes the reference frame used for the nacelle results discussion.

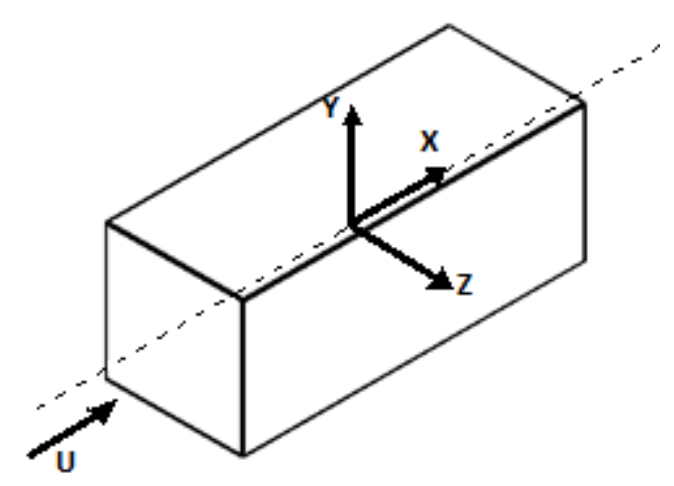

Figure 5.47 Reference Frame for Nacelle Cases

The origin was located at the center of the nacelle. The dimensions of the cuboid and cylinder nacelle were normalized with the width $W_{C u b}$ and diameter $D_{c y l}$ respectively. The normalized $\mathrm{X}$ displacement $x$ is parallel and flows the direction of the inflow wind while the normalized Y displacement $y$, and normalized $\mathrm{Z}$ displacement $z$ is perpendicular to $x$ and the axial direction of the nacelle. Figure 5.47 showed a cuboid nacelle, similar reference frame used for the cylinder nacelle.

\subsubsection{General Flow Characteristics}

Some general flow characteristics were observed in all the cases simulated. Figure 5.48 and Figure 5.49 showed the time-averaged normalized $\mathrm{X}$ and $\mathrm{Y}$ velocity $\left(v_{x}\right.$ and $\left.v_{y}\right)$, and Figure 5.50 showed the time-averaged velocity vectors plot for the cuboid nacelle Case 2 (sharp shaped) $\left(R e_{c u b}=\right.$ $6.71 \mathrm{E}+06)$. Figure 5.51 showed the corresponding instantaneous velocity vectors plot. Figure 5.53 and Figure 5.54 showed the time-averaged normalized $\mathrm{X}$ and $\mathrm{Y}$ velocity, and Figure 5.55 showed the time-averaged velocity vectors plot for the cuboid nacelle Case 11 (smooth shaped). Figure 5.56 showed the corresponding instantaneous velocity vectors plot.

For both shape and smooth shaped nacelle, the wind velocity was reduced upwind of the nacelle before being sped up around the nacelle. Velocity 
deficit and mean reversed flow was observed at the rear end of the nacelle. Differences in the fluid flow were observed between the two edge profiles. The sharp shaped nacelle experienced turbulent flow separation and shear layer instabilities. Reattachment of the flow occurred as the flow progresses and a significant wake width was observed, which was mainly a result of the flow separation. The smooth shaped nacelle did not experience flow separation. Instead, there was a tendency for the flow to stay attached to the curved surface, which is also known as the Coandă effect. After which, the boundary layer developed across the surface of the nacelle and towards the rear of the nacelle. The wake width was almost similar to the width of the nacelle due to the lack of flow separation.

Similar observations were seen for the cylinder nacelle Case 2 as shown in Figure 5.58 to Figure 5.66. The figures for the remaining cases are presented in Appendix G and Appendix H.

While the flow was similar across the radial direction (YZ plane) for the cylinder nacelle (Figure 5.62 and Figure 5.67), different flow characteristics was observed for the cuboid nacelle. For the sharp shaped cuboid nacelle, the flow first separated in a circular manner as seen for $x=-0.5 L_{c y l}$ to $x=$ 0 in Figure 5.52. As the flow reattached to the nacelle surface further downstream, the resultant wake developed with a square trend that is rotated $45^{\circ}$. For the smooth shaped cuboid nacelle (Figure 5.57), as flow separation did not occur, the resultant flow and wake followed the shape of the cuboid and developed with a square trend. 


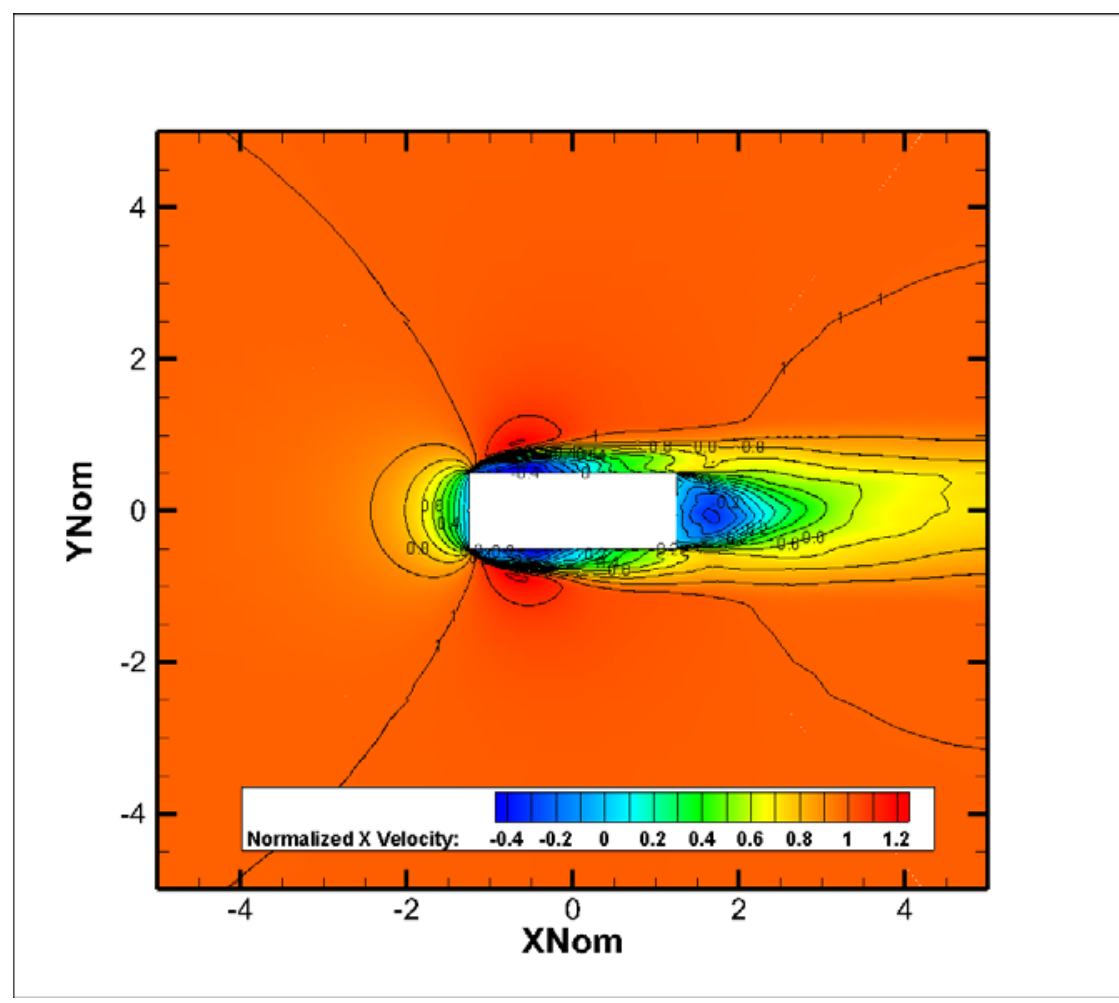

Figure 5.48 Mean Normalized X Velocity for Cuboid Nacelle Case 2: $R e_{c u b}=$ $6.71 \mathrm{E}+06$

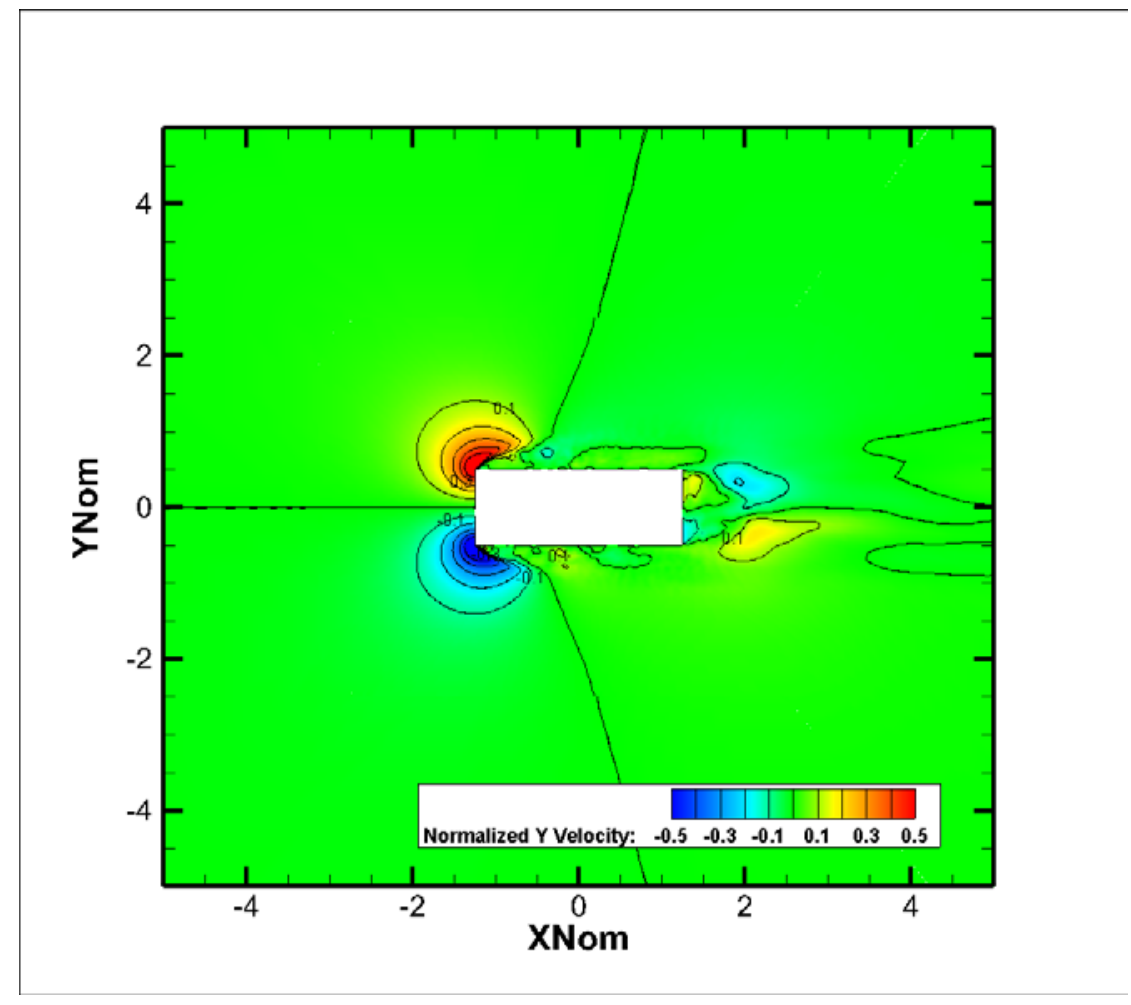

Figure 5.49 Mean Normalized Y Velocity for Cuboid Nacelle Case 2: $R e_{c u b}=$ $6.71 \mathrm{E}+06$ 

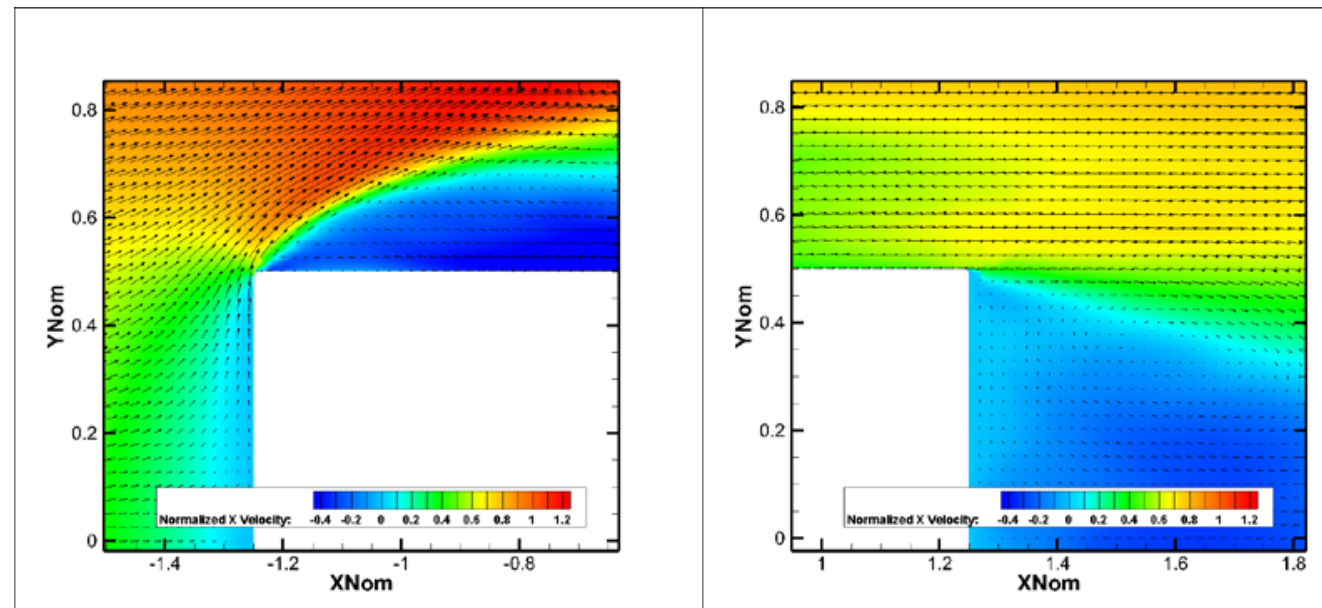

Figure 5.50 Mean Normalized Velocity Vectors for Cuboid Nacelle Case 2: $R e_{c u b}=6.71 \mathrm{E}+06$

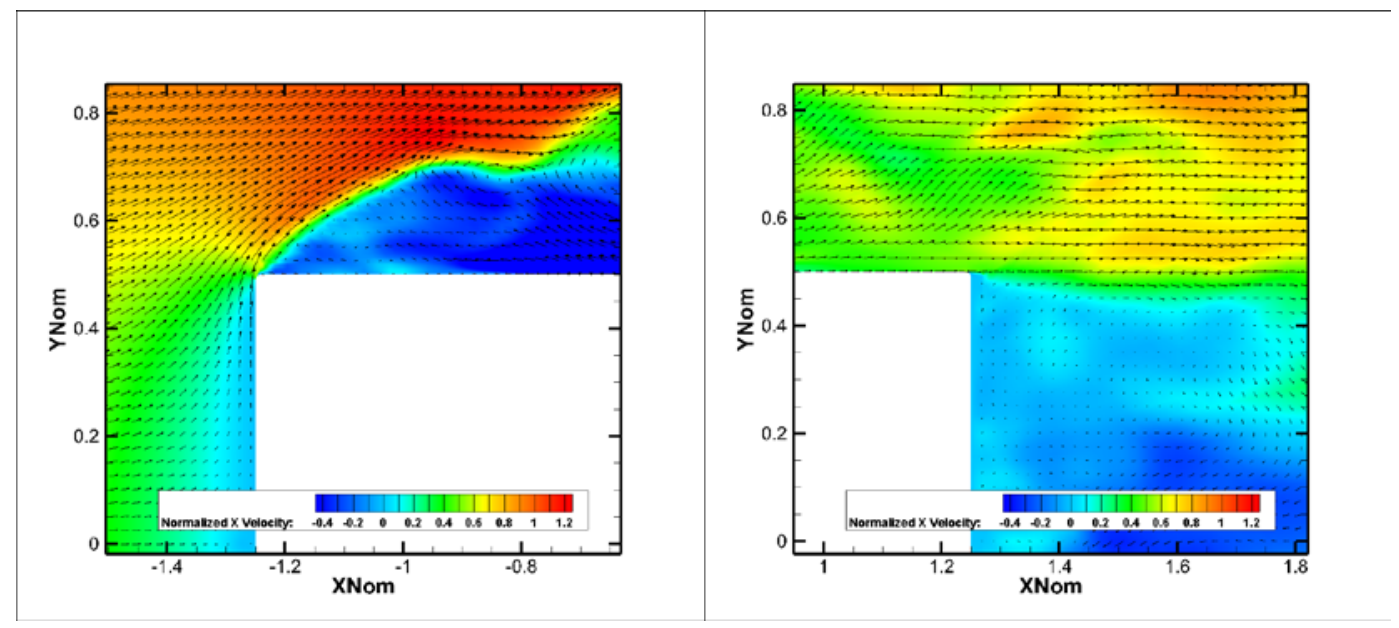

Figure 5.51 Instantaneous Normalized Velocity Vectors for Cuboid Nacelle Case 2: $R e_{c u b}=6.71 \mathrm{E}+06$ 


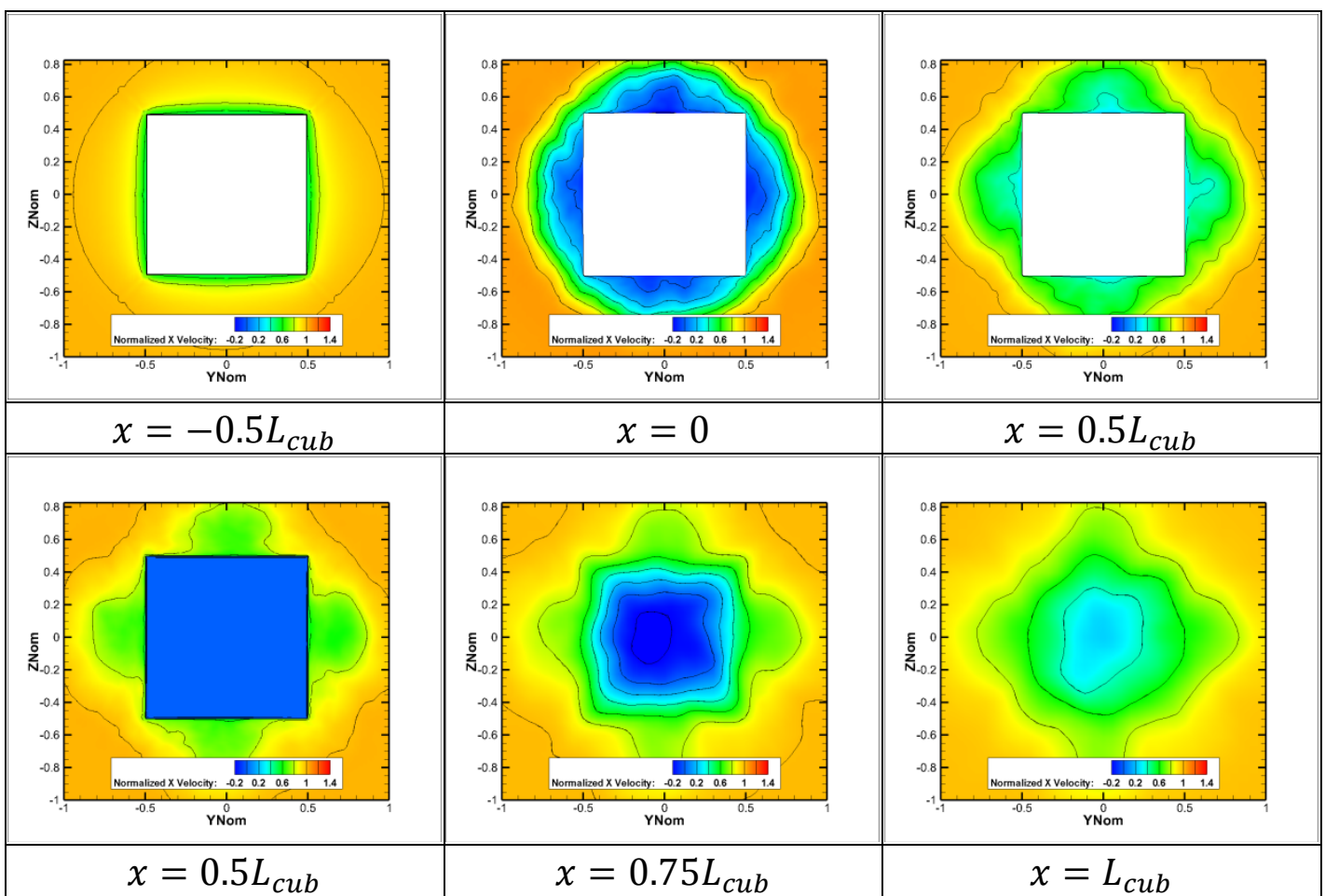

Figure 5.52 Mean Normalized Velocity X on YZ Plane for Cuboid Nacelle Case 2: $R e_{c u b}=6.71 \mathrm{E}+06$

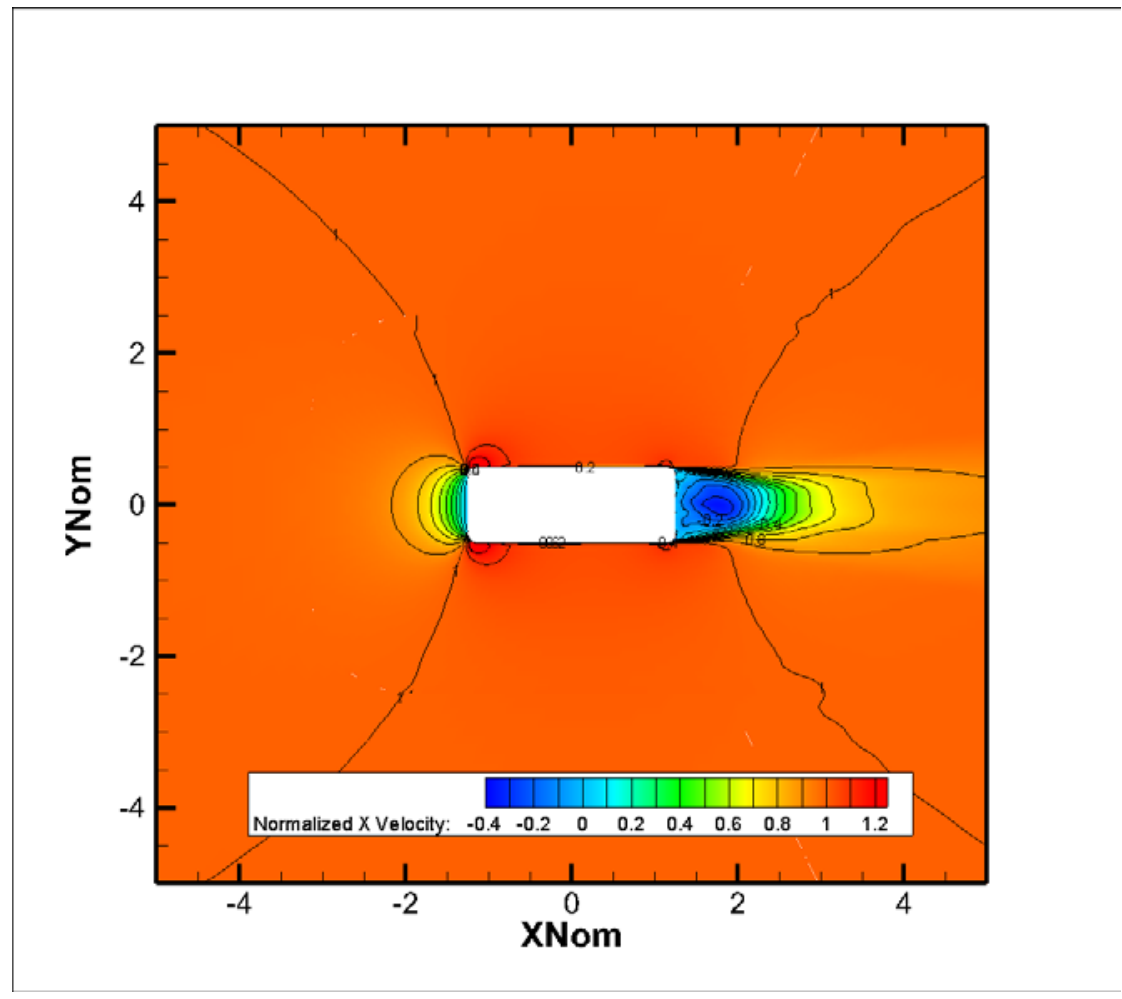

Figure 5.53 Mean Normalized X Velocity for Cuboid Nacelle Case 11: $R e_{c u b}=$ $6.71 \mathrm{E}+06$ 


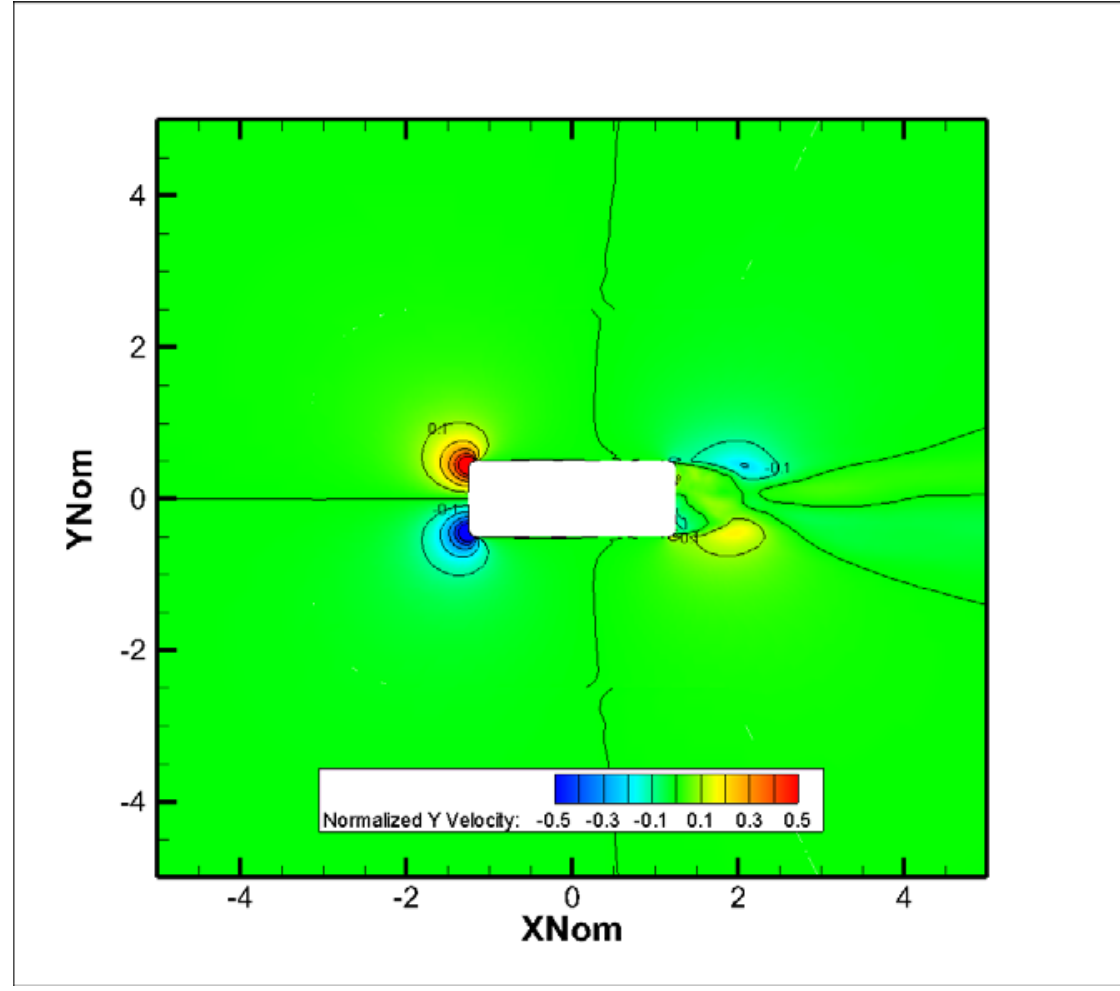

Figure 5.54 Mean Normalized Y Velocity for Cuboid Nacelle Case 11: $R e_{c u b}=$ $6.71 \mathrm{E}+06$

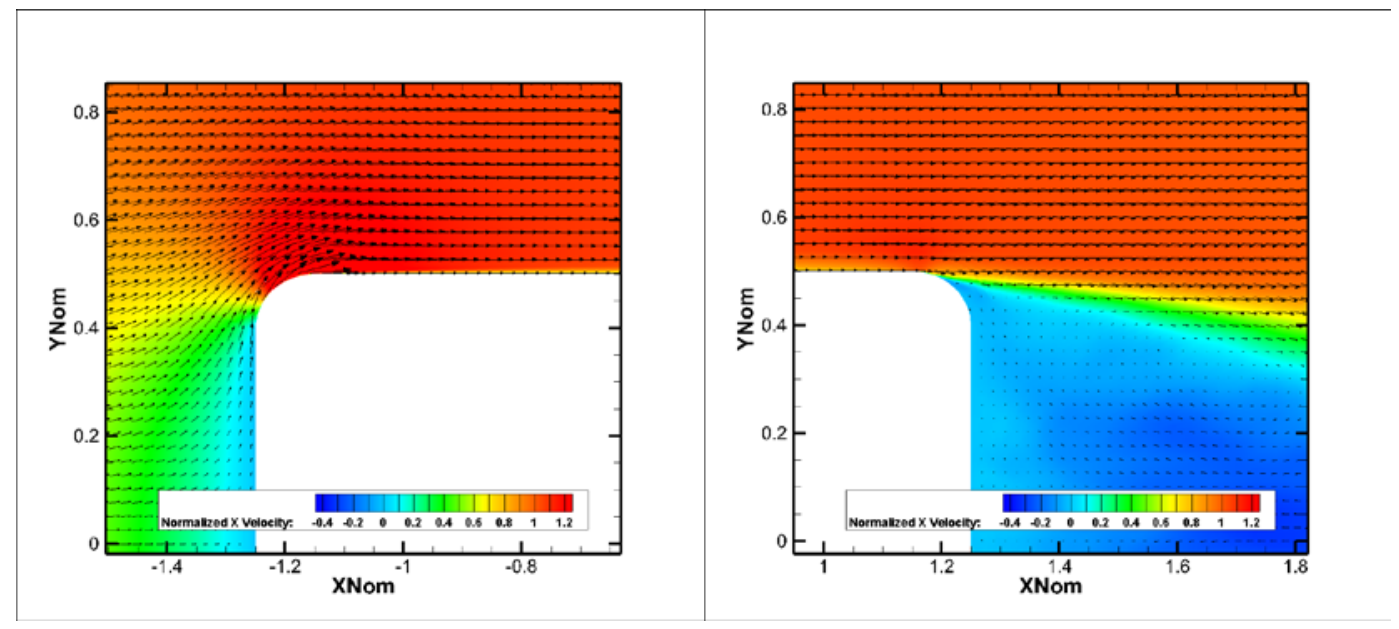

Figure 5.55 Mean Normalized Velocity Vectors for Cuboid Nacelle Case 11: $R e_{c u b}=6.71 \mathrm{E}+06$ 

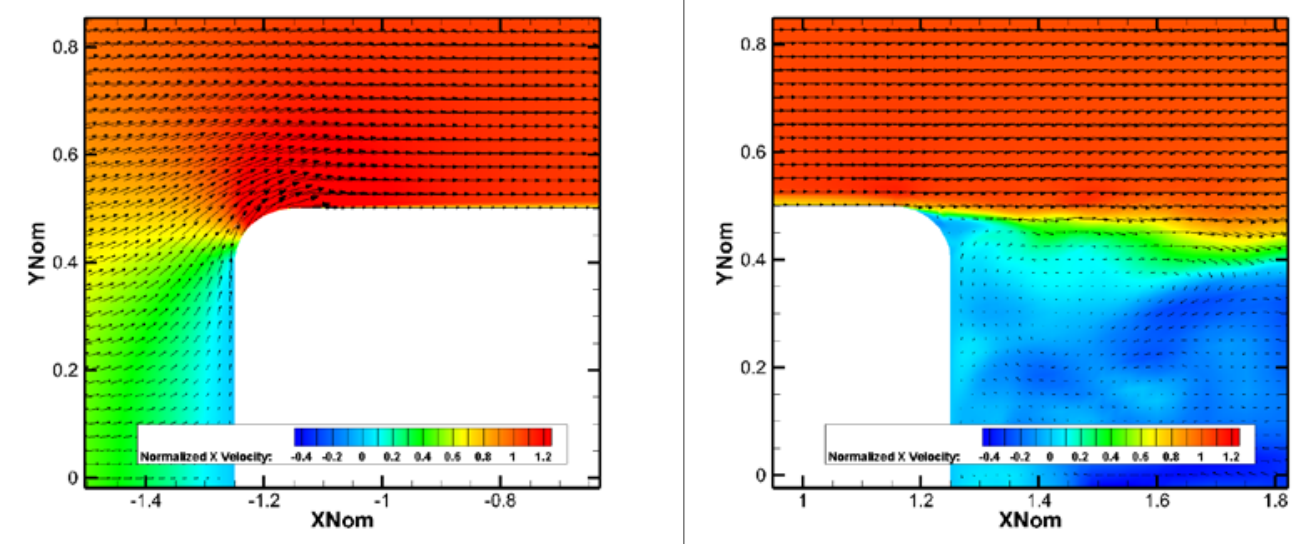

Figure 5.56 Instantaneous Normalized Velocity Vectors for Cuboid Nacelle Case 11: $R e_{c u b}=6.71 \mathrm{E}+06$

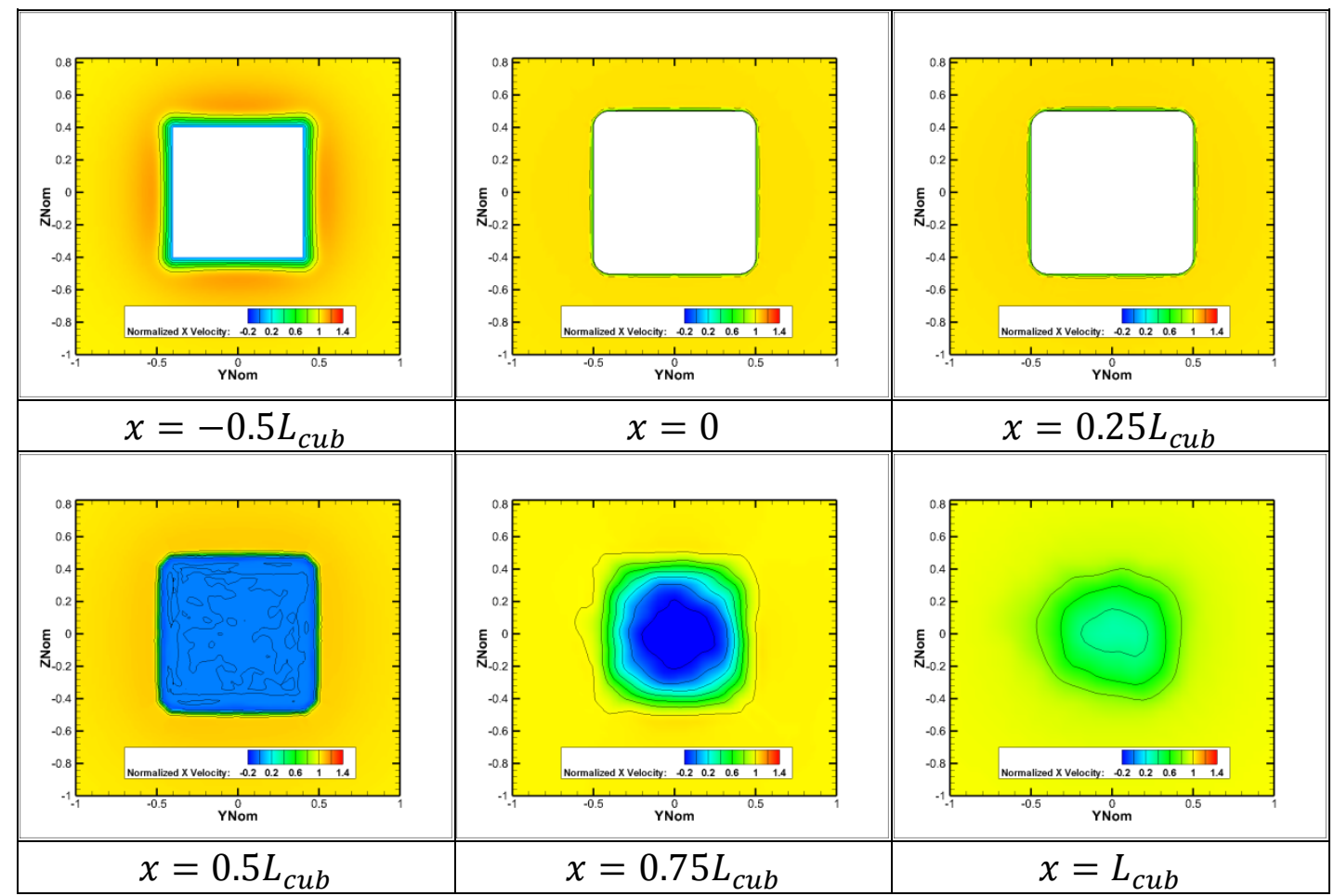

Figure 5.57 Mean Normalized Velocity X on YZ Plane for Cuboid Nacelle Case 11: $R e_{c u b}=6.71 \mathrm{E}+06$ 


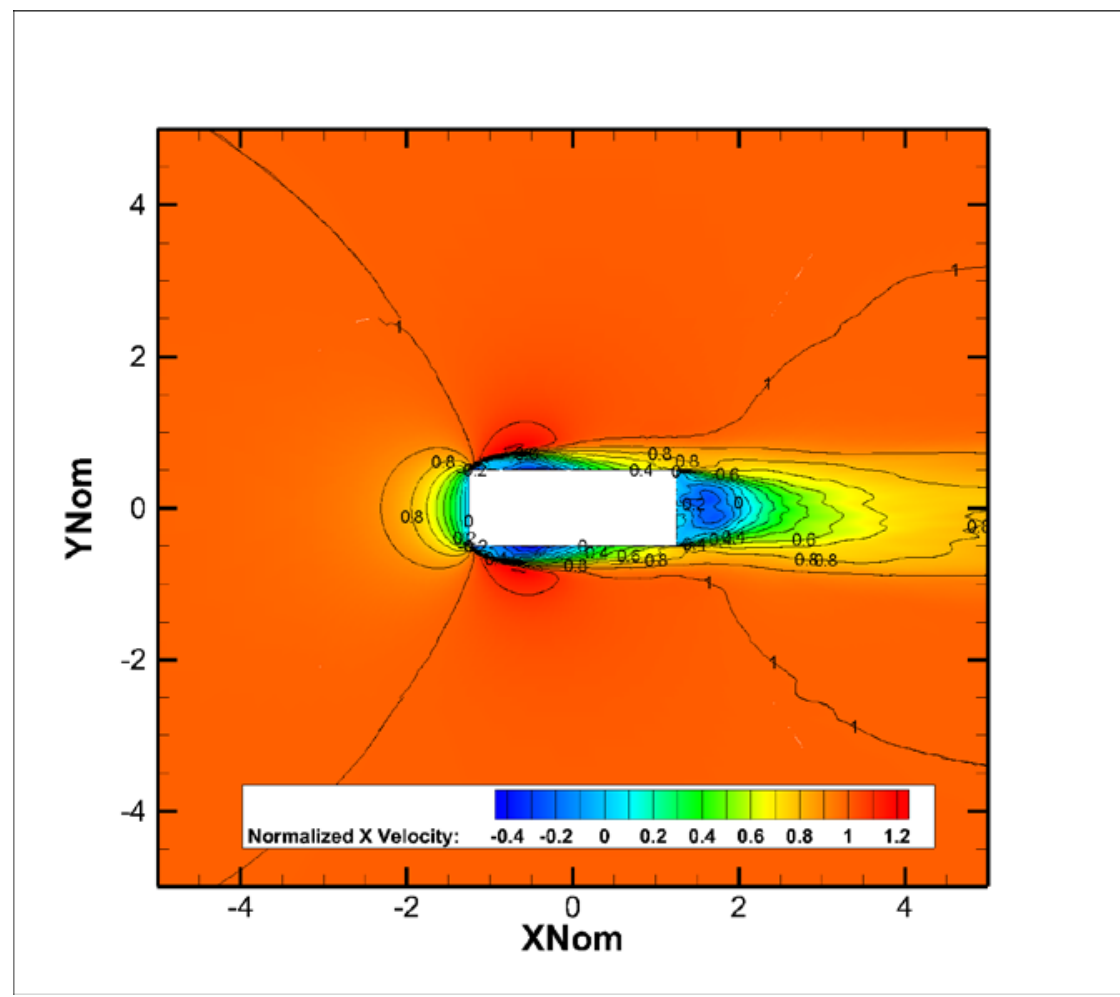

Figure 5.58 Mean Normalized X Velocity for Cylinder Nacelle Case 2: $R e_{c y l}=$ $6.71 \mathrm{E}+06$

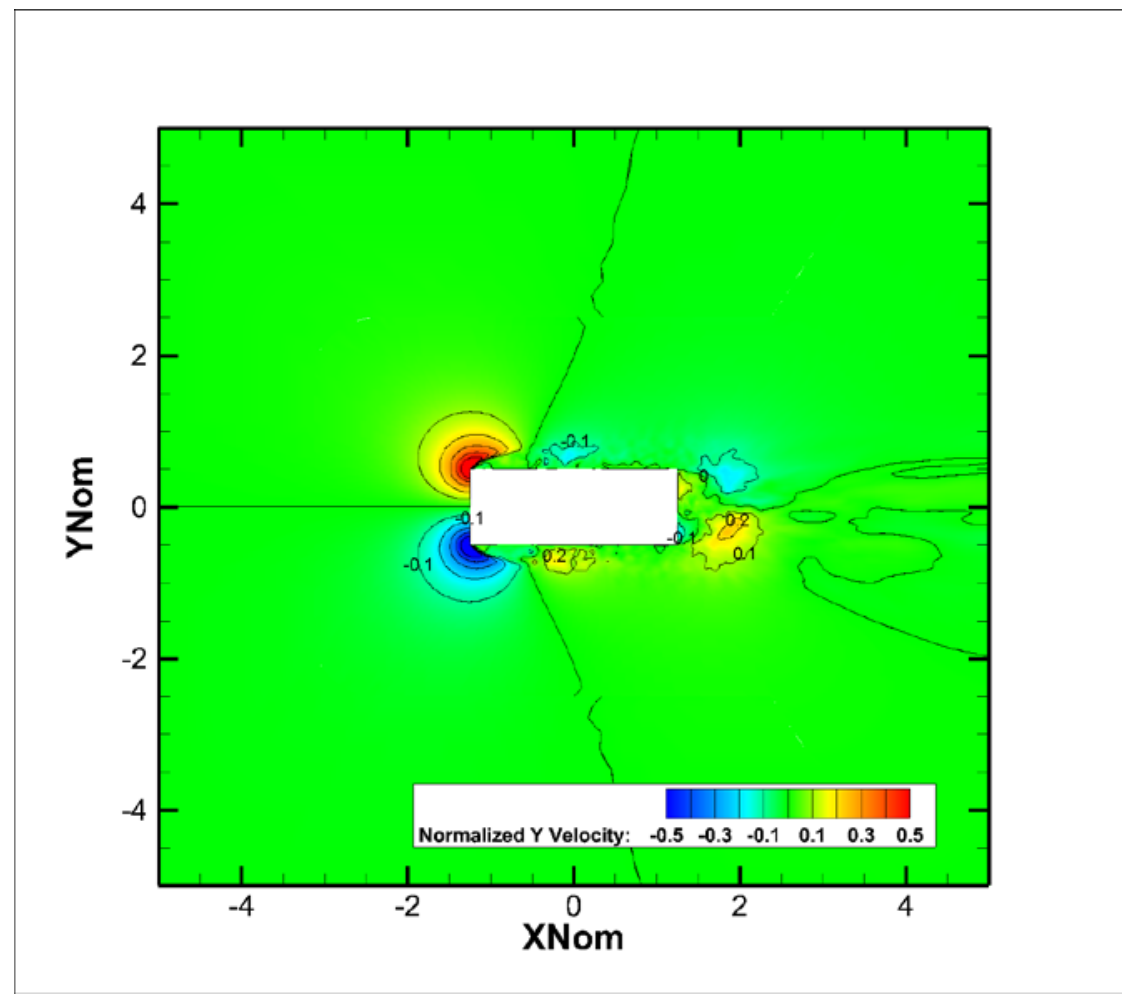

Figure 5.59 Mean Normalized Y Velocity for Cylinder Nacelle Case 2: $R e_{c y l}=$ $6.71 \mathrm{E}+06$ 

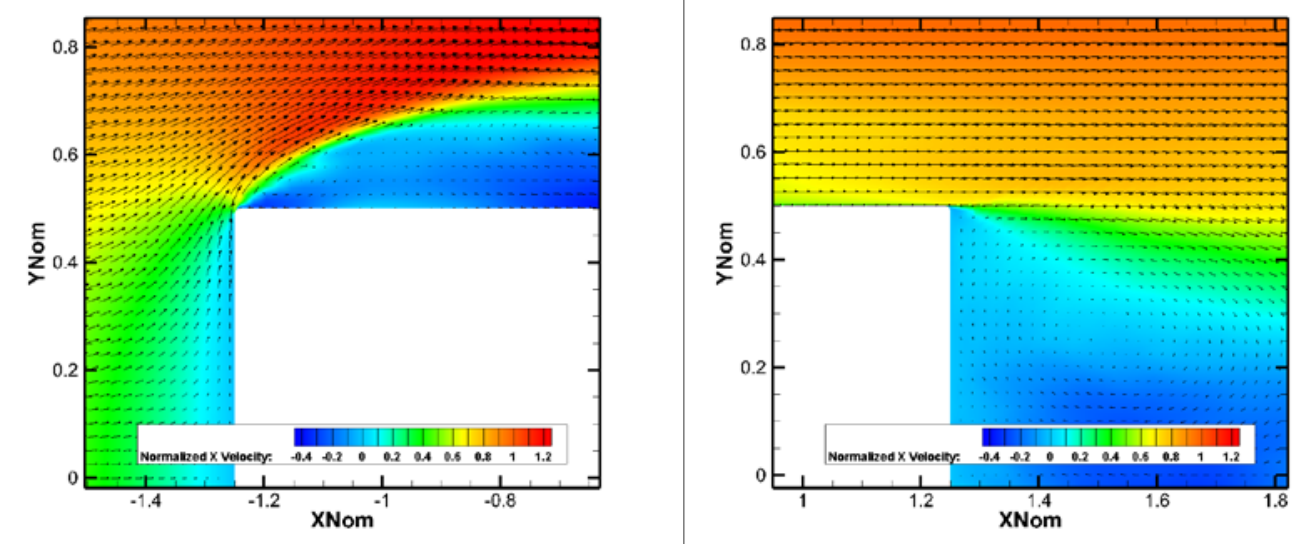

Figure 5.60 Mean Normalized Velocity Vectors for Cylinder Nacelle Case 2: $R e_{c y l}=6.71 \mathrm{E}+06$
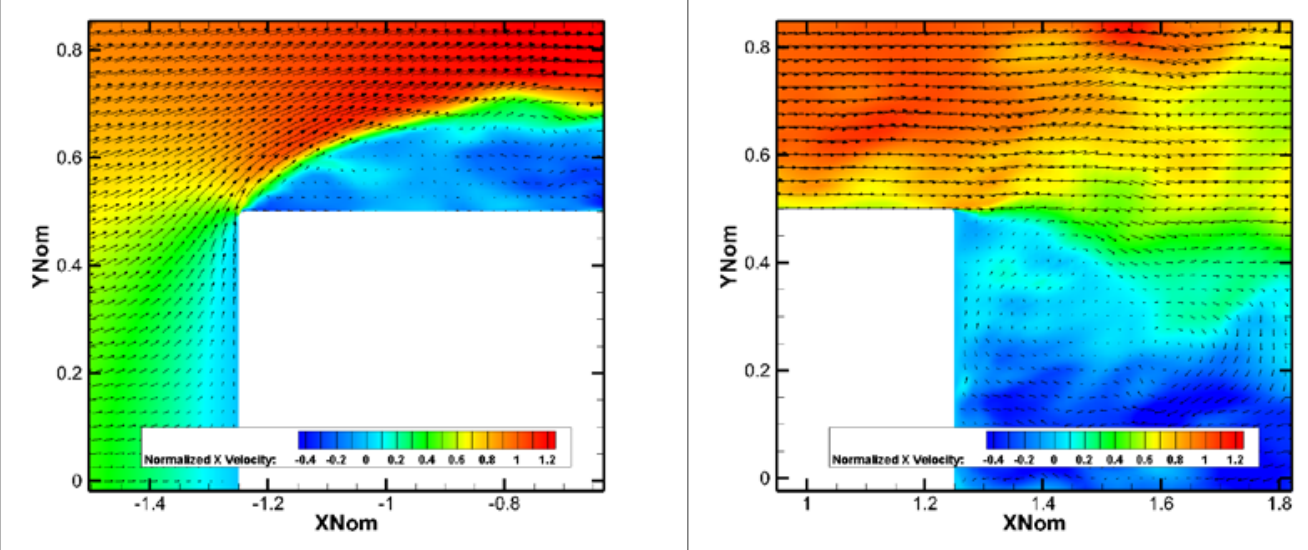

Figure 5.61 Instantaneous Normalized Velocity Vectors for Cylinder Nacelle Case 2: $\mathfrak{R}_{c y l}=6.71 \mathrm{E}+06$ 


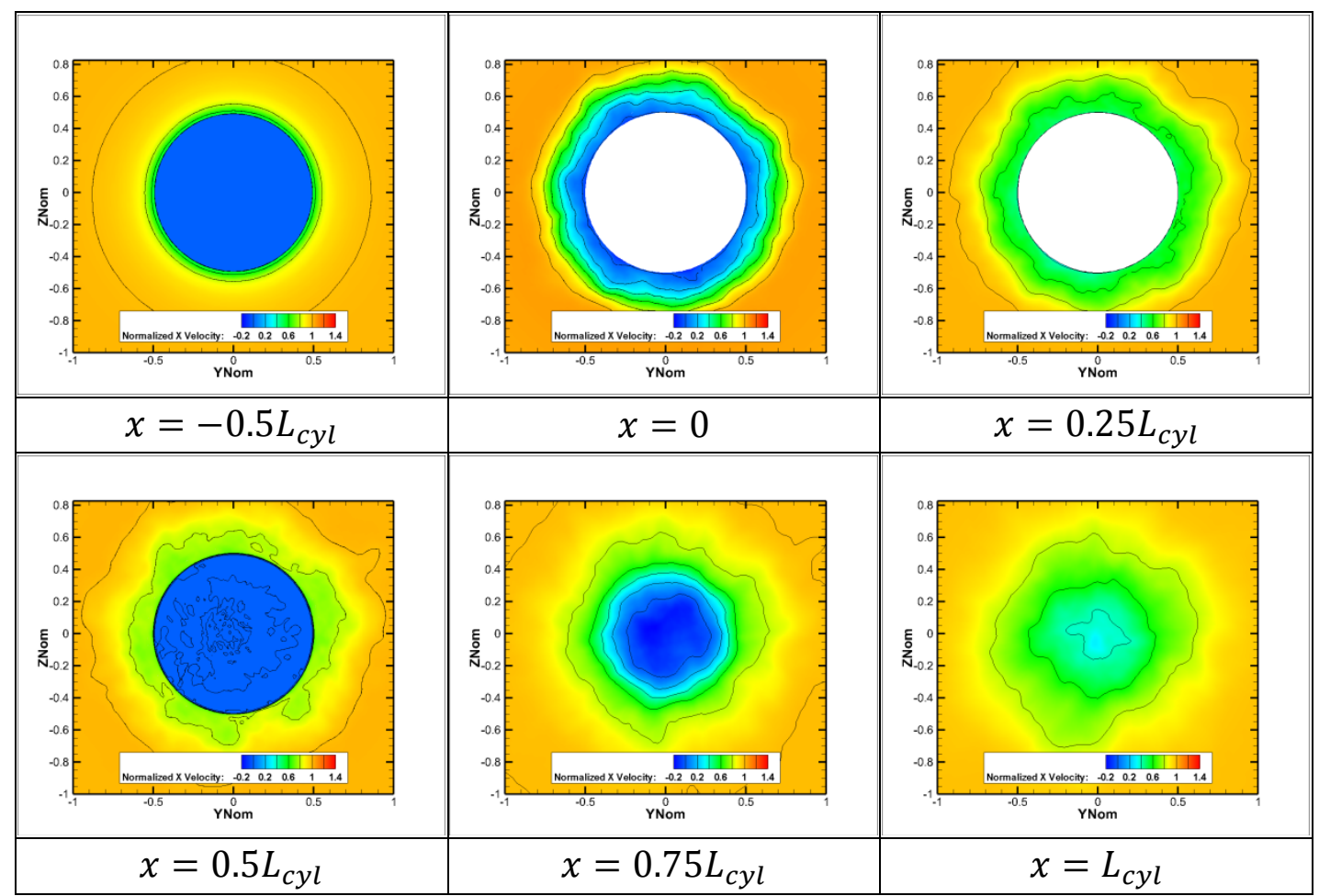

Figure 5.62 Mean Normalized Velocity X on YZ Plane for Cylinder Nacelle Case 2: $\mathfrak{R}_{\text {cub }}=6.71 \mathrm{E}+06$

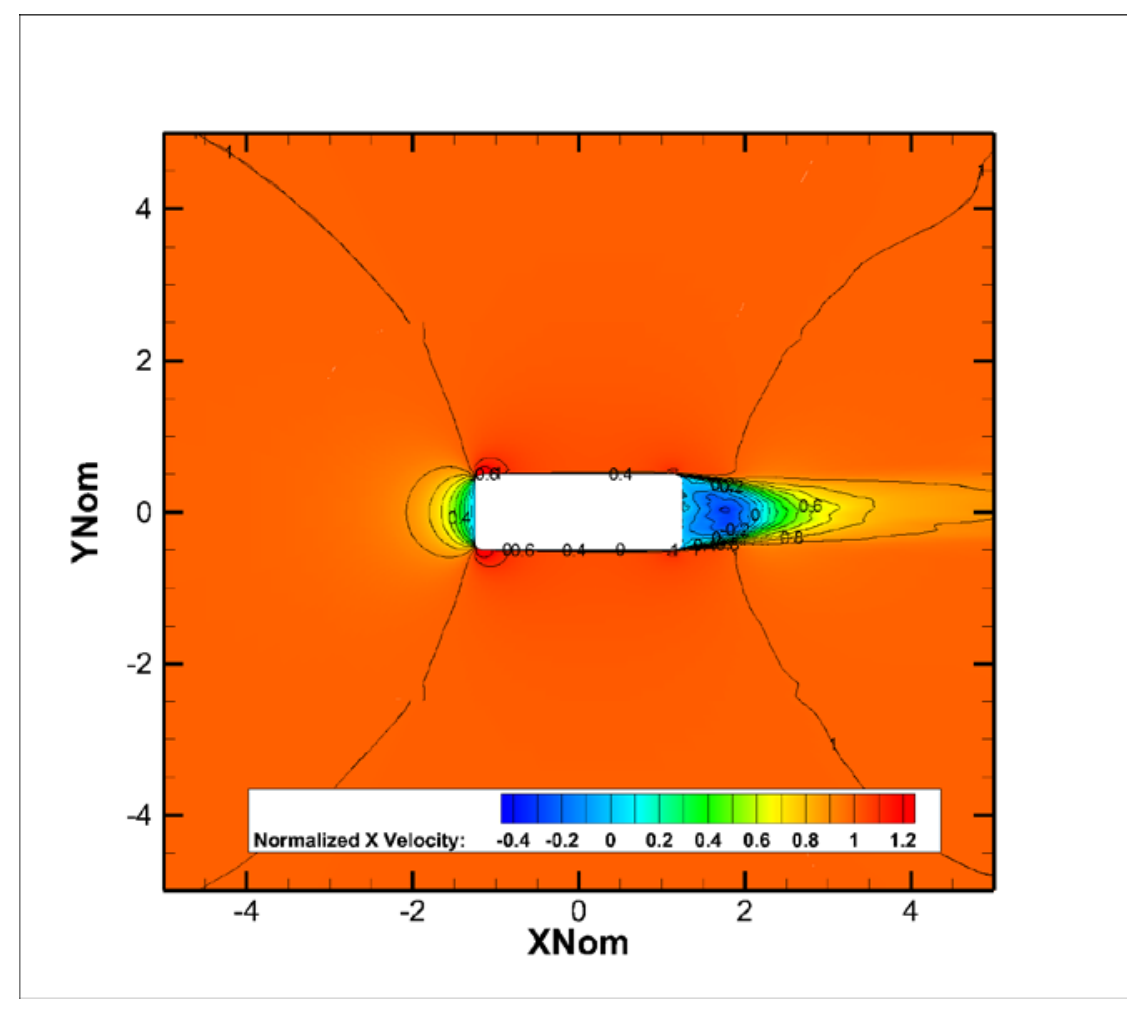

Figure 5.63 Mean Normalized X Velocity for Cylinder Nacelle Case 11: $\mathfrak{R}_{c y l}=$ $6.71 \mathrm{E}+06$ 


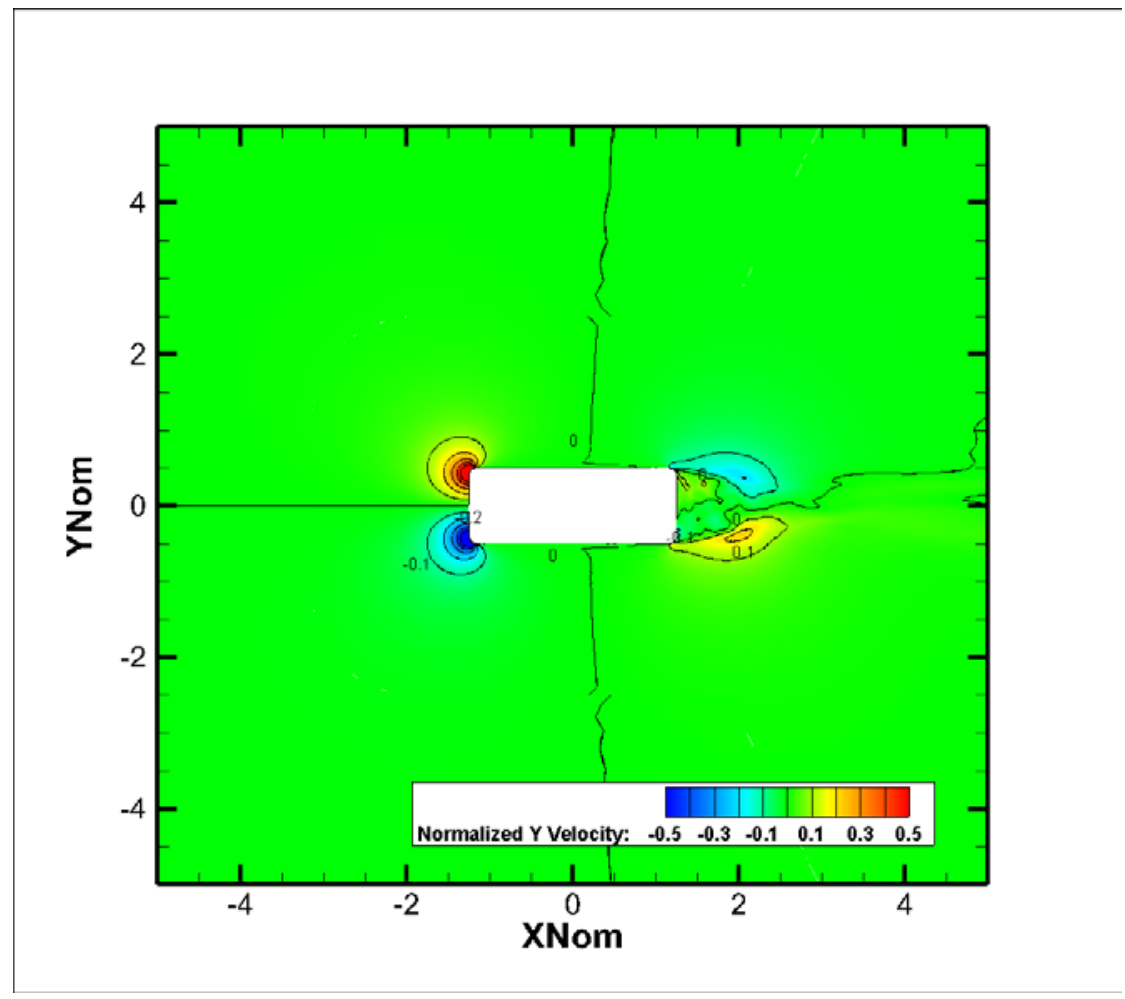

Figure 5.64 Mean Normalized Y Velocity for Cylinder Nacelle Case 11: $\mathfrak{R}_{c y l}=$ $6.71 \mathrm{E}+06$

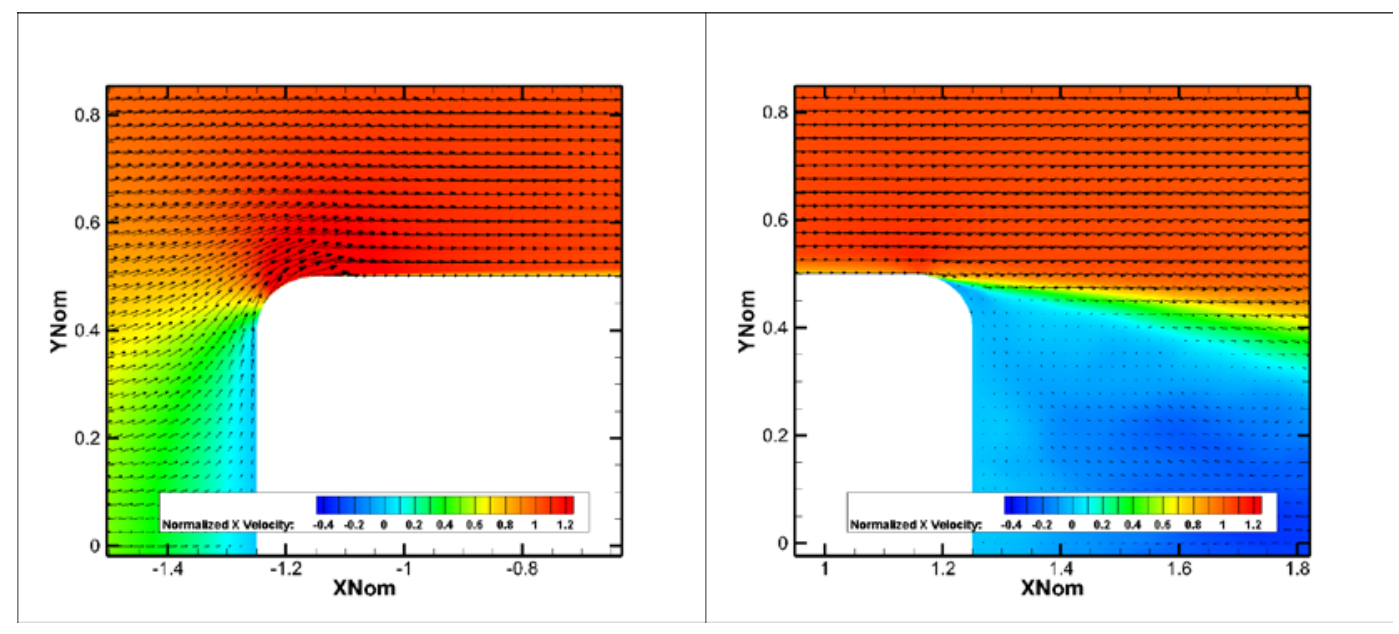

Figure 5.65 Mean Normalized Velocity Vectors for Cylinder Nacelle Case 11: $\mathfrak{R}_{\text {cyl }}=6.71 \mathrm{E}+06$ 

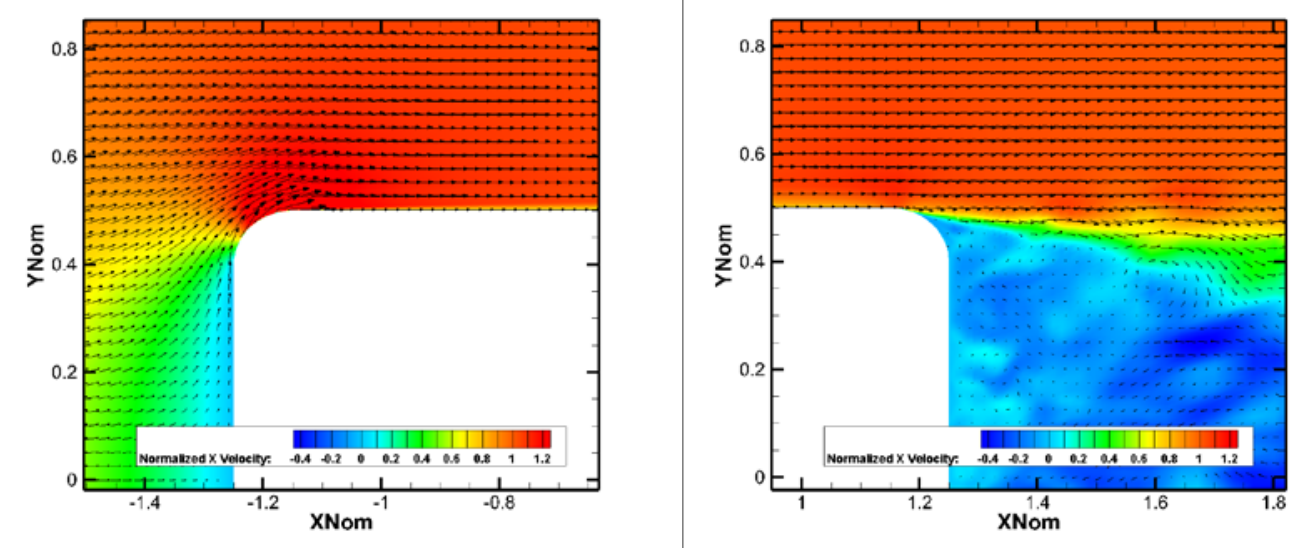

Figure 5.66 Instantaneous Normalized Velocity Vectors for Cylinder Nacelle Case 11: $\mathfrak{R}_{c y l}=6.71 \mathrm{E}+06$

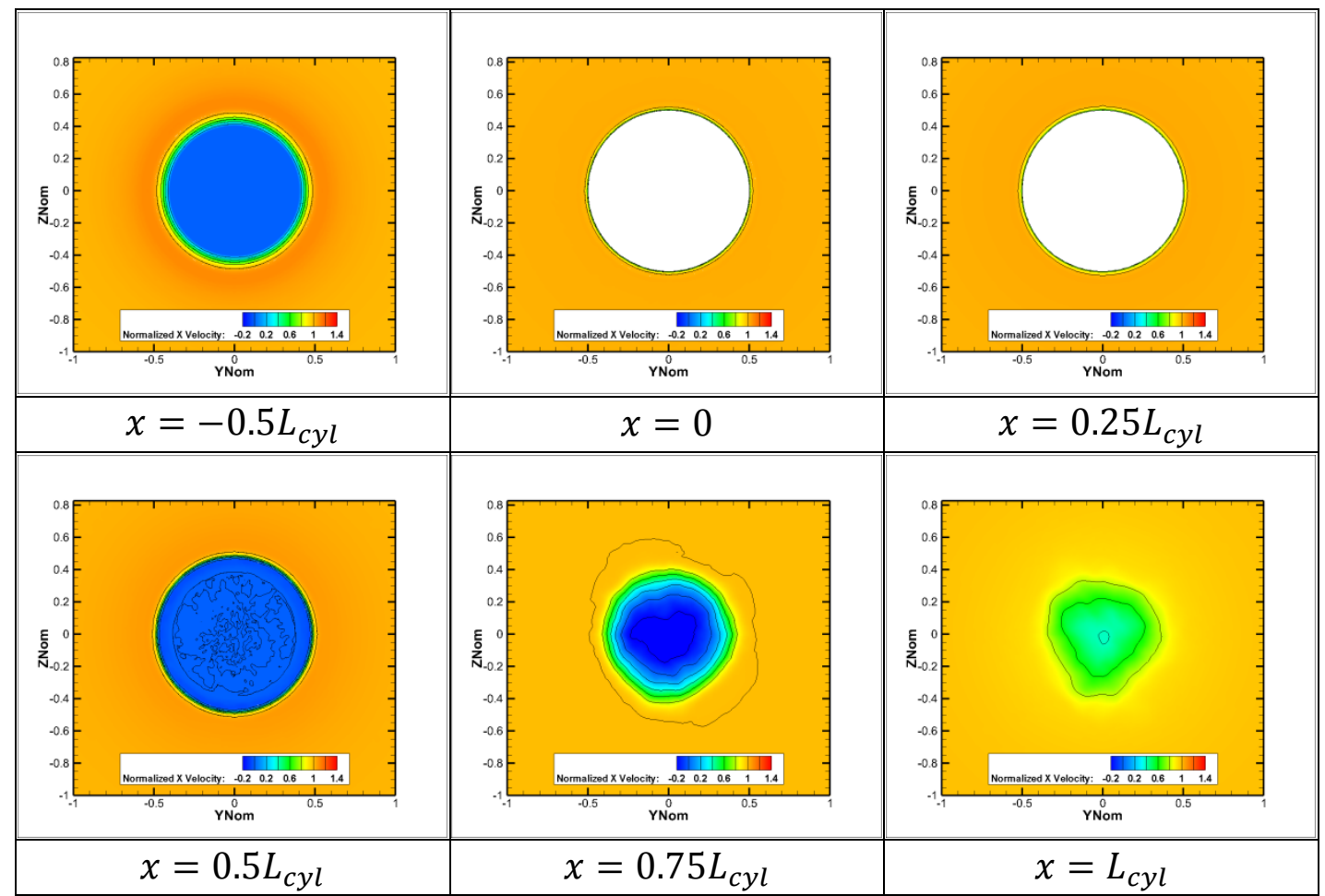

Figure 5.67 Mean Normalized Velocity X on YZ Plane for Cylinder Nacelle Case 11: $\mathfrak{R}_{\text {cub }}=6.71 \mathrm{E}+06$

When comparing the flow field along $x$ across various $R e_{c y l}$ and $R e_{c u b}$ in Figure 5.68 to Figure 5.71, the flow velocity along cuboid and cylinder shaped nacelle and in the wake did not show any trends or dependency on $R e_{c y l}$ or $R e_{c u b}$. 


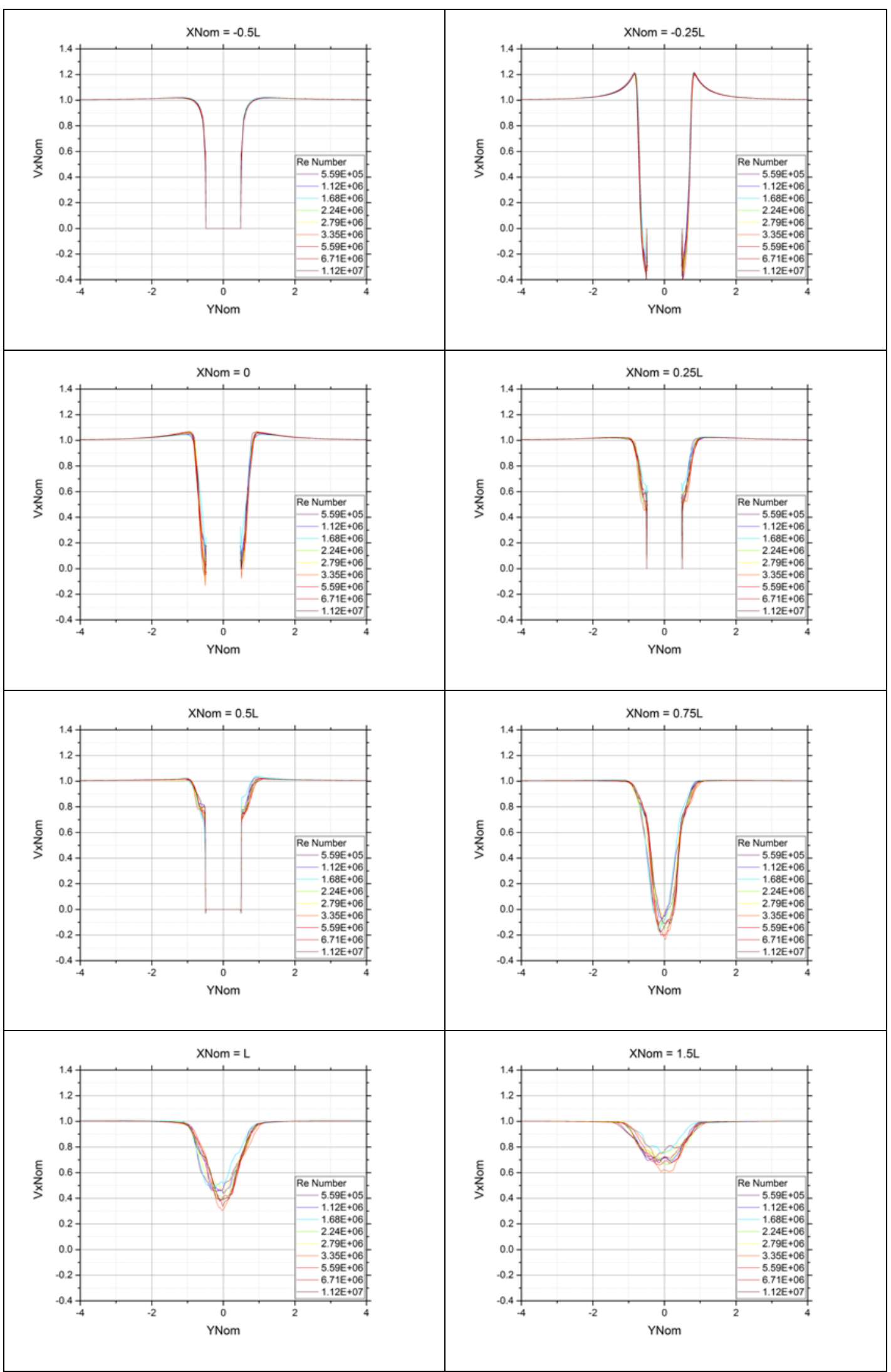

Figure 5.68 $v_{x}$ vs $y$ Plot of Various Re Values for Sharp Cylinder Cases 


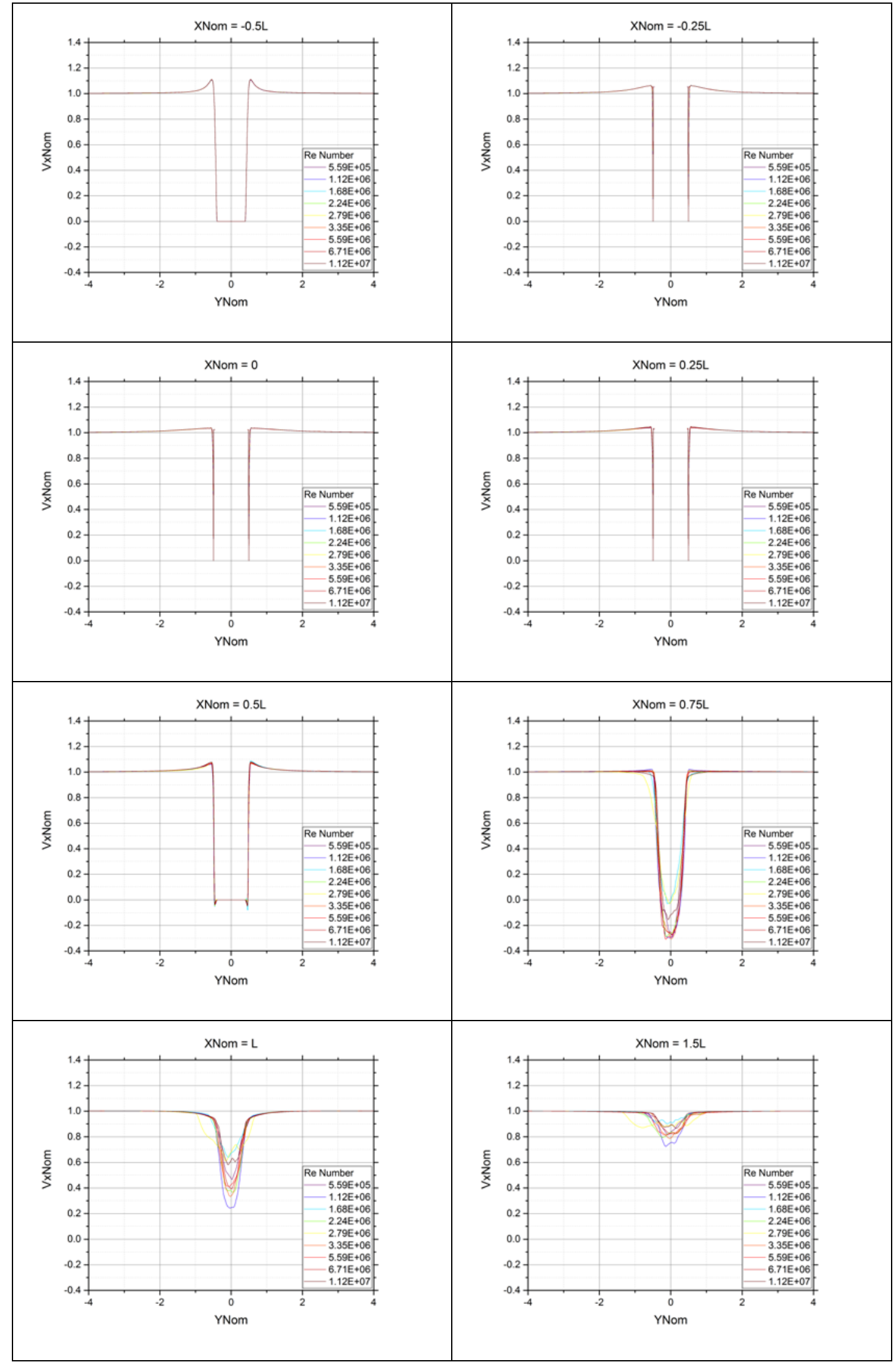

Figure 5.69 $v_{x}$ vs $y$ Plot of Various Re Values for Smooth Cylinder Cases 


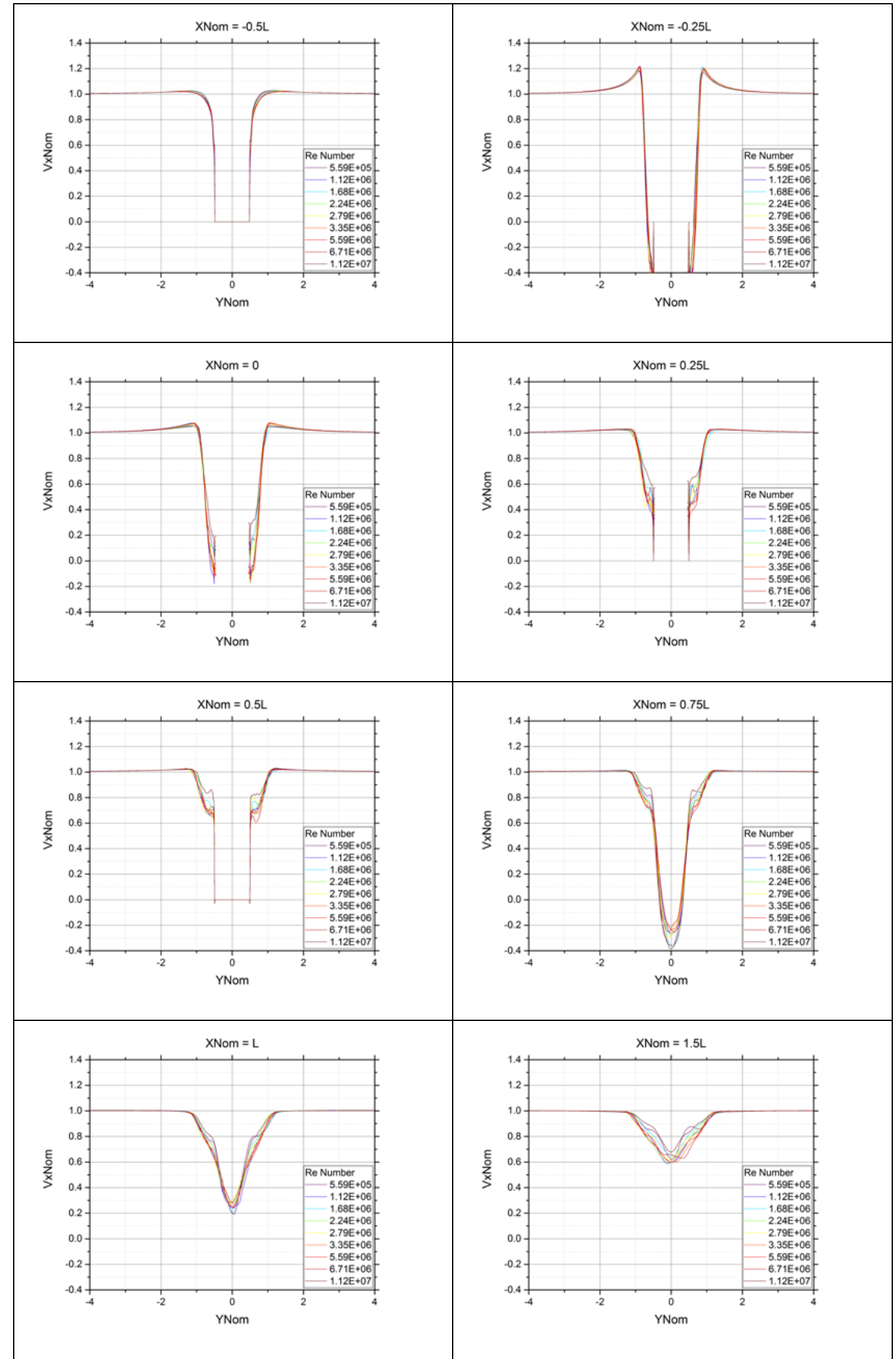

Figure 5.70 $v_{x}$ vs $y$ Plot of Various Re Values for Sharp Cuboid Cases 


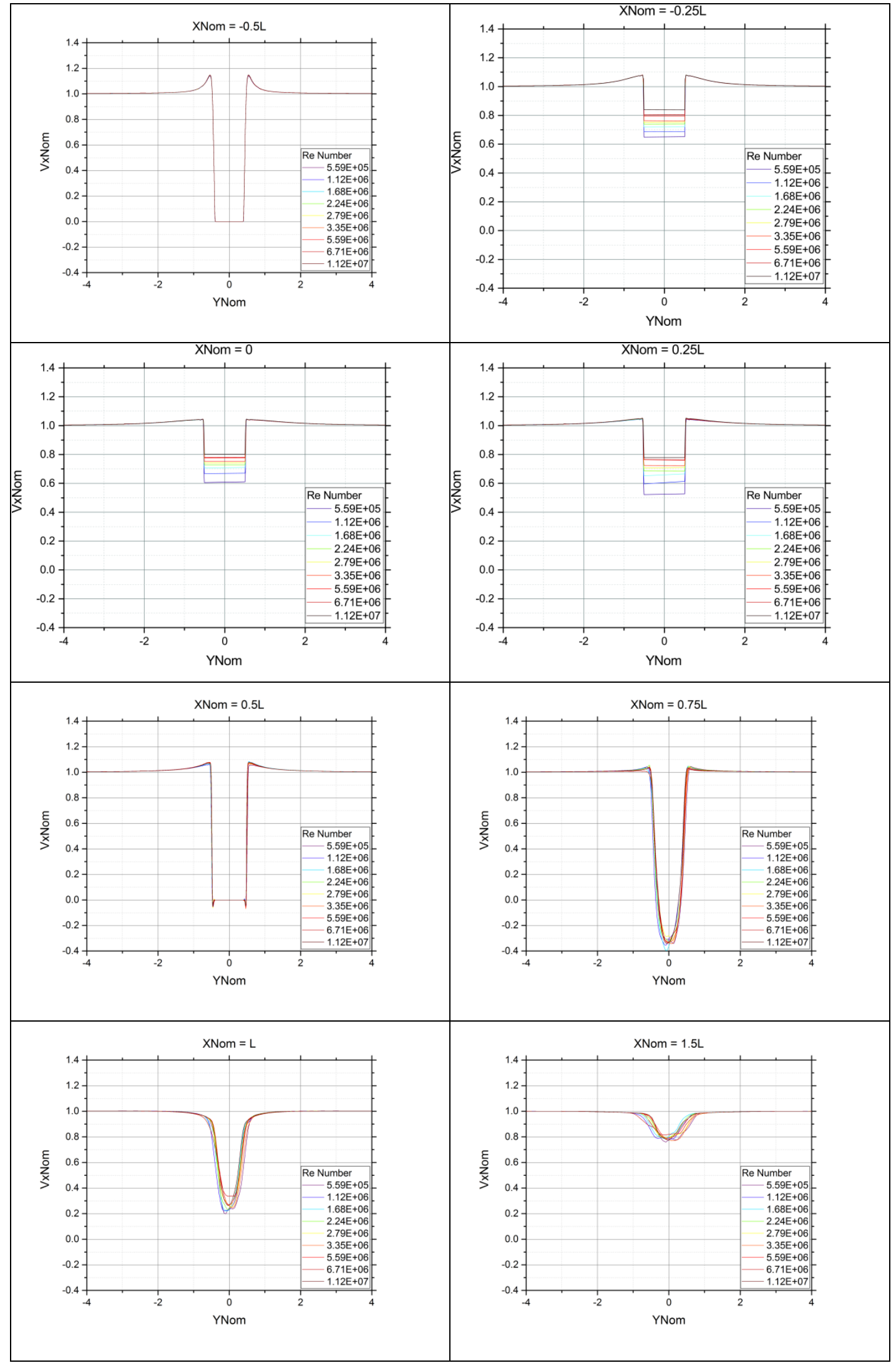

Figure 5.71 $v_{x}$ vs $y$ Plot of Various Re Values for Smooth Cuboid Cases 


\subsubsection{Comparison with Results from Literature}

In contrast with the earlier study in Section 5.4.3.3, there have been very few experimental studies that focused on axial flow over circular cylinder. The number of studies that focused on high $R e_{c y l}$ was further limited. No comparative experimental results could be found for flow over a cuboid and a smooth cylinder. The studies described in Table 5.7 were used to compare and validate the CFD results for the sharp cylinder cases.

Table 5.7 Literatures Used for Nacelle Results Comparison

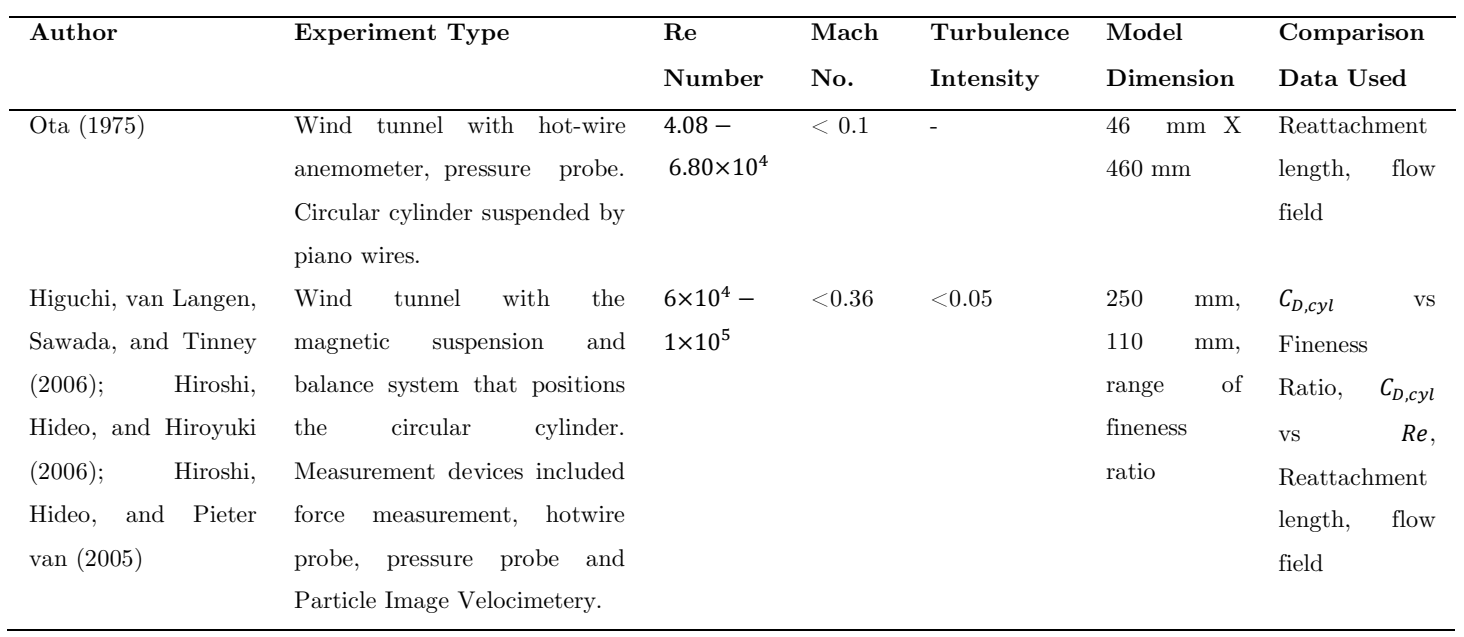

$C_{D, c y l}$ vs $R e_{c y l}$

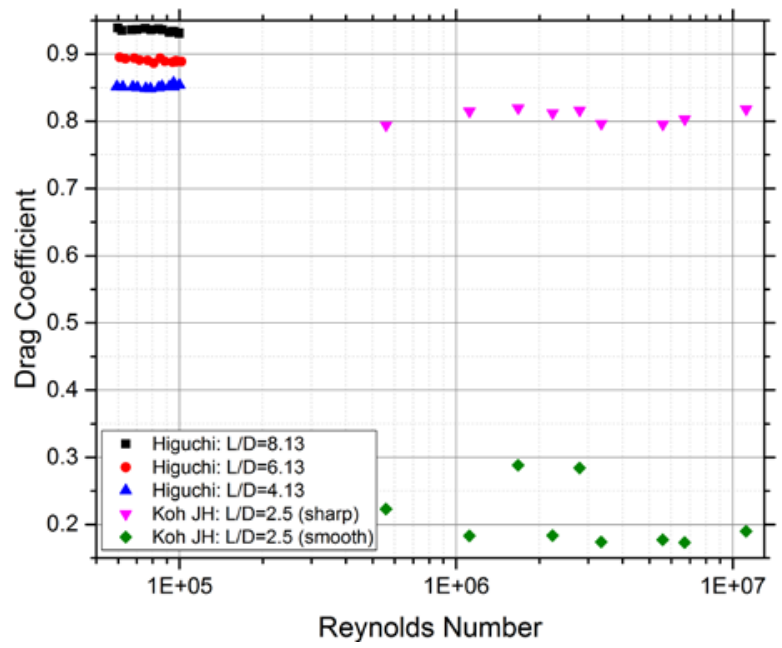

Figure 5.72 Comparison graph between experimental and simulation data for

$$
C_{D, c y l} \text { vs } \mathbf{R e}_{\text {cyl }}
$$


While there was no overlap of dataset for similar $R e_{c y l}$, the experiment and the CFD results showed minor variations with $R e_{c y l}$ and there was no quantifiable dependency for mean drag coefficient of cylinder $\left(C_{D, c y l}\right)$ on $R e_{c y l}$ as showed in Figure 5.72. In addition, having a smooth shaped cylinder reduced the $C_{D, c y l}$ significantly due to the Coandă effect.

\section{$C_{D, c y l}$ vs Fineness Ratio}

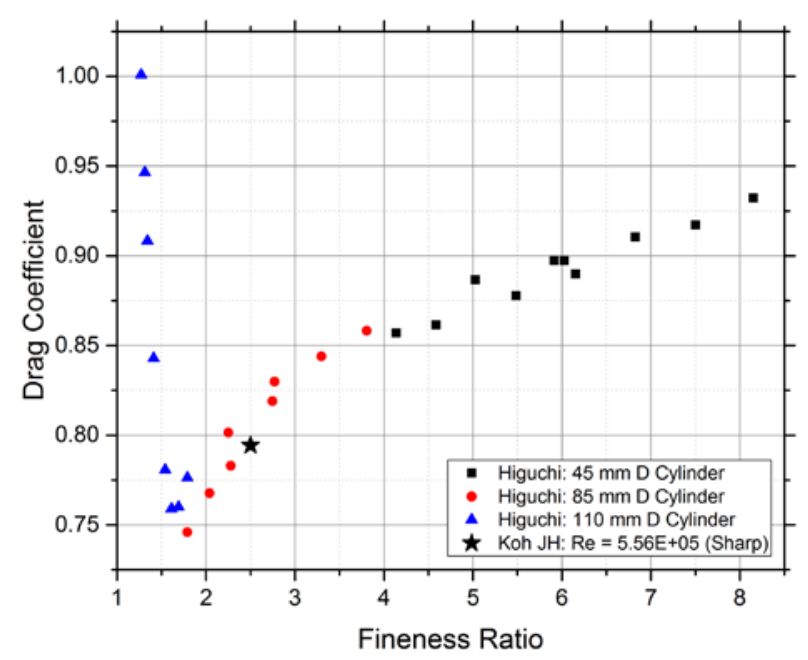

Figure 5.73 Comparison graph between experimental and simulation data for $C_{D, c y l}$ vs Fineness ratio

Figure 5.73 showed the scatter plot for $C_{D, c y l}$ vs fineness ratio. In this study, a single fineness ratio of 2.5 was used and the $C_{D, c y l}$ value simulated in CFD fitted very well with the general trend observed in the scatter plot. 


\section{$C_{p}$ vs Distance from front of cylinder}

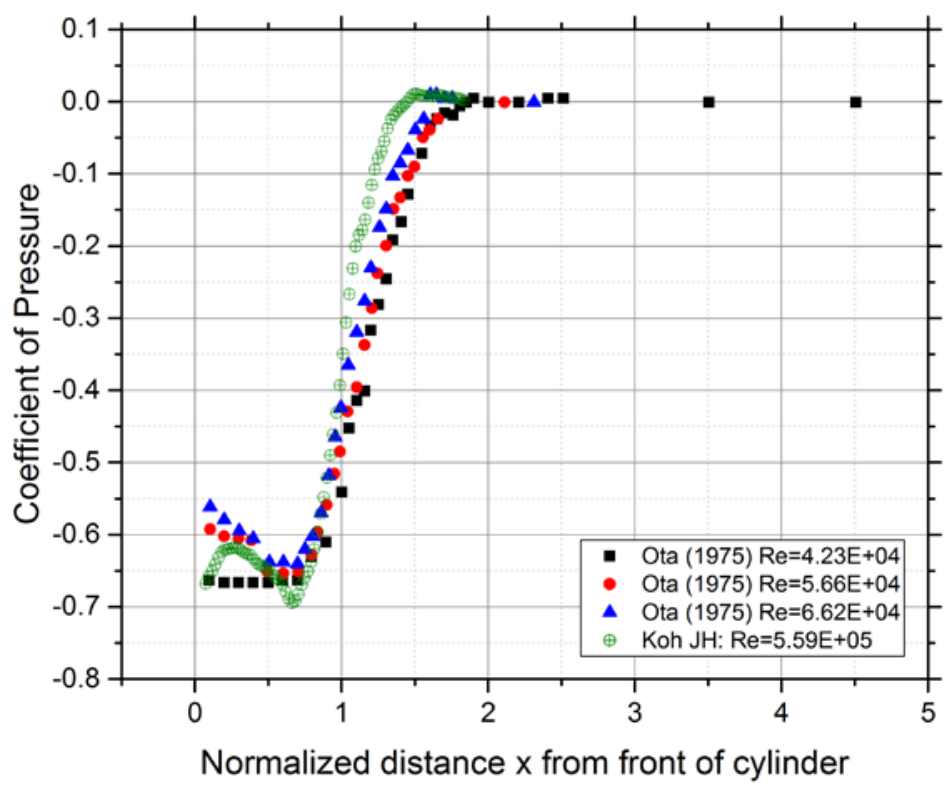

Figure 5.74 Comparison graph between experimental and simulation data for $\boldsymbol{C}_{\boldsymbol{P}}$ vs normalized distance (diameter) from leading edge of cylinder

Figure 5.74 shows the scatter plot for $C_{P}$ vs normalized distance (with diameter) from the leading edge of the cylinder. Even though the CFD results were compared with experiment results with relatively lower $R e_{c y l}$, the $C_{P}$ value followed trend of the experiment results closely along the length of the cylinder. This plot is useful in determining the reattachment length, as described in the next section. 


\section{Reattachment Length vs $\boldsymbol{R e}_{c y l}$}

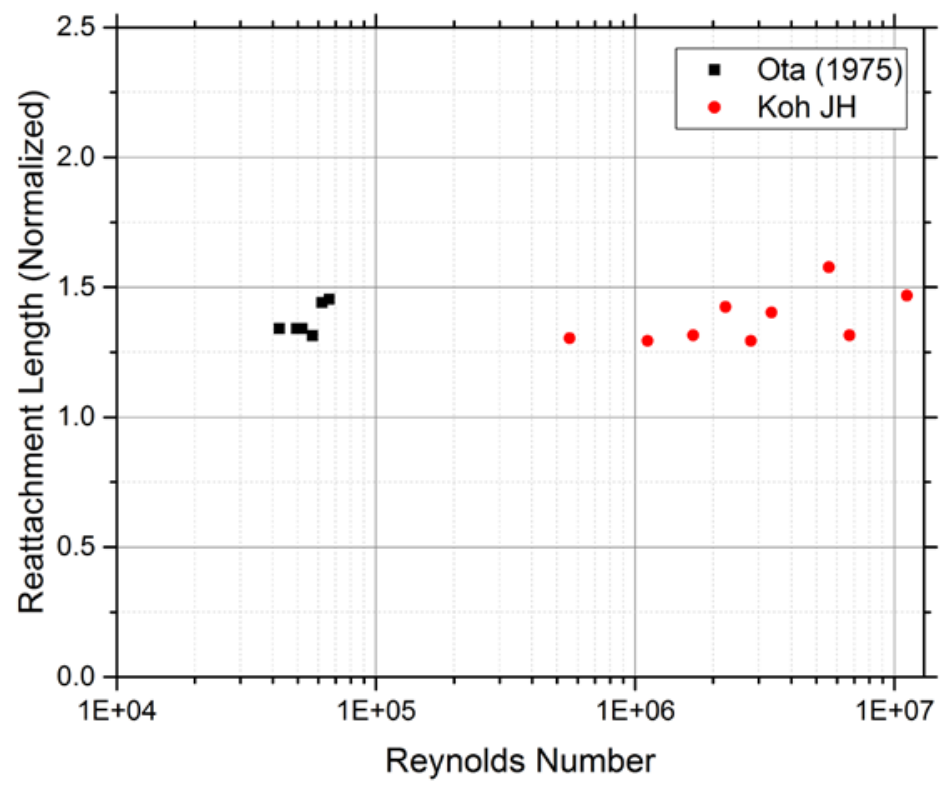

Figure 5.75 Comparison graph between experimental and simulation data for Reattachment length vs $\boldsymbol{R e}_{c y l}$

The normalized reattachment length (with diameter) in experiment study can be defined by a number of different methods. For this comparison, the reattachment length was determined by the point in which the pressure coefficient increases sharply from negative to positive value. Figure 5.75 showed the reattachment length for various $R e_{c y l}$. While there is no overlap of dataset for similar $R e_{c y l}$, the experiment and the CFD results showed minor variations with $R e_{c y l}$ and there was no quantifiable dependency for reattachment length on $R e_{c y l}$. 


\section{Velocity flow fields}

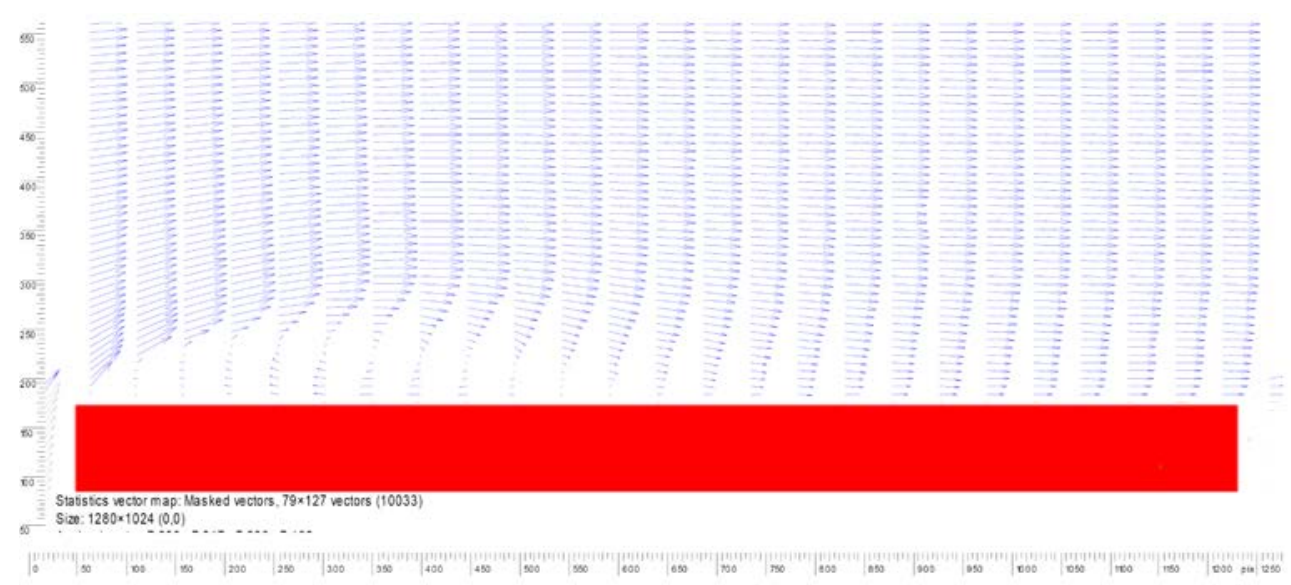

Figure 5.76 Mean velocity with fineness ratio $=3$ cylinder at $R e_{c y l}=1.00 \mathrm{E}+04$ from experiment (Hiroshi et al., 2005)

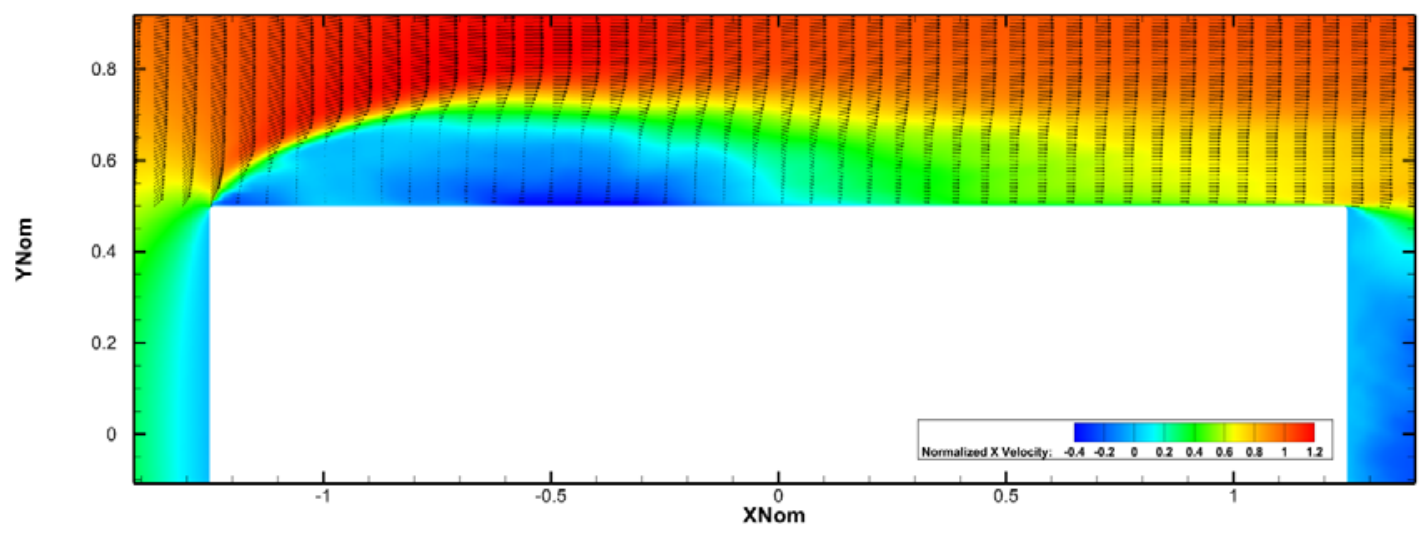

Figure 5.77 Mean velocity with fineness ratio $=2.5$ cylinder at $R e_{c y l}=5.59 \mathrm{E}+05$ from CFD simulation

Figure 5.76 shows the mean velocity flow field with fineness ratio of 3.0 at $R e_{c y l}=1.00 \mathrm{E}+04$ from experiment and Figure 5.77 shows the mean velocity flow field with fineness ratio of 2.5 at $R e_{c y l}=5.59 \mathrm{E}+05$ from experiment. From the CFD results, clear observations of separated and reattachment of shear layer, and recirculating flow in the separation bubble was noted. These observations were also in line with the experiment results.

\subsubsection{Limitations}

With the good comparative results between the experiment and CFD simulations, it gives increased confidence in the computation mesh, CFD setup and turbulence models used to model high $R e_{c y l}$ flow over cylinder. 
While no experiment comparison was done with cuboid shaped models and smooth cylinder models, it is very likely that similar computation mesh, CFD setup and turbulence models can yield results that are reasonably representative of actual flow conditions.

Although the next logical step is to develop empirical influence models, similar the process in Section 5.4.5, it is important to note that a key weakness in this study that the design is limited to a fixed fineness ratio, two different shapes and two different edge radiuses. Ideally, the user of the wind turbine simulation tool will only need to input nacelle parameters such as the dimensions and edge radius to the tool. Therefore, the analysis needs to be expanded to a larger range of nacelle design parameters before development of the nacelle influence model, which is not conducted in this study.

\subsection{Chapter Conclusion}

In this chapter, the author developed a novel meshing and modeling method to conduct computational fluid dynamics analysis efficiently for high Reynolds number flow over bluff bodies that can produce reasonable predictions of turbulent wake profile. CFD analysis was conducted for both the tower and nacelle separately. 18 test cases were generated to analyze the flow across a wind turbine tower, while 9 test cases were generated to analyze the flow across a wind turbine nacelle based on each permutation of smooth- and sharp-edged nacelles, and cylindrical or cuboid shaped nacelles.

For the tower cases, the CFD simulation and experiment results generally showed good comparison, and further comparison was made with existing tower influence models. The strength and weaknesses of existing tower influence models were noted, and a new tower influence model was proposed by the author based on the CFD simulation results.

The new semi-empirical tower influence model proposed by the author showed significant improvement over the models used in FAST and Bladed. 
The amount of flow speed up along the side of the tower is dependent on $R e$, and the negative $v_{x}$ behind the tower is also present, which better represents the tower shadow effect. In addition, the new formulation can now model the mean $v_{y}$ trends that are present due to vortex shedding in the wake of the tower. It is important to note that the new model by the author based entirely on the CFD analysis conducted, and is valid for the range of $R e_{\text {tower }}$ that is being tested for. This can be further expanded by further simulation and comparison, as future work. In addition, the new model proposed currently utilizes mean velocity adjustments, while the actual physical flow perturbations consist of vortex shedding and turbulence generated by the flow over the cylinder.

For the nacelle cases, due to the lack of experiment results for high $R e$ flow, only the CFD simulation for the sharp cylindrical nacelle was compared. The comparison data set had relatively lower $R e_{c y l}$. The CFD simulation and experiment results showed very good comparisons over various flow parameters. Three key findings were noted, (1) having a smooth shaped nacelle can significantly decrease the $C_{D, c y l}$ or $C_{D, c u b} ;(2)$ there is no significant observations that indicates that the flow characteristics is influenced by $R e_{c y l}$ or $R e_{c u b}$; (3) the good comparative results between the experiment and CFD simulations for the sharp cylindrical nacelle gives increased confidence in the computation mesh, CFD setup and turbulence models used.

It is important to note that a key weakness in the nacelle study is that the design was limited to a small number of design parameter. Therefore, the analysis needs to be expanded to a larger range of nacelle design parameters before development of the nacelle influence model. 


\section{CONCLUSION AND FUTURE WORK}

\subsection{Conclusion}

In this study, the author has presented a thorough literature review to support the motivation of this work. This helped to formulate the following objectives of this study:

- To build a reliable aero-hydro-servo-elastic FAST numerical model of the SWAY prototype downwind floating wind turbine;

- $\quad$ To verify, calibrate and validate the FAST numerical model of the SWAY prototype downwind floating wind turbine with open sea test data;

- To modify and improve the FAST numerical code based on validation analysis;

- To conduct a computational fluid dynamics (CFD) analysis to analyze the flow fields around tower and nacelle; and

- Compare and improve/develop correction models for tower and nacelle influence using CFD simulations over various wind speed, size and shapes.

The author has achieved all of the objectives except to develop the correction models for the nacelle influence.

A FAST model of the spar-type $1 / 6.5^{\text {th }}$-scale prototype SWAY system was built with design descriptions made available by SWAY AS. Different modeling strategies and assumptions were used to enable the model to work without major changes to the FAST tool. Changes were made to the FAST code to account for the wind loading on each tower element in each simulation time-step.

The FAST model was calibrated and tuned through one static equilibrium test, one roll free-decay test and one pitch free-decay test. After calibration, the comparison of the FAST simulations and experiment results matched 
with minor discrepancies because of the simplifying assumptions made in modeling the wind turbine.

To validate the model, two non-operating test cases and two operating test cases (one in Control Region II and one in Control Region III) were selected from the available data sets. From the results, it was shown that wind loading on the tower did have a significant effect on the dynamics of the wind turbine system when it was not operating in high wind speeds and a less significant effect when the system is operating, as the dominant wind loading occurs on the turbine blades. Including the wind loading on the tower increased the accuracy of the simulation results in comparison with the experimental results, particularly in the wind-loading direction.

For the blade loads, the flapwise and edgewise bending structural frequencies as well as the $1 \mathrm{P}, 2 \mathrm{P}$, and $3 \mathrm{P}$ frequencies were similar for both experiment and simulation results. However, there were significant differences in the magnitude of the loads. It is likely that the tower shadow model does not accurately simulate the tower blade loads that occurring when the blade passes behind the tower. Other possibilities include that either the units of the blade loads were incorrect or the sensor connection

Although mixed results were observed in comparing the system behavior in the experiment and FAST simulations, this study was useful in building competencies, learning and understanding the key issues and challenges in an open-sea validation study, and identifying some limitations in the modeling approach. Unlike laboratory testing, the complex nature of the environment and the inability to fully characterize its influence on the system is one of the key challenges in validating a model using open-sea data.

The author developed a novel meshing and modeling method to conduct computational fluid dynamics analysis robustly and efficiently for high Reynolds number flow over bluff bodies. Reasonable predictions of turbulent 
wake profile were achieved for different type of flows that includes, (1) flow around a circular cylinder, (2) axial flow across a 3D cylinder, (2) axial flow across a 3D cuboid.

CFD analysis was conducted for both the tower and nacelle separately. 18 test cases were generated to analyze the flow across a wind turbine tower, while 9 test cases were generated to analyze the flow across a wind turbine nacelle based on each permutation of smooth- and sharp-edged nacelles, and cylindrical or cuboid shaped nacelles.

For the tower cases, the CFD simulation and experiment results generally showed good comparison, and further comparison was made with existing tower influence models. The strength and weaknesses of existing tower influence models were noted, and a new tower influence model was proposed by the author based on the CFD simulation results.

The new semi-empirical tower influence model proposed by the author showed significant improvement over the models used in FAST and Bladed. The amount of flow speed up along the side of the tower is dependent on $R e$, and the negative $v_{x}$ behind the tower is also present, which better represents the tower shadow effect. In addition, the new formulation can now model the mean $v_{y}$ trends that are present due to vortex shedding in the wake of the tower. It is important to note that the new model by the author based entirely on the CFD analysis conducted, and is valid for the range of $R e_{\text {tower }}$ that is being tested for.

For the nacelle cases, due to the lack of experiment results for high $R e$ flow, only the CFD simulation for the sharp cylindrical nacelle was compared. The comparison data set had relatively lower $R e_{c y l}$. The CFD simulation and experiment results showed very good comparisons over various flow parameters. Three key findings were noted, (1) having a smooth shaped nacelle can significantly decrease the $C_{D, c y l}$ or $C_{D, c u b} ;(2)$ there is no significant observations that indicates that the flow characteristics is 
influenced by $R e_{c y l}$ or $R e_{c u b}$; (3) the good comparative results between the experiment and CFD simulations for the sharp cylindrical nacelle gives increased confidence in the computation mesh, CFD setup and turbulence models used.

It is important to note that a key weakness in the nacelle study is that the design was limited to a small number of design parameter. Therefore, the analysis needs to be expanded to a larger range of nacelle design parameters before development of the nacelle influence model.

Although the objective of developing a new nacelle influence model was not achieved in this study, the analysis results and limitation has been reported. CFD analysis of smooth- and sharp-edged nacelles, and cylindrical or cuboid shaped nacelles showed reasonable predictions over various flow parameters. Key findings were also noted that support in the development of nacelle influence models.

\subsection{Future Work}

\subsubsection{Study-Specific}

This study opens a number of interesting directions in which further work and research can be pursued upon.

- FAST has its limitation to representing multi-directional waves which may occur at near-shore locations since complicated wave forms may form due to reflection of the waves from the shore. Improvements to the wave modeling in FAST will be useful to develop test cases in such conditions.

- The lack of a control system model significantly limited the ability to accurately represent the turbine behavior. Increasing the model fidelity, running the SWAY study in the latest version of FAST 8 and setting up proper control algorithm in the model very likely improve the comparison simulation cases. 
- Running the validation study with other numerical tools such Bladed is advisable to check if the model performs differently across other tools, and identify potential areas of improvement.

- The improved tower influence model is only valid based on the range of Reynolds number that is being simulated in. This can be further expanded by running CFD simulations over a larger range of Reynolds number.

- The CFD simulations conducted in this study is limited to a single CFD software and turbulence modeling setup. While this study does not seek to compare performance of different software or turbulence models, running other software and models for high Reynolds number flows is useful to refine the flow characteristics, results and influence models. This will help to increase the confidence in the results and models since experiment data from such challenging flow conditions are extremely limited.

- A weakness of the flow analysis for the nacelle is that the design is limited to a fixed fineness ratio, two different shapes and two different edge radiuses. Ideally, the user of the wind turbine simulation tool will only need to input nacelle parameters such as the dimensions and edge radius to the tool. Therefore, the model can be further expanded by running simulations over a larger range of nacelle design parameters. There are further opportunities to improve on the model, such as to include turbulence and vortices effects from the tower.

- Once the independent and separate models proposed for the tower and nacelle, the next logical step will be to determine the flow conditions at the areas that are influenced by both the tower and the nacelle by introducing a blending strategy to merge both models

- As the combined model analysis only seek to develop a primitive methodology to incorporate the two different influence models, further work is required to reduce the shortcomings and increase the 
robustness of the model. This can be done by considering various combinations of nacelle and tower design parameters, and nacelle overhang length.

- Incorporation of the tower and nacelle influence model into numerical simulation tools will useful to compare the pre- and postimprovement simulation result changes.

- Comparative studies for upwind and downwind floating wind turbines with the new models will be useful to more accurately quantify the loads and dynamics differences. In particular, the ultimate/fatigue load analysis on blades and yaw bearing, blade-tower clearance, and dynamic motion of rotor-nacelle assembly will be of most interest.

\subsubsection{Others}

The following areas of future work and research are not directly relevant to this study but are critical to improving the popularity and viability of downwind offshore wind turbines:

- As discussed in Section 2.2.4, individual pitch control has been suggested for downwind turbines to reduce fatigue and loads, yawing of the system and mitigate effects of the tower wake. Further development and implementation of these advanced control algorithms in both simulation and actual downwind turbines remain a key challenge for researchers.

- An issue identified (Jamieson, 2011a) with downwind rotors is the unique situation encountered during an emergency stop of the wind turbine. For large wind turbines, the usual operating procedure in emergency braking is to pitch the blades towards fine pitch. This causes a negative angle of attack that reverses the direction of the lift and thrust of the rotor, causing the blades to deflect towards the tower. This may become more problematic if flexible blades are used. A direct solution would be to increase the capabilities of the rotor brake with correct synchronization with the blade pitch system. 
Another less-used solution would be to utilize "active stall" control, where the blade is pitched towards the stall position to limit the power (Spruce, 2004). Together with other conditions such as wind gust and fault conditions, control strategies and solutions for these unique situations would need to be studied carefully to ensure the safety in the operation of downwind wind turbines.

- As discussed in Section 2.2.7, using a downwind configuration and eliminating issues with blade to tower clearance provides designers opportunity to develop innovative tower designs that reduce cost and loads to the system. Further research and development can be conducted that are focused on designing the tower to reduce the tower shadow effect on the wind turbine blades.

- With the new design possibilities described in Section 2.2, there are plenty of opportunities for optimization of various components on the wind turbine system. For example, wind turbine blades that can be made more flexible can be re-designed and optimized for structural strength and cost. Innovative design for towers can also help to reduce fatigue and loads on the tower, and minimize the tower shadow effect. A full system design optimization of an offshore downwind wind turbine would also be very useful to incorporate all design elements, especially the substructure and foundations to reduce the cost of the system (DNV GL, 2013).

- Detailed cost analysis conducted by organizations (Fingersh, Hand, \& Laxson, 2006; Greenacre, Gross, \& Heptonstall, 2010; International Renewable Energy Agency, 2012; Tegen et al., 2013; The Crown Estate, 2012) and researchers (Ashuri, Zaaijer, Martins, van Bussel, \& van Kuik, 2014; Kaiser \& Snyder, 2013; Laura \& Vicente, 2014; Myhr et al., 2014) are available based on traditional upwind offshore turbine designs. These data and cost algorithms have also been refined with the experience gained from the wind turbine projects over the years. To quantify the potential cost savings for a downwind 
offshore wind turbine, a detailed cost analysis would need to be conducted, ideally based on an actual project. Projections of cost reduction (direct and indirect) for all wind turbine components will require extensive studies that may involve inputs from researchers, turbine manufactures and designers. When the cost analysis studies of downwind turbines are available, they will become very useful for turbine manufactures, investors and key decision makers in deciding the direction for the research and development of offshore wind turbine technology. 


\section{REFERENCES}

2-B Energy. Key Features - 2-B Energy. Retrieved 17 March 2015, 2015, from http://2benergy.com/windturbine/key-features/

Achenbach, E. (1968). Distribution of local pressure and skin friction around a circular cylinder in cross-flow up to $\mathrm{Re}=5 \times 106$. Journal of Fluid Mechanics, 34(04), 625-639. doi: doi:10.1017/S0022112068002120

Adrian, T. (2012). EP 2436924. European Patent Office.

Aerodyn Energiesystems GmbH. (2014a). Offshore Family: 6.0 MW - 8.0 MW. Büdelsdorf, Germany.

Aerodyn Energiesystems GmbH. (2014b). SCDnezzy 8.0 MW. Büdelsdorf, Germany.

Ahlström, A. (2006). Influence of wind turbine flexibility on loads and power production. Wind Energy, 9(3), 237-249. doi: http://dx.doi.org/10.1002/we.167

ANSYS. (2013). ANSYS Fluent Theory Guide. Canonsbury, PA: ANSYS Inc.

Areva. (2013). Factsheet: Trianel Windpark Borkum.

Ashuri, T. (2012). Beyond classical upscaling: integrated aeroservoelastic design and optimization of large offshore wind turbines. (Masters Dissertation), Delft University of Technology, The Netherlands.

Ashuri, T., Zaaijer, M. B., Martins, J. R. R. A., van Bussel, G. J. W., \& van Kuik, G. A. M. (2014). Multidisciplinary design optimization of offshore wind turbines for minimum levelized cost of energy. Renewable Energy, 68(0), 893-905. doi: http://dx.doi.org/10.1016/j.renene.2014.02.045

Azegami, K., Hasegawa, T., Matsunobu, T., Sakamoto, K., \& Yanagibashi, T. (2012). WO2012105034. World Intellectual Property Organization.

Azegami, K., Sakamoto, K., Yanagibashi, T., \& Matsunobu, T. (2014). United States Patent No. US20140103656.

Bak, C., Madsen, H. A., \& Johansen, J. (2001, 2001). Influence from bladetower interaction on fatigue loads and dynamics (poster). Paper presented at the European Wind Energy Conference and Exhibition 2001, Copenhagen, Denmark.

Bergami, L., Madsen, H. A., \& Rasmussen, F. (2014). A Two-Bladed Teetering Hub configuration for the DTU $10 \mathrm{MW}$ RWT: loads considerations. Paper presented at the European Wind Energy Conference \& Exhibition 2014.

Bir, G. S. (2007). User's Guide to BModes (Software for Computing Rotating Beam Coupled Modes). Golden, Colorado: National Renewable Energy Laboratory.

Blevins, R. D. (2001). Flow-induced vibration (2 ed.). Malabar, Florida: Krieger Publishing Company.

Blue H Group Technology Ltd. (2013). Blue H - Products - Phase 1. Retrieved 7 May 2013, from http://www.bluehgroup.com/product/phase1.php 
Borgen, E. (2007). United States Patent No. US7156037.

Bossanyi, E. A. (2003). Individual Blade Pitch Control for Load Reduction. Wind Energy, 6(2), 119-128. doi: http://dx.doi.org/10.1002/we.76

Bossanyi, E. A. (2005). Further load reductions with individual pitch control. Wind Energy, 8(4), 481-485. doi: http://dx.doi.org/10.1002/we.166

Bracchi, T. (2014). Downwind Rotor: Studies on yaw Stability and Design of a Suitable Thin Airfoil. (PhD Dissertation), Norwegien University of Science and Technology, Norway.

Bracchi, T., \& Krogstad, P.-A. (2012). Yaw Moments of a Three-axis Wind Turbine with Yaw Error. Paper presented at the The Twenty-second International Offshore and Polar Engineering Conference, Rhodes, Greece.

British Petroleum. (2015). BP Energy Outlook 2035: BP London, UK.

Brown, C. J., Graham, J. M. R., \& Giannakidis, G. (2000). Aerodynamic Interaction Betweeen a Turbine Blade and the tower : Computation and Experiment. Paper presented at the European Congress on Computational Methods in Applied Science and Engineering, Barcelona, Spain.

Browning, J. R., \& Goupee, A. J. (2012). Calibration and Validation of a Spar-Type Floating Offshore Wind Turbine Model using the FAST Dynamic Simulation Tool Preprint. Paper presented at the The Science of Making Torque From Wind (TORQUE), Oldenburg, Germany.

Butterfield, C. P., Musial, W., \& Jonkman, J. (2007). Overview of Offshore Wind Technology. Paper presented at the Chinese Renewable Energy Industry Association WindPower Shanghai Conference, Shanghai, China.

Butterfield, S., Musial, W., \& Jonkman, J. (2005). Engineering Challenges for Floating Offshore Wind Turbines. Paper presented at the 2005 Copenhagen Offshore Wind Conference, Copenhagen, Denmark.

C-Power. (2011). Thornton Bank Offshore Wind Farm: Annual Activity Report 2011. Oostende, Belgium.

Caduff, M., Huijbregts, M. A. J., Althaus, H.-J., Koehler, A., \& Hellweg, S. (2012). Wind Power Electricity: The Bigger the Turbine, The Greener the Electricity? Environmental Science \& Technology, 46(9), 4725-4733. doi: http://dx.doi.org/10.1021/es204108n

Cantwell, B., \& Coles, D. (1983). An experimental study of entrainment and transport in the turbulent near wake of a circular cylinder. Journal of Fluid Mechanics, 136, 321-374. doi: doi:10.1017/S0022112083002189

Carter, J. W. (2001). United States Patent No. US6327957.

Chayesteh, S. (2014). Vestas JV seeks green light to test largest ever wind turbine. Reuters. http://www.reuters.com/article/2014/07/11/vestaswind-turbine-idUSL6N0PK2S320140711

Chen, L., Ponta, F. L., \& Lago, L. I. (2011). Perspectives on innovative concepts in wind-power generation. Energy for Sustainable Development, 15(4), 398-410. doi: http://dx.doi.org/10.1016/j.esd.2011.06.006

Cheney, M. C. (1997, June 15-18 1997). Reducing Rotor Weight. Paper presented at the American Wind Energy Association WINDPOWER '97, Austin, Texas. 
China Ming Yang Wind Power Group Limited. (2014). MY Expects to Erect A 6MW Off-Shore SCD Wind Turbine Generator in Norway. http://ir.mywind.com.cn/phoenix.zhtml?c $=238508 \& \mathrm{p}=$ irol$\underline{\text { newsArticle\&ID }=1939579}$

Cokljat, D., Caridi, D., Link, G., Lechner, R., \& Menter, F. R. (2009). Embedded LES Methodology for General-Purpose CFD Solvers. Paper presented at the Turbulent Shear Flow Phenomena, 6th Int. Symp. Turbulence and Shear Flow Phenomena, Seoul, Korea.

Cordle, A., Jonkman, J., \& Hassan, G. L. G. (2011, 2011). State of the Art in Floating Wind Turbine Design Tools. Paper presented at the 21st International Offshore and Polar Engineering Conference, Maui, Hawaii.

Coulling, A. J., Goupee, A. J., Robertson, A., Jonkman, J. M., \& Dagher, H. J. (2013). Validation of a FAST semi-submersible floating wind turbine numerical model with DeepCwind test data. Journal of Renewable and Sustainable Energy, 5(2), 023116-023116. doi: $10.1063 / 1.4796197$

DanTysk Offshore Wind GmbH. (2012). DanTysk offshore wind farm: harnessing the power of the North Sea wind. Hamburg, Germany.

Davidson, L. (2011). Fluid mechanics, turbulent flow and turbulence modeling Chalmers University of Technology, Goteborg, Sweden (Nov 2011)

Davydov, B. (1961). On statistical dynamics of an incompressible turbulent fluid. Paper presented at the Soviet Physics Doklady.

De, B. A., Duffey, T., \& Picot, N. (2012). WO2012123019. World Intellectual Property Organization.

De Vries, E. (2011). Development of two-bladed offshore wind turbine Wind Stats Report (Vol. 24).

Delany, N. K., \& Sorensen, N. E. (1953). Low-speed drag of cylinders of various shapes. Moffett Field, CA, United States: National Advisory Committee for Aeronautics.

Det Norske Veritas AS. (2014). Design of Offshore Wind Turbine Structures. Høvik, Norway.

Deutsche Offshore-Testfeld und Infrastruktur GmbH \& Co. KG (DOTI). (2010). The Building of an Offshore Wind Farm. Oldenburg, Germany.

DNV GL. (2013). Project FORCE - Offshore Wind Cost Reduction through Integrated Design: Technical Report.

DONG energy. (2008). The Construction of Nysted Offshore Wind Farm. Nysted, Denmark.

DONG energy. (2013). Anholt Offshore Wind Farm. Federicia, Denmark.

DONG energy. (2015). Horns Rev 2: One of the World's Largest Offshore Wind Farms. Fredericia, Denmark.

Drake, D. G. (2005). United States Patent No. US6979175.

Driscoll, F., Jonkman, J., Robertson, A., Sirnivas, S., Skaare, B., \& Nielsen, F. G. (2016). Validation of a FAST Model of the Statoil-Hywind Demo Floating Wind Turbine. Paper presented at the 13th Deep Sea Offshore Wind R\&D Conference, Trondheim, Norway. 
Du, Z., \& Selig, M. S. (1998). A 3-D stall-delay model for horizontal axis wind turbine performance prediction. Paper presented at the 36th AIAA Aerospace Sciences Meeting and Exhibit, Reno, Nevada.

Edzard, H. (1999). Germany Patent No. DE29912737.

Eggenspieler, G. (2011). Turbulence modeling. ANSYS Inc.

Eggers, A. J., \& Digumarthi, R. V. (1992, 1992). Approximate Scaling of Rotational Effects on Mean Aerodynamic Moments and Power Generated by CER Blades Operating in Deep-Stalled Flow. Paper presented at the 11th ASME Wind Energy Symposium, Houston, Texas.

Engels, W., Obdam, T., \& Savenije, F. (2009). Current developments in wind - 2009: Energy Research Centre at the Netherlands.

Engineering Sciences Data Unit. (1986). ESDU 80025 Mean forces, pressures and flow field velocities for circular cylindrical structures: single cylinder with two-dimensional flow: Engineering Sciences Data Unit.

European Committee for Standardization. (2005). EN 1991-1-4:2005 Eurocode 1. Actions on structures. General actions. Wind actions.

European Wind Energy Association. (2011). UpWind: Design Limits and Solutions for Very Large Wind Turbines.

European Wind Energy Association. (2013). The European Wind InitiativeWind Power Research and Development to 2020.

Ferziger, J. H., \& Peric, M. (2012). Computational methods for fluid dynamics: Springer Science \& Business Media.

Fingersh, L. J., Hand, M. M., \& Laxson, A. S. (2006). Wind turbine design cost and scaling model.

Foster, M. (2015). Hitachi to supply 5MW turbine to Murakami project. Windpower

Offshore. http://www.windpoweroffshore.com/article/1332841/hitachi-supply-5mwturbine-murakami-project

Frau, E., Kress, C., Chokani, N., \& Abhari, R. S. (2015). Comparison of Performance and Unsteady Loads of Multimegawatt Downwind and Upwind Turbines. Journal of Solar Energy Engineering, 137(4), 041004041004. doi: 10.1115/1.4030314

Fukushima Offshore Wind Consortium. (2013). Fukushima Floating Offshore Wind Farm Demonstration Project (Fukushima FORWARD). Tokyo, Japan.

Fukushima Offshore Wind Consortium. (2014). Fukushima Floating Offshore Wind Farm Demonstration Project (Fukushima FORWARD) Construction of Phase I -.

Gamesa Corporación Tecnológica. (2014). Ending of the Azimut project that will enable the development of a 15 MW offshore wind turbine in 2020 . http://www.gamesacorp.com/en/communication/news/ending-of-theazimut-project-that-will-enable-the-development-of-a-15-mw-offshorewind-turbine-in-2020.html?idCategoria $=60$

Garrad Hassan \& Partners Ltd. (2013). Bladed Multibody dynamics V4.5 Theory Manual. Bristol: Garrad Hassan \& Partners Ltd. 
GE Wind Energy LLC. (2006). Advanced Wind Turbine Program Next Generation Turbine Development Project: June 17, 1997--April 30, 2005. Golden, CO: National Renewable Energy Laboratory.

Glasgow, J. C., Miller, D. R., \& Corrigan, R. D. (1981). Comparison of upwind and downwind rotor operation of the DOE/NASA 100-kW MOD$O$ wind turbine. Paper presented at the Second DOE/NASA Wind Turbine Dynamics Workshop, Cleveland, Ohio. http://adsabs.harvard.edu/abs/1981wtd..nasa..225G

Global Tech I Offshore Wind GmbH. (2014). Pioneering performance in the North Sea: The offshore wind farm Global Tech I. Hamburg, Germany.

Global Wind Energy Council. (2014). Global Wind Statistics 2014. Brussels, Belgium.

Görtler, H. (1942). Berechnung von Aufgaben der freien Turbulenz auf Grund eines neuen Näherungsansatzes. ZAMM - Journal of Applied Mathematics and Mechanics / Zeitschrift für Angewandte Mathematik und Mechanik, 22(5), 244-254. doi: 10.1002/zamm.19420220503

Goupee, A. J., Koo, B. J., Kimball, R. W., Lambrakos, K. F., \& Dagher, H. J. (2014). Experimental Comparison of Three Floating Wind Turbine Concepts. Journal of Offshore Mechanics and Arctic Engineering, 136(2), 020906-020906. doi: 10.1115/1.4025804

Graham, J., \& Brown, C. (1999). Aerodynamics of a horizontal axis rotor interacting with the tower. Paper presented at the 1999 European Wind Energy Conference, Nice, France.

Greenacre, P., Gross, R., \& Heptonstall, P. (2010). Great Expectations: The cost of offshore wind in UK waters-.

Griffith, D. T., \& Ashwill, T. D. (2011). The Sandia 100-meter all-glass baseline wind turbine blade: SNL100-00.

Hanjalic, K. (1970). Two-dimensional asymmetric turbulent flow in ducts. $\mathrm{Ph}$. D. Thesis, University of London.

Hansen, C., \& Schoenefeldt, D. (2007). Germany Patent No. DE 10061916.

Harlow, F. H., \& Nakayama, P. I. (1968). Transport of turbulence energy decay rate: Los Alamos Scientific Lab., N. Mex.

Henderson, A. R., Zaaijer, M. B., Bulder, B., Pierik, J., Huijsmans, R., van Hees, M., . . Wolf, M. J. (2004). Floating windfarms for shallow offshore sites. Paper presented at the 14th International Offshore and Polar Engineering Conference, Toulon, France.

Henkes, R. A. W. M. (1996). Comparison of turbulence models for attached boundary layers relevant to aeronautics. Applied Scientific Research, 57(1), 43-65. doi: 10.1007/bf02528763

Higuchi, H., van Langen, P., Sawada, H., \& Tinney, C. E. (2006). Axial flow over a blunt circular cylinder with and without shear layer reattachment. Journal of Fluids and Structures, 22(6-7), 949-959. doi: http://dx.doi.org/10.1016/j.jfluidstructs.2006.04.020

Hiroshi, H., Hideo, S., \& Hiroyuki, K. (2006). Separated Flow Field over Blunt Circular Cylinder Suspended Magnetically in Free-Stream Direction 36th AIAA Fluid Dynamics Conference and Exhibit. American Institute of Aeronautics and Astronautics. 
Hiroshi, H., Hideo, S., \& Pieter van, L. (2005). Flow over a Magnetically Suspended Cylinder in an Axial Free Stream 43rd AIAA Aerospace Sciences Meeting and Exhibit: American Institute of Aeronautics and Astronautics.

Hitachi Ltd. (2013). Construction of Demonstration Prototype Of 5-MW Offshore Wind Turbine with Downwind Rotor. http://www.hitachi.com/New/cnews/131126b.html

Holmas, T. (2010a). Design of SWAY Alu Tower. Sandsli, Norway: Virtual Prototyping AS.

Holmas, T. (2010b). SWAY Tower Dimensions. Rådal ,Norway: Virtual Prototyping AS.

Holmes, J. D. (2007). Wind loading of structures (2nd ed.). London: Taylor $\&$ Francis.

Hoogedoorn, E., Jacobs, G. B., \& Beyene, A. (2010). Aero-elastic behavior of a flexible blade for wind turbine application: A 2D computational study. Energy, 35(2), 778-785. http://dx.doi.org/10.1016/j.energy.2009.08.030

Hubbard, H. H., Grosveld, F. W., \& Shepherd, K. P. (1983). Noise characteristics of large wind turbine generators. Noise Control Engineering Journal.

Hubbard, H. H., \& Shepherd, K. P. (1982). Noise Measurements for Single and Multiple Operation of $50 \mathrm{~kW}$ Wind Turbine Generators. United States: National Aeronautics and Space Administration.

Hubbard, H. H., \& Shepherd, K. P. (1990). Wind Turbine Acoustics: National Aeronautical and Space Administration.

Inamura, S., Shigenaga, E. Y., \& Kiyoki, S. (2013). Development of 2-MW Downwind Wind Power Generation System Hitachi Review (Vol. 62, pp. $60)$.

International Electrotechnical Commission. (2009). Wind Turbines-Part 3: Design requirements for offshore wind turbines.

International Electrotechnical Commission. (2014). Wind Turbines-Part 1: Design requirements (3.1 ed.).

International Energy Agency. (2013). Technology Roadmap: Wind Energy 2013 Edition. Paris, France.

International Energy Agency. (2014a). Key World Energy Statistics 2014: International Energy Agency.

International Energy Agency. (2014b). World Energy Outlook 2014: International Energy Agency.

International Organization for Standardization. (2009). ISO 4354:2009 Wind actions on structures (pp. 68).

International Renewable Energy Agency. (2012). Renewable Energy Technologies: Cost Analysis Series Wind Power. Abu Dhabi, United Arab Emirates.

Ishida, S., Kokubun, K., Nimura, T., Utsunomiya, T., Sato, I., \& Yoshida, S. (2013). At-sea experiment of a hybrid spar type offshore wind turbine. Paper presented at the ASME 2013 32nd International Conference on Ocean, Offshore and Arctic Engineering. 
James, W. D., Paris, S. W., \& Malcolm, G. N. (1980). Study of Viscous Crossflow Effects on Circular Cylinders at High Reynolds Numbers. AIAA Journal, 18(9), 1066-1072. doi: 10.2514/3.50855

Jamieson, P. (2009). Light-Weight, High-Speed Rotors for Offshore. Paper presented at the European Offshore Wind Conference and Exhibition 2009, Stockholm, Sweden. http://scholar.google.com/scholar?hl=en\&btnG=Search\&q=intitle:LightWeight,+ High-Speed + Rotors + for + Offshore $\# 0$

Jamieson, P. (2011a). Innovation in Wind Turbine Design: Chichester, West Sussex.

Jamieson, P. (2011b). Offshore Wind Turbines Innovation in Wind Turbine Design (pp. 131-142): John Wiley \& Sons, Ltd.

Janajreh, I., Qudaih, R., Talab, I., \& Ghenai, C. (2010). Aerodynamic flow simulation of wind turbine: Downwind versus upwind configuration. Energy Conversion and Management, 51(8), 1656-1663. doi: http://dx.doi.org/10.1016/j.enconman.2009.12.013

Janajreh, I., Talab, I., \& Macpherson, J. (2010). Numerical Simulation of Tower Rotor Interaction for Downwind Wind Turbine. Modelling and Simulation in Engineering, 2010, 1-11. doi: http://dx.doi.org/10.1155/2010/860814

Jin, K. K., Ghulam, M., Kim, J. H., Ha, S. K., López, B. M., \& Gorostidi, A. (2009). Life Prediction of Wind Turbine Blades. Paper presented at the 17th International Conference on Composite Materials, Edinburgh, UK.

John, F. M., David, M., \& Daniel, S. (2013). Creep/Fatigue Behavior of Resin Infused Biaxial Glass Fabric Laminates. Paper presented at the 54th AIAA/ASME/ASCE/AHS/ASC Structures, Structural Dynamics, and Materials Conference, Boston, Massachusetts.

Johnson, G. L. (2001). Wind energy systems

Jones Jr, G. W., Cincotta, J. J., \& Walker, R. W. (1969). Aerodynamic forces on a stationary and oscillating circular cylinder at high Reynolds numbers. Moffett Field, CA, United States: National Advisory Committee for Aeronautics.

Jones, W. P., \& Launder, B. E. (1972). The prediction of laminarization with a two-equation model of turbulence. International journal of heat and mass transfer, 15(2), 301-314.

Jonkman, B. J., \& Jonkman, J. (2013). Addendum to the User's Guides for FAST, A2AD, and AeroDyn. Golden, Colorado: National Renewable Energy Laboratory.

Jonkman, B. J., \& Jonkman, J. (2016). Guide to Changes in FAST v8: National Renewable Energy Laboratory.

Jonkman, B. J., \& Kilcher, L. (2012). TurbSim User's Guide: Version 1.06.00. Golden, CO: National Renewable Energy Laboratory.

Jonkman, J. (2007). Dynamics Modeling and Loads Analysis of an Offshore Floating Wind Turbine. (Ph.D. dissertation), University of Colorado, United States. 
Jonkman, J. (2009). Dynamics of offshore floating wind turbines-model development and verification. Wind Energy(June), 459-492. doi: $10.1002 /$ we

Jonkman, J. (2014). FAST Theory Manual. Golden, Colorado (to be published) National Renewable Energy Laboratory.

Jonkman, J., \& Buhl, M. L. (2007, 2007). Loads Analysis of a Floating Offshore Wind Turbine Using Fully Coupled Simulation. Paper presented at the WindPower 2007 Conference \& Exhibition, Los Angeles, California.

Jonkman, J., \& Buhl, M. L. J. (2005). FAST User's Guide. Golden, Colorado: National Renewable Energy Laboratory.

Jonkman, J., \& Musial, W. (2010). Offshore Code Comparison Collaboration ( OC3 ) for IEA Task 23 Offshore Wind Technology and Deployment. Golden, Colorado: National Renewable Energy Laboratory.

Kaiser, M. J., \& Snyder, B. F. (2013). Modeling offshore wind installation costs on the U.S. Outer Continental Shelf. Renewable Energy, 50(0), 676691. doi: http://dx.doi.org/10.1016/j.renene.2012.07.042

Kaldellis, J. K., \& Zafirakis, D. P. (2012). Trends, Prospects, and R\&D Directions in Wind Turbine Technology. In A. Sayigh (Ed.), Comprehensive Renewable Energy (pp. 671-724). Oxford: Elsevier.

Kapsali, M., \& Kaldellis, J. K. (2012). Offshore Wind Power Basics. In A. Sayigh (Ed.), Comprehensive Renewable Energy (pp. 431-468). Oxford: Elsevier.

Kelley, N. D., McKenna, H. E., \& Hemphill, R. R. (1985). Acoustic Noise Associated with the MOD-1 Wind Turbine: Its Source, Impact, and Control. Golden, Colorado: US Department of Energy.

Kittilä, E.-M. (2012). Implementation and Evaluation of Wind Turbine Control Concepts. (Masters Dissertation), Tampere University of Technology, Germany.

Koh, J. H., Robertson, A., Jonkman, J., Driscoll, F., \& Ng, E. Y. K. (2013). Building and Calibration of a FAST Model of the SWAY Prototype Floating Wind Turbine. Paper presented at the International Conference on Renewable Energy Research and Applications (ICRERA) 2013, Madrid, Spain.

Koh, J. H., Robertson, A., Jonkman, J., Driscoll, F., \& Ng, E. Y. K. (2015). Validation of SWAY Wind Turbine Response in FAST, with a Focus on the Influence of Tower Wind Loads. Paper presented at the The Twentyfifth International Offshore and Polar Engineering Conference.

Kolmogorov, A. N. (1941). The local Structure of turbulence in incompressible viscous fluid for very large Reynolds numbers /In Russian]. Paper presented at the Dokl. Akad. Nauk SSSR.

Kolmogorov, A. N. (1942). Equations of Turbulent Motion of an Incompressible Fluid. Izvestia Academy of Sciences, USSR; Physics, $6(1$ and 2).

Koo, B. J., Goupee, A. J., Kimball, R. W., \& Lambrakos, K. F. (2014). Model Tests for a Floating Wind Turbine on Three Different Floaters. 
Journal of Offshore Mechanics and Arctic Engineering, 136(2), 020907020907. doi: 10.1115/1.4024711

Kress, C., Chokani, N., \& Abhari, R. S. (2015). Downwind wind turbine yaw stability and performance. Renewable Energy, 83(0), 1157-1165. doi: http://dx.doi.org/10.1016/j.renene.2015.05.040

Kress, C., Chokani, N., Abhari, R. S., Hashimoto, T., Watanabe, M., Sano, T., \& Saeki, M. (2015). Economical and operational merits of offshore multi-megawatt downwind turbines. Paper presented at the EWEA Offshore 2015, Copenhagen, Denmark.

Kristiansen, L. M. R. (2014). LM Wind Power to Develop Flexible Blade Concept that Reduces Cost of Energy by up to $10 \%$. http://www.lmwindpower.com/Media/Media-Kit/Press-

Releases/2014/03/LM-Wind-Power-to-develop-flexible-blade-conceptthat-reduces-cost-of-energy-by-up-to-10-percent

Laino, D. J. (2001). Analysis of wind turbine rotor tower clearance. Paper presented at the 20th 2001 ASME Wind Energy Symposium, Reno, Nevada.

Laino, D. J., \& Hansen, A. C. (2001). User's Guide to the Computer Software Routines AeroDyn Interface for ADAMSB: Windward Engineering.

Laino, D. J., \& Hansen, A. C. (2002). Aerodyn user's guide: National Renewable Energy Laboratory.

Larsen, T. J., Madsen, H. A., \& Thomsen, K. (2005). Active load reduction using individual pitch, based on local blade flow measurements. Wind Energy, 8(1), 67-80. doi: http://dx.doi.org/10.1002/we.141

Launder, B. E., \& Sharma, B. I. (1974). Application of the energydissipation model of turbulence to the calculation of flow near a spinning disc. Letters in heat and mass transfer, 1(2), 131-137.

Launder, B. E., \& Spalding, D. B. (1972). Mathematical Models of Turbulence. London: Academic Press.

Laura, C.-S., \& Vicente, D.-C. (2014). Life-cycle cost analysis of floating offshore wind farms. Renewable Energy, $66(0)$, 41-48. doi: http://dx.doi.org/10.1016/j.renene.2013.12.002

Lauritsen, P. (2004). United States Patent No. US20040103655.

Le Gourieres, D. (2014). Wind power plants: theory and design: Elsevier.

Lefranc, M. (2011). United States Patent No. US20110006539.

Leung, D. Y. C., \& Yang, Y. (2012). Wind energy development and its environmental impact: A review. Renewable and Sustainable Energy Reviews, 16(1), 1031-1039. doi: http://dx.doi.org/10.1016/j.rser.2011.09.024

Li, W., Sun, H. L., Xing, Z. X., \& Chen, L. (2012). The Study of Individual Pitch Control on Reducing The Load Fluctuation of Tower Shadow. Advanced Materials Research, 347, 2260-2267.

Liu, K. S. (2012). United States Patent No. US20120269628.

London Array Limited. (2013). London Array: The world's largest offshore wind farm. London, UK. 
Long, H., \& Moe, G. (2012). Preliminary Design of Bottom-Fixed Lattice Offshore Wind Turbine Towers in the Fatigue Limit State by the Frequency Domain Method. Journal of Offshore Mechanics and Arctic Engineering, 134(3), 031902-031902. doi: http://dx.doi.org/10.1115/1.4005200

Loth, E., \& Selig, M. S. (2014). WO2014205348. World Intellectual Property Organization.

Loth, E., Steele, A., Ichter, B., Selig, M. S., \& Moriarty, P. (2012). Segmented Ultralight Pre-Aligned Rotor for Extreme-Scale Wind Turbines. Paper presented at the 50th AIAA Aerospace Sciences Meeting including the New Horizons Forum and Aerospace Exposition, Nashville, Tennessee.

Madsen, H. A., Johansen, J., Sørensen, N. N., Larsen, G. C., \& Hansen, M. H. (2007). Simulation of Low Frequency Noise from a Downwind Wind Turbine Rotor. Paper presented at the 45th AIAA Aerospace Sciences Meeting and Exhibit, Reno, Nevada.

Manwell, J. F., McGowan, J. G., \& Rogers, A. L. (2009). Wind Energy Explained. Chichester, UK: John Wiley \& Sons, Ltd.

Mathey, F., Cokljat, D., Bertoglio, J.-P., \& Sergent, E. (2006). Specification of LES inlet boundary condition using vortex method. Progress in Computational Fluid Dynamics, 6, 58-67.

Matsunobu, T., Hasegawa, T., Isogawa, M., Sato, K., Futami, M., \& Kato, H. (2009). Development of 2-MW Downwind Turbine Tailored to Japanese Conditions Hitachi Review (Vol. 58, pp. 213).

Matsunobu, T., Saeki, M., Hasegawa, T., \& Kato, H. (2012). United States Patent No. US20120057976.

McNerney, G. M., van Dam, C. P., \& Yen-Nakafuji, D. T. (2003). BladeWake Interaction Noise for Turbines With Downwind Rotors. Journal of Solar Energy Engineering, 125(4), 497-497. doi: http://dx.doi.org/10.1115/1.1627830

Menter, F. R. (1994). Two-equation eddy-viscosity turbulence models for engineering applications. AIAA Journal, 32(8), 1598-1605. doi: $10.2514 / 3.12149$

Miller, B. (2014). 2-B Energy to launch two-blade $6 \mathrm{MW}$ offshore turbine in 2015.

Windpower

Offshore. http://www.windpoweroffshore.com/article/1226740/2-b-energy-launchtwo-blade-6mw-offshore-turbine-2015

Mingyang Wind Power. (2012). New Generation Super Compact Drive Wind Energy Convertor: From onshore to offshore, multi-megawatt wind turbine. Guangdong, China.

Mizuno, E. (2014). Overview of wind energy policy and development in Japan. Renewable and Sustainable Energy Reviews, 40(0), 999-1018. doi: http://dx.doi.org/10.1016/j.rser.2014.07.184

Moe, G. (2007). What is the optimum size for a wind turbine? Paper presented at the ASME 2007 26th International Conference on Offshore Mechanics and Arctic Engineering. 
Mohseni, K., Kosović, B., Shkoller, S., \& Marsden, J. E. (2003). Numerical simulations of the Lagrangian averaged Navier-Stokes equations for homogeneous isotropic turbulence. Physics of Fluids, 15(2), 524-544. doi: doi:http://dx.doi.org/10.1063/1.1533069

Moriarty, P., \& Hansen, A. C. (2005). AeroDyn Theory Manual.

Munduate, X., Coton, F. N., \& Galbraith, R. A. M. (2004). An Investigation of the Aerodynamic Response of a Wind Turbine Blade to Tower Shadow. Journal of Solar Energy Engineering, 126(4), 1034-1034. doi: http://dx.doi.org/10.1115/1.1765683

Musial, W., \& Butterfield, S. (2004, 2004). Future for Offshore Wind Energy in the United States. Paper presented at the EnergyOcean 2004, Palm Beach, Florida.

Musial, W., Butterfield, S., \& Ram, B. (2006). Energy from Offshore Wind. Paper presented at the Offshore Technology Conference 2006, Houston, Texas.

Musial, W., \& Ram, B. (2010). Large-scale offshore wind power in the United States: Assessment of opportunities and barriers. Golden, Colorado: National Renewable Energy Laboratory.

Muskulus, M. (2012). The Full-height Lattice Tower Concept. Energy Procedia, 24(0), 371-377. doi: http://dx.doi.org/10.1016/j.egypro.2012.06.120

Myhr, A., Bjerkseter, C., Agotnes, A., \& Nygaard, T. A. (2014). Levelised cost of energy for offshore floating wind turbines in a life cycle perspective. Renewable Energy, 66(0), 714-728. doi: http://dx.doi.org/10.1016/j.renene.2014.01.017

Namik, H., \& Stol, K. (2010). Individual blade pitch control of floating offshore wind turbines. Wind Energy, 13(1), 74-85. doi: http://dx.doi.org/10.1002/we.332

Navalkar, S. T., Wingerden, J. W. v., \& Kuik, G. A. M. v. (2014). Individual blade pitch for yaw control. Journal of Physics: Conference Series, 524(1), 012057.

Nichols, J. ( 2011). SWAY 1:6 Scale Tower Wind Turbine Controller Pitch System Interface to Turbine Controller. Glasgow, Scotland: Garrad Hassan \& Partners Ltd.

Nicoud, F., \& Ducros, F. (1999). Subgrid-scale stress modelling based on the square of the velocity gradient tensor. Flow, Turbulence and Combustion, $62(3), 183-200$.

Nygaard, T. A., \& Grorud, C. (2010). US20100202881.

O'Connor, K., Loth, E., \& Selig, M. S. (2013). Design of a 2-D Fairing for a Wind Turbine Tower. Paper presented at the 31st AIAA Applied Aerodynamics Conference, San Diego, California.

O'Connor, K., Loth, E., \& Selig, M. S. (2015). Experiments on Fairing Design for a Wind Turbine Tower. Paper presented at the 33rd Wind Energy Symposium, Kissimmee, Florida.

O'Connor, K. (2014). Development and Testing of a Fairing for a Wind Turbine Tower. (Masters Dissertation), University of Virginia, United States. 
Ota, T. (1975). An Axisymmetric Separated and Reattached Flow on a Longitudinal Blunt Circular Cylinder. Journal of Applied Mechanics, 42(2), 311-315. doi: 10.1115/1.3423573

Parkinson, G. V., \& Jandali, T. (1970). A wake source model for bluff body potential flow. Journal of Fluid Mechanics, 40(03), 577-594. doi: doi:10.1017/S0022112070000320

Perveen, R., Kishor, N., \& Mohanty, S. R. (2014). Off-shore wind farm development: Present status and challenges. Renewable and Sustainable $\begin{array}{llll}\text { Energy } & \text { Reviews, } & \text { 29(0), } & \text { 780-792. }\end{array}$ http://dx.doi.org/10.1016/j.rser.2013.08.108

Pope, S. B. (2000). Turbulent Flows: Cambridge University Press.

Popko, W., Vorpahl, F., Zuga, A., Kohlmeier, M., Jonkman, J., Robertson, A., . . . Okstad, K. M. (2012). Offshore Code Comparison Collaboration Continuation (OC4), Phase I-Results of Coupled Simulations of an Offshore Wind Turbine with Jacket Support Structure. Paper presented at the 22nd International Society of Offshore and Polar Engineers Conference, Rhodes, Greece.

Popko, W., Vorpahl, F., Zuga, A., Kohlmeier, M., Jonkman, J., Robertson, A., . . . Okstad, K. M. (2014). Offshore Code Comparison Collaboration Continuation (OC4), Phase I-Results of Coupled Simulations of an Offshore Wind Turbine with Jacket Support Structure. Journal of Ocean and Wind Energy, 1(1), 1-11.

Powles, S. R. J. (1983). The effects of tower shadow on the dynamics of a horizontal-axis wind turbine. Wind Engineering, 7, 26-42.

Prowell, I., Stewart, G. M., \& Goupee, A. J. (2013). Numerical Prediction of Experimentally Observed Behavior of a Scale Model of an Offshore Wind Turbine Supported by a Tension-Leg Platform Preprint. Paper presented at the 2013 Offshore Technology Conference, Houston, Texas.

Publicover, B. (2014). Hitachi finishes installing 5MW turbine in Japan after repeated delays. Recharge News. http://www.rechargenews.com/wind/1395182/hitachi-finishes-installing5mw-turbine-in-japan-after-repeated-delays

Reichardt, H. (1942). Gesetzmässigkeiten der freien Turbulenz. Berlin.

Reiso, M. (2010). Root flapwise moment on downwind and upwind rotors with truss and tubular towers. Paper presented at the 6th PhD Seminar on Wind Energy in Europe, Trondheim, Norway.

Reiso, M., Hagen, T. R., \& Muskulus, M. (2013). A calibration method for downwind wake models accounting for the unsteady behaviour of the wind turbine tower shadow behind monopile and truss towers. Journal of Wind Engineering and Industrial Aerodynamics, 121(0), 29-38. doi: http://dx.doi.org/10.1016/j.jweia.2013.07.016

Reiso, M., \& Muskulus, M. (2013). The simultaneous effect of a fairing tower and increased blade flexibility on a downwind mounted rotor. Journal of Renewable and Sustainable Energy, 5(3). doi: http://dx.doi.org/10.1063/1.4803749

Reiso, M., Muskulus, M., \& Moe, G. (2011). Tower shadow-Experiment comparing wake behind tubular and truss towers. Paper presented at the 
22nd International Offshore and Polar Engineering Conference, Maui, Hawaii.

Ren, N., Li, Y., \& Ou, J. (2012). The wind-wave tunnel test of a tension-leg platform type floating offshore wind turbine. Journal of Renewable and Sustainable Energy, 4(6), 063117-063117. doi: 10.1063/1.4767928

reNEWS. (2014). 2-B Energy bags $€ 26.5 \mathrm{~m}$ war chest. http://renews.biz/63037/decc-pours-4m-into-offshore-rd/

Richardson, L. F. (1922). Weather prediction by numerical process: Cambridge University Press.

Robertson, A. (2015). Introduction to the OC5 Project, an IEA Task Focused on Validating Offshore Wind Modeling Tools. Paper presented at the 12th Deep Sea Offshore Wind R\&D Conference, Trondheim, Norway.

Robertson, A., Jonkman, J., Musial, W., Popko, W., \& Vorpahl, F. (2014). Final Technical Report: IEA Wind Task 30 Offshore Code Comparison Collaboration Continued: IEA Wind.

Robertson, A., Jonkman, J., Musial, W., Vorpahl, F., \& Popko, W. (2013). Offshore Code Comparison Collaboration, Continuation: Phase II Results of a Floating Semisubmersible Wind System. Paper presented at the EWEA Offshore 2013, Frankfurt, Germany.

Robertson, A., Jonkman, J., Vorpahl, F., Popko, W., Qvist, J., Frøyd, L., . . - Soares, C. G. (2014). Offshore code comparison collaboration continuation within IEA Wind Task 30: Phase II results regarding a floating semisubmersible wind system. Paper presented at the ASME 2014 33rd International Conference on Ocean, Offshore and Arctic Engineering.

Robertson, A., Wendt, F. F., Jonkman, J., Popko, W., Vorpahl, F., Stansberg, C. T., . . . de Vaal, J. B. (2015). OC5 Project Phase I: Validation of Hydrodynamic Loading on a Fixed Cylinder. Paper presented at the The Twenty-fifth International Offshore and Polar Engineering Conference.

Roddier, D., Cermelli, C., Aubault, A., \& Weinstein, A. (2010). WindFloat: A floating foundation for offshore wind turbines. Journal of Renewable and Sustainable Energy, 2(3), 033104-033104. doi: http://dx.doi.org/10.1063/1.3435339

Rodi, W., \& Scheuerer, G. (1986). Scrutinizing the k- $\varepsilon$ Turbulence Model Under Adverse Pressure Gradient Conditions. Journal of Fluids Engineering, 108(2), 174-179. doi: 10.1115/1.3242559

Roshko, A. (1961). Experiments on the flow past a circular cylinder at very high Reynolds number. Journal of Fluid Mechanics, 10(03), 345-356. doi: doi:10.1017/S0022112061000950

Saeki, M., Sano, A., Kato, H., Owada, M., \& Yoshida, S. (2014). Concept of The HITACHI 5MW Offshore Downwind Turbine (Poster). Paper presented at the European Wind Energy Conference and Exhibition 2014, Barcelona, Spain.

Saeki, M., Tobinaga, I., Sugino, J., \& Shiraishi, T. (2014). Development of 5-MW Offshore Wind Turbine and 2-MW Floating Offshore Wind Turbine Technology Hitachi Review (Vol. 63): Hitachi Ltd. 
Saffman, P. G. (1970). A Model for Inhomogeneous Turbulent Flow. Proceedings of the Royal Society of London A: Mathematical, Physical and Engineering Sciences, 317(1530), 417-433. doi: 10.1098/rspa.1970.0125

Saidur, R., Islam, M. R., Rahim, N. A., \& Solangi, K. H. (2010). A review on global wind energy policy. Renewable and Sustainable Energy Reviews, 14(7), 1744-1762. doi: http://dx.doi.org/10.1016/j.rser.2010.03.007

Sato, I., Asano, H., Utsunomiya, T., \& Yoshida, S. (2014). Japan Patent No. JP2013-050180.

Schewe, G. (1983). On the force fluctuations acting on a circular cylinder in crossflow from subcritical up to transcritical Reynolds numbers. Journal of Fluid Mechanics, 133, 265-285.

Schlichting, H., \& Gersten, K. (2000). Boundary-layer theory: Springer Science \& Business Media.

Scholte-Wassink, H. (2011). United States Patent No. US20110074155.

Scruton, C. (1981). An introduction to wind effects on structures. London: Oxford University Press for the Design Council, the British Standards Institution and the Council of Engineering Institution.

Scruton, C., Rogers, E. W. E., Menzies, J. B., \& Scorer, R. S. (1971). Steady and Unsteady Wind Loading of Buildings and Structures [and Discussion]. Philosophical Transactions of the Royal Society of London. Series A, Mathematical and Physical Sciences, 269(1199), 353-383. doi: $10.1098 /$ rsta. 1971.0038

Selvam, K. (2007). Individual pitch control for large scale wind turbines: Energy research Centre of the Netherlands.

Selvam, K., Kanev, S., van Wingerden, J. W., van Engelen, T., \& Verhaegen, M. (2009). Feedback-feedforward individual pitch control for wind turbine load reduction. International Journal of Robust and Nonlinear Control, 19(1), 72-91. doi: http://dx.doi.org/10.1002/rnc.1324

Sescu, A., \& Afjeh, A. A. (2012). Evaluation of Aerodynamic Infrasound Radiating From Upwind and Downwind HAWTS. Paper presented at the ASME 2012 International Mechanical Engineering Congress and Exposition.

Shih, W. C. L., Wang, C., Coles, D., \& Roshko, A. (1993). Experiments on flow past rough circular cylinders at large Reynolds numbers. Journal of Wind Engineering and Industrial Aerodynamics, 49(1-3), 351-368. doi: http://dx.doi.org/10.1016/0167-6105(93)90030-R

Shikha, Bhatti, T. S., \& Kothari, D. P. (2003). Aspects of Technological Development of Wind Turbines. Journal of Energy Engineering, 129(3), 81-95. doi: http://dx.doi.org/10.1061/(ASCE)0733-9402(2003)129:3(81)

Siegfriedsen, S. (2007). United States Patent No. US20070098555.

Siegfriedsen, S. (2013). United States Patent No. US8426993.

Skaare, B., Nielsen, F. G., Hanson, T. D., Yttervik, R., Havmøller, O., \& Rekdal, A. (2014). Analysis of measurements and simulations from the Hywind Demo floating wind turbine. Wind Energy, n/a-n/a. doi: $10.1002 /$ we. 1750 
Smagorinsky, J. (1963). General Circulation Experiments with the Primitvie Equations. Monthly Weather Review, 91(3), 99-164. doi: doi:10.1175/1520-0493(1963)091<0099:GCEWTP $>2.3$.CO;2

Smith, P. (2014). Ming Yang installs 6.5MW prototype. Windpower Offshore. $\quad$ http://www.windpoweroffshore.com/article/1320170/mingyang-installs-65mw-prototype

Snel, H., \& Schepers, J. (1995). Joint investigation of dynamic inflow effects and implementation of an engineering method: Netherlands Energy Research Foundation ECN.

Snieckus, D. (2014a). IN DEPTH: Aerodyn's monster turbine. Recharge News. $\quad$ http://www.rechargenews.com/wind/offshore/1377824/INDEPTH-Aerodyns-monster-turbine

Snieckus, D. (2014b). IN DEPTH: The two-bladed revolution. Recharge News. http://www.rechargenews.com/wind/offshore/article1364056.ece

Snieckus, D. (2014c). Norway trial for Ming Yang 6MW. Recharge News. http://www.rechargenews.com/wind/offshore/article1365752.ece

Snieckus, D. (2015). IN DEPTH: Ming Yang's two-blader gears up to cut costs offshore. Recharge News.

http://www.rechargenews.com/wind/1389697/in-depth-ming-yangs-twoblader-gears-up-to-cut-costs-offshore

Spruce, C. (2004). Power control of active stall wind turbines. Paper presented at the European Wind Energy Conference, London, UK.

Statoil ASA. (2012). Hywind by Statoil - The Floating Wind Turbine.

Steele, A., Ichter, B., Qin, C., Loth, E., Selig, M. S., \& Moriarty, P. (2013). Aerodynamics of an Ultralight Load-Aligned Rotor for Extreme-Scale Wind Turbines. Paper presented at the 51st AIAA Aerospace Sciences Meeting including the New Horizons Forum and Aerospace Exposition, Grapevine, Texas.

Stewart, G., Lackner, M., \& Goupee, A. (2012). Calibration and Validation of a FAST Floating Wind Turbine Model of the DeepCwind Scaled Tension-Leg Platform. Paper presented at the 22nd International Offshore and Polar Engineering Conference, Rhodes, Greece.

Stoer, R. D. (2011). Germany Patent No. DE102010009435.

Sun, X., Huang, D., \& Wu, G. (2012). The current state of offshore wind energy technology development. Energy, 41(1), 298-312. doi: http://dx.doi.org/10.1016/j.energy.2012.02.054

SWAY AS. (2013). SWAY Concept. Retrieved 25 Aug 2014, from http://www.sway.no/?page $=166$

Tang, Y.-g., Song, K., \& Wang, B. (2015). Experiment study of dynamics response for wind turbine system of floating foundation. China Ocean Engineering, 29(6), 835-846. doi: 10.1007/s13344-015-0059-2

Tangler, J. L. (2000). The Evolution of Rotor and Blade Design. Paper presented at the WindPower 2000, Palm Springs, California.

Tegen, S., Lantz, E., Hand, M., Maples, B., Smith, A., \& Schwabe, P. (2013). 2011 Cost of Wind Energy Review: National Renewable Energy Laboratory (NREL), Golden, CO. 
The Crown Estate. (2012). Offshore Wind Cost Reduction: Pathways Study. London, UK.

Tomasicchio, G. R., D’Alessandro, F., Musci, E., Fonseca, N., Mavrakos, S. A., Kirkegaard, J., . . . Wolbring, J. (2014). Physical Model Experiments on Floating Off-Shore Wind Turbines. Paper presented at the Proceedings of the HYDRALAB IV Joint User Meeting. Lisbon.

Utsunomiya, T., Sato, I., Yoshida, S., Ookubo, H., \& Ishida, S. (2013). Dynamic response analysis of a floating offshore wind turbine during severe typhoon event. Paper presented at the ASME 2013 32nd International Conference on Ocean, Offshore and Arctic Engineering.

Utsunomiya, T., Shiraishi, T., Sato, I., Inui, E., \& Ishida, S. (2014, 7-10 April 2014). Floating offshore wind turbine demonstration project at Goto Islands, Japan. Paper presented at the OCEANS 2014 - TAIPEI.

Van Engelen, T., \& Van der Hooft, E. (2005). Individual pitch control inventory: Energy research Centre of the Netherlands.

van Solingen, E., \& van Wingerden, J. W. (2015). Linear individual pitch control design for two-bladed wind turbines. Wind Energy, 18(4), 677697. doi: http://dx.doi.org/10.1002/we. 1720

Vargo, D. J. (1974). Wind energy developments in the 20th century. NASA STI/Recon Technical Report A, 75, 17503.

Vattenfall. (2010). Horns Rev 1 Offshore Wind Farm. Stockholm, Sweden.

Vattenfall. (2012). Ormonde Offshore Wind Farm: Media Pack. Stockholm, Sweden.

Verelst, D. (2009). Flexible wind turbine blades: a FEM-BEM coupled model approach. (Masters Dissertation), Delft University of Technology, The Netherlands.

Viterna, L. A., \& Janetzke, D. C. (1982). Theoretical and experimental power from large horizontal-axis wind turbines. United States, : National Aeronautics and Space Administration.

Vølund, P. (2005). Concrete is the future for offshore foundations. Wind Engineering, 29(6), 531-539.

Vries, E. d. (2014). Windtech: Aerodyn two-blade floating 8MW SCD. Windpower Monthly. http://www.windpowermonthly.com/article/1318520/windtech-aerodyntwo-blade-floating-8mw-scd

WAMIT User Manual Version 7.0. (1999). Dept. of Ocean Engineering, Massachusetts Institute of ....

Watson, G., Hill, B., Courtney, F., \& Goldman, P. (2005). A Framework for Offshore Wind Energy Development in the United States: Massachusetts Technology Collaborative (MTC).

Weaver, L. E. (2014). United States Patent No. US8740543.

Wenxin, Z., \& Karl, S. (2007). Individual Blade Pitch for Active Yaw Control of a Horizontal-Axis Wind Turbine. Paper presented at the 45th AIAA Aerospace Sciences Meeting and Exhibit, Reno, Navada.

Weston, D. (2015). EWEA Offshore: Aerodyn 6MW connected to grid. Windpower

Monthly. 
http://www.windpowermonthly.com/article/1337932/ewea-offshoreaerodyn-6mw-connected-grid

Wieselsberger, C. (1921). Neuere Feststellungen über die Gesetze des Flüssigkeits- und Luftwiderstandes. Phys. Z, 22, 321-328.

Wilcox, D. C. (1988). Reassessment of the scale-determining equation for advanced turbulence models. AIAA Journal, 26(11), 1299-1310. doi: $10.2514 / 3.10041$

Wilcox, D. C. (1993). Comparison of two-equation turbulence models for boundary layers with pressure gradient. AIAA Journal, 31(8), 1414-1421. doi: $10.2514 / 3.11790$

Wilcox, D. C. (2006). Turbulence Modling for CFD (3rd ed.). California: D $\mathrm{C}$ W Industries.

Wilmshurst, S. M. B., Powles, S. J. R., \& Wilson, D. M. A. (1985, 27-29 March 1985). The problem of tower shadow. Paper presented at the 7th British Wind Energy Association Conference, Oxford.

Windpower Monthly. (2016). The 10 Biggest Turbines in the World. http://www.windpowermonthly.com/10-biggest-turbines

Wood, E. (2010). Wind Farms: Are All the Best Spots Taken? Renewable Energy World Magazine. http://www.renewableenergyworld.com/rea/news/article/2010/05/windfarms-are-all-the-best-spots-taken

World Wind Energy Association. (2009). WWEA 2014 Half-year Report. Bonn, Germany.

Wright, A. D. Modern control design for flexible wind turbines.

Zahle, F., Sorensen, N. N., \& Johansen, J. (2009). Wind Turbine RotorTower Interaction Using an Incompressible Overset Grid Method. Wind Energy, 12(6), 594-619. doi: http://dx.doi.org/10.1002/We.327

Zhao, Q., Sheng, C., \& Afjeh, A. (2014). Computational Aerodynamic Analysis of Offshore Upwind and Downwind Turbines. Journal of Aerodynamics, 2014, 13. doi: http://dx.doi.org/10.1155/2014/860637 


\section{APPENDIX A}

\section{A.1. Instrumentation}

Turbine data were acquired through two independent data acquisition systems (DAS). A Bachman MPC240 controller and SCADA system were used to operate the turbine and gather information from a set of sensors. The other DAS was a National Instruments (NI) PXI system running LabVIEW. The PXI was chosen because of its ability to (1) rapidly interrogate sensors that are widely distributed and (2) use global positioning system (GPS) time to tightly coordinate and synchronize measurements.

The PXI was installed by NREL in the tower base. Select data sets from the Bachman controller were acquired through a real-time DeviceNet link between the PXI and controller operating at 100 hertz (Hz). Each controller variable was acquired by the Bachman hardware through a variety of analog and digital interfaces. Controller variables were scaled into engineering units by SWAY's Bachman control code according to calibrations derived by SWAY documentation. The Bachman controller was installed before NREL joined the project, and NREL was provided no information on calibration; therefore, data from the Bachman controller were used with caution.

NREL installed several instruments, each of which internally measured and processed its data. Those data were acquired by the PXI via R232/422 serial communication and synchronized to either a GPS time reference using onboard GPS receivers or an external GPS-synchronized trigger pulse. Data acquired by the PXI system were stored locally in the NI TDMS file format in files that are one-hour long. A personal computer (PC) housed in a nearby onshore data shed automatically synchronized new PXI data files with a local data archive every hour. The wave and current measurements were provided by an Acoustic Wave and Current (AWAC) unit where data were logged internally on the instrument and manually synchronized with the computer in the data shed through an underwater serial data cable. 
Data consolidated by all instruments were able to be remotely accessed via a file transfer protocol link, but poor bandwidth to the test site limited the amount of data that could be downloaded. This data acquisition system was monitored and administered remotely at the NWTC.

\section{Measurements}

The wind speed and direction were measured with two three-axis ATI $\mathrm{SATI} / 3 \mathrm{~K}$ ultrasonic anemometers. One was mounted on a horizontal boom that extended upwind and perpendicular to the tower. NREL installed this boom to try to obtain an undisturbed measurement of the wind inflow to the turbine. The other anemometer was mounted by SWAY near the base of the nacelle.

The platform motions were measured with two motion reference units (MRU). A Teledyne DMS-05 motion reference unit was located in the tower near the centerline just above the waterline, and an Xsens MTi-G motion tracker unit was located near the end of the anemometer boom at nacelle height. A dual antenna Hemisphere V101 GPS was used to provide position and heading.

\section{Data Collection}

The NREL DAS was deployed in late June 2012 and started to collect data on June 20, 2012. The turbine was decommissioned and removed in December 2013. The NREL DAS operated nearly continuously for 2012; however, the PC stopped functioning in September, resulting in a data loss from September 22 through October 20. In early 2013, the NREL DAS began to exhibit increasing instability, and it required periodic rebooting and eventual reinstallation of the software. As a result, the dataset has many gaps, many of them for durations exceeding one month. The last data were recorded on September 27, 2013. 
During the deployment period, the SWAY turbine was infrequently operated, which resulted in very limited operational data. Table A.1 summarizes the number of one-hour datasets measured by wind speed and wave height.

Table A.1 Data Summary of Deployment Period

\begin{tabular}{|c|c|c|c|c|c|}
\hline \multicolumn{6}{|c|}{ Significant Wave Height (m) } \\
\hline & 0.25 & 0.5 & 0.75 & 1 & 1.25 \\
\hline 1 & 209 & 2 & & & \\
\hline 2 & 587 & 5 & & & \\
\hline 3 & 596 & 7 & & & \\
\hline 4 & 431 & 7 & & & \\
\hline 5 & 298 & 30 & & & \\
\hline 6 & 163 & 58 & 1 & & \\
\hline 7 & 98 & 83 & 1 & & \\
\hline 8 & 39 & 84 & & & \\
\hline 9 & 17 & 86 & 2 & & \\
\hline 10 & 2 & 36 & 2 & & \\
\hline 11 & & 24 & 6 & & \\
\hline 12 & & 5 & 12 & & \\
\hline 13 & & 5 & 10 & & \\
\hline 14 & 1 & 1 & 10 & 3 & \\
\hline 15 & & & & 3 & \\
\hline
\end{tabular}




\section{APPENDIX B}

\section{B.1. WAMIT Setup Log File}

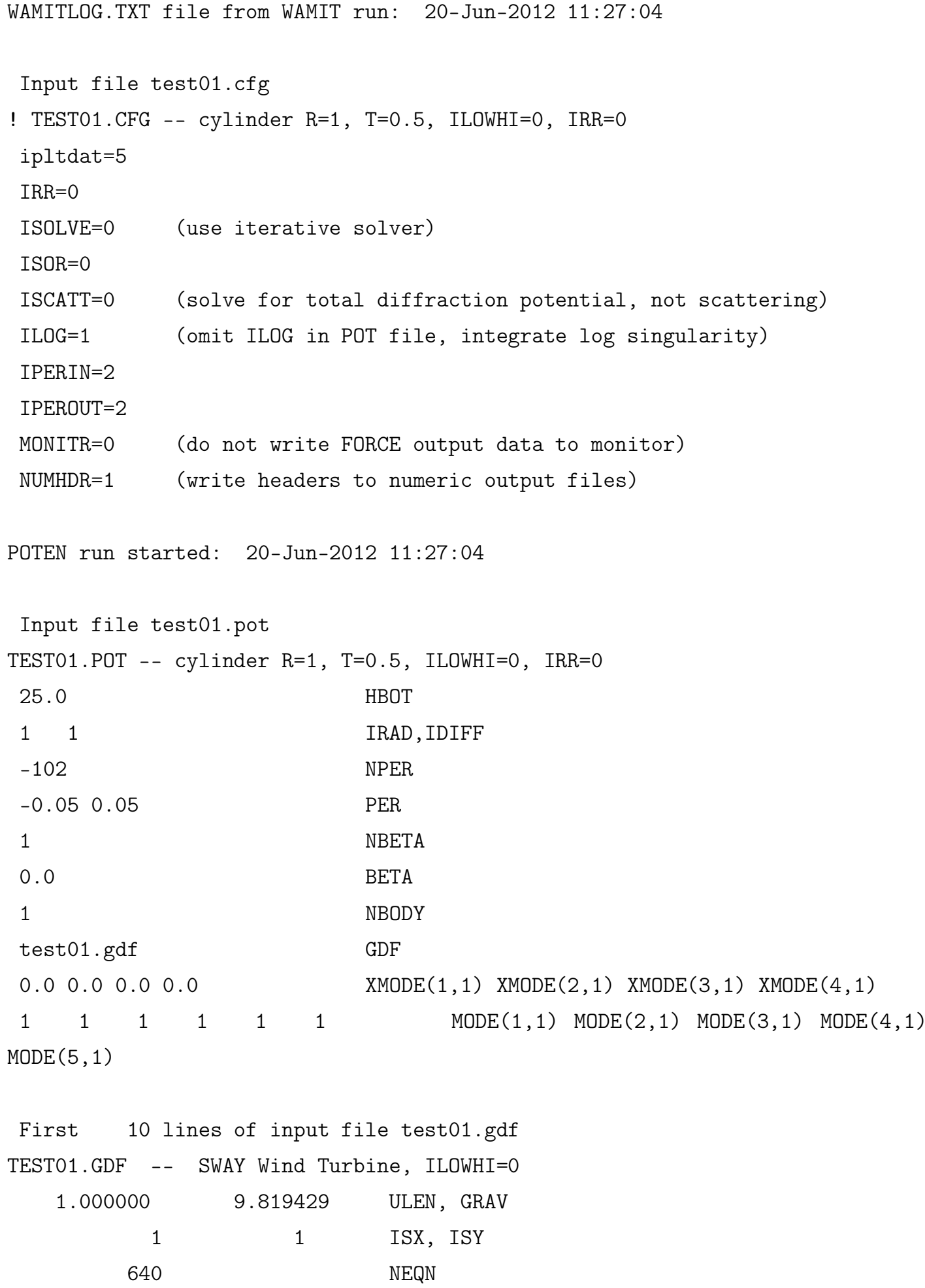




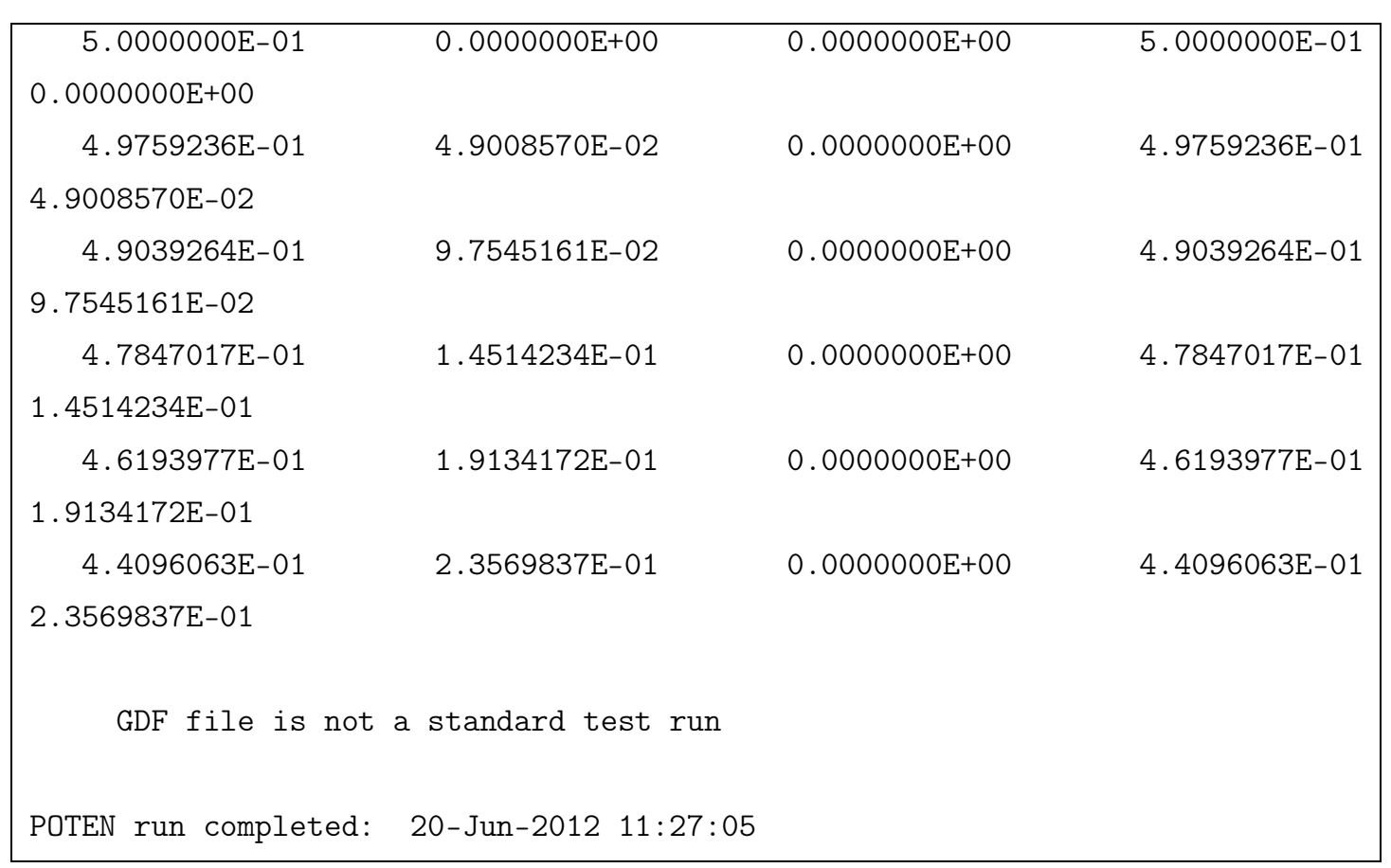




\section{APPENDIX C}

\section{C.1. Airfoil-Data File - Cylinder.dat}

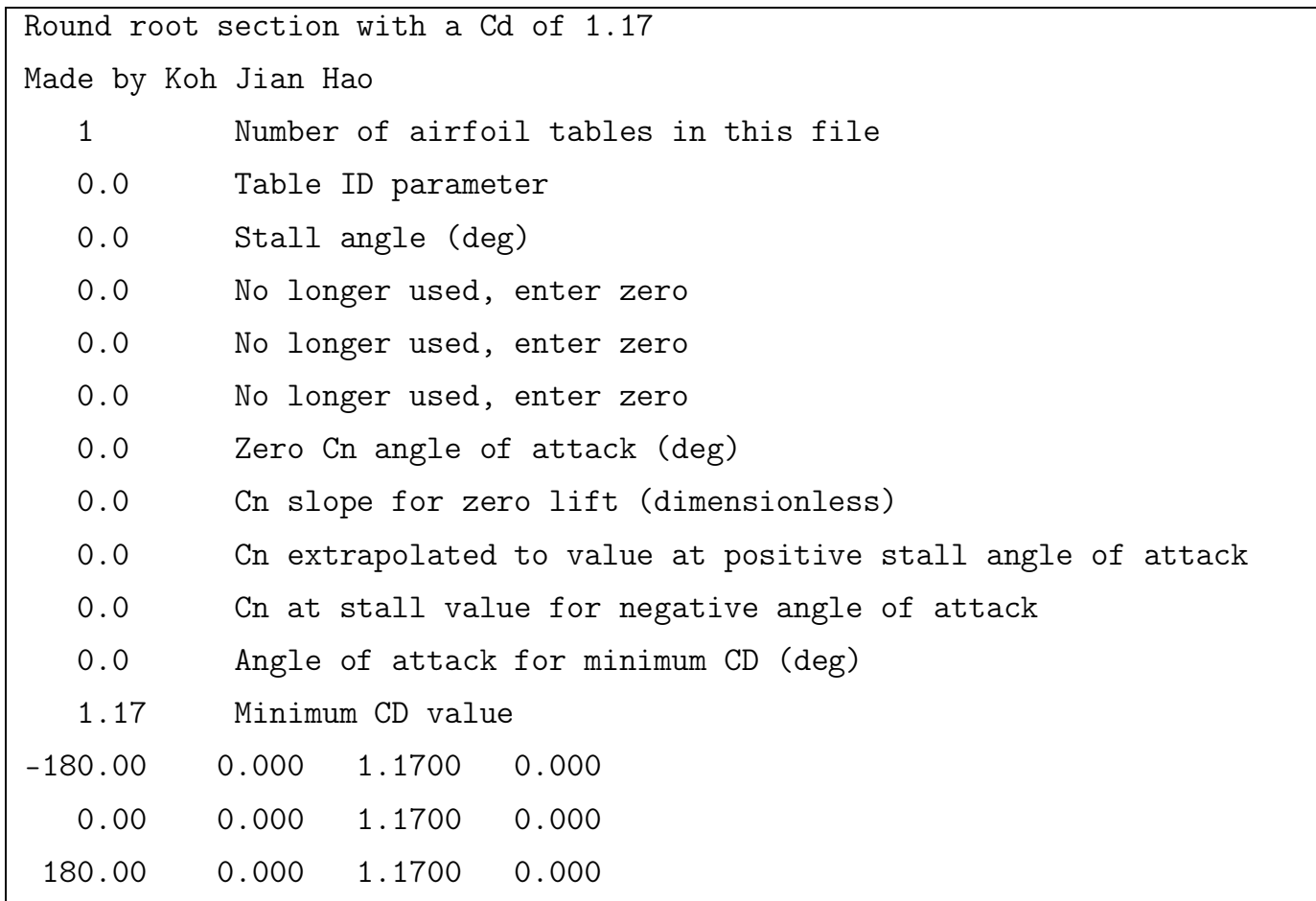

\section{C.2. Airfoil-Data File - Transition.dat}

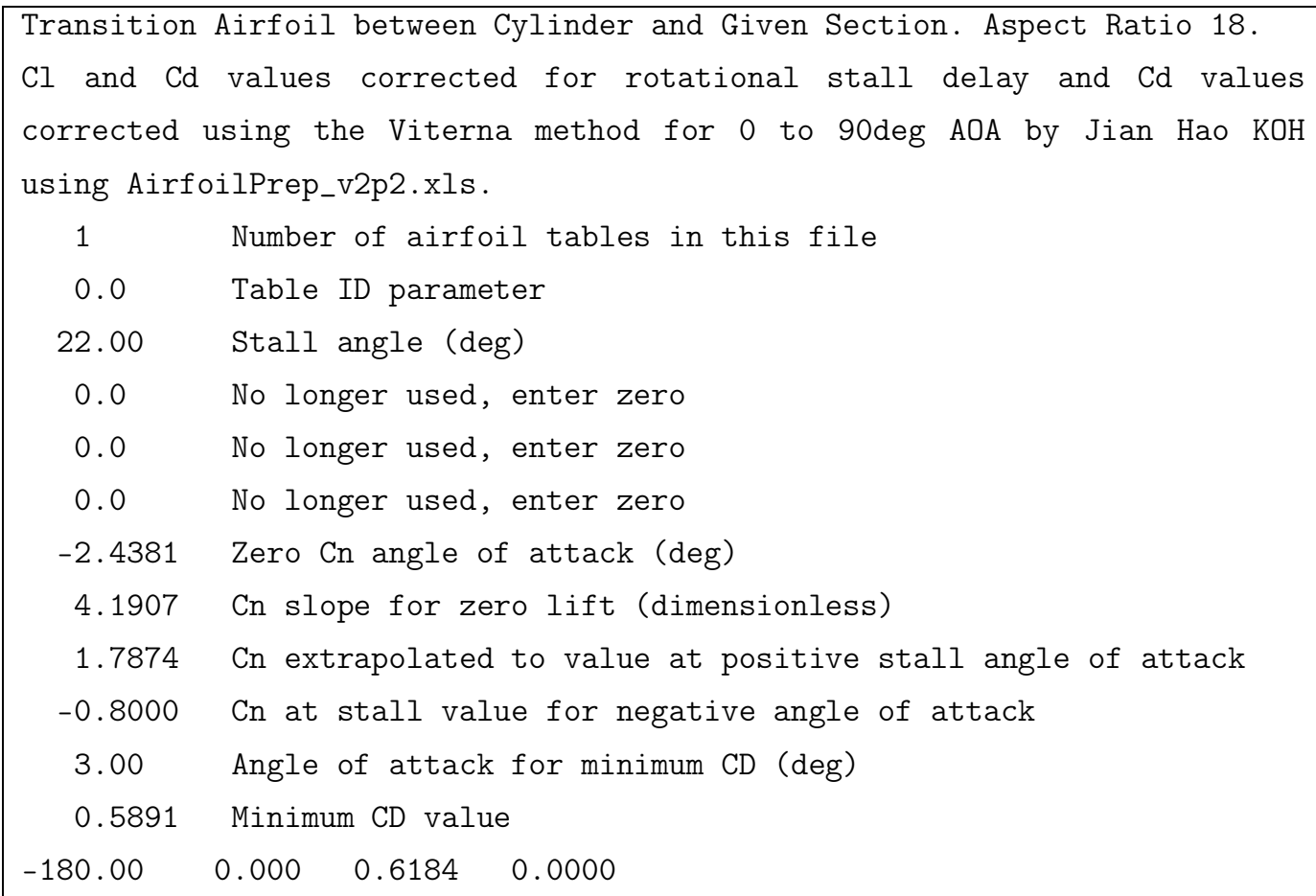




\begin{tabular}{|c|c|c|c|}
\hline-170.00 & 0.335 & 0.6522 & 0.4000 \\
\hline-160.00 & 0.670 & 0.7488 & -0.0167 \\
\hline-150.00 & 0.637 & 0.8940 & 0.0673 \\
\hline-140.00 & 0.617 & 1.0662 & 0.1350 \\
\hline-130.00 & 0.567 & 1.2390 & 0.1959 \\
\hline-120.00 & 0.474 & 1.3847 & 0.2481 \\
\hline-110.00 & 0.339 & 1.4777 & 0.2876 \\
\hline-100.00 & 0.176 & 1.4981 & 0.3103 \\
\hline-90.00 & 0.000 & 1.4340 & 0.3134 \\
\hline-80.00 & -0.176 & 1.4981 & 0.3524 \\
\hline-70.00 & -0.339 & 1.4777 & 0.3745 \\
\hline-60.00 & -0.474 & 1.3847 & 0.3796 \\
\hline-50.00 & -0.567 & 1.2390 & 0.3707 \\
\hline-40.00 & -0.617 & 1.0662 & 0.3539 \\
\hline-30.00 & -0.637 & 0.8940 & 0.3417 \\
\hline-20.00 & -0.670 & 0.7488 & 0.3695 \\
\hline-10.00 & -0.290 & 0.6366 & 0.2319 \\
\hline-6.00 & -0.196 & 0.5917 & -0.0444 \\
\hline-5.00 & -0.134 & 0.5910 & -0.0441 \\
\hline-4.00 & -0.072 & 0.5903 & -0.0437 \\
\hline-3.00 & -0.010 & 0.5899 & -0.0449 \\
\hline-2.00 & 0.052 & 0.5896 & -0.0461 \\
\hline-1.00 & 0.115 & 0.5896 & -0.0473 \\
\hline 0.00 & 0.179 & 0.5896 & -0.0485 \\
\hline 1.00 & 0.242 & 0.5894 & -0.0503 \\
\hline 2.00 & 0.306 & 0.5892 & -0.0521 \\
\hline 3.00 & 0.365 & 0.5891 & -0.0539 \\
\hline 4.00 & 0.425 & 0.5892 & -0.0558 \\
\hline 5.00 & 0.481 & 0.5894 & -0.0574 \\
\hline 6.00 & 0.538 & 0.5898 & -0.0590 \\
\hline 7.00 & 0.588 & 0.5911 & -0.0602 \\
\hline 8.00 & 0.638 & 0.5928 & -0.0614 \\
\hline 9.00 & 0.677 & 0.5960 & -0.0651 \\
\hline 10.00 & 0.716 & 0.6001 & -0.0688 \\
\hline 11.00 & 0.748 & 0.6089 & -0.0694 \\
\hline 12.00 & 0.781 & 0.6187 & -0.0701 \\
\hline 13.00 & 0.817 & 0.6301 & -0.0721 \\
\hline 14.00 & 0.852 & 0.6424 & -0.0742 \\
\hline 15.00 & 0.881 & 0.6578 & -0.0750 \\
\hline 16.00 & 0.902 & 0.6733 & -0.0758 \\
\hline 17.00 & 0.921 & 0.6905 & -0.0779 \\
\hline 18.00 & 0.938 & 0.7084 & -0.0800 \\
\hline
\end{tabular}




\begin{tabular}{|rrrr|}
\hline 19.00 & 0.949 & 0.7283 & -0.0811 \\
20.00 & 0.958 & 0.7488 & -0.0822 \\
30.00 & 0.910 & 0.8940 & -0.1841 \\
40.00 & 0.882 & 1.0662 & -0.2567 \\
50.00 & 0.810 & 1.2390 & -0.3147 \\
60.00 & 0.676 & 1.3847 & -0.3590 \\
70.00 & 0.485 & 1.4777 & -0.3888 \\
80.00 & 0.251 & 1.4981 & -0.4036 \\
90.00 & 0.000 & 1.4340 & -0.4036 \\
100.00 & -0.176 & 1.4981 & -0.4426 \\
110.00 & -0.339 & 1.4777 & -0.4647 \\
120.00 & -0.474 & 1.3847 & -0.4698 \\
130.00 & -0.567 & 1.2390 & -0.4609 \\
140.00 & -0.617 & 1.0662 & -0.4441 \\
150.00 & -0.637 & 0.8940 & -0.4318 \\
160.00 & -0.670 & 0.7488 & -0.4597 \\
170.00 & -0.335 & 0.6522 & -0.5000 \\
180.00 & 0.000 & 0.6184 & 0.0000 \\
\hline
\end{tabular}

\section{C.3. Airfoil-Data File - Main 1.dat}

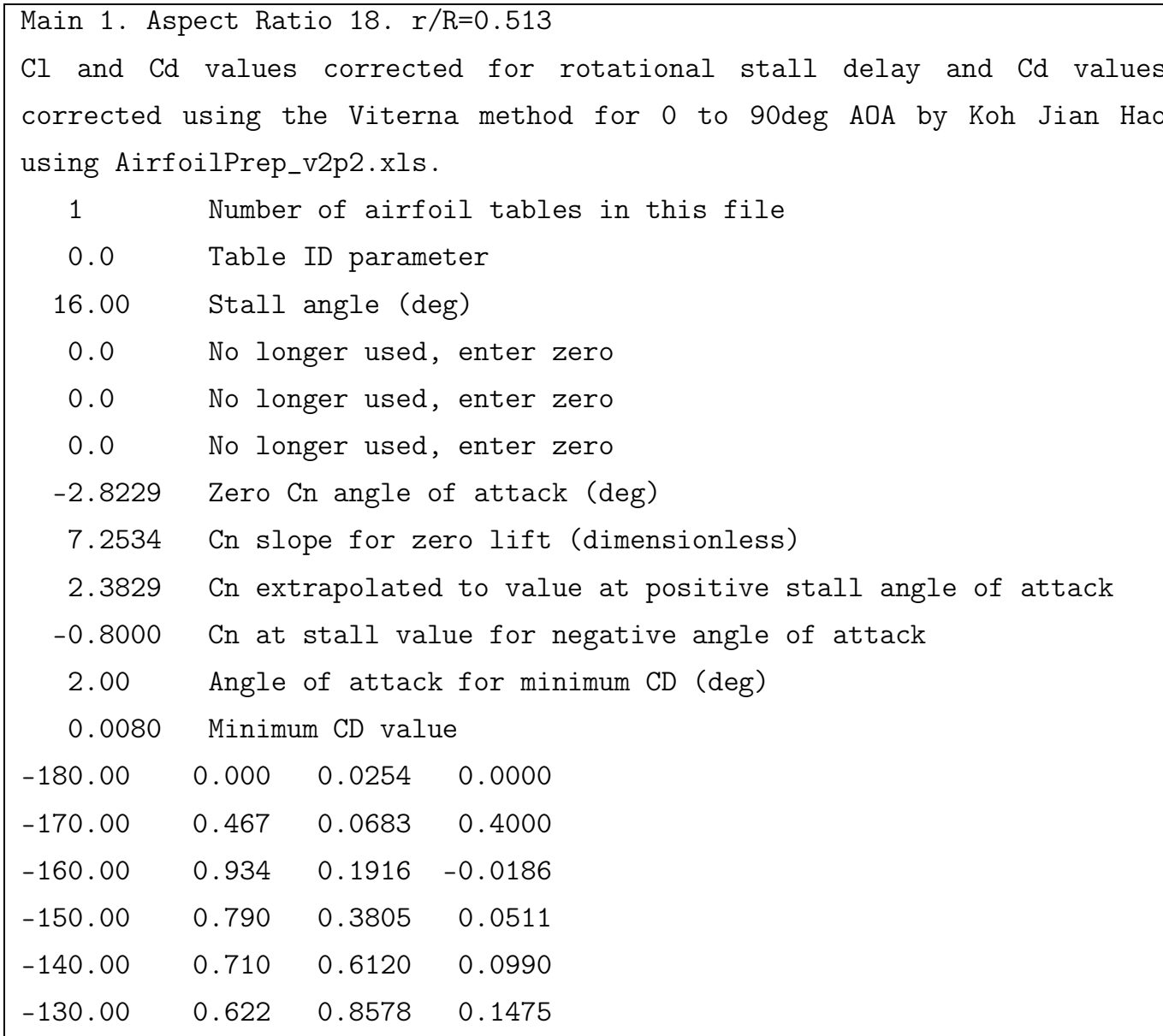




\begin{tabular}{|c|c|c|c|}
\hline-120.00 & 0.503 & 1.0882 & 0.1977 \\
\hline-110.00 & 0.352 & 1.2749 & 0.2458 \\
\hline-100.00 & 0.179 & 1.3952 & 0.2861 \\
\hline-90.00 & 0.000 & 1.4340 & 0.3134 \\
\hline-80.00 & -0.179 & 1.3952 & 0.3262 \\
\hline-70.00 & -0.352 & 1.2749 & 0.3233 \\
\hline-60.00 & -0.503 & 1.0882 & 0.3090 \\
\hline-50.00 & -0.622 & 0.8578 & 0.2907 \\
\hline-40.00 & -0.710 & 0.6120 & 0.2795 \\
\hline-30.00 & -0.790 & 0.3805 & 0.2957 \\
\hline-20.00 & -0.934 & 0.1916 & 0.3998 \\
\hline-10.00 & -0.462 & 0.0641 & 0.2851 \\
\hline-6.00 & -0.391 & 0.0130 & -0.0444 \\
\hline-5.00 & -0.268 & 0.0118 & -0.0441 \\
\hline-4.00 & -0.145 & 0.0107 & -0.0437 \\
\hline-3.00 & -0.022 & 0.0101 & -0.0449 \\
\hline-2.00 & 0.101 & 0.0094 & -0.0461 \\
\hline-1.00 & 0.230 & 0.0092 & -0.0473 \\
\hline 0.00 & 0.359 & 0.0090 & -0.0485 \\
\hline 1.00 & 0.488 & 0.0085 & -0.0503 \\
\hline 2.00 & 0.616 & 0.0080 & -0.0521 \\
\hline 3.00 & 0.730 & 0.0082 & -0.0539 \\
\hline 4.00 & 0.844 & 0.0086 & -0.0558 \\
\hline 5.00 & 0.948 & 0.0092 & -0.0574 \\
\hline 6.00 & 1.052 & 0.0099 & -0.0590 \\
\hline 7.00 & 1.133 & 0.0120 & -0.0602 \\
\hline 8.00 & 1.214 & 0.0143 & -0.0614 \\
\hline 9.00 & 1.253 & 0.0183 & -0.0651 \\
\hline 10.00 & 1.292 & 0.0226 & -0.0688 \\
\hline 11.00 & 1.310 & 0.0342 & -0.0694 \\
\hline 12.00 & 1.327 & 0.0462 & -0.0701 \\
\hline 13.00 & 1.355 & 0.0601 & -0.0721 \\
\hline 14.00 & 1.382 & 0.0743 & -0.0742 \\
\hline 15.00 & 1.385 & 0.0917 & -0.0750 \\
\hline 16.00 & 1.386 & 0.1091 & -0.0758 \\
\hline 17.00 & 1.382 & 0.1286 & -0.0779 \\
\hline 18.00 & 1.377 & 0.1483 & -0.0800 \\
\hline 19.00 & 1.356 & 0.1699 & -0.0811 \\
\hline 20.00 & 1.334 & 0.1916 & -0.0822 \\
\hline 30.00 & 1.128 & 0.3805 & -0.1736 \\
\hline 40.00 & 1.015 & 0.6120 & -0.2251 \\
\hline 50.00 & 0.888 & 0.8578 & -0.2689 \\
\hline
\end{tabular}




\begin{tabular}{|rrrr|}
\hline 60.00 & 0.719 & 1.0882 & -0.3098 \\
70.00 & 0.503 & 1.2749 & -0.3474 \\
80.00 & 0.256 & 1.3952 & -0.3795 \\
90.00 & 0.000 & 1.4340 & -0.4036 \\
100.00 & -0.179 & 1.3952 & -0.4164 \\
110.00 & -0.352 & 1.2749 & -0.4135 \\
120.00 & -0.503 & 1.0882 & -0.3992 \\
130.00 & -0.622 & 0.8578 & -0.3810 \\
140.00 & -0.710 & 0.6120 & -0.3697 \\
150.00 & -0.790 & 0.3805 & -0.3859 \\
160.00 & -0.934 & 0.1916 & -0.4900 \\
170.00 & -0.467 & 0.0683 & -0.5000 \\
180.00 & 0.000 & 0.0254 & 0.0000 \\
\hline
\end{tabular}

\section{C.4. Airfoil-Data File - Main 2.dat}

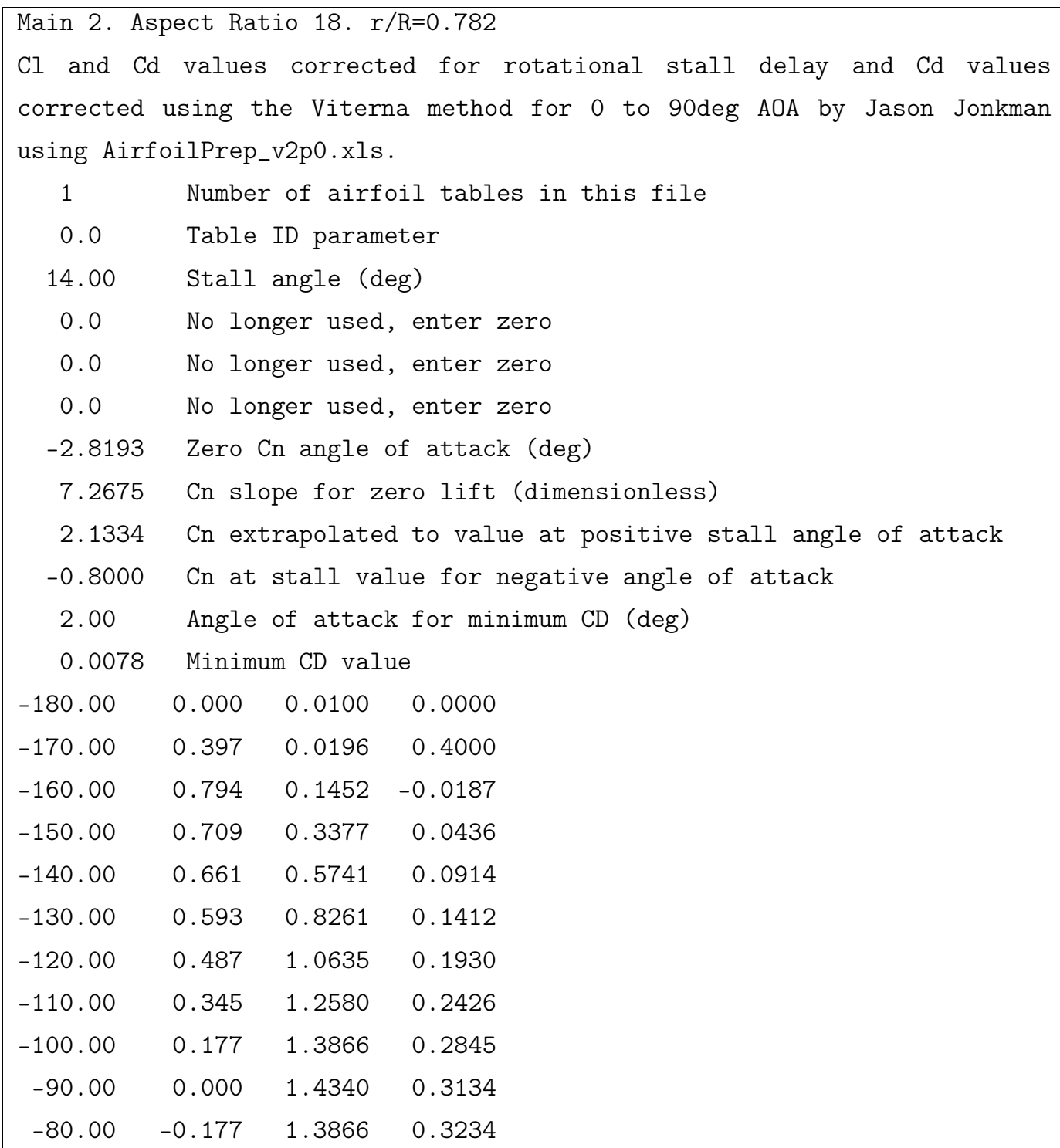




\begin{tabular}{|c|c|c|c|}
\hline-70.00 & -0.345 & 1.2580 & 0.3173 \\
\hline-60.00 & -0.487 & 1.0635 & 0.2991 \\
\hline-50.00 & -0.593 & 0.8261 & 0.2755 \\
\hline-40.00 & -0.661 & 0.5741 & 0.2561 \\
\hline-30.00 & -0.709 & 0.3377 & 0.2575 \\
\hline-20.00 & -0.794 & 0.1452 & 0.3266 \\
\hline-10.00 & -0.422 & 0.0507 & 0.2511 \\
\hline-6.00 & -0.391 & 0.0129 & -0.0444 \\
\hline-5.00 & -0.268 & 0.0118 & -0.0441 \\
\hline-4.00 & -0.145 & 0.0107 & -0.0437 \\
\hline-3.00 & -0.022 & 0.0101 & -0.0449 \\
\hline-2.00 & 0.101 & 0.0095 & -0.0461 \\
\hline-1.00 & 0.230 & 0.0092 & -0.0473 \\
\hline 0.00 & 0.359 & 0.0089 & -0.0485 \\
\hline 1.00 & 0.489 & 0.0084 & -0.0503 \\
\hline 2.00 & 0.618 & 0.0078 & -0.0521 \\
\hline 3.00 & 0.730 & 0.0083 & -0.0539 \\
\hline 4.00 & 0.843 & 0.0087 & -0.0558 \\
\hline 5.00 & 0.943 & 0.0094 & -0.0574 \\
\hline 6.00 & 1.043 & 0.0100 & -0.0590 \\
\hline 7.00 & 1.118 & 0.0120 & -0.0602 \\
\hline 8.00 & 1.192 & 0.0139 & -0.0614 \\
\hline 9.00 & 1.218 & 0.0170 & -0.0651 \\
\hline 10.00 & 1.244 & 0.0200 & -0.0688 \\
\hline 11.00 & 1.246 & 0.0296 & -0.0694 \\
\hline 12.00 & 1.247 & 0.0390 & -0.0701 \\
\hline 13.00 & 1.260 & 0.0498 & -0.0721 \\
\hline 14.00 & 1.272 & 0.0605 & -0.0742 \\
\hline 15.00 & 1.257 & 0.0733 & -0.0750 \\
\hline 16.00 & 1.243 & 0.0860 & -0.0758 \\
\hline 17.00 & 1.225 & 0.1004 & -0.0779 \\
\hline 18.00 & 1.207 & 0.1147 & -0.0800 \\
\hline 19.00 & 1.171 & 0.1300 & -0.0811 \\
\hline 20.00 & 1.135 & 0.1452 & -0.0822 \\
\hline 30.00 & 1.013 & 0.3377 & -0.1636 \\
\hline 40.00 & 0.944 & 0.5741 & -0.2155 \\
\hline 50.00 & 0.847 & 0.8261 & -0.2615 \\
\hline 60.00 & 0.696 & 1.0635 & -0.3046 \\
\hline 70.00 & 0.493 & 1.2580 & -0.3440 \\
\hline 80.00 & 0.253 & 1.3866 & -0.3779 \\
\hline 90.00 & 0.000 & 1.4340 & -0.4036 \\
\hline 100.00 & -0.177 & 1.3866 & -0.4136 \\
\hline
\end{tabular}




\begin{tabular}{|llll|}
\hline 110.00 & -0.345 & 1.2580 & -0.4075 \\
120.00 & -0.487 & 1.0635 & -0.3893 \\
130.00 & -0.593 & 0.8261 & -0.3657 \\
140.00 & -0.661 & 0.5741 & -0.3463 \\
150.00 & -0.709 & 0.3377 & -0.3477 \\
160.00 & -0.794 & 0.1452 & -0.4168 \\
170.00 & -0.397 & 0.0196 & -0.5000 \\
180.00 & 0.000 & 0.0100 & 0.0000 \\
\hline
\end{tabular}

\section{C.5. Airfoil-Data File - Main 3.dat}

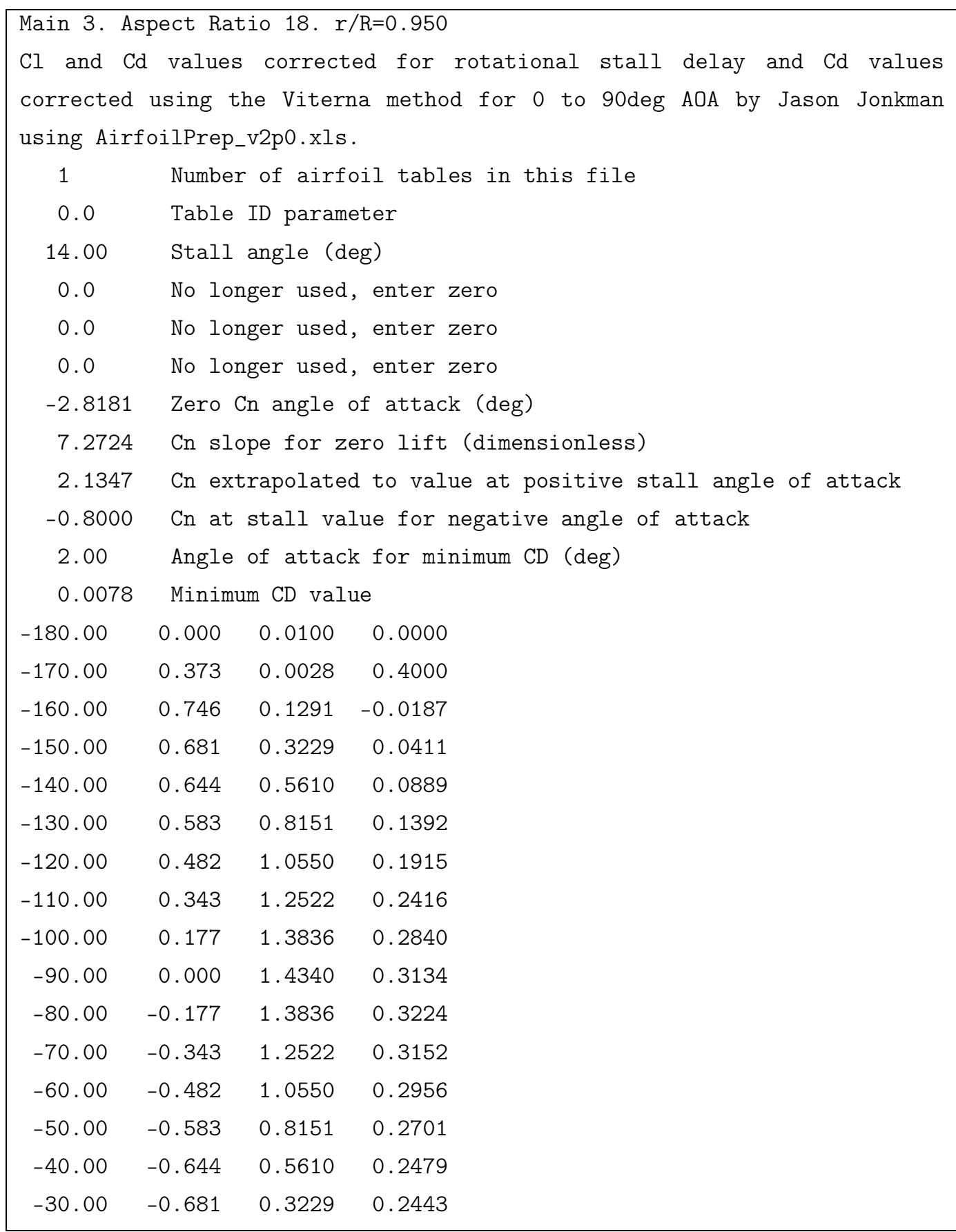




\begin{tabular}{|c|c|c|c|}
\hline-20.00 & -0.746 & 0.1291 & 0.3012 \\
\hline-10.00 & -0.408 & 0.0461 & 0.2391 \\
\hline-6.00 & -0.390 & 0.0129 & -0.0444 \\
\hline-5.00 & -0.268 & 0.0118 & -0.0441 \\
\hline-4.00 & -0.145 & 0.0107 & -0.0437 \\
\hline-3.00 & -0.022 & 0.0101 & -0.0449 \\
\hline-2.00 & 0.101 & 0.0096 & -0.0461 \\
\hline-1.00 & 0.230 & 0.0092 & -0.0473 \\
\hline 0.00 & 0.360 & 0.0089 & -0.0485 \\
\hline 1.00 & 0.489 & 0.0083 & -0.0503 \\
\hline 2.00 & 0.618 & 0.0078 & -0.0521 \\
\hline 3.00 & 0.730 & 0.0083 & -0.0539 \\
\hline 4.00 & 0.842 & 0.0087 & -0.0558 \\
\hline 5.00 & 0.941 & 0.0094 & -0.0574 \\
\hline 6.00 & 1.040 & 0.0101 & -0.0590 \\
\hline 7.00 & 1.112 & 0.0120 & -0.0602 \\
\hline 8.00 & 1.184 & 0.0137 & -0.0614 \\
\hline 9.00 & 1.206 & 0.0165 & -0.0651 \\
\hline 10.00 & 1.228 & 0.0191 & -0.0688 \\
\hline 11.00 & 1.224 & 0.0279 & -0.0694 \\
\hline 12.00 & 1.219 & 0.0365 & -0.0701 \\
\hline 13.00 & 1.227 & 0.0463 & -0.0721 \\
\hline 14.00 & 1.234 & 0.0557 & -0.0742 \\
\hline 15.00 & 1.213 & 0.0669 & -0.0750 \\
\hline 16.00 & 1.194 & 0.0781 & -0.0758 \\
\hline 17.00 & 1.170 & 0.0907 & -0.0779 \\
\hline 18.00 & 1.148 & 0.1031 & -0.0800 \\
\hline 19.00 & 1.107 & 0.1162 & -0.0811 \\
\hline 20.00 & 1.066 & 0.1291 & -0.0822 \\
\hline 30.00 & 0.973 & 0.3229 & -0.1603 \\
\hline 40.00 & 0.920 & 0.5610 & -0.2123 \\
\hline 50.00 & 0.833 & 0.8151 & -0.2590 \\
\hline 60.00 & 0.689 & 1.0550 & -0.3029 \\
\hline 70.00 & 0.490 & 1.2522 & -0.3430 \\
\hline 80.00 & 0.252 & 1.3836 & -0.3774 \\
\hline 90.00 & 0.000 & 1.4340 & -0.4036 \\
\hline 100.00 & -0.177 & 1.3836 & -0.4126 \\
\hline 110.00 & -0.343 & 1.2522 & -0.4054 \\
\hline 120.00 & -0.482 & 1.0550 & -0.3858 \\
\hline 130.00 & -0.583 & 0.8151 & -0.3603 \\
\hline 140.00 & -0.644 & 0.5610 & -0.3381 \\
\hline 150.00 & -0.681 & 0.3229 & -0.3345 \\
\hline
\end{tabular}




\begin{tabular}{|rrrr|}
\hline 160.00 & -0.746 & 0.1291 & -0.3915 \\
170.00 & -0.373 & 0.0028 & -0.5000 \\
180.00 & 0.000 & 0.0100 & 0.0000 \\
\hline
\end{tabular}




\section{APPENDIX D}

D.1. Tower Case 1 - Normalized X Velocity

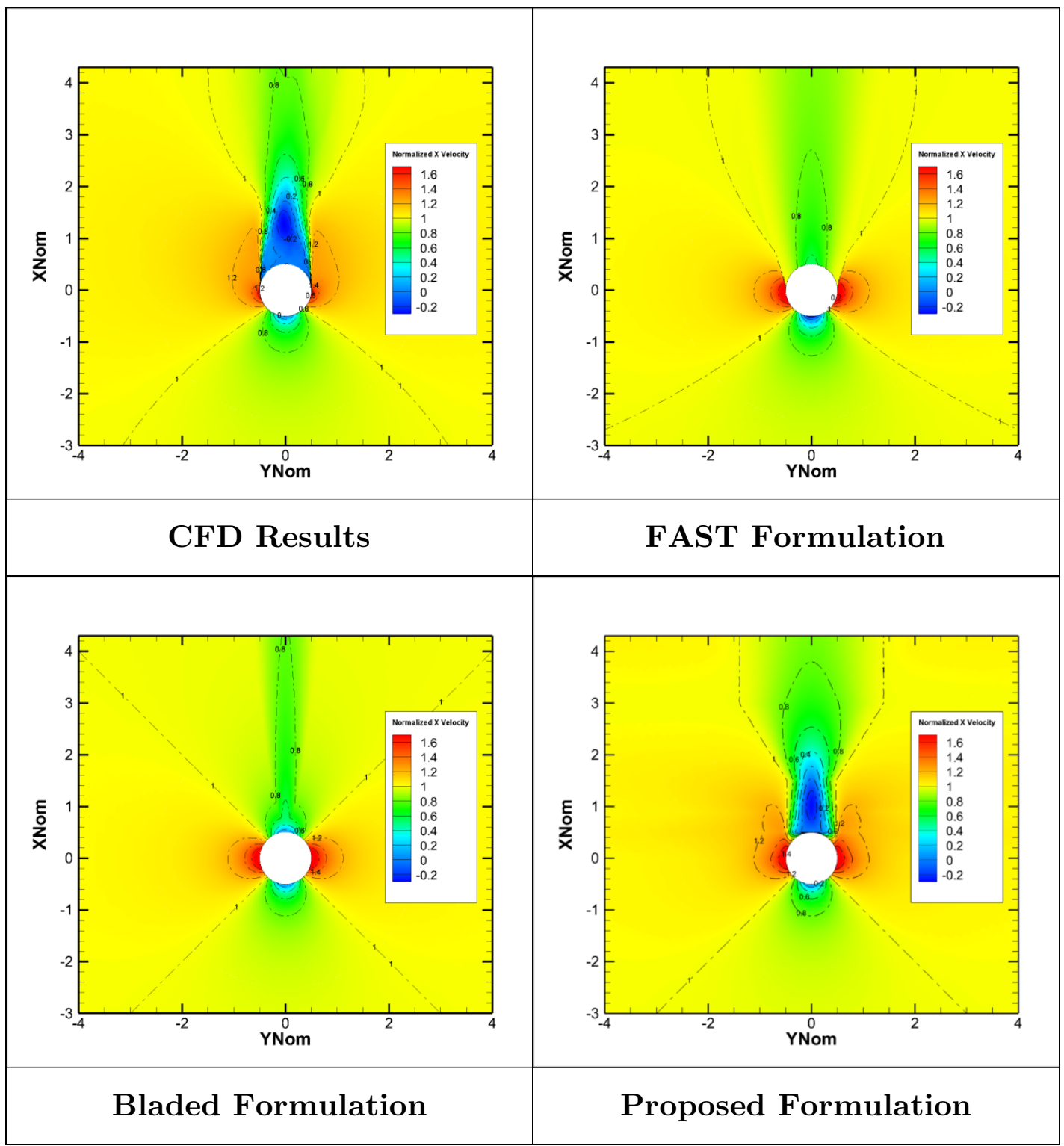




\section{D.2. Tower Case 1 - Normalized Y Velocity}

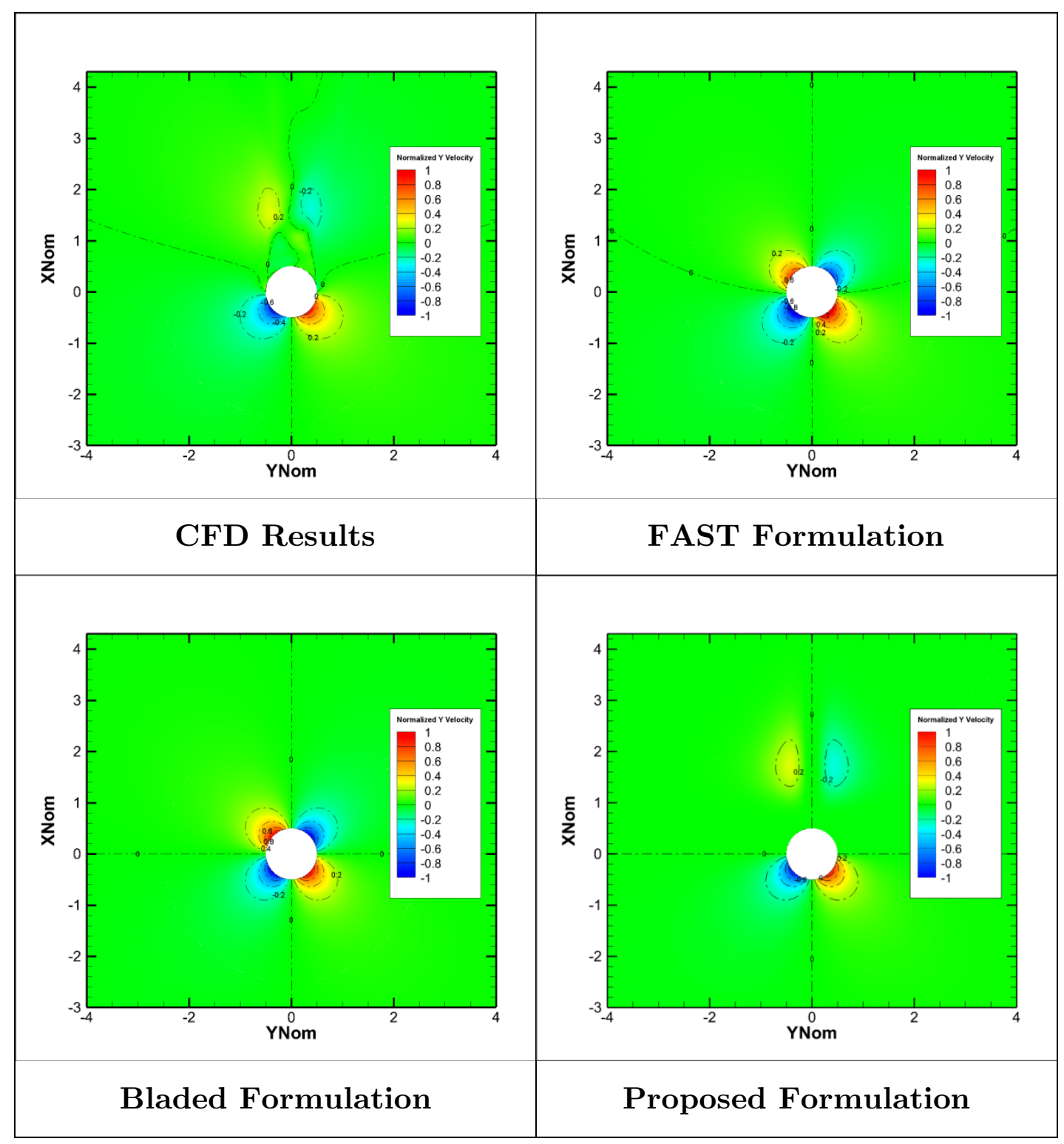




\section{D.3. Tower Case 2 - Normalized X Velocity}

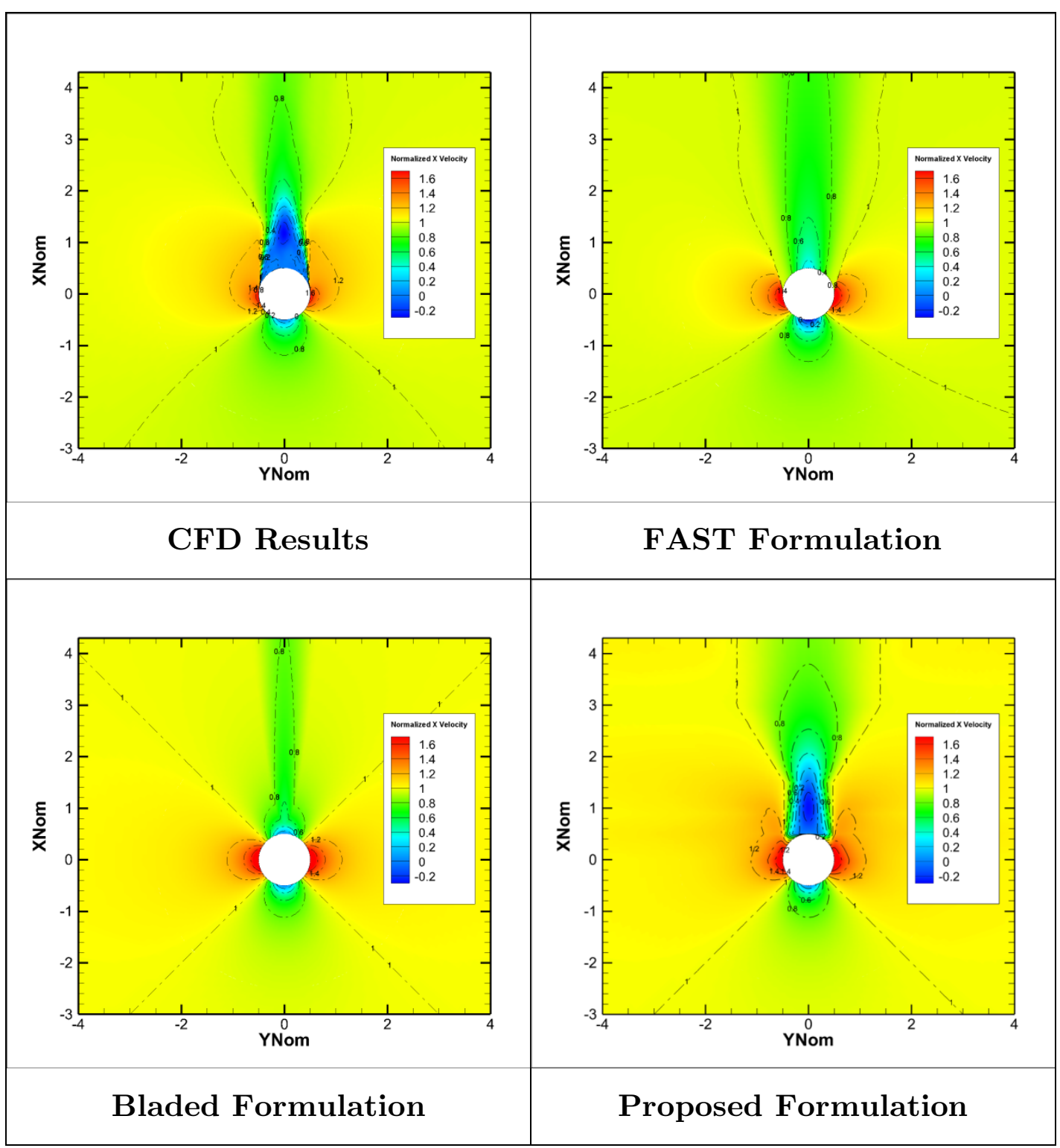




\section{D.4. Tower Case 2 - Normalized Y Velocity}

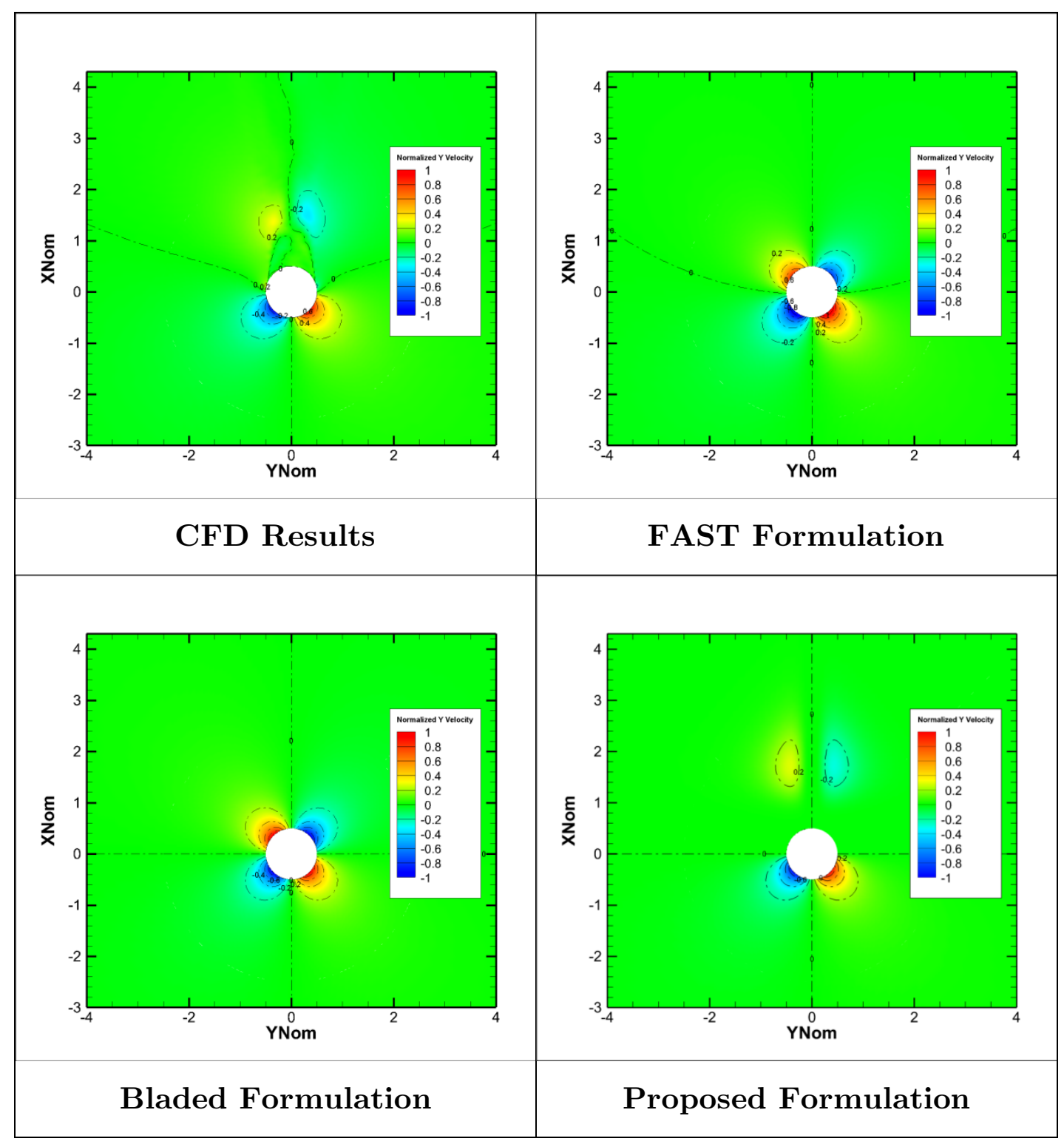




\section{D.5. Tower Case 3 - Normalized X Velocity}

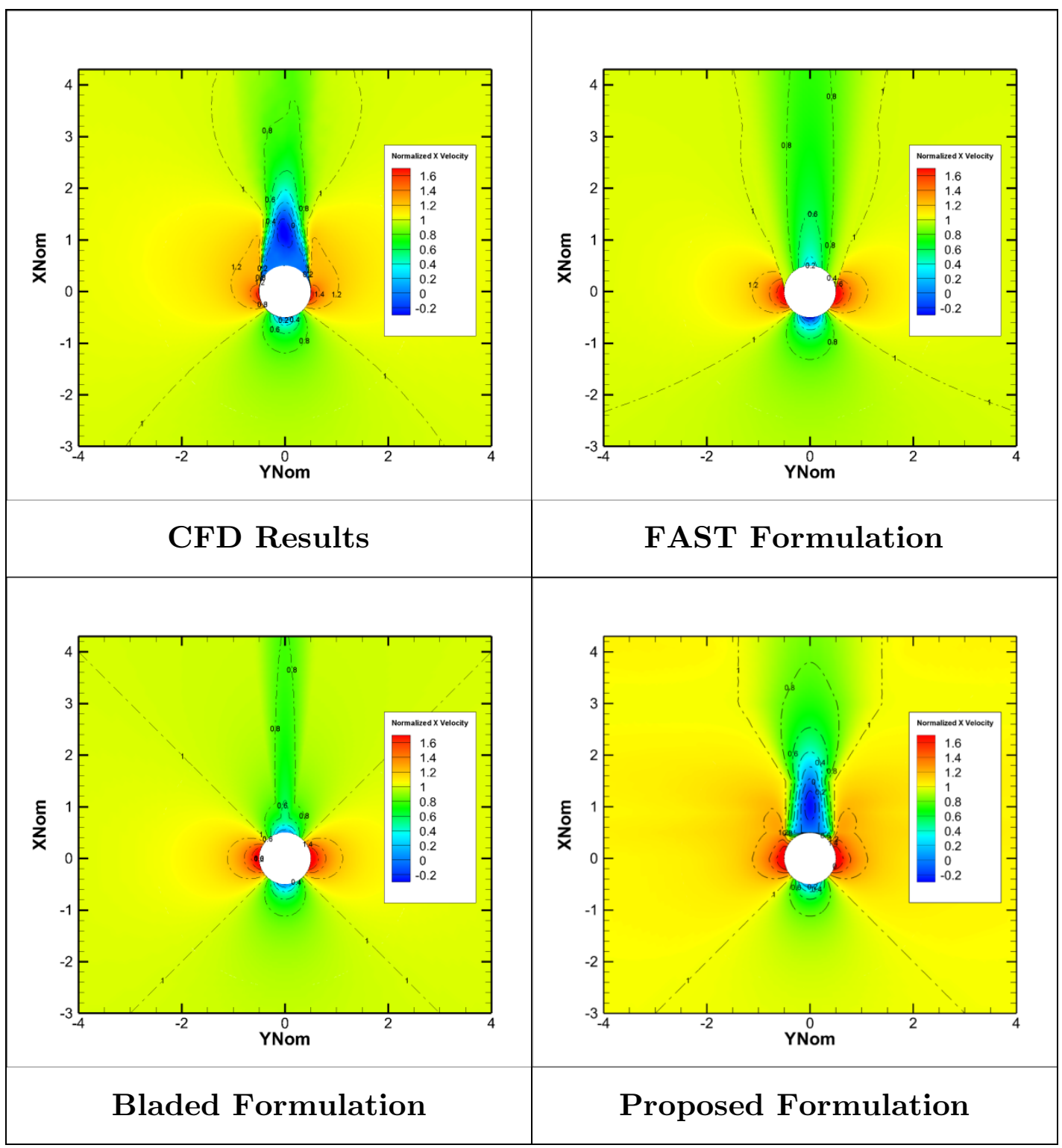




\section{D.6. Tower Case 3 - Normalized Y Velocity}

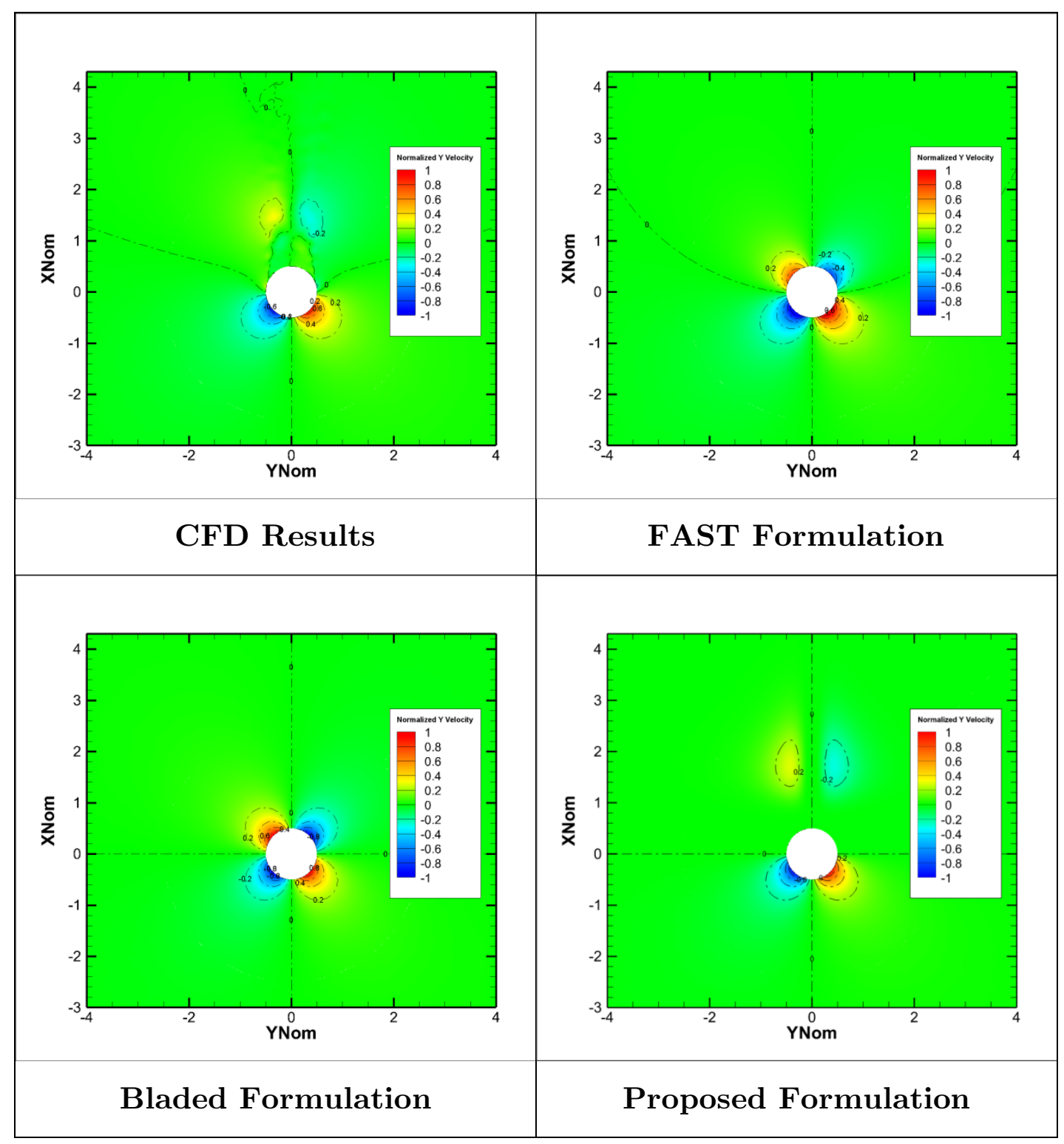




\section{D.7. Tower Case 4 - Normalized X Velocity}

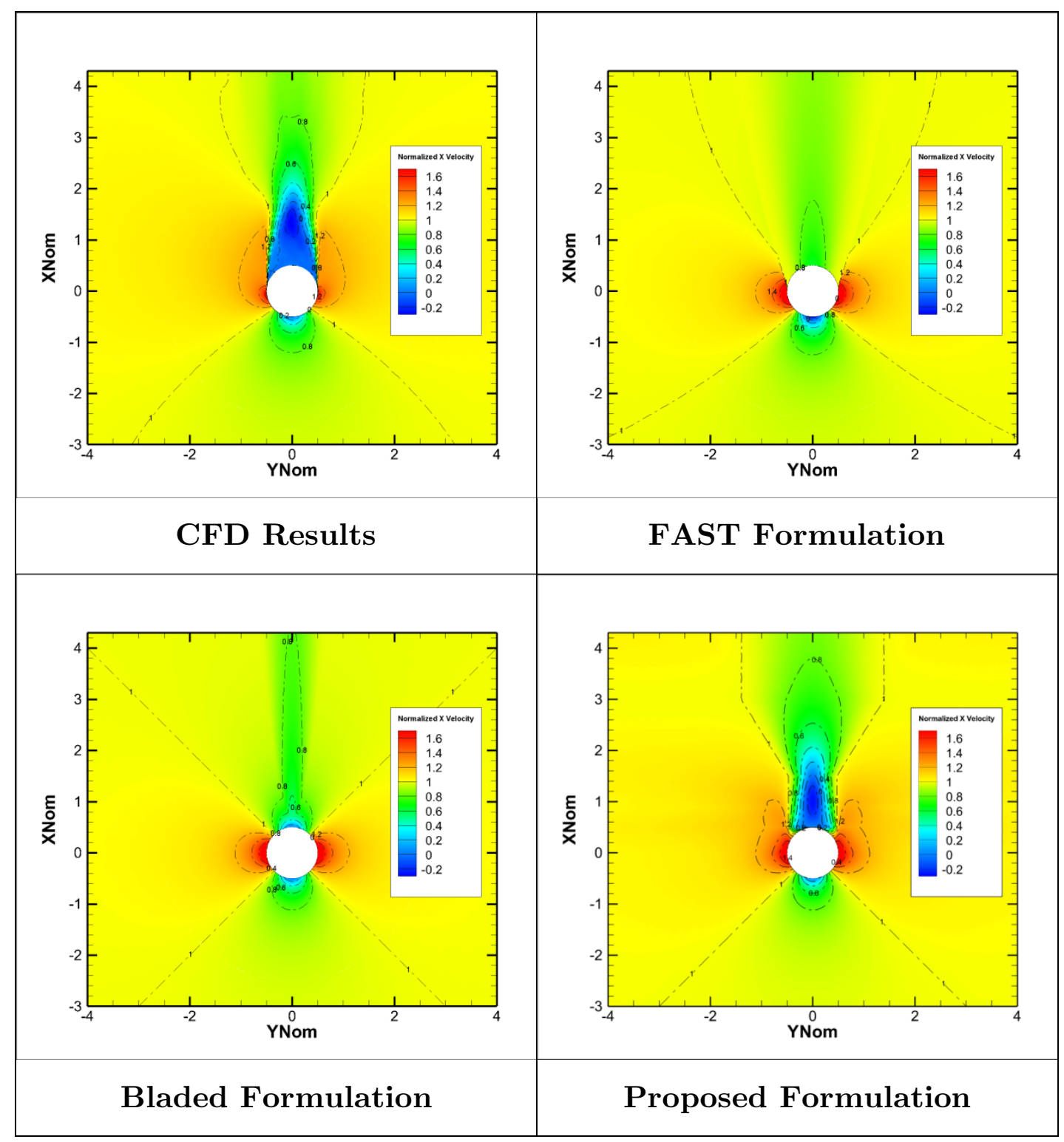




\section{D.8. Tower Case 4 - Normalized Y Velocity}

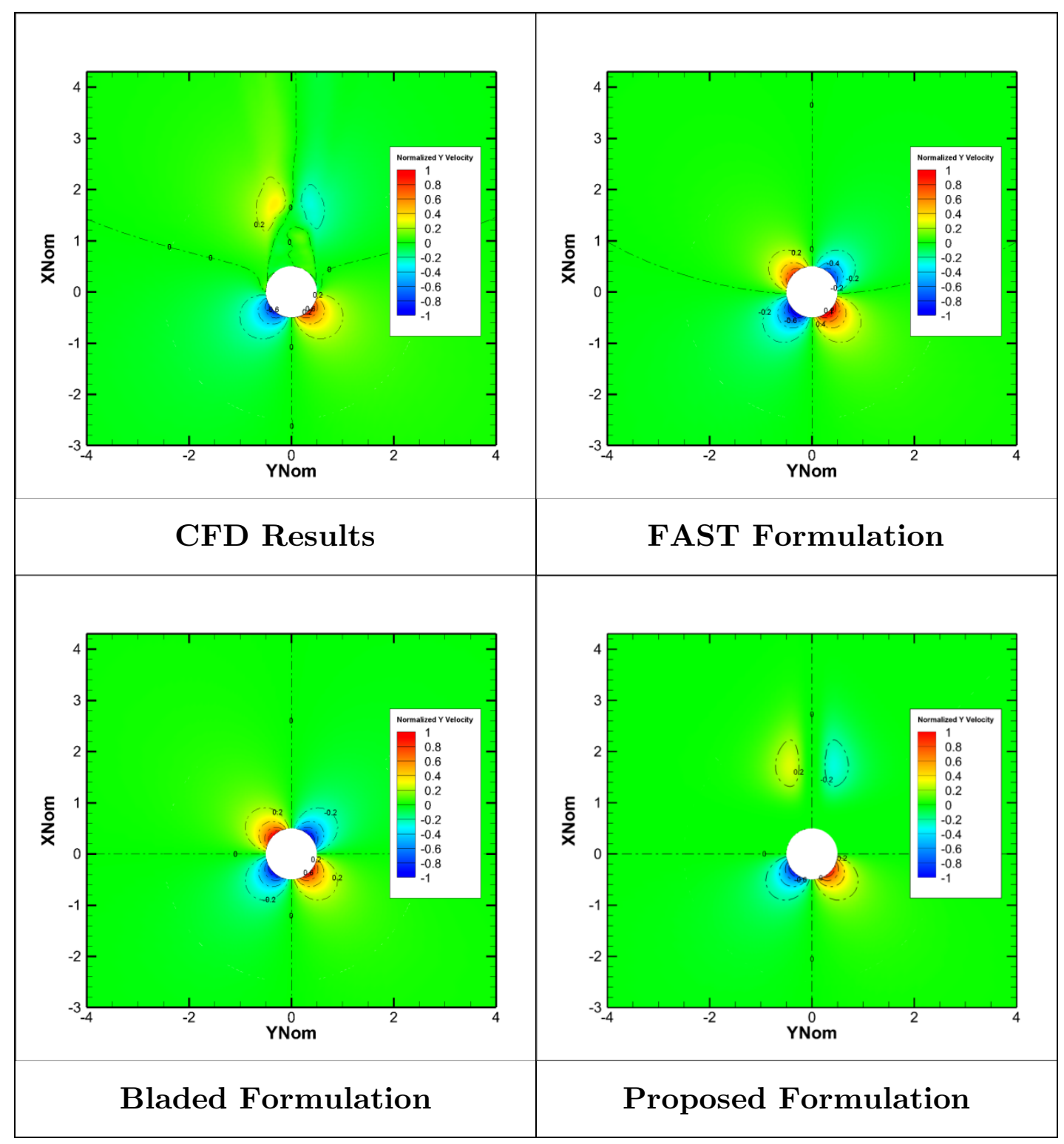




\section{D.9. Tower Case 5 - Normalized X Velocity}

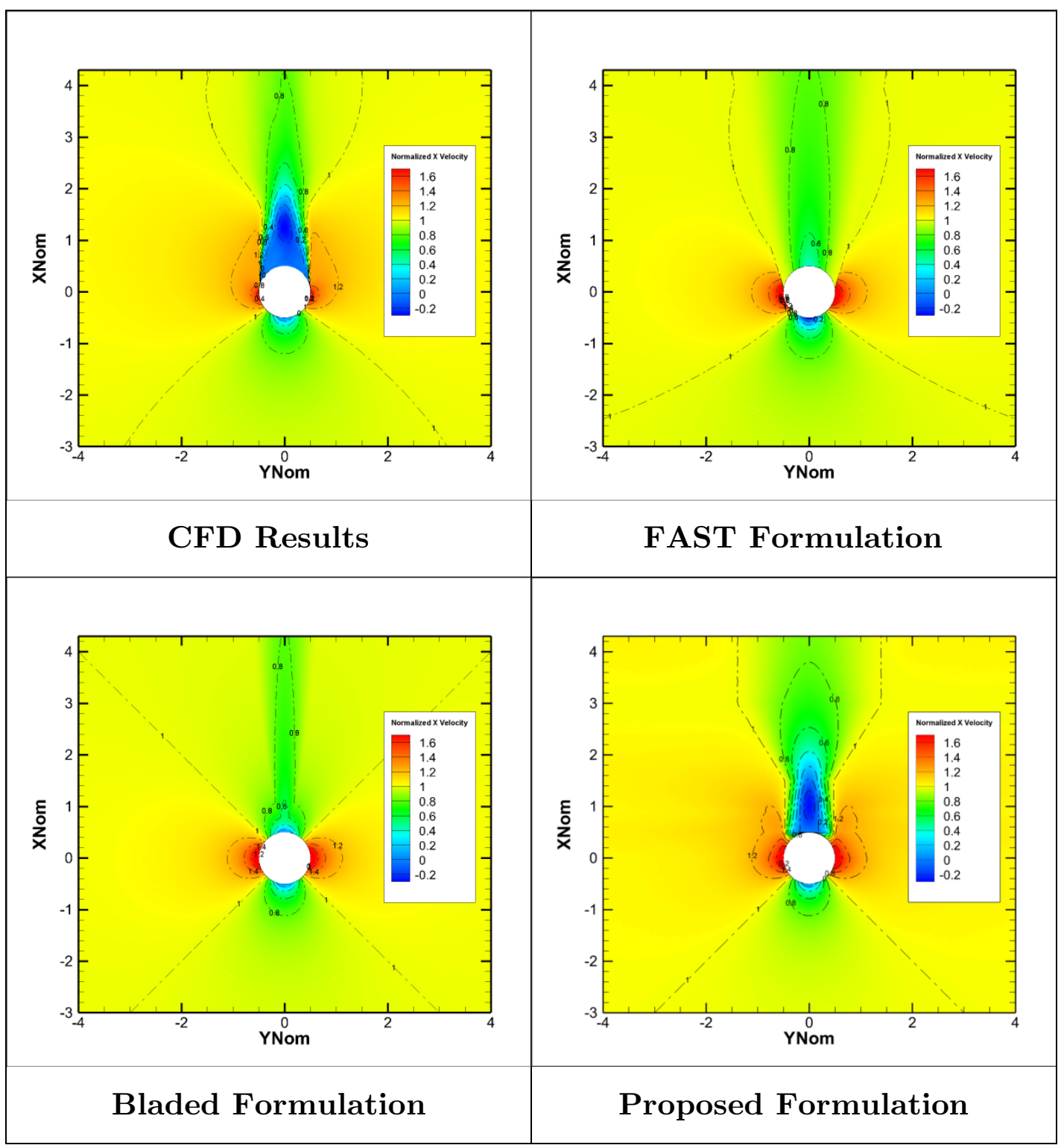


D.10. Tower Case 5 - Normalized Y Velocity

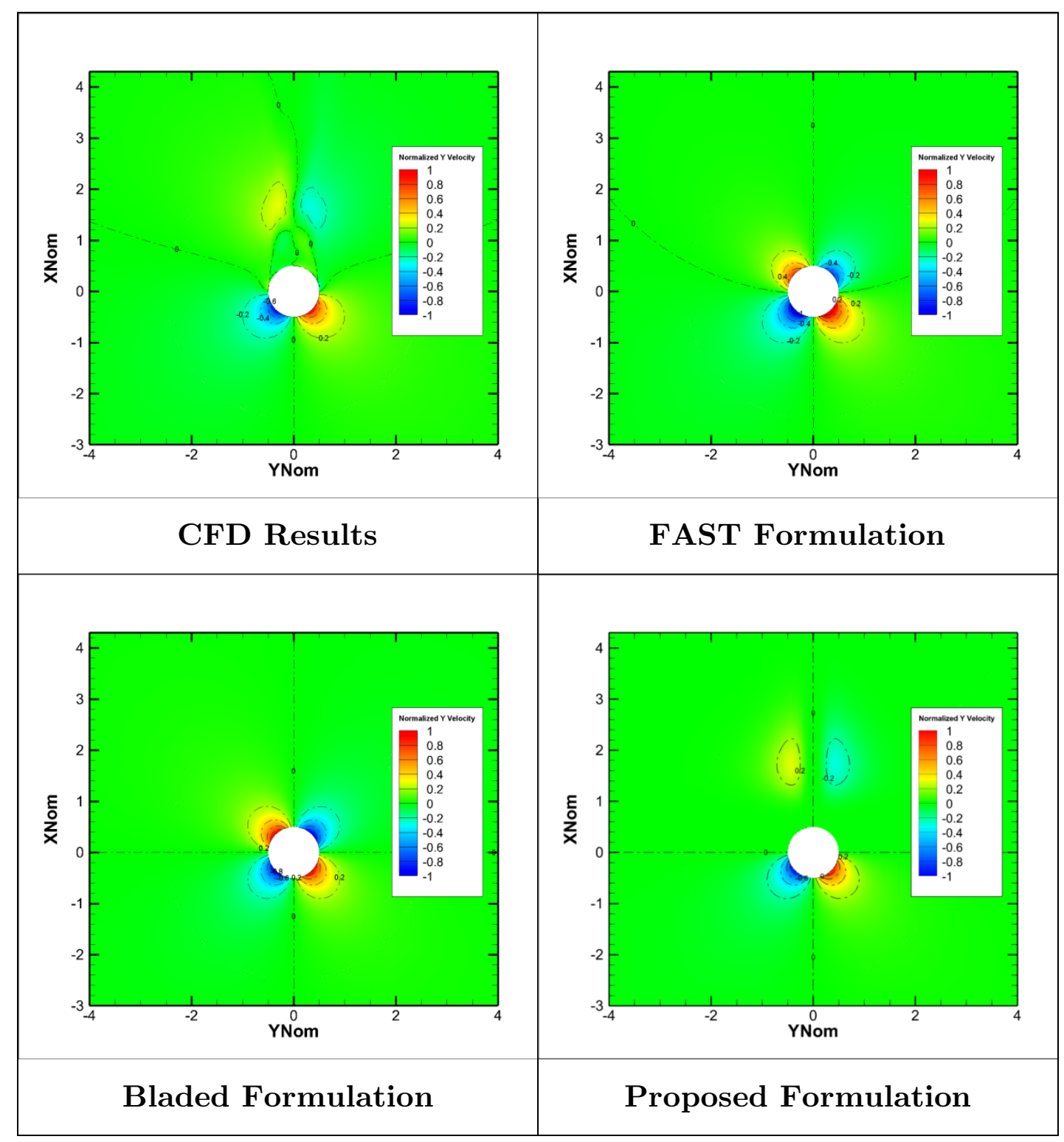


D.11. Tower Case 6 - Normalized X Velocity

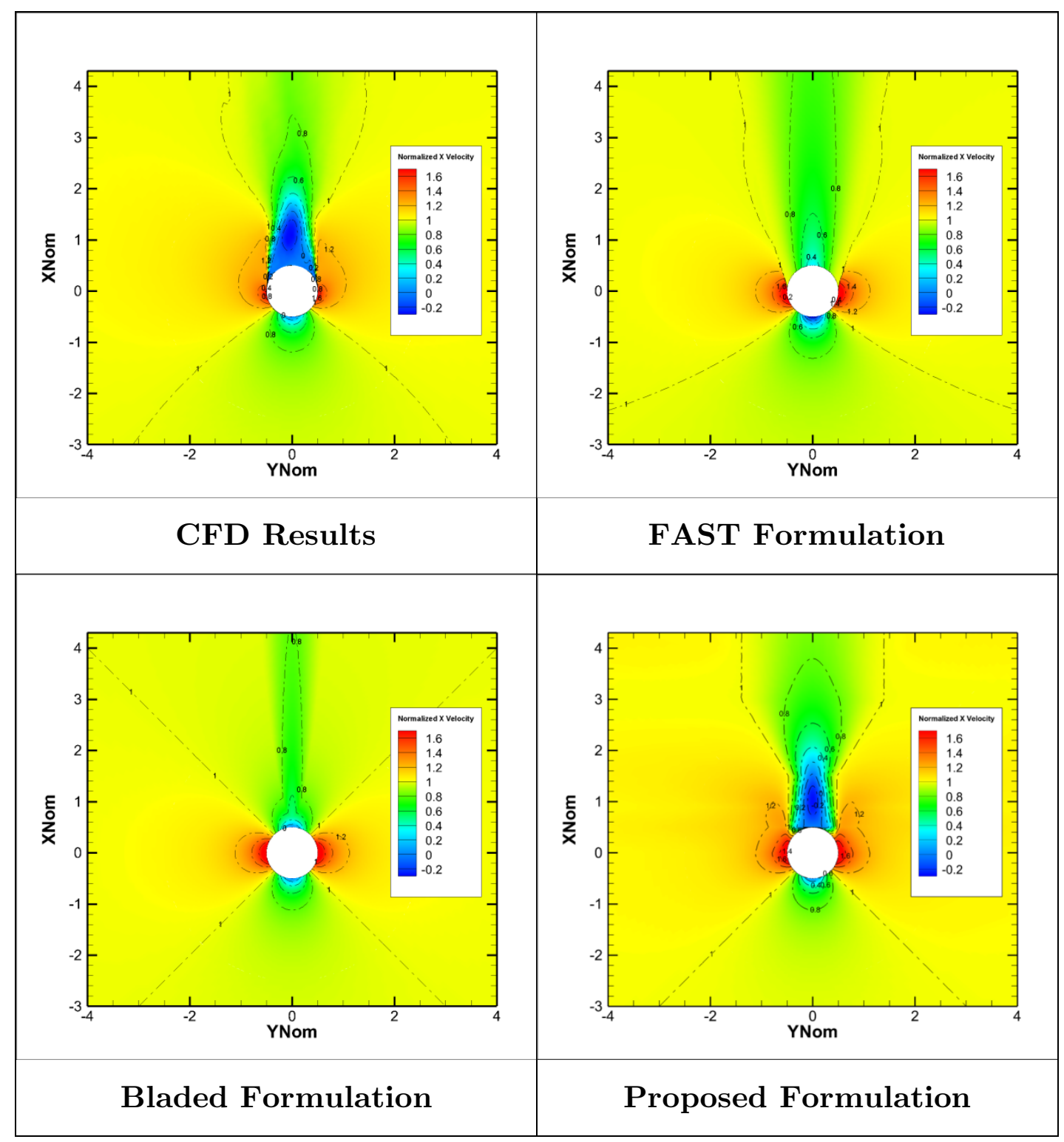


D.12. Tower Case 6 - Normalized Y Velocity

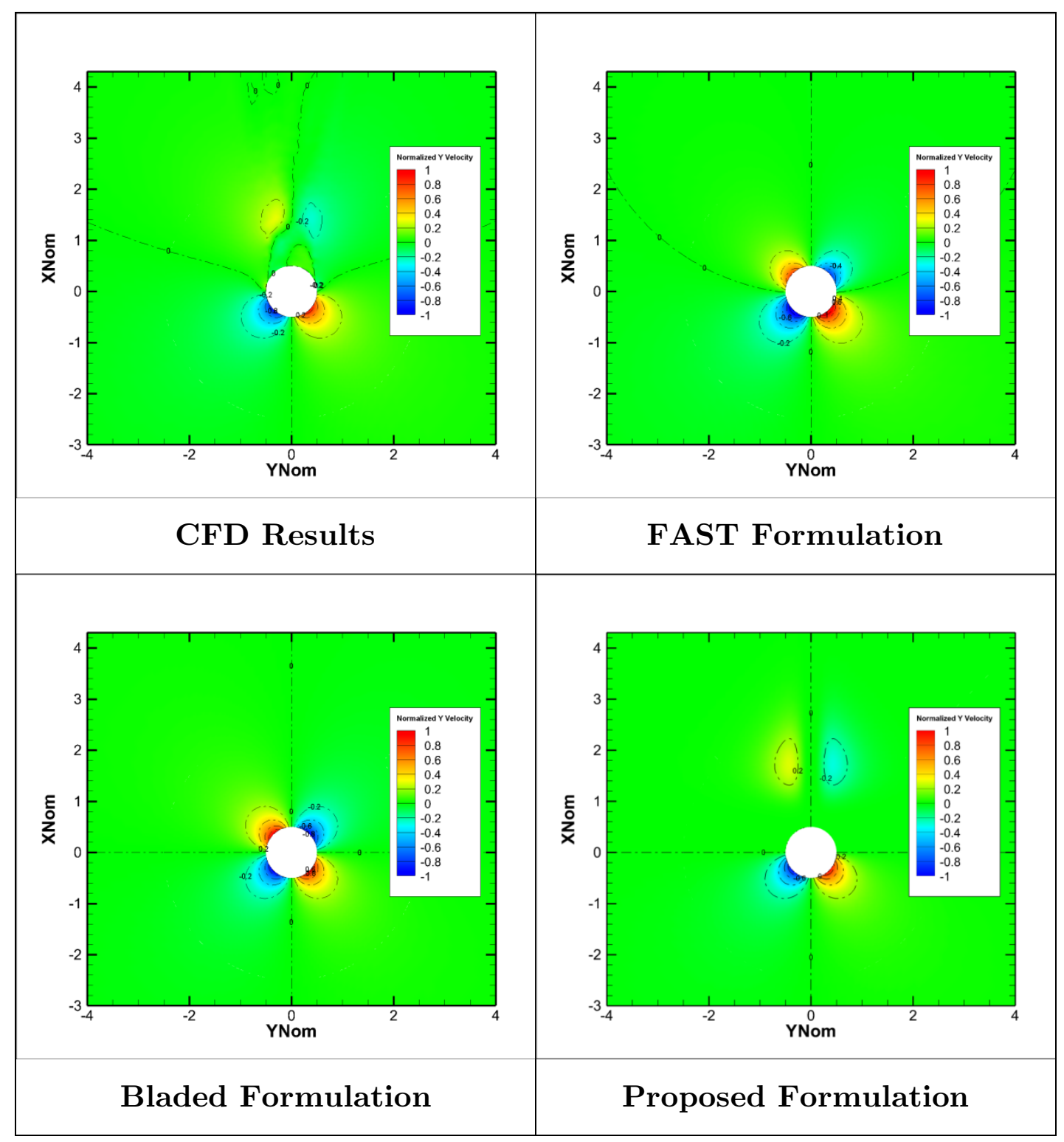




\section{D.13. Tower Case 7 - Normalized X Velocity}

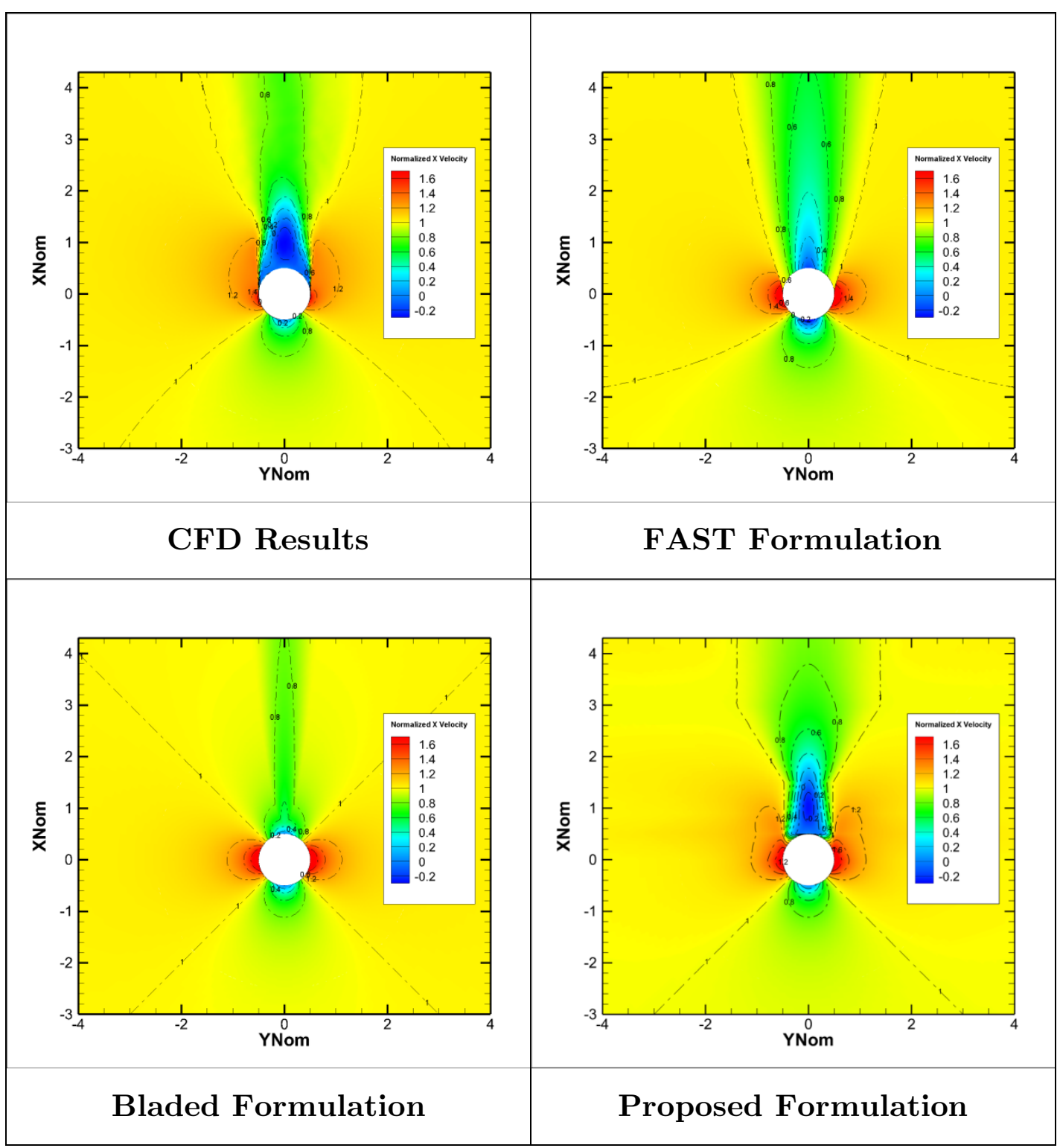




\section{D.14. Tower Case 7 - Normalized Y Velocity}

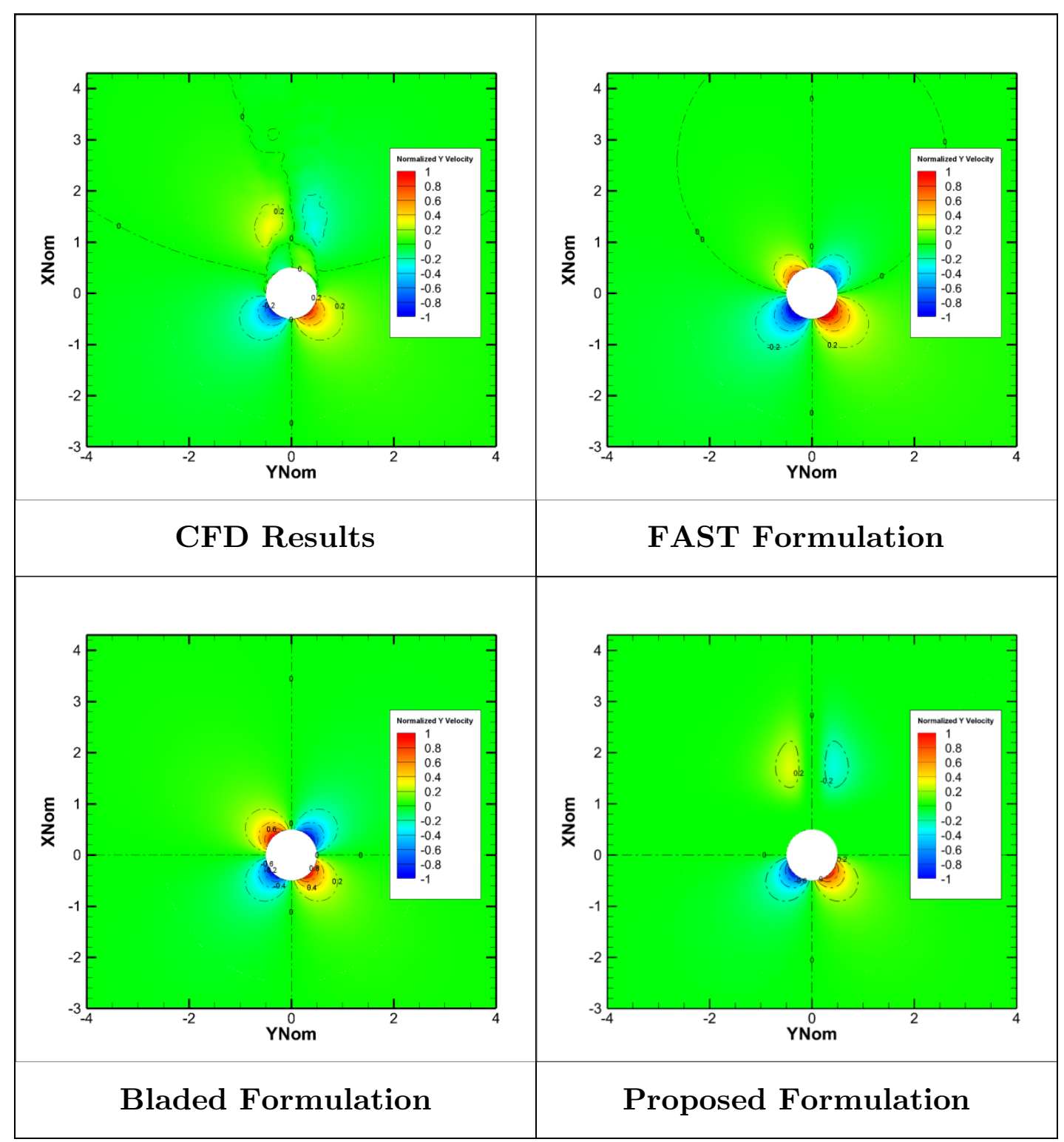


D.15. Tower Case 8 - Normalized X Velocity

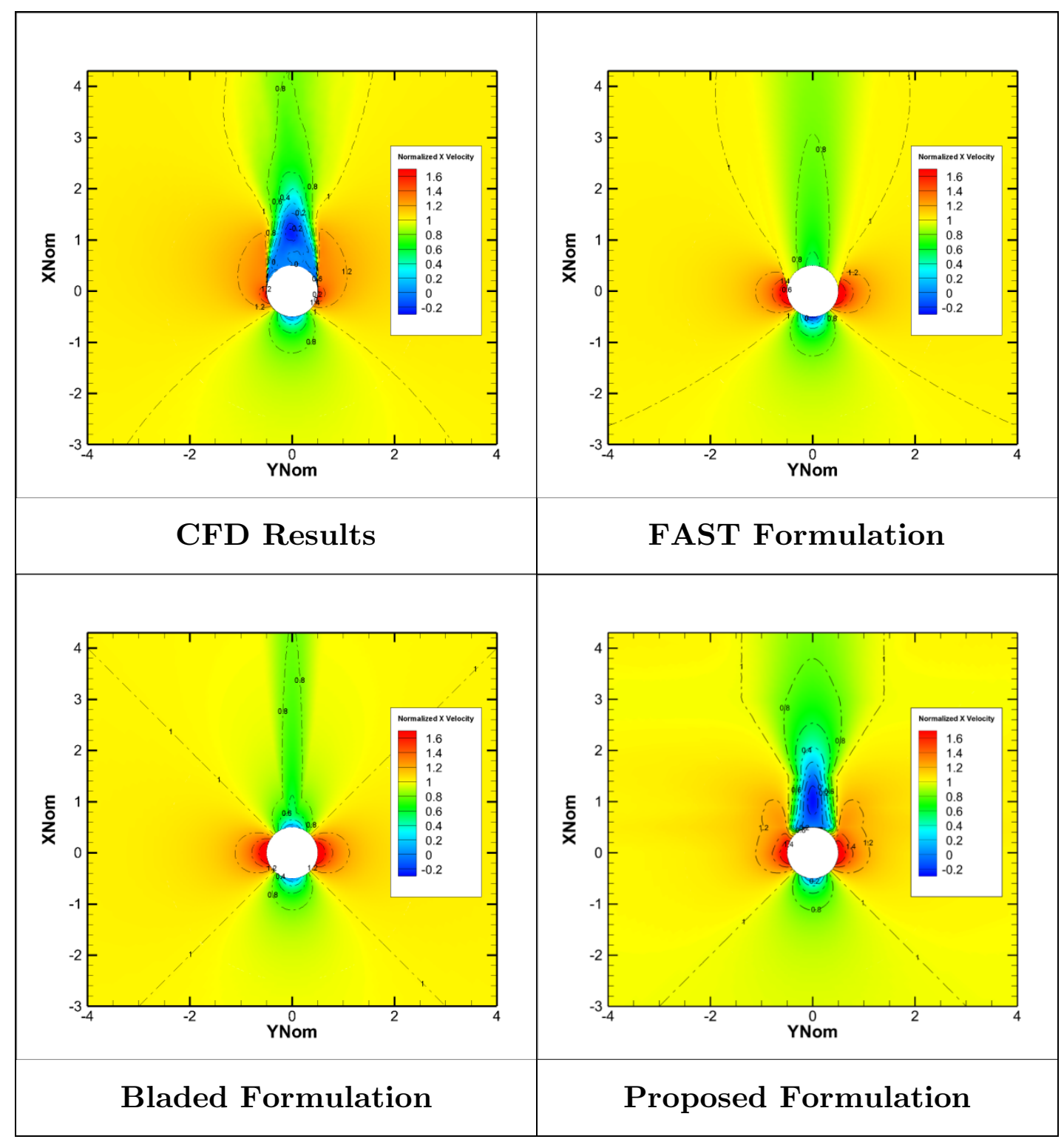


D.16. Tower Case 8 - Normalized Y Velocity

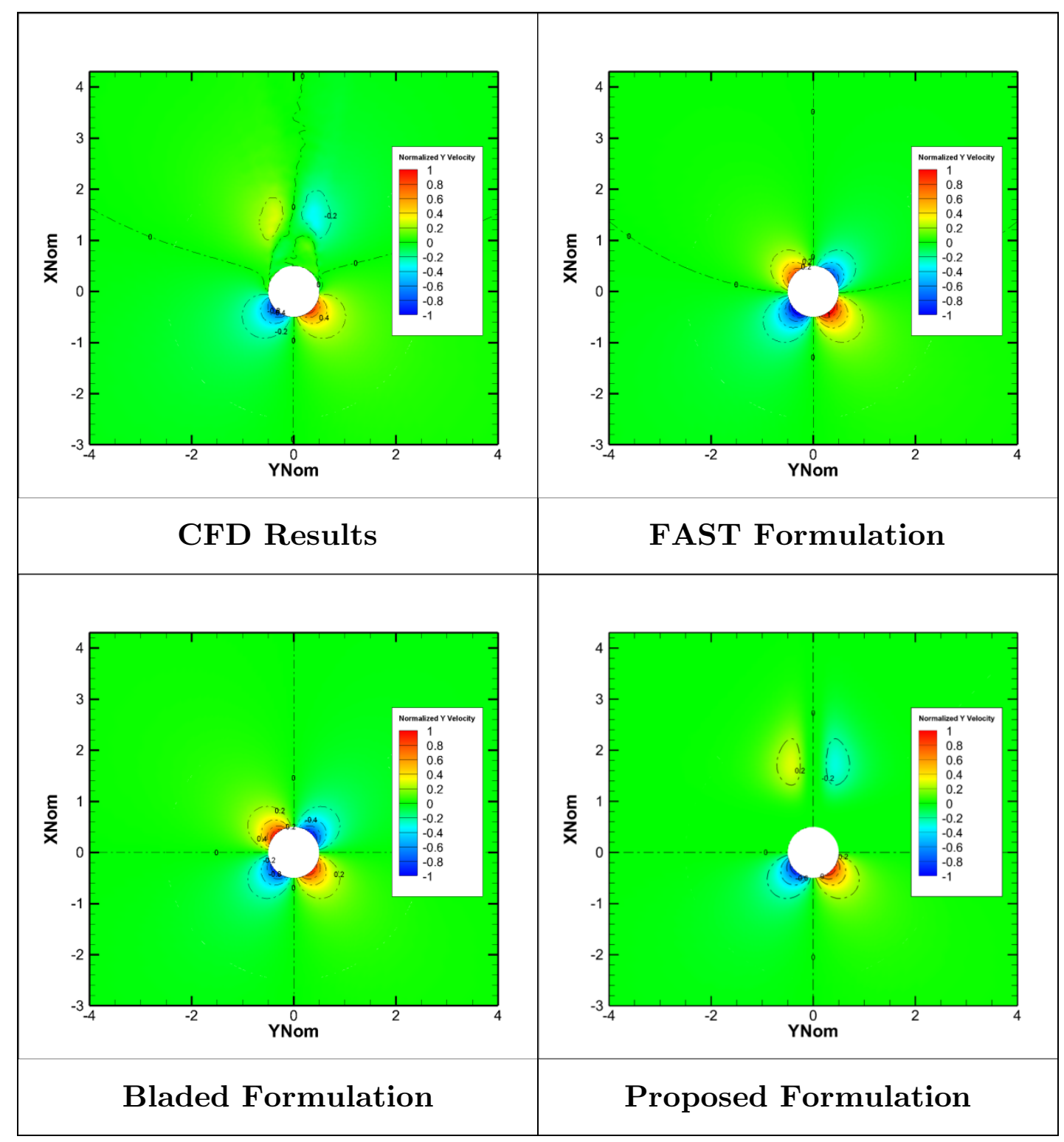


D.17. Tower Case 9 - Normalized X Velocity

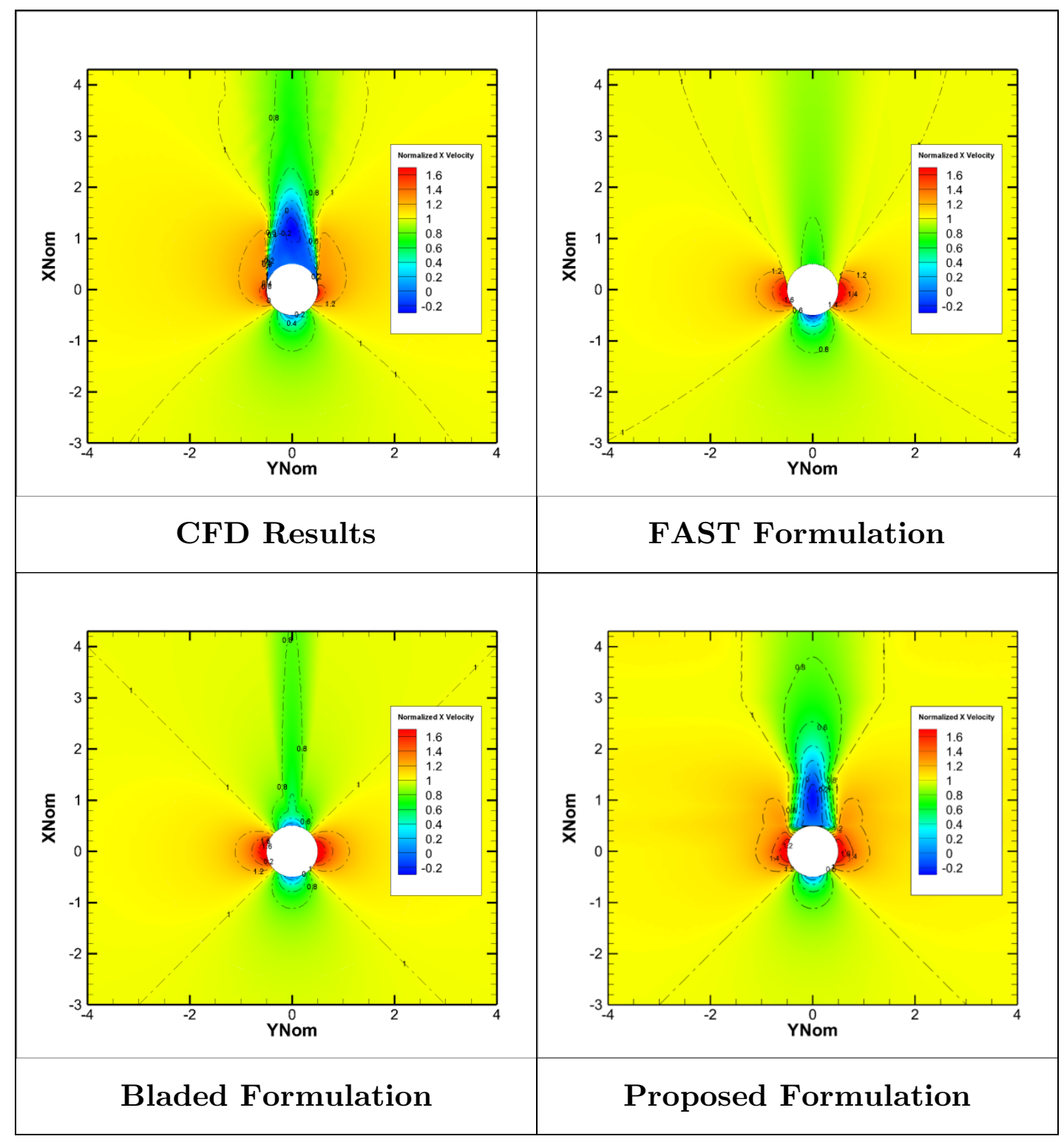


D.18. Tower Case 9 - Normalized Y Velocity

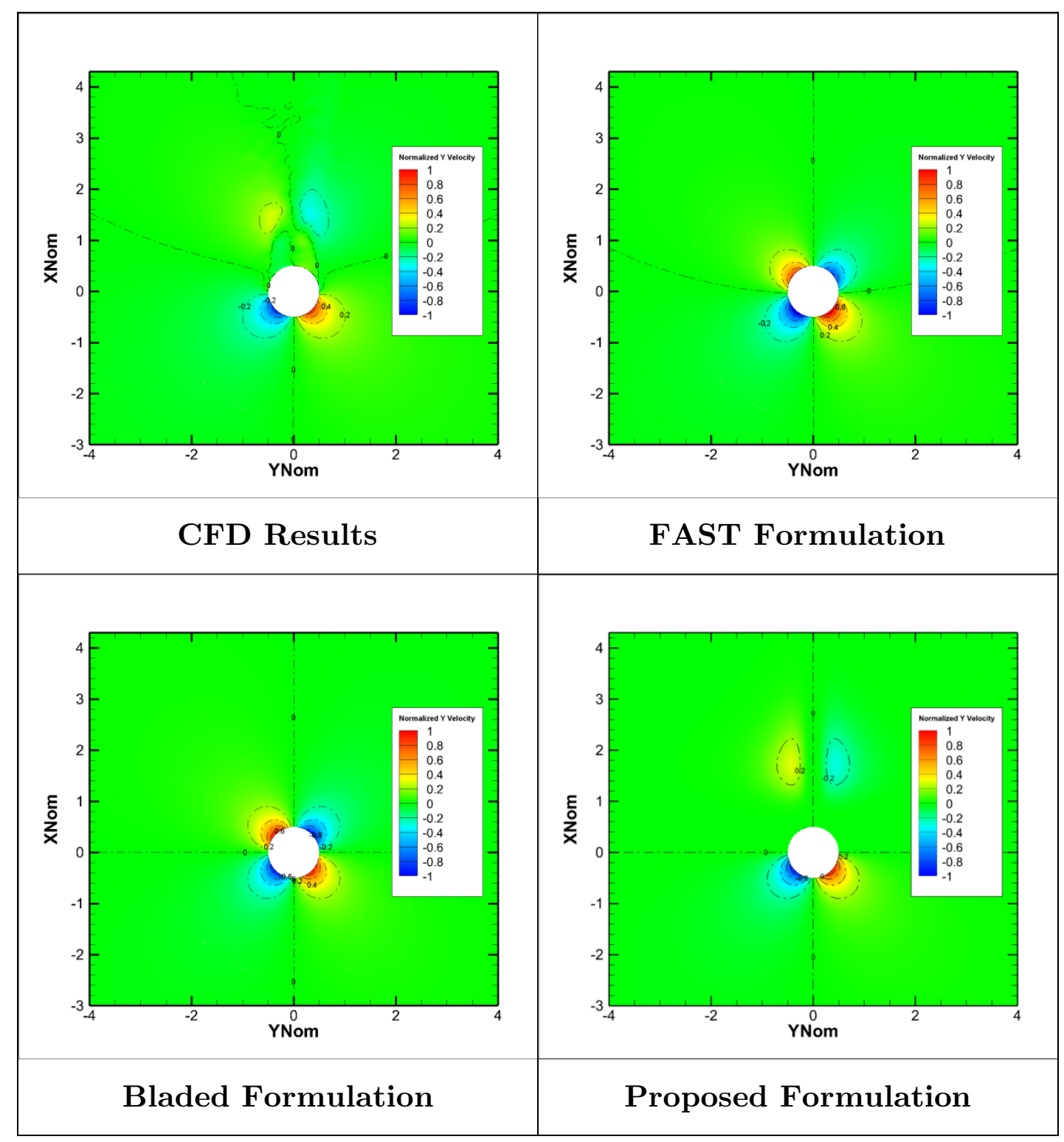




\section{APPENDIX E}

\section{E.1. FFT of $C_{l}$ for Tower Case 1 in Log and Linear Scale}
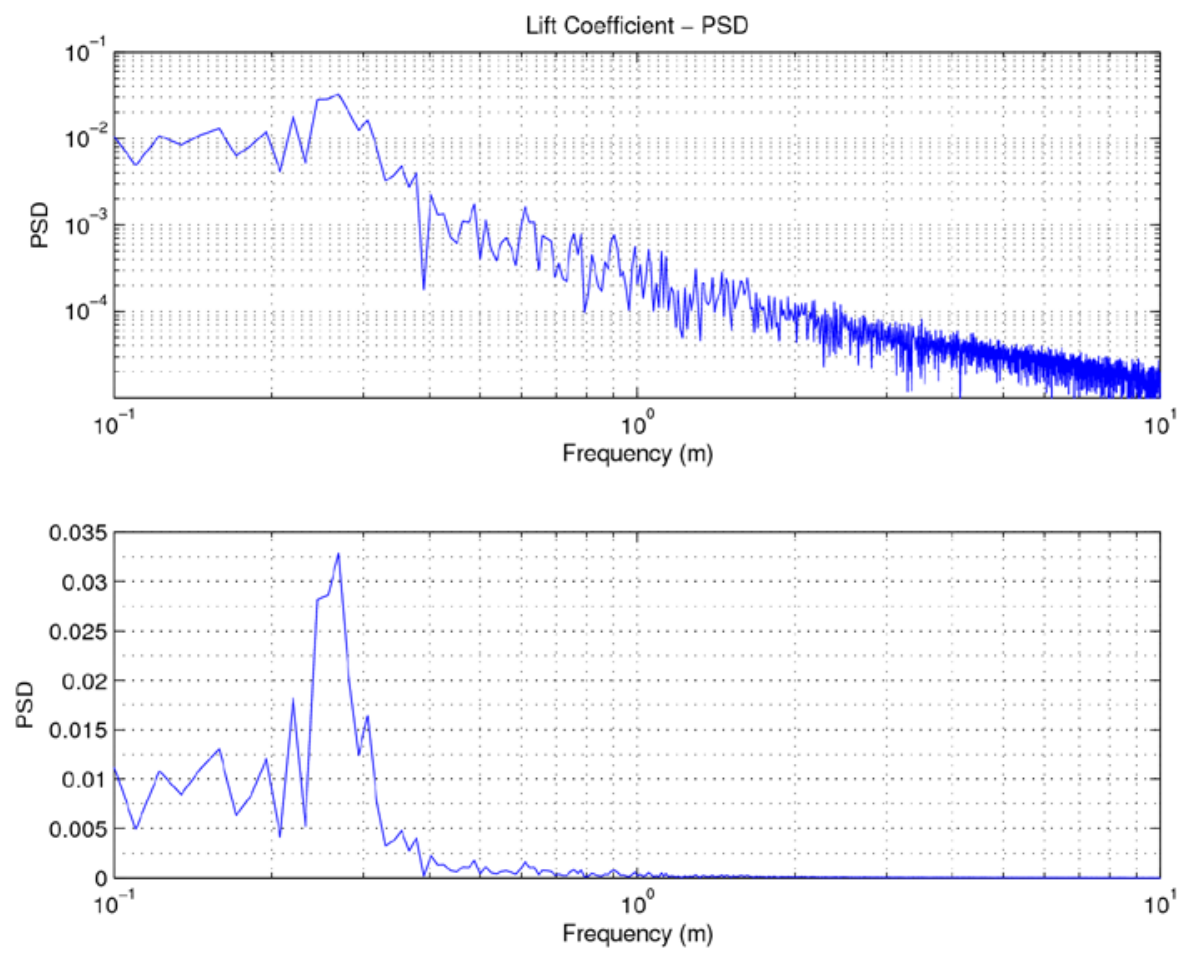

E.2. FFT of $C_{l}$ for Tower Case 2 in Log and Linear Scale
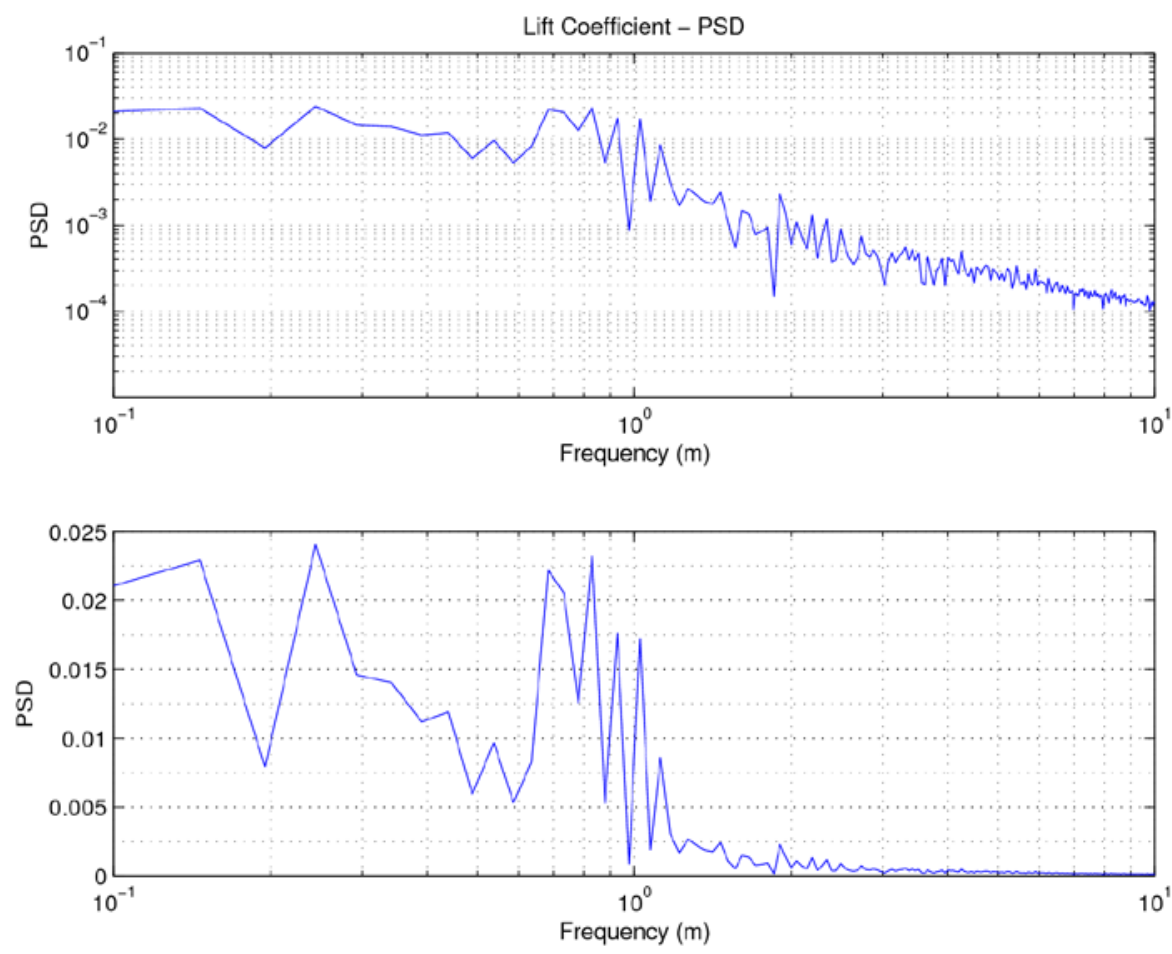


\section{E.3. FFT of $C_{l}$ for Tower Case 3 in Log and Linear Scale}
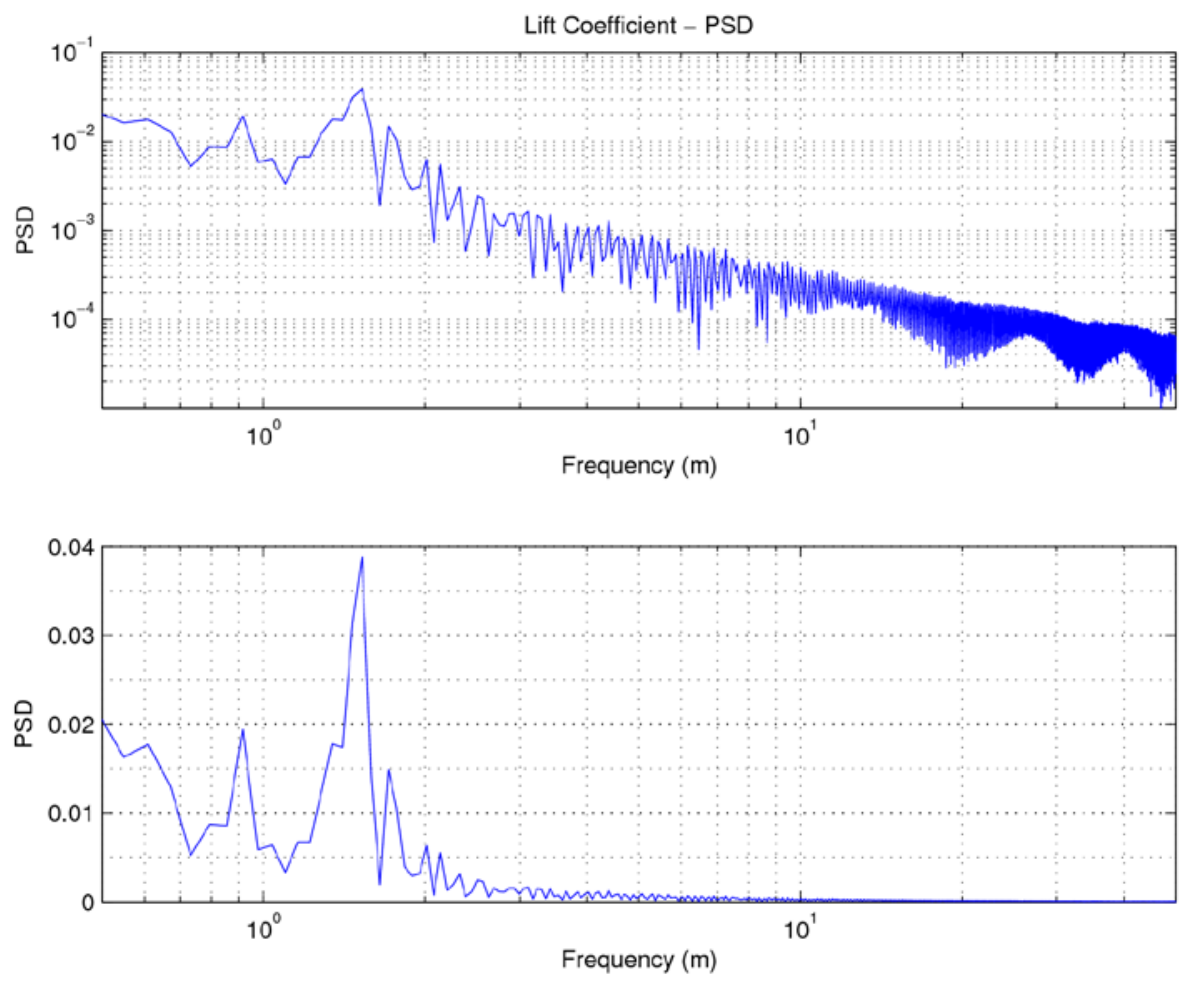

E.4. FFT of $C_{l}$ for Tower Case 4 in Log and Linear Scale
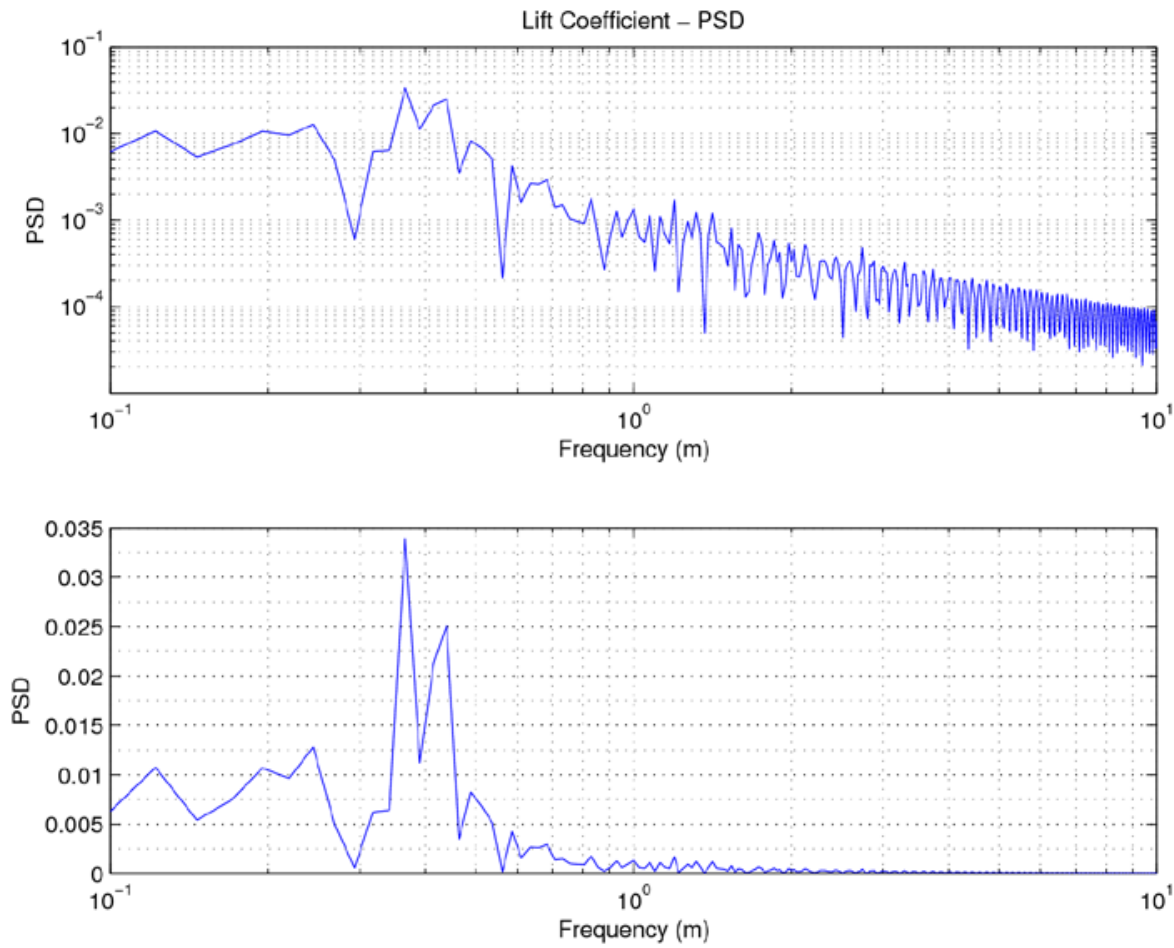


\section{E.5. FFT of $C_{l}$ for Tower Case 5 in $\log$ and Linear Scale}
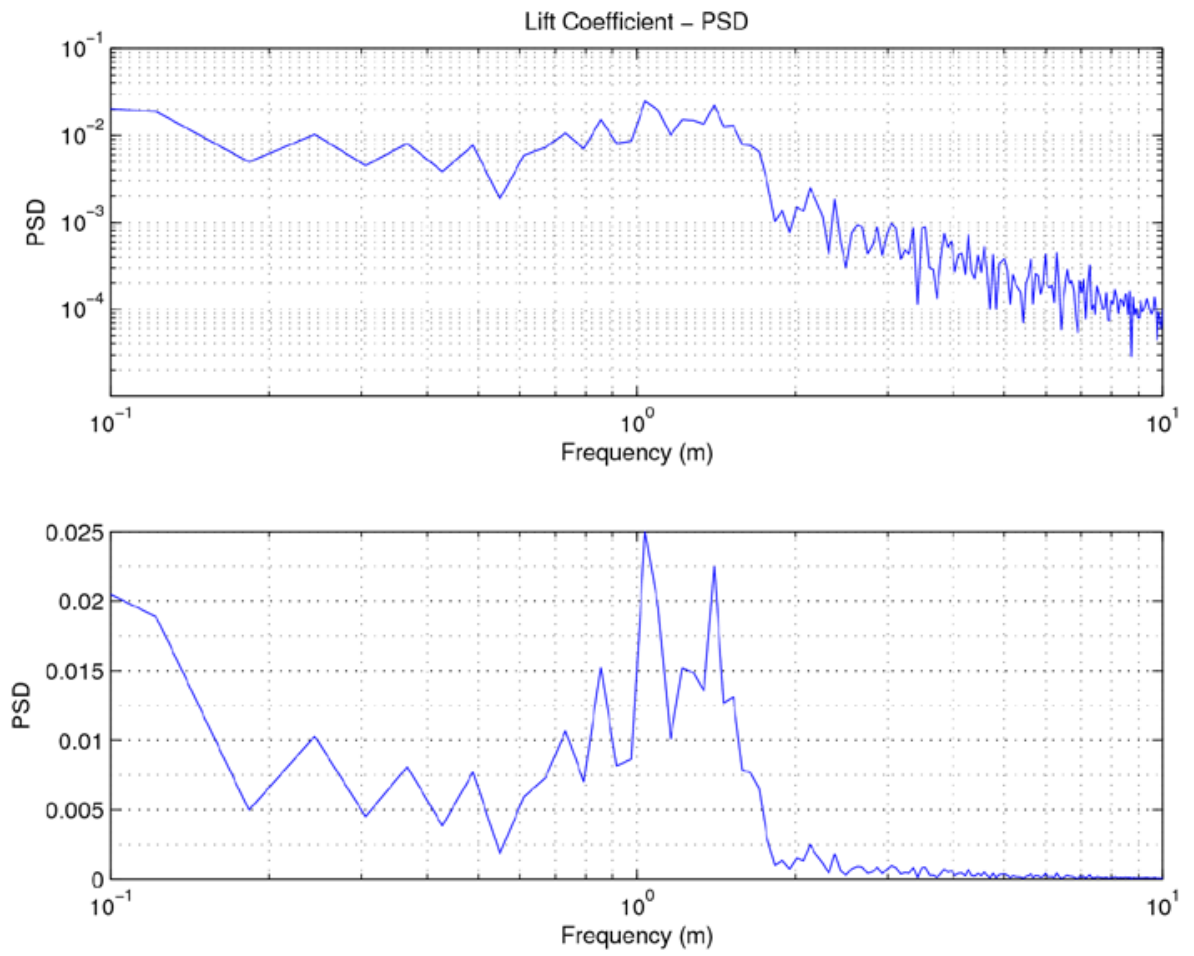

E.6. FFT of $C_{l}$ for Tower Case 6 in Log and Linear Scale
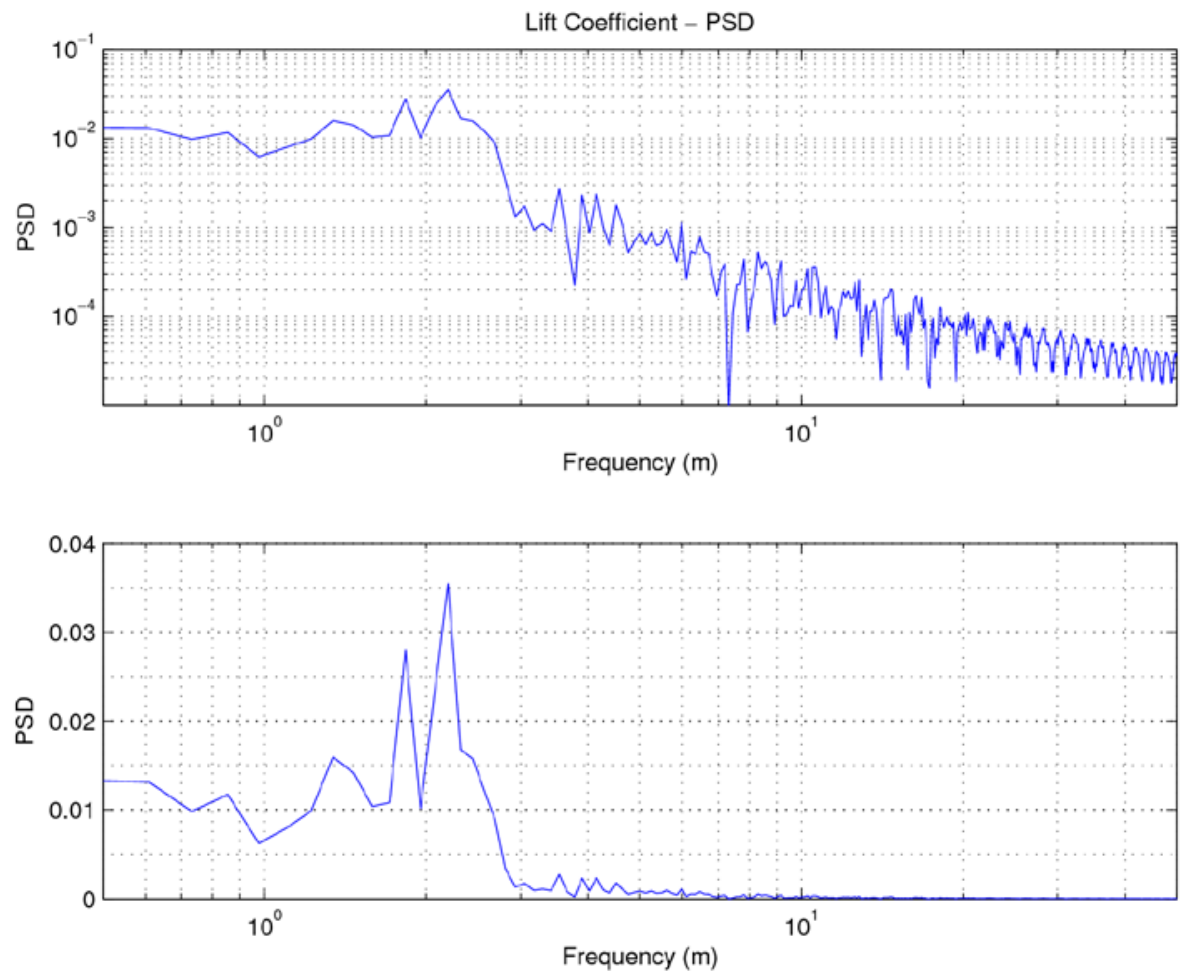


\section{E.7. FFT of $C_{l}$ for Tower Case 7 in Log and Linear Scale}
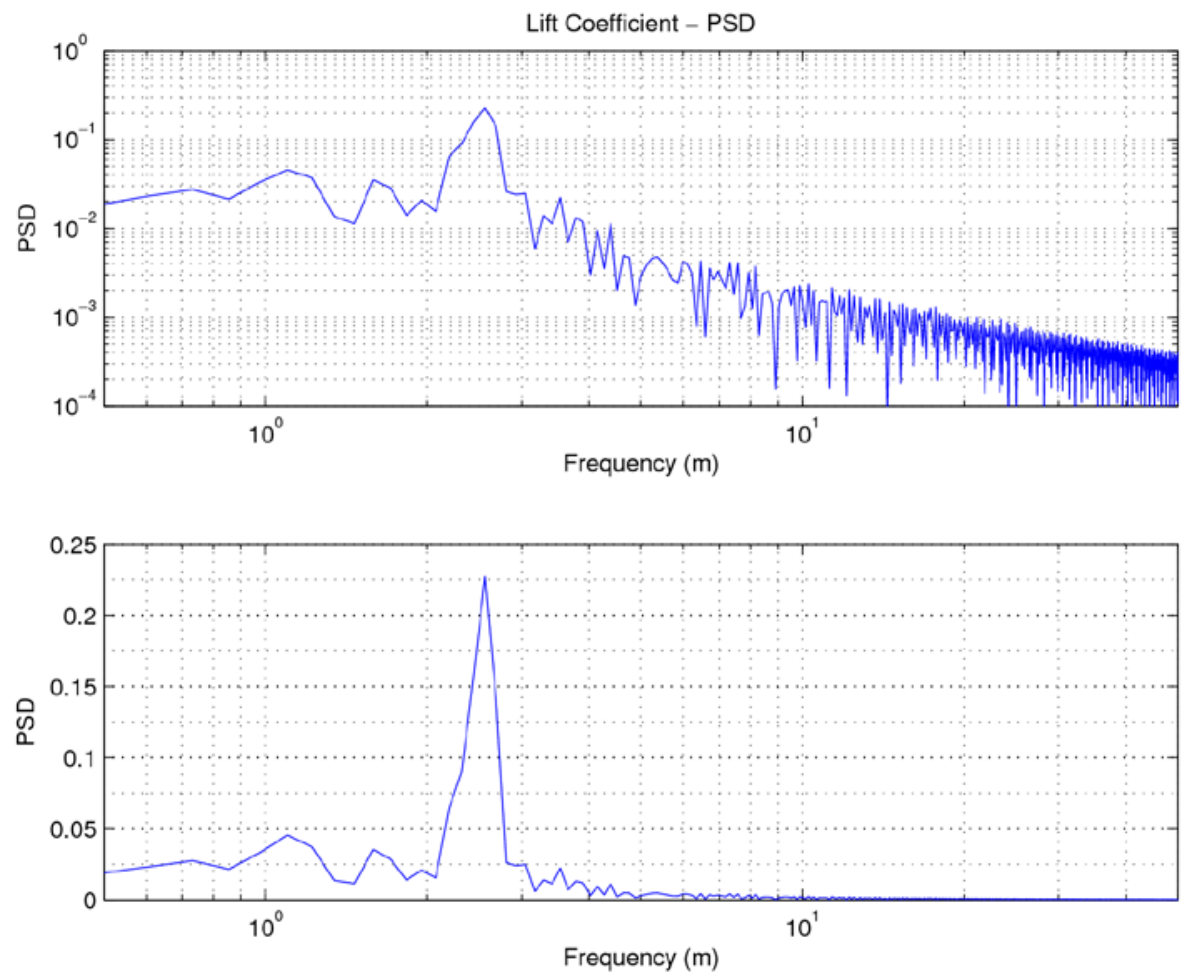

\section{E.8. FFT of $C_{l}$ for Tower Case 8 in Log and Linear Scale}
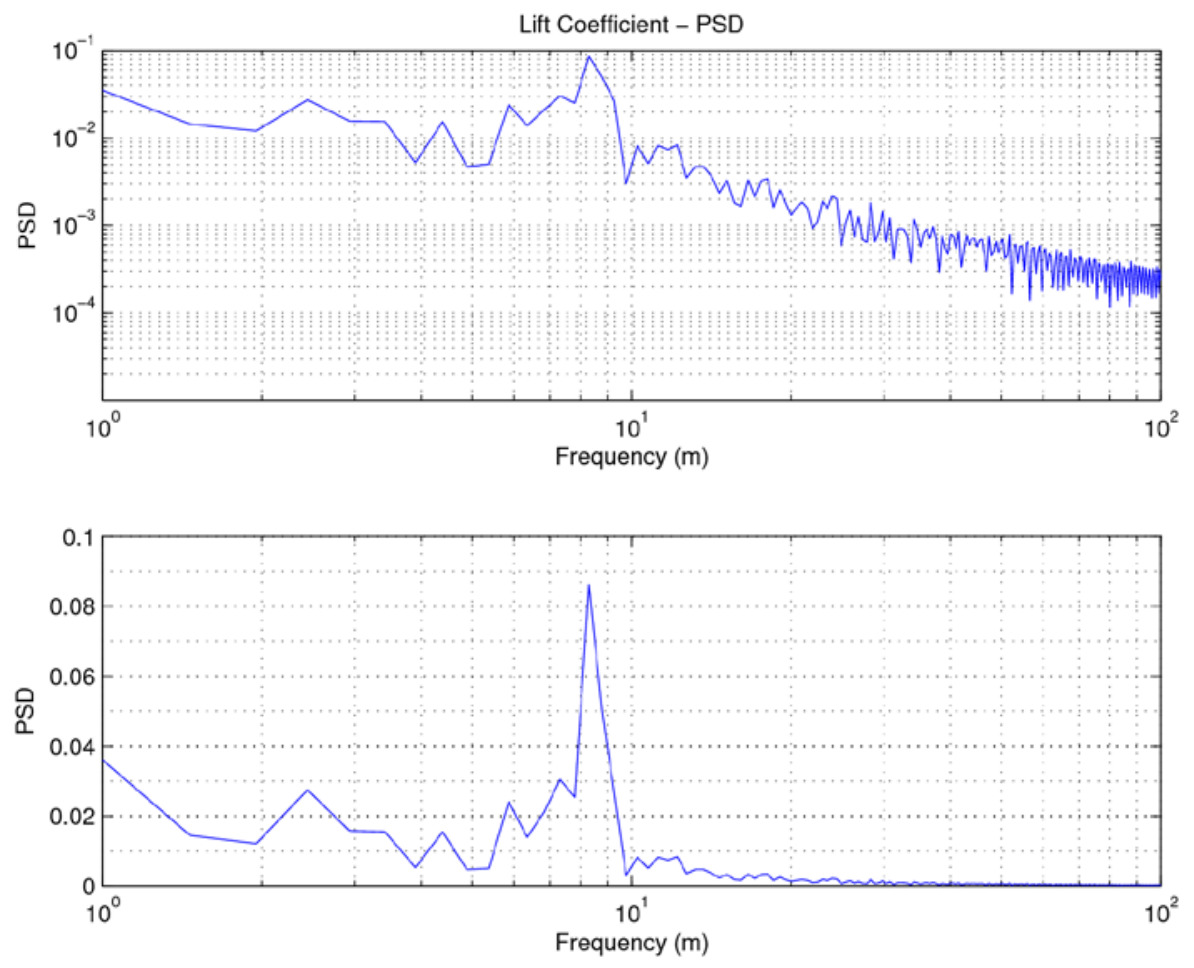
E.9. FFT of $C_{l}$ for Tower Case 9 in Log and Linear Scale
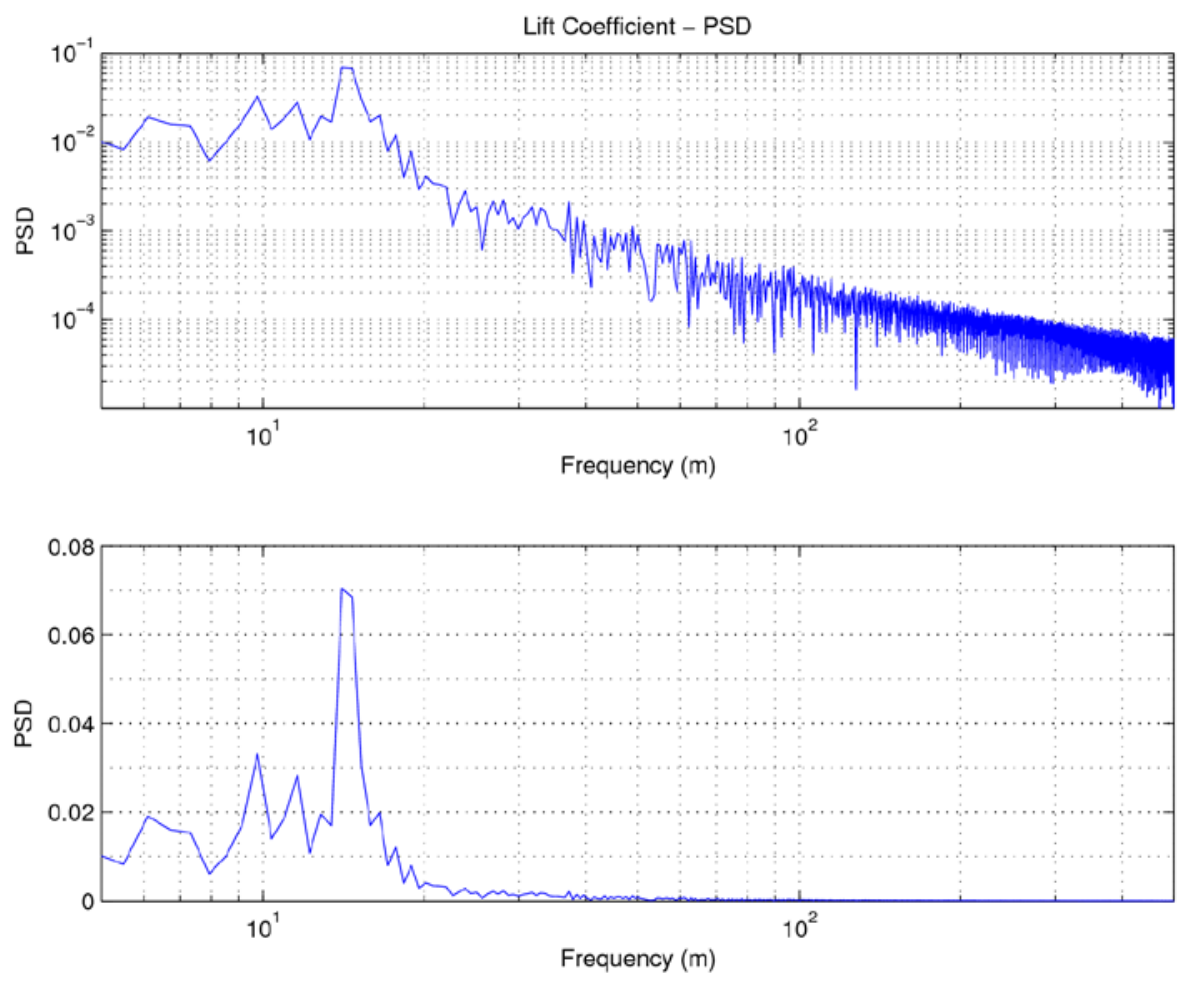


\section{APPENDIX F}

F.1. $\quad v_{x}$ vs $y$ Plot of Various $x$ Values for Tower Case 1

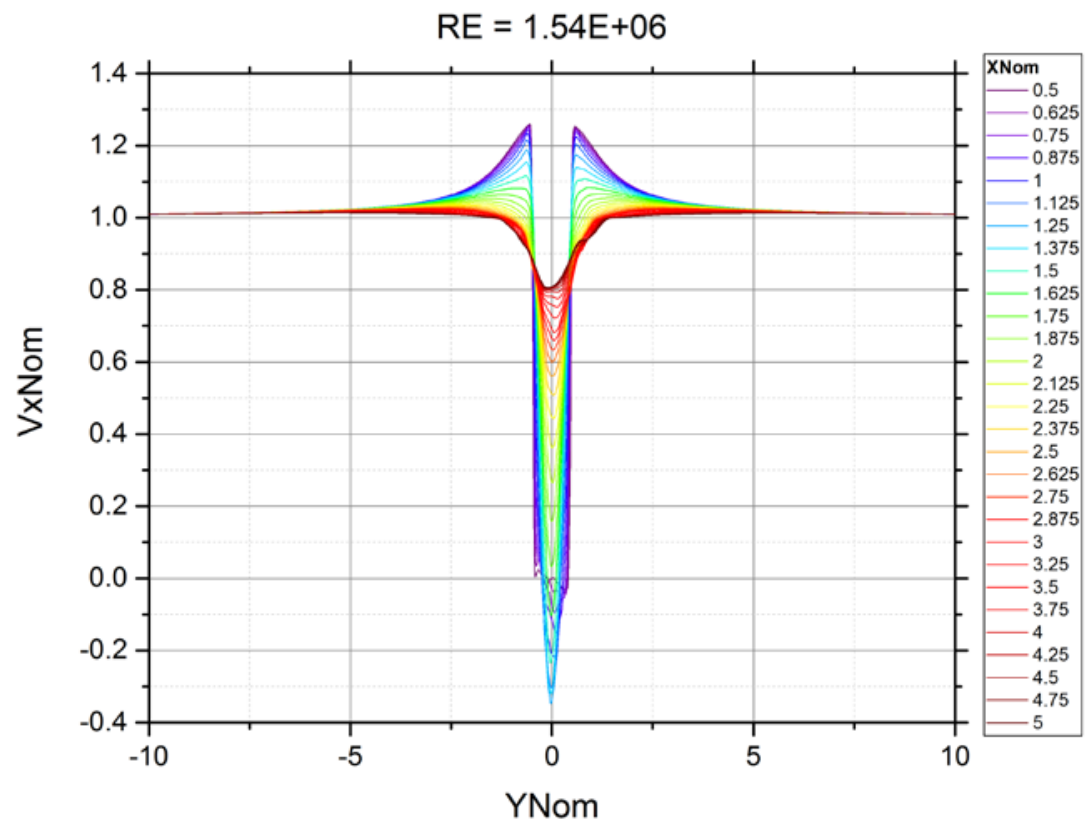

F.2. $\quad v_{x}$ vs $y$ Plot of Various $x$ Values for Tower Case 2

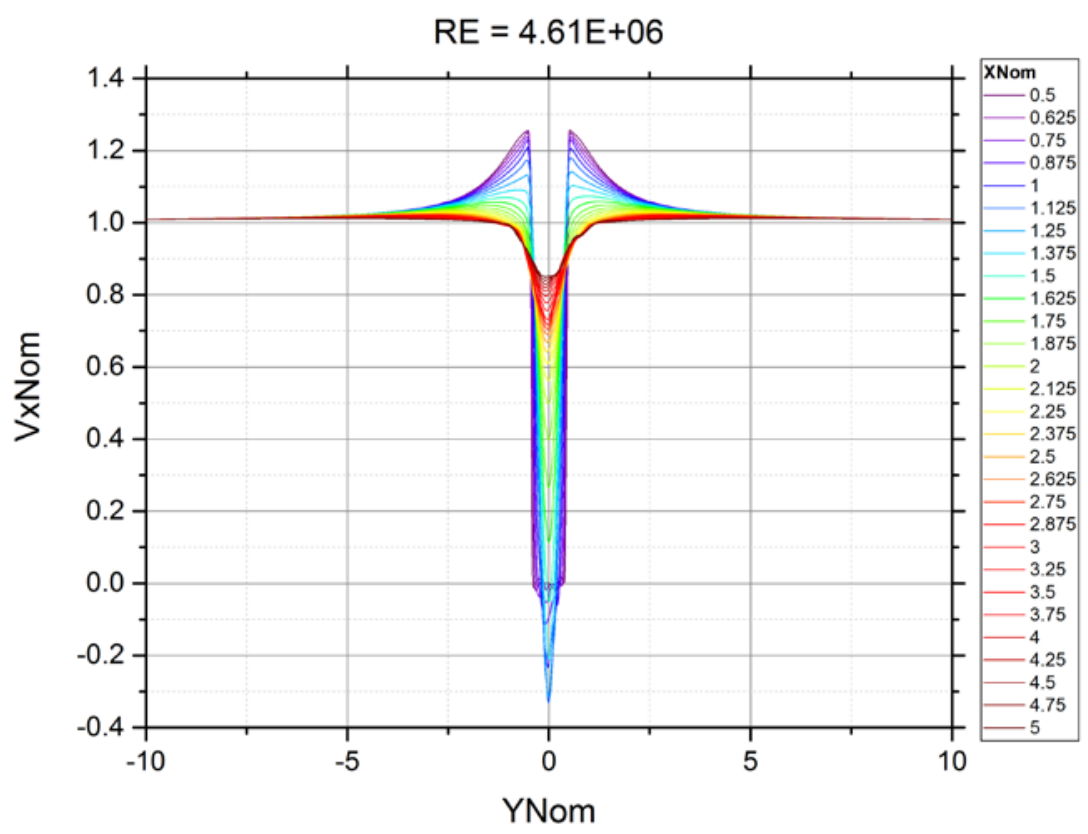


F.3. $\quad v_{x}$ vs $y$ Plot of Various $x$ Values for Tower Case 3

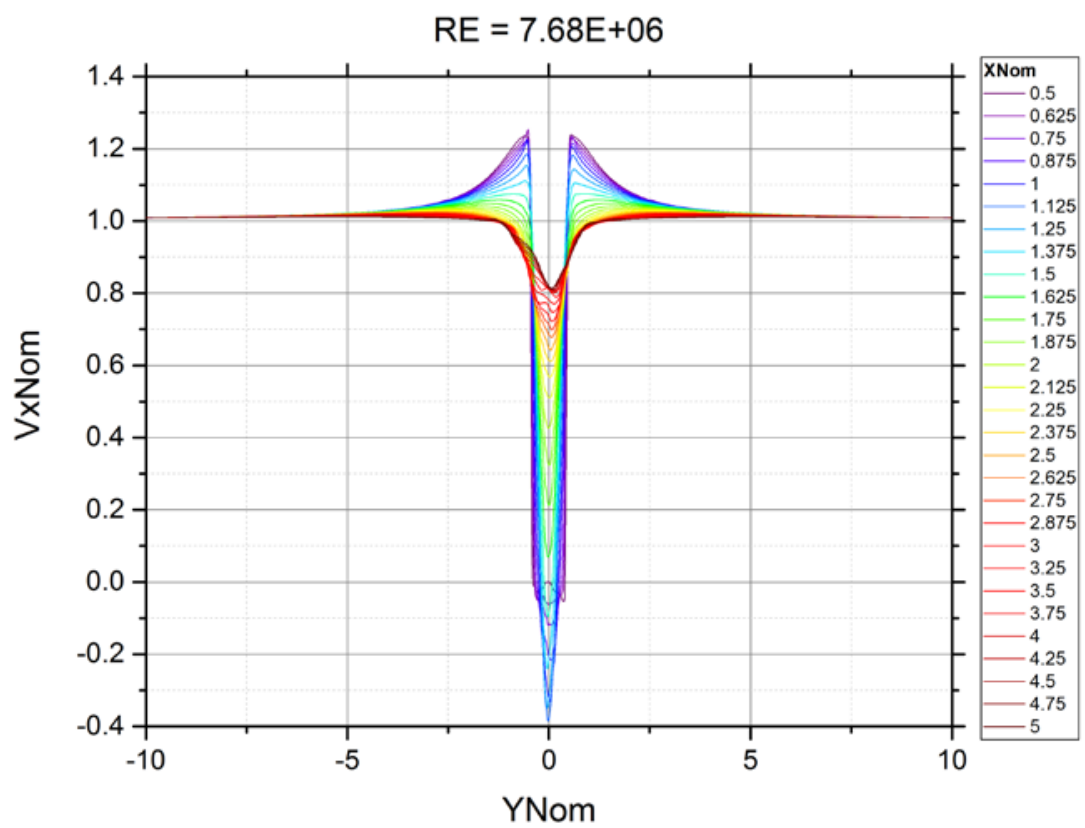

F.4. $v_{x}$ vs $y$ Plot of Various $x$ Values for Tower Case 4

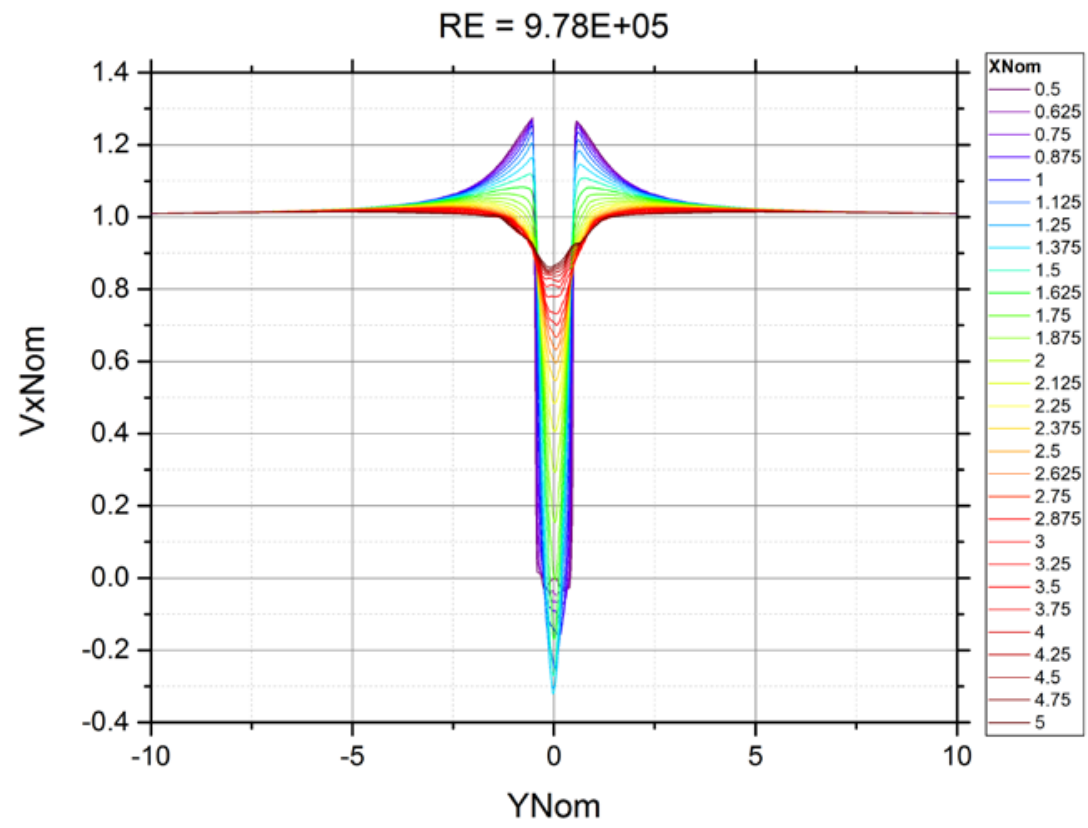


F.5. $\quad v_{x}$ vs $y$ Plot of Various $x$ Values for Tower Case 5

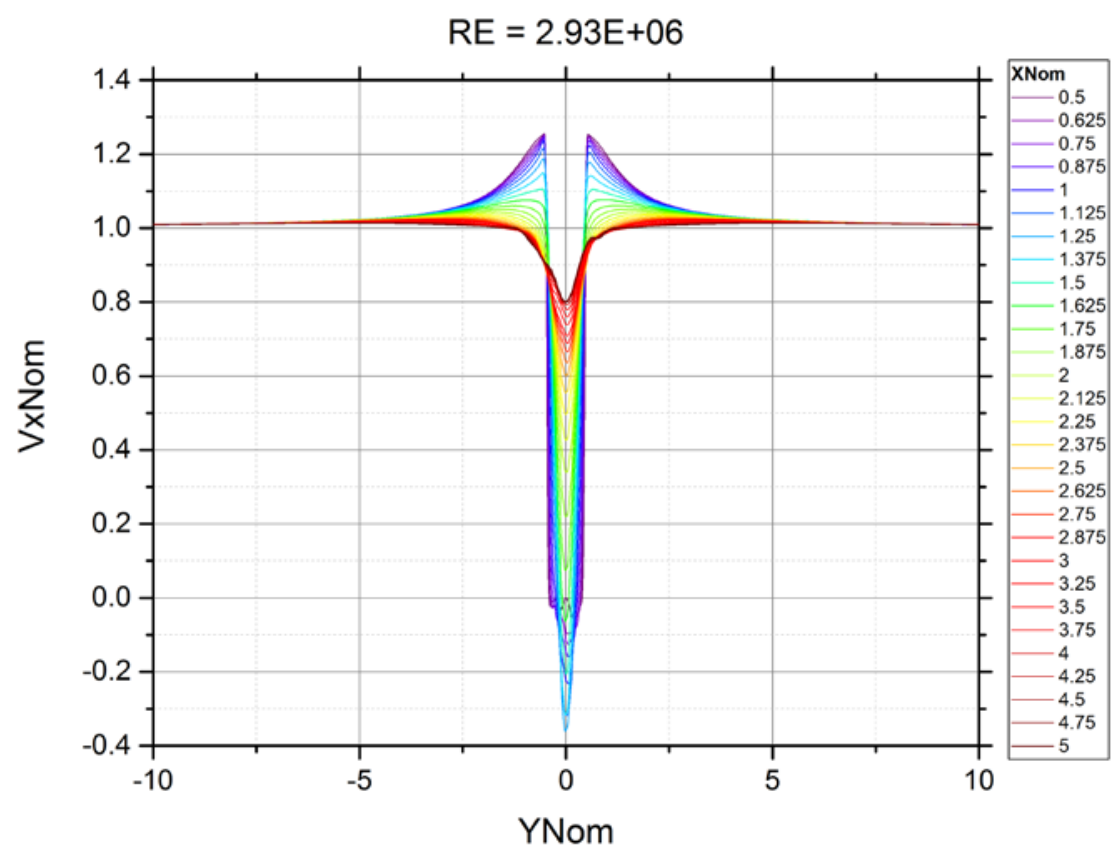

F.6. $\quad v_{x}$ vs $y$ Plot of Various $x$ Values for Tower Case 6

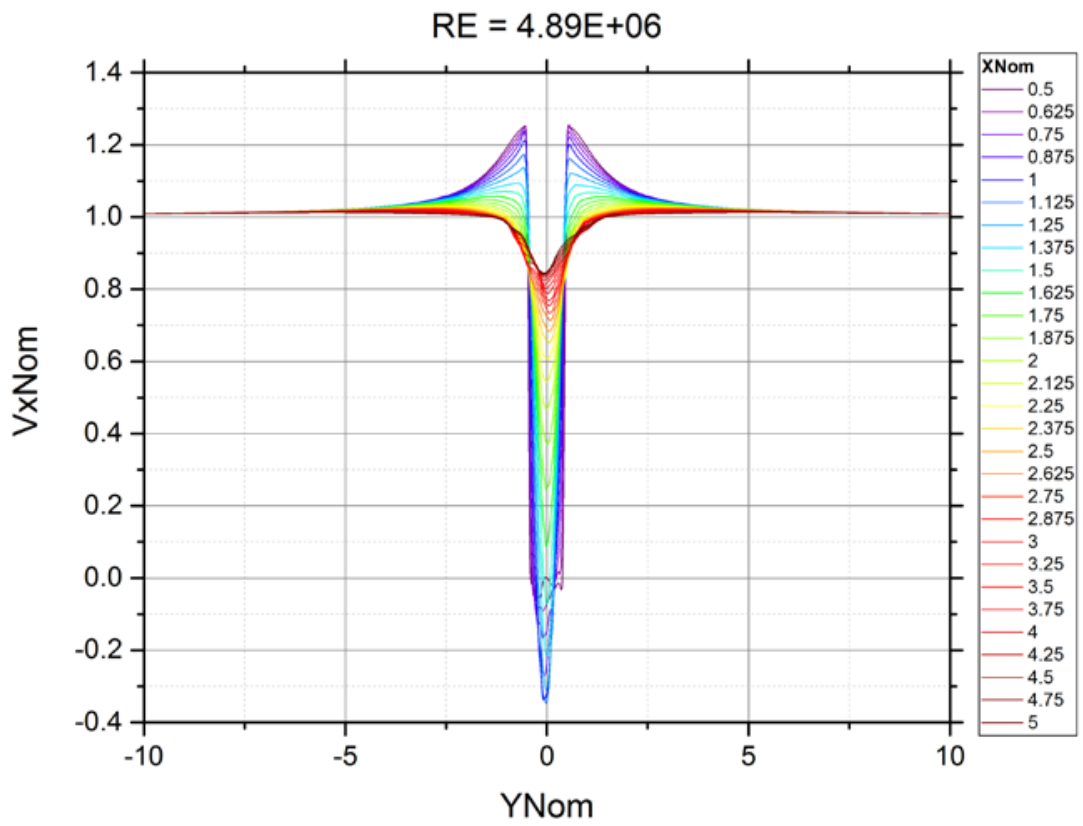


F.7. $v_{x}$ vs $y$ Plot of Various $x$ Values for Tower Case 7

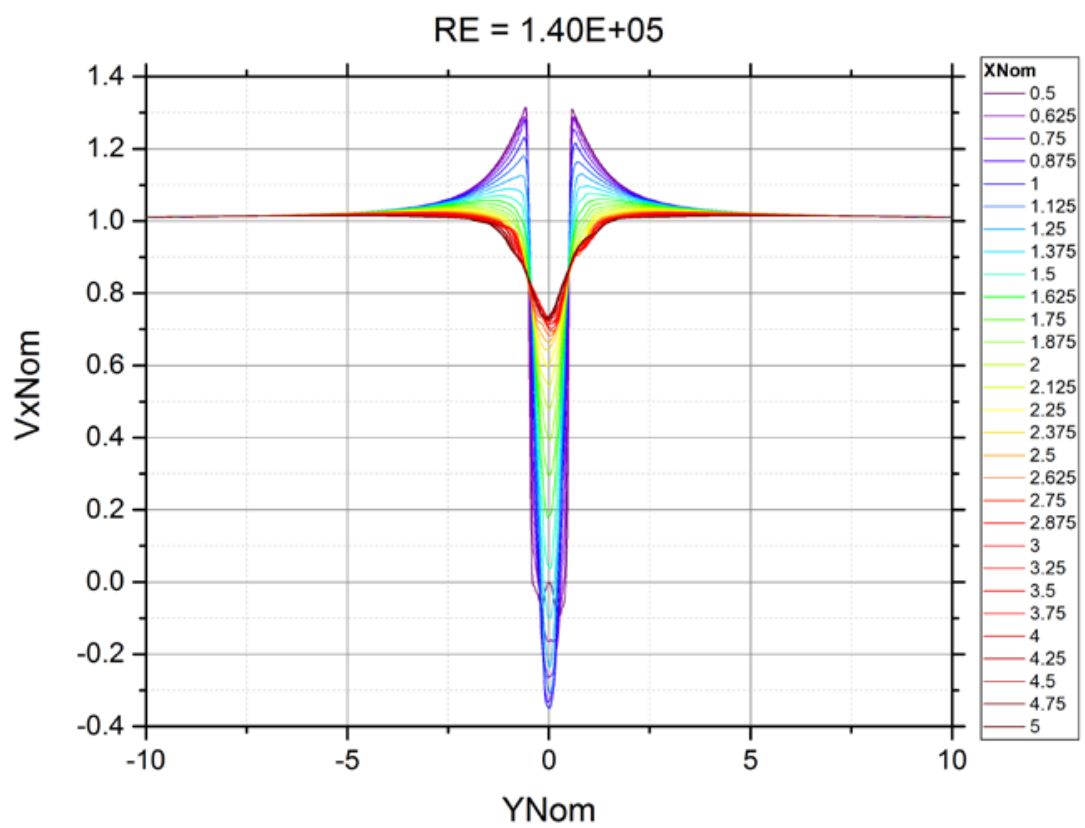

F.8. $v_{x}$ vs $y$ Plot of Various $x$ Values for Tower Case 8

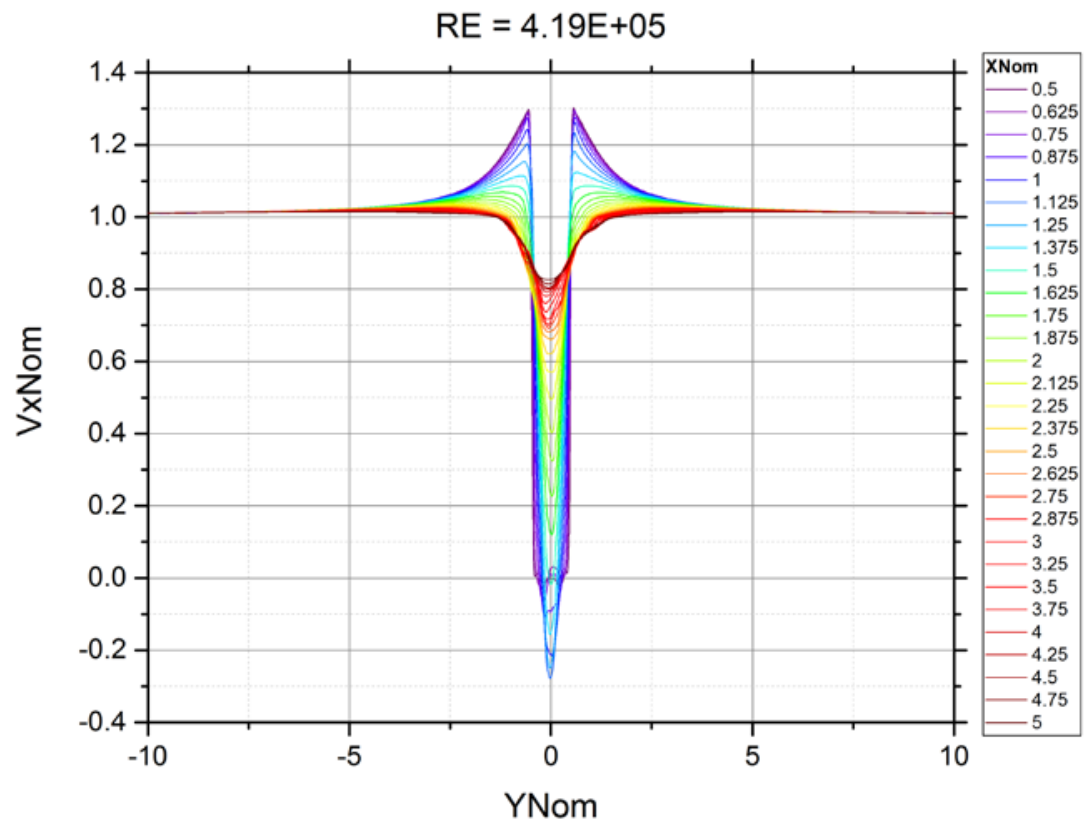


F.9. $v_{x}$ vs $y$ Plot of Various $x$ Values for Tower Case 9

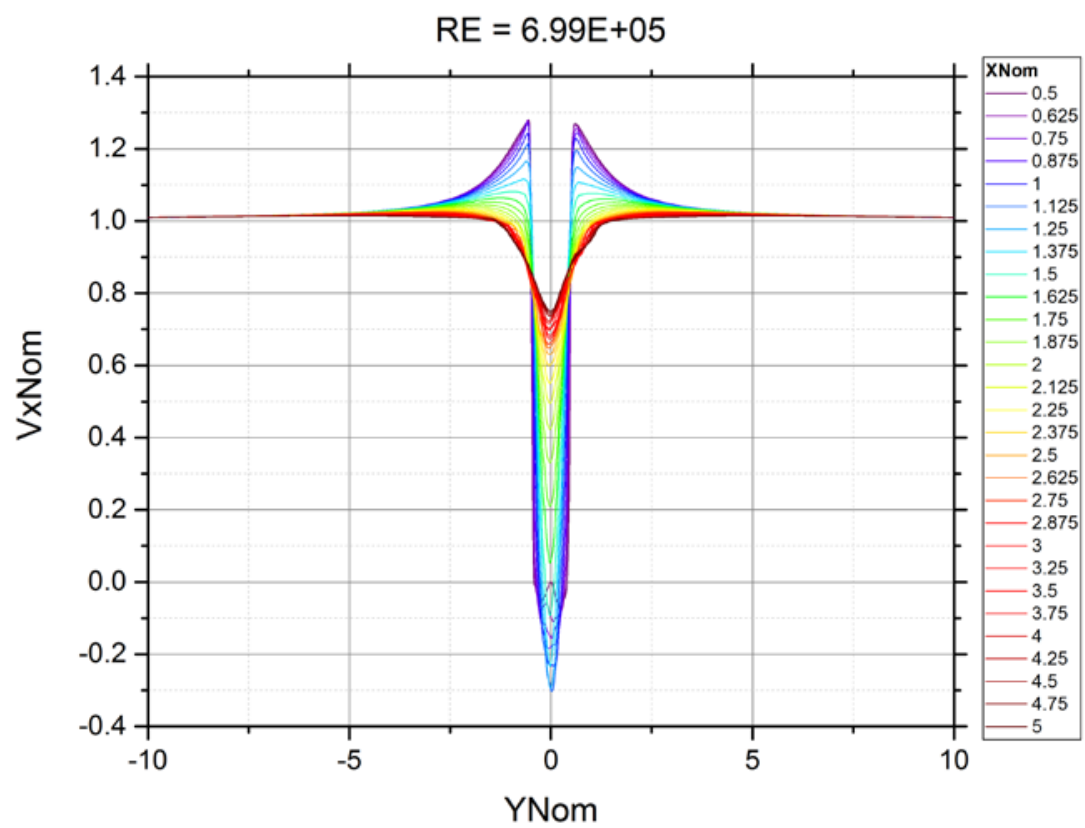




\section{APPENDIX G}

\section{G.1. Cuboid Nacelle Case 1 (Sharp)}

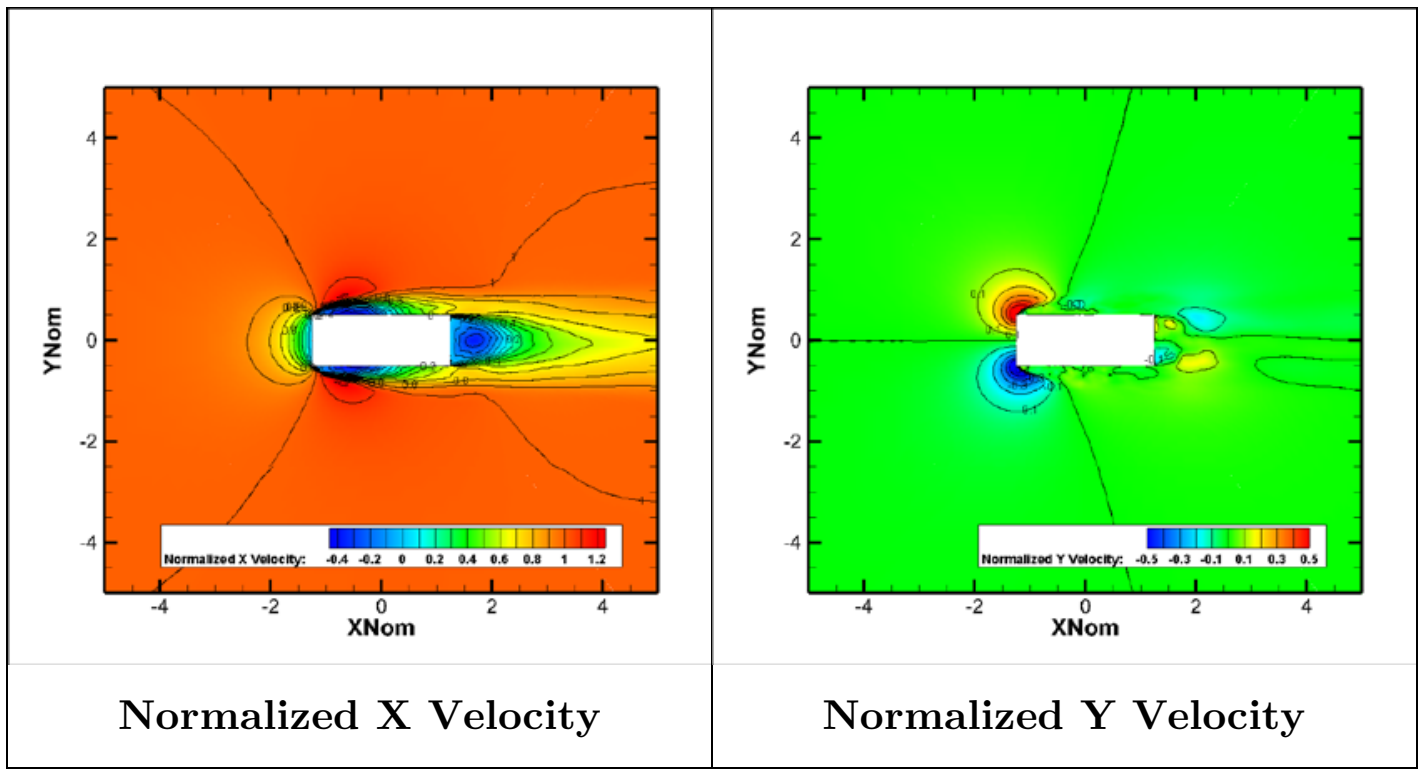

G.2. Cuboid Nacelle Case 10 (Smooth)

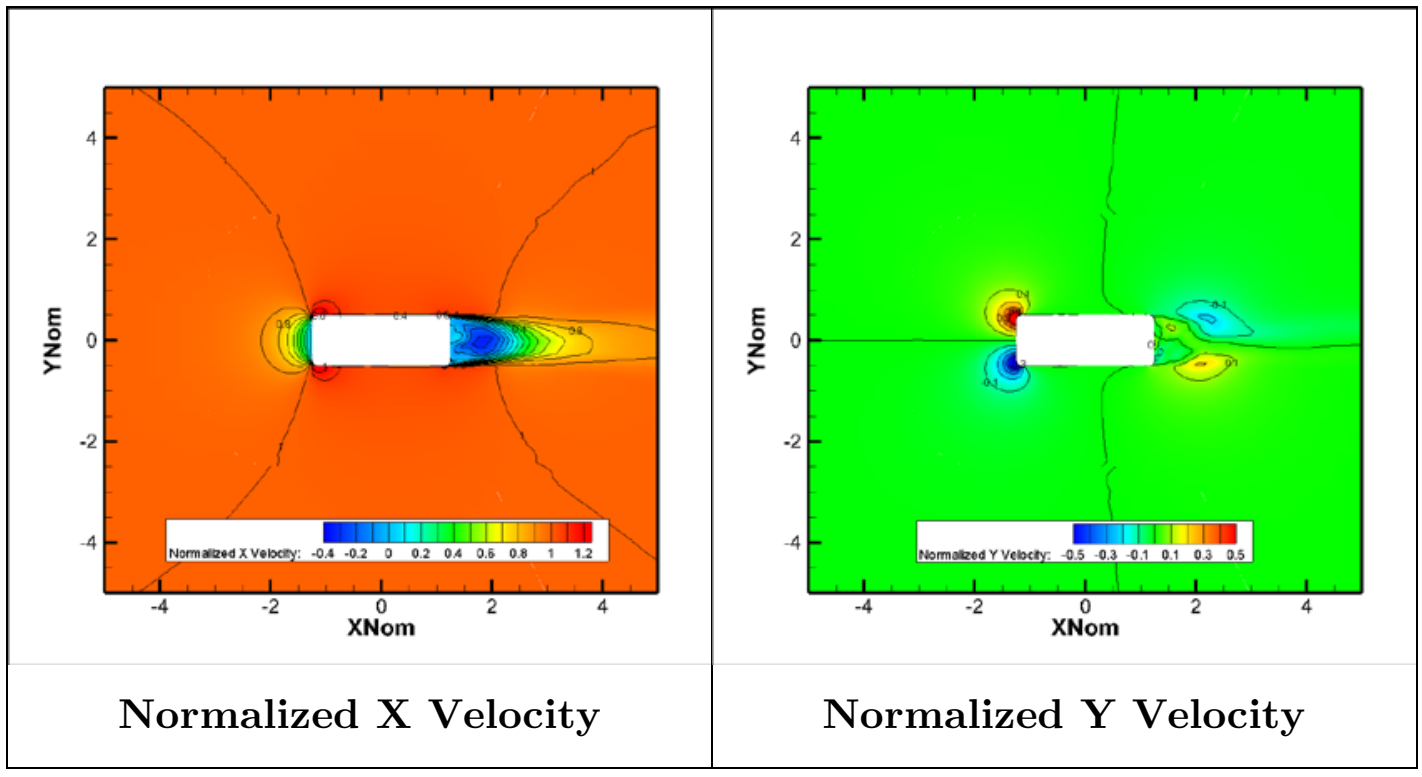




\section{G.3. Cuboid Nacelle Case 2 (Sharp)}

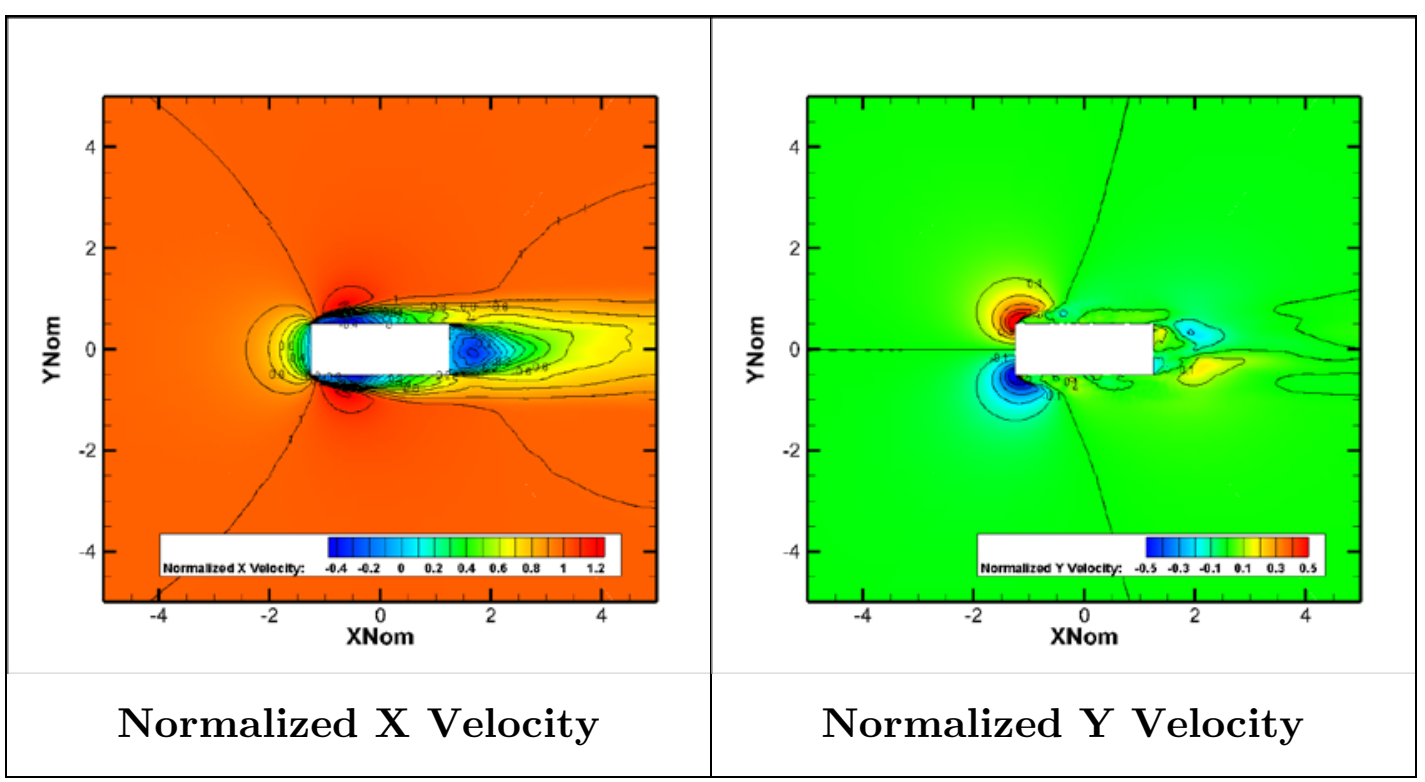

\section{G.4. Cuboid Nacelle Case 11 (Smooth)}

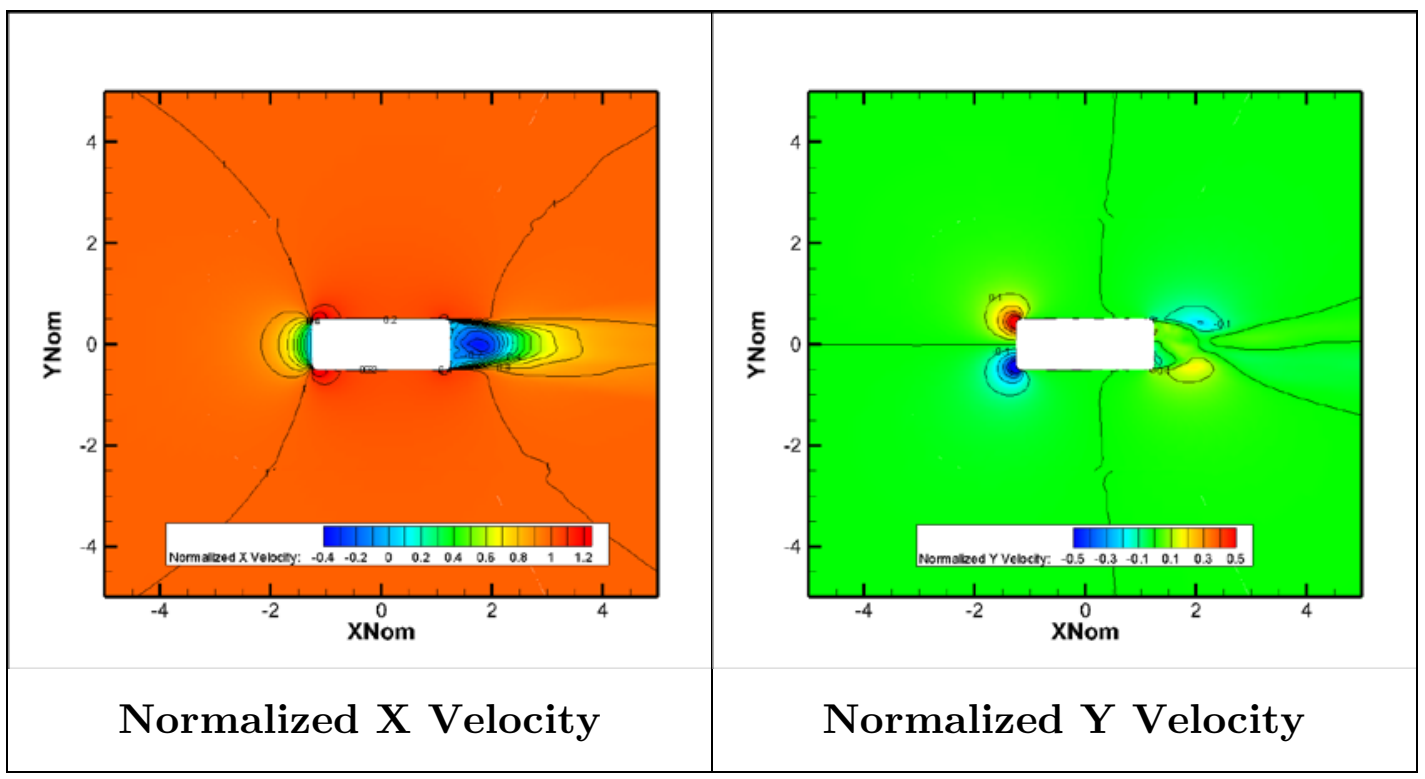




\section{G.5. Cuboid Nacelle Case 3 (Sharp)}

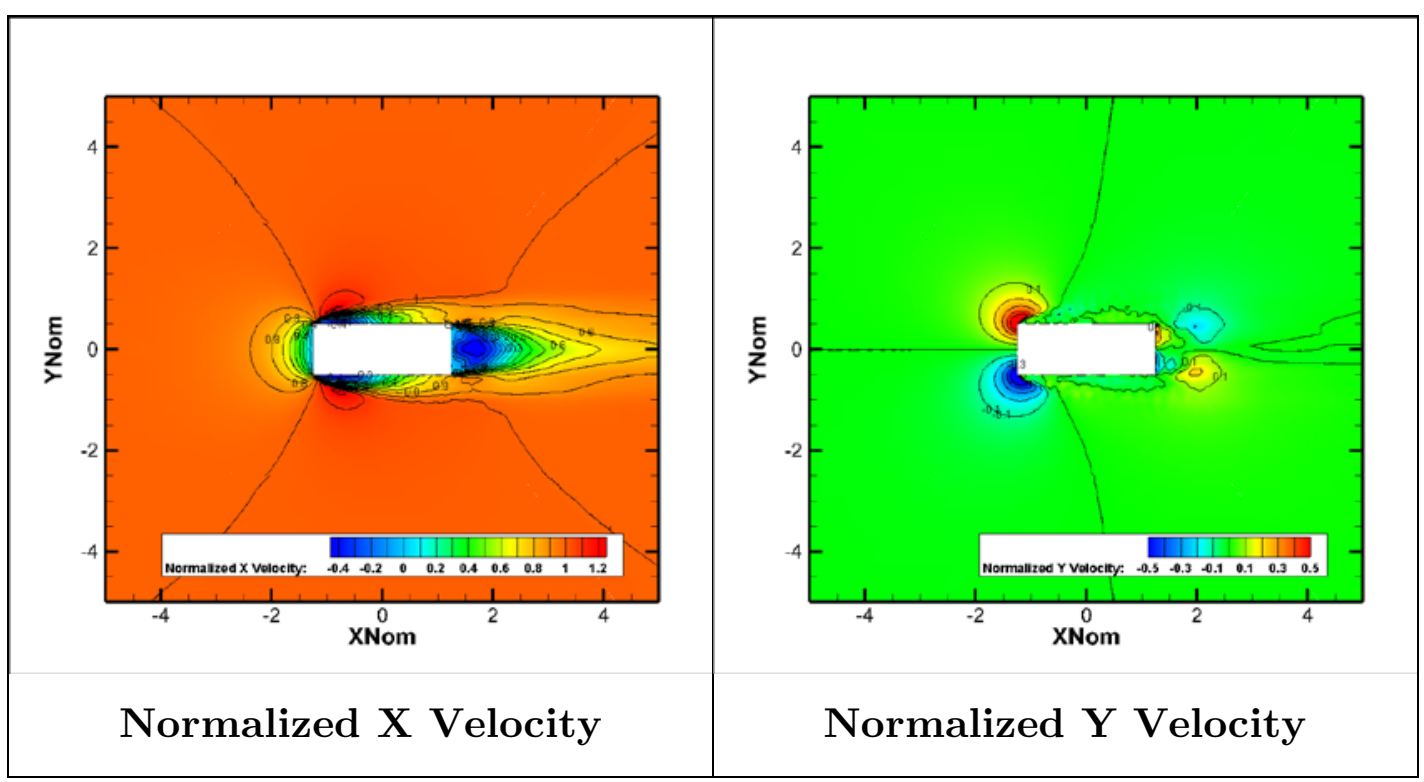

G.6. Cuboid Nacelle Case 12 (Smooth)

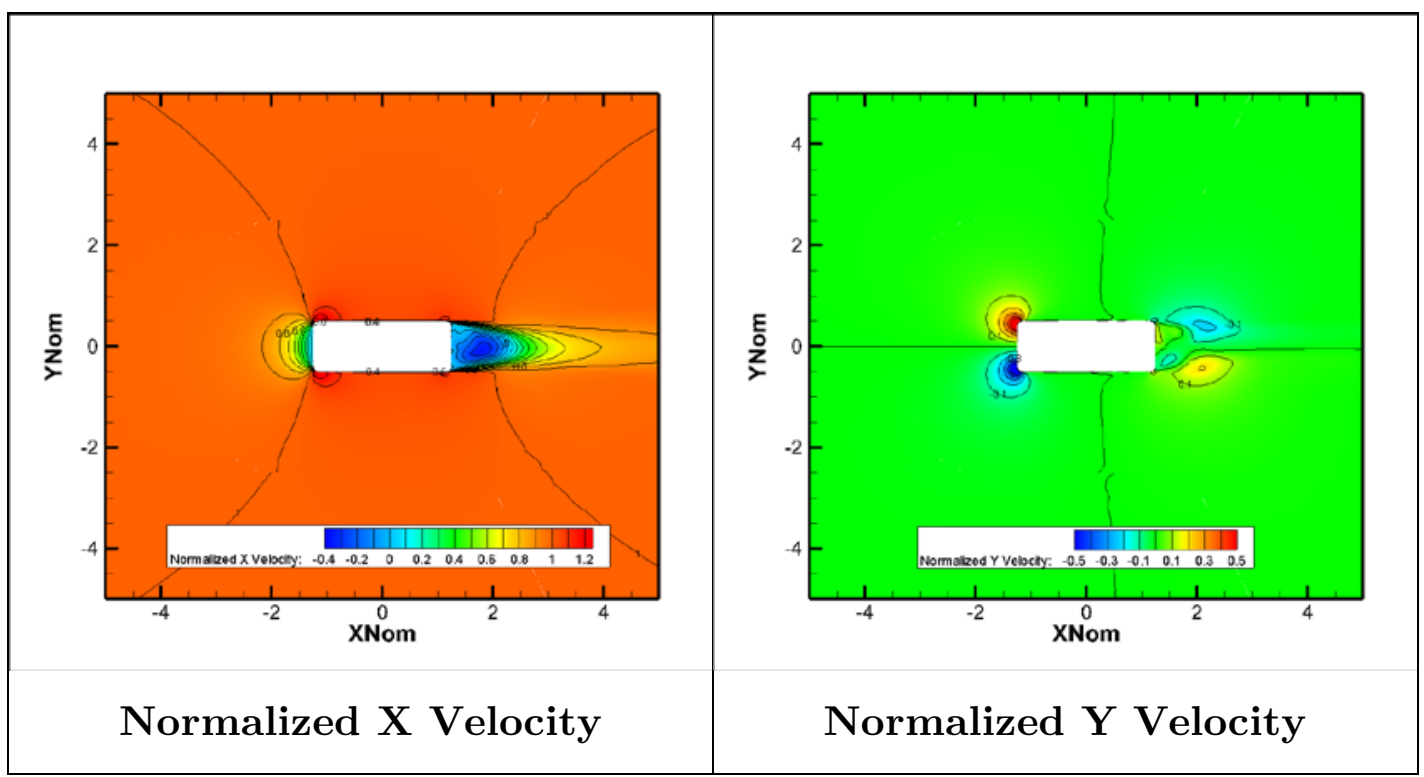




\section{G.7. Cuboid Nacelle Case 4 (Sharp)}

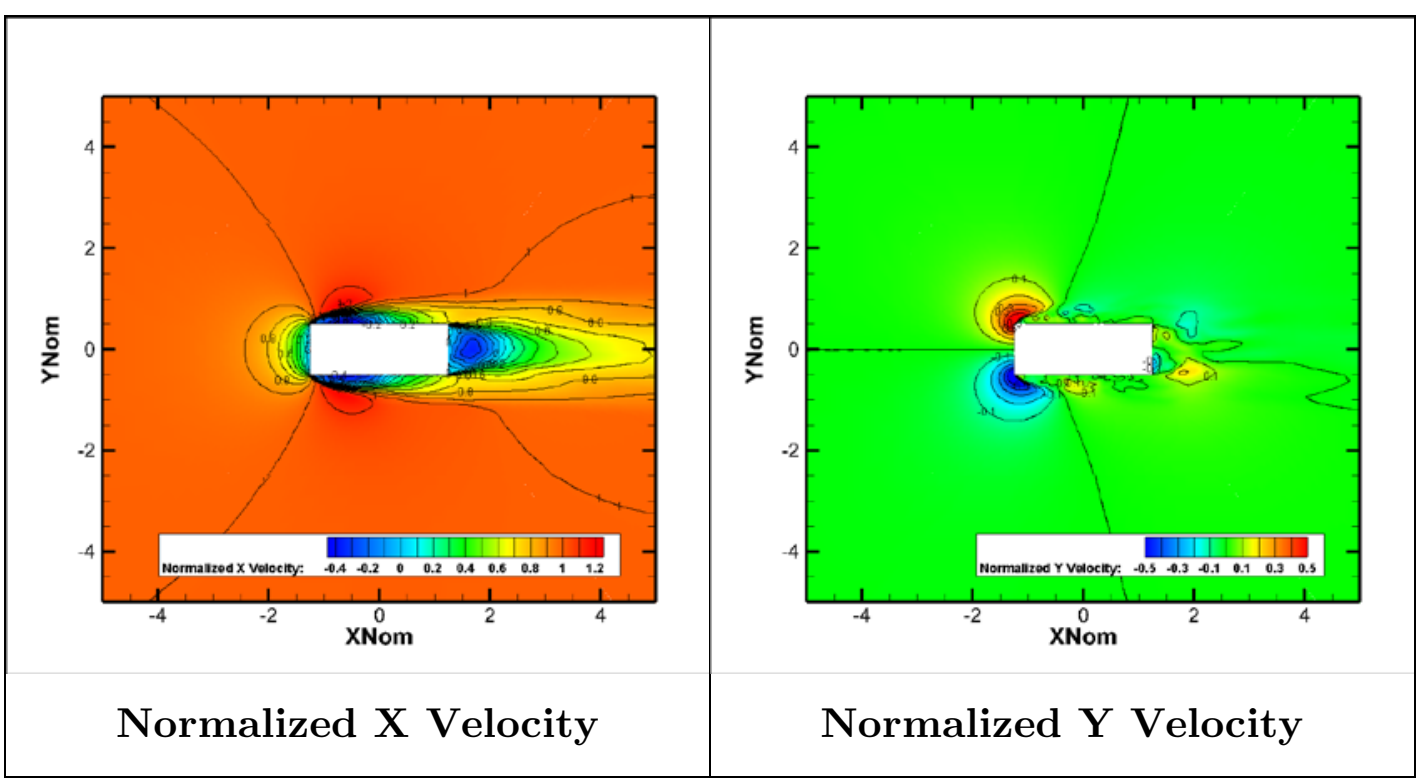

G.8. Cuboid Nacelle Case 13 (Smooth)

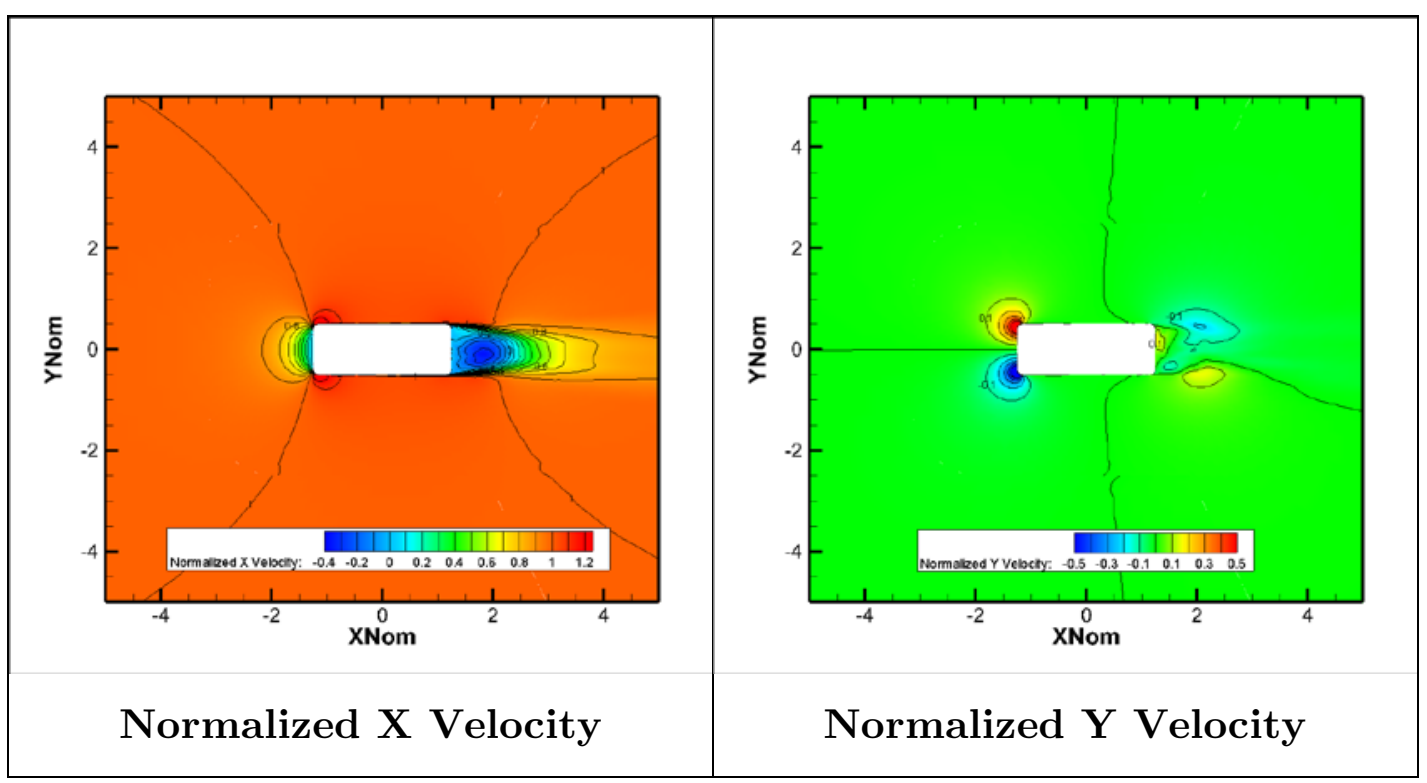




\section{G.9. Cuboid Nacelle Case 5 (Sharp)}

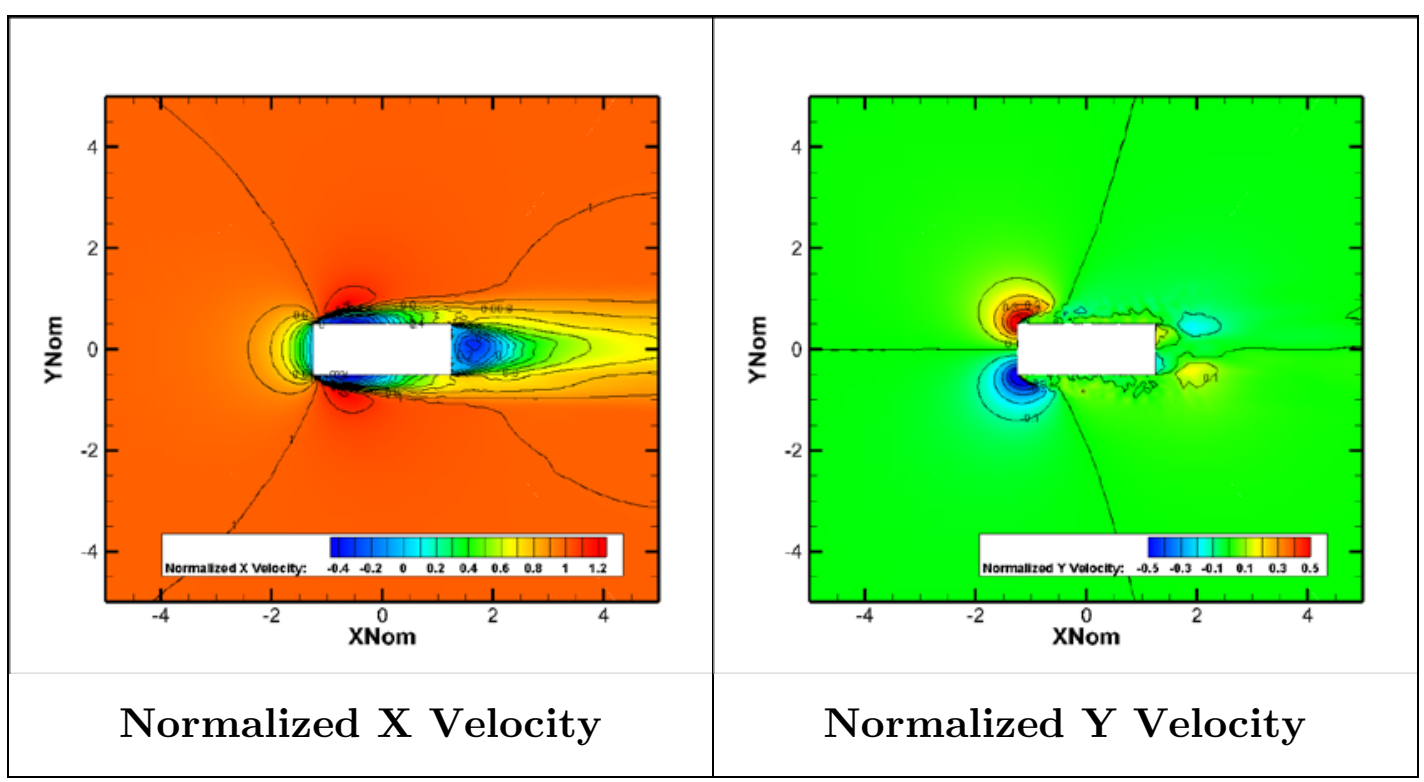

G.10. Cuboid Nacelle Case 14 (Smooth)

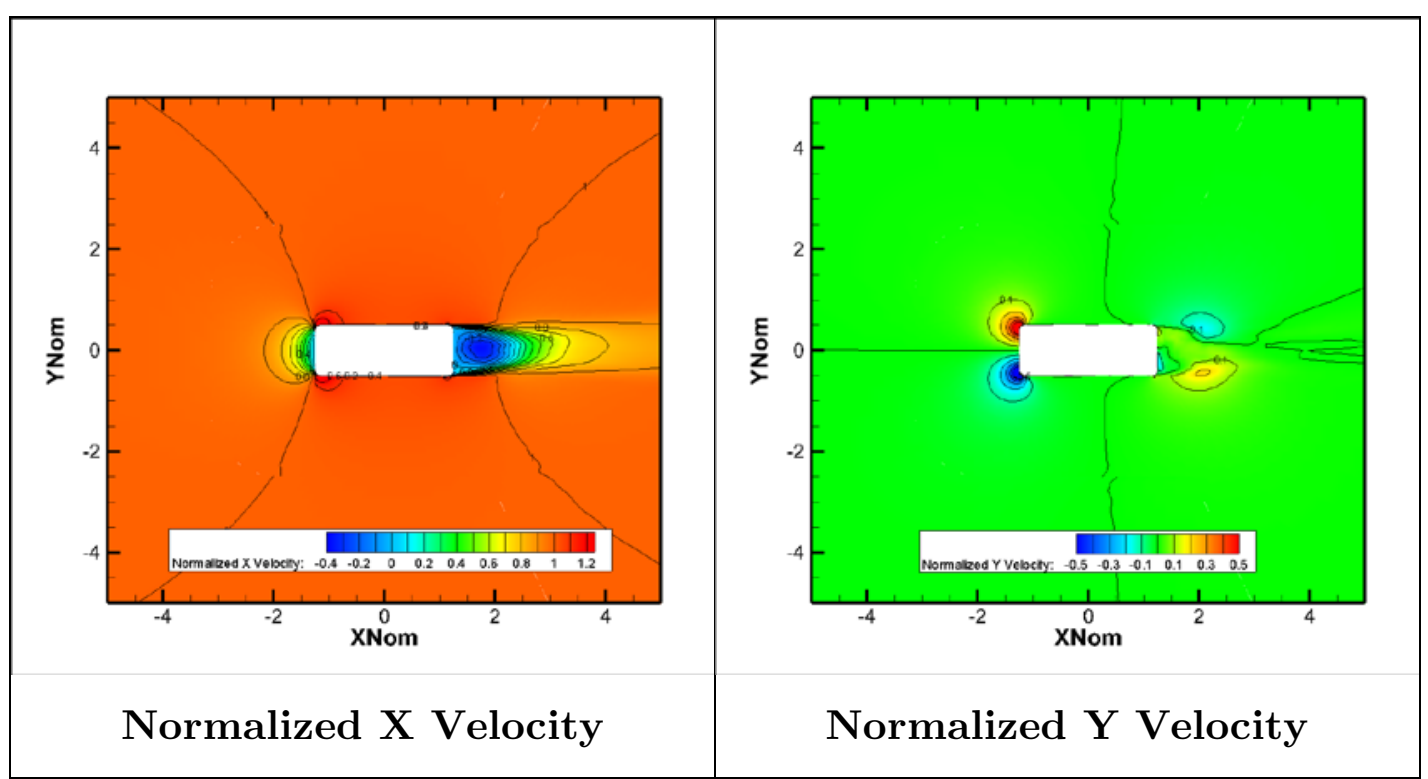


G.11. Cuboid Nacelle Case 6 (Sharp)

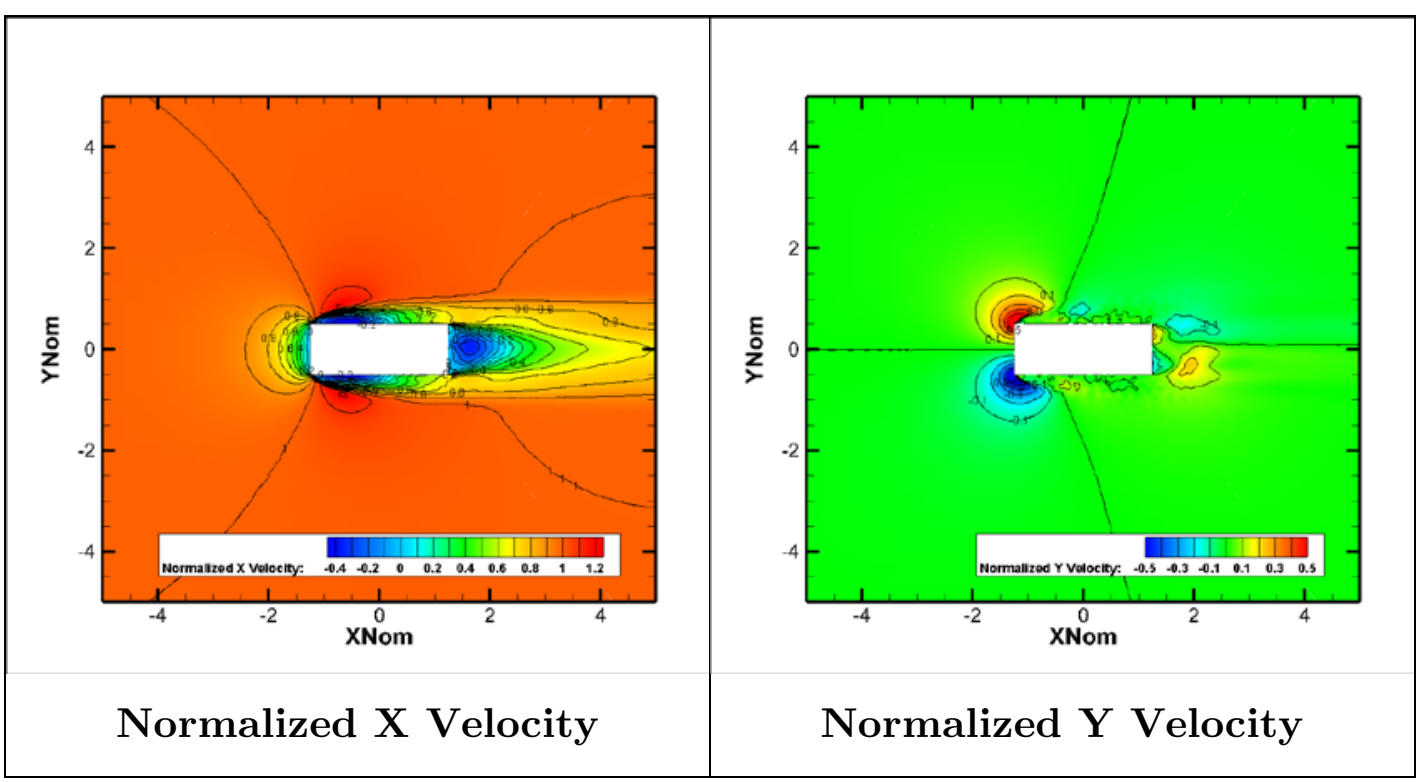

G.12. Cuboid Nacelle Case 15 (Smooth)

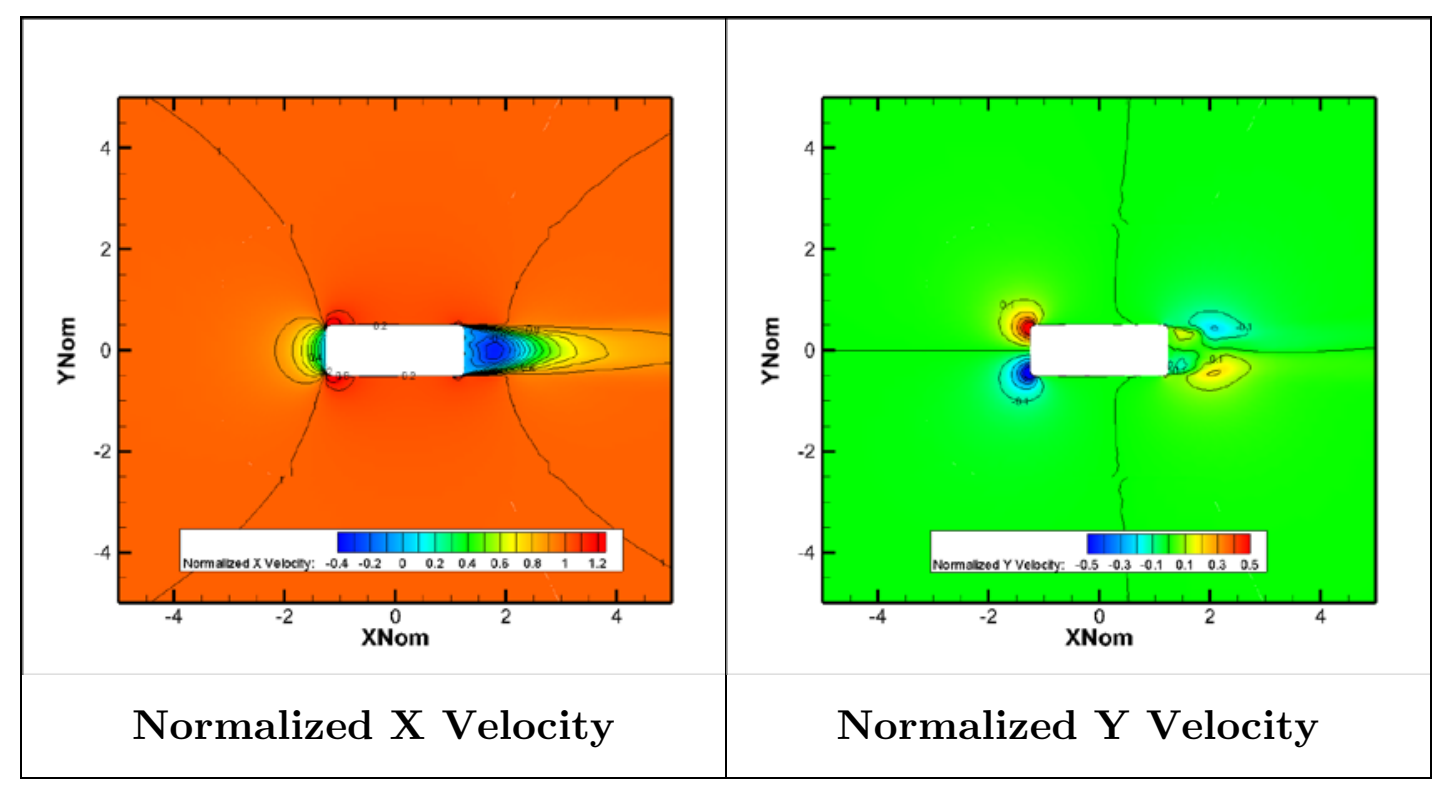




\section{G.13. Cuboid Nacelle Case 7 (Sharp)}

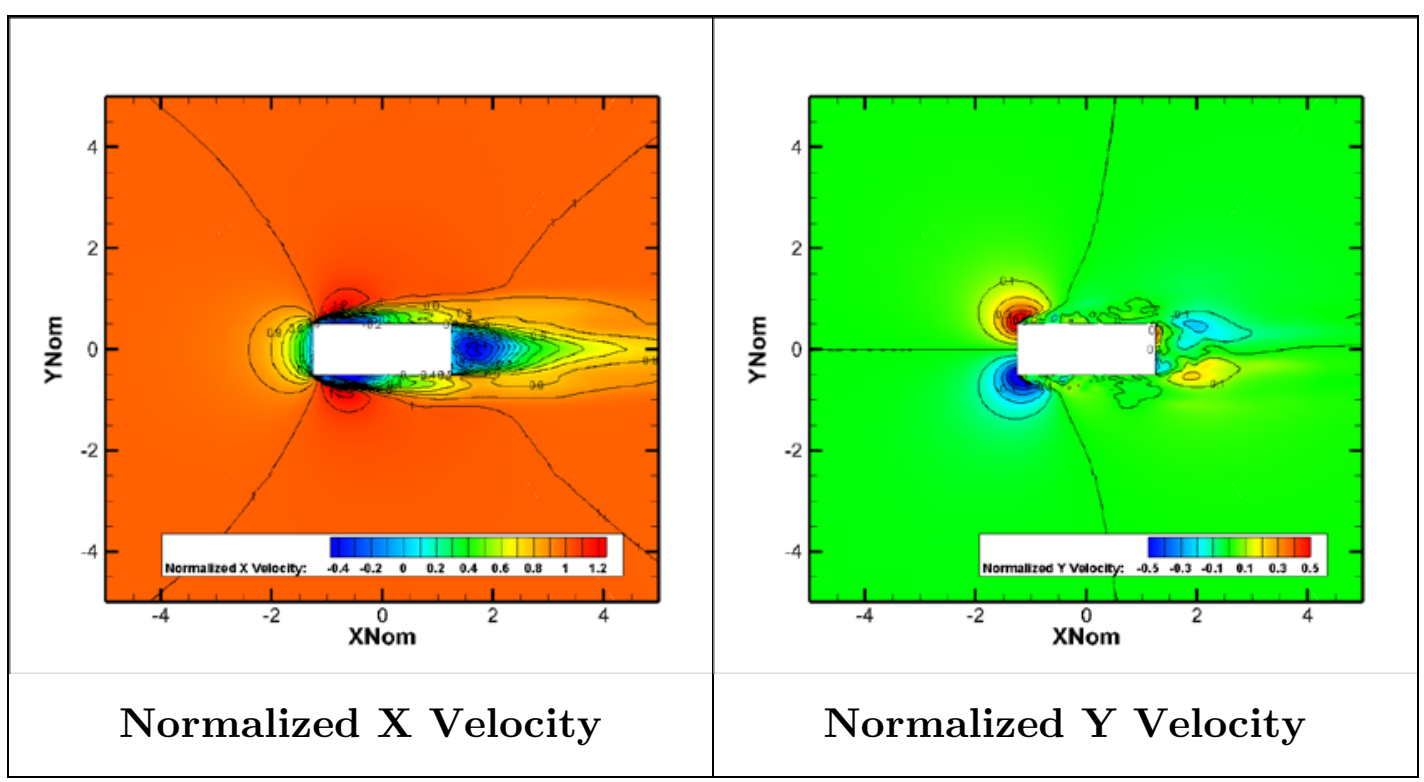

G.14. Cuboid Nacelle Case 16 (Smooth)

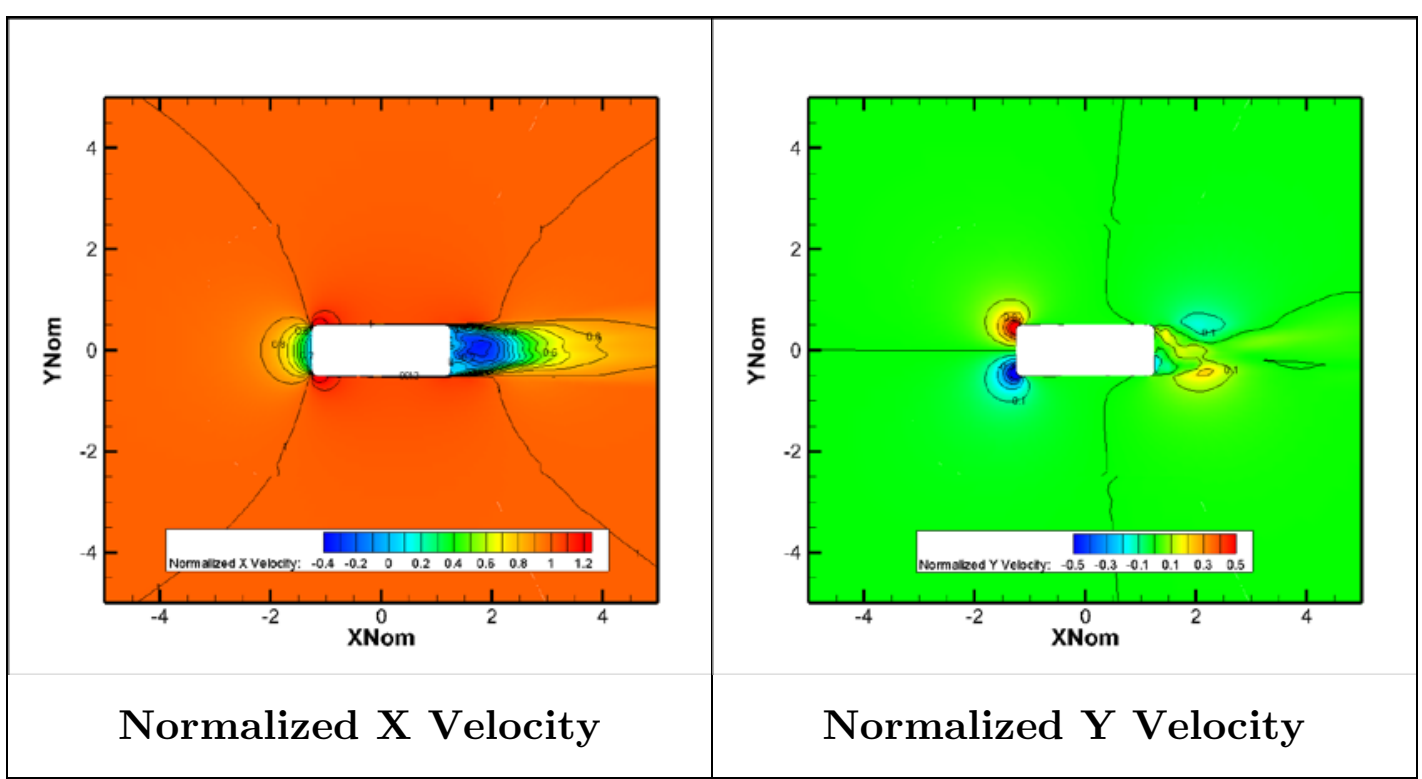




\section{G.15. Cuboid Nacelle Case 8 (Sharp)}

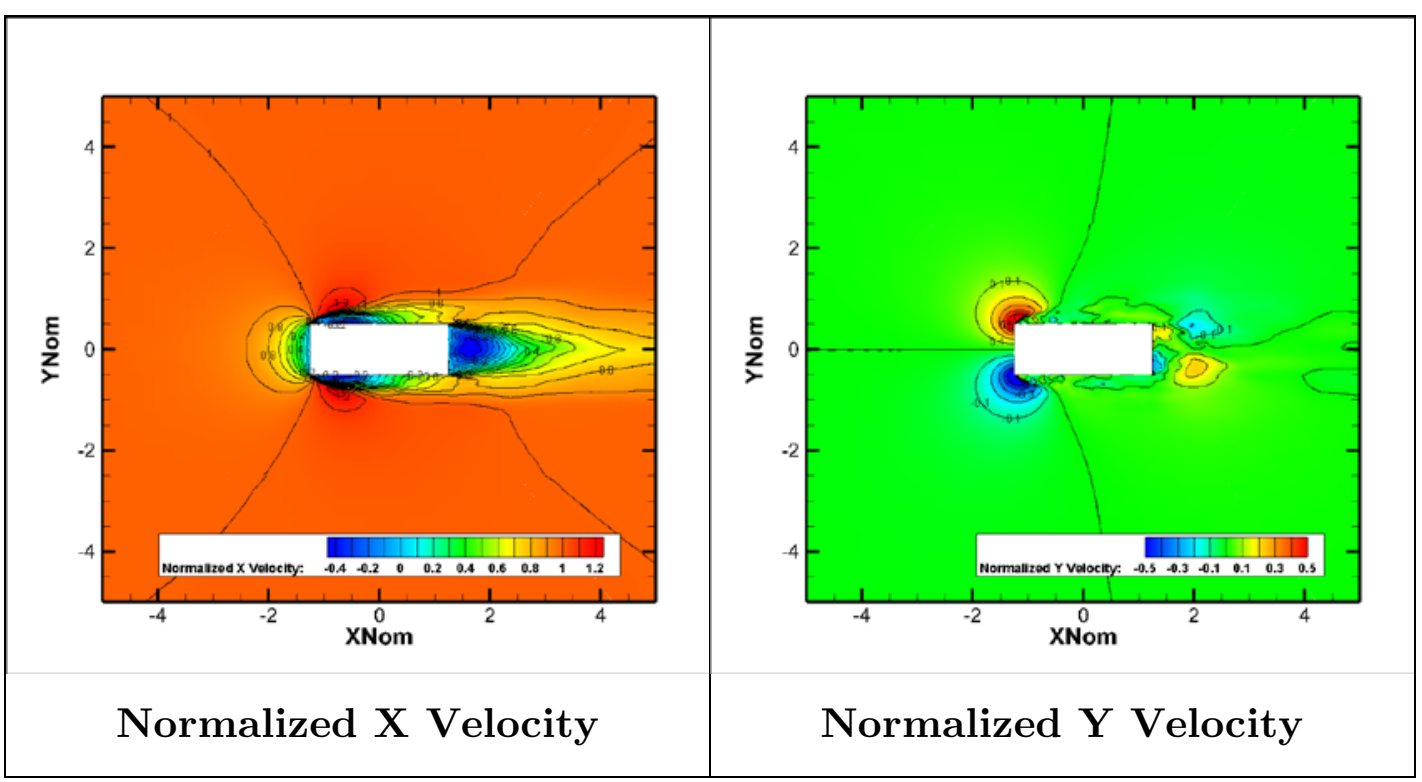

G.16. Cuboid Nacelle Case 17 (Smooth)

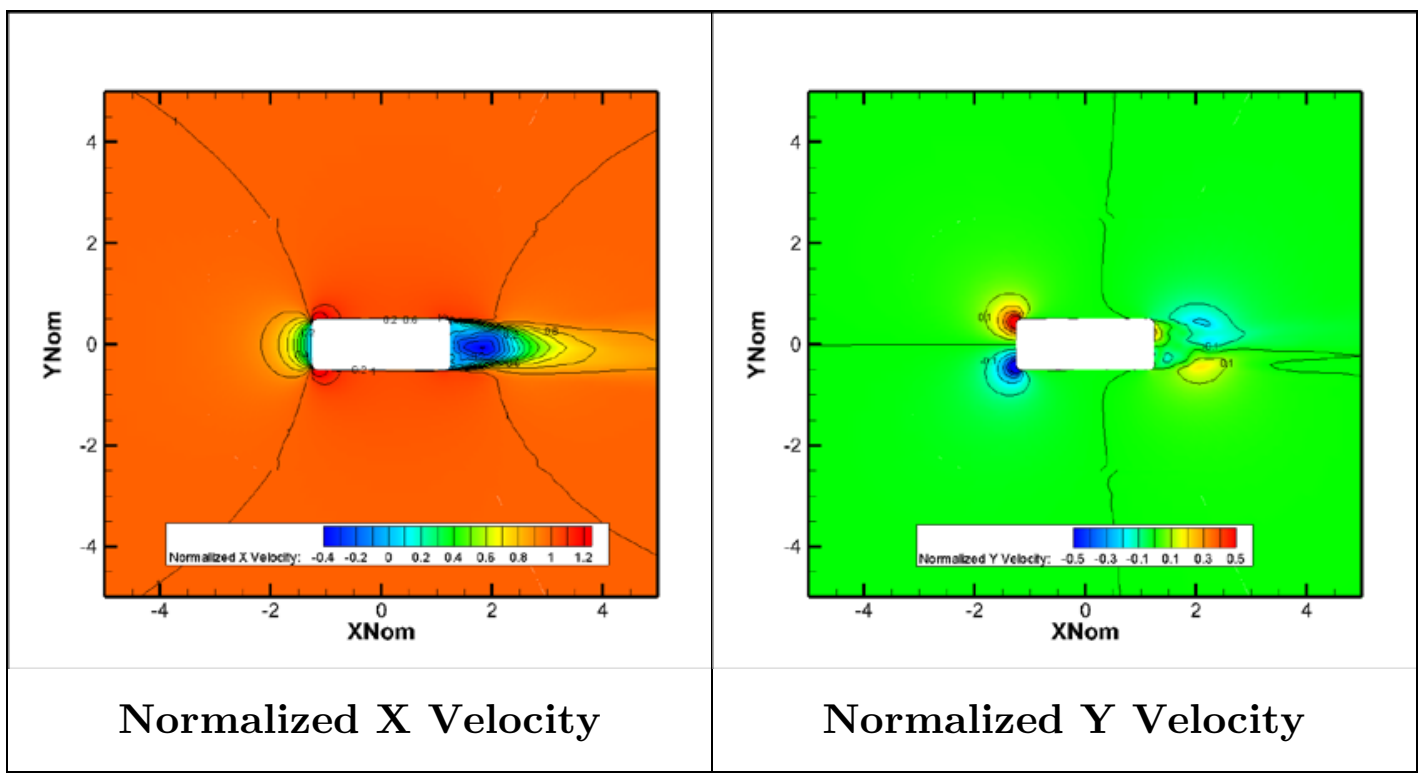


G.17. Cuboid Nacelle Case 9 (Sharp)

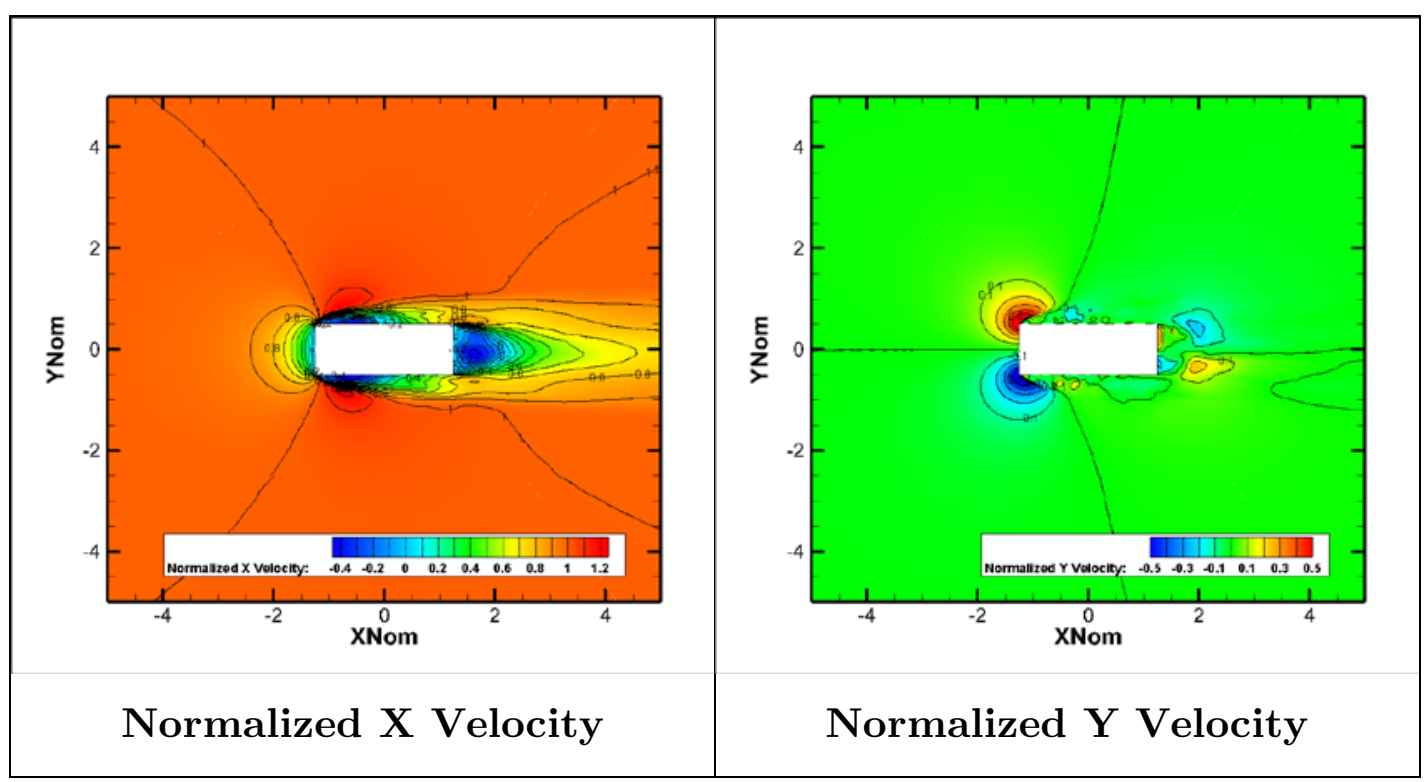

G.18. Cuboid Nacelle Case 18 (Smooth)

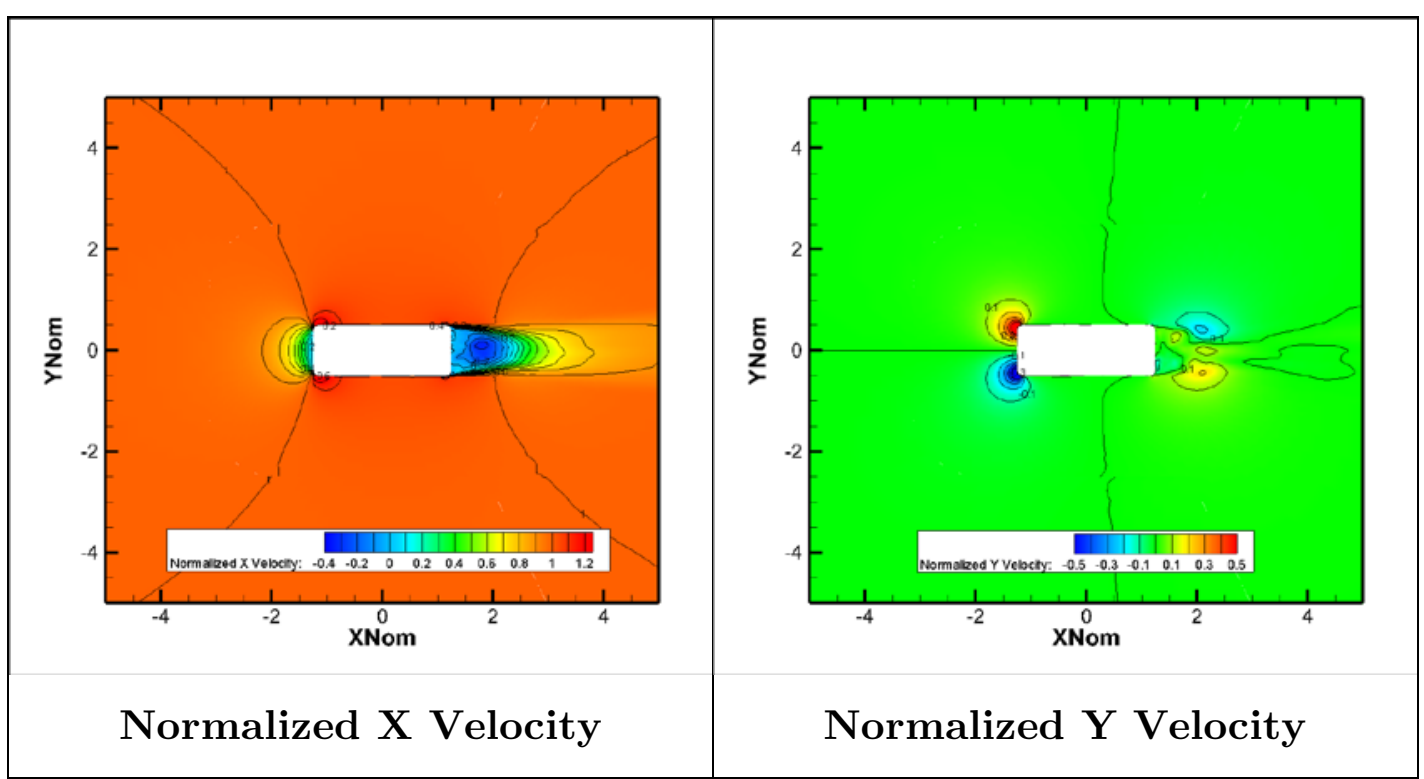




\section{APPENDIX H}

H.1. Cylinder Nacelle Case 1 (Sharp)

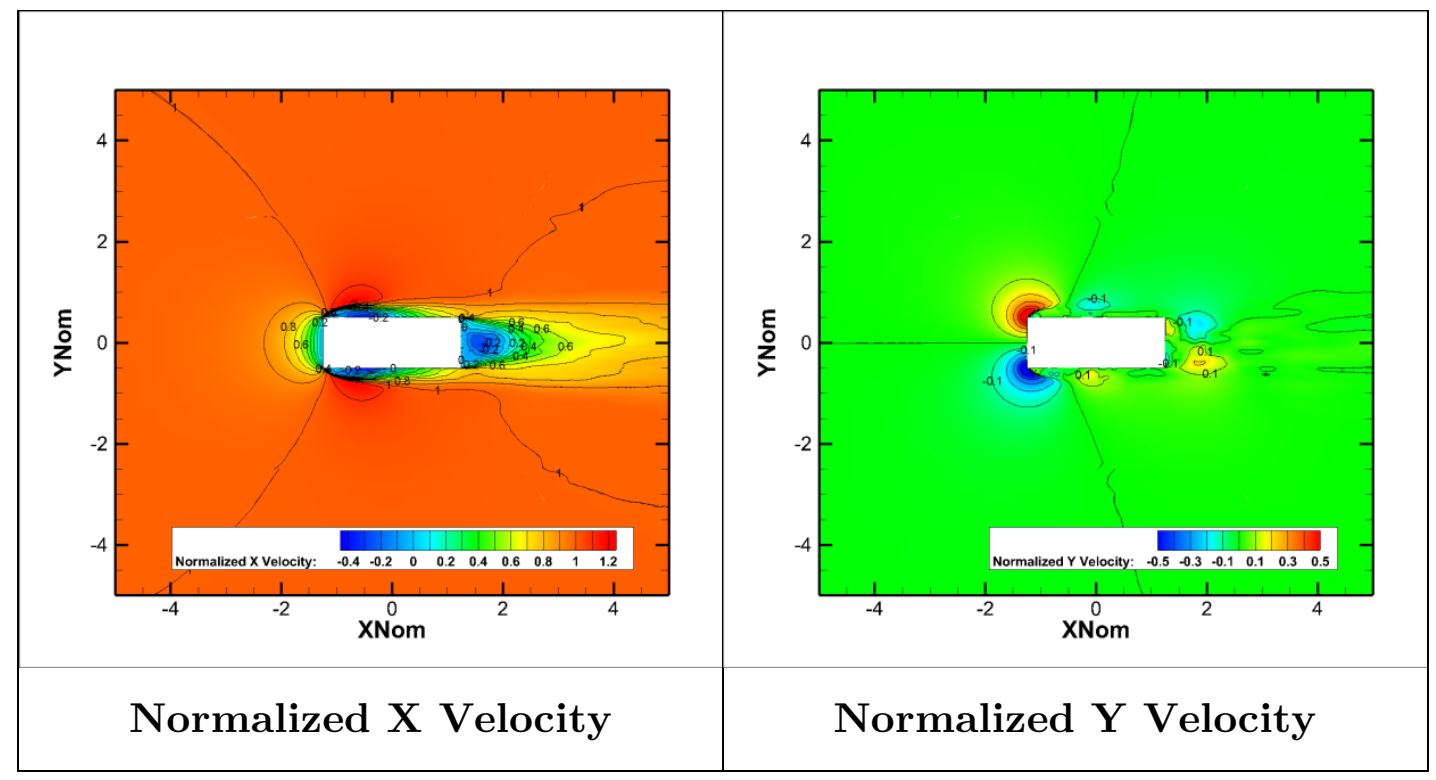

H.2. Cylinder Nacelle Case 10 (Smooth)

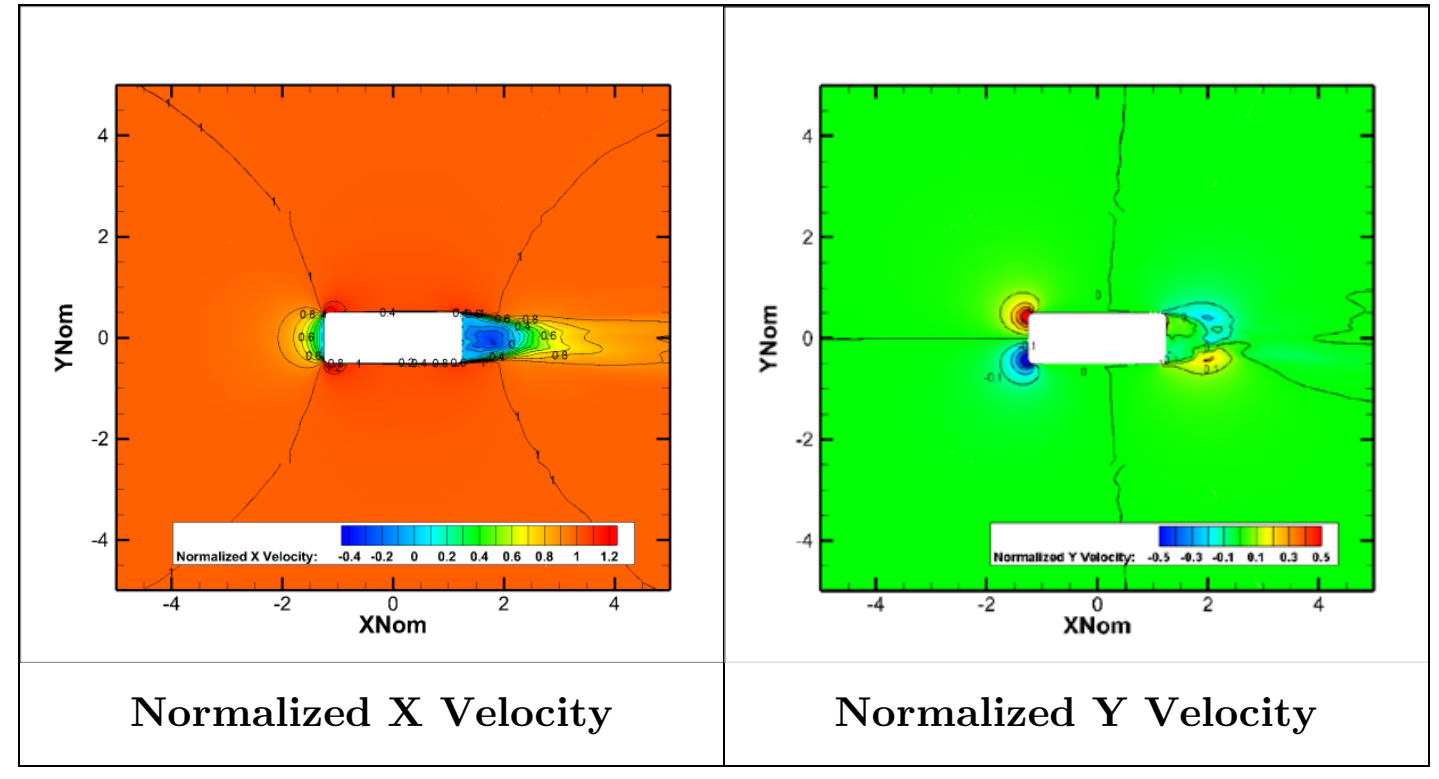


H.3. Cylinder Nacelle Case 2 (Sharp)

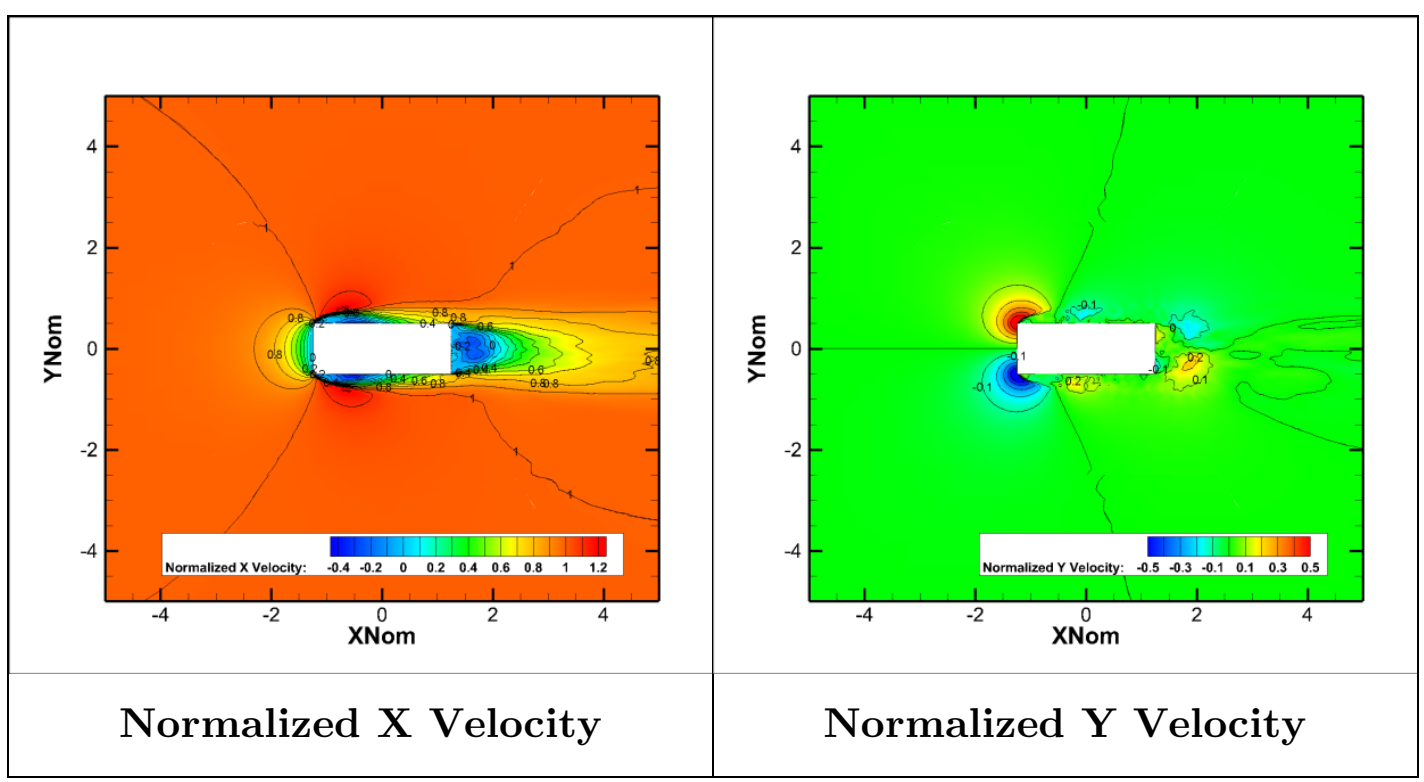

H.4. Cylinder Nacelle Case 11 (Smooth)

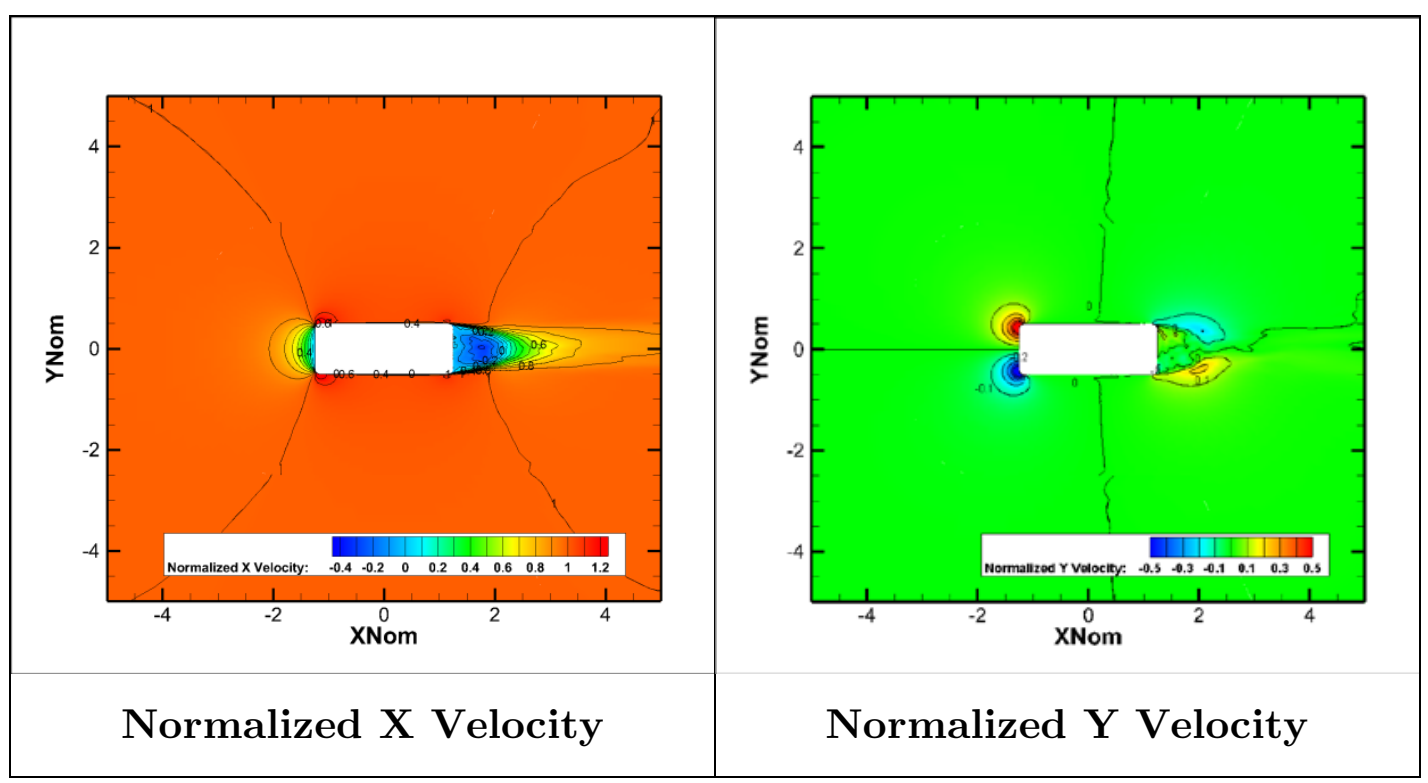




\section{H.5. Cylinder Nacelle Case 3 (Sharp)}

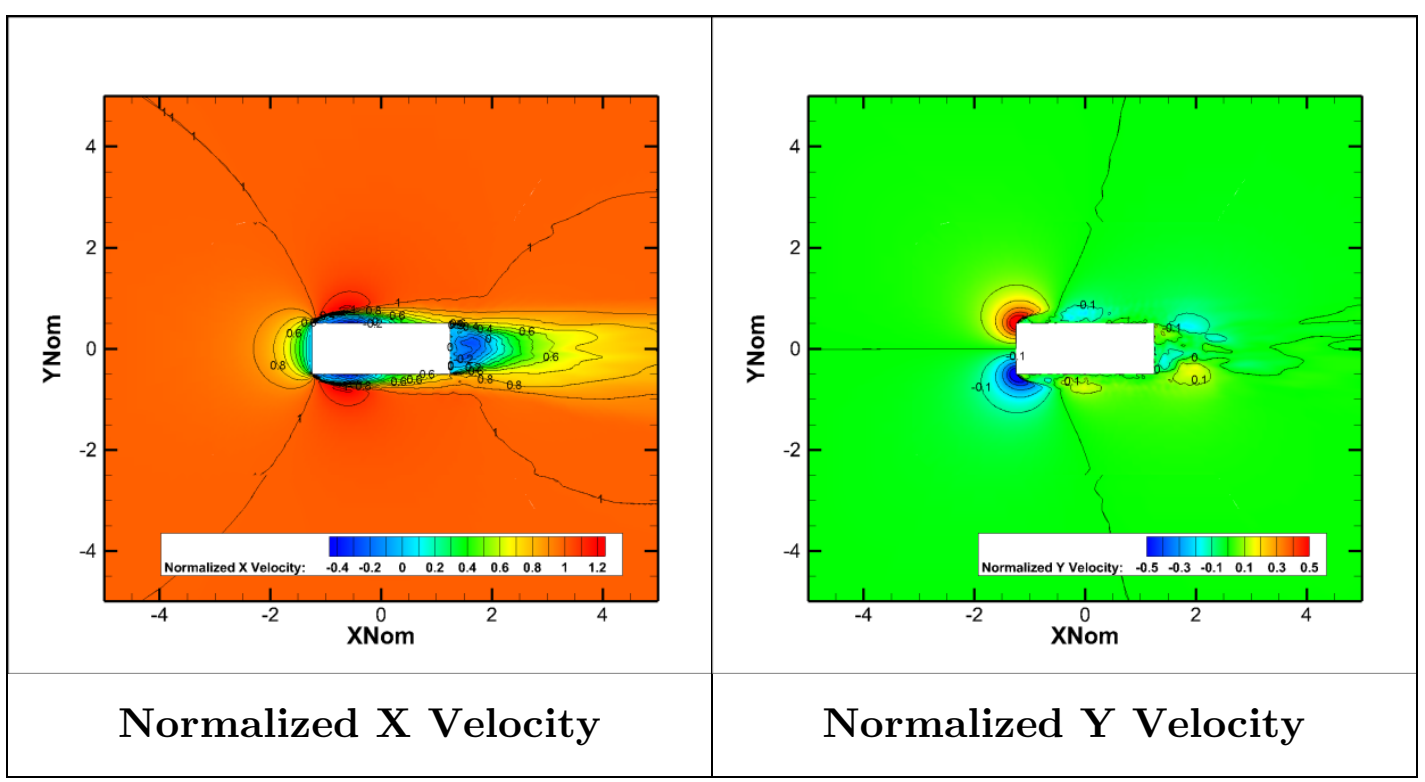

\section{H.6. Cylinder Nacelle Case 12 (Smooth)}

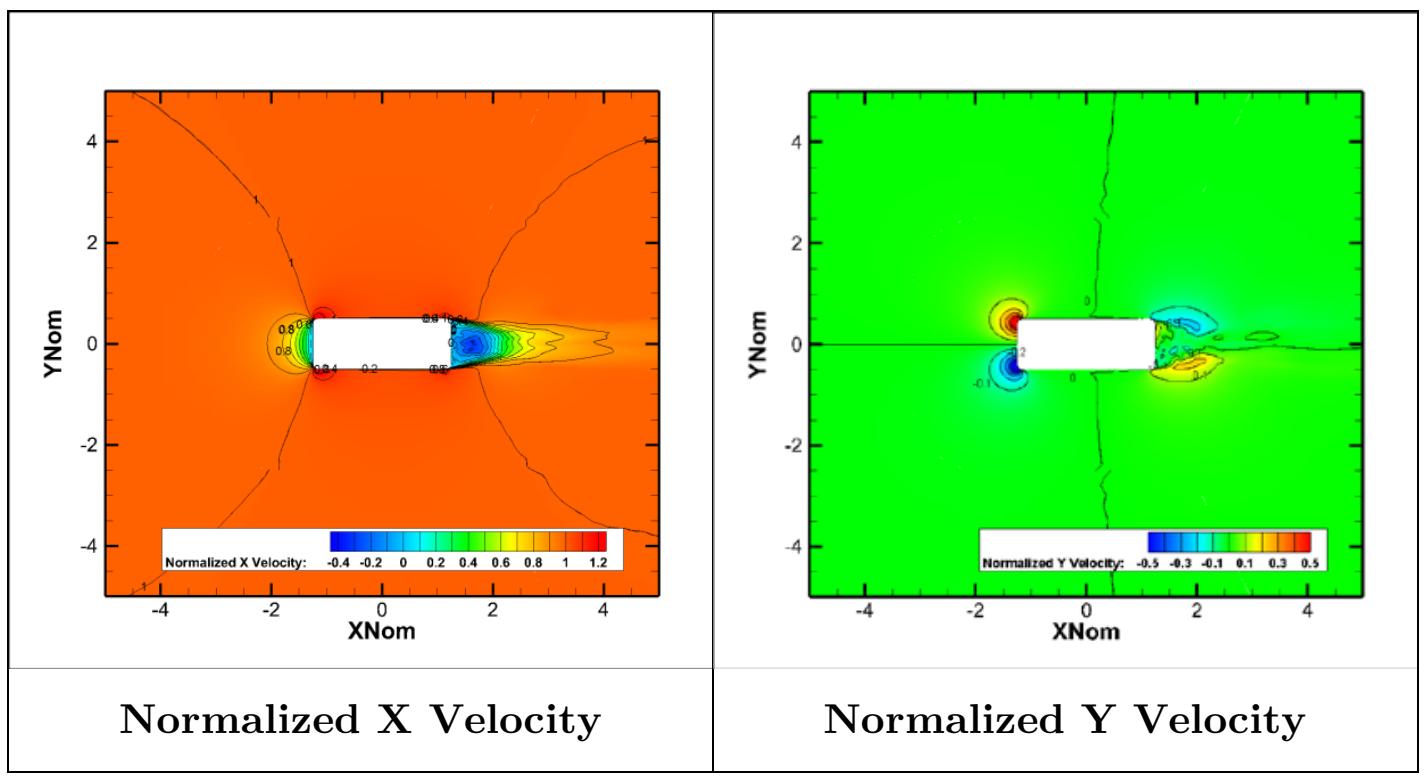


H.7. Cylinder Nacelle Case 4 (Sharp)

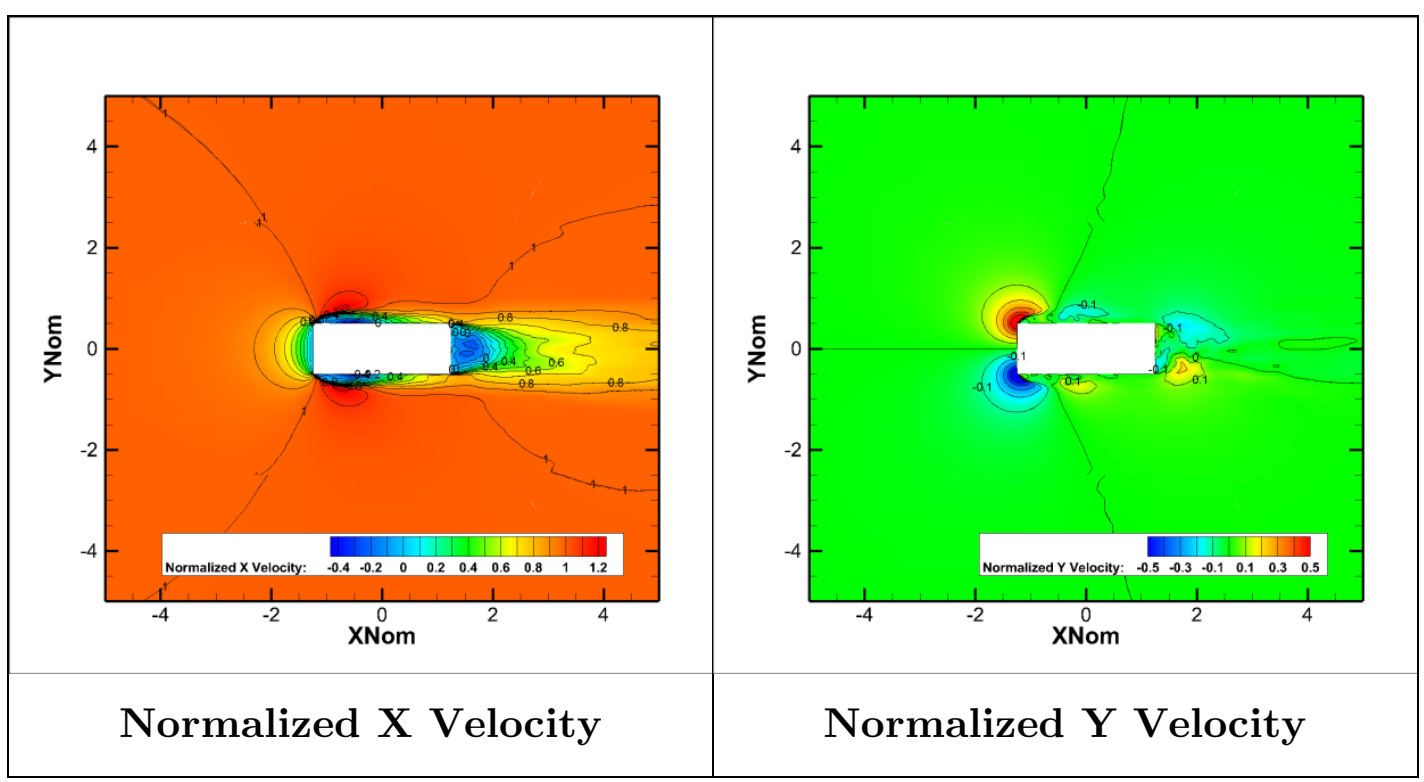

H.8. Cylinder Nacelle Case 13 (Smooth)

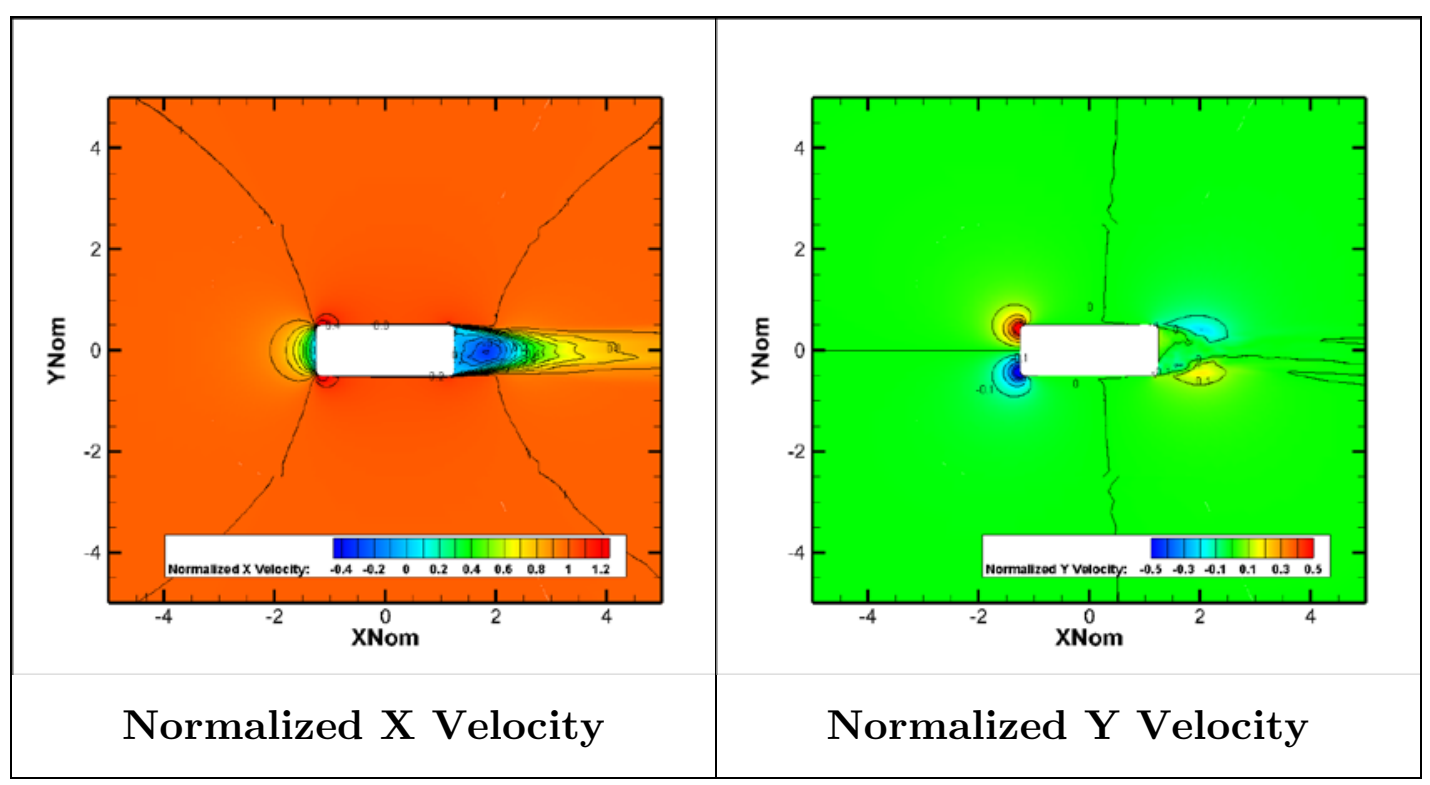




\section{H.9. Cylinder Nacelle Case 5 (Sharp)}

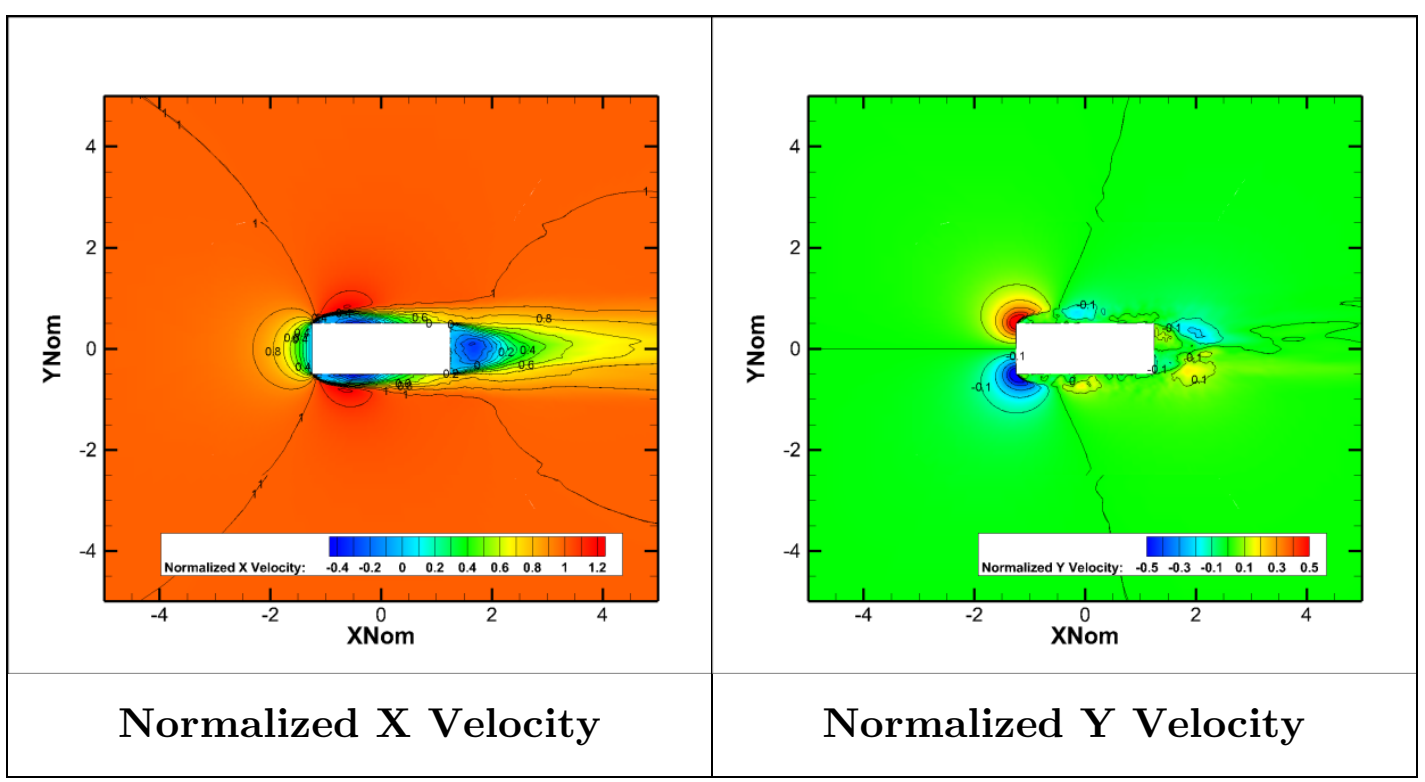

\section{H.10. Cylinder Nacelle Case 14 (Smooth)}

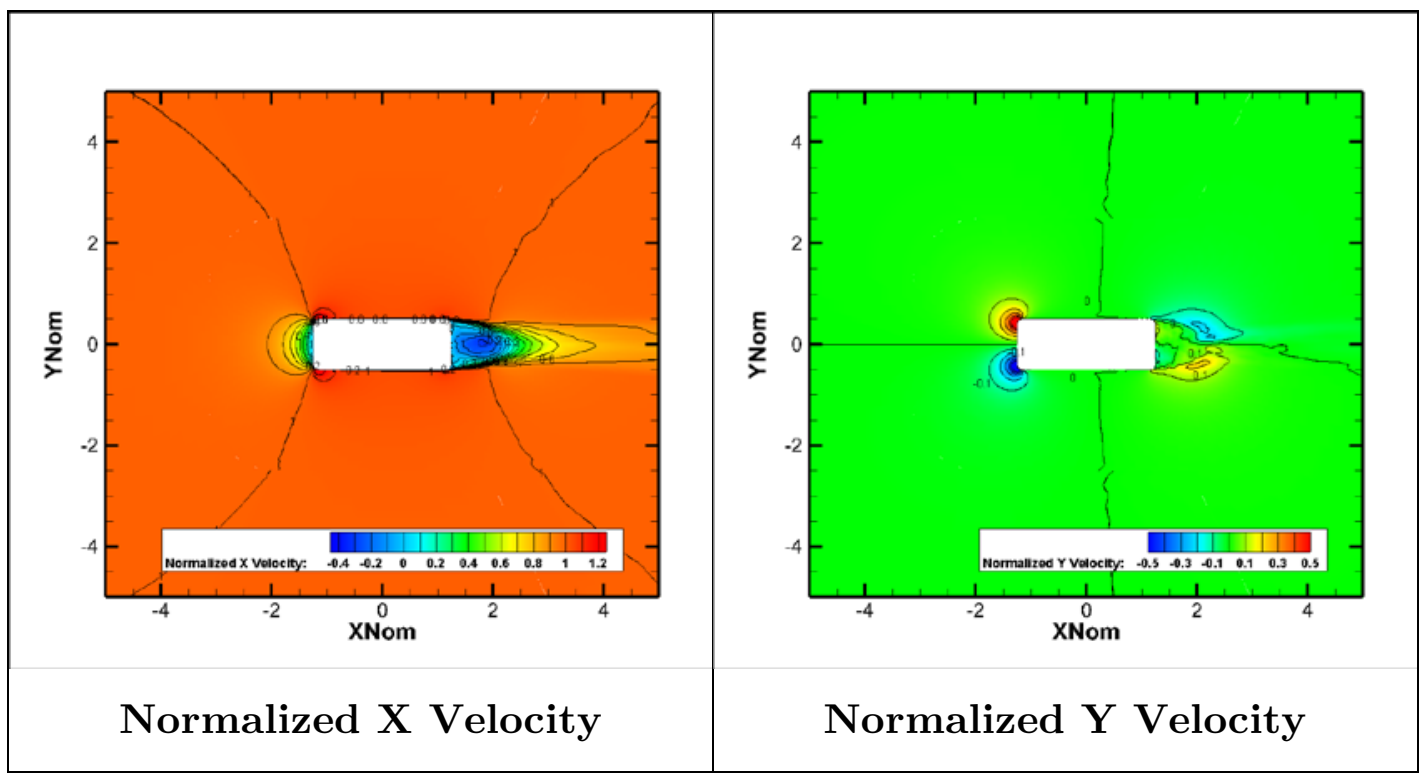


H.11. Cylinder Nacelle Case 6 (Sharp)

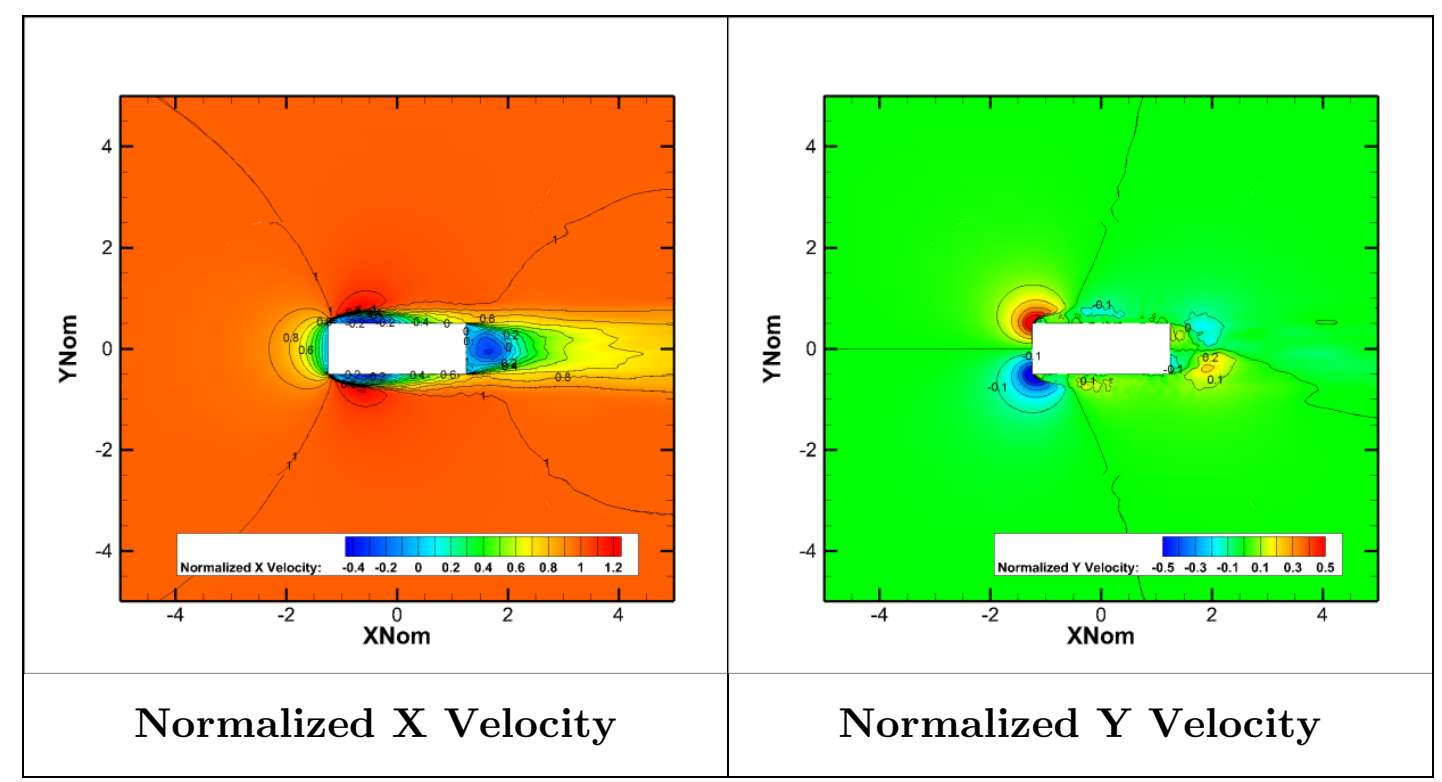

H.12. Cylinder Nacelle Case 15 (Smooth)

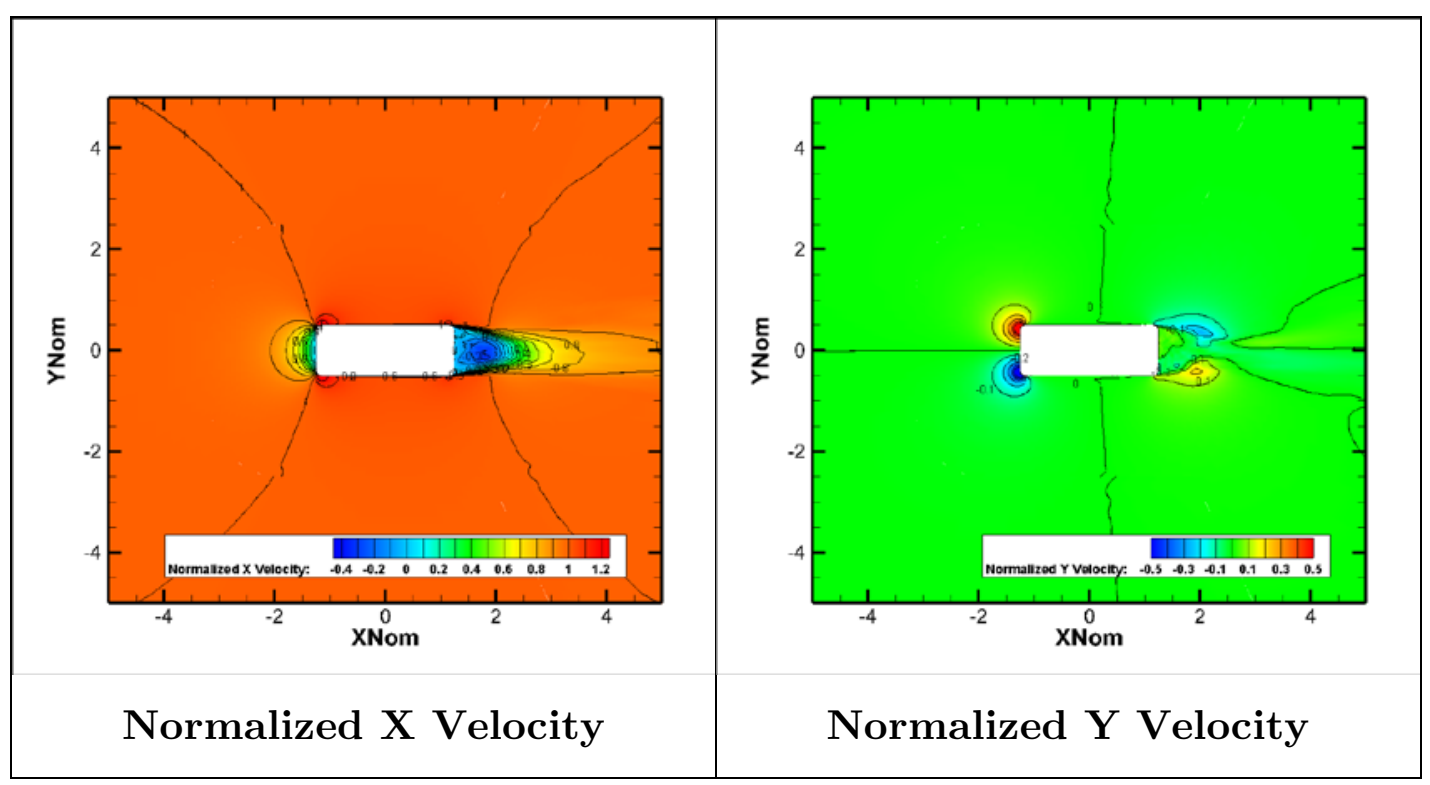




\section{H.13. Cylinder Nacelle Case 7 (Sharp)}

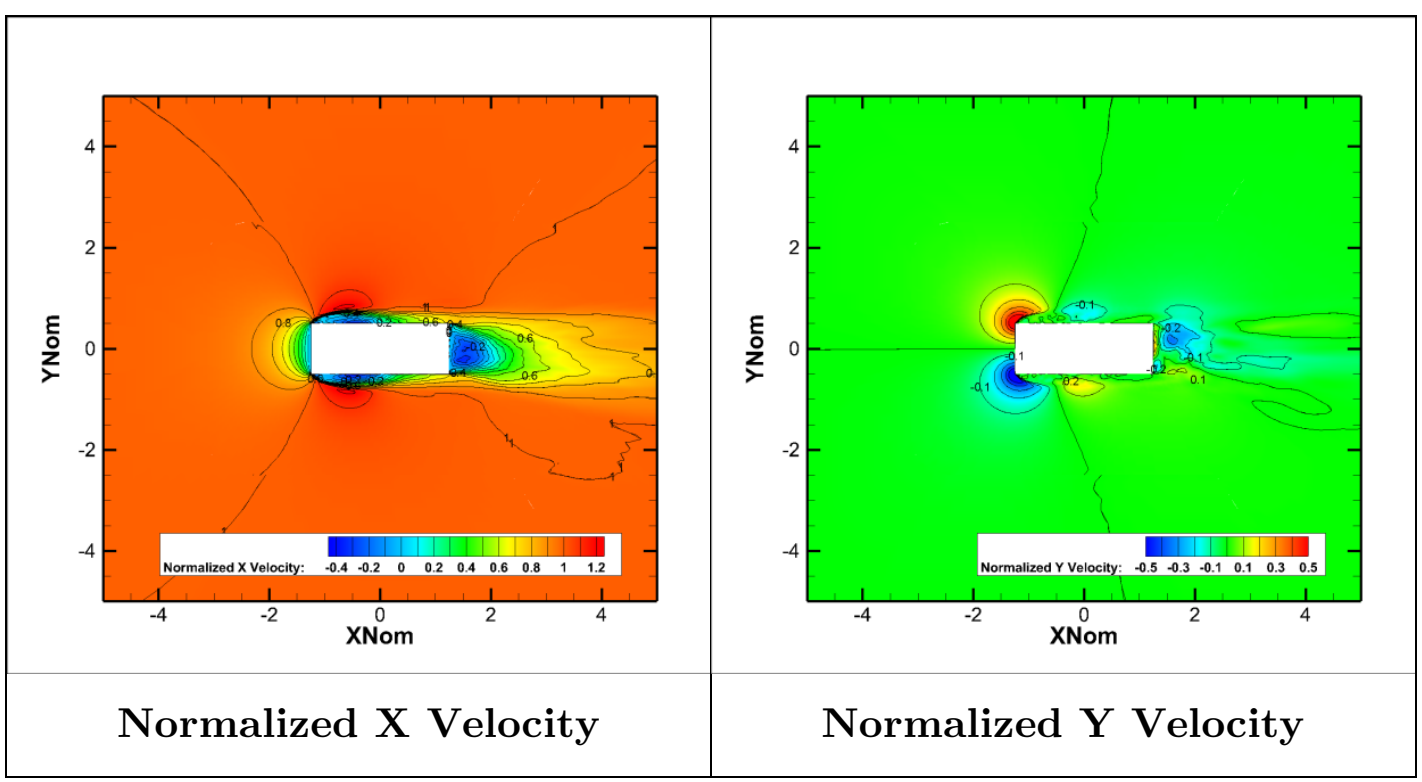

\section{H.14. Cylinder Nacelle Case 16 (Smooth)}

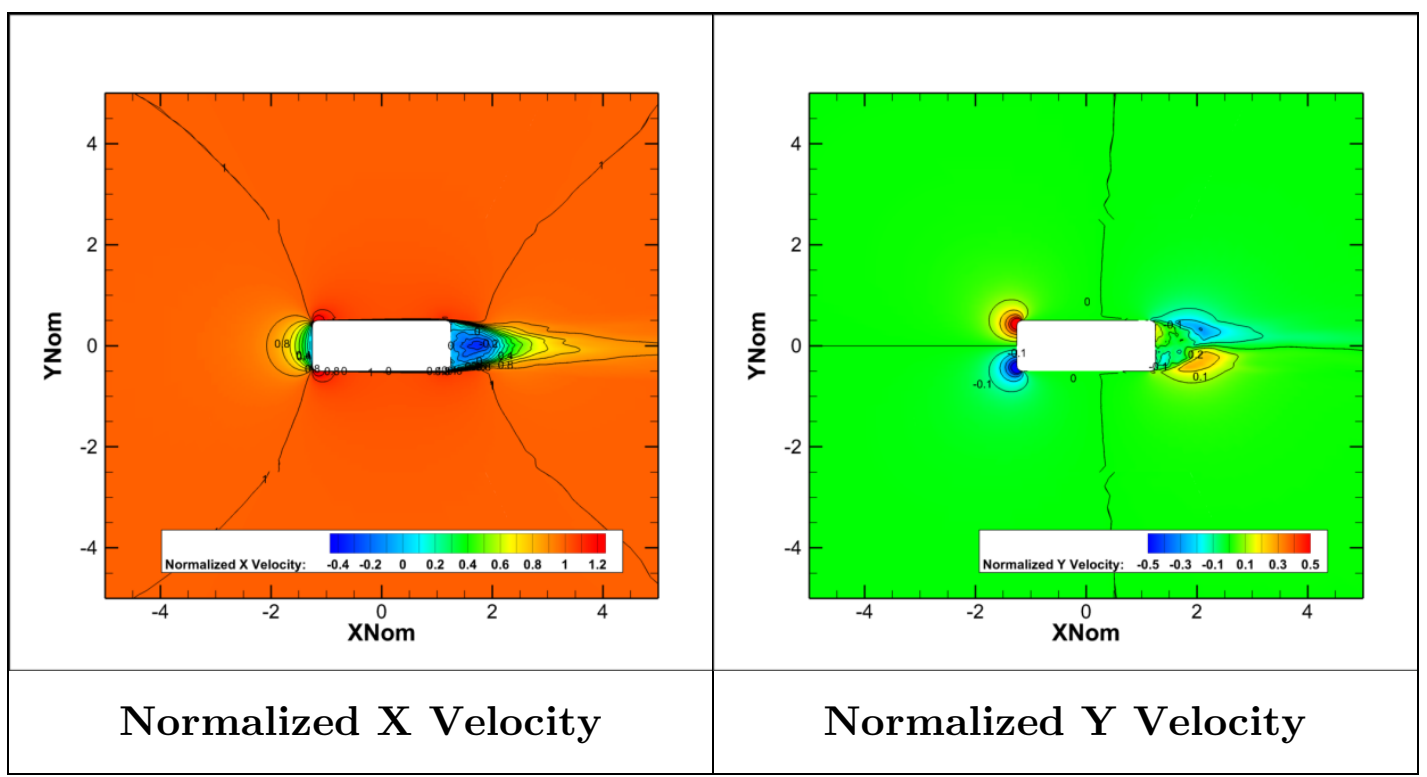




\section{H.15. Cylinder Nacelle Case 8 (Sharp)}

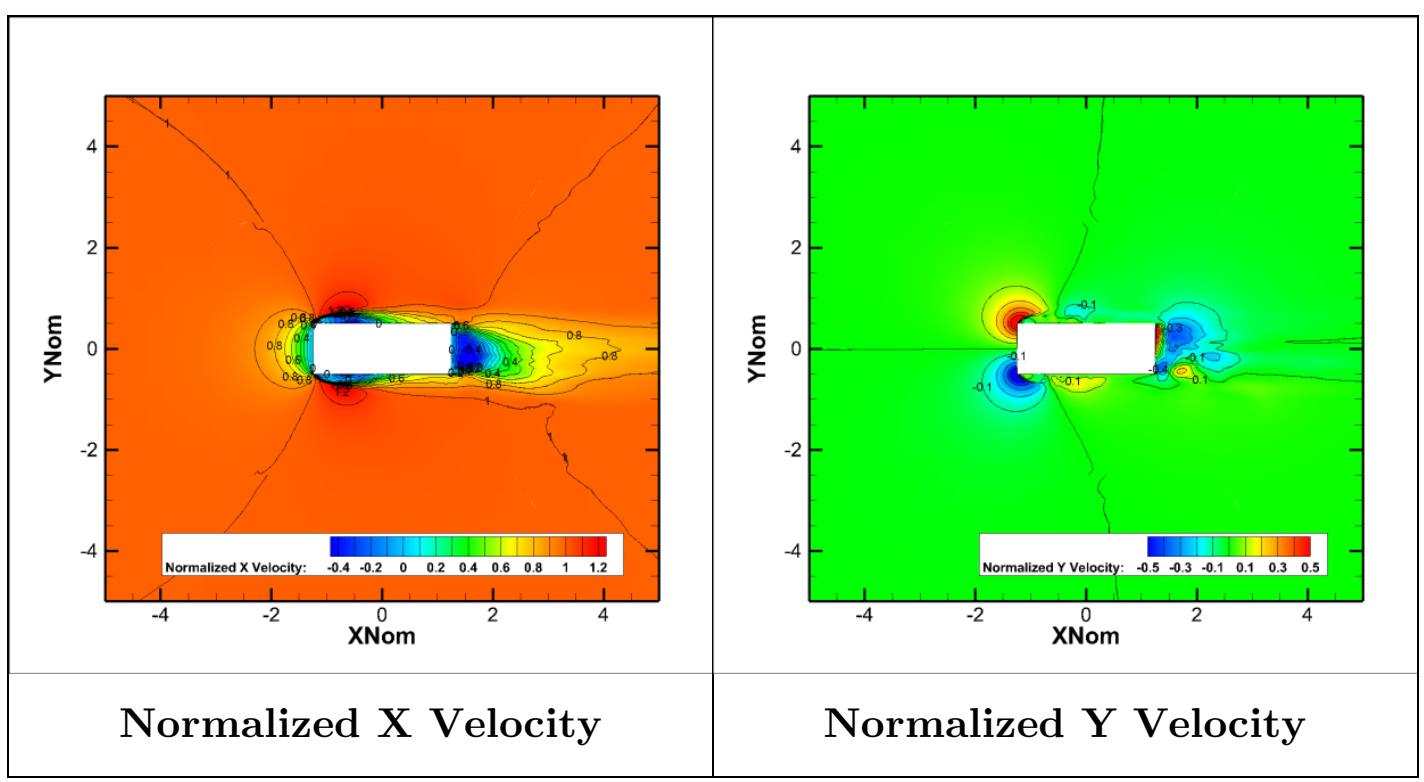

H.16. Cylinder Nacelle Case 17 (Smooth)

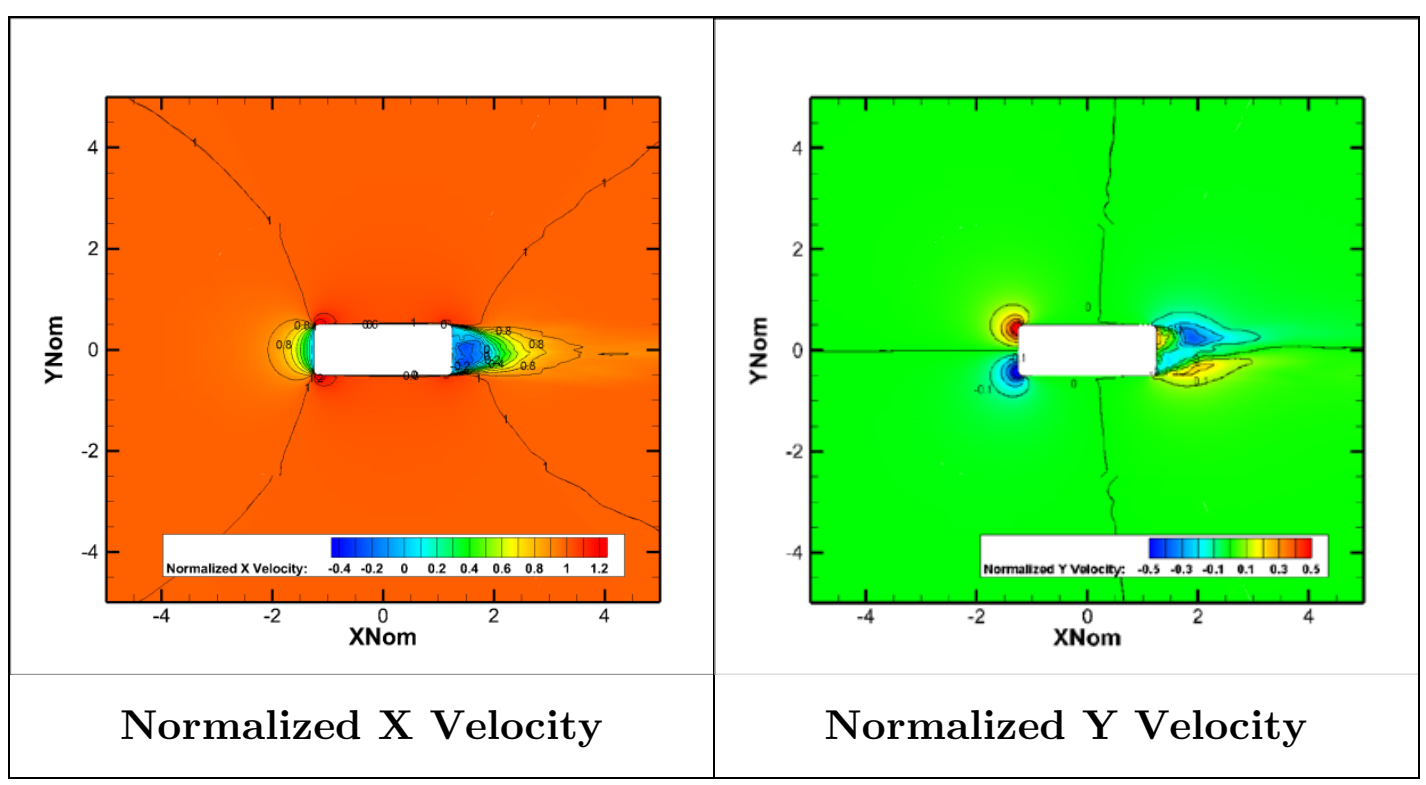




\section{H.17. Cylinder Nacelle Case 9 (Sharp)}

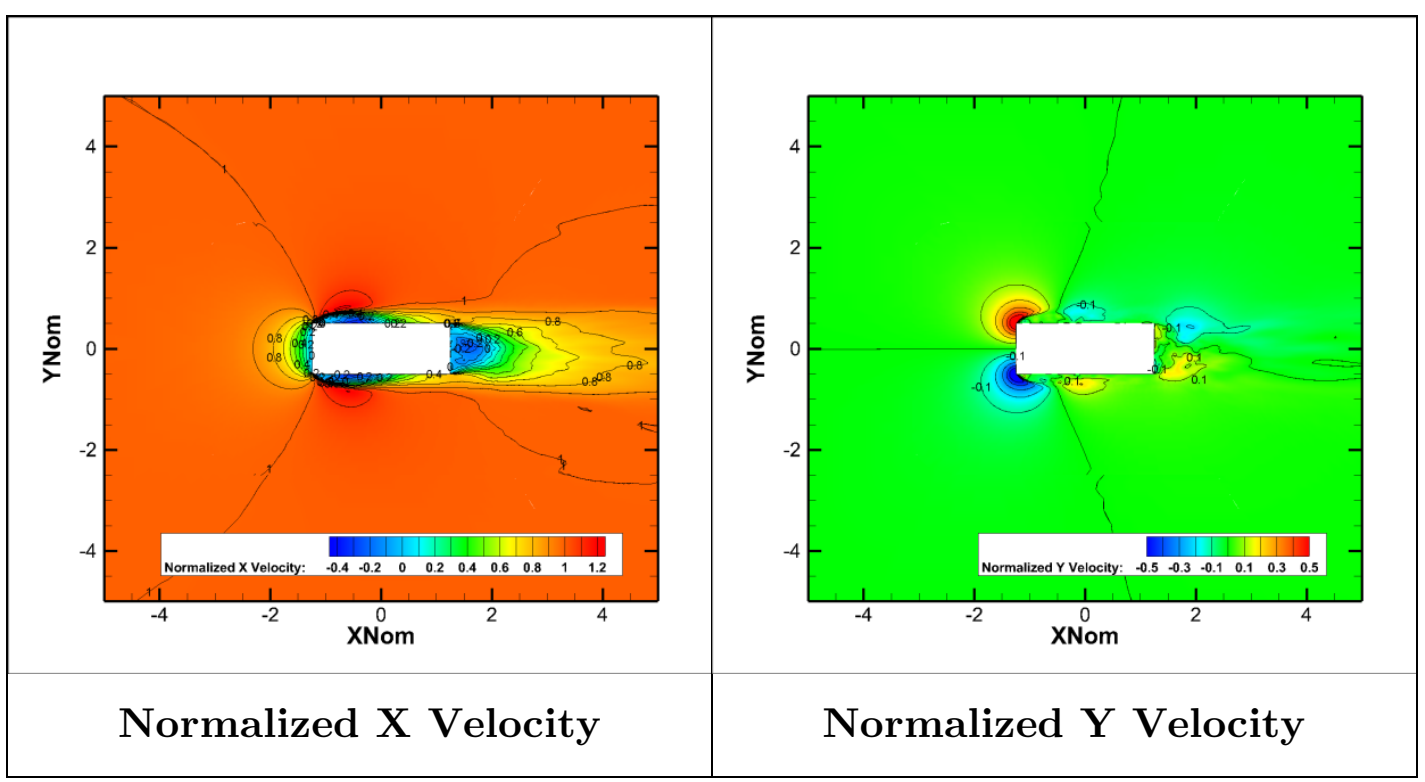

\section{H.18. Cylinder Nacelle Case 18 (Smooth)}

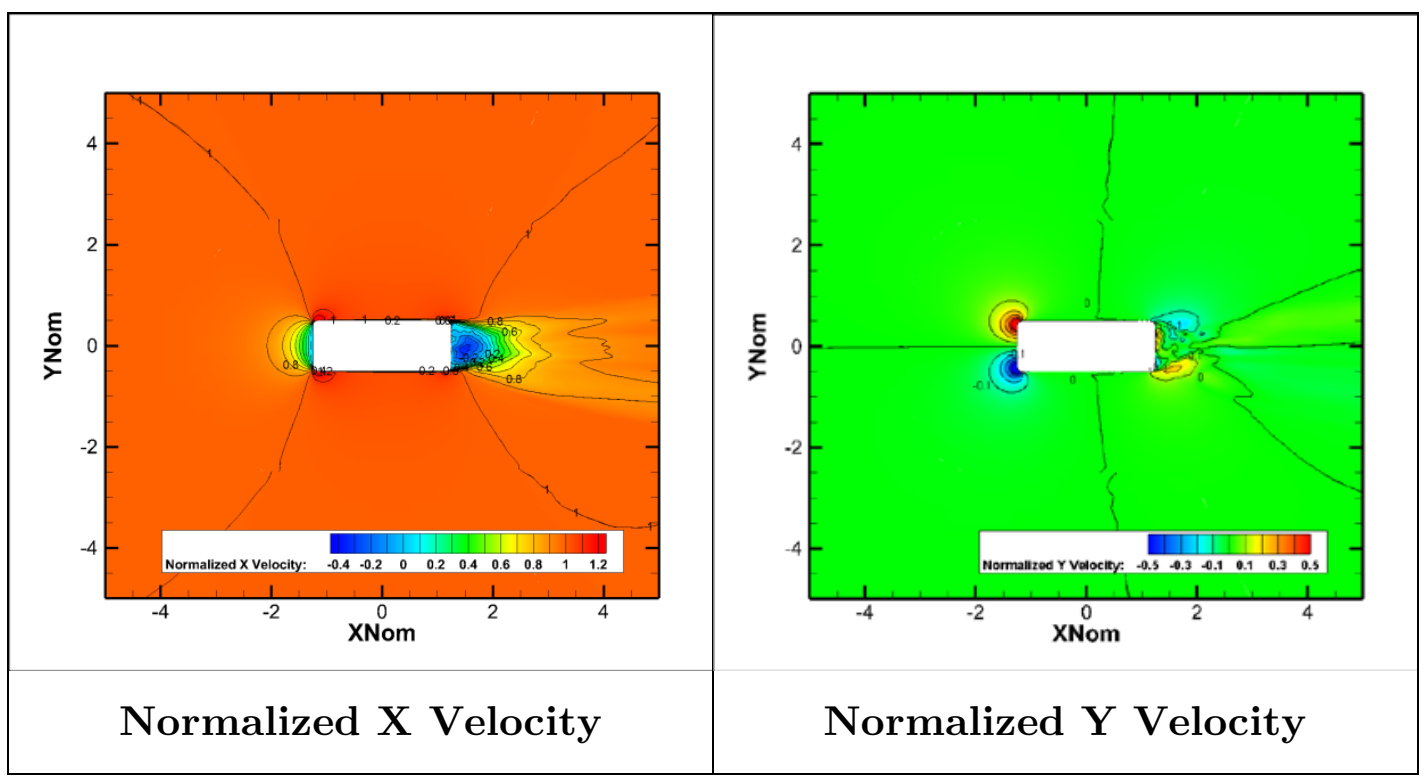

